\title{
The NUBASE evaluation of nuclear and decay properties*
}

\author{
G. Audi ${ }^{\mathrm{a},}$, O. Bersillon ${ }^{\mathrm{b}}$, J. Blachot ${ }^{\mathrm{b}}$ and A.H. Wapstra ${ }^{\mathrm{c}}$ \\ a Centre de Spectrométrie Nucléaire et de Spectrométrie de Masse, CSNSM, IN2P3-CNRS\&UPS, Bâtiment 108, \\ F-91405 Orsay Campus, France \\ b Service de Physique Nucléaire, CEA, B.P. 12, F-91680 Bruyères-le-Châtel, France \\ ${ }^{\mathrm{c}}$ National Institute of Nuclear Physics and High-Energy Physics, NIKHEF, PO Box 41882, 1009DB Amsterdam, \\ The Netherlands
}

\begin{abstract}
This paper presents the NUBASE evaluation of nuclear and decay properties of nuclides in their ground- and isomeric-states. All nuclides for which some experimental information is known are considered. NUBASE uses extensively the information given by the "Evaluated Nuclear Structure Data Files" and includes the masses from the "Atomic Mass Evaluation" (AME, second part of this issue). But it also includes information from recent literature and is meant to cover all experimental data along with their references. In case no experimental data is available, trends in the systematics of neighboring nuclides have been used, whenever possible, to derive estimated values (labeled in the database as non-experimental). Adopted procedures and policies are presented.
\end{abstract}

AMDC: http://csnwww.in2p3.fr/AMDC/

\section{Introduction}

The present evaluation responds to the needs expressed by the nuclear physics community, from fundamental physics to applied nuclear sciences, for a database which contains values for the main basic nuclear properties such as masses, excitation energies of isomers, half-lives, spins and parities, decay modes and their intensities. A

* This work has been undertaken with the encouragement of the IUPAP Commission on Symbols, Units, Nomenclature, Atomic Masses and Fundamental Constants (SUN-AMCO).

$\S$ Corresponding author. E-mail address: audi@csnsm.in2p3.fr (G. Audi). 
requirement is that all the information should be properly referenced in that database to allow checks on their validity.

One of the applications of such a database is the "Atomic Mass Evaluation" (AME) in which it is essential to have clear identification of the states involved in a decay, a reaction or a mass-spectrometric line. This is the main reason for which these two evaluations are coupled in the present issue. Furthermore, calculations requiring radioactive parameters for nuclear applications (e.g. reactors, waste management, nuclear astrophysics) need to access this basic information on any nuclide. In the preparation of a nuclear physics experiment, such a database could also be quite useful.

Most of the data mentioned above are in principle already present in two evaluated files: the "Evaluated Nuclear Structure Data Files" (ENSDF) [1] and the "Atomic Mass Evaluation" (AME2003, second part of this issue). The demand for a database as described above could be thus partially fulfilled by combining them in a 'horizontal' structure (which exists in the AME, but not in ENSDF). NUBASE is therefore, at a first level, a critical compilation of these two evaluations.

While building NUBASE, we found it necessary to examine the literature, firstly, to revise several of the collected results in ENSDF and ensure that the mentioned data are presented in a more consistent way; secondly, to have as far as possible all the available experimental data included, not only the recent ones (updating requirement), but also those missed in ENSDF (completeness requirement). This implied some evaluation work, which appears in the remarks added in the NUBASE table and in the discussions below. Full references are given for all of the added experimental information (cf. Section 2.7).

There is no strict cut-off date for the data from literature used in the present NUBASE2003 evaluation: all data available to us until the material was sent (November $19,2003)$ to the publisher have been included. Those which could not be included for special reasons, like the need for a heavy revision of the evaluation at a too late stage, are added in remarks to the relevant data.

The contents of NUBASE are described below, along with some of the policies adopted in this work. Updating procedures of NUBASE are presented in Section 3. Finally, the electronic distribution of NUBASE and an interactive display of its contents with a World Wide Web Java program or with a PC-program are described in Section 4.

The present publication updates and includes all the information given in the previous and very first evaluation of NUBASE [2], published in 1997.

\section{Contents of NUBASE}

NUBASE contains experimentally known nuclear properties together with some values estimated by extrapolation of experimental data for 3177 nuclides. NUBASE also 
contains data on isomeric states. We presently know 977 nuclides having one or more excited isomers according to our definition below. In the present evaluation we extended the definition of isomers compared to NUBASE'97 where only states with half-lives greater than 1 millisecond were considered. In present mass spectrometric experiments performed at accelerators, with immediate detection of the produced nuclei, isomers with half-lives as short as $100 \mathrm{~ns}$ may be present in the detected signals. We aimed at including as much as possible all those which play or might play in the near future a rôle in such experiments. We include also the description of those states that are involved in mass measurements and thus enter the AME2003.

For each nuclide $(A, Z)$, and for each state (ground or excited isomer), the following quantities have been compiled, and when necessary evaluated: mass excess, excitation energy of the excited isomeric states, half-life, spin and parity, decay modes and intensities for each mode, isotopic abundances of the stable nuclei, and references for all experimental values of the above items.

In the description below, references to papers that are also quoted in the NUBASE table are given with the same Nuclear Structure Reference key number style [3]. They are listed at the end of this issue (AME2003, Part II, p. 579).

In NUBASE'97, the names and the chemical symbols used for elements 104 to 109 were those recommended then by the Commission on Nomenclature of Inorganic Chemistry of the International Union of Pure and Applied Chemistry (IUPAC). Since then, unfortunately for the resulting confusion, the names were changed and moreover two of them were displaced [4] (see also AME2003, Part I, Section 6.5). The user should therefore be careful when comparing results between NUBASE'97 and the present NuBASE2003 for nuclides with $Z \geq 104$. The finally adopted names and symbols are: 104 rutherfordium (Rf), 105 dubnium (Db), 106 seaborgium (Sg), 107 bohrium (Bh), 108 hassium (Hs), and 109 meitnerium (Mt), while the provisional symbols $\mathrm{Ea}, \mathrm{Eb}, \ldots, \mathrm{Ei}$ are used for elements $110,111, \ldots, 118$.

Besides considering all nuclides for which at least one piece of information is experimentally available, we also included unknown nuclides - for which we give estimated properties - in order to ensure continuity of the set of the considered nuclides at the same time in $N$, in $Z$, in $A$ and in $N-Z$. The chart of the nuclides defined this way has a smooth contour.

As far as possible, one standard deviations $(1 \sigma)$ are given to represent the uncertainties connected with the experimental values. Unfortunately, authors do not always define the meaning of the uncertainties they quote; under such circumstances, the uncertainties are assumed to be one standard deviations. In many cases, the uncertainties are not given at all; we then estimated them on the basis of the limitations of the method of measurement.

Values and errors that are given in the NUBASE table have been rounded, even if unrounded values were found in ENSDF or in the literature. In cases where the two 
furthest-left significant digit in the error were larger than a given limit (30 for the energies, to maintain strict identity with AME2003, and 25 for all other quantities), values and errors were rounded off (see examples in the 'Explanation of table'). In very few cases, when essential for traceability, we added a remark with the original value.

When no experimental data exist for a nuclide, values can often be estimated from observed trends in the systematics of experimental data. In the AME2003, masses estimated from systematic trends were already flagged with the symbol '\#'. The use of this symbol has been extended in NUBASE to all other quantities and has the same meaning of indicating non-experimental information.

\subsection{Mass excess}

The mass excess is defined as the difference between the atomic mass (in mass units) and the mass number, and is given in $\mathrm{keV}$ for each nuclear state, together with its one standard deviation uncertainty. The mass excess values given in NUBASE are exactly those of the AME2003 evaluation, given in the second part of this issue.

It sometimes happens that knowledge of masses can yield information on the decay modes, in particular regarding nucleon-stability. Such information has been used here, as can be seen in the table for ${ }^{10} \mathrm{He},{ }^{19} \mathrm{Na},{ }^{39} \mathrm{Sc},{ }^{62} \mathrm{As}$ or ${ }^{63} \mathrm{As}$. In some cases we rejected claimed observation of decay modes, when not allowed by energetic consideration. As an example, ENSDF2000 compiles for ${ }^{142} \mathrm{Ba}$ five measurements of delayed neutron decay intensities, whereas $Q\left(\beta^{-} \mathrm{n}\right)=-2955(7) \mathrm{keV}$.

Figure 1 complements the main table in displaying the precisions on the masses, in a color-coded chart, as a function of $N$ and $Z$.

\subsection{Isomers}

In the first version of NUBASE in 1997 [2], a simple definition for the excited isomers was adopted: they were states that live longer than 1 millisecond. Already in NUBASE97, we noticed that such a simple definition had several drawbacks, particularly for alpha and proton decaying nuclides: whereas for $\beta$-decay a limit of 1 millisecond was acceptable (the shortest-lived known $\beta$-decaying nuclide $\left({ }^{35} \mathrm{Na}\right.$ ) has a half-life of 1.5 millisecond), for $\alpha$ or proton decay, several cases are known where an isomer with a half-life far below 1 millisecond lives still longer than the ground-state.

As mentioned earlier, the definition of isomers is now extended to include a large number of excited states, with half-lives as short as $100 \mathrm{~ns}$, that are of interest for mass spectrometric works at accelerators. Isomers are given in order of increasing excitation energy and identified by appending ' $m$ ', ' $n$ ', ' $p$ ' or ' $q$ ' to the nuclide name, e.g. ${ }^{90} \mathrm{Nb}$ for the ground-state, ${ }^{90} \mathrm{Nb}^{m}$ for the first excited isomer, ${ }^{90} \mathrm{Nb}^{n}$ for the second 


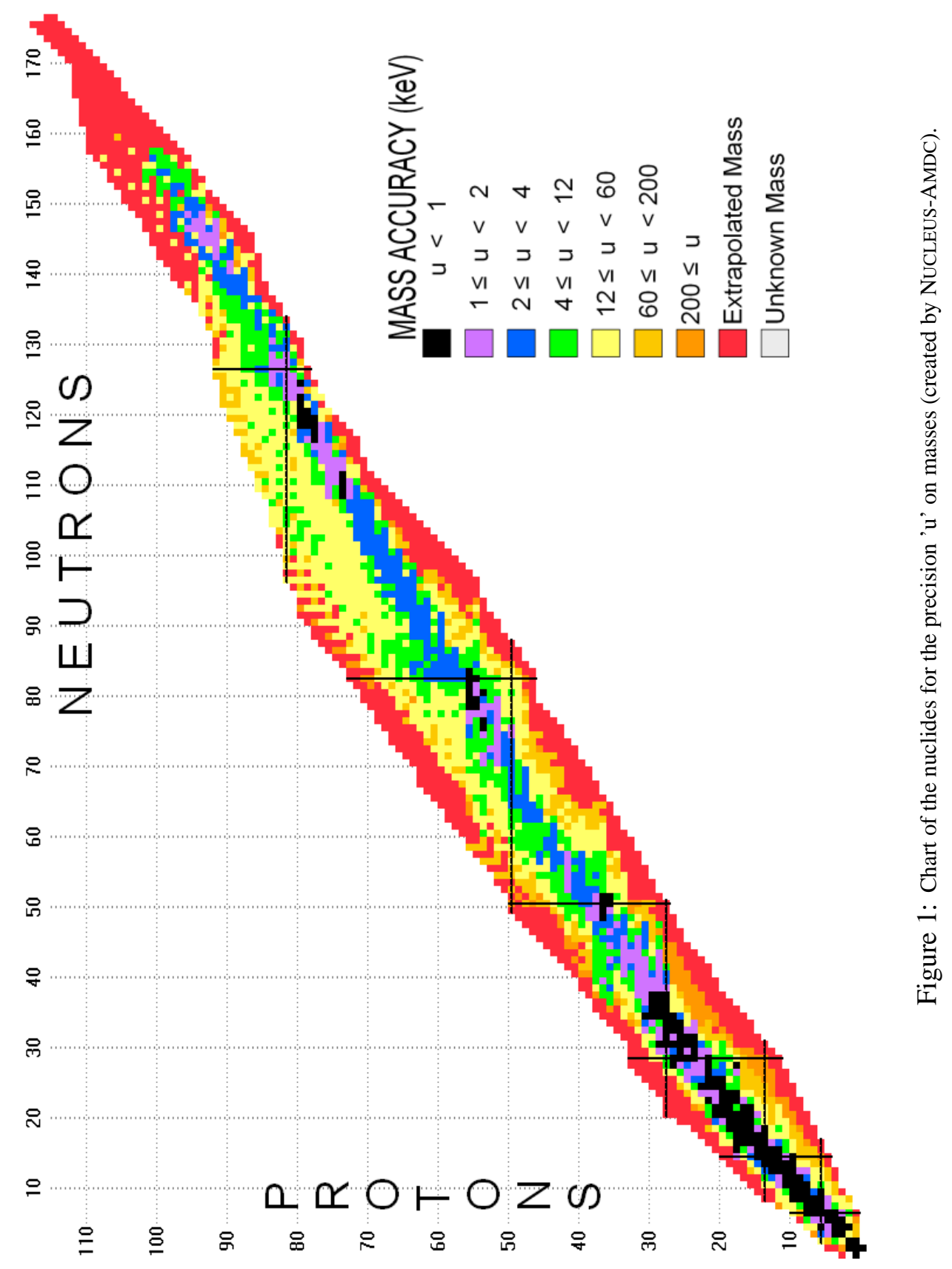


one, ${ }^{90} \mathrm{Nb}^{p}$ and ${ }^{90} \mathrm{Nb}^{q}$ for respectively the third and fourth. In NUBASE97 we could not report in a normal way the third excited isomer of ${ }^{178} \mathrm{Ta}$ with half-life $59 \mathrm{~ms}$, because of poorness of notation; the new notation adopted here removes also such a limitation.

The excitation energy can be derived from a number of different experimental methods. When this energy is derived from a method other than $\gamma$-ray spectrometry, the origin is indicated by a two-letter code and the numerical value is taken from AME. Otherwise, the code is left blank and the numerical value is taken from ENSDF or from literature update.

When the existence of an isomer is under discussion (e.g. ${ }^{141} \mathrm{~Tb}^{m}$ ) it is flagged with 'EU' in the origin field to mean "existence uncertain". A comment is generally added to indicate why its existence is questioned, or where this matter has been discussed. Depending on the degree of our confidence in this existence, we can still give a mass excess value and an excitation energy, or omit them altogether (e.g. $\left.{ }^{138} \mathrm{Pm}^{n}\right)$. In the latter case, the mention "non-existent" appears in place of that excitation energy.

When an isomer has been reported, and later proved not to exist (e.g. ${ }^{184} \mathrm{Lu}^{m}$ ), it is flagged with 'RN' in the origin field to mean "reported, non-existent". In such case we give of course no mass excess value and no excitation energy, and, as in the case of the 'EU's above, they are replaced by the same mention "non-existent".

Note: we have extended the use of the two flags 'EU' and 'RN' to cases where the discovery of a nuclide (e.g. ${ }^{260} \mathrm{Fm}$ ) is questioned. In this case however we always give an estimate, derived from systematic trends, for the ground state masses.

In several cases, ENSDF gives a lower and a higher limit for an isomeric excitation energy. A uniform distribution of probabilities has been assumed which yields a value at the middle of the range and a $1 \sigma$ uncertainty of $29 \%$ of that range (cf. Appendix B of the AME2003, Part I, for a complete description of this procedure). An example is ${ }^{136} \mathrm{La}$ for which it is known that the excited isomer lies above the level at $230.1 \mathrm{keV}$, but, as explained in ENSDF, there are good experimental indications that the difference between these two levels lies between 10 and $40 \mathrm{keV}$. We present this information as $E=255(9) \mathrm{keV}$. However, if that difference would have been derived from theory or from systematics, the resulting $E$ is considered as non-experimental and the value flagged with the '\#' symbol.

In case that the uncertainty $\sigma$ on the excitation energy $E$ is relatively large compared to the value, the assignment to ground state and isomeric state is uncertain. If $\sigma>E / 2$ a flag is added in the NUBASE table.

As a result of this work, the orderings of several ground-states and isomeric-states have been reversed compared to those in ENSDF. They are flagged in the NUBASE table with the ' $\&$ ' symbol. In several cases we found evidence for a state below the adopted ENSDF ground-state. Also, in many other cases, the systematics of nuclides with the same parities in $N$ and $Z$ strongly suggest that such a lower state should exist. 
They have been added in the NUBASE table and can be located easily, since they are also flagged with the ' $\&$ ' symbol. In a few cases, new information on masses can also lead to reversal of the level ordering. Thanks to the coupling of the NUBASE and the AME evaluations, all changes in level ordering are carefully synchronized.

\section{News on isomeric excitation energies}

Interestingly, the technique of investigating proton decay of very proton-rich nuclides gives information on isomeric excitation energies. Thus, such work on ${ }^{167} \mathrm{Ir}$ [1997Da07] shows that it has an isomeric excitation energy $E=175.3(2.2) \mathrm{keV}$. This information is displayed by the 'p' symbol following the excitation energy. In addition, study of the $\alpha$-decay series of these activities not only showed that a number of $\alpha$ lines earlier assigned to ground-states belong in reality to isomers, but also allowed to derive values for their excitation energies.

Another case of such a change is ${ }^{181} \mathrm{~Pb}$. The $\alpha$ decay half-life that was previouly assigned to ${ }^{181} \mathrm{~Pb}^{m}$ is now assigned to the ground-state, following the work of Toth et al. [1996 To01] who showed, first, that contrary to a previous work, there is no $\alpha$ line at higher energy than the one just mentioned, and second, that the observed $\alpha$ is in correlation with the decay of the daughter ${ }^{177} \mathrm{Hg}$, which is also most probably a $5 / 2^{-}$ state.

\subsection{Half-life}

For some light nuclei, the half-life $\left(T_{1 / 2}\right)$ is deduced from the level total width $\left(\Gamma_{\mathrm{cm}}\right)$ by the equation $\Gamma_{\mathrm{cm}} T_{1 / 2} \simeq \hbar \ln 2$ :

$$
T_{1 / 2}(\mathrm{~s}) \simeq 4.56210^{-22} / \Gamma_{\mathrm{cm}}(\mathrm{MeV}) .
$$

Quite often uncertainties for half-lives are given asymmetrically $T_{-b}^{+a}$. If these uncertainties are used in some applications, they need to be symmetrized. Earlier (cf. AME'95) a rough symmetrization was used: take the central value to be the midvalue between the upper and lower $1 \sigma$-equivalent limits $T+(a-b) / 2$, and define the uncertainty to be the average of the two uncertainties $(a+b) / 2$. A strict statistical derivation (see Appendix) shows that a better approximation for the central value is obtained by using $T+0.64 \times(a-b)$. The exact expression for the uncertainty is given in the Appendix.

When two or more independent measurements have been reported, they are averaged, while being weighed by their reported precision. While doing this, we consider the NORMALIZED CHI, $\chi_{n}$ (or 'consistency factor' or 'Birge ratio'), as defined in AmE2003, Part I, Section 5.2. Only when $\chi_{n}$ is beyond 2.5, do we depart from the statistical result, and adopt the external error for the average, following the same 
policy as discussed and adopted in AME2003, Part I, Section 5.4. Very rarely, when the Birge ratio $\chi_{n}$ is so large that we consider all errors given as non-relevant, do we adopt the arithmetic average (unweighed) for the result and the corresponding error (based on the dispersion of values). In all such cases, a remark is added to the data, giving the list of values that were averaged, and, when relevant, the value of the Birge ratio $\chi_{n}$ and the reason for our choice.

In the case of experiments in which extremely rare events are observed, and where the results are very asymmetric, we did not average directly the half-lives derived from different works, but instead, when the information given in the papers was sufficient (e.g. ${ }^{264} \mathrm{Hs}$ or ${ }^{269} \mathrm{Hs}$ ), we combined the delay times of the individual events, as prescribed by Schmidt et al [1984Sc13].

Some measurements are reported as a range of values with most probable lower and upper limits. They are treated, as explained above (cf. Section 2.2), as a uniform distribution of probabilities with a value at the middle of the range and a $1 \sigma$ uncertainty of $29 \%$ of that range (cf. Appendix B of the AME2003 for a complete description of this procedure).

For some nuclides identified by using a time-of-flight spectrometer, an upper or a lower limit on the half-life is given.

i) For observed species, we give this important but isolated piece of information (lower limit) in place of the uncertainty on the half-life, and within brackets (e.g. ${ }^{36} \mathrm{Mg}$, p. 34). The user of our table should be careful in that this limit can be very far below the eventually measured half-life. To help to avoid confusion, we now give, in addition, an estimate (as always in the present two evaluations, flagged with \#) for the half-life derived from trends in systematics.

ii) For nuclides sought for but not observed, we give the found upper limit in place of the half-life. Upper limits for undetected nuclides have been evaluated for NUBASE by F. Pougheon [1993Po.A], based on the time-of-flight of the experimental setup and the yields expected from the trends in neighboring nuclides (e.g. ${ }^{19} \mathrm{Na}$ ).

When half-lives for nuclides with the same parities in $Z$ and $N$ are found to vary smoothly (see Fig. 2), interpolation or extrapolation is used to obtain reasonable estimates.

\subsection{Spin and parity}

As in ENSDF, values are presented without and with parentheses based upon strong and weak assignment arguments, respectively (see the introductory pages of Ref. [5]). Unfortunately, the latter include estimates from systematics or theory. Where we can distinguish them, we use parentheses if the so-called "weak" argument is an experimental one, but the symbol '\#' in the other cases. The survey might have not been complete, and the reader might still find non-flagged non-experimental cases (the 


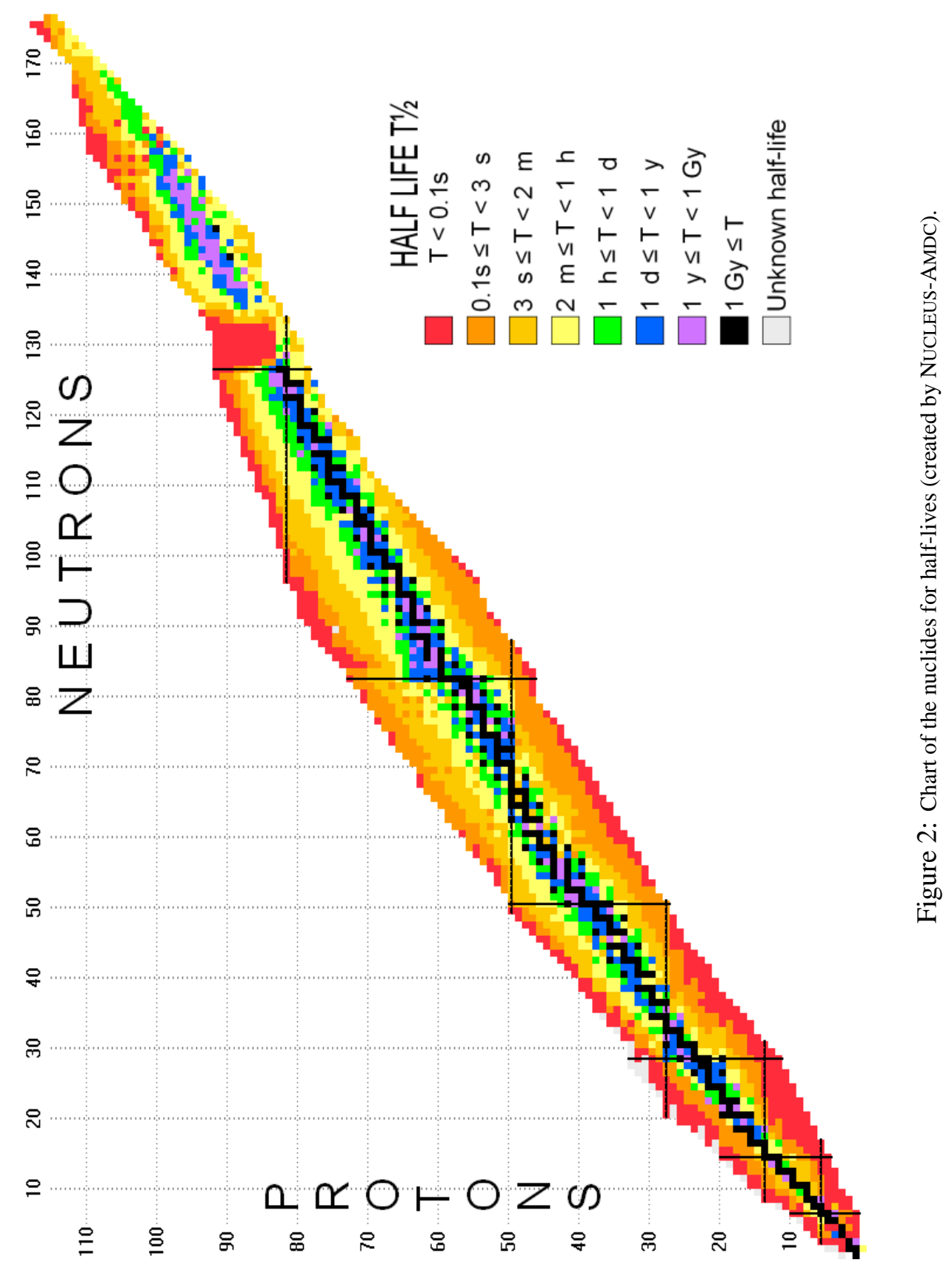


authors will gratefully appreciate mention of such cases to improve future versions of NUBASE).

If spin and parity are not known from experiment, they can be estimated, in some cases, from systematic trends in neighboring nuclides with the same parities in $N$ and $Z$. This is often true for odd- $A$ nuclides (see Fig. 3 and Fig. 4), but also, not so rarely, for odd-odd ones, as can be seen in Fig. 5. These estimated values are also flagged with the '\#' symbol. In several cases we replaced the ENSDF systematics by our own.

The review of nuclear radii and moments of Otten [1989Ot.A], in which the spins were compiled, was used to check and complete the spin values in NUBASE.

\subsection{Decay modes and intensities}

The most important policy, from our point of view, in coding the information for the decay modes, is in establishing a very clear distinction between a decay mode that is energetically allowed but not yet experimentally observed (represented by a question mark alone, which thus refers to the decay mode itself), and a decay mode that is actually observed but for which the intensity could not be determined (represented by ' $=$ ?', the question mark referring here to the quantity after the equal sign).

As in ENSDF, no corrections have been made to normalize the primary intensities to $100 \%$.

Besides direct updates from the literature, we also made use of partial evaluations by other authors (with proper quotation). They are mentioned below, when discussing some particular decay modes.

\section{The $\beta^{+}$decay}

In the course of our work we refined some definitions and notations for the $\beta^{+}$ decay, in order to present more clearly the available information. We denote with $\beta^{+}$ the decay process that includes both electron capture, denoted $\varepsilon$, and the decay by positron emission, denoted $\mathrm{e}^{+}$. One can then symbolically write: $\beta^{+}=\varepsilon+\mathrm{e}^{+}$. As is well known, for an available energy below $1022 \mathrm{keV}$, only electron capture $\varepsilon$ is allowed; above that value both processes compete.

Remark: this notation is not the same as the one implicitly used in ENSDF, where the combination of both modes is denoted "EC+B+".

When both modes compete, the separated intensities are not always available from experiment. Most of the time, separated values in ENSDF are calculated ones. In continuation of one of our general policies, in which we retain whenever possible only experimental information, we decided not to retain ENSDF's calculated separated values (which are scarce and not always updated). Most often, it is in some very particular cases that the distinction is of importance, like in the case of rare or extremely rare processes (e.g. $\left.{ }^{91} \mathrm{Nb},{ }^{54} \mathrm{Mn},{ }^{119} \mathrm{Te}^{m}\right)$. Then, the use of our notation is useful. 


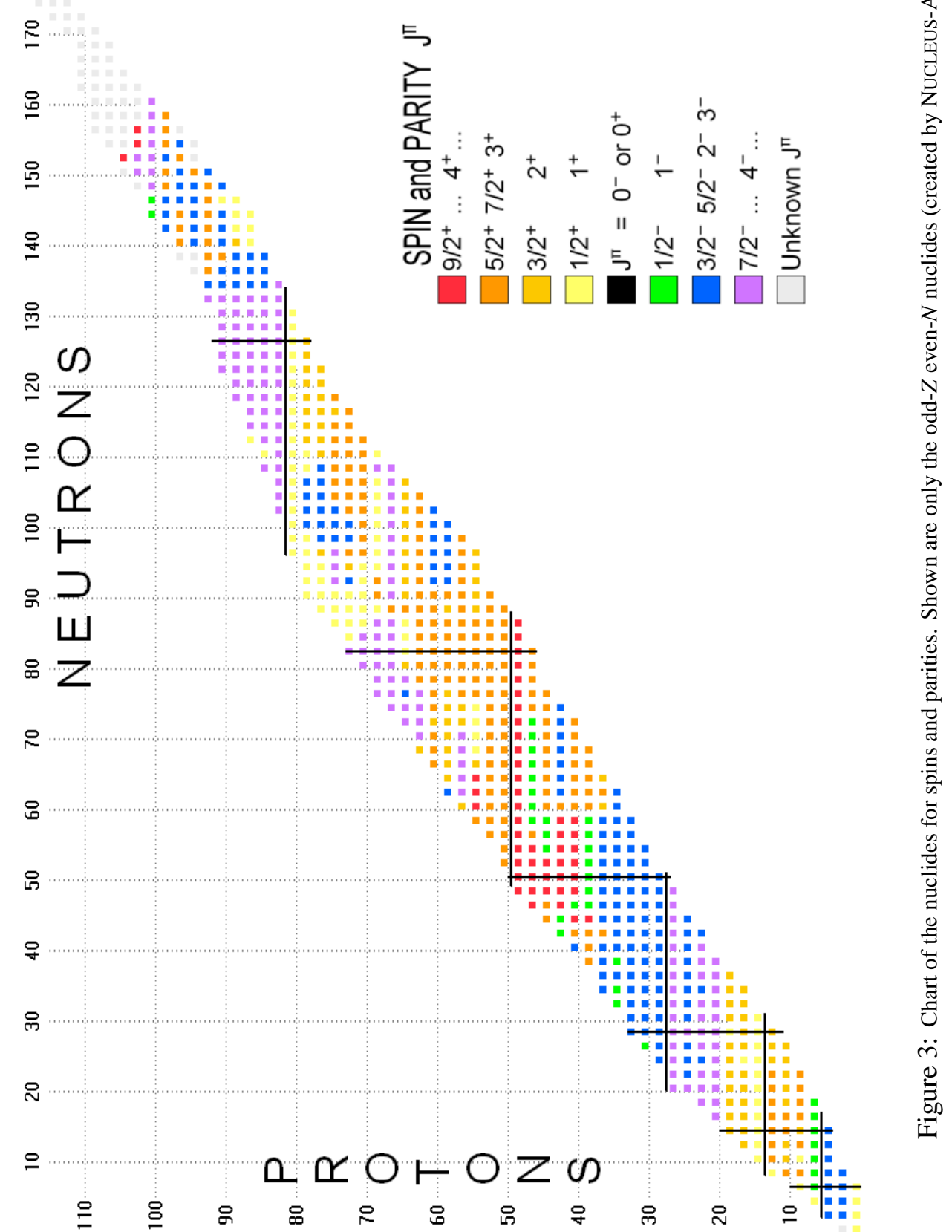




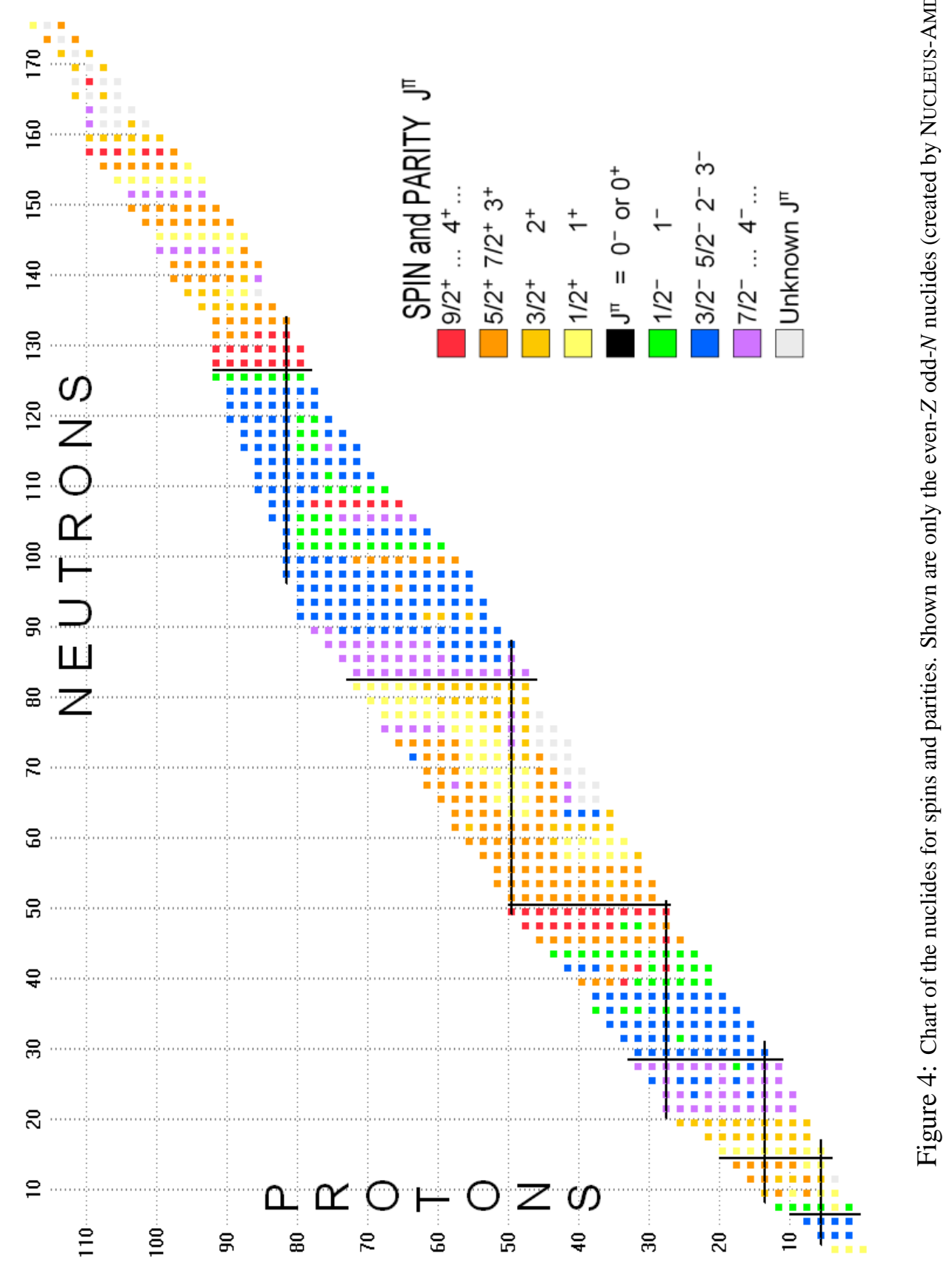




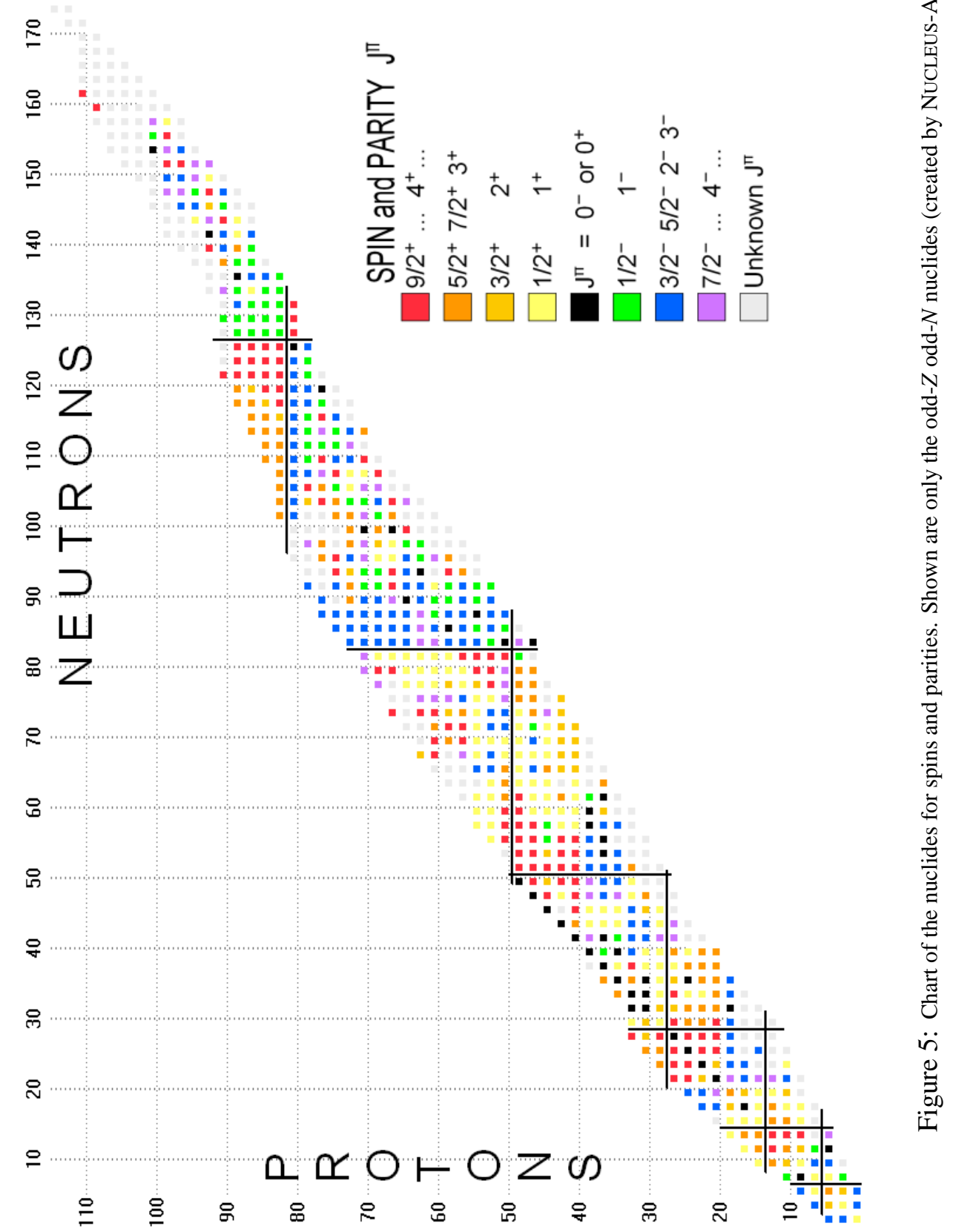


In the same line, we give both electron capture $\varepsilon$-delayed fission and the positron $e^{+}$-delayed fission with the same symbol $\beta^{+} \mathrm{SF}$.

\section{The double- $\beta$ decay}

In the course of our work we found that half-lives for double- $\beta$ decay were not always given in a consistent way in ENSDF. For NUBASE we decided to give only half-life values or upper-limits related to the dominant process, which is in general the two-neutrino gs-gs transition (one exception may be ${ }^{98} \mathrm{Mo}$, for which the neutrinoless decay is predicted to be faster, see [2002Tr04]). No attempt was made to convert to the same statistical confidence level (CL) upper limit results given by different authors.

The excellent recent compilation of Tretyak and Zdesenko [2002Tr04] was of great help in this part of our work.

\section{The $\beta$-delayed decays}

For delayed decays, intensities have to be considered carefully. By definition, the intensity of a decay mode is the percentage of decaying nuclei in that mode. But traditionally, the intensities of the pure $\beta$ decay and of those of the delayed ones are summed to give an intensity that is assigned to the pure $\beta$ decay. For example, if the $(A, Z)$ nuclide has a decay described, according to the tradition, by ' $\beta^{-}=100$; $\beta^{-} \mathrm{n}=20^{\prime}$, this means that for 100 decays of the parent $(A, Z), 80(A, Z+1)$ and $20(A-$ $1, Z+1)$ daughter nuclei are produced and that 100 electrons and 20 delayed-neutrons are emitted. A strict notation, following the definition above, would have been in this case ' $\beta^{-}=80 ; \beta^{-} \mathrm{n}=20$ '. However we decided to follow the tradition and use in our work the notation: ' $\beta^{-}=100 ; \beta^{-} \mathrm{n}=20$ '.

This also holds for more complex delayed emissions. A decay described by: ' $\beta^{-}=100 ; \beta^{-} \mathrm{n}=30 ; \beta^{-} 2 \mathrm{n}=20 ; \beta^{-} \alpha=10^{-}$' corresponds to the emission of 100 electrons, $(30+2 \times 20=70)$ delayed-neutrons and 10 delayed- $\alpha$ particles; and in terms of residual nuclides, to $40(A, Z+1), 30(A-1, Z+1), 20(A-2, Z+1)$ and $10(A-4, Z-1)$. More generally, $P_{\mathrm{n}}$, the number of emitted neutrons per 100 decays, can be written:

$$
P_{\mathrm{n}}=\sum_{i} i \times \beta_{i \mathrm{n}}^{-} ;
$$

and similar expressions for $\alpha$ or proton emission. The number of residual $\beta$ daughter $(A, Z+1)$ is:

$$
\beta^{-}-\sum_{i} \beta_{i \mathrm{n}}^{-}-\sum_{j} \beta_{j \alpha}^{-}-\ldots
$$

Another special remark concerns the intensity of a particular $\beta$-delayed mode. The primary $\beta$-decay populates several excited states in the $\beta$-daughter, that will further decay by particle emission. However, in the case where the daughter's ground state also decays by the same particle emission, some authors included its decay 
in the value for the concerned $\beta$-delayed intensity. We decided not to do so for two reasons. Firstly, because the energies of the particles emitted from the excited states are generally much higher than that from the ground-state, implying different subsequent processes. Secondly, because the characteristic times for the decays from the excited states are related to the parent, whereas those for the decays from the daughter's ground state are due to the daughter. For example ${ }^{9} \mathrm{C}$ decays through $\beta^{+}$ mode with an intensity of $100 \%$ of which $12 \%$ and $11 \%$ to two excited p-emitting states in ${ }^{9} \mathrm{~B}$, and $17 \%$ to an $\alpha$-emitting state. We give thus $\beta^{+} \mathrm{p}=23 \%$ and $\beta^{+} \alpha=17 \%$, from which the user of our table can derive a $60 \%$ direct feeding of the ground-state of ${ }^{9} \mathrm{~B}$. In a slightly different example, ${ }^{8} \mathrm{~B}$ decays only to two excited states in ${ }^{8} \mathrm{Be}$ which in turn decay by $\alpha$ and $\gamma$ emission, but not to the ${ }^{8} \mathrm{Be}$ ground-state. We write thus $\beta^{+}=100 \%$ and $\beta^{+} \alpha=100 \%$, the difference of which leaves $0 \%$ for the feeding of the daughter's ground state.

Finally, we want to draw to the attention of the user of our table, that the percentages are, by definition, related to 100 decaying nuclei, not to the primary beta-decay fraction. An illustrative example is given by the decay of ${ }^{228} \mathrm{~Np}$, for which the delayed-fission probability is given in the original paper as $0.020(9) \%$ [1994Kr13], but this number is relative to the $\varepsilon$ process, the intensity of which is $59(7) \%$. We thus renormalized the delayed-fission intensity to $0.012(6) \%$ of the total decay.

In collecting the delayed proton and $\alpha$ activities, the remarkable work of Hardy and Hagberg [1989Ha.A], in which this physics was reviewed and discussed, was an appreciable help in our work. The review of Honkanen, Äystö and Eskola [6] on delayed-protons has also been verified.

Similarly, the review of delayed neutron emission by Hansen and Jonson [1989Ha.B] was carefully examined and used in our table, as well as the evaluation of Rudstam, Aleklett and Sihver [1993Ru01].

\subsection{Isotopic abundances}

Isotopic abundances are taken from the compilation of K.J.R. Rosman and P.D.P. Taylor [1998Ro45] and are listed in the decay field with the symbol IS. They are displayed as given in [1998Ro45], i.e. we did not even apply our rounding policy.

\subsection{References}

The year of the archival file is indicated for the nuclides evaluated in ENSDF; otherwise, this entry is left blank.

References for all of the experimental updates are given by the NSR key number [3], and listed at the end of this issue (p. 579). They are followed by one, two or three one-letter codes which specify the added or modified physical quantities (see the 


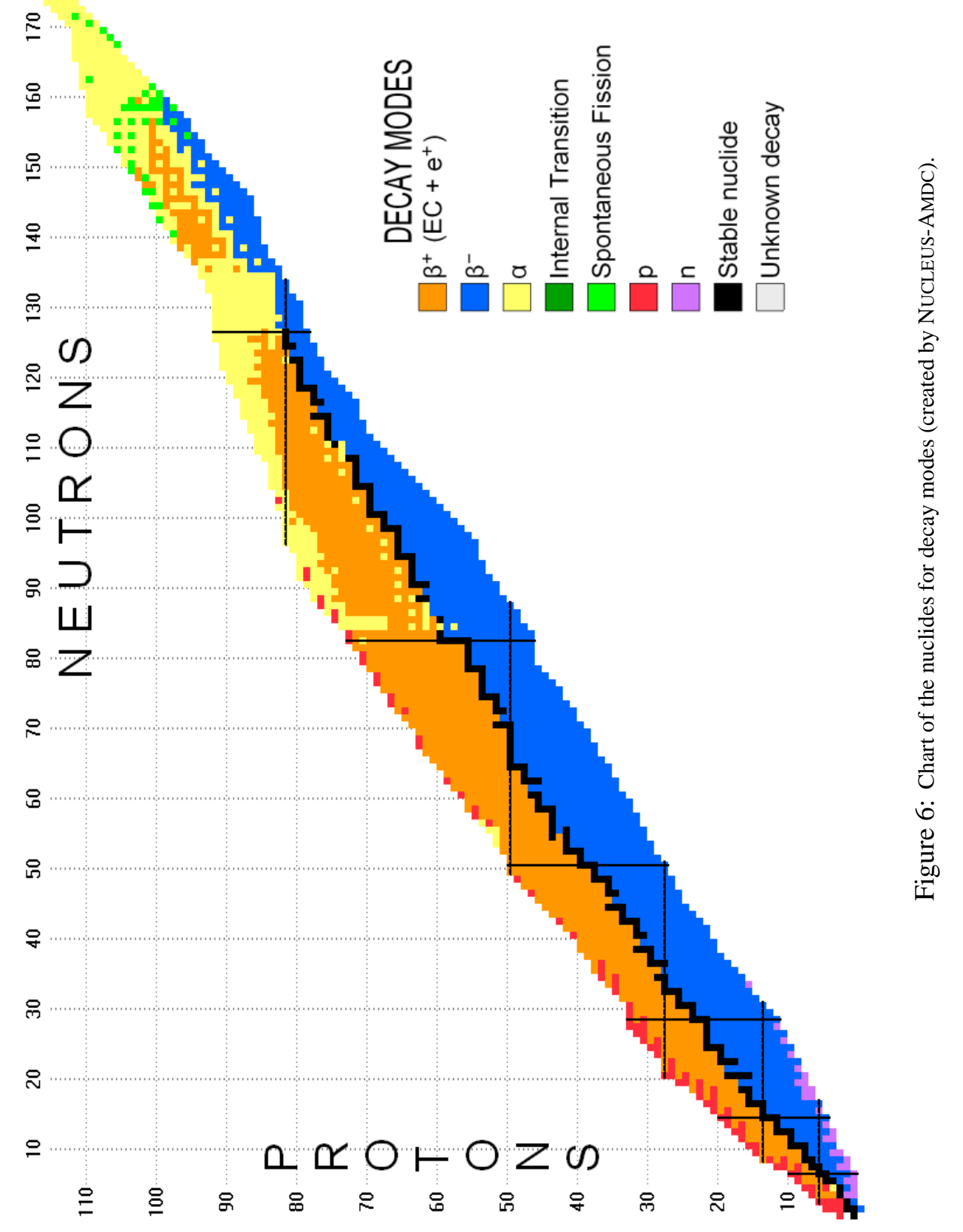


Explanation of Table). In cases where more than one reference is needed to describe the updates, they are given in a remark. No reference is given for systematic values. The ABBW reference key is used in cases where it may not appear unambiguously that re-interpretations of the data were made by the present authors.

\section{Updating procedure}

NUBASE is updated via two routes: from ENSDF after each new $A$-chain evaluation (or from the bi-annual releases), and directly from the literature.

ENSDF files are retrieved from NNDC using the on-line service [1] and transferred through the Internet. Two of the present authors [7] developed programs to successively:

- check that each $Z$ in the $A$-chain has an 'adopted levels' data set; if not, a corresponding data set is generated from the 'decay' or 'reaction' data set,

- extract the 'adopted levels' data sets from ENSDF,

- extract from these data sets the required physical quantities, and convert them into a format similar to the NUBASE format.

The processed data are used to update manually the previous version of NUBASE. This step is done separately by the four authors and cross-checked until full agreement is reached.

The ENSDF is updated generally by $A$-chains, and, more recently, also by individual nuclides. Its contents however is very large, since it encompasses all the complex nuclear structure and decay properties. This is a huge effort, and it is no wonder that some older data (including annual reports, conference proceedings, and theses) are missing, and that some recent data have not yet been included. Where we notice such missing data, they are analyzed and evaluated, as above, independently by the four authors and the proposed updatings are compared. Most often these new data are included in the next ENSDF evaluation and the corresponding references can be removed from the NUBASE database.

\section{Distribution and displays of NUBASE}

Full content of the present evaluation is accessible on-line at the web site of the Atomic Mass Data Center (AMDC) [8] through the World Wide Web. An electronic AscII file for the NUBASE table, for use with computer programs, is also distributed by the AMDC. This file will not be updated, to allow stable reference data for calculations. Any work using that file should make reference to the present paper and not to the electronic file.

The contents of NUBASE can be displayed by a Java program JVNUBASE [9] through the World Wide Web and also with a PC-program called "NuCLEUS" [10]. Both can 
be accessed or downloaded from the AMDC. They will be updated regularly to allow the user to check for the latest available information in NUBASE.

\section{Conclusions}

A 'horizontal' evaluated database has been developed which contains most of the main properties of the nuclides in their ground and isomeric states. These data originate from a critical compilation of two evaluated datasets: the ENSDF, updated and completed from the literature, and the AME. The guidelines in setting up this database were to cover as completely as possible all the experimental data, and to provide proper reference for those used in NUBASE and not already included in ENSDF; this traceability allows any user to check the recommended data and, if necessary, undertake a re-evaluation.

As a result of this 'horizontal' work, a greater homogeneity in data handling and presentation has been obtained for all of the nuclides. Furthermore, isomeric assignments and excitation energies have been reconsidered on a firmer basis and their data improved.

It is expected to follow up this second version of NUBASE with improved treatments. Among them, we plan to complete the extension due to the new definition of isomer to states with half-lives between $100 \mathrm{~ns}$ and 1 millisecond that are available at the large-scale facilities. Another foreseeable implementation would be to provide the main $\alpha, \gamma$, conversion and X-ray lines accompanying the decays. NUBASE could also be extended to other nuclear properties: energies of the first $2^{+}$states in even-even nuclides, radii, moments ... An interesting feature that is already implemented, but not yet checked sufficiently to be included here, is to give for each nuclide, in ground or isomeric-state, the year of its discovery.

\section{Acknowledgements}

We wish to thank our many colleagues who answered our questions about their experiments and those who sent us preprints of their papers. Continuous interest, discussions, suggestions and help in the preparation of the present publication by C. Thibault were highly appreciated. We appreciate the help provided by J.K. Tuli in solving some of the puzzles we encountered. Special thanks are due to S. Audi for the preparation of the color figures from the NUCLEUS program, and to C. Gaulard and D. Lunney for careful reading of the manuscript. A.H.W. expresses his gratitude to the NIKHEF-K laboratory and especially to Mr. K. Huyser for his continual help, and J.B. to the ISN-Grenoble and DRFMC-Grenoble laboratories for permission to use their facilities. 


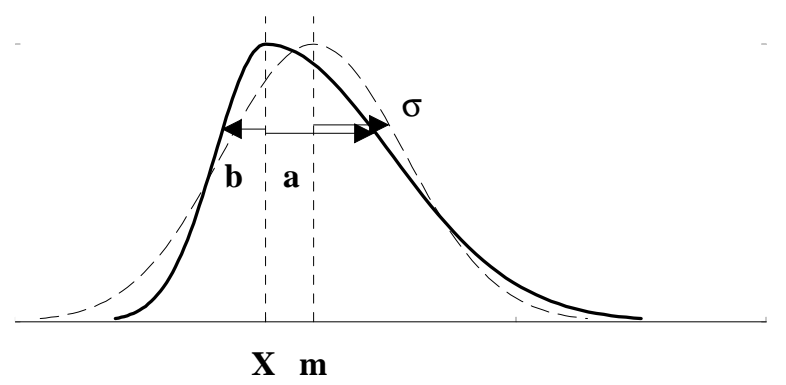

Figure 7: Simulated asymmetric probability density function (heavy solid line) and the equivalent symmetric one (dashed line).

\section{Appendix A. Symmetrization of asymmetric uncertainties}

Experimental data are sometimes given with asymmetric uncertainties, $X_{-b}^{+a}$. If these data are to be used with other ones in some applications, their uncertainties may need to be symmetrized. A simple method (Method 1), used earlier, consisted in taking the central value to be the mid-value between the upper and lower $1 \sigma$-equivalent limits $X+(a-b) / 2$, and define the uncertainty to be the average of the two uncertainties $(a+b) / 2$.

An alternative method (Method 2) is to consider the random variable $x$ associated with the measured quantity. For this random variable, we assume the probability density function to be an asymmetric normal distribution having a modal (most probable) value of $x=X$, a standard deviation $b$ for $x<X$, and a standard deviation $a$ for $x>X$ (Fig. 7). Then the average value of this distribution is

$$
\langle x\rangle=X+\sqrt{2 / \pi}(a-b),
$$

with variance

$$
\sigma^{2}=(1-2 / \pi)(a-b)^{2}+a b
$$

The median value $m$ which divides the distribution into two equal areas is given, for $a>b$, by

$$
\operatorname{erf}\left(\frac{m-X}{\sqrt{2} a}\right)=\frac{a-b}{2 a}
$$

and by a similar expression for $b>a$.

We define the equivalent symmetric normal distribution we are looking for as a distribution having a mean value equal to the median value $m$ of the previous distribution with same variance $\sigma$. 
Table A. Examples of treatment of asymmetric uncertainties for half-lives. Method 1 is the classical method, used previously, as in the AME'95. Method 2 is the one developed in this Appendix and used for half-lives and intensities of the decay modes.

\begin{tabular}{ccccc}
\hline Nuclide & Original $T_{1 / 2}$ & Method 1 & Method 2 \\
& & & & \\
${ }^{76} \mathrm{Ni}$ & $240+550-190 \mathrm{~ms}$ & $420 \pm 370$ & $470 \pm 390$ \\
${ }^{222} \mathrm{U}$ & $1.0+1.0-0.4$ & $\mu \mathrm{s}$ & $1.3 \pm 0.7$ & $1.4 \pm 0.7$ \\
${ }^{264} \mathrm{Hs}$ & $327+448-120 \quad \mu \mathrm{s}$ & $490 \pm 280$ & $540 \pm 300$ \\
${ }^{266} \mathrm{Mt}$ & $1.01+0.47-0.24 \mathrm{~ms}$ & $1.1 \pm 0.4$ & $1.2 \pm 0.4$ \\
\hline
\end{tabular}

If the shift $m-X$ of the central value is small compared to $a$ or $b$, expression (2) can be written [11]:

$$
m-X \simeq \sqrt{\pi / 8}(a-b) \simeq 0.6267(a-b) .
$$

In order to allow for a small non-linearity that appears for higher values of $m-X$, we adopt for Method 2 the relation

$$
m-X=0.64(a-b) .
$$

Table A illustrates the results from both methods. In NUBASE, Method 2 is used for the symmetrization of asymmetric half-lives and of asymmetric decay intensities.

\section{References}

References quoted in the text as [1993Po.A] or [2002Tr04] (NSR style) are listed under "References used in the AME2003 and the NUBASE2003 evaluations", p. 579.

[1] T.W. Burrows, Nucl. Instrum. Meth. 286 (1990) 595; http://www.nndc.bnl.gov/

[2] G. Audi, O. Bersillon, J. Blachot and A.H. Wapstra, Nucl. Phys. A 624 (1997) 1; http://csnwww.in2p3.fr/AMDC/nubase/nubase97.pdf

[3] Nuclear Structure Reference (NSR): a computer file of indexed references maintained by NNDC, Brookhaven National Laboratory; http://www2.nndc.bnl.gov/nsr/

[4] Commission on Nomenclature of Inorganic Chemistry, Pure and Applied Chemistry 69 (1997) 2471.

[5] General Policies, Nuclear Data Sheets, 71(1994) $v$. 
[6] J. Honkanen, J. Äystö and K. Eskola, Phys. Scr. 34 (1986) 608.

[7] O. Bersillon and J. Blachot, NEANDC(E) 246/L, INDC(FR) 071/L, September 1991.

[8] The NUBASE2003 files in the electronic distribution and complementary information can be retrieved from the Atomic Mass Data Center (AMDC) through the Web: http://csnwww.in2p3.fr/amdc/

[9] E. Durand, Report CsNSM 97-09, July 1997; http://csnwww.in2p3.fr/AMDC/nucleus/stg-durand.doc

[10] B. Potet, J. Duflo and G. Audi, Proceedings Enam'95 conference, Arles, June 1995, p. 151; http://csnwww.in2p3.fr/AMDC/nucleus/arlnucleus.ps

[11] R.D. Evans, The Atomic Nucleus (McGraw-Hill, New York, 1955) p. 766. 


\section{Table I. Table of nuclear and decay properties}

\section{EXPLANATION OF TABLE}

Data are presented in groups ordered according to increasing mass number $A$.

Nuclide

Nuclidic name: mass number $A=N+Z$ and element symbol (for $Z>109$ see Section 2). Element indications with suffix ' $m$ ', ' $n$ ', ' $p$ ' or ' $q$ ' indicate assignments to excited isomeric states (defined, see text, as upper states with half-lives larger than $100 \mathrm{~ns}$ ). Suffixes ' $p$ ' and ' $q$ ' indicate also non-isomeric levels, of use in the AME2003. Suffix ' $r$ ' indicates a state from a proton resonance occurring in $(\mathrm{p}, \gamma)$ reactions (e.g. ${ }^{28} \mathrm{Si}^{r}$ ). Suffix ' $x$ ' applies to mixtures of levels (with relative ratio $R$, given in the 'Half-life'column), e.g. occurring in spallation reactions (indicated 'spmix' in the ' $J$ ', column) or fission ('fsmix').

Mass excess

Mass excess $[M(\mathrm{in} \mathrm{u})-A]$, in $\mathrm{keV}$, and its one standard deviation uncertainty as given in the 'Atomic Mass Evaluation' (AME2003, second part of this volume).

Rounding policy: in cases where the furthest-left significant digit in the error is larger than 3, values and errors are rounded off, but not to more than tens of keV. (Examples: $2345.67 \pm 2.78 \rightarrow 2345.7 \pm 2.8,2345.67 \pm 4.68 \rightarrow 2346 \pm 5$, but $2346.7 \pm 468.2 \rightarrow 2350 \pm 470)$.

\# in place of decimal point: value and uncertainty derived not from purely experimental data, but at least partly from systematic trends (cf. AME2003).

Excitation energy

For excited isomers only: energy difference, in $\mathrm{keV}$, between levels adopted as higher level isomer and ground state isomer, and its one standard deviation uncertainty, as given in AME2003 when derived from the AME, otherwise as given by ENSDF. The rounding policy is the same as for the mass excess (see above).

\# in place of decimal point: value and uncertainty derived from systematic trends. The excitation energy is followed by its origin code when derived from a method other than $\gamma$-ray spectrometry:

MD Mass doublet

RQ Reaction energy difference

AD $\alpha$ energy difference

BD $\beta$ energy difference

$\mathrm{p} \quad$ proton decay

XL L X-rays

$\mathrm{Nm}$ estimated value derived with help of Nilsson model

When the existence of an isomer is questionable the following codes are used:

EU existence of isomer is under discussion (e.g. ${ }^{141} \mathrm{~Tb}^{m}$ ). If existence is strongly doubted, no excitation energy and no mass are given. They are replaced by the mention "non-existent" (e.g. $\left.{ }^{138} \mathrm{Pm}^{n}\right)$.

$\mathrm{RN}$ isomer is proved not to exist (e.g. $\left.{ }^{184} \mathrm{Lu}^{m}\right)$. Excitation energy and mass are replaced by the mention "non-existent".

Remark: codes EU and RN are also used when the discovery of a nuclide (e.g. ${ }^{260} \mathrm{Fm}$ ) is questioned. In this case however we always give an estimate, derived from systematic trends, for the ground state mass.

Isomeric assignment:

* In case the uncertainty $\sigma$ on the excitation energy $E$ is larger than half that energy $(\sigma>E / 2)$, these quantities are followed by an asterix (e.g. ${ }^{130} \mathrm{In}$ and ${ }^{130} \operatorname{In}^{m}$ )

\& In case the ordering of the ground- and isomeric-states are reversed compared to ENSDF, an ampersand sign is added (e.g. ${ }^{90} \mathrm{Tc}$ and ${ }^{90} \mathrm{Tc}^{m}$ ). 
Half-life $\quad \mathrm{s}=$ seconds; $\mathrm{m}=$ minutes; $\mathrm{h}=$ hours; $\mathrm{d}=$ days; $\mathrm{y}=$ years;

$1 \mathrm{y}=31556926 \mathrm{~s} \quad$ or $365.2422 \mathrm{~d}$

adopted values for NUBASE (see text)

STABLE $=$ stable nuclide or nuclide for which no finite value for half-life has been found.

\# value estimated from systematic trends in neighboring nuclides with the same $Z$ and $N$ parities.

subunits:
ms: $10^{-3} \mathrm{~s}$ millisecond
$\mu \mathrm{s}: 10^{-6} \mathrm{~s}$ microsecond
ky: $10^{3}$ y kiloyear
ns : $10^{-9} \mathrm{~s}$ nanosecond
My: $10^{6}$ y megayear
ps : $10^{-12}$ s picosecond
Gy: $10^{9}$ y gigayear
fs : $10^{-15} \mathrm{~s}$ femtosecond
Ty: $10^{12}$ y terayear
as : $10^{-18} \mathrm{~s}$ attosecond
Py: $10^{15}$ y petayear
zs : $10^{-21} \mathrm{~s}$ zeptosecond
Ey: $10^{18}$ y exayear
ys : $10^{-24} \mathrm{~s}$ yoctosecond
$\mathrm{Zy}: 10^{21}$ y zettayear
Yy: $10^{24}$ y yottayear

For isomeric mixtures: $R$ is the production ratio of excited isomeric state to ground-state.

Spin and parity:

() uncertain spin and/or parity.

\# values estimated from systematic trends in neighboring nuclides with the same $Z$ and $N$ parities.

high high spin.

low low spin.

am same $J^{\pi}$ as $\alpha$-decay parent;

For isomeric mixtures: mix (spmix and fsmix if coming from spallation and fission respectively).

Ens $\quad$ Year of the archival file of the ENSDF

(in order to reduce the width of the Table, the two digits for the centuries are omitted).

Reference Reference keys:

(in order to reduce the width of the Table, the two digits for the centuries are omitted; at the end of this volume however, the full reference key-number is given: $1992 \mathrm{~Pa} 05$ and not 92Pa05)

92Pa05 Updates to ENSDF derived from regular journal. These keys are taken from Nuclear Data Sheets. Where not yet available, the style 03Ya.1 is provisionally adopted.

95Am.A Updates to ENSDF derived from abstract, preprint, private communication, conference, thesis or annual report.

ABBW Re-interpretation by the present authors.

The reference key-numbers are followed by one, two or three letter codes which specifies the added or modified physical quantities:

$\mathrm{T}$ for half-life

J for spin and/or parity

E for the isomer excitation energy

D for decay mode and/or intensity

I for identification 
Decay modes Decay modes followed by their intensities (in \%), and their one standard deviation

and

intensities uncertainties. The special notation $1.8 \mathrm{e}-12$ stands for $1.8 \times 10^{-12}$.

The uncertainties are given - only in this field - in the ENSDF-style: $\alpha=25.923$ stands for $\alpha=25.9 \pm 2.3 \%$

The ordering is according to decreasing intensities.

$\begin{array}{ll}\alpha & \alpha \text { emission } \\ \mathrm{p} \mathrm{2p} & \text { proton emission } \quad \text { 2-proton emission } \\ \mathrm{n} 2 \mathrm{n} & \text { neutron emission } \quad \text { 2-neutron emission } \\ \varepsilon & \text { electron capture } \\ \mathrm{e}^{+} & \text {positron emission } \\ \beta^{+} & \beta^{+} \text {decay } \quad\left(\beta^{+}=\varepsilon+\mathrm{e}^{+}\right) \\ \beta^{-} & \beta^{-} \text {decay } \\ 2 \beta^{-} & \text {double } \beta^{-} \text {decay } \\ 2 \beta^{+} & \text {double } \beta^{+} \text {decay } \\ \beta^{-} \mathrm{n} & \beta^{-} \text {delayed neutron emission } \\ \beta^{-} 2 \mathrm{n} & \beta^{-} \text {delayed 2-neutron emission } \\ \beta^{+} \mathrm{p} & \beta^{+} \text {delayed proton emission } \\ \beta^{+} 2 \mathrm{p} & \beta^{+} \text {delayed 2-proton emission } \\ \beta^{-} \alpha & \beta^{-} \text {delayed } \alpha \text { emission } \\ \beta^{+} \alpha & \beta^{+} \text {delayed } \alpha \text { emission } \\ \beta^{-} \mathrm{d} & \beta^{-} \text {delayed deuteron emission } \\ \mathrm{IT} & \text { internal transition } \\ \mathrm{SF} & \text { spontaneous fission } \\ \beta^{+} \mathrm{SF} & \beta^{+} \text {delayed fission } \\ \beta^{-} \mathrm{SF} & \beta^{-} \text {delayed fission } \\ 24 \mathrm{Ne} & \text { heavy cluster emission } \\ \cdots & \text { list is continued in a remark, at the end of the } A \text {-group }\end{array}$

For long-lived nuclides:

ist is continued in a remark, at the end of the $A$-group

* A remark on the corresponding nuclide is given below the block of data corresponding to the same $A$.

Remarks. For nuclides indicated with an asterix at the end of the line, remarks have been added. They are collected in groups at the end of each block of data corresponding to the same $A$. They start with a code letter, like the ones following the reference key-number, as given above, indicating to which quantity the remark applies. They give:

i) Continuation for the list of decays. In this case, the remark starts with three dots.

ii) Information explaining how a value has been derived.

iii) Reasons for changing a value or its uncertainty as given by the authors or for rejecting it.

iv) Complementary references for updated data.

v) Separate values entering an adopted average. 


\begin{tabular}{|c|c|c|c|c|c|c|c|c|c|c|c|c|}
\hline Nuclide & \multicolumn{2}{|c|}{$\begin{array}{l}\text { Mass excess } \\
(\mathrm{keV})\end{array}$} & $\begin{array}{l}\text { Excitation } \\
\text { energy }(\mathrm{keV})\end{array}$ & \multicolumn{3}{|c|}{ Half-life } & \multirow{2}{*}{$\begin{array}{c}J^{\pi} \\
1 / 2^{+}\end{array}$} & \multirow{2}{*}{$\begin{array}{c}\text { Ens } \\
00\end{array}$} & \multicolumn{2}{|c|}{ Reference } & \multicolumn{2}{|l|}{$\begin{array}{l}\text { Decay modes and } \\
\text { intensities }(\%)\end{array}$} \\
\hline${ }^{1} \mathrm{n}$ & 8071.3171 & 0.0005 & & 613.9 & $\mathrm{~s}$ & 0.6 & & & $02 \mathrm{PaDG}$ & $\mathrm{T}$ & $\beta^{-}=100$ & \\
\hline${ }^{1} \mathrm{H}$ & 7288.9705 & 0.0001 & & STABLE & & & $1 / 2^{+}$ & 00 & 98Ro45 & $\mathrm{D}$ & IS $=99.988570$ & * \\
\hline$*{ }^{1} \mathrm{H}$ & $\mathrm{D}:$ all isotop & pic abundan & $\mathrm{s}$ in NUBASE a & from $98 \mathrm{Ro}$ & & & & & & & & $* *$ \\
\hline${ }^{2} \mathrm{H}$ & 13135.7216 & 0.0003 & & STABLE & & & $1^{+}$ & 99 & & & IS $=0.011570$ & \\
\hline${ }^{3} \mathrm{H}$ & 14949.8060 & 0.0023 & & 12.32 & $\mathrm{y}$ & 0.02 & $1 / 2^{+}$ & 00 & & & $\beta^{-}=100$ & \\
\hline${ }^{3} \mathrm{He}$ & 14931.2148 & 0.0024 & & STABLE & & & $1 / 2^{+}$ & 98 & & & $\mathrm{IS}=0.0001373$ & \\
\hline${ }^{3} \mathrm{Li}$ & $28670 \#$ & $2000 \#$ & RN & p-unstable & & & & 98 & & & p ? & \\
\hline${ }^{4} \mathrm{H}$ & 25900 & 100 & & 139 & ys & 10 & $2^{-}$ & 98 & $03 \mathrm{Me} 11$ & $\mathrm{~T}$ & $\mathrm{n}=100$ & * \\
\hline${ }^{4} \mathrm{He}$ & 2424.9156 & 0.0001 & & STABLE & & & $0^{+}$ & 98 & & & $\mathrm{IS}=99.9998633$ & \\
\hline${ }^{4} \mathrm{Li}$ & 25320 & 210 & & $\begin{array}{l}91 \\
91\end{array}$ & ys & 9 & $2^{-}$ & 98 & $65 \mathrm{Ce} 02$ & $\mathrm{~T}$ & $\mathrm{p}=100$ & \\
\hline$*^{4} \mathrm{H}$ & $\mathrm{T}:$ width $=3$. & $.28(0.23) \mathrm{M}$ & ; also 91Go19 & $4.7(1.0)$ out & Neigl & hed, $n$ & not used & & & & & $* *$ \\
\hline${ }^{5} \mathrm{H}$ & 32890 & 100 & & $>910$ & ys & & $\left(1 / 2^{+}\right)$ & 02 & 03Go11 & $\mathrm{T}$ & $2 n=100$ & * \\
\hline${ }^{5} \mathrm{He}$ & 11390 & 50 & & 700 & ys & 30 & $3 / 2^{-}$ & 02 & & & $\mathrm{n}=100$ & \\
\hline${ }^{5} \mathrm{Li}$ & 11680 & 50 & & 370 & ys & 30 & $3 / 2^{-}$ & 02 & & & $\mathrm{p}=100$ & \\
\hline${ }^{5} \mathrm{Be}$ & 38000\# & $4000 \#$ & & & & & $1 / 2^{+} \#$ & 02 & & & p? & \\
\hline$*{ }^{5} \mathrm{H}$ & $\mathrm{T}$ : from wid & th $<0.5 \mathrm{M}$ & ; at variance $\mathrm{w}$ & th $01 \mathrm{Ko} 52=$ & 2800 & 50) ys & s, width $=$ & $=1.9(($ & $0.4)$ & & & $* *$ \\
\hline$*^{5} \mathrm{H}$ & $\mathrm{T}: \quad(\mathrm{sam}$ & e authors) $b$ & with instrume & tal resolutio & $\mathrm{n}=1.3$ & $3 \mathrm{MeV}$ & & & & & & $* *$ \\
\hline$*^{5} \mathrm{H}$ & $\mathrm{T}: \quad$ other & rs $91 \mathrm{Go} 19=$ & (25) ys $95 \mathrm{Al} 3$ & $=110 \mathrm{ys}$ prob & ably & for hi & iigher stat & & & & & $* *$ \\
\hline$*{ }^{5} \mathrm{H}$ & $\mathrm{J}$ : from angu & ular distribu & on consistent $\mathrm{x}$ & th $l=0$ & & & & & & & & $* *$ \\
\hline${ }^{6} \mathrm{H}$ & 41860 & 260 & & 290 & ys & 70 & $2^{-} \#$ & 02 & & & $\mathrm{n} ? ; 3 \mathrm{n} ?$ & \\
\hline${ }^{6} \mathrm{He}$ & 17595.1 & 0.8 & & 806.7 & ms & 1.5 & $0^{+}$ & 02 & 90Ri01 & $\mathrm{D}$ & $\beta^{-}=100 ; \beta^{-} \mathrm{d}=0.000285$ & \\
\hline${ }^{6} \mathrm{Li}$ & 14086.793 & 0.015 & & STABLE & & & $1^{+}$ & 02 & & & $\mathrm{IS}=7.594$ & \\
\hline $\begin{array}{l}{ }^{6} \mathrm{Be} \\
{ }^{6} \mathrm{Be}\end{array}$ & 18375 & 5 & & $\begin{array}{l}5.0 \\
5.0\end{array}$ & zs & 0.3 & $0^{+}$ & 02 & & & $2 \mathrm{p}=100$ & \\
\hline${ }^{6} \mathrm{~B}$ & $43600 \#$ & $700 \#$ & & p-unstable\# & & & $2^{-} \#$ & & & & $2 \mathrm{p}$ ? & \\
\hline${ }^{7} \mathrm{H}$ & 49140\# & $1010 \#$ & & 23 & ys & 6 & $1 / 2^{+} \#$ & & 03Ko11 & $\mathrm{T}$ & $2 n ?$ & * \\
\hline${ }^{7} \mathrm{He}$ & 26101 & 17 & & 2.9 & $\mathrm{Zs}$ & 0.5 & $(3 / 2)^{-}$ & 03 & $02 \mathrm{Me} 07$ & $\mathrm{~T}$ & $\mathrm{n}=100$ & $*$ \\
\hline${ }^{7} \mathrm{Li}$ & 14908.14 & 0.08 & & StABLE & & & $3 / 2^{-}$ & 03 & & & $\mathrm{IS}=92.414$ & \\
\hline${ }^{7} \mathrm{Be}$ & 15770.03 & 0.11 & & 53.22 & $\mathrm{~d}$ & 0.06 & $3 / 2^{-}$ & 03 & & & $\varepsilon=100$ & \\
\hline${ }^{7} \mathrm{~B}$ & 27870 & 70 & & 350 & ys & $\begin{array}{c}0.00 \\
50\end{array}$ & $\left(3 / 2^{-}\right)$ & 03 & & & $\mathrm{p}=100$ & \\
\hline$*^{7} \mathrm{H}$ & $\mathrm{T}$ : from esti & imated widtl & $20(5) \mathrm{MeV}$ in $\mathrm{F}$ & g. 5 & & & & & & & & $* *$ \\
\hline$*^{7} \mathrm{He}$ & $\mathrm{T}$ : from 159 & (28) $\mathrm{keV}$, a & rage $02 \mathrm{Me} 07=$ & $50(80) 69 \mathrm{St}$ & $2=1$ & $60(30$ & & & & & & $* *$ \\
\hline${ }^{8} \mathrm{He}$ & 31598 & 7 & & 119.0 & $\mathrm{~ms}$ & 1.5 & $0^{+}$ & 99 & 88Aj01 & $\mathrm{D}$ & $\beta^{-}=100 ; \beta^{-} \mathrm{n}=161 ; \beta^{-} \mathrm{t}=0.91$ & * \\
\hline${ }^{8} \mathrm{Li}$ & 20946.84 & 0.09 & & 840.3 & $\mathrm{~ms}$ & 0.9 & $2^{+}$ & 99 & 90 Sa16 & $\mathrm{T}$ & $\beta^{-}=100 ; \beta^{-} \alpha=100$ & * \\
\hline${ }^{8} \mathrm{Be}$ & 4941.67 & 0.04 & & 67 & as & 17 & $0^{+}$ & 99 & & & $\alpha=100$ & \\
\hline${ }^{8} \mathrm{~B}$ & 22921.5 & 1.0 & & 770 & $\mathrm{~ms}$ & 3 & $2^{+}$ & 99 & 88Aj01 & $\mathrm{D}$ & $\beta^{+}=100 ; \beta^{+} \alpha=100$ & * \\
\hline${ }^{8} \mathrm{C}$ & 35094 & 23 & & 2.0 & $\mathrm{zs}$ & 0.4 & $0^{+}$ & 99 & & & $2 \mathrm{p}=100$ & \\
\hline$*^{8} \mathrm{He}$ & $\mathrm{D}: \beta^{-} \mathrm{n}$ inte & ensity is fror & $88 \mathrm{Aj} 01 ; \beta^{-} \mathrm{t}$ ir & ensity from & $86 \mathrm{Bc}$ & 041 & & & & & & $* *$ \\
\hline$*^{8} \mathrm{Li}$ & $\mathrm{D}: \beta^{-}$decay & $y$ to first $2^{+}$ & ate in ${ }^{8} \mathrm{Be}$, wh & h decays 10 & $0 \%$ i & in $2 \alpha$ & & & & & & $* *$ \\
\hline$*^{8} \mathrm{~B}$ & $\mathrm{D}: \beta^{+}$to $2 \mathrm{e}$ & excited state & in ${ }^{8} \mathrm{Be}$, then $\alpha$ & and $\gamma$, but no & $t$ to ${ }^{8}$ & ${ }^{8} \mathrm{Be} \mathrm{gl}^{1}$ & round-sta & & & & & $* *$ \\
\hline${ }^{9} \mathrm{He}$ & 40939 & 29 & & 7 & zs & 4 & $1 / 2^{(-\#)}$ & 99 & $99 \mathrm{Bo} 26$ & $\mathrm{~T}$ & $\mathrm{n}=100$ & * \\
\hline${ }^{9} \mathrm{Li}$ & 24954.3 & 1.9 & & 178.3 & $\mathrm{~ms}$ & 0.4 & $3 / 2^{-}$ & 99 & 95Re.A & $\mathrm{D}$ & $\beta^{-}=100 ; \beta^{-} \mathrm{n}=50.82$ & * \\
\hline${ }^{9} \mathrm{Be}$ & 11347.6 & 0.4 & & STABLE & & & $3 / 2^{-}$ & 99 & & & $\mathrm{IS}=100$ & \\
\hline${ }^{9} \mathrm{~B}$ & 12415.7 & 1.0 & & 800 & zs & 300 & $3 / 2^{-}$ & 99 & & & $\mathrm{p}=100$ & \\
\hline${ }^{9} \mathrm{C}$ & 28910.5 & 2.1 & & 126.5 & $\mathrm{~ms}$ & 0.9 & $\left(3 / 2^{-}\right)$ & 99 & 88Aj01 & $\mathrm{D}$ & $\beta^{+}=100 ; \beta^{+} \mathrm{p}=23 ; \beta^{+} \alpha=17$ & $*$ \\
\hline$*{ }^{9} \mathrm{He}$ & $\mathrm{T}:$ derived $\mathrm{f}$ & from width & $0(60) \mathrm{keV}$ & $\mathrm{J}:$ from 0 & Ch3 & & & & & & & $* *$ \\
\hline$*^{9} \mathrm{Li}$ & $\mathrm{D}:$ also $92 \mathrm{~T}$ & $\mathrm{e} 03 \beta^{-} \mathrm{n}=5$ & 1) $\% 81 \mathrm{La} 11=$ & (5) outweig & ned, & not us & & & & & & $* *$ \\
\hline$*^{9} \mathrm{C}$ & $\mathrm{D}: \beta^{+}=12 \%$ & $b$ and $11 \% \mathrm{t}$ & 2 excited p-em & ting states is & ${ }^{9} \mathrm{~B}$ & and 1 & $17 \%$ to $\alpha$ & $x$ emi & itter & & & $* *$ \\
\hline
\end{tabular}




\begin{tabular}{|c|c|c|c|c|c|c|c|c|c|c|c|c|c|}
\hline \multirow{2}{*}{$\begin{array}{l}\text { Nuclide } \\
{ }^{10} \mathrm{He}\end{array}$} & \multicolumn{2}{|c|}{$\begin{array}{l}\text { Mass excess } \\
(\mathrm{keV})\end{array}$} & \multicolumn{3}{|c|}{$\begin{array}{l}\text { Excitation } \\
\text { energy(keV) }\end{array}$} & \multicolumn{3}{|c|}{ Half-life } & \multirow{2}{*}{$\begin{array}{c}J^{\pi} \\
0^{+}\end{array}$} & \multirow{2}{*}{$\begin{array}{l}\text { Ens } \\
99\end{array}$} & \multicolumn{2}{|c|}{ Reference } & $\begin{array}{l}\text { Decay modes and } \\
\text { intensities }(\%)\end{array}$ \\
\hline & 48810 & 70 & & & & 2.7 & zs & 1.8 & & & 940s04 & $\mathrm{T}$ & $2 n=100$ \\
\hline${ }^{10} \mathrm{Li}$ & 33051 & 15 & & & & 2.0 & $\mathrm{zs}$ & 0.5 & $\left(1^{-}, 2^{-}\right)$ & 99 & 94 Yo01 & $\mathrm{TJ}$ & $\mathrm{n}=100$ \\
\hline${ }^{10} \mathrm{Li}^{m}$ & 33250 & 40 & 200 & 40 & RQ & 3.7 & $\mathrm{zs}$ & 1.5 & $1^{+}$ & & $97 \mathrm{Zi04}$ & $\mathrm{T}$ & $\mathrm{IT}=100$ \\
\hline${ }^{10} \mathrm{Li}^{n}$ & 33530 & 40 & 480 & 40 & RQ & 1.35 & $\mathrm{zs}$ & 0.24 & $2^{+}$ & & 94 Yo01 & $\mathrm{T}$ & $\mathrm{IT}=100$ \\
\hline${ }^{10} \mathrm{Be}$ & 12606.7 & 0.4 & & & & 1.51 & My & 0.06 & $0^{+}$ & 99 & & & $\beta^{-}=100$ \\
\hline${ }^{10} \mathrm{~B}$ & 12050.7 & 0.4 & & & & StABLE & & & $3^{+}$ & 99 & & & $\mathrm{IS}=19.97$ \\
\hline${ }^{10} \mathrm{C}$ & 15698.7 & 0.4 & & & & 19.290 & s & 0.012 & $0^{+}$ & 99 & $90 \mathrm{Ba} 02$ & $\mathrm{~T}$ & $\beta^{+}=100$ \\
\hline${ }^{10} \mathrm{~N}$ & 38800 & 400 & & & & 200 & ys & 140 & $\left(2^{-}\right)$ & 99 & 02Le16 & TJ & $\mathrm{p}$ ? \\
\hline$*{ }^{10} \mathrm{He}$ & $\mathrm{D}:$ most & ably 2 & on em & tter $\mathrm{f}$ & rom & $=-1070$ & 70) $\mathrm{k}$ & $\mathrm{eV}$ & & & & & \\
\hline$*{ }^{10} \mathrm{Li}^{m}$ & $\mathrm{~T}:$ avera & $7 \mathrm{Zi04}=$ & -100 & 0) 94 & Yo0 & $100(70) \mathrm{l}$ & $\mathrm{eV}$ & & & & & & \\
\hline$*^{10} \mathrm{Li}^{n}$ & $\mathrm{~T}:$ avera & $4 \mathrm{Yo01}=$ & 23) 93 & $\mathrm{Bo} 03$ & $=150$ & 0) $\mathrm{keV}$, B & irge & ratio $B=$ & $=2.8$ & & & & \\
\hline
\end{tabular}

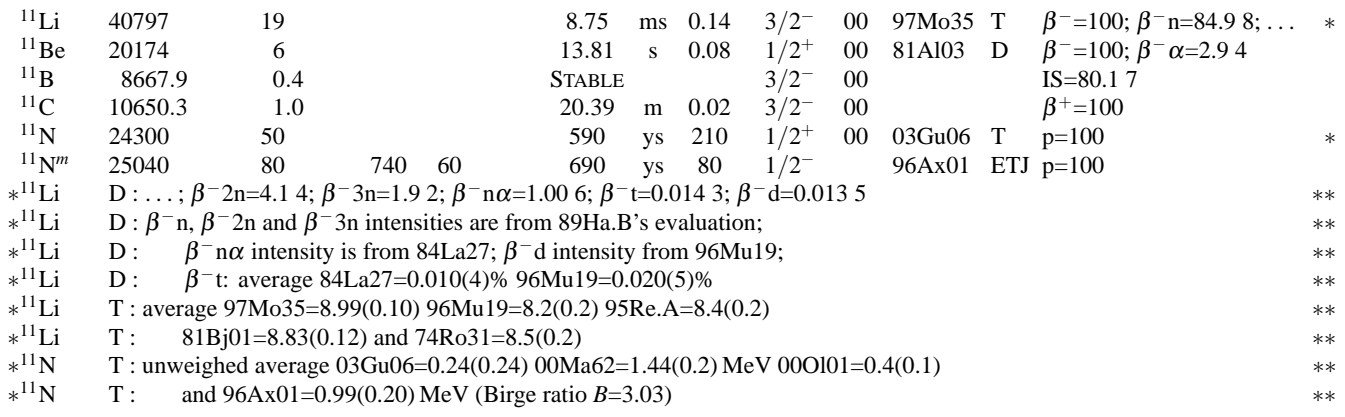

\begin{tabular}{|c|c|c|c|c|c|c|c|c|c|c|}
\hline${ }^{12} \mathrm{Li}$ & $50100 \#$ & $1000 \#$ & $<10$ & $\mathrm{~ns}$ & & & 00 & 74Bo05 & I & $\mathrm{n}$ ? \\
\hline${ }^{12} \mathrm{Be}$ & 25077 & 15 & 21.50 & $\mathrm{~ms}$ & 0.04 & $0^{+}$ & 00 & $01 \mathrm{Be} 53$ & $\mathrm{~T}$ & $\beta^{-}=100 ; \beta^{-} \mathrm{n}=0.503$ \\
\hline${ }^{12} \mathrm{~B}$ & 13368.9 & 1.4 & 20.20 & $\mathrm{~ms}$ & 0.02 & $1^{+}$ & 00 & $66 \mathrm{Sc} 23$ & $\mathrm{D}$ & $\beta^{-}=100 ; \beta^{-} \alpha=1.63$ \\
\hline${ }^{12} \mathrm{C}$ & 0.0 & 0.0 & StABLE & & & $0^{+}$ & 00 & & & IS $=98.938$ \\
\hline${ }^{12} \mathrm{~N}$ & 17338.1 & 1.0 & 11.000 & $\mathrm{~ms}$ & 0.016 & $1^{+}$ & 00 & $66 \mathrm{Sc} 23$ & $\mathrm{D}$ & $\beta^{+}=100 ; \beta^{+} \alpha=3.55$ \\
\hline${ }^{12} \mathrm{O}$ & 32048 & 18 & 580 & ys & 30 & $0^{+}$ & 00 & $95 \mathrm{Kr} 03$ & $\mathrm{~T}$ & $2 \mathrm{p}=6030 ; \beta^{+} ?$ \\
\hline${ }^{2} \mathrm{Be}$ & $\mathrm{D}$ : fror & 53. & eighed, & not & ssed & & & & & \\
\hline
\end{tabular}

\begin{tabular}{|c|c|c|c|c|c|c|c|c|c|c|c|c|c|c|}
\hline${ }^{13} \mathrm{Be}$ & 33250 & 70 & & & & 0.5 & ns & 0.1 & $\left(1 / 2^{+}\right)$ & & 01 Th01 & TJ & $\mathrm{n}$ ? & \\
\hline${ }^{13} \mathrm{Be}^{p}$ & 33950 & 90 & 700 & 120 & RQ & 2.7 & zs & 1.8 & $\left(1 / 2^{-}\right)$ & 00 & & & & \\
\hline${ }^{13} \mathrm{Be}^{q}$ & 35160 & 50 & 1910 & 90 & RQ & & & & $\left(5 / 2^{+}\right)$ & & & & & \\
\hline${ }^{13} \mathrm{~B}$ & 16562.2 & 1.1 & & & & 17.33 & $\mathrm{~ms}$ & 0.17 & $3 / 2^{-}$ & 00 & & & $\beta^{-}=100 ; \beta^{-} \mathrm{n}=0.284$ & \\
\hline${ }^{13} \mathrm{C}$ & 3125.0113 & 0.0009 & & & & StABLE & & & $1 / 2^{-}$ & 01 & & & $\mathrm{IS}=1.078$ & \\
\hline${ }^{13} \mathrm{~N}$ & 5345.48 & 0.27 & & & & 9.965 & $\mathrm{~m}$ & 0.004 & $1 / 2^{-}$ & 00 & & & $\beta^{+}=100$ & \\
\hline${ }^{13} \mathrm{O}$ & 23112 & 10 & & & & 8.58 & $\mathrm{~ms}$ & 0.05 & $\left(3 / 2^{-}\right)$ & 00 & $70 \mathrm{Es} 03$ & $\mathrm{D}$ & $\beta^{+}=100 ; \beta^{+} \mathrm{p}=10.920$ & \\
\hline${ }^{14} \mathrm{Be}$ & 39950 & 130 & & & & 4.35 & $\mathrm{~ms}$ & 0.17 & $0^{+}$ & 01 & 02Je11 & $\mathrm{D}$ & $\beta^{-}=100 ; \beta^{-} \mathrm{n}=982 ; \ldots$ & $*$ \\
\hline${ }^{14} \mathrm{Be}^{p}$ & 41470 & 60 & 1520 & 150 & & & & & $\left(2^{+}\right)$ & & 95Bo10 & & & \\
\hline${ }^{14} \mathrm{~B}$ & 23664 & 21 & & & & 12.5 & $\mathrm{~ms}$ & 0.5 & $2^{-}$ & 01 & 95Re.A & $\mathrm{D}$ & $\beta^{-}=100 ; \beta^{-} \mathrm{n}=6.0423$ & \\
\hline${ }^{14} \mathrm{C}$ & 3019.893 & 0.004 & & & & 5.70 & ky & 0.03 & $0^{+}$ & 01 & & & $\beta^{-}=100$ & \\
\hline${ }^{14} \mathrm{~N}$ & 2863.4170 & 0.0006 & & & & STABLE & & & $1^{+}$ & 01 & & & IS $=99.6327$ & \\
\hline${ }^{14} \mathrm{O}$ & 8007.36 & 0.11 & & & & 70.598 & $\mathrm{~s}$ & 0.018 & $0^{+}$ & 01 & $01 \mathrm{Ga} 59$ & $\mathrm{~T}$ & $\beta^{+}=100$ & * \\
\hline${ }^{14} \mathrm{~F}$ & $32660 \#$ & $400 \#$ & & & & & & & $2^{-} \#$ & & & & $\mathrm{p}$ ? & \\
\hline$*{ }^{14} \mathrm{Be}$ & $\mathrm{D}: \ldots ; \beta^{-} 2$ & $\mathrm{n}=0.808 ; \beta$ & $-3 n=0$ & $.22 ;$ & $\beta^{-} t^{-}$ & $021 ; \beta^{-} \alpha$ & $x<0$ & .004 & & & & & & $* *$ \\
\hline$*^{14} \mathrm{Be}$ & D : supersed & s 99Be53, s & same $g$ & roup & & & & & & & & & & $* *$ \\
\hline$*^{14} \mathrm{O}$ & $\mathrm{T}:$ average 0 & $1 \mathrm{Ga} 59=70.5$ & $560(0$. & 049) 7 & $8 \mathrm{WiO}$ & $=70.613(0$ & 0.025 & 5) $73 \mathrm{Cl}$ & $12=70.590$ & $0(0.0$ & 30) & & & $* *$ \\
\hline
\end{tabular}




\begin{tabular}{|c|c|c|c|c|c|c|c|c|c|}
\hline Nuclide & \multicolumn{2}{|c|}{$\begin{array}{l}\text { Mass excess } \\
\quad(\mathrm{keV})\end{array}$} & $\begin{array}{c}\text { Excitation } \\
\text { energy }(\mathrm{keV})\end{array}$ & \multicolumn{2}{|c|}{ Half-life } & $J^{\pi}$ & Ens & Reference & $\begin{array}{l}\text { Decay modes and } \\
\text { intensities }(\%)\end{array}$ \\
\hline${ }^{15} \mathrm{Be}$ & 49800\# & $500 \#$ & & $<200$ & ns & & & 03Ba47 I & $\mathrm{n}$ ? \\
\hline${ }^{15} \mathrm{~B}$ & 28972 & 22 & & 9.87 & ms 0.07 & $3 / 2^{-}$ & 93 & 95Re.A TD & $\beta^{-}=100 ; \beta^{-} \mathrm{n}=93.612 ; \beta^{-} 2 \mathrm{n}=0.42$ \\
\hline${ }^{15} \mathrm{C}$ & 9873.1 & 0.8 & & 2.449 & s 0.005 & $1 / 2^{+}$ & 94 & & $\beta^{-}=100$ \\
\hline${ }^{15} \mathrm{~N}$ & 101.4380 & 0.0007 & & STABLE & & $1 / 2^{-}$ & 94 & & $\mathrm{IS}=0.3687$ \\
\hline${ }^{15} \mathrm{O}$ & 2855.6 & 0.5 & & 122.24 & s 0.16 & $1 / 2^{-}$ & 94 & & $\beta^{+}=100$ \\
\hline${ }^{15} \mathrm{~F}$ & 16780 & 130 & & 410 & ys 60 & $\left(1 / 2^{+}\right)$ & 93 & 01Ze.A T & $\mathrm{p}=100$ \\
\hline$*^{15} \mathrm{~B}$ & $\mathrm{D}: \beta^{-} 2 \mathrm{n}$ int & tensity is fro & m 89Re.A & $\mathrm{J}:$ given & in $91 \mathrm{Aj} 0$ & & & & \\
\hline$*^{15} \mathrm{~B}$ & $\mathrm{~T}$ : four othe & r outweighe & results, see El & SDF'93, ra & anging 10 . & $.1-10.8$ & & & \\
\hline$*{ }^{15} \mathrm{~F}$ & $\mathrm{~T}$ : average $\mathrm{C}$ & $01 \mathrm{Ze} \cdot \mathrm{A}=1.2$ & $(0.22) \mathrm{MeV} 78$ & $\mathrm{e} 16=1.2$ & $0.3) 78 \mathrm{Ke}$ & $06=0.8 \mathrm{c}$ & $(0.3)$ & & \\
\hline
\end{tabular}

\begin{tabular}{|c|c|c|c|c|c|c|c|c|c|c|c|}
\hline${ }^{16} \mathrm{Be}$ & $57680 \#$ & $500 \#$ & $<200$ & ns & & $0^{+}$ & & 03Ba47 & I & $2 \mathrm{n} ?$ & $*$ \\
\hline${ }^{16} \mathrm{~B}$ & 37080 & 60 & $<190$ & ps & & $0^{-}$ & 99 & & & $\mathrm{n}$ ? & \\
\hline${ }^{16} \mathrm{C}$ & 13694 & 4 & 747 & $\mathrm{~ms}$ & 8 & $0^{+}$ & 99 & 89Re.A & $\mathrm{D}$ & $\beta^{-}=100 ; \beta^{-} \mathrm{n}=97.923$ & \\
\hline${ }^{16} \mathrm{~N}$ & 5683.7 & 2.6 & 7.13 & $\mathrm{~s}$ & 0.02 & $2^{-}$ & 99 & $74 \mathrm{Ne} 10$ & $\mathrm{D}$ & $\beta^{-}=100 ; \beta^{-} \alpha=0.001007$ & \\
\hline${ }^{16} \mathrm{O}$ & -4737.0014 & 0.0001 & STABLE & & & $0^{+}$ & 99 & & & $\mathrm{IS}=99.75716$ & \\
\hline${ }^{16} \mathrm{~F}$ & 10680 & 8 & 11 & zs & 6 & $0^{-}$ & 99 & & & $\mathrm{p}=100$ & \\
\hline${ }^{16} \mathrm{Ne}$ & 23996 & 20 & 9 & $\mathrm{zs}$ & & $0^{+}$ & 99 & & & $2 p=100$ & \\
\hline$*^{16} \mathrm{Be}$ & I : 100 event & s expected, none observed & & & & & & & & & ** \\
\hline${ }^{17} \mathrm{~B}$ & 43770 & 170 & 5.08 & $\mathrm{~ms}$ & 0.05 & $\left(3 / 2^{-}\right)$ & 99 & 88Du09 & $\mathrm{D}$ & $\beta^{-}=100 ; \beta^{-} \mathrm{n}=631 ; \ldots$ & * \\
\hline${ }^{17} \mathrm{C}$ & 21039 & 17 & 193 & $\mathrm{~ms}$ & 5 & $\left(3 / 2^{+}\right)$ & 99 & 01Ma08 & $\mathrm{J}$ & $\beta^{-}=100 ; \beta^{-} \mathrm{n}=28.413$ & * \\
\hline${ }^{17} \mathrm{~N}$ & 7871 & 15 & 4.173 & $\mathrm{~s}$ & 0.004 & $1 / 2^{-}$ & 99 & 94Do08 & $\mathrm{D}$ & $\beta^{-}=100 ; \beta^{-} \mathrm{n}=951 ; \ldots$ & $*$ \\
\hline${ }^{17} \mathrm{O}$ & -808.81 & 0.11 & STABLE & & & $5 / 2^{+}$ & 99 & & & $\mathrm{IS}=0.0381$ & \\
\hline${ }^{17} \mathrm{~F}$ & 1951.70 & 0.25 & 64.49 & $\mathrm{~s}$ & 0.16 & $5 / 2^{+}$ & 99 & & & $\beta^{+}=100$ & \\
\hline${ }^{17} \mathrm{Ne}$ & 16461 & 27 & 109.2 & $\mathrm{~ms}$ & 0.6 & $1 / 2^{-}$ & 99 & $88 \mathrm{Bo} 39$ & $\mathrm{D}$ & $\beta^{+}=100 ; \beta^{+} \mathrm{p}=96.09 ; \beta^{+} \alpha=2.79$ & \\
\hline$*{ }^{17} \mathrm{~B}$ & $\mathrm{D}: \ldots ; \beta^{-2}$ & $\mathrm{n}=117 ; \beta^{-} 3 \mathrm{n}=3.57 ; \beta^{-4}$ & $=0.43$ & & & & & & & & ** \\
\hline$*{ }^{17} \mathrm{C}$ & $\mathrm{T}:$ average 9 & $5 \mathrm{Sc} 03=193(6) 95 \mathrm{Re} . \mathrm{A}=18$ & (10) $86 \mathrm{C}$ & $\mathrm{u} 01=$ & $=202($ & & & & & & $* *$ \\
\hline$*{ }^{17} \mathrm{C}$ & $\mathrm{D}: \beta^{-} \mathrm{n}$ inte & nsity is from 95Re.A & & & & & & & & & ** \\
\hline$*^{17} \mathrm{~N}$ & $\mathrm{D}: \ldots ; \beta^{-} 0$ & $x=0.00254$ & & & & & & & & & k* \\
\hline
\end{tabular}

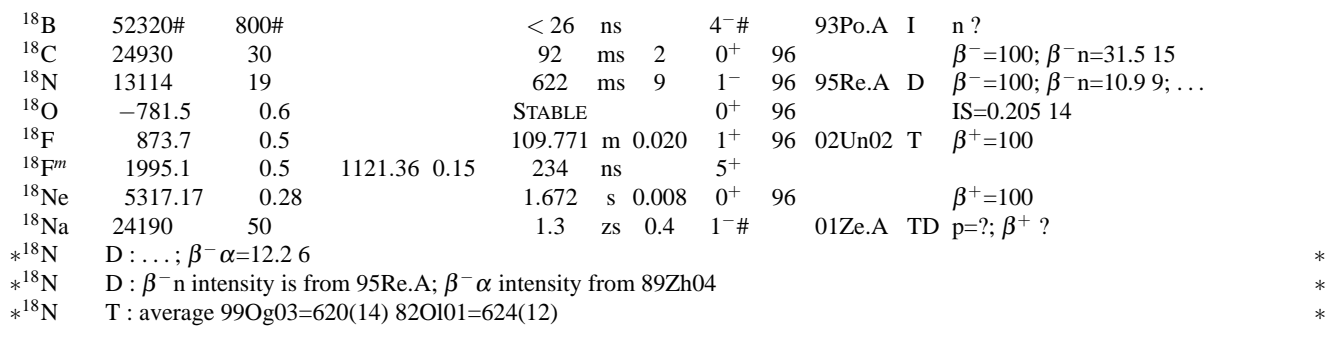

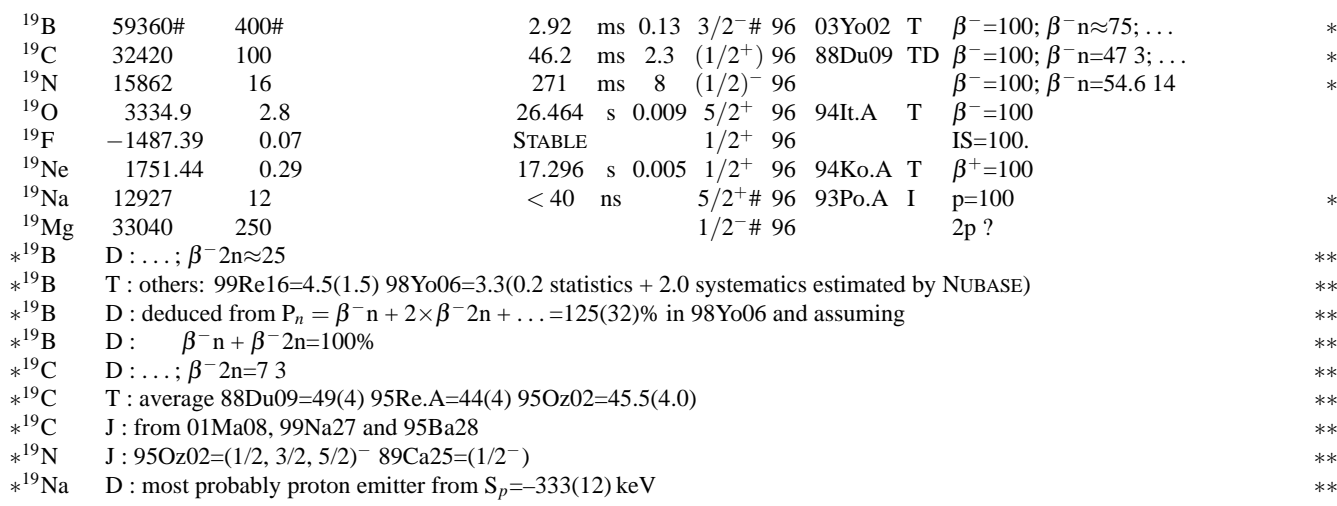




\begin{tabular}{|c|c|c|c|c|c|c|c|c|c|c|c|}
\hline Nuclide & \multicolumn{2}{|c|}{$\begin{array}{l}\text { Mass excess } \\
\quad(\mathrm{keV})\end{array}$} & $\begin{array}{l}\text { Excitation } \\
\text { energy }(\mathrm{keV})\end{array}$ & \multicolumn{3}{|c|}{ Half-life } & $J^{\pi}$ & Ens & \multicolumn{2}{|c|}{ s Reference } & $\begin{array}{l}\text { Decay modes and } \\
\text { intensities }(\%)\end{array}$ \\
\hline${ }^{20} \mathrm{C}$ & 37560 & 240 & & 16 & $\mathrm{~ms}$ & 3 & $0^{+}$ & 98 & $90 \mathrm{Mu} 06 \mathrm{~T}$ & & $\beta^{-}=100 ; \beta^{-} \mathrm{n}=7214$ \\
\hline${ }^{20} \mathrm{~N}$ & 21770 & 60 & & 130 & $\mathrm{~ms}$ & 7 & & 98 & 95Re.A & TD & $\beta^{-}=100 ; \beta^{-} \mathrm{n}=57.025$ \\
\hline${ }^{20} \mathrm{O}$ & 3797.5 & 1.1 & & 13.51 & $\mathrm{~s}$ & 0.05 & $0^{+}$ & 98 & & & $\beta^{-}=100$ \\
\hline${ }^{20} \mathrm{~F}$ & -17.40 & 0.08 & & 11.163 & $\mathrm{~s}$ & 0.008 & $2^{+}$ & 98 & 98Ti06 & $\mathrm{T}$ & $\beta^{-}=100$ \\
\hline${ }^{20} \mathrm{Ne}$ & -7041.9313 & 0.0018 & & Stable & & & $0^{+}$ & 98 & & & $\mathrm{IS}=90.483$ \\
\hline${ }^{20} \mathrm{Na}$ & 6848 & 7 & & 447.9 & $\mathrm{~ms}$ & 2.3 & $2^{+}$ & 98 & $89 \mathrm{Cl02} \mathrm{I}$ & $\mathrm{D}$ & $\beta^{+}=100 ; \beta^{+} \alpha=25.04$ \\
\hline${ }^{20} \mathrm{Mg}$ & 17570 & 27 & & 90 & $\mathrm{~ms}$ & 6 & $0^{+}$ & 98 & $95 \mathrm{Pi03}$ & TD & $\beta^{+}=100 ; \beta^{+} \mathrm{p}=30.416$ \\
\hline$*^{20} \mathrm{C}$ & $\mathrm{T}:$ average 90 & $9 \mathrm{Mu} 06=14($ & 6-5) 95Re.A 1 & $7(3.5)$ & & & & & & & \\
\hline$*^{20} \mathrm{Mg}$ & $\mathrm{T}:$ average 95 & $5 \mathrm{Pi} 03=95(3$ & $92 \mathrm{Go} 10=82(4)$ & with Birg & rati & tio $B=2$ & & & & & \\
\hline
\end{tabular}

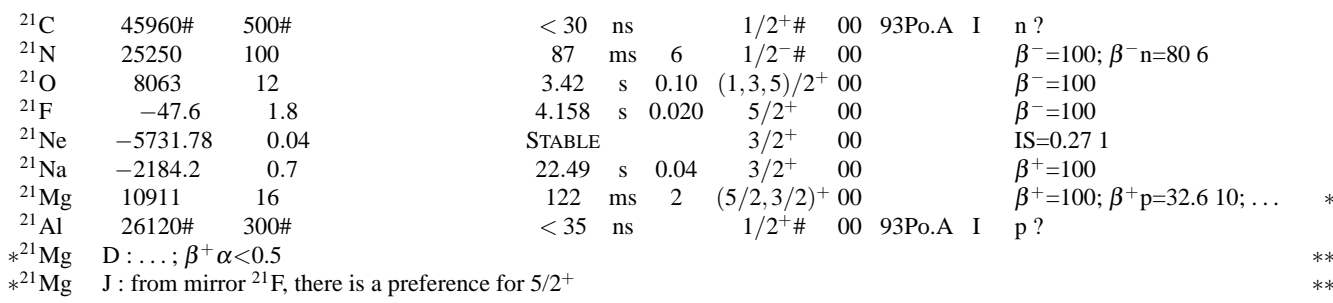

\begin{tabular}{|c|c|c|c|c|c|c|c|c|c|c|c|c|}
\hline${ }^{22} \mathrm{C}$ & 53280\# & $900 \#$ & & & 6.2 & $\mathrm{~ms}$ & 1.3 & $0^{+}$ & 00 & 03Yo02 & & $\beta^{-}=100 ; \beta^{-} \mathrm{n}=9939 ; \ldots$ \\
\hline${ }^{22} \mathrm{~N}$ & 32040 & 190 & & & 13.9 & $\mathrm{~ms}$ & 1.4 & & 00 & 03 Yo02 & $\mathrm{T}$ & $\beta^{-}=100 ; \beta^{-} \mathrm{n}=355$ \\
\hline${ }^{22} \mathrm{O}$ & 9280 & 60 & & & 2.25 & $\mathrm{~s}$ & 0.15 & $0^{+}$ & 00 & & & $\beta^{-}=100 ; \beta^{-} \mathrm{n}<22$ \\
\hline${ }^{22} \mathrm{~F}$ & 2793 & 12 & & & 4.23 & s & 0.04 & $4^{+},\left(3^{+}\right)$ & 00 & & & $\beta^{-}=100 ; \beta^{-} \mathrm{n}<11$ \\
\hline${ }^{22} \mathrm{Ne}$ & -8024.715 & 0.018 & & & STABLE & & & $0^{+}$ & 00 & & & $\mathrm{IS}=9.253$ \\
\hline${ }^{22} \mathrm{Na}$ & -5182.4 & 0.4 & & & 2.6019 & $\mathrm{y}$ & 0.0004 & $3^{+}$ & 00 & & & $\beta^{+}=100$ \\
\hline${ }^{22} \mathrm{Na}^{m}$ & -4599.4 & 0.4 & 583.03 & 0.09 & 244 & ns & 6 & $1^{+}$ & 00 & & & $\mathrm{IT}=100$ \\
\hline${ }^{22} \mathrm{Mg}$ & -397.0 & 1.3 & & & 3.857 & s & 0.009 & $0^{+}$ & 00 & & & $\beta^{+}=100$ \\
\hline${ }^{22} \mathrm{Al}$ & 18180\# & 90\# & & & 59 & $\mathrm{~ms}$ & 3 & $(3)^{+}$ & 00 & $97 \mathrm{~B} 103$ & $\mathrm{D}$ & $\beta^{+}=100 ; \beta^{+} \mathrm{p}=443 ; \ldots$ \\
\hline${ }^{22} \mathrm{Si}$ & 32160\# & $200 \#$ & & & 29 & $\mathrm{~ms}$ & 2 & $0^{+}$ & 00 & $96 \mathrm{~B} 111$ & $\mathrm{D}$ & $\beta^{+}=100 ; \beta^{+} \mathrm{p}=324$ \\
\hline$*^{22} \mathrm{C}$ & $\mathrm{D}: \ldots ; \beta^{-} 2 \mathrm{r}$ & & $\mathrm{D}$ : from & 98Yo06 & & & & & & & & \\
\hline${ }^{22} \mathrm{~N}$ & D : from $90 \mathrm{M}$ & Lu06 & & & & & & & & & & \\
\hline$*^{22} \mathrm{Al}$ & $\mathrm{D}: \ldots ; \beta^{+} 2_{\mathrm{p}}$ & $=095$. & $\alpha=0,3$ & & & & & & & & & \\
\hline
\end{tabular}

\begin{tabular}{|c|c|c|c|c|c|c|c|c|c|c|c|}
\hline${ }^{23} \mathrm{~N}$ & 38400\# & $300 \#$ & 14.5 & $\mathrm{~ms}$ & 2.4 & $1 / 2^{-} \#$ & 00 & 98Yo06 & $\mathrm{T}$ & $\beta^{-}=100 ; \beta^{-} \mathrm{n}=8021 ; \beta^{-} 2 \mathrm{n} ?$ & * \\
\hline${ }^{23} \mathrm{O}$ & 14610 & 120 & 90 & $\mathrm{~ms}$ & 40 & $1 / 2^{+} \#$ & 00 & $90 \mathrm{Mu} 06$ & $\mathrm{~T}$ & $\beta^{-}=100 ; \beta^{-} \mathrm{n}=317$ & \\
\hline${ }^{23} \mathrm{~F}$ & 3330 & 80 & 2.23 & $\mathrm{~s}$ & 0.14 & $(3 / 2,5 / 2)^{+}$ & 00 & & & $\beta^{-}=100 ; \beta^{-} \mathrm{n}<14$ & \\
\hline${ }^{23} \mathrm{Ne}$ & -5154.05 & 0.10 & 37.24 & $\mathrm{~s}$ & 0.12 & $5 / 2^{+}$ & 00 & & & $\beta^{-}=100$ & \\
\hline${ }^{23} \mathrm{Na}$ & -9529.8536 & 0.0027 & STABLE & & & $3 / 2^{+}$ & 00 & & & IS $=100$. & \\
\hline${ }^{23} \mathrm{Mg}$ & -5473.8 & 1.3 & 11.317 & $\mathrm{~s}$ & 0.011 & $3 / 2^{+}$ & 00 & & & $\beta^{+}=100$ & \\
\hline${ }^{23} \mathrm{Al}$ & 6770 & 19 & 470 & $\mathrm{~ms}$ & 30 & $5 / 2^{+} \#$ & 00 & 95Ti08 & D & $\beta^{+}=100 ; \beta^{+} \mathrm{p}=84$ & $*$ \\
\hline${ }^{23} \mathrm{Si}$ & 23770\# & $200 \#$ & 42.3 & $\mathrm{~ms}$ & 0.4 & $3 / 2^{+} \#$ & 00 & $97 \mathrm{~B} 104$ & TD & $\beta^{+}=100 ; \beta^{+} \mathrm{p} \approx 88$ & $*$ \\
\hline$*^{23} \mathrm{~N}$ & $\mathrm{~T}$ : statistical & error 1.4 , & imated by & y Nu & BASE & & & & & & ** \\
\hline$*^{23} \mathrm{Al}$ & $\mathrm{D}: \beta^{+} \mathrm{p}=3.5$ & $1.9) \%$ from & $.5 \times 4.8 / 2$ & $2=7$ & & & & & & & ** \\
\hline$*^{23} \mathrm{Si}$ & $\mathrm{D}: \ldots ; \beta^{+} 2 \mathrm{p}$ & $=3.63$ & & & & & & & & & ** \\
\hline
\end{tabular}

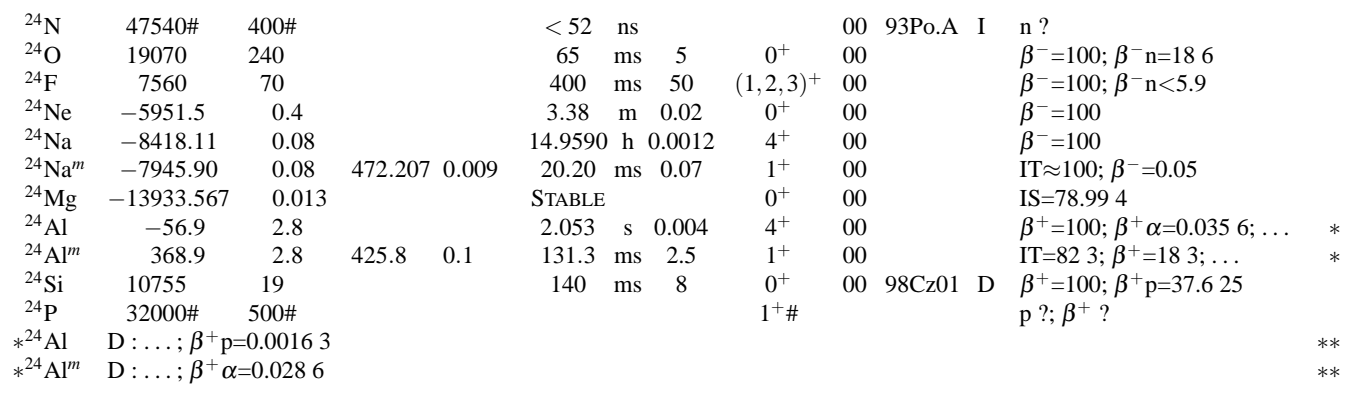




\begin{tabular}{|c|c|c|c|c|c|c|c|c|c|c|}
\hline Nuclide & \multicolumn{2}{|c|}{$\begin{array}{l}\text { Mass excess } \\
\quad(\mathrm{keV})\end{array}$} & $\begin{array}{c}\text { Excitation } \\
\text { energy }(\mathrm{keV})\end{array}$ & \multicolumn{3}{|c|}{ Half-life } & $J^{\pi}$ & Ens & Reference & $\begin{array}{l}\text { Decay modes and } \\
\text { intensities }(\%)\end{array}$ \\
\hline${ }^{25} \mathrm{~N}$ & $56500 \#$ & $500 \#$ & & $<260$ & ns & & $1 / 2^{-} \#$ & & 99Sa06 ID & $\mathrm{n} ? ; 2 \mathrm{n} ? ; \beta^{-}=0$ \\
\hline${ }^{25} \mathrm{O}$ & $27440 \#$ & $260 \#$ & & $<50$ & $\mathrm{~ns}$ & & $3 / 2^{+} \#$ & 00 & 93Po.A I & n? \\
\hline${ }^{25} \mathrm{~F}$ & 11270 & 100 & & 50 & $\mathrm{~ms}$ & 6 & $5 / 2^{+} \#$ & 00 & & $\beta^{-}=100 ; \beta^{-} \mathrm{n}=145$ \\
\hline${ }^{25} \mathrm{Ne}$ & -2108 & 26 & & 602 & $\mathrm{~ms}$ & 8 & $(3 / 2)^{+}$ & 00 & & $\beta^{-}=100$ \\
\hline${ }^{25} \mathrm{Na}$ & -9357.8 & 1.2 & & 59.1 & $\mathrm{~s}$ & 0.6 & $5 / 2^{+}$ & 00 & & $\beta^{-}=100$ \\
\hline${ }^{25} \mathrm{Mg}$ & -13192.83 & 0.03 & & STABLE & & & $5 / 2^{+}$ & 00 & & $\mathrm{IS}=10.001$ \\
\hline${ }^{25} \mathrm{Al}$ & -8916.2 & 0.5 & & 7.183 & $\mathrm{~s}$ & 0.012 & $5 / 2^{+}$ & 00 & & $\beta^{+}=100$ \\
\hline${ }^{25} \mathrm{Si}$ & 3824 & 10 & & 220 & $\mathrm{~ms}$ & 3 & $5 / 2^{+}$ & 00 & & $\beta^{+}=100 ; \beta^{+} \mathrm{p}=36.815$ \\
\hline${ }^{25} \mathrm{P}$ & 18870\# & $200 \#$ & & $<30$ & $\mathrm{~ns}$ & & $1 / 2^{+} \#$ & 00 & 93Po.A I & $\mathrm{p}$ ? \\
\hline
\end{tabular}

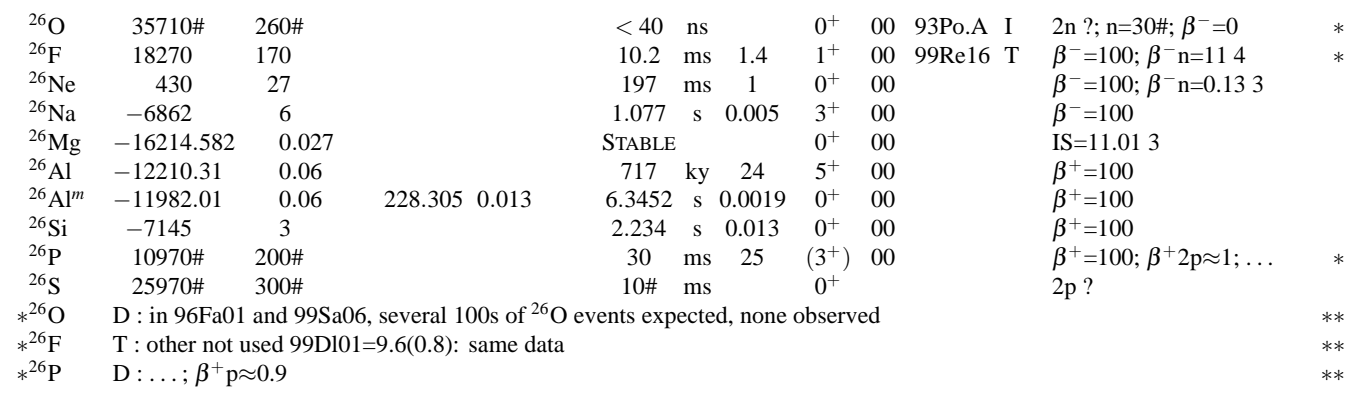

\begin{tabular}{|c|c|c|c|c|c|c|c|c|c|}
\hline${ }^{27} \mathrm{O}$ & 44950\# & $500 \#$ & $<260$ & ns & & $3 / 2^{+} \#$ & $99 \mathrm{Sa} 06 \mathrm{I}$ & $\mathrm{n} ? ; 2 \mathrm{n} ?$ & \\
\hline${ }^{27} \mathrm{~F}$ & 24930 & 380 & 4.9 & $\mathrm{~ms}$ & 0.2 & $5 / 2^{+} \# 01$ & 98No.A T & $\beta^{-}=100 ; \beta^{-} \mathrm{n}=7721$ & $*$ \\
\hline${ }^{27} \mathrm{Ne}$ & 7070 & 110 & 32 & $\mathrm{~ms}$ & 2 & $3 / 2^{+} \# 01$ & & $\beta^{-}=100 ; \beta^{-} \mathrm{n}=2.05$ & \\
\hline${ }^{27} \mathrm{Na}$ & -5517 & 4 & 301 & $\mathrm{~ms}$ & 6 & $5 / 2^{+} \quad 01$ & $84 \mathrm{Gu} 19 \mathrm{D}$ & $\beta^{-}=100 ; \beta^{-} \mathrm{n}=0.134$ & \\
\hline${ }^{27} \mathrm{Mg}$ & -14586.65 & 0.05 & 9.458 & $\mathrm{~m}$ & 0.012 & $1 / 2^{+} \quad 01$ & & $\beta^{-}=100$ & \\
\hline${ }^{27} \mathrm{Al}$ & -17196.66 & 0.12 & STABLE & & & $5 / 2^{+} \quad 01$ & & IS $=100$ & \\
\hline${ }^{27} \mathrm{Si}$ & -12384.30 & 0.15 & 4.16 & $\mathrm{~s}$ & 0.02 & $5 / 2^{+} \quad 01$ & & $\beta^{+}=100$ & \\
\hline${ }^{27} \mathrm{P}$ & -717 & 26 & 260 & $\mathrm{~ms}$ & 80 & $1 / 2^{+} \quad 01$ & & $\beta^{+}=100 ; \beta^{+} \mathrm{p}=0.07$ & \\
\hline${ }^{27} \mathrm{~S}$ & 17540\# & $200 \#$ & 21 & $\mathrm{~ms}$ & 4 & $\left(5 / 2^{+}\right) 01$ & & $\beta^{+}=100 ; \beta^{+} 2 \mathrm{p}=2.010 ; \ldots$ & $*$ \\
\hline$*^{27} \mathrm{~F}$ & \multicolumn{8}{|c|}{$6.5(1.1)$ and $97 \mathrm{Ta} 22=5.3(0.9)$ outweighed; and } & ** \\
\hline$*^{27} \mathrm{~F}$ & \multicolumn{8}{|c|}{$\mathrm{T}: \quad 99 \mathrm{Dl} 101=5.2(0.3)$ same data as in $99 \operatorname{Re} 16$} & $*$ \\
\hline$*^{27} \mathrm{~S}$ & \multicolumn{8}{|c|}{$\mathrm{D}: \ldots ; \beta^{+} \mathrm{p}=?$} & $* *$ \\
\hline
\end{tabular}

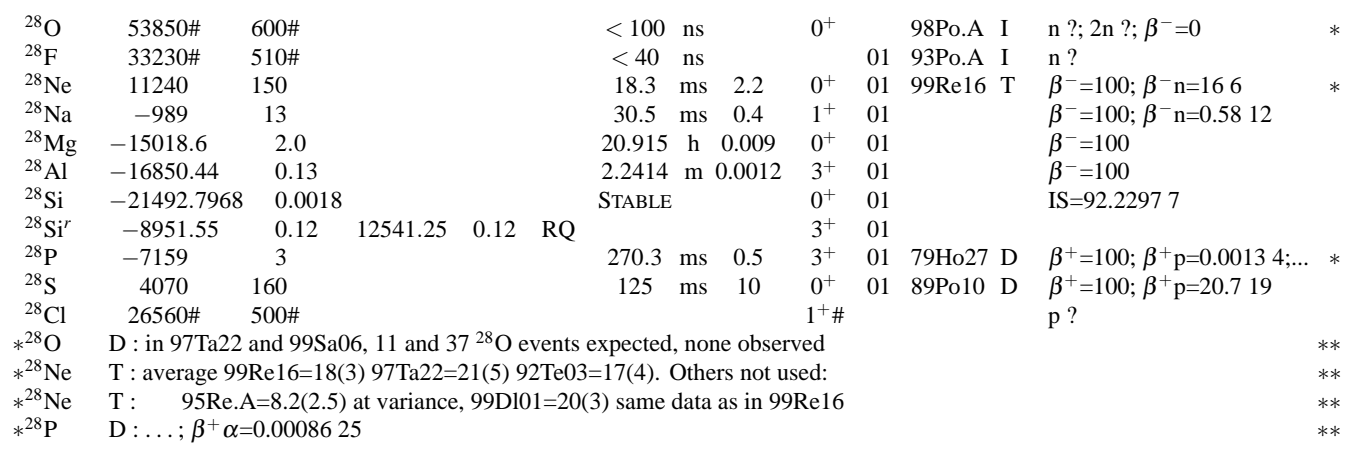




\begin{tabular}{|c|c|c|c|c|c|c|c|c|c|c|c|}
\hline Nuclide & \multicolumn{2}{|c|}{$\begin{array}{l}\text { Mass excess } \\
\quad(\mathrm{keV})\end{array}$} & $\begin{array}{c}\text { Excitation } \\
\text { energy }(\mathrm{keV})\end{array}$ & \multicolumn{3}{|c|}{ Half-life } & $J^{\pi}$ & Ens & Reference & \multicolumn{2}{|l|}{$\begin{array}{l}\text { Decay modes and } \\
\text { intensities }(\%)\end{array}$} \\
\hline${ }^{29} \mathrm{~F}$ & $40300 \#$ & $580 \#$ & & 2.6 & $\mathrm{~ms}$ & 0.3 & $5 / 2^{+} \#$ & 01 & $99 \operatorname{Re} 16 \mathrm{~T}$ & $\beta^{-}=100 ; \beta^{-} \mathrm{n}=6040$ & $*$ \\
\hline${ }^{29} \mathrm{Ne}$ & 18060 & 270 & & 15.6 & $\mathrm{~ms}$ & 0.5 & $3 / 2^{+} \#$ & 01 & 01Be53 D & $\beta^{-}=100 ; \beta^{-} \mathrm{n}=194$ & * \\
\hline${ }^{29} \mathrm{Na}$ & 2665 & 13 & & 44.9 & $\mathrm{~ms}$ & 1.2 & $3 / 2^{(+\#)}$ & 01 & 95Re.A D & $\beta^{-}=100 ; \beta^{-} \mathrm{n}=25.923$ & * \\
\hline${ }^{29} \mathrm{Mg}$ & -10619 & 14 & & 1.30 & $\mathrm{~s}$ & 0.12 & $3 / 2^{+}$ & 01 & & $\beta^{-}=100$ & \\
\hline${ }^{29} \mathrm{Al}$ & -18215.3 & 1.2 & & 6.56 & $\mathrm{~m}$ & 0.06 & $5 / 2^{+}$ & 01 & & $\beta^{-}=100$ & \\
\hline${ }^{29} \mathrm{Si}$ & -21895.046 & 0.021 & & STABLE & & & $1 / 2^{+}$ & 01 & & $\mathrm{IS}=4.68325$ & \\
\hline${ }^{29} \mathrm{P}$ & -16952.6 & 0.6 & & 4.142 & s & 0.015 & $1 / 2^{+}$ & 01 & & $\beta^{+}=100$ & \\
\hline${ }^{29} \mathrm{~S}$ & -3160 & 50 & & 187 & $\mathrm{~ms}$ & 4 & $5 / 2^{+}$ & 01 & 79Vi01 D & $\beta^{+}=100 ; \beta^{+} \mathrm{p}=46.410$ & \\
\hline${ }^{29} \mathrm{Cl}$ & 13140\# & $200 \#$ & & $<20$ & ns & & $3 / 2^{+} \#$ & 01 & 93Po.A I & $\mathrm{p}$ ? & \\
\hline$*^{29} \mathrm{~F}$ & \multicolumn{10}{|c|}{$\mathrm{D}: \ldots ; \beta^{-} 2 \mathrm{n} ?$} & $* *$ \\
\hline$*^{29} \mathrm{~F}$ & \multicolumn{10}{|c|}{$\mathrm{T}:$ average $99 \operatorname{Re} 16=2.9(0.8) 98 \mathrm{No} . \mathrm{A}=2.6(0.4) 97 \mathrm{Ta} 22=2.4(0.8)$. Others not } & $* *$ \\
\hline$*^{29} \mathrm{~F}$ & \multicolumn{10}{|c|}{$\mathrm{T}: \quad$ used: $99 \mathrm{D} 101=2.4(0.4)$ same data as in $99 \operatorname{Re} 16$} & $* *$ \\
\hline$*^{29} \mathrm{~F}$ & \multicolumn{10}{|c|}{$\mathrm{D}: \beta^{-} \mathrm{n}$ from $99 \mathrm{Dl} 01=100(80) \%$} & $* *$ \\
\hline$*^{29} \mathrm{Ne}$ & \multicolumn{10}{|c|}{$\mathrm{D}: \ldots ; \beta^{-} 2 \mathrm{n}<2.2$} & ** \\
\hline$*^{29} \mathrm{Ne}$ & \multicolumn{10}{|c|}{ D : average 01Be53=17 $599 \operatorname{Re} 16=27$ 9; other not used: $99 \mathrm{D} 101=27(9) \%$, same } & $* *$ \\
\hline$*^{29} \mathrm{Ne}$ & \multicolumn{10}{|c|}{ D : data as in $99 \operatorname{Re} 16 . \beta^{-} 2 \mathrm{n}$ limit is from $01 \mathrm{Be} 53$} & $* *$ \\
\hline$*^{29} \mathrm{Na}$ & \multicolumn{10}{|c|}{$\mathrm{D}: \beta^{-} \mathrm{n}:$ average $95 \mathrm{Re} . \mathrm{A}=27.1(1.6) \% 84 \mathrm{La} 03=21.5(3.0) \%$} & $* *$ \\
\hline
\end{tabular}

\begin{tabular}{|c|c|c|c|c|c|c|c|c|c|c|}
\hline${ }^{30} \mathrm{~F}$ & 48900\# & $600 \#$ & $<260$ & ns & & & & 99Sa06 & I & $\mathrm{n}$ ? \\
\hline${ }^{30} \mathrm{Ne}$ & 23100 & 570 & 5.8 & $\mathrm{~ms}$ & 0.2 & $0^{+}$ & 01 & 99D101 & $\mathrm{D}$ & $\beta^{-}=100 ; \beta^{-} \mathrm{n}=138$ \\
\hline${ }^{30} \mathrm{Na}$ & 8361 & 25 & 48.4 & $\mathrm{~ms}$ & 1.7 & $2^{+}$ & 01 & 99D101 & $\mathrm{T}$ & $\beta^{-}=100 ; \beta^{-} \mathrm{n}=304$ \\
\hline${ }^{30} \mathrm{Mg}$ & -8911 & 8 & 335 & $\mathrm{~ms}$ & 17 & $0^{+}$ & 01 & 84La03 & $\mathrm{D}$ & $\beta^{-}=100 ; \beta^{-} \mathrm{n}<0.06$ \\
\hline${ }^{30} \mathrm{Al}$ & -15872 & 14 & 3.60 & s & 0.06 & $3^{+}$ & 01 & & & $\beta^{-}=100$ \\
\hline${ }^{30} \mathrm{Si}$ & -24432.928 & 0.030 & Stable & & & $0^{+}$ & 01 & & & IS $=3.08725$ \\
\hline${ }^{30} \mathrm{P}$ & -20200.6 & 0.3 & 2.498 & $\mathrm{~m}$ & 0.004 & $1^{+}$ & 01 & & & $\beta^{+}=100$ \\
\hline${ }^{30} \mathrm{~S}$ & -14063 & 3 & 1.178 & $\mathrm{~s}$ & 0.005 & $0^{+}$ & 01 & & & $\beta^{+}=100$ \\
\hline${ }^{30} \mathrm{Cl}$ & 4440\# & $200 \#$ & $<30$ & ns & & $3^{+} \#$ & 01 & 93Po.A & I & $\mathrm{p}$ ? \\
\hline${ }^{30} \mathrm{Ar}$ & 20080\# & $300 \#$ & $<20$ & ns & & $0^{+}$ & & 93Po.A & I & $2 p ?$ \\
\hline$*^{30} \mathrm{Ne}$ & $\mathrm{D}:$ from $9(17$ & 7)\% & & & & & & & & \\
\hline$*^{30} \mathrm{Na}$ & $\mathrm{D}: \ldots ; \beta^{-} 2$ & $\mathrm{n}=1.1716 ; \beta^{-} \alpha=5.5 \mathrm{e}-520$ & & & & & & & & \\
\hline$*^{30} \mathrm{Na}$ & $\mathrm{T}$ : average 9 & $9 \mathrm{D} 101=50(4) 97 \mathrm{Ta} 22=48(5)$ & $84 \mathrm{La02}=$ & $=4 \delta(2$ & & & & & & \\
\hline$*^{30} \mathrm{P}$ & D : first obse & rved radionuclide, in 1934 & & & & & & & & \\
\hline
\end{tabular}

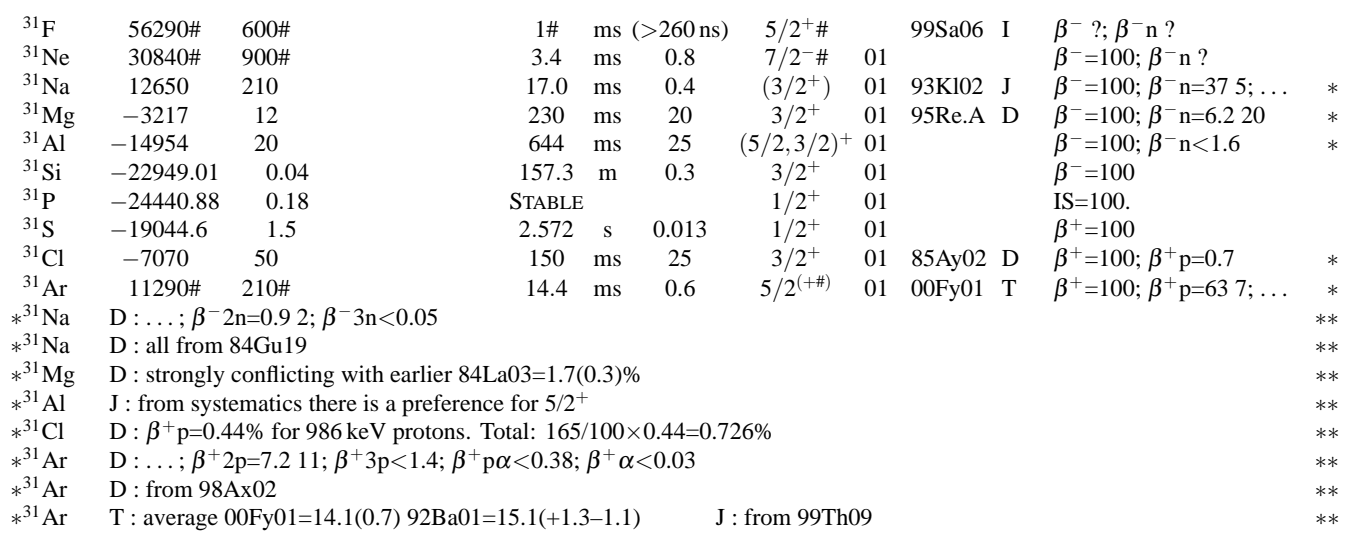

\begin{tabular}{|c|c|c|c|c|c|c|c|c|c|c|}
\hline${ }^{32} \mathrm{Ne}$ & 37280\# & 800\# & & 3.5 & $\mathrm{~ms}$ & 0.9 & $0^{+}$ & 01 & & $\beta^{-}=100 ; \beta^{-} \mathrm{n} ?$ \\
\hline${ }^{32} \mathrm{Na}$ & 19060 & 360 & & 12.9 & $\mathrm{~ms}$ & 0.7 & $\left(3^{-}, 4^{-}\right)$ & 01 & $93 \mathrm{~K} 102 \mathrm{~J}$ & $\beta^{-}=100 ; \beta^{-} \mathrm{n}=247 ;$ \\
\hline${ }^{32} \mathrm{Mg}$ & -955 & 18 & & 95 & $\mathrm{~ms}$ & 16 & $0^{+}$ & 01 & & $\beta^{-}=100 ; \beta^{-} \mathrm{n}=2.45$ \\
\hline${ }^{32} \mathrm{Al}$ & -11060 & 90 & & 31.7 & $\mathrm{~ms}$ & 0.8 & $1^{+}$ & 01 & 95Re.A TD & $\beta^{-}=100 ; \beta^{-} \mathrm{n}=0.75$ \\
\hline${ }^{32} \mathrm{Al}^{m}$ & -10100 & 90 & $955.7 \quad 0.4$ & 200 & $\mathrm{~ns}$ & 20 & $\left(4^{+}\right)$ & 01 & 96Ro02 ETJ & \\
\hline${ }^{32} \mathrm{Si}$ & -24080.91 & 0.05 & & 132 & $\mathrm{y}$ & 13 & $0^{+}$ & 01 & & $\beta^{-}=100$ \\
\hline${ }^{32} \mathrm{Si}^{m}$ & -18497.9 & 1.0 & $5583.0 \quad 1.0$ & 27 & ns & 2 & $\left(5^{-}\right)$ & & 97Fo01 ETJ & \\
\hline
\end{tabular}




\begin{tabular}{|c|c|c|c|c|c|c|}
\hline Nuclide & $\begin{array}{l}\text { Mass excess } \\
(\mathrm{keV})\end{array}$ & $\begin{array}{l}\text { Excitation } \\
\text { energy }(\mathrm{keV})\end{array}$ & Half-life & $J^{\pi}$ & Ens Reference & $\begin{array}{l}\text { Decay modes and } \\
\text { intensities }(\%)\end{array}$ \\
\hline
\end{tabular}

\begin{tabular}{|c|c|c|c|c|c|c|c|c|c|c|c|c|}
\hline \multicolumn{13}{|c|}{$A$-group continued ... } \\
\hline${ }^{32} \mathrm{P}$ & -24305.22 & 0.19 & & & 14.263 & d & 0.003 & $1^{+}$ & 01 & 02Un02 & $\mathrm{T}$ & $\beta^{-}=100$ \\
\hline${ }^{32} \mathrm{~S}$ & -26015.70 & 0.14 & & & StaBle & & & $0^{+}$ & 01 & & & IS $=94.9331$ \\
\hline${ }^{32} \mathrm{Cl}$ & -13330 & 7 & & & 298 & $\mathrm{~ms}$ & 1 & $1^{+}$ & 01 & 79 Но27 & $\mathrm{D}$ & $\beta^{+}=100 ; \beta^{+} \alpha=0.0548 ; \ldots$ \\
\hline${ }^{32} \mathrm{Ar}$ & -2200.2 & 1.8 & & & 98 & $\mathrm{~ms}$ & 2 & $0^{+}$ & 01 & & & $\beta^{+}=100 ; \beta^{+} \mathrm{p}=433$ \\
\hline${ }^{32} \mathrm{Ar}^{m}$ & $3400 \#$ & $100 \#$ & $5600 \#$ & $100 \#$ & & & & $5^{-} \#$ & & & & IT ? \\
\hline${ }^{32} \mathrm{~K}$ & 20420\# & $500 \#$ & & & & & & $1^{+} \#$ & & & & $\mathrm{p} ?$ \\
\hline${ }^{32} \mathrm{~K}^{m}$ & 21370\# & $510 \#$ & 950\# & $100 \#$ & & & & $4^{+} \#$ & & & & $\mathrm{p}$ ? \\
\hline$*^{32} \mathrm{Na}$ & $\mathrm{D}: \ldots ; \beta^{-}$ & $2 n=82$ & & & & & & & & & & \\
\hline$*^{32} \mathrm{Na}$ & $\mathrm{T}$ : average & 98No.A & $=11.5(0$. & ) $84 \mathrm{La}$ & $3.2(0.4)$ & & & & & & & \\
\hline$*^{32} \mathrm{Cl}$ & $\mathrm{D}: \ldots ; \beta^{+}$ & $\mathrm{p}=0.02$ & & & & & & & & & & \\
\hline
\end{tabular}

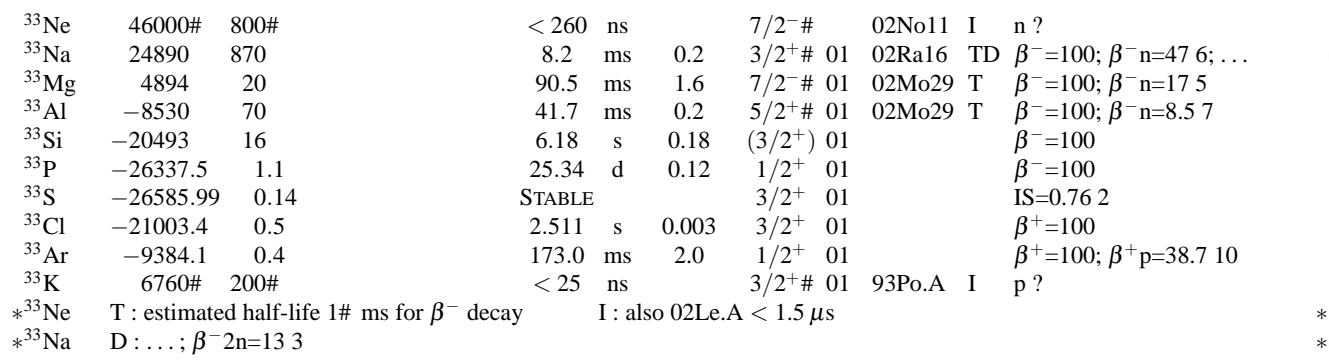

\begin{tabular}{|c|c|c|c|c|c|c|c|c|c|c|c|c|c|}
\hline${ }^{34} \mathrm{Ne}$ & $53120 \#$ & $810 \#$ & & & $1 \#$ & $\mathrm{~ms}$ & $>1.5 \mu \mathrm{s})$ & $0^{+}$ & & 02Le.A & I & $\beta^{-} ? ; \beta^{-} \mathrm{n} ?$ & * \\
\hline${ }^{34} \mathrm{Na}$ & 32760\# & $900 \#$ & & & 5.5 & $\mathrm{~ms}$ & 1.0 & $1^{+}$ & 01 & ABBW & $\mathrm{D}$ & $\beta^{-}=100 ; \beta^{-} 2 \mathrm{n} \approx 50 ; \beta^{-} \mathrm{n} \approx 15$ & * \\
\hline${ }^{34} \mathrm{Mg}$ & 8810 & 230 & & & 20 & $\mathrm{~ms}$ & 10 & $0^{+}$ & 01 & & & $\beta^{-}=100 ; \beta^{-} \mathrm{n} ?$ & \\
\hline${ }^{34} \mathrm{Al}$ & -2930 & 110 & & & 56.3 & $\mathrm{~ms}$ & 0.5 & $4^{-} \#$ & 01 & $01 \mathrm{Nu} 01$ & $\mathrm{~T}$ & $\beta^{-}=100 ; \beta^{-} \mathrm{n}=12.525$ & * \\
\hline${ }^{34} \mathrm{Si}$ & -19957 & 14 & & & 2.77 & $\mathrm{~s}$ & 0.20 & $0^{+}$ & 01 & & & $\beta^{-}=100$ & \\
\hline${ }^{34} \mathrm{P}$ & -24558 & 5 & & & 12.43 & s & 0.08 & $1^{+}$ & 01 & & & $\beta^{-}=100$ & \\
\hline${ }^{34} \mathrm{~S}$ & -29931.79 & 0.11 & & & STABLE & & & $0^{+}$ & 01 & & & $\mathrm{IS}=4.2928$ & \\
\hline${ }^{34} \mathrm{Cl}$ & -24439.78 & 0.18 & & & 1.5264 & $\mathrm{~s}$ & 0.0014 & $0^{+}$ & 01 & & & $\beta^{+}=100$ & \\
\hline${ }^{34} \mathrm{Cl}^{m}$ & -24293.42 & 0.18 & 146.36 & 0.03 & 32.00 & $\mathrm{~m}$ & 0.04 & $3^{+}$ & 01 & & & $\beta^{+}=55.46 ; \mathrm{IT}=44.66$ & \\
\hline${ }^{34} \mathrm{Ar}$ & -18377.2 & 0.4 & & & 845 & $\mathrm{~ms}$ & 3 & $0^{+}$ & 01 & & & $\beta^{+}=100$ & \\
\hline${ }^{34} \mathrm{~K}$ & $-1480 \#$ & $300 \#$ & & & $<40$ & ns & & $1^{+} \#$ & 01 & 93Po.A & I & $\mathrm{p}$ ? & \\
\hline${ }^{34} \mathrm{Ca}$ & 13150\# & $300 \#$ & & & $<35$ & ns & & $0^{+}$ & 01 & 93Po.A & I & $2 p ?$ & \\
\hline$*^{34} \mathrm{Ne}$ & \multirow{5}{*}{\multicolumn{12}{|c|}{$\begin{array}{l}\mathrm{D}: \beta^{-} \mathrm{n} \approx 15 \%, \beta^{-} 2 \mathrm{n} \approx 50 \% \text { estimated from } \mathrm{P}_{n}=\beta^{-} \mathrm{n}+2 \times \beta^{-} 2 \mathrm{n}=115(20) \% \text { in } 84 \mathrm{La} 03 \\
\mathrm{D}: \quad \text { assuming } \beta^{-} \mathrm{n} / \beta^{-} 2 \mathrm{n}=0.3 \text { from trends in the }{ }^{30} \mathrm{Na}^{-33} \mathrm{Na} \text { series: } 264134 \\
\mathrm{D}: \text { from } 95 \mathrm{Re} . \mathrm{A} \text {; strongly conflicting with } 89 \mathrm{Ba} 0=27(5) \% \text { and } 88 \mathrm{Mu} 08=54(12) \% \\
\text { T : also } 95 \operatorname{Re} . \mathrm{A}=42(6) \mathrm{ms}\end{array}$}} & ** \\
\hline$*^{34} \mathrm{Na}$ & & & & & & & & & & & & & ** \\
\hline$*^{34} \mathrm{Na}$ & & & & & & & & & & & & & ** \\
\hline$*^{34} \mathrm{Al}$ & & & & & & & & & & & & & $* *$ \\
\hline$*^{34} \mathrm{Al}$ & & & & & & & & & & & & & $* *$ \\
\hline
\end{tabular}

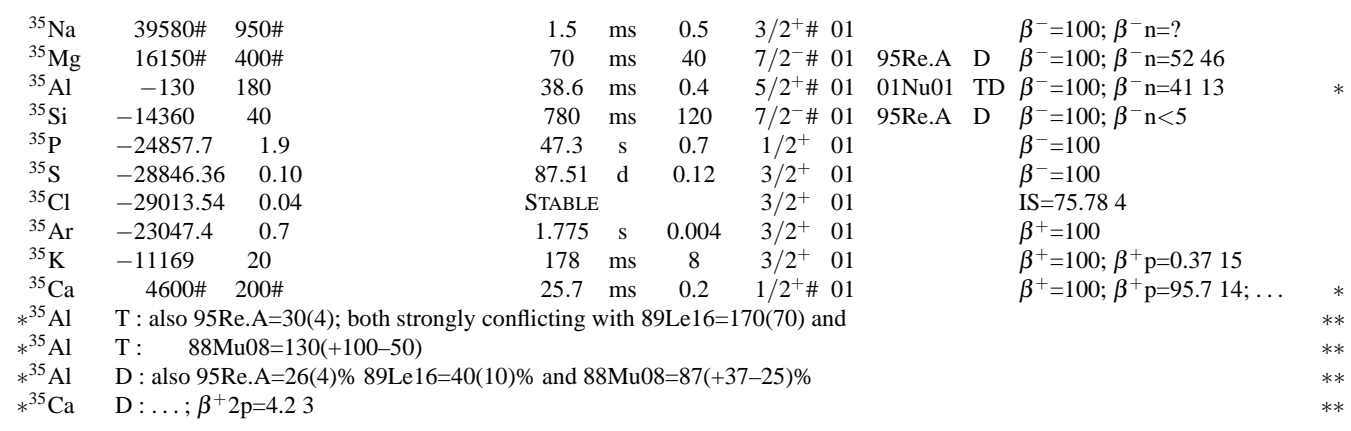




\begin{tabular}{|c|c|c|c|c|c|c|c|c|c|c|}
\hline Nuclide & \multicolumn{2}{|c|}{$\begin{array}{l}\text { Mass excess } \\
\quad(\mathrm{keV})\end{array}$} & $\begin{array}{c}\text { Excitation } \\
\text { energy }(\mathrm{keV})\end{array}$ & \multicolumn{3}{|c|}{ Half-life } & $J^{\pi}$ & \multicolumn{2}{|c|}{ Ens Reference } & $\begin{array}{l}\text { Decay modes and } \\
\text { intensities }(\%)\end{array}$ \\
\hline${ }^{36} \mathrm{Na}$ & 47950\# & $950 \#$ & & $<260$ & ns & & & & 02No11 I & $\mathrm{n}$ ? \\
\hline${ }^{36} \mathrm{Mg}$ & 21420\# & $500 \#$ & & $5 \#$ & $\mathrm{~ms}(>$ & $200 \mathrm{~ns})$ & $0^{+}$ & 01 & 89Gu03 I & $\beta^{-}$? \\
\hline${ }^{36} \mathrm{Al}$ & 5780 & 210 & & 90 & $\mathrm{~ms}$ & 40 & & 01 & & $\beta^{-}=100 ; \beta^{-} \mathrm{n}<30$ \\
\hline${ }^{36} \mathrm{Si}$ & -12480 & 120 & & 450 & $\mathrm{~ms}$ & 60 & $0^{+}$ & 01 & 95Re.A D & $\beta^{-}=100 ; \beta^{-} \mathrm{n}=125$ \\
\hline${ }^{36} \mathrm{P}$ & -20251 & 13 & & 5.6 & $\mathrm{~s}$ & 0.3 & $4^{-} \#$ & 01 & & $\beta^{-}=100$ \\
\hline${ }^{36} \mathrm{~S}$ & -30664.07 & 0.19 & & STABLE & & & $0^{+}$ & 01 & & $\mathrm{IS}=0.021$ \\
\hline${ }^{36} \mathrm{Cl}$ & -29521.86 & 0.07 & & 301 & ky & 2 & $2^{+}$ & 01 & & $\beta^{-}=98.11 ; \beta^{+}=1.91$ \\
\hline${ }^{36} \mathrm{Ar}$ & -30231.540 & 0.027 & & STABLE & & & $0^{+}$ & 01 & & $\mathrm{IS}=0.336530 ; 2 \beta^{+} ?$ \\
\hline${ }^{36} \mathrm{~K}$ & -17426 & 8 & & 342 & $\mathrm{~ms}$ & 2 & $2^{+}$ & 01 & & $\beta^{+}=100 ; \beta^{+} \mathrm{p}=0.04814 ; \ldots$ \\
\hline${ }^{36} \mathrm{Ca}$ & -6440 & 40 & & 102 & $\mathrm{~ms}$ & 2 & $0^{+}$ & 01 & $95 \operatorname{Tr} 02 \mathrm{D}$ & $\beta^{+}=100 ; \beta^{+} \mathrm{p}=56.813$ \\
\hline${ }^{36} \mathrm{Sc}$ & 13900\# & $500 \#$ & & & & & & & & p ? \\
\hline$*^{36} \mathrm{Na}$ & I : also 02Le. & $\mathrm{A}<1.5 \mu \mathrm{s}$ & & & & & & & & $* *$ \\
\hline$*^{36} \mathrm{~K}$ & $\mathrm{D}: \ldots ; \beta^{+} \alpha$ & $t=0.003413$ & & & & & & & & $* *$ \\
\hline
\end{tabular}

$\begin{array}{lcc}{ }^{37} \mathrm{Na} & 55280 \# & 960 \# \\ { }^{37} \mathrm{Mg} & 29250 \# & 900 \# \\ { }^{37} \mathrm{Al} & 9950 & 330 \\ { }^{37} \mathrm{Si} & -6580 & 170 \\ { }^{37} \mathrm{P} & -18990 & 40 \\ { }^{37} \mathrm{~S} & -26896.36 & 0.20 \\ { }^{37} \mathrm{Cl} & -31761.53 & 0.05 \\ { }^{37} \mathrm{Ar} & -30947.66 & 0.21 \\ { }^{37} \mathrm{~K} & -24800.20 & 0.09 \\ { }^{37} \mathrm{Ca} & -13162 & 22 \\ { }^{37} \mathrm{Sc} & 2840 \# & 300 \# \\ { }^{37} \mathrm{Na} & \mathrm{I}: \text { also } 02 \mathrm{No} 11>260\end{array}$

\begin{tabular}{|c|c|c|c|c|c|c|}
\hline $1 \#$ & $\mathrm{~ms}($ & $(>1.5 \mu \mathrm{s})$ & $3 / 2^{+} \#$ & & 02Le.A I & $\beta^{-} ? ; \beta^{-} \mathrm{n} ?$ \\
\hline $40 \#$ & $\mathrm{~ms}($ & $(>260 \mathrm{~ns})$ & $7 / 2^{-} \#$ & 01 & 96Sa34 I & $\beta^{-} ? ; \beta^{-} \mathrm{n} ?$ \\
\hline $20 \#$ & $\mathrm{~ms}$ & $(>1 \mu \mathrm{s})$ & $3 / 2^{+} \#$ & 01 & 910r01 I & $\beta^{-} ?$ \\
\hline 90 & $\mathrm{~ms}$ & 60 & $7 / 2^{-} \#$ & 01 & 95Re.A D & $\beta^{-}=100 ; \beta^{-} \mathrm{n}=1713$ \\
\hline 2.31 & $\mathrm{~s}$ & 0.13 & $1 / 2^{+} \#$ & 01 & & $\beta^{-}=100$ \\
\hline 5.05 & $\mathrm{~m}$ & 0.02 & $7 / 2^{-}$ & 01 & & $\beta^{-}=100$ \\
\hline STABLE & & & $3 / 2^{+}$ & 01 & & IS $=24.224$ \\
\hline 35.04 & $\mathrm{~d}$ & 0.04 & $3 / 2^{+}$ & 01 & & $\varepsilon=100$ \\
\hline 1.226 & $\mathrm{~s}$ & 0.007 & $3 / 2^{+}$ & 01 & & $\beta^{+}=100$ \\
\hline 181.1 & $\mathrm{~ms}$ & 1.0 & $\begin{array}{l}\left(3 / 2^{+}\right) \\
7 / 2^{-} \#\end{array}$ & 01 & $95 \operatorname{Tr} 03 \mathrm{D}$ & $\begin{array}{l}\beta^{+}=100 ; \beta^{+} \mathrm{p}=82.17 \\
\mathrm{p} ?\end{array}$ \\
\hline
\end{tabular}

$\begin{array}{llllllll}{ }^{38} \mathrm{Mg} & 35000 \# & 500 \# & & & 1 \# & \mathrm{~ms}(>260 \mathrm{~ns}) \\ { }^{38} \mathrm{Al} & 16050 & 730 & & & 40 \# & \mathrm{~ms}(>200 \mathrm{~ns}) \\ { }^{38} \mathrm{Si} & -4070 & 140 & & & 90 \# \mathrm{~ms}(>1 \mu \mathrm{s}) \\ { }^{38} \mathrm{P} & -14760 & 100 & & & 640 \mathrm{~ms} & 140 \\ { }^{38} \mathrm{~S} & -26861 & 7 & & & 170.3 \mathrm{~m} & 0.7 \\ { }^{38} \mathrm{Cl} & -29798.10 & 0.10 & & & 37.24 \mathrm{~m} & 0.05 \\ { }^{38} \mathrm{Cl} & -29126.74 & 0.10 & 671.361 & 0.008 & 715 \mathrm{~ms} & 3 \\ { }^{38} \mathrm{Ar} & -34714.6 & 0.3 & & & \text { STABLE } & \\ { }^{38} \mathrm{~K} & -28800.7 & 0.4 & & & 7.636 \mathrm{~m} & 0.018 \\ { }^{38} \mathrm{~K}^{m} & -28670.2 & 0.4 & 130.50 & 0.28 & \mathrm{RQ} & 923.9 \mathrm{~ms} & 0.6 \\ { }^{38} \mathrm{~K}^{n} & -25342.7 & 0.4 & 3458.0 & 0.2 & 21.98 \mu \mathrm{s} & 0.11 \\ { }^{38} \mathrm{Ca} & -22059 & 5 & & & 440 \mathrm{~ms} & 8 \\ { }^{38} \mathrm{Sc} & -4940 \# & 300 \# & & & <300 \mathrm{~ns} & \\ { }^{38} \mathrm{Sc} c^{m} & -4270 \# & 320 \# & 670 \# & 100 \# & & & \\ { }^{38} \mathrm{Ti} & 9100 \# & 250 \# & & & <120 \mathrm{~ns} & \end{array}$

$\begin{array}{cccl}0^{+} & 01 & 97 \mathrm{Sa} 14 \mathrm{I} & \beta^{-} ? \\ & 01 & 89 \mathrm{Gu} 03 \mathrm{I} & \beta^{-} ? \\ 0^{+} & 01 & 91 \mathrm{Zh} 24 \mathrm{I} & \beta^{-} ? ; \beta^{-} \mathrm{n} ? \\ & 01 & 95 \mathrm{Re} . \mathrm{A} \mathrm{D} & \beta^{-}=100 ; \beta^{-} \mathrm{n}=125 \\ 0^{+} & 01 & & \beta^{-}=100 \\ 2^{-} & 01 & & \beta^{-}=100 \\ 5^{-} & 01 & & \mathrm{IT}=100 \\ 0^{+} & 01 & & \mathrm{IS}=0.06325 \\ 3^{+} & 01 & & \beta^{+}=100 \\ 0^{+} & 01 & & \beta^{+}=100 \\ \left(7^{+}\right),\left(5^{+}\right) & 01 & \mathrm{IT}=100 \\ 0^{+} & 01 & & \beta^{+}=100 \\ 2^{-} \# & 01 & 94 \mathrm{~B} 110 \mathrm{I} & \mathrm{p} ? \\ 5^{-\#} & 01 & & \mathrm{IT} ? ; \mathrm{p} ? \\ 0^{+} & 01 & 96 \mathrm{~B} 121 \mathrm{I} & 2 \mathrm{p} ?\end{array}$

$*^{38} \mathrm{Mg} \quad \mathrm{I}: 18$ events reported

\begin{tabular}{|c|c|c|c|c|c|c|}
\hline$<260$ & & & $7 / 2^{-} \#$ & & 02No11 I & $\mathrm{n}$ ? \\
\hline $10 \#$ & $\mathrm{~ms}$ & $(>200 \mathrm{~ns})$ & $3 / 2^{+} \#$ & 01 & 89Gu03 I & $\beta^{-} ?$ \\
\hline $90 \#$ & $\mathrm{~ms}$ & $(>1 \mu \mathrm{s})$ & $7 / 2^{-} \#$ & 01 & 90Au.A I & $\beta^{-}$? \\
\hline 190 & $\mathrm{~ms}$ & 50 & $1 / 2^{+} \#$ & 01 & 95Re.A TD & $\beta^{-}=100 ; \beta^{-} \mathrm{n}=26$ \\
\hline 11.5 & $\mathrm{~s}$ & 0.5 & $(3,5,7) / 2^{-}$ & 01 & & $\beta^{-}=100$ \\
\hline 55.6 & $\mathrm{~m}$ & 0.2 & $3 / 2^{+}$ & 01 & & $\beta^{-}=100$ \\
\hline 269 & $\mathrm{y}$ & 3 & $7 / 2^{-}$ & 01 & & $\beta^{-}=100$ \\
\hline STABLE & & & $3 / 2^{+}$ & 01 & & IS $=93.258144$ \\
\hline 859.6 & $\mathrm{~ms}$ & 1.4 & $3 / 2^{+}$ & 01 & & $\beta^{+}=100$ \\
\hline$<300$ & ns & & $7 / 2^{-} \#$ & 01 & $94 \mathrm{~B} 110 \mathrm{I}$ & $\mathrm{p}=100$ \\
\hline 31 & $\mathrm{~ms}$ & 4 & $3 / 2^{+} \#$ & 01 & 90De43 TD & $\beta^{+}=100$ \\
\hline
\end{tabular}

$\begin{array}{lcc}{ }^{39} \mathrm{Mg} & 43570 \# & 510 \# \\ { }^{39} \mathrm{Al} & 21400 & 1470 \\ { }^{39} \mathrm{Si} & 1930 & 340 \\ { }^{39} \mathrm{P} & -12870 & 100 \\ { }^{39} \mathrm{~S} & -23160 & 50 \\ { }^{39} \mathrm{Cl} & -29800.2 & 1.7 \\ { }^{39} \mathrm{Ar} & -33242 & 5 \\ { }^{39} \mathrm{~K} & -33807.01 & 0.19 \\ { }^{39} \mathrm{Ca} & -27274.4 & 1.9 \\ { }^{39} \mathrm{Sc} & -14168 & 24 \\ { }^{39} \mathrm{Ti} & 1500 \# & 210 \#\end{array}$

${ }^{39} \mathrm{Mg} \quad \mathrm{T}$ : estimated half-life $1 \# \mathrm{~ms}$ for $\beta^{-}$decay

$*^{39} \mathrm{Sc} \quad \mathrm{D}:$ most probably proton emitter from $\mathrm{S}_{p}=-602(24) \mathrm{keV}$

$*^{39} \mathrm{Ti} \quad \mathrm{D}: \ldots ; \beta^{+} \mathrm{p}=8515 ; \beta^{+} 2 \mathrm{p}=15 \# \quad \mathrm{D}: \beta^{+} 2 \mathrm{p}$ decay observed by $92 \mathrm{Mo} 15$

$*^{39} \mathrm{Ti} \quad \mathrm{T}:$ average 90De43=26(+8-7) $01 \mathrm{Gi} 01=31(+6-4)$ 


\begin{tabular}{|c|c|c|c|c|c|c|c|c|c|c|c|}
\hline Nuclide & \multicolumn{2}{|c|}{$\begin{array}{l}\text { Mass excess } \\
\quad(\mathrm{keV})\end{array}$} & $\begin{array}{c}\text { Excitation } \\
\text { energy }(\mathrm{keV})\end{array}$ & \multicolumn{3}{|c|}{ Half-life } & $J^{\pi}$ & Ens & Reference & \multicolumn{2}{|l|}{$\begin{array}{l}\text { Decay modes and } \\
\text { intensities }(\%)\end{array}$} \\
\hline${ }^{40} \mathrm{Mg}$ & 50240\# & $900 \#$ & & $1 \#$ & $\mathrm{~ms}$ & & $0^{+}$ & & 02No11 I & $\beta^{-} ? ; \beta^{-} \mathrm{n} ?$ & $*$ \\
\hline${ }^{40} \mathrm{Al}$ & 29300\# & $700 \#$ & & $10 \#$ & $\mathrm{~ms}$ & $(>260 \mathrm{~ns})$ & & 02 & 97Sa14 I & $\beta^{-} ? ; \beta^{-} \mathrm{n}$ ? & * \\
\hline${ }^{40} \mathrm{Si}$ & 5470 & 560 & & $20 \#$ & $\mathrm{~ms}$ & $(>200 \mathrm{~ns})$ & $0^{+}$ & 02 & $89 \mathrm{Gu} 03 \mathrm{I}$ & $\beta^{-}$?; $\beta^{-} \mathrm{n}$ ? & \\
\hline${ }^{40} \mathrm{P}$ & -8110 & 140 & & 153 & $\mathrm{~ms}$ & 8 & $\left(2^{-}, 3^{-}\right)$ & 02 & & $\beta^{-}=100 ; \ldots$ & * \\
\hline${ }^{40} \mathrm{~S}$ & -22870 & 140 & & 8.8 & $\mathrm{~s}$ & 2.2 & $0^{+}$ & 02 & & $\beta^{-}=100$ & \\
\hline${ }^{40} \mathrm{Cl}$ & -27560 & 30 & & 1.35 & $\mathrm{~m}$ & 0.02 & $2^{-}$ & 02 & & $\beta^{-}=100$ & \\
\hline${ }^{40} \mathrm{Ar}$ & -35039.8960 & 0.0027 & & STABLE & & & $0^{+}$ & 02 & & $\mathrm{IS}=99.600330$ & \\
\hline${ }^{40} \mathrm{~K}$ & -33535.20 & 0.19 & & 1.251 & Gy & 0.011 & $4^{-}$ & 02 & & IS $=0.01171 ; \ldots$ & $*$ \\
\hline${ }^{40} \mathrm{~K}^{m}$ & -31891.56 & 0.19 & 1643.6390 .011 & 336 & ns & 12 & $0^{+}$ & 02 & & $\mathrm{IT}=100$ & \\
\hline${ }^{40} \mathrm{Ca}$ & -34846.27 & 0.21 & & STABLE & E $\quad($ & $(>5.9 \mathrm{Zy})$ & $0^{+}$ & 01 & 99Be64 T & $\mathrm{IS}=96.941156 ; 2 \beta^{+}$? & \\
\hline${ }^{40} \mathrm{Sc}$ & -20523.2 & 2.8 & & 182.3 & $\mathrm{~ms}$ & 0.7 & $4^{-}$ & 02 & & $\beta^{+}=100 ; \ldots$ & $*$ \\
\hline${ }^{40} \mathrm{Ti}$ & -8850 & 160 & & 53.3 & $\mathrm{~ms}$ & 1.5 & $0^{+}$ & 02 & & $\beta^{+}=100 ; \beta^{+} \mathrm{p}=100$ & \\
\hline${ }^{40} \mathrm{~V}$ & 10330\# & $500 \#$ & & & & & $2^{-} \#$ & & & p? & \\
\hline$*^{40} \mathrm{Mg}$ & I : one event $\mathrm{e}$ & xpected, nc & one observed; simil & earch in & 02Le & & & & & & $* *$ \\
\hline$*^{40} \mathrm{Al}$ & I : 34 events $r$ & eported in & 75a14; also one ev & in $96 \mathrm{Sa}$ & & & & & & & $* *$ \\
\hline$*^{40} \mathrm{P}$ & $\mathrm{D}: \ldots ; \beta^{-} \mathrm{n}=$ & 15.821 & & & & & & & & & $* *$ \\
\hline$*^{40} \mathrm{~K}$ & $\mathrm{D}: \ldots ; \beta^{-}=8$ & $9.2813 ; \beta$ & ${ }^{+}=10.7213$ & & & & & & & & $* *$ \\
\hline$*^{40} \mathrm{Sc}$ & $\mathrm{D}: \ldots ; \beta^{+} \mathrm{p}=$ & $=0.447 ; \beta^{+}$ & $\alpha=0.0175$ & & & & & & & & $* *$ \\
\hline
\end{tabular}

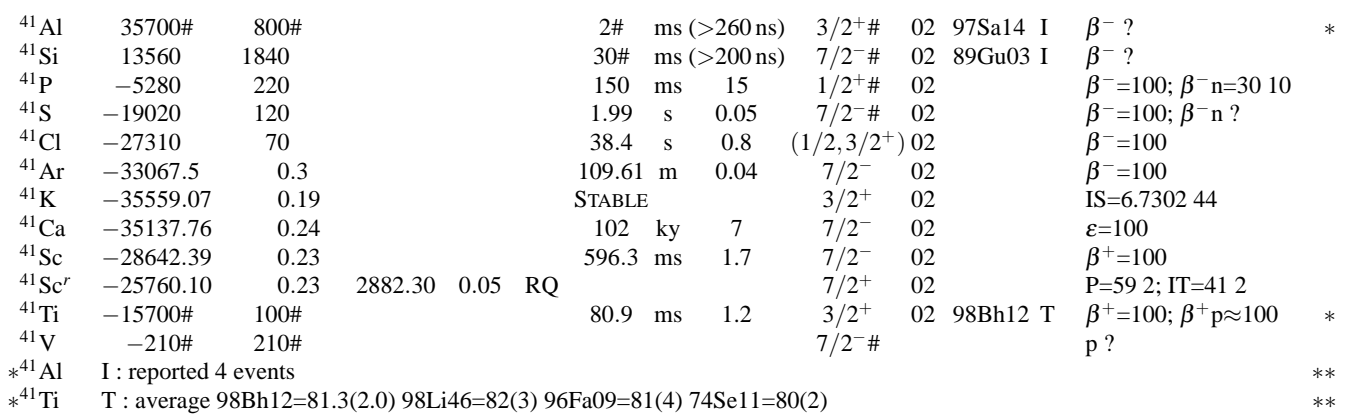

\begin{tabular}{|c|c|c|c|c|c|c|c|c|c|c|c|}
\hline${ }^{42} \mathrm{Al}$ & 43680\# & $900 \#$ & & & $1 \#$ & $\mathrm{~ms}$ & & & & & $\beta^{-} ? ; \beta^{-} \mathrm{n} ?$ \\
\hline${ }^{42} \mathrm{Si}$ & 18430\# & $500 \#$ & & & $5 \#$ & $\mathrm{~ms}$ & $>200 \mathrm{~ns})$ & $0^{+}$ & 01 & 90Le03 I & $\beta^{-} ? ; \beta^{-} \mathrm{n}$ ? \\
\hline${ }^{42} \mathrm{P}$ & 940 & 450 & & & 120 & $\mathrm{~ms}$ & 30 & & 01 & 89Le16 T & $\beta^{-}=100 ; \beta^{-} \mathrm{n}=5020$ \\
\hline${ }^{42} \mathrm{~S}$ & -17680 & 120 & & & 1.013 & $\mathrm{~s}$ & 0.015 & $0^{+}$ & 01 & & $\beta^{-}=100 ; \beta^{-} \mathrm{n}<4$ \\
\hline${ }^{42} \mathrm{Cl}$ & -24910 & 140 & & & 6.8 & $\mathrm{~s}$ & 0.3 & & 01 & & $\beta^{-}=100$ \\
\hline${ }^{42} \mathrm{Ar}$ & -34423 & 6 & & & 32.9 & $\mathrm{y}$ & 1.1 & $0^{+}$ & 01 & & $\beta^{-}=100$ \\
\hline${ }^{42} \mathrm{~K}$ & -35021.56 & 0.22 & & & 12.360 & $\mathrm{~h}$ & 0.012 & $2^{-}$ & 01 & & $\beta^{-}=100$ \\
\hline${ }^{42} \mathrm{Ca}$ & -38547.07 & 0.25 & & & STABLE & & & $0^{+}$ & 01 & & IS $=0.64723$ \\
\hline${ }^{42} \mathrm{Sc}$ & -32121.24 & 0.27 & & & 681.3 & $\mathrm{~ms}$ & 0.7 & $0^{+}$ & 01 & & $\beta^{+}=100$ \\
\hline${ }^{42} \mathrm{Sc}^{m}$ & -31504.96 & 0.28 & 616.28 & 0.06 & 61.7 & $\mathrm{~s}$ & 0.4 & $(7,5,6)^{+}$ & 01 & & $\beta^{+}=100$ \\
\hline${ }^{42} \mathrm{Sc}^{r}$ & -26044.91 & 0.26 & 6076.33 & $0.08 \mathrm{RQ}$ & & & & $\left(1^{+} t o 4^{+}\right)$ & 01 & & $\mathrm{IT}=100$ \\
\hline${ }^{42} \mathrm{Ti}$ & -25122 & 5 & & & 199 & $\mathrm{~ms}$ & 6 & $0^{+}$ & 01 & & $\beta^{+}=100$ \\
\hline${ }^{42} \mathrm{~V}$ & $-8170 \#$ & $200 \#$ & & & $<55$ & $\mathrm{~ns}$ & & $2^{-} \#$ & 01 & 92Bo37 I & $\mathrm{p}$ ? \\
\hline${ }^{42} \mathrm{Cr}$ & $5990 \#$ & $300 \#$ & & & 14 & $\mathrm{~ms}$ & 3 & $0^{+}$ & 01 & 01Gi01 TD & $\beta^{+} \approx 100 ; \beta^{+} \mathrm{p}=? ; 2 \mathrm{p} ?$ \\
\hline$*^{42} \mathrm{Si}$ & TD : ENSDF & ports pre & minary val & ues from 98 & Yo.A: ha & alf-lif & $=20 \mathrm{~ms} 10$ & 0 and & & & \\
\hline$*^{42} \mathrm{Si}$ & $\mathrm{TD}: \quad \% \beta$ & $n=10348$ & subject to & further analy & ysis acco & ording & to the aut & hors & & & \\
\hline${ }^{43} \mathrm{Si}$ & 26700\# & $700 \#$ & & & $15 \#$ & $\mathrm{~ms}$ & $>260 \mathrm{~ns})$ & $3 / 2^{-} \#$ & & 02No11 I & $\beta^{-} ? ; \beta^{-} \mathrm{n} ?$ \\
\hline${ }^{43} \mathrm{P}$ & 5770 & 970 & & & 33 & $\mathrm{~ms}$ & 3 & $1 / 2^{+} \#$ & 01 & & $\beta^{-}=100 ; \beta^{-} \mathrm{n}=100$ \\
\hline${ }^{43} \mathrm{~S}$ & -11970 & 200 & & & 260 & $\mathrm{~ms}$ & 15 & $3 / 2^{-} \#$ & 01 & 98Wi.A T & $\beta^{-}=100 ; \beta^{-} \mathrm{n}=4010$ \\
\hline${ }^{43} \mathrm{~S}^{m}$ & -11650 & 200 & 319 & 5 & 480 & ns & 50 & $\left(7 / 2^{-}\right)$ & 01 & 00Sa21 EJ & $\mathrm{IT}=100$ \\
\hline${ }^{43} \mathrm{Cl}$ & -24170 & 160 & & & 3.07 & $\mathrm{~s}$ & 0.07 & $3 / 2^{+} \#$ & 01 & & $\beta^{-}=100 ; \beta^{-} \mathrm{n} ?$ \\
\hline${ }^{43} \mathrm{Ar}$ & -32010 & 5 & & & 5.37 & $\mathrm{~m}$ & 0.06 & $\left(5 / 2^{-}\right)$ & 01 & & $\beta^{-}=100$ \\
\hline${ }^{43} \mathrm{~K}$ & -36593 & 9 & & & 22.3 & $\mathrm{~h}$ & 0.1 & $3 / 2^{+}$ & 01 & & $\beta^{-}=100$ \\
\hline${ }^{43} \mathrm{Ca}$ & -38408.6 & 0.3 & & & STABLE & & & $7 / 2^{-}$ & 01 & & $\mathrm{IS}=0.13510$ \\
\hline
\end{tabular}

... A-group is continued on next page ... 


\begin{tabular}{|c|c|c|c|c|c|c|c|c|c|c|c|c|}
\hline Nuclide & \multicolumn{2}{|c|}{$\begin{array}{l}\text { Mass excess } \\
\quad(\mathrm{keV})\end{array}$} & \multicolumn{2}{|c|}{$\begin{array}{l}\text { Excitation } \\
\text { energy }(\mathrm{keV})\end{array}$} & \multicolumn{3}{|c|}{ Half-life } & $J^{\pi}$ & Ens & Reference & \multicolumn{2}{|l|}{$\begin{array}{l}\text { Decay modes and } \\
\text { intensities }(\%)\end{array}$} \\
\hline \multicolumn{13}{|c|}{$\ldots A$-group continued $\ldots$} \\
\hline${ }^{43} \mathrm{Sc}$ & -36187.9 & 1.9 & & & 3.891 & $\mathrm{~h}$ & 0.012 & $7 / 2^{-}$ & 01 & & $\beta^{+}=100$ & \\
\hline${ }^{43} \mathrm{Sc}^{m}$ & -36036.5 & 1.9 & 151.4 & 0.2 & 438 & $\mu \mathrm{s}$ & 7 & $3 / 2^{+}$ & 01 & & $\mathrm{IT}=100$ & \\
\hline${ }^{43} \mathrm{Ti}$ & -29321 & 7 & & & 509 & $\mathrm{~ms}$ & 5 & $7 / 2^{-}$ & 01 & & $\beta^{+}=100$ & \\
\hline${ }^{43} \mathrm{Ti}^{m}$ & -29008 & 7 & 313.0 & 1.0 & 12.6 & $\mu \mathrm{s}$ & 0.6 & $\left(3 / 2^{+}\right)$ & 01 & & $\mathrm{IT}=100$ & \\
\hline${ }^{43} \mathrm{Ti}^{n}$ & -26255 & 7 & 3066.4 & 1.0 & 560 & ns & 6 & $\left(19 / 2^{-}\right)$ & 01 & & $\mathrm{IT}=100$ & \\
\hline${ }^{43} \mathrm{~V}$ & $-18020 \#$ & 230\# & & & $80 \#$ & $\mathrm{~ms}$ & & $7 / 2^{-} \#$ & 01 & & $\beta^{+} ?$ & $*$ \\
\hline${ }^{43} \mathrm{Cr}$ & $-2130 \#$ & $220 \#$ & & & 21.6 & $\mathrm{~ms}$ & 0.7 & $\left(3 / 2^{+}\right)$ & 01 & & $\beta^{+}=100 ; \beta^{+} \mathrm{p}=236 ; \ldots$ & * \\
\hline$*^{43} \mathrm{~S}^{m}$ & \multicolumn{11}{|c|}{$\mathrm{J}$ : from comparison of $B(\mathrm{E} 2)$ and half-life with theoretical ones } & $* *$ \\
\hline$*^{43} \mathrm{~V}$ & \multicolumn{11}{|c|}{$\mathrm{T}:>800 \mathrm{~ms}$ reported by $92 \mathrm{Bo} 37$ and adopted in ENSDF'01. To be confirmed. } & $* *$ \\
\hline$*{ }^{43} \mathrm{Cr}$ & \multicolumn{11}{|c|}{$\mathrm{D}: \ldots ; \beta^{+} 2 \mathrm{p}=65 ; \beta^{+} \alpha ?$} & ** \\
\hline
\end{tabular}

\begin{tabular}{|c|c|c|c|c|c|c|c|c|c|c|c|c|}
\hline${ }^{44} \mathrm{Si}$ & $32840 \#$ & $800 \#$ & & & & $10 \#$ & $\mathrm{~ms}$ & & $0^{+}$ & & & $\beta^{-} ? ; \beta^{-} \mathrm{n} ?$ \\
\hline${ }^{44} \mathrm{P}$ & $12100 \#$ & $700 \#$ & & & & $30 \#$ & $\mathrm{~ms}$ & $>200 \mathrm{~ns})$ & & 99 & 89Gu03 I & $\beta^{-}$? \\
\hline${ }^{44} \mathrm{~S}$ & -9120 & 390 & & & & 123 & $\mathrm{~ms}$ & 10 & $0^{+}$ & 99 & & $\beta^{-}=100 ; \beta^{-} \mathrm{n}=183$ \\
\hline${ }^{44} \mathrm{Cl}$ & -20230 & 110 & & & & 560 & $\mathrm{~ms}$ & 110 & & 99 & & $\beta^{-}=100 ; \beta^{-} \mathrm{n}<8$ \\
\hline${ }^{44} \mathrm{Ar}$ & -32673.1 & 1.6 & & & & 11.87 & $\mathrm{~m}$ & 0.05 & $0^{+}$ & 99 & & $\beta^{-}=100$ \\
\hline${ }^{44} \mathrm{~K}$ & -35810 & 40 & & & & 22.13 & $\mathrm{~m}$ & 0.19 & $2^{-}$ & 99 & & $\beta^{-}=100$ \\
\hline${ }^{44} \mathrm{Ca}$ & -41468.5 & 0.4 & & & & STABLE & & & $0^{+}$ & 99 & & $\mathrm{IS}=2.086110$ \\
\hline${ }^{44} \mathrm{Sc}$ & -37816.1 & 1.8 & & & & 3.97 & $\mathrm{~h}$ & 0.04 & $2^{+}$ & 99 & & $\beta^{+}=100$ \\
\hline${ }^{44} \mathrm{Sc}^{m}$ & -37545.2 & 1.8 & 270.95 & 0.20 & & 58.61 & $\mathrm{~h}$ & 0.10 & $6^{+}$ & 99 & & $\mathrm{IT}=98.807 ; \beta^{+}=1.207$ \\
\hline${ }^{44} \mathrm{Sc}^{n}$ & -37669.9 & 1.8 & 146.224 & 0.022 & & 50.4 & $\mu \mathrm{s}$ & 0.7 & $0^{-}$ & 99 & & \\
\hline${ }^{44} \mathrm{Ti}$ & -37548.5 & 0.7 & & & & 60.0 & $\mathrm{y}$ & 1.1 & $0^{+}$ & 99 & & $\varepsilon=100$ \\
\hline${ }^{44} \mathrm{~V}$ & -24120 & 120 & & & * & 111 & $\mathrm{~ms}$ & 7 & $\left(2^{+}\right)$ & 99 & & $\beta^{+}=100 ; \beta^{+} \alpha=?$ \\
\hline${ }^{44} \mathrm{~V}^{m}$ & $-23850 \#$ & $160 \#$ & $270 \#$ & $100 \#$ & $*$ & 150 & $\mathrm{~ms}$ & 3 & $\left(6^{+}\right)$ & 99 & & $\beta^{+}=100$ \\
\hline${ }^{44} \mathrm{~V}^{n}$ & $-23970 \#$ & $160 \#$ & $150 \#$ & $100 \#$ & & & & & $0^{-} \#$ & & & \\
\hline${ }^{44} \mathrm{Cr}$ & $-13460 \#$ & $50 \#$ & & & & 54 & $\mathrm{~ms}$ & 4 & $0^{+}$ & 99 & $96 \mathrm{Fa} 09 \mathrm{D}$ & $\beta^{+}=100 ; \beta^{+} \mathrm{p}=73$ \\
\hline${ }^{44} \mathrm{Mn}$ & $6400 \#$ & $500 \#$ & & & & $<105$ & ns & & $2^{-} \#$ & 99 & & p ? \\
\hline${ }^{14} \mathrm{Ti}$ & $\mathrm{T}:$ also 01 & $\mathrm{Ha} 21=5$ & & & & & & & & & & \\
\hline
\end{tabular}

\begin{tabular}{|c|c|c|c|c|c|c|c|c|c|c|c|c|c|c|}
\hline${ }^{45} \mathrm{P}$ & 17900\# & $800 \#$ & & & & $8 \#$ & $\mathrm{~ms}$ & $>200 \mathrm{~ns})$ & $1 / 2^{+} \#$ & 93 & 90Le03 & I & $\beta^{-} ?$ & \\
\hline${ }^{45} \mathrm{~S}$ & -3250 & 1740 & & & & 82 & $\mathrm{~ms}$ & 13 & $3 / 2^{-} \#$ & 97 & & & $\beta^{-}=100 ; \beta^{-} \mathrm{n}=54$ & \\
\hline${ }^{45} \mathrm{Cl}$ & -18360 & 120 & & & & 400 & $\mathrm{~ms}$ & 40 & $3 / 2^{+} \#$ & 95 & & & $\beta^{-}=100 ; \beta^{-} \mathrm{n}=244$ & \\
\hline${ }^{45} \mathrm{Ar}$ & -29770.6 & 0.5 & & & & 21.48 & $\mathrm{~s}$ & 0.15 & $(1,3,5) / 2^{-}$ & 95 & & & $\beta^{-}=100$ & $*$ \\
\hline${ }^{45} \mathrm{~K}$ & -36608 & 10 & & & & 17.3 & $\mathrm{~m}$ & 0.6 & $3 / 2^{+}$ & 95 & & & $\beta^{-}=100$ & \\
\hline${ }^{45} \mathrm{Ca}$ & -40812.0 & 0.4 & & & & 162.67 & $\mathrm{~d}$ & 0.25 & $7 / 2^{-}$ & 95 & 94Lo04 & $\mathrm{T}$ & $\beta^{-}=100$ & \\
\hline${ }^{45} \mathrm{Sc}$ & -41067.8 & 0.8 & & & & STABLE & & & $7 / 2^{-}$ & 95 & & & $\mathrm{IS}=100$ & \\
\hline${ }^{45} \mathrm{Sc}^{m}$ & -41055.4 & 0.8 & 12.40 & 0.05 & & 318 & $\mathrm{~ms}$ & 7 & $3 / 2^{+}$ & 95 & & & $\mathrm{IT}=100$ & \\
\hline${ }^{45} \mathrm{Ti}$ & -39005.7 & 1.0 & & & & 184.8 & $\mathrm{~m}$ & 0.5 & $7 / 2^{-}$ & 95 & & & $\beta^{+}=100$ & \\
\hline${ }^{45} \mathrm{~V}$ & -31880 & 17 & & & & 547 & $\mathrm{~ms}$ & 6 & $7 / 2^{-}$ & 95 & & & $\beta^{+}=100$ & \\
\hline${ }^{45} \mathrm{Cr}$ & -18970 & 500 & & & $*$ & 50 & $\mathrm{~ms}$ & 6 & $7 / 2^{-} \#$ & 95 & & & $\beta^{+}=100 ; \beta^{+} \mathrm{p}>27$ & \\
\hline${ }^{45} \mathrm{Cr}^{m}$ & $-18920 \#$ & $510 \#$ & $50 \#$ & $100 \#$ & $*$ & 1\# & $\mathrm{ms}$ & & $3 / 2^{+} \#$ & & & & IT $? ; \beta^{+} ?$ & \\
\hline${ }^{45} \mathrm{Mn}$ & $-5110 \#$ & $300 \#$ & & & & $<70$ & ns & & $7 / 2^{-} \#$ & 97 & $92 \mathrm{Bo} 37$ & I & $\mathrm{p} ?$ & \\
\hline${ }^{45} \mathrm{Fe}$ & $13580 \#$ & $220 \#$ & & & & 4.9 & $\mathrm{~ms}$ & 1.5 & $3 / 2^{+} \#$ & 97 & 02Gi09 & TD & $2 p=755 ; \beta^{+}=255 ; \ldots$ & * \\
\hline${ }^{5} \mathrm{Ar}$ & $: 7 / 2^{-} \# \mathrm{i}$ & expec & from $t$ & eory and & & systen & atics. & ee ENSDI & & & & & & $* *$ \\
\hline${ }^{5} \mathrm{Fe}$ & $: \ldots ; \beta$ & $=255$ & & & & & & & & & & & & $*$ \\
\hline${ }^{45} \mathrm{Fe}$ & $\mathrm{T}:$ average & e $02 \mathrm{Gi} 09$ & $4.7(+3$ & 1.4) 021 & $02=$ & $3.2(+2.6$ & $6-1.0$ & & $: \beta^{+} \mathrm{p}$ fro & 010 & ii01 & & & $*$ \\
\hline
\end{tabular}

\begin{tabular}{|c|c|c|c|c|c|c|c|}
\hline${ }^{46} \mathrm{P}$ & $25500 \#$ & $900 \#$ & & & $4 \#$ & $\mathrm{~ms}$ & $(>200 \mathrm{~ns})$ \\
\hline${ }^{46} \mathrm{~S}$ & 700\# & 700\# & & & $30 \#$ & $\mathrm{~ms}$ & $(>200 \mathrm{~ns})$ \\
\hline${ }^{46} \mathrm{Cl}$ & -14710 & 720 & & & 220 & $\mathrm{~ms}$ & 40 \\
\hline${ }^{46} \mathrm{Ar}$ & -29720 & 40 & & & 8.4 & $\mathrm{~s}$ & 0.6 \\
\hline${ }^{46} \mathrm{~K}$ & -35418 & 16 & & & 105 & $\mathrm{~s}$ & 10 \\
\hline${ }^{46} \mathrm{Ca}$ & -43135.1 & 2.3 & & & StABLE & & $(>100 \mathrm{Ey})$ \\
\hline${ }^{46} \mathrm{Sc}$ & -41757.1 & 0.8 & & & 83.79 & d & 0.04 \\
\hline${ }^{46} \mathrm{Sc}^{m}$ & -41614.6 & 0.8 & 142.528 & 0.007 & 18.75 & $\mathrm{~s}$ & 0.04 \\
\hline${ }^{46} \mathrm{Ti}$ & -44123.4 & 0.8 & & & Stable & & \\
\hline${ }^{46} \mathrm{~V}$ & -37073.0 & 1.0 & & & 422.50 & $\mathrm{~ms}$ & 0.11 \\
\hline${ }^{46} \mathrm{~V}^{m}$ & -36271.5 & 1.0 & 801.46 & 0.10 & 1.02 & $\mathrm{~ms}$ & 0.07 \\
\hline
\end{tabular}

... A-group is continued on next page ...

: $\beta^{+} \mathrm{p}$ from $01 \mathrm{Gi} 01$ 


\begin{tabular}{|c|c|c|c|c|c|c|c|c|c|c|c|c|c|}
\hline Nuclide & $\begin{array}{l}\text { Mass excess } \\
\quad(\mathrm{keV})\end{array}$ & \multicolumn{3}{|c|}{$\begin{array}{l}\text { Excitation } \\
\text { energy }(\mathrm{keV})\end{array}$} & \multicolumn{3}{|c|}{ Half-life } & $J^{\pi}$ & Ens & \multicolumn{2}{|c|}{ Reference } & \multicolumn{2}{|l|}{$\begin{array}{l}\text { Decay modes and } \\
\text { intensities }(\%)\end{array}$} \\
\hline \multicolumn{14}{|c|}{. A-group continued } \\
\hline${ }^{46} \mathrm{Cr}$ & $-29474 \quad 20$ & & & & 260 & $\mathrm{~ms}$ & 60 & $0^{+}$ & 00 & & & $\beta^{+}=100$ & \\
\hline${ }^{46} \mathrm{Mn}$ & $-12370 \# \quad 110 \#$ & & & * & 37 & $\mathrm{~ms}$ & 3 & $\left(4^{+}\right)$ & 00 & 92Bo37 & TD & $\beta^{+}=100 ; \beta^{+} \mathrm{p}=222 ; \ldots$ & * \\
\hline${ }^{46} \mathrm{Mn}^{m}$ & $-12220 \# 150 \#$ & $150 \#$ & $100 \#$ & * & $1 \#$ & $\mathrm{~ms}$ & & $1^{-} \#$ & & & & $\beta^{+} ?$ & \\
\hline${ }^{46} \mathrm{Fe}$ & $760 \# 350 \#$ & & & & 9 & $\mathrm{~ms}$ & 4 & $0^{+}$ & 00 & $01 \mathrm{Gi} 01$ & $\mathrm{TD}$ & $\beta^{+}=100 ; \beta^{+} \mathrm{p}=3620$ & \\
\hline$*^{46} \mathrm{Ca}$ & \multicolumn{12}{|c|}{$\mathrm{T}$ : limit is for neutrinoless $\beta \beta$ decay } & $* *$ \\
\hline$*{ }^{46} \mathrm{Mn}$ & \multicolumn{12}{|c|}{$\mathrm{D}: \ldots ; \beta^{+} 2 \mathrm{p} \approx 18 ; \beta^{+} \alpha ?$} & $* *$ \\
\hline$*^{46} \mathrm{Mn}$ & \multicolumn{12}{|c|}{$\mathrm{T}:$ average $92 \mathrm{Bo} 37=41(+7-6) 01 \mathrm{Gi} 01=34.0(+4.5-3.5)$} & $* *$ \\
\hline$*^{46} \mathrm{Mn}$ & \multicolumn{12}{|c|}{$\mathrm{D}: \beta^{+} 2 \mathrm{p} \approx 18 \%$ estimated from $\mathrm{P}_{p}=\beta^{+} \mathrm{p}+2 \times \beta^{+} 2 \mathrm{p}=58(9) \%$ in $01 \mathrm{Gi} 01$} & $* *$ \\
\hline
\end{tabular}

$\begin{array}{lrrrr}{ }^{47} \mathrm{~S} & 8000 \# & 800 \# & & \\ { }^{47} \mathrm{Cl} & -10520 \# & 600 \# & & \\ { }^{47} \mathrm{Ar} & -25910 & 100 & & \\ { }^{47} \mathrm{~K} & -35696 & 8 & & \\ { }^{47} \mathrm{Ca} & -42340.1 & 2.3 & & \\ { }^{47} \mathrm{Sc} & -44332.1 & 2.0 & & \\ { }^{47} \mathrm{Sc}^{m} & -43565.3 & 2.0 & 766.83 & 0.09 \\ { }^{47} \mathrm{Ti} & -44932.4 & 0.8 & & \\ { }^{47} \mathrm{~V} & -42002.1 & 0.8 & & \\ { }^{47} \mathrm{Cr} & -34558 & 14 & & \\ { }^{47} \mathrm{Mn} & -2260 \# & 160 \# & & \\ { }^{47} \mathrm{Fe} & -6620 \# & 260 \# & & \\ { }^{47} \mathrm{Fe} & -5850 \# & 280 \# & 770 \# & 100 \# \\ { }^{47} \mathrm{Co} & 10700 \# & 500 \# & & \\ { }^{47} \mathrm{Ar} & \mathrm{D}: \text { from } 95 \mathrm{SoO} 03 & \end{array}$

\begin{tabular}{|c|c|c|c|c|c|c|c|}
\hline $20 \#$ & $\mathrm{~ms}$ & $(>200 \mathrm{~ns})$ & $3 / 2^{-} \#$ & 95 & $89 \mathrm{Gu} 03$ & I & $\beta^{-} ?$ \\
\hline 200\# & $\mathrm{ms}$ & $(>200 \mathrm{~ns})$ & $3 / 2^{+} \#$ & 95 & $89 \mathrm{Gu} 03$ & I & $\beta^{-}=100 ; \beta^{-} \mathrm{n}<3$ \\
\hline 580 & $\mathrm{~ms}$ & 120 & $3 / 2^{-} \#$ & 95 & 89Ba.B & $\mathrm{T}$ & $\beta^{-}=100 ; \beta^{-} \mathrm{n}<1$ \\
\hline 17.50 & $\mathrm{~s}$ & 0.24 & $1 / 2^{+}$ & 95 & & & $\beta^{-}=100$ \\
\hline 4.536 & $\mathrm{~d}$ & 0.003 & $7 / 2^{-}$ & 95 & & & $\beta^{-}=100$ \\
\hline 3.3492 & $\mathrm{~d}$ & 0.0006 & $7 / 2^{-}$ & 95 & & & $\beta^{-}=100$ \\
\hline 272 & ns & 8 & $(3 / 2)^{+}$ & 95 & & & $\mathrm{IT}=100$ \\
\hline STABLE & & & $5 / 2^{-}$ & 95 & & & $\mathrm{IS}=7.442$ \\
\hline 32.6 & $\mathrm{~m}$ & 0.3 & $3 / 2^{-}$ & 95 & & & $\beta^{+}=100$ \\
\hline 500 & $\mathrm{~ms}$ & 15 & $3 / 2^{-}$ & 95 & & & $\beta^{+}=100$ \\
\hline 100 & $\mathrm{~ms}$ & 50 & $5 / 2^{-} \#$ & 95 & $96 \mathrm{Fa} 09$ & TD & $\beta^{+}=100 ; \beta^{+} \mathrm{p}=3.49$ \\
\hline 21.8 & $\mathrm{~ms}$ & 0.7 & $\begin{array}{l}7 / 2^{-} \# \\
3 / 2^{+} \# \\
7 / 2^{-} \#\end{array}$ & 97 & 01Gi01 & $\mathrm{TD}$ & $\begin{array}{l}\beta^{+}=100 ; \beta^{+} \mathrm{p}=877 \\
\text { IT ? } \\
\mathrm{p} \text { ? }\end{array}$ \\
\hline
\end{tabular}

$\begin{array}{lcc}{ }^{48} \mathrm{~S} & 13200 \# & 900 \# \\ { }^{48} \mathrm{Cl} & -4700 \# & 700 \# \\ { }^{48} \mathrm{Ar} & -23720 \# & 300 \# \\ { }^{48} \mathrm{~K} & -32124 & 24 \\ { }^{48} \mathrm{Ca} & -44214 & 4 \\ { }^{48} \mathrm{Sc} & -44496 & 5\end{array}$

$\begin{array}{cccc}10 \# & \mathrm{~ms} & (>200 \mathrm{~ns}) & 0 \\ 100 \# & \mathrm{~ms} & (>200 \mathrm{~ns}) & \\ 500 \# & \mathrm{~ms} & & 0 \\ 6.8 & \mathrm{~s} & 0.2 & (2 \\ 53 & \mathrm{Ey} & 17 & 0 \\ 43.67 & \mathrm{~h} & 0.09 & 6 \\ \text { STABLE } & & & 0 \\ 15.9735 & \mathrm{~d} & 0.0025 & 4 \\ 21.56 & \mathrm{~h} & 0.03 & 0 \\ 158.1 & \mathrm{~ms} & 2.2 & 4 \\ 44 & \mathrm{~ms} & 7 & 0 \\ & & & 6^{+} \\ 10 \# & \mathrm{~ms} & (>500 \mathrm{~ns}) & 0\end{array}$

$\begin{array}{ll} & \\ 0^{+} & \\ \left(2^{-}\right) & 95 \\ 0^{+} & 95 \\ 6^{+} & 95 \\ 0^{+} & 95 \\ 4^{+} & 95 \\ 0^{+} & 95 \\ 4^{+} & 97 \\ 0^{+} & 95 \\ 6^{+} \# & \\ 0^{+} & 01\end{array}$

$90 \mathrm{Le} 03$ I $\quad \beta^{-}$?

$89 \mathrm{Gu} 03$ I $\beta^{-}$?

$\beta^{-}$?

$\beta^{-}=100 ; \beta^{-} \mathrm{n}=1.1415$

$95 \quad 00 \mathrm{Br} 63 \quad \mathrm{~T} \quad \mathrm{IS}=0.18721 ; \ldots$

$95 \quad \beta^{-}=100$

IS $=73.723$

$\beta^{+}=100$

$\beta^{+}=100$

$-44475.4 \quad 2.6$

$-29320 \quad 110$

$-18160 \# 70 \#$

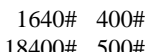

$\mathrm{D}: \ldots ; 2 \beta^{-}=$?; $\beta^{-}$?

$10 \#$ ms $(>500 \mathrm{~ns})$

$*^{*}{ }^{48} \mathrm{Ca} \quad \mathrm{T}:$ average $00 \mathrm{Br} 63=42(33-13) 96 \mathrm{Ba} 80=43(+24-11$ statistics +14 systematics $)$

$*^{48} \mathrm{Ca} \quad \mathrm{T}:$ also $T>36$ Ey from $70 \mathrm{Ba} 61$. Single $\beta^{-}$decay: $T>6$ Ey $(95 \% \mathrm{CL})$, from $85 \mathrm{Al} 17$

$*^{48} \mathrm{Mn} \quad \mathrm{D}: \ldots ; \beta^{+} \alpha=6 \mathrm{e}-4$

$*^{48} \mathrm{Mn} \quad \mathrm{D}$ : one $\beta^{+} \alpha$ event was observed, versus $437 \beta^{+} \mathrm{p}$, in fig. 4 of $87 \mathrm{Se} 07$

$\begin{array}{lrc}{ }^{49} \mathrm{~S} & 22000 \# & 950 \# \\ { }^{49} \mathrm{Cl} & 300 \# & 800 \# \\ { }^{49} \mathrm{Ar} & -18150 \# & 500 \# \\ { }^{49} \mathrm{~K} & -30320 & 70 \\ { }^{49} \mathrm{Ca} & -41289 & 4 \\ { }^{49} \mathrm{Sc} & -46552 & 4 \\ { }^{49} \mathrm{Ti} & -48558.8 & 0.8 \\ { }^{49} \mathrm{~V} & -47956.9 & 1.2 \\ { }^{49} \mathrm{Cr} & -45330.5 & 2.4 \\ { }^{49} \mathrm{Mn} & -37616 & 24 \\ { }^{49} \mathrm{Fe} & -24580 \# & 150 \# \\ { }^{49} \mathrm{Co} & -9580 \# & 260 \# \\ { }^{49} \mathrm{Ni} & 9000 \# & 400 \#\end{array}$

$\begin{array}{ccccclll}<200 & \mathrm{~ns} & & 3 / 2^{-\#} & 97 & 90 \mathrm{Le} 03 & \mathrm{I} & \mathrm{n} ? \\ 50 \# & \mathrm{~ms} & (>200 \mathrm{~ns}) & 3 / 2^{+} \# & 95 & 89 \mathrm{Gu} 03 & \mathrm{I} & \beta^{-} ? \\ 170 & \mathrm{~ms} & 50 & 3 / 2^{-} \# & 95 & 03 \mathrm{We} 09 & \mathrm{TD} & \beta^{-}=100 ; \beta^{-} \mathrm{n}=6520 \\ 1.26 & \mathrm{~s} & 0.05 & \left(3 / 2^{+}\right) & 95 & & & \beta^{-}=100 ; \beta^{-} \mathrm{n}=869 \\ 8.718 & \mathrm{~m} & 0.006 & 3 / 2^{-} & 95 & & & \beta^{-}=100 \\ 57.2 & \mathrm{~m} & 0.2 & 7 / 2^{-} & 95 & & & \beta^{-}=100 \\ \mathrm{STABLE} & & & 7 / 2^{-} & 95 & & & \mathrm{IS}=5.412 \\ 330 & \mathrm{~d} & 15 & 7 / 2^{-} & 95 & & & \varepsilon=100 \\ 42.3 & \mathrm{~m} & 0.1 & 5 / 2^{-} & 95 & & & \beta^{+}=100 \\ 382 & \mathrm{~ms} & 7 & 5 / 2^{-} & 01 & & & \beta^{+}=100 \\ 70 & \mathrm{~ms} & 3 & \left(7 / 2^{-}\right) & 01 & 96 \mathrm{Fa} 09 & \mathrm{~J} & \beta^{+}=100 ; \beta^{+} \mathrm{p}=5210 \\ <35 & \mathrm{~ns} & & 7 / 2^{-} \# 97 & 94 \mathrm{~B} 110 & \mathrm{I} & \mathrm{p} ? \\ 13 & \mathrm{~ms} & 4 & 7 / 2^{-} \# & 97 & 01 \mathrm{Gi01} & \mathrm{TD} & \beta^{+}=100 ; \beta^{+} \mathrm{p}=?\end{array}$

$*^{49} \mathrm{~S} \quad \mathrm{I}$ : statistics precludes any conclusion, say authors 


\begin{tabular}{|c|c|c|c|c|c|c|c|c|c|c|c|}
\hline Nuclide & \multicolumn{2}{|c|}{$\begin{array}{l}\text { Mass excess } \\
\quad(\mathrm{keV})\end{array}$} & \multicolumn{2}{|c|}{$\begin{array}{c}\text { Excitation } \\
\text { energy }(\mathrm{keV})\end{array}$} & \multicolumn{3}{|c|}{ Half-life } & $J^{\pi}$ & Ens & Reference & $\begin{array}{l}\text { Decay modes and } \\
\text { intensities }(\%)\end{array}$ \\
\hline${ }^{50} \mathrm{Cl}$ & 7300\# & $900 \#$ & & & $20 \#$ & $\mathrm{~ms}$ & & & & & $\beta^{-} ?$ \\
\hline${ }^{50} \mathrm{Ar}$ & $-14500 \#$ & $700 \#$ & & & 85 & $\mathrm{~ms}$ & 30 & $0^{+}$ & 95 & 03We09 TD & $\beta^{-}=100 ; \beta^{-} \mathrm{n}=3510$ \\
\hline${ }^{50} \mathrm{~K}$ & -25350 & 280 & & & 472 & $\mathrm{~ms}$ & 4 & $\left(0^{-}, 1,2^{-}\right)$ & 95 & & $\beta^{-}=100 ; \beta^{-} \mathrm{n}=293$ \\
\hline${ }^{50} \mathrm{Ca}$ & -39571 & 9 & & & 13.9 & $\mathrm{~s}$ & 0.6 & $0^{+}$ & 95 & & $\beta^{-}=100$ \\
\hline${ }^{50} \mathrm{Sc}$ & -44537 & 16 & & & 102.5 & $\mathrm{~s}$ & 0.5 & $5^{+}$ & 95 & & $\beta^{-}=100$ \\
\hline${ }^{50} \mathrm{Sc}^{m}$ & -44280 & 16 & 256.895 & 0.010 & 350 & $\mathrm{~ms}$ & 40 & $2^{+}, 3^{+}$ & 95 & & $\mathrm{IT}>97.5 ; \beta^{-}<2.5$ \\
\hline${ }^{50} \mathrm{Ti}$ & -51426.7 & 0.8 & & & STABLE & & & $0^{+}$ & 95 & & $\mathrm{IS}=5.182$ \\
\hline${ }^{50} \mathrm{~V}$ & -49221.6 & 1.0 & & & 150 & Py & 40 & $6^{+}$ & 95 & & $\mathrm{IS}=0.2504 ; \beta^{+}=8311 ; \ldots *$ \\
\hline${ }^{50} \mathrm{Cr}$ & -50259.5 & 1.0 & & & STABLE & & (>1.3 Ey) & $0^{+}$ & 95 & 03Bi05 $\mathrm{T}$ & $\mathrm{IS}=4.34513 ; 2 \beta^{+} ?$ \\
\hline${ }^{50} \mathrm{Mn}$ & -42626.8 & 1.0 & & & 283.9 & $\mathrm{~ms}$ & 0.5 & $0^{+}$ & 95 & & $\beta^{+}=100$ \\
\hline${ }^{50} \mathrm{Mn}^{m}$ & -42398 & 7 & 229 & 7 & 1.75 & $\mathrm{~m}$ & 0.03 & $5^{+}$ & 95 & & $\beta^{+}=100$ \\
\hline${ }^{50} \mathrm{Fe}$ & -34480 & 60 & & & 155 & $\mathrm{~ms}$ & 11 & $0^{+}$ & 01 & & $\beta^{+}=100 ; \beta^{+} \mathrm{p} \approx 0$ \\
\hline${ }^{50} \mathrm{Co}$ & $-17200 \#$ & $170 \#$ & & & 44 & $\mathrm{~ms}$ & 4 & $\left(6^{+}\right)$ & 01 & $96 \mathrm{Fa} 09 \quad J D$ & $\beta^{+}=100 ; \beta^{+} \mathrm{p}=5412$ \\
\hline${ }^{50} \mathrm{Ni}$ & $-3790 \#$ & $260 \#$ & & & 9.1 & $\mathrm{~ms}$ & 1.8 & $0^{+}$ & 97 & 01Ma.A T & $\beta^{+}$? \\
\hline$*^{50} \mathrm{~V}$ & $\mathrm{D}: \ldots ; \beta^{-}$ & $=1711$ & & & & & & & & & ** \\
\hline
\end{tabular}

$\begin{array}{cccccccl}2 \# & \mathrm{~ms}(>200 \mathrm{~ns}) & 3 / 2^{+} \# & 97 & 90 \mathrm{Le} 03 & \mathrm{I} & \beta^{-} ? \\ 60 \# & \mathrm{~ms}(>200 \mathrm{~ns}) & 3 / 2^{-} \# & 97 & 89 \mathrm{Gu} 03 & \mathrm{I} & \beta^{-} ? \\ 365 & \mathrm{~ms} & 5 & 3 / 2^{+} \# & 97 & & & \beta^{-}=100 ; \beta^{-} \mathrm{n}=475 \\ 10.0 & \mathrm{~s} & 0.8 & 3 / 2^{-} \# & 97 & & & \beta^{-}=100 ; \beta^{-} \mathrm{n} ? \\ 12.4 & \mathrm{~s} & 0.1 & (7 / 2)^{-} & 97 & & & \beta^{-}=100 \\ 5.76 & \mathrm{~m} & 0.01 & 3 / 2^{-} & 97 & & & \beta^{-}=100 \\ \text { STABLE } & & & 7 / 2^{-} & 97 & & & \mathrm{IS}=99.7504 \\ 27.7025 & \mathrm{~d} & 0.0024 & 7 / 2^{-} & 97 & & & \varepsilon=100 \\ 46.2 & \mathrm{~m} & 0.1 & 5 / 2^{-} & 97 & & & \beta^{+}=100 \\ 305 & \mathrm{~ms} & 5 & 5 / 2^{-} & 97 & & & \beta^{+}=100 \\ 60 \# & \mathrm{~ms}(>200 \mathrm{~ns}) & 7 / 2^{-} \# & 97 & 87 P 004 & \mathrm{I} & \beta^{+} ? \\ 30 \# & \mathrm{~ms}(>200 \mathrm{~ns}) & 7 / 2^{-} \# & 97 & 87 P o 04 & \mathrm{I} & \beta^{+} ?\end{array}$

$\begin{array}{ccc}10 \# & \mathrm{~ms} & \\ 105 & \mathrm{~ms} & 5 \\ 4.6 & \mathrm{~s} & 0.3 \\ 8.2 & \mathrm{~s} & 0.2 \\ 1.7 & \mathrm{~m} & 0.1 \\ 3.743 & \mathrm{~m} & 0.005 \\ \text { STABLE } & & \\ 5.591 & \mathrm{~d} & 0.003 \\ 21.1 & \mathrm{~m} & 0.2 \\ 8.275 & \mathrm{~h} & 0.008 \\ 45.9 & \mathrm{~s} & 0.6 \\ 115 & \mathrm{~ms} & 23 \\ 104 & \mathrm{~ms} & 11 \\ 38 & \mathrm{~ms} & 5\end{array}$

00

$\begin{array}{lll}00 & & \beta^{-} ? \\ 00 & \text { ABBW } \quad \mathrm{D} \quad & \beta^{-}=100 ; \beta^{-} \mathrm{n} \approx 64 ; \ldots \\ 00 & & \beta^{-}=100 ; \beta^{-} \mathrm{n}<2 \\ 00 & & \beta^{-}=100 \\ 00 & & \beta^{-}=100 \\ 00 & \beta^{-}=100 \\ 00 & \mathrm{IS}=83.78918 \\ 00 & \beta^{+}=100 \\ 00 & \beta^{+}=98.255 ; \mathrm{IT}=1.755 \\ 00 & \beta^{+}=100 \\ 00 & \beta^{+} \approx 100 ; \mathrm{IT}<0.004 \\ 00 & \beta^{+}=100 \\ & 97 \mathrm{Ha} 04 \mathrm{TD} \beta^{+}=? ; \mathrm{IT} ? \\ 00 & \beta^{+}=100 ; \beta^{+} \mathrm{p}=17.014 \\ 00 & \mathrm{p} ?\end{array}$

${ }^{52} \mathrm{Ar}-3000 \# \quad 900 \#$

${ }^{52} \mathrm{Ca} \quad-32510 \quad 700$

${ }^{52} \mathrm{Sc} \quad-40360 \quad 190$

${ }^{52} \mathrm{Ti}-49465 \quad 7$

$\begin{array}{lll}{ }^{52} \mathrm{~V} & -51441.3 & 1.0 \\ { }^{52} \mathrm{Cr} & -55416.9 & 0.8\end{array}$

${ }^{52} \mathrm{Mn} \quad-50705.4 \quad 2.0$

$\begin{array}{lllll}{ }^{52} \mathrm{Mn}^{m} & -50327.7 & 2.0 & 377.749 & 0.005\end{array}$

${ }^{52} \mathrm{Fe} \quad-48332 \quad 7$

${ }^{52} \mathrm{Fe}^{m}-41520 \quad 130 \quad 6810 \quad 130 \quad$

${ }^{52} \mathrm{Co}-33920 \# \quad 70 \#$

${ }^{52} \mathrm{Co}^{m}-33540 \# \quad 120 \# \quad 380 \# \quad 100 \#$

${ }^{52} \mathrm{Ni}-22650 \# \quad 80 \#$

${ }^{52} \mathrm{Cu} \quad-2630 \# \quad 260 \#$

${ }^{52} \mathrm{~K} \quad \mathrm{D}: \beta^{-} \mathrm{n} \approx 64 \%, \beta^{-} 2 \mathrm{n} \approx 21 \%$ estimated from $\mathrm{P}_{n}=\beta^{-} \mathrm{n}+2 \times \beta^{-} 2 \mathrm{n}=107(20) \%$ in $83 \mathrm{La} 23$

$*^{52} \mathrm{~K} \quad \mathrm{D}$ : $\quad$ and assuming $\beta^{-} \mathrm{n} / \beta^{-} 2 \mathrm{n}=3$ as in ${ }^{32} \mathrm{Na}$

$*^{52} \mathrm{Co}^{m} \quad \mathrm{I}$ : tentative: no specific evidence for ${ }^{52} \mathrm{Co}^{m}$, say authors in $97 \mathrm{Ha} 04$ 


\begin{tabular}{|c|c|c|c|c|c|c|c|c|c|c|c|c|c|c|}
\hline \multirow{2}{*}{$\begin{array}{l}\text { Nuclide } \\
{ }^{53} \mathrm{Ar}\end{array}$} & \multicolumn{2}{|c|}{$\begin{array}{l}\text { Mass excess } \\
\quad(\mathrm{keV})\end{array}$} & \multicolumn{3}{|c|}{$\begin{array}{l}\text { Excitation } \\
\text { energy }(\mathrm{keV})\end{array}$} & \multicolumn{3}{|c|}{ Half-life } & \multirow{2}{*}{$\begin{array}{c}J^{\pi} \\
5 / 2^{-} \#\end{array}$} & \multirow{2}{*}{$\begin{array}{c}\text { Ens } \\
99\end{array}$} & \multicolumn{2}{|c|}{ Reference } & \multicolumn{2}{|l|}{$\begin{array}{l}\text { Decay modes and } \\
\text { intensities }(\%)\end{array}$} \\
\hline & $4600 \#$ & $1000 \#$ & & & & 3\# & $\mathrm{ms}$ & & & & & & $\beta^{-} ? ; \beta^{-} \mathrm{n} ?$ & \\
\hline${ }^{53} \mathrm{~K}$ & $-12000 \#$ & $700 \#$ & & & & 30 & $\mathrm{~ms}$ & 5 & $3 / 2^{+} \#$ & 99 & ABBW & $\mathrm{D}$ & $\beta^{-}=100 ; \beta^{-} \mathrm{n} \approx 67 ; \ldots$ & $*$ \\
\hline${ }^{53} \mathrm{Ca}$ & $-27900 \#$ & $500 \#$ & & & & 90 & $\mathrm{~ms}$ & 15 & $3 / 2^{-} \#$ & 99 & $83 \mathrm{La} 23$ & $\mathrm{D}$ & $\beta^{-}=100 ; \beta^{-} \mathrm{n}>30$ & * \\
\hline${ }^{53} \mathrm{Sc}$ & $-37620 \#$ & $300 \#$ & & & & $>3$ & $\mathrm{~s}$ & & $7 / 2^{-} \#$ & 99 & 98So03 & TD & $\beta^{-}=100 ; \beta^{-} \mathrm{n} ?$ & \\
\hline${ }^{53} \mathrm{Ti}$ & -46830 & 100 & & & & 32.7 & $\mathrm{~s}$ & 0.9 & $(3 / 2)^{-}$ & 99 & & & $\beta^{-}=100$ & \\
\hline${ }^{53} \mathrm{~V}$ & -51849 & 3 & & & & 1.60 & $\mathrm{~m}$ & 0.04 & $7 / 2^{-}$ & 99 & & & $\beta^{-}=100$ & \\
\hline${ }^{53} \mathrm{Cr}$ & -55284.7 & 0.8 & & & & STABLE & & & $3 / 2^{-}$ & 99 & & & $\mathrm{IS}=9.50117$ & \\
\hline${ }^{53} \mathrm{Mn}$ & -54687.9 & 0.8 & & & & 3.7 & My & 0.4 & $7 / 2^{-}$ & 99 & & & $\varepsilon=100$ & \\
\hline${ }^{53} \mathrm{Fe}$ & -50945.3 & 1.8 & & & & 8.51 & $\mathrm{~m}$ & 0.02 & $7 / 2^{-}$ & 99 & & & $\beta^{+}=100$ & \\
\hline${ }^{53} \mathrm{Fe}^{m}$ & -47904.9 & 1.8 & 3040.4 & 0.3 & & 2.526 & $\mathrm{~m}$ & 0.024 & $19 / 2^{-}$ & 99 & $97 \mathrm{Ge} 11$ & $\mathrm{~T}$ & $\mathrm{IT}=100$ & * \\
\hline${ }^{53} \mathrm{Co}$ & -42645 & 18 & & & & 242 & $\mathrm{~ms}$ & 8 & $7 / 2^{-} \#$ & 99 & 02Lo13 & $\mathrm{T}$ & $\beta^{+}=100$ & $*$ \\
\hline${ }^{53} \mathrm{Co}^{m}$ & -39447 & 22 & 3197 & 29 & $\mathrm{p}$ & 247 & $\mathrm{~ms}$ & 12 & $\left(19 / 2^{-}\right)$ & 99 & & & $\beta^{+} \approx 98.5 ; \mathrm{p} \approx 1.5$ & \\
\hline${ }^{53} \mathrm{Ni}$ & $-29370 \#$ & $160 \#$ & & & & 45 & $\mathrm{~ms}$ & 15 & $7 / 2^{-} \#$ & 99 & 76Vi02 & $\mathrm{D}$ & $\beta^{+}=100 ; \beta^{+} \mathrm{p} \approx 45$ & \\
\hline${ }^{53} \mathrm{Cu}$ & $-13460 \#$ & $260 \#$ & & & & $<300$ & ns & & $3 / 2^{-} \#$ & 99 & 93Bl.A & I & $\mathrm{p}$ ?; $\beta^{+}$? & \\
\hline$*^{53} \mathrm{~K}$ & $\mathrm{D}: \ldots ; \beta$ & $2 \mathrm{n} \approx 17$ & & & & & & & & & & & & ** \\
\hline$*^{53} \mathrm{~K}$ & $\mathrm{D}: \beta^{-} \mathrm{n} \approx$ & $67 \%, \beta^{-}$ & $2 \mathrm{n} \approx 17 \%$ & estin & ated & om $\mathrm{P}_{n}=$ & $=\beta^{-} \mathrm{n}$ & $2 \times \beta^{-}$ & $=100(30)^{c}$ & $\%$ in 8 & $33 \mathrm{La} 23$ & & & ** \\
\hline$*^{53} \mathrm{~K}$ & $\mathrm{D}: \quad$ an & assumi & $\lg \beta^{-} \mathrm{n} /$ & $3-2 n=$ & 4 as & ${ }^{33} \mathrm{Na}$ & & & & & & & & ** \\
\hline$*^{53} \mathrm{Ca}$ & $\mathrm{D}: \beta^{-} \mathrm{n}=4$ & $0(10) \%$ & $s$ a lowe & limi & (see & (NSDF) & & & & & & & & ** \\
\hline$*^{53} \mathrm{Ca}$ & $\mathrm{T}$ : expect & $T=2 \#$ & $\mathrm{~s}$ from & stem & tics & Ca isoto & opes & & & & & & & $* *$ \\
\hline$*^{53} \mathrm{Fe}^{m}$ & $\mathrm{~T}$ : averag & $97 \mathrm{Ge} 11$ & $=2.48(0$ & 05) 6 & $\mathrm{De} 2$ & $=2.51(0.0$ & 02) $67 \mathrm{E}$ & $06=2.5$ & (0.03) & & & & & $* *$ \\
\hline$*^{53} \mathrm{Co}$ & $\mathrm{T}$ : averag & $02 \mathrm{Lo} 13$ & $=240(9$ & $89 \mathrm{Ho}$ & $13=2$ & $0(20) 73 \mathrm{~K}$ & Ko10= & $62(25)$ & & & & & & $* *$ \\
\hline
\end{tabular}

\begin{tabular}{|c|c|c|c|c|c|c|c|c|c|c|c|c|c|}
\hline${ }^{54} \mathrm{~K}$ & $-5400 \#$ & $900 \#$ & & & 10 & $\mathrm{~ms}$ & 5 & $2^{-} \#$ & 01 & & & $\beta^{-}=100 ; \beta^{-} \mathrm{n}=?$ & \\
\hline${ }^{54} \mathrm{Ca}$ & $-23890 \#$ & 700\# & & & $50 \#$ & $\mathrm{~ms}$ & $(>300 \mathrm{~ns})$ & $0^{+}$ & 01 & 97Be70 & I & $\beta^{-} ? ; \beta^{-} \mathrm{n} ?$ & \\
\hline${ }^{54} \mathrm{Sc}$ & -34220 & 370 & & & 260 & $\mathrm{~ms}$ & 30 & $3^{+} \#$ & 01 & $02 \mathrm{Ja} 16$ & $\mathrm{~T}$ & $\beta^{-}=100 ; \beta^{-} \mathrm{n} ?$ & $\uparrow$ \\
\hline${ }^{54} \mathrm{Sc}^{m}$ & -34110 & 370 & 110 & 3 & 7 & $\mu \mathrm{s}$ & 5 & $\left(5^{+}\right)$ & 01 & $98 \mathrm{Gr} 14$ & EJ & $\mathrm{IT}=100$ & \\
\hline${ }^{54} \mathrm{Ti}$ & -45590 & 120 & & & 1.5 & $\mathrm{~s}$ & 0.4 & $0^{+}$ & 01 & & & $\beta^{-}=100$ & \\
\hline${ }^{54} \mathrm{~V}$ & -49891 & 15 & & & 49.8 & s & 0.5 & $3^{+}$ & 01 & & & $\beta^{-}=100$ & \\
\hline${ }^{54} \mathrm{~V}^{m}$ & -49783 & 15 & 108 & 3 & 900 & ns & 500 & $\left(5^{+}\right)$ & & $98 \mathrm{Gr} 14$ & EJ & $\mathrm{IT}=100$ & \\
\hline${ }^{54} \mathrm{Cr}$ & -56932.5 & 0.8 & & & STABLE & & & $0^{+}$ & 01 & & & $\mathrm{IS}=2.3657$ & \\
\hline${ }^{54} \mathrm{Mn}$ & -55555.4 & 1.3 & & & 312.03 & $\mathrm{~d}$ & 0.03 & $3^{+}$ & 01 & 02Un02 & $\mathrm{T}$ & $\varepsilon=100 ; \beta^{-}<2.9 \mathrm{e}-4 ; \ldots$ & $*$ \\
\hline${ }^{54} \mathrm{Fe}$ & -56252.5 & 0.7 & & & STABLE & & & $0^{+}$ & 01 & & & $\mathrm{IS}=5.84535 ; 2 \beta^{+} ?$ & \\
\hline${ }^{54} \mathrm{Fe}^{m}$ & -49725.6 & 0.9 & 6526.9 & 0.6 & 364 & $\mathrm{~ns}$ & 7 & $10^{+}$ & 01 & & & $\mathrm{IT}=100$ & \\
\hline${ }^{54} \mathrm{Co}$ & -48009.5 & 0.7 & & & 193.23 & $\mathrm{~ms}$ & 0.14 & $0^{+}$ & 01 & & & $\beta^{+}=100$ & \\
\hline${ }^{54} \mathrm{Co}^{m}$ & -47812.1 & 0.9 & 197.4 & 0.5 & 1.48 & $\mathrm{~m}$ & 0.02 & $(7)^{+}$ & 01 & & & $\beta^{+}=100$ & \\
\hline${ }^{54} \mathrm{Ni}$ & -39210 & 50 & & & 104 & $\mathrm{~ms}$ & 7 & $0^{+}$ & 01 & 02Lo13 & $\mathrm{T}$ & $\beta^{+}=100$ & * \\
\hline${ }^{54} \mathrm{Cu}$ & $-21690 \#$ & $210 \#$ & & & $<75$ & ns & & $3^{+} \#$ & 01 & $94 \mathrm{~B} 110$ & I & $\mathrm{p}$ ? & \\
\hline${ }^{54} \mathrm{Zn}$ & $-6570 \#$ & $400 \#$ & & & & & & $0^{+}$ & & & & $2 p ?$ & \\
\hline$*^{54} \mathrm{Sc}$ & \multicolumn{12}{|c|}{$\mathrm{T}:$ average $02 \mathrm{Ja} 16=360(60) 98 \mathrm{So} 03=225(40)$} & $* *$ \\
\hline$*^{54} \mathrm{Mn}$ & \multicolumn{12}{|l|}{$\mathrm{D}: \ldots ; \mathrm{e}^{+}=$} & $* *$ \\
\hline$*^{54} \mathrm{Mn}$ & \multicolumn{12}{|c|}{$\mathrm{D}: \mathrm{e}^{+}$average $98 \mathrm{Wu} 01=1.20(0.26) 97 \mathrm{Za} 07=2.2(0.9)$} & ** \\
\hline$*^{54} \mathrm{Ni}$ & \multicolumn{12}{|c|}{$\mathrm{T}:$ average $02 \mathrm{Lo} 13=103(9) 99 \operatorname{Re} 06=106(12)$} & $* *$ \\
\hline
\end{tabular}

\begin{tabular}{|c|c|c|c|c|c|c|c|c|c|c|c|}
\hline${ }^{55} \mathrm{~K}$ & $-270 \#$ & $1000 \#$ & $3 \#$ & $\mathrm{~ms}$ & & $3 / 2^{+} \#$ & & & & $\beta^{-} ? ; \beta^{-} \mathrm{n} ?$ & \\
\hline${ }^{55} \mathrm{Ca}$ & $-18120 \#$ & 700\# & $30 \#$ & $\mathrm{~ms}$ & $(>300 \mathrm{~ns})$ & $5 / 2^{-} \#$ & & $97 \mathrm{Be} 70$ & I & $\beta^{-} ?$ & \\
\hline${ }^{55} \mathrm{Sc}$ & -29580 & 740 & 120 & $\mathrm{~ms}$ & 40 & $7 / 2^{-} \#$ & 01 & & & $\beta^{-}=100 ; \beta^{-} \mathrm{n} ?$ & \\
\hline${ }^{55} \mathrm{Ti}$ & -41670 & 150 & 490 & $\mathrm{~ms}$ & 90 & $3 / 2^{-} \#$ & 01 & 98Am04 & $\mathrm{T}$ & $\beta^{-}=100$ & * \\
\hline${ }^{55} \mathrm{~V}$ & -49150 & 100 & 6.54 & $\mathrm{~s}$ & 0.15 & $7 / 2^{-} \#$ & 01 & & & $\beta^{-}=100$ & \\
\hline${ }^{55} \mathrm{Cr}$ & -55107.5 & 0.8 & 3.497 & $\mathrm{~m}$ & 0.003 & $3 / 2^{-}$ & 01 & & & $\beta^{-}=100$ & \\
\hline${ }^{55} \mathrm{Mn}$ & -57710.6 & 0.7 & StABLE & & & $5 / 2^{-}$ & 01 & & & $\mathrm{IS}=100$ & \\
\hline${ }^{55} \mathrm{Fe}$ & -57479.4 & 0.7 & 2.737 & $\mathrm{y}$ & 0.011 & $3 / 2^{-}$ & 01 & & & $\varepsilon=100$ & \\
\hline${ }^{55} \mathrm{Co}$ & -54027.6 & 0.7 & 17.53 & $\mathrm{~h}$ & 0.03 & $7 / 2^{-}$ & 01 & & & $\beta^{+}=100$ & \\
\hline${ }^{55} \mathrm{Ni}$ & -45336 & 11 & 204.7 & $\mathrm{~ms}$ & 1.7 & $7 / 2^{-}$ & 01 & 02Lo13 & $\mathrm{T}$ & $\beta^{+}=100$ & * \\
\hline${ }^{55} \mathrm{Cu}$ & $-31620 \#$ & $300 \#$ & $40 \#$ & $\mathrm{~ms}$ & $(>200 \mathrm{~ns})$ & $3 / 2^{-} \#$ & 01 & 87Po04 & I & $\beta^{+} ? ; \mathrm{p} ?$ & \\
\hline${ }^{55} \mathrm{Zn}$ & $-14920 \#$ & $250 \#$ & $20 \#$ & $\mathrm{~ms}$ & $(>1.6 \mu \mathrm{s})$ & $5 / 2^{-} \#$ & 01 & 01Gi10 & I & $\beta^{+} ?$ & \\
\hline$*^{55} \mathrm{Ti}$ & $\mathrm{T}$ : unweig & hed aver & $96 \mathrm{Do} 23$ & $=600$ & (40) and 95 & o. $A=54$ & $5(95)$ & & & & $* *$ \\
\hline$*^{55} \mathrm{Ti}$ & $\mathrm{T}: \quad(\mathrm{Bi}$ & rge ratio & & & & & & & & & $* *$ \\
\hline$*^{55} \mathrm{Ni}$ & $\mathrm{T}:$ average & $02 \mathrm{Lo} 13$ & (3) $87 \mathrm{~Hz}$ & $A=2$ & $12.1(3.8)$ & $\mathrm{Ay} 01=2$ & $8(5)$ & & & & ** \\
\hline$*^{55} \mathrm{Ni}$ & and & $177 \mathrm{Ho} 2$ & $(6) ; 97 \mathrm{~V}$ & $006=$ & $=204(3)$ supe & rseded b & y $99 R$ & $\mathrm{e} 06$ & & & $\leqslant *$ \\
\hline
\end{tabular}




\begin{tabular}{|c|c|c|c|c|c|c|c|c|c|c|c|c|}
\hline Nuclide & \multicolumn{2}{|c|}{$\begin{array}{l}\text { Mass excess } \\
\quad(\mathrm{keV})\end{array}$} & \multirow[t]{2}{*}{$\begin{array}{l}\text { Excitation } \\
\text { energy }(\mathrm{keV})\end{array}$} & \multicolumn{3}{|c|}{ Half-life } & $J^{\pi}$ & Ens & \multicolumn{2}{|c|}{ Reference } & \multicolumn{2}{|l|}{$\begin{array}{l}\text { Decay modes and } \\
\text { intensities }(\%)\end{array}$} \\
\hline${ }^{56} \mathrm{Ca}$ & $-13440 \#$ & $900 \#$ & & $10 \#$ & $\mathrm{~ms}$ & $(>300 \mathrm{~ns})$ & $0^{+}$ & 99 & $97 \mathrm{Be} 70$ & I & $\beta^{-} ?$ & \\
\hline${ }^{56} \mathrm{Sc}$ & $-25270 \#$ & $700 \#$ & & $80 \#$ & $\mathrm{~ms}$ & $(>300 \mathrm{~ns})$ & $3^{+} \#$ & 99 & $97 \mathrm{Be} 70$ & I & $\beta^{-}$? & \\
\hline${ }^{56} \mathrm{Ti}$ & -38940 & 200 & & 164 & $\mathrm{~ms}$ & 24 & $0^{+}$ & 99 & 98Am04 & $\mathrm{TD}$ & $\beta^{-}=100 ; \beta^{-} \mathrm{n} ?$ & $*$ \\
\hline${ }^{56} \mathrm{~V}$ & -46080 & 200 & & 216 & $\mathrm{~ms}$ & 4 & $\left(1^{+}\right)$ & 99 & $03 \mathrm{Ma} 02$ & $\mathrm{TJ}$ & $\beta^{-}=100 ; \beta^{-} \mathrm{n} ?$ & \\
\hline${ }^{56} \mathrm{Cr}$ & -55281.2 & 1.9 & & 5.94 & $\mathrm{~m}$ & 0.10 & $0^{+}$ & 99 & & & $\beta^{-}=100$ & \\
\hline${ }^{56} \mathrm{Mn}$ & -56909.7 & 0.7 & & 2.5789 & $\mathrm{~h}$ & 0.0001 & $3^{+}$ & 99 & & & $\beta^{-}=100$ & \\
\hline${ }^{56} \mathrm{Fe}$ & -60605.4 & 0.7 & & STABLE & & & $0^{+}$ & 99 & & & $\mathrm{IS}=91.75436$ & \\
\hline${ }^{56} \mathrm{Co}$ & -56039.4 & 2.1 & & 77.23 & $\mathrm{~d}$ & 0.03 & $4^{+}$ & 99 & & & $\beta^{+}=100$ & \\
\hline${ }^{56} \mathrm{Ni}$ & -53904 & 11 & & 6.075 & $\mathrm{~d}$ & 0.010 & $0^{+}$ & 99 & & & $\beta^{+}=100$ & \\
\hline${ }^{56} \mathrm{Cu}$ & $-38600 \#$ & $140 \#$ & & 93 & $\mathrm{~ms}$ & 3 & $\left(4^{+}\right)$ & 99 & 01Bo54 & TJD & $\beta^{+}=100 ; \beta^{+} \mathrm{p}=0.4012$ & \\
\hline${ }^{56} \mathrm{Zn}$ & $-25730 \#$ & $260 \#$ & & 36 & $\mathrm{~ms}$ & 10 & $0^{+}$ & 01 & 95Wa.A & $\mathrm{T}$ & $\beta^{+}$?; $\beta^{+} \mathrm{p}$ ? & $*$ \\
\hline${ }^{56} \mathrm{Ga}$ & $-4740 \#$ & $260 \#$ & & & & & $3^{+} \#$ & & & & p? & \\
\hline$*^{56} \mathrm{Ti}$ & $\mathrm{T}$ : average & $98 \mathrm{Amo}$ & $0(40) 96 \mathrm{Do} 23$ & $0(30)$ & & & & & & & & $* *$ \\
\hline$*^{56} \mathrm{Zn}$ & $\mathrm{T}$ : half-life & is deri & rom experimen & $\mathrm{p}, \mathrm{n}) \mathrm{cros}$ & ss sec & tions & & & & & & $* *$ \\
\hline$*^{56} \mathrm{Zn}$ & I : identifie & by tim & flight $01 \mathrm{Gi} 10$ & $T>1.6$ & & & & & & & & $* *$ \\
\hline
\end{tabular}

\begin{tabular}{|c|c|c|c|c|c|c|c|c|c|c|}
\hline${ }^{57} \mathrm{Ca}$ & $-7120 \#$ & 1000\# & $5 \#$ & $\mathrm{~ms}$ & & $5 / 2^{-} \#$ & & & & $\beta^{-} ? ; \beta^{-} \mathrm{n} ?$ \\
\hline${ }^{57} \mathrm{Sc}$ & $-20690 \#$ & $700 \#$ & 13 & $\mathrm{~ms}$ & 4 & $7 / 2^{-} \#$ & 98 & 02So.A & $\mathrm{TD}$ & $\beta^{-}=100 ; \beta^{-} \mathrm{n}=33 \#$ \\
\hline${ }^{57} \mathrm{Ti}$ & -33540 & 460 & 60 & $\mathrm{~ms}$ & 16 & $5 / 2^{-} \#$ & 98 & 99So20 & $\mathrm{T}$ & $\beta^{-}=100 ; \beta^{-} \mathrm{n}=0.3 \#$ \\
\hline${ }^{57} \mathrm{~V}$ & -44190 & 230 & 350 & $\mathrm{~ms}$ & 10 & $\left(3 / 2^{-}\right)$ & 98 & $03 \mathrm{Ma} 02$ & $\mathrm{TJ}$ & $\beta^{-}=100 ; \beta^{-} \mathrm{n}=0.4 \#$ \\
\hline${ }^{57} \mathrm{Cr}$ & -52524.1 & 1.9 & 21.1 & $\mathrm{~s}$ & 1.0 & $\left(3 / 2^{-}\right)$ & 98 & & & $\beta^{-}=100$ \\
\hline${ }^{57} \mathrm{Mn}$ & -57486.8 & 1.8 & 85.4 & $\mathrm{~s}$ & 1.8 & $5 / 2^{-}$ & 98 & & & $\beta^{-}=100$ \\
\hline${ }^{57} \mathrm{Fe}$ & -60180.1 & 0.7 & STABLE & & & $1 / 2^{-}$ & 98 & & & $\mathrm{IS}=2.11910$ \\
\hline${ }^{57} \mathrm{Co}$ & -59344.2 & 0.7 & 271.74 & $\mathrm{~d}$ & 0.06 & $7 / 2^{-}$ & 98 & & & $\varepsilon=100$ \\
\hline${ }^{57} \mathrm{Ni}$ & -56082.0 & 1.8 & 35.60 & $\mathrm{~h}$ & 0.06 & $3 / 2^{-}$ & 98 & & & $\beta^{+}=100$ \\
\hline${ }^{57} \mathrm{Cu}$ & -47310 & 16 & 196.3 & $\mathrm{~ms}$ & 0.7 & $3 / 2^{-}$ & 98 & & & $\beta^{+}=100$ \\
\hline${ }^{57} \mathrm{Zn}$ & $-32800 \#$ & $100 \#$ & 38 & $\mathrm{~ms}$ & 4 & $7 / 2^{-} \#$ & 98 & 02Lo13 & $\mathrm{T}$ & $\beta^{+}=100 ; \beta^{+} \mathrm{p} \approx 65$ \\
\hline${ }^{57} \mathrm{Ga}$ & $-15900 \#$ & $260 \#$ & & & & $1 / 2^{-} \#$ & & & & $\mathrm{p}$ ? \\
\hline$*^{57} \mathrm{Ti}$ & $\mathrm{T}$ : averag & 99So2 & ; $98 \mathrm{Am}$ & $\mathrm{n} 04=$ & $0(30)$ & ariance $\mathrm{n}$ & lot & & & \\
\hline$*{ }^{57} \mathrm{Zn}$ & $\mathrm{T}:$ averag & 02Lo13 & & & & & & & & \\
\hline
\end{tabular}

\begin{tabular}{|c|c|c|c|c|c|c|c|c|c|c|c|c|c|}
\hline${ }^{58} \mathrm{Sc}$ & $-15170 \#$ & $800 \#$ & & & 12 & $\mathrm{~ms}$ & 5 & $3^{+} \#$ & & 02So.A & $\mathrm{TD}$ & $\beta^{-}=100$ & \\
\hline${ }^{58} \mathrm{Ti}$ & $-30770 \#$ & $700 \#$ & & & 54 & $\mathrm{~ms}$ & 7 & $0^{+}$ & 97 & 99 So 20 & TD & $\beta^{-}=100$ & * \\
\hline${ }^{58} \mathrm{~V}$ & -40210 & 250 & & & 191 & $\mathrm{~ms}$ & 8 & $3^{+} \#$ & 97 & $03 \mathrm{Ma} 02$ & TD & $\beta^{-}=100 ; \beta^{-} \mathrm{n} ?$ & * \\
\hline${ }^{58} \mathrm{Cr}$ & -51830 & 200 & & & 7.0 & $\mathrm{~s}$ & 0.3 & $0^{+}$ & 97 & & & $\beta^{-}=100$ & \\
\hline${ }^{58} \mathrm{Mn}$ & -55910 & 30 & & & 3.0 & $\mathrm{~s}$ & 0.1 & $1^{+}$ & 97 & & & $\beta^{-}=100$ & \\
\hline${ }^{58} \mathrm{Mn}^{m}$ & -55840 & 30 & 71.78 & 0.05 & 65.2 & $\mathrm{~s}$ & 0.5 & $(4)^{+}$ & 97 & & & $\beta^{-}=? ; \mathrm{IT}=20 \#$ & \\
\hline${ }^{58} \mathrm{Fe}$ & -62153.4 & 0.7 & & & STABLE & & & $0^{+}$ & 97 & & & $\mathrm{IS}=0.2824$ & \\
\hline${ }^{58} \mathrm{Co}$ & -59845.9 & 1.2 & & & 70.86 & d & 0.06 & $2^{+}$ & 00 & & & $\beta^{+}=100$ & \\
\hline${ }^{58} \mathrm{Co}^{m}$ & -59821.0 & 1.2 & 24.95 & 0.06 & 9.04 & $\mathrm{~h}$ & 0.11 & $5^{+}$ & 00 & & & $\mathrm{IT}=100$ & \\
\hline${ }^{58} \mathrm{Co}^{n}$ & -59792.8 & 1.2 & 53.15 & 0.07 & 10.4 & $\mu \mathrm{s}$ & 0.3 & $4^{+}$ & 00 & & & $\mathrm{IT}=100$ & \\
\hline${ }^{58} \mathrm{Ni}$ & -60227.7 & 0.6 & & & STABLE & & (>700Ey) & $0^{+}$ & 01 & & & $\mathrm{IS}=68.076989 ; 2 \beta^{+} ?$ & $*$ \\
\hline${ }^{58} \mathrm{Cu}$ & -51662.1 & 1.6 & & & 3.204 & $\mathrm{~s}$ & 0.007 & $1^{+}$ & 01 & & & $\beta^{+}=100$ & \\
\hline${ }^{58} \mathrm{Zn}$ & -42300 & 50 & & & 84 & $\mathrm{~ms}$ & 9 & $0^{+}$ & 99 & 02Lo13 & $\mathrm{T}$ & $\beta^{+}=100 ; \beta^{+} \mathrm{p}<3$ & $*$ \\
\hline${ }^{58} \mathrm{Ga}$ & $-23990 \#$ & $210 \#$ & & & * & & & $2^{+} \#$ & & & & $\mathrm{p}$ ? & \\
\hline${ }^{58} \mathrm{Ga}^{m}$ & $-23960 \#$ & $230 \#$ & $30 \#$ & $100 \#$ & $*$ & & & $5^{+} \#$ & & & & $\mathrm{p}$ ? & \\
\hline${ }^{58} \mathrm{Ge}$ & $-8370 \#$ & $320 \#$ & & & & & & $0^{+}$ & & & & $2 p ?$ & \\
\hline$*^{58} \mathrm{Ti}$ & $\mathrm{T}:$ average & 02So.A & $=59(9)$ & 99 So $20=$ & (10) & & & & & & & & ** \\
\hline$*^{58} \mathrm{~V}$ & $\mathrm{~T}$ : average & $03 \mathrm{Ma} 0$ & $2=185($ & 0) $98 \mathrm{Am}$ & $4=200(20) 98$ & So03 & $=205(20)$ & & & & & & $* *$ \\
\hline$*^{58} \mathrm{Ni}$ & $\mathrm{T}:>400 \mathrm{E}$ & $y$ to $2^{+}$ & level of & $58 \mathrm{Fe},>7$ & 0 Ey to groun & d-sta & & & & & & & ** \\
\hline$*^{58} \mathrm{Zn}$ & $\mathrm{T}$ : average & 02Lo13 & $=83(10$ & $98 \mathrm{Jo} 18=$ & $6(18)$ & & & & & & & & $*$ \\
\hline
\end{tabular}




\begin{tabular}{|c|c|c|c|c|c|c|c|c|c|c|c|c|}
\hline \multirow{2}{*}{$\begin{array}{l}\text { Nuclide } \\
{ }^{59} \mathrm{Sc}\end{array}$} & \multicolumn{2}{|c|}{$\begin{array}{l}\text { Mass excess } \\
\quad(\mathrm{keV})\end{array}$} & \multicolumn{2}{|c|}{$\begin{array}{c}\text { Excitation } \\
\text { energy }(\mathrm{keV})\end{array}$} & \multicolumn{3}{|c|}{ Half-life } & \multirow{2}{*}{$\begin{array}{c}J^{\pi} \\
7 / 2^{-\#}\end{array}$} & \multirow[t]{2}{*}{ Ens } & Reference & \multicolumn{2}{|l|}{$\begin{array}{l}\text { Decay modes and } \\
\text { intensities }(\%)\end{array}$} \\
\hline & $-10040 \#$ & $900 \#$ & & & $10 \#$ & $\mathrm{~ms}$ & & & & & $\beta^{-} ? ; \beta^{-} \mathrm{n} ?$ & \\
\hline${ }^{59} \mathrm{Ti}$ & $-25220 \#$ & $700 \#$ & & & 30 & $\mathrm{~ms}$ & 3 & $5 / 2^{-} \#$ & 02 & 02So.A $\mathrm{T}$ & $\beta^{-}=100$ & * \\
\hline${ }^{59} \mathrm{~V}$ & -37070 & 310 & & & 75 & $\mathrm{~ms}$ & 7 & $7 / 2^{-} \#$ & 02 & & $\beta^{-}=100 ; \beta^{-} \mathrm{n} ?$ & \\
\hline${ }^{59} \mathrm{Cr}$ & -47890 & 240 & & & 460 & $\mathrm{~ms}$ & 50 & $5 / 2^{-} \#$ & 02 & & $\beta^{-}=100$ & \\
\hline${ }^{59} \mathrm{Cr}^{m}$ & -47390 & 240 & 503.0 & 1.7 & 96 & $\mu \mathrm{s}$ & 20 & $\left(9 / 2^{+}\right)$ & 02 & & $\mathrm{IT}=100$ & \\
\hline${ }^{59} \mathrm{Mn}$ & -55480 & 30 & & & 4.59 & $\mathrm{~s}$ & 0.05 & $(5 / 2)^{-}$ & 02 & & $\beta^{-}=100$ & \\
\hline${ }^{59} \mathrm{Fe}$ & -60663.1 & 0.7 & & & 44.495 & d & 0.009 & $3 / 2^{-}$ & 02 & & $\beta^{-}=100$ & \\
\hline${ }^{59} \mathrm{Co}$ & -62228.4 & 0.6 & & & STABLE & & & $7 / 2^{-}$ & 02 & & $\mathrm{IS}=100$ & \\
\hline${ }^{59} \mathrm{Ni}$ & -61155.7 & 0.6 & & & 101 & ky & 13 & $3 / 2^{-}$ & 02 & 94Ru19 T & $\beta^{+}=100$ & * \\
\hline${ }^{59} \mathrm{Cu}$ & -56357.2 & 0.8 & & & 81.5 & $\mathrm{~s}$ & 0.5 & $3 / 2^{-}$ & 02 & & $\beta^{+}=100$ & \\
\hline${ }^{59} \mathrm{Zn}$ & -47260 & 40 & & & 182.0 & $\mathrm{~ms}$ & 1.8 & $3 / 2^{-}$ & 02 & & $\beta^{+}=100 ; \beta^{+} \mathrm{p}=0.103$ & \\
\hline${ }^{59} \mathrm{Ga}$ & $-34120 \#$ & $170 \#$ & & & & & & $3 / 2^{-} \#$ & & & p? & \\
\hline${ }^{59} \mathrm{Ge}$ & $-17000 \#$ & $280 \#$ & & & & & & $7 / 2^{-} \#$ & & & $2 \mathrm{p} ?$ & \\
\hline$*^{59} \mathrm{Ti}$ & $\mathrm{T}$ : superse & des 99 & So $20=58$ & 7) same & oup & & & & & & & ** \\
\hline$*^{59} \mathrm{Ni}$ & $\mathrm{T}$ : unweig & hed av & erage 94 & $19=108$ & 3) $94 \mathrm{Ru} 1$ & 19(me & orite $)=$ & (22) $81 \mathrm{~N}$ & $8=10$ & & & $* *$ \\
\hline$*^{59} \mathrm{Ni}$ & $\mathrm{T}: \quad(\mathrm{Bi}$ & rge rati & o $B=2.0$ & & & & & & & & & ** \\
\hline
\end{tabular}

\begin{tabular}{|c|c|c|c|c|c|c|c|c|c|c|c|c|c|}
\hline${ }^{60} \mathrm{Sc}$ & $-4000 \#$ & $900 \#$ & & & $3 \#$ & $\mathrm{~ms}$ & & $3^{+} \#$ & & & & $\beta^{-} ?$ & \\
\hline${ }^{60} \mathrm{Ti}$ & $-21650 \#$ & $800 \#$ & & & 22 & $\mathrm{~ms}$ & 2 & $0^{+}$ & & 02So.A & TD & $\beta^{-}=100$ & \\
\hline${ }^{60} \mathrm{~V}$ & -32580 & 470 & & & 122 & $\mathrm{~ms}$ & 18 & $3^{+} \#$ & 97 & 99So20 & TD & $\beta^{-}=100 ; \beta^{-} \mathrm{n} ?$ & * \\
\hline${ }^{60} \mathrm{~V}^{m}$ & $-32580 \#$ & 490\# & 0\# & $150 \#$ & 40 & $\mathrm{~ms}$ & 15 & $1^{+} \#$ & & 03So02 & $\mathrm{TD}$ & $\beta^{-}=? ;$ IT ? & \\
\hline${ }^{60} \mathrm{~V}^{n}$ & -32480 & 470 & 101 & 1 & & & $(>400 \mathrm{~ns})$ & & & 99So20 & EI & $\mathrm{IT}=100$ & \\
\hline${ }^{60} \mathrm{Cr}$ & -46500 & 210 & & & 560 & $\mathrm{~ms}$ & 60 & $0^{+}$ & 93 & $96 \mathrm{Do} 23$ & $\mathrm{~T}$ & $\beta^{-}=100$ & $*$ \\
\hline${ }^{60} \mathrm{Mn}$ & -53180 & 90 & & & 51 & $\mathrm{~s}$ & 6 & $0^{+}$ & 94 & & & $\beta^{-}=100$ & \\
\hline${ }^{60} \mathrm{Mn}^{m}$ & -52910 & 90 & 271.90 & 0.10 & 1.77 & $\mathrm{~s}$ & 0.02 & $3^{+}$ & 94 & 92Sc.A & E & $\beta^{-}=88.58 ; \mathrm{IT}=11.58$ & \\
\hline${ }^{60} \mathrm{Fe}$ & -61412 & 3 & & & 1.5 & My & 0.3 & $0^{+}$ & 93 & & & $\beta^{-}=100$ & \\
\hline${ }^{60} \mathrm{Co}$ & -61649.0 & 0.6 & & & 5.2713 & $\mathrm{y}$ & 0.0008 & $5^{+}$ & 00 & & & $\beta^{-}=100$ & \\
\hline${ }^{60} \mathrm{Co}^{m}$ & -61590.4 & 0.6 & 58.59 & 0.01 & 10.467 & $\mathrm{~m}$ & 0.006 & $2^{+}$ & 00 & & & $\mathrm{IT} \approx 100 ; \beta^{-}=0.243$ & \\
\hline${ }^{60} \mathrm{Ni}$ & -64472.1 & 0.6 & & & STABLE & & & $0^{+}$ & 96 & & & $\mathrm{IS}=26.223177$ & \\
\hline${ }^{60} \mathrm{Cu}$ & -58344.1 & 1.7 & & & 23.7 & $\mathrm{~m}$ & 0.4 & $2^{+}$ & 93 & & & $\beta^{+}=100$ & \\
\hline${ }^{60} \mathrm{Zn}$ & -54188 & 11 & & & 2.38 & $\mathrm{~m}$ & 0.05 & $0^{+}$ & 02 & & & $\beta^{+}=100$ & \\
\hline${ }^{60} \mathrm{Ga}$ & $-40000 \#$ & $110 \#$ & & & 70 & $\mathrm{~ms}$ & 10 & $\left(2^{+}\right)$ & 02 & $01 \mathrm{Ma} 96$ & $\mathrm{TJ}$ & $\beta^{+}=100 ; \beta^{+} \mathrm{p}=1.67 ; \ldots$ & * \\
\hline${ }^{60} \mathrm{Ge}$ & $-27770 \#$ & $230 \#$ & & & $30 \#$ & $\mathrm{~ms}$ & & $0^{+}$ & & & & $\beta^{+} ?$ & \\
\hline${ }^{60} \mathrm{As}$ & $-6400 \#$ & $600 \#$ & & & & & & $5^{+} \#$ & & & & p ? & \\
\hline${ }^{60} \mathrm{As}^{m}$ & $-6340 \#$ & $600 \#$ & $60 \#$ & $20 \#$ & & & & $2^{+} \#$ & & & & p? & \\
\hline$*{ }^{60} \mathrm{~V}$ & $\mathrm{~T}:$ also 98 & $\mathrm{BAm04}=$ & $=200(40)$, & not used & & & & & & & & & ** \\
\hline$*^{60} \mathrm{Cr}$ & $\mathrm{T}$ : weighe & ed avera & ge $96 \mathrm{Do}$ & $23=510(1$ & $88 \mathrm{Bo} 0$ & $=570$ & (60); oth & Am. & $0(30$ & & & & $* *$ \\
\hline$*^{60} \mathrm{Ga}$ & $\mathrm{D}: \ldots ; \beta$ & $+\alpha<0.0$ & 02320 & & & & & & & & & & ** \\
\hline$*^{60} \mathrm{Ga}$ & $\mathrm{T}$ : averag & 02Lo1 & $13=70(13$ & $01 \mathrm{Ma} 96$ & $0(15)$ & & & & & & & & $* *$ \\
\hline
\end{tabular}

\begin{tabular}{|c|c|c|c|c|c|c|c|c|c|c|c|c|c|}
\hline${ }^{61} \mathrm{Ti}$ & $-15650 \#$ & $900 \#$ & & & $10 \#$ & $\mathrm{~ms}$ & $(>300 \mathrm{~ns})$ & $1 / 2^{-} \#$ & 99 & $97 \mathrm{Be} 70$ & I & $\beta^{-} ? ; \beta^{-} \mathrm{n} ?$ & \\
\hline${ }^{61} \mathrm{~V}$ & $-29360 \#$ & $400 \#$ & & & 47.0 & $\mathrm{~ms}$ & 1.2 & $7 / 2^{-} \#$ & 99 & 03So02 & TD & $\beta^{-}=100 ; \beta^{-} \mathrm{n}<6$ & \\
\hline${ }^{61} \mathrm{Cr}$ & -42180 & 250 & & & 261 & $\mathrm{~ms}$ & 15 & $5 / 2^{-} \#$ & 99 & 99 So20 & TD & $\beta^{-}=100 ; \beta^{-} \mathrm{n} ?$ & $*$ \\
\hline${ }^{61} \mathrm{Mn}$ & -51560 & 230 & & & 670 & $\mathrm{~ms}$ & 40 & $(5 / 2)^{-}$ & 99 & $99 \mathrm{Ha} 05$ & $\mathrm{D}$ & $\beta^{-}=100 ; \beta^{-} \mathrm{n}=$ ? & \\
\hline${ }^{61} \mathrm{Fe}$ & -58921 & 20 & & & 5.98 & $\mathrm{~m}$ & 0.06 & $3 / 2^{-}, 5 / 2^{-}$ & 99 & & & $\beta^{-}=100$ & \\
\hline${ }^{61} \mathrm{Fe}^{m}$ & -58060 & 20 & 861 & 3 & 250 & $\mathrm{~ns}$ & 10 & $9 / 2^{+} \#$ & 99 & $98 \mathrm{Gr} 14$ & $\mathrm{E}$ & $\mathrm{IT}=100$ & \\
\hline${ }^{61} \mathrm{Co}$ & -62898.4 & 0.9 & & & 1.650 & $\mathrm{~h}$ & 0.005 & $7 / 2^{-}$ & 99 & & & $\beta^{-}=100$ & \\
\hline${ }^{61} \mathrm{Ni}$ & -64220.9 & 0.6 & & & StABLE & & & $3 / 2^{-}$ & 99 & & & $\mathrm{IS}=1.13996$ & \\
\hline${ }^{61} \mathrm{Cu}$ & -61983.6 & 1.0 & & & 3.333 & $\mathrm{~h}$ & 0.005 & $3 / 2^{-}$ & 99 & & & $\beta^{+}=100$ & \\
\hline${ }^{61} \mathrm{Zn}$ & -56345 & 16 & & & 89.1 & $\mathrm{~s}$ & 0.2 & $3 / 2^{-}$ & 99 & & & $\beta^{+}=100$ & \\
\hline${ }^{61} \mathrm{Zn}^{m}$ & -56257 & 16 & 88.4 & 0.1 & $<430$ & $\mathrm{~ms}$ & & $1 / 2^{-}$ & 99 & & & $\mathrm{IT}=100$ & \\
\hline${ }^{61} \mathrm{Zn}^{n}$ & -55927 & 16 & 418.10 & 0.15 & 140 & $\mathrm{~ms}$ & 70 & $3 / 2^{-}$ & 99 & & & $\mathrm{IT}=100$ & \\
\hline${ }^{61} \mathrm{Zn}^{p}$ & -55589 & 16 & 756.02 & 0.18 & $<130$ & $\mathrm{~ms}$ & & $5 / 2^{-}$ & 99 & & & $\mathrm{IT}=100$ & \\
\hline${ }^{61} \mathrm{Ga}$ & -47090 & 50 & & & 168 & $\mathrm{~ms}$ & 3 & $3 / 2^{-}$ & 99 & 02We07 & $\mathrm{TD}$ & $\beta^{+}=100 ; \beta^{+} \mathrm{p} \approx 0$ & \\
\hline${ }^{61} \mathrm{Ga}^{m}$ & $-47000 \#$ & $110 \#$ & $90 \#$ & $100 \#$ & & & & $1 / 2^{-} \#$ & & & & & \\
\hline${ }^{61} \mathrm{Ge}$ & $-33730 \#$ & $300 \#$ & & & 39 & $\mathrm{~ms}$ & 12 & $3 / 2^{-} \#$ & 99 & 02Lo13 & $\mathrm{T}$ & $\beta^{+}=100 ; \beta^{+} \mathrm{p} \approx 80$ & $*$ \\
\hline${ }^{61} \mathrm{As}$ & $-18050 \#$ & $600 \#$ & & & & & & $3 / 2^{-} \#$ & & & & p ? & \\
\hline$*{ }^{61} \mathrm{Cr}$ & $\mathrm{T}$ : averag & e $99 \mathrm{So}^{2}$ & $20=251$ & 2) $98 \mathrm{Am}$ & $270(20)$ & & & & & & & & ** \\
\hline$*^{61} \mathrm{Ge}$ & $\mathrm{T}:$ averag & e $02 \mathrm{Lo}$ & $13=36(21$ & ) $87 \mathrm{HoO}$ & $0(15)$ & & & & & & & & ** \\
\hline
\end{tabular}




\begin{tabular}{|c|c|c|c|c|c|c|c|c|c|c|c|c|c|c|}
\hline Nuclide & \multicolumn{2}{|c|}{$\begin{array}{l}\text { Mass excess } \\
\quad(\mathrm{keV})\end{array}$} & \multicolumn{3}{|c|}{$\begin{array}{l}\text { Excitation } \\
\text { energy }(\mathrm{keV})\end{array}$} & \multicolumn{3}{|c|}{ Half-life } & $J^{\pi}$ & Ens & \multicolumn{2}{|c|}{ Reference } & \multicolumn{2}{|l|}{$\begin{array}{l}\text { Decay modes and } \\
\text { intensities }(\%)\end{array}$} \\
\hline${ }^{62} \mathrm{Ti}$ & $-11650 \#$ & $900 \#$ & & & & $10 \#$ & $\mathrm{~ms}$ & & $0^{+}$ & & & & $\beta^{-}$? & \\
\hline${ }^{62} \mathrm{~V}$ & $-24420 \#$ & $500 \#$ & & & & 33.5 & $\mathrm{~ms}$ & 2.0 & $3^{+} \#$ & 01 & 03So02 & TD & $\beta^{-}=100$ & \\
\hline${ }^{62} \mathrm{Cr}$ & -40410 & 340 & & & & 199 & $\mathrm{~ms}$ & 9 & $0^{+}$ & 01 & 02So.A & TD & $\beta^{-}=100 ; \beta^{-} \mathrm{n} ?$ & $*$ \\
\hline${ }^{62} \mathrm{Mn}$ & -48040 & 220 & & & $*$ & 671 & $\mathrm{~ms}$ & 5 & $\left(3^{+}\right)$ & 01 & $99 \mathrm{Ha} 05$ & TD & $\beta^{-}=100 ; \beta^{-} \mathrm{n}=?$ & \\
\hline${ }^{62} \mathrm{Mn}^{m}$ & $-48040 \#$ & $270 \#$ & 0\# & $150 \#$ & $*$ & 92 & $\mathrm{~ms}$ & 13 & $\left(1^{+}\right)$ & & 99So20 & TJD & $\beta^{-}=100 ; \beta^{-} \mathrm{n} \approx 0$ & \\
\hline${ }^{62} \mathrm{Fe}$ & -58901 & 14 & & & & 68 & $\mathrm{~s}$ & 2 & $0^{+}$ & 01 & & & $\beta^{-}=100$ & \\
\hline${ }^{62} \mathrm{Co}$ & -61432 & 20 & & & & 1.50 & $\mathrm{~m}$ & 0.04 & $2^{+}$ & 01 & & & $\beta^{-}=100$ & \\
\hline${ }^{62} \mathrm{Co}^{m}$ & -61410 & 21 & 22 & 5 & & 13.91 & $\mathrm{~m}$ & 0.05 & $5^{+}$ & 01 & & & $\beta^{-}>99 ; \mathrm{IT}<1$ & \\
\hline${ }^{62} \mathrm{Ni}$ & -66746.1 & 0.6 & & & & STABLE & & & $0^{+}$ & 01 & & & $\mathrm{IS}=3.634517$ & \\
\hline${ }^{62} \mathrm{Cu}$ & -62798 & 4 & & & & 9.673 & $\mathrm{~m}$ & 0.008 & $1^{+}$ & 01 & 02Un02 & $\mathrm{T}$ & $\beta^{+}=100$ & * \\
\hline${ }^{62} \mathrm{Zn}$ & -61171 & 10 & & & & 9.186 & $\mathrm{~h}$ & 0.013 & $0^{+}$ & 01 & & & $\beta^{+}=100$ & \\
\hline${ }^{62} \mathrm{Ga}$ & -52000 & 28 & & & & 115.99 & $\mathrm{~ms}$ & 0.17 & $0^{+}$ & 01 & 03Нy02 & $\mathrm{T}$ & $\beta^{+}=100$ & * \\
\hline${ }^{62} \mathrm{Ga}^{m}$ & -51183 & 28 & 817.5 & 0.5 & & 4.6 & $\mathrm{~ns}$ & 0.5 & $\left(3^{+}\right)$ & 01 & $98 \mathrm{Vi06}$ & ETJ & $\mathrm{IT}=100$ & \\
\hline${ }^{62} \mathrm{Ge}$ & $-42240 \#$ & $140 \#$ & & & & 130 & $\mathrm{~ms}$ & 40 & $0^{+}$ & 01 & $02 \mathrm{Lo} 13$ & TD & $\beta^{+}=100$ & * \\
\hline${ }^{62} \mathrm{As}$ & $-24960 \#$ & $300 \#$ & & & & & & & $1^{+} \#$ & 01 & & & $\mathrm{p}$ ? & * \\
\hline$*^{62} \mathrm{Cr}$ & \multicolumn{13}{|c|}{$\mathrm{T}:$ average 02So. $\mathrm{A}=209(12)$ 99So20 $=187(15) 98 \mathrm{Am} 04=190(30)$} & $* *$ \\
\hline$*^{62} \mathrm{Cu}$ & \multicolumn{13}{|c|}{$\mathrm{T}:$ others $97 \mathrm{Zi06}(\mathrm{LS}$ method $)=9.68(0.04) 97 \mathrm{Zi06}(\mathrm{IC}$ method $)=9.673(0.026)$} & $* *$ \\
\hline$*^{62} \mathrm{Cu}$ & \multirow{2}{*}{\multicolumn{13}{|c|}{$\mathrm{T}: \quad 69 \mathrm{Jo} 07=9.73(0.02) 69 \mathrm{Bo} 11=9.7(0.1) 65 \mathrm{Li1} 1=9.79(0.06) 65 \mathrm{~Eb} 01=9.76(0.02)$}} & $* *$ \\
\hline$*^{62} \mathrm{Ga}$ & & \multirow{2}{*}{\multicolumn{12}{|c|}{$\mathrm{T}:$ average $03 \mathrm{Hy} 02=115.84(0.25) 79 \mathrm{Da} 04=116.34(0.35) 78 \mathrm{Al} 23=115.95(0.30)$}} & ** \\
\hline$*^{62} \mathrm{Ge}$ & \multicolumn{12}{|c|}{ I : $T=113(+6-5) \mathrm{ms}$ in $93 \mathrm{Wi03}$ (table 1 ) is a misprint for ${ }^{62} \mathrm{Ga}$} & & ** \\
\hline$*^{62} \mathrm{As}$ & \multicolumn{13}{|c|}{$\mathrm{D}: \mathrm{p}$-unstable from estimated $\mathrm{S}_{p}=-1476 \#(422 \#) \mathrm{keV}$} & ** \\
\hline
\end{tabular}

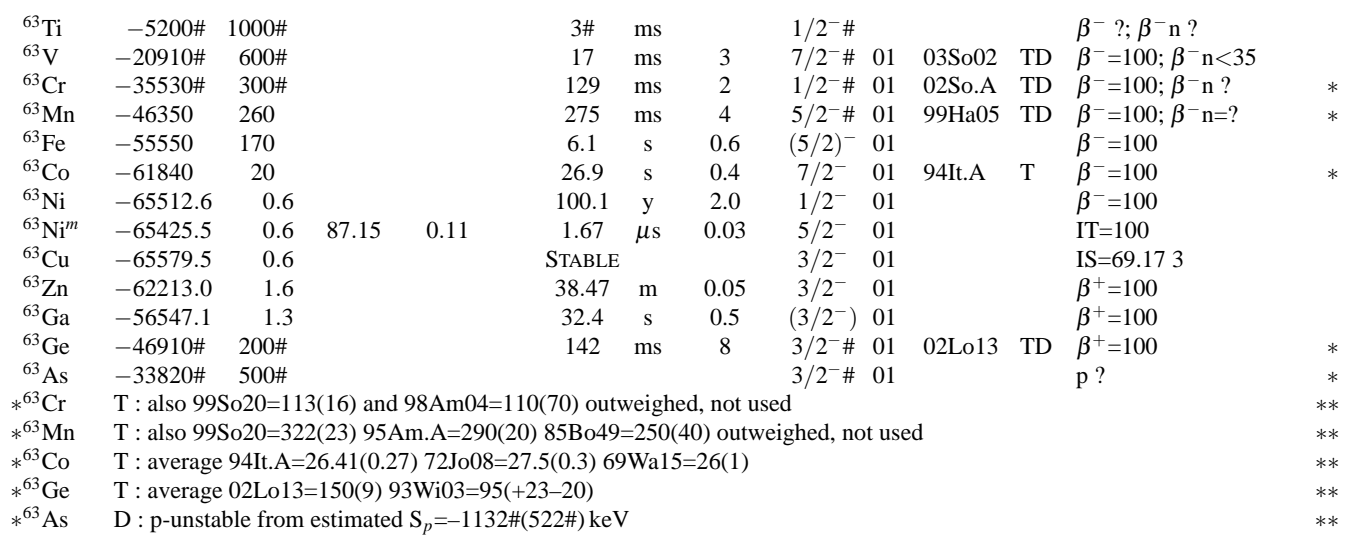

\begin{tabular}{|c|c|c|c|c|c|c|c|c|c|c|c|c|}
\hline${ }^{64} \mathrm{~V}$ & $-15400 \#$ & $700 \#$ & & & $10 \#$ & $\mathrm{~ms}$ & $(>300 \mathrm{~ns})$ & & 97 & $97 \mathrm{Be} 70$ & I & $\beta^{-} ?$ \\
\hline${ }^{64} \mathrm{Cr}$ & $-33150 \#$ & $400 \#$ & & & 43 & $\mathrm{~ms}$ & 1 & $0^{+}$ & & 02So.A & $\mathrm{TD}$ & $\beta^{-}=100$ \\
\hline${ }^{64} \mathrm{Mn}$ & -42620 & 270 & & & 88.8 & $\mathrm{~ms}$ & 2.5 & $\left(1^{+}\right)$ & 96 & 99So20 & TJD & $\beta^{-}=100 ; \beta^{-} \mathrm{n}=?$ \\
\hline${ }^{64} \mathrm{Mn}^{m}$ & -42490 & 270 & 135 & 3 & $>100$ & $\mu \mathrm{s}$ & & & & $98 \mathrm{Gr} 14$ & ET & $\mathrm{IT}=100$ \\
\hline${ }^{64} \mathrm{Fe}$ & -54770 & 280 & & & 2.0 & $\mathrm{~s}$ & 0.2 & $0^{+}$ & 96 & & & $\beta^{-}=100$ \\
\hline${ }^{64} \mathrm{Co}$ & -59793 & 20 & & & 300 & $\mathrm{~ms}$ & 30 & $1^{+}$ & 96 & & & $\beta^{-}=100$ \\
\hline${ }^{64} \mathrm{Ni}$ & -67099.3 & 0.6 & & & STABLE & & & $0^{+}$ & 96 & & & IS $=0.92569$ \\
\hline${ }^{64} \mathrm{Cu}$ & -65424.2 & 0.6 & & & 12.700 & $\mathrm{~h}$ & 0.002 & $1^{+}$ & 96 & & & $\beta^{+}=61.03 ; \beta^{-}=39.03$ \\
\hline${ }^{64} \mathrm{Zn}$ & -66003.6 & 0.7 & & & STABLE & & (>2.3 Еy) & $0^{+}$ & 96 & $85 \mathrm{No} 03$ & $\mathrm{~T}$ & $\mathrm{IS}=48.6360 ; 2 \beta^{+} ?$ \\
\hline${ }^{64} \mathrm{Ga}$ & -58834.3 & 2.0 & & & 2.627 & $\mathrm{~m}$ & 0.012 & $0^{(+\#)}$ & 96 & & & $\beta^{+}=100$ \\
\hline${ }^{64} \mathrm{Ga}^{m}$ & -58791.5 & 2.0 & 42.85 & 0.08 & 21.9 & $\mu \mathrm{s}$ & 0.7 & $2^{+}$ & 96 & 99Ta29 & TJ & $\mathrm{IT}=100$ \\
\hline${ }^{64} \mathrm{Ge}$ & -54350 & 30 & & & 63.7 & $\mathrm{~s}$ & 2.5 & $0^{+}$ & 96 & & & $\beta^{+}=100$ \\
\hline${ }^{64} \mathrm{As}$ & $-39520 \#$ & $360 \#$ & & & 40 & $\mathrm{~ms}$ & 30 & $0^{+} \#$ & & 02Lo13 & TD & $\beta^{+}=100$ \\
\hline$*{ }^{64} \mathrm{Cr}$ & $\mathrm{T}:$ also $99 \mathrm{~s}$ & $20=44$ & 2) $\mathrm{o}$ & & & & & & & & & \\
\hline$*{ }^{64} \mathrm{Mn}$ & $\mathrm{T}:$ average & o.A & 99 & $20=8$ & $\mathrm{Ha} 05=8$ & 9(4); & $98 \mathrm{Am} 04=1$ & $40(30)$ & ot u & & & \\
\hline
\end{tabular}




\begin{tabular}{|c|c|c|c|c|c|c|c|c|c|c|c|c|}
\hline Nuclide & \multicolumn{2}{|c|}{$\begin{array}{l}\text { Mass excess } \\
\quad(\mathrm{keV})\end{array}$} & \multicolumn{2}{|c|}{$\begin{array}{l}\text { Excitation } \\
\text { energy }(\mathrm{keV})\end{array}$} & \multicolumn{3}{|c|}{ Half-life } & $J^{\pi}$ & Ens & \multicolumn{2}{|c|}{ Reference } & $\begin{array}{l}\text { Decay modes and } \\
\text { intensities }(\%)\end{array}$ \\
\hline${ }^{65} \mathrm{~V}$ & $-11250 \#$ & $800 \#$ & & & $10 \#$ & $\mathrm{~ms}$ & & $5 / 2^{-} \#$ & & & & $\beta^{-} ? ; \beta^{-} \mathrm{n} ?$ \\
\hline${ }^{65} \mathrm{Cr}$ & $-27800 \#$ & $500 \#$ & & & 27 & $\mathrm{~ms}$ & 3 & $1 / 2^{-} \#$ & 97 & 02So.A & $\mathrm{TD}$ & $\beta^{-}=100 ; \beta^{-} \mathrm{n} ?$ \\
\hline${ }^{65} \mathrm{Mn}$ & -40670 & 540 & & & 92 & $\mathrm{~ms}$ & 1 & $5 / 2^{-} \#$ & 93 & 02So.A & $\mathrm{TD}$ & $\beta^{-}=100 ; \beta^{-} \mathrm{n}=$ ? \\
\hline${ }^{65} \mathrm{Fe}$ & -50880 & 240 & & & 1.3 & $\mathrm{~s}$ & 0.3 & $1 / 2^{-} \#$ & 93 & 99So20 & $\mathrm{T}$ & $\beta^{-}=100$ \\
\hline${ }^{65} \mathrm{Fe}^{m}$ & -50520 & 240 & 364 & 3 & 430 & ns & 130 & $\left(5 / 2^{-}\right)$ & & 98Gr14 & ETJ & $\mathrm{IT}=100$ \\
\hline${ }^{65} \mathrm{Co}$ & -59170 & 13 & & & 1.20 & $\mathrm{~s}$ & 0.06 & $(7 / 2)^{-}$ & 93 & & & $\beta^{-}=100$ \\
\hline${ }^{65} \mathrm{Ni}$ & -65126.1 & 0.6 & & & 2.5172 & $\mathrm{~h}$ & 0.0003 & $5 / 2^{-}$ & 97 & & & $\beta^{-}=100$ \\
\hline${ }^{65} \mathrm{Ni}^{m}$ & -64113.1 & 1.2 & 1013 & 1 & 26.7 & ns & 1.0 & $9 / 2^{+}$ & & $95 \mathrm{~B} 101$ & ETJ & \\
\hline${ }^{65} \mathrm{Cu}$ & -67263.7 & 0.7 & & & STABLE & & & $3 / 2^{-}$ & 93 & & & $\mathrm{IS}=30.833$ \\
\hline${ }^{65} \mathrm{Zn}$ & -65911.6 & 0.7 & & & 244.06 & $\mathrm{~d}$ & 0.10 & $5 / 2^{-}$ & 00 & & & $\beta^{+}=100$ \\
\hline${ }^{65} \mathrm{Zn}^{m}$ & -65857.7 & 0.7 & 53.928 & 0.010 & 1.6 & $\mu \mathrm{s}$ & 0.6 & $(1 / 2)^{-}$ & 00 & & & $\mathrm{IT}=100$ \\
\hline${ }^{65} \mathrm{Ga}$ & -62657.2 & 0.8 & & & 15.2 & $\mathrm{~m}$ & 0.2 & $3 / 2^{-}$ & 93 & & & $\beta^{+}=100$ \\
\hline${ }^{65} \mathrm{Ge}$ & -56410 & 100 & & & 30.9 & $\mathrm{~s}$ & 0.5 & $(3 / 2)^{-}$ & 93 & $87 \mathrm{Vi01}$ & $\mathrm{D}$ & $\beta^{+}=100 ; \beta^{+} \mathrm{p}=0.0113$ \\
\hline${ }^{65}$ As & $-46980 \#$ & $300 \#$ & & & 170 & $\mathrm{~ms}$ & 30 & $3 / 2^{-} \#$ & 93 & 02Lo13 & $\mathrm{T}$ & $\beta^{+}=100$ \\
\hline${ }^{65} \mathrm{Se}$ & $-32920 \#$ & $600 \#$ & & & $<50$ & $\mathrm{~ms}$ & & $3 / 2^{-} \#$ & 93 & 94Mo.A & $\mathrm{T}$ & $\beta^{+}=100 ; \beta^{+} \mathrm{p}=?$ \\
\hline$*^{65} \mathrm{Mn}$ & \multicolumn{12}{|c|}{$\mathrm{T}:$ others $99 \mathrm{Ha} 05=88(4) 99 \mathrm{So} 20=100(8) 98 \mathrm{Am} 04=110(20)$ outweighed, not used } \\
\hline$*^{65} \mathrm{Fe}$ & \multicolumn{12}{|c|}{$\mathrm{T}: 95 \mathrm{Am} . \mathrm{A}=760(50) \mathrm{ms}$ supersedes $94 \mathrm{Cz} 02=450(150)$ from same group, none used } \\
\hline$*^{65} \mathrm{As}$ & \multicolumn{12}{|c|}{$\mathrm{T}:$ average $02 \mathrm{Lo} 13=126(16) 95 \mathrm{Mo} 26=190(11)$ with Birge ratio $B=3.3$} \\
\hline$*^{65} \mathrm{Se}$ & \multicolumn{12}{|c|}{$\mathrm{D}$ : from $93 \mathrm{Ba} 12$} \\
\hline
\end{tabular}

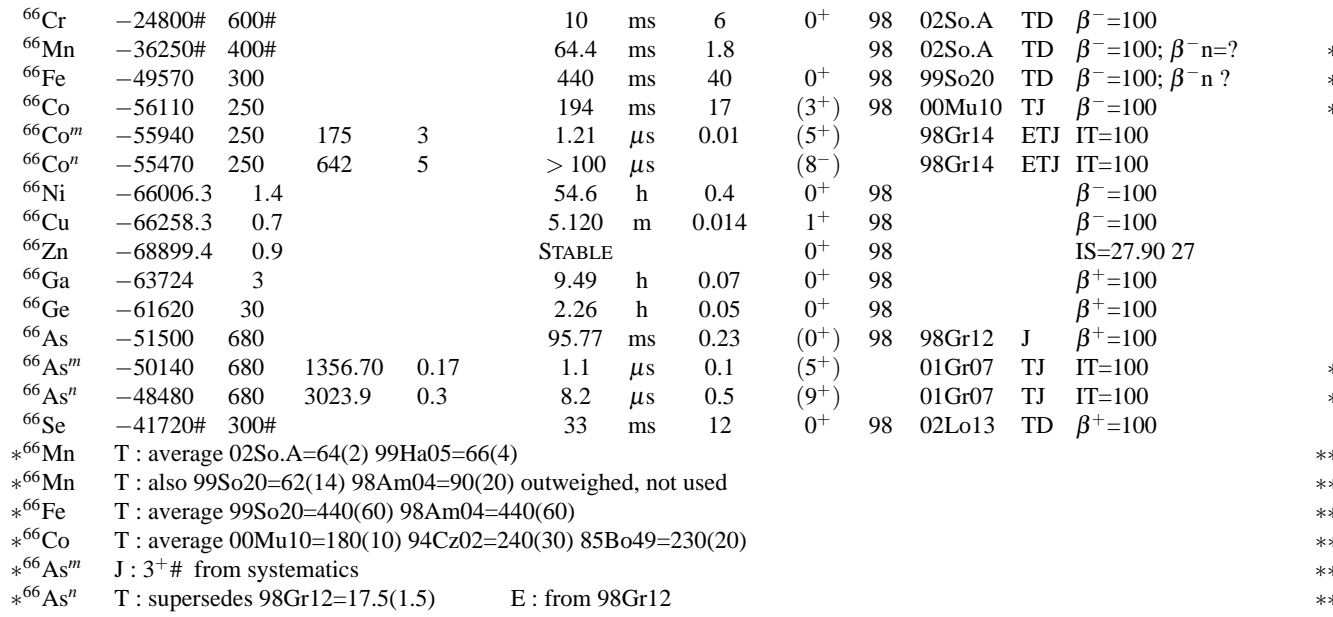

$\begin{array}{lllll}{ }^{67} \mathrm{Cr} & -19050 \# & 700 \# & & \\ { }^{67} \mathrm{Mn} & -33400 \# & 500 \# & & \\ { }^{67} \mathrm{Fe} & -45690 & 420 & & \\ { }^{67} \mathrm{Fe}^{m} & -45320 & 420 & 367 & 3 \\ { }^{67} \mathrm{Co} & -55060 & 320 & & \\ { }^{67} \mathrm{Ni} & -63742.7 & 2.9 & & \\ { }^{67} \mathrm{Ni} & -62736 & 4 & 1007 & 3 \\ { }^{67} \mathrm{Cu} & -67318.8 & 1.2 & & \\ { }^{67} \mathrm{Zn} & -67880.4 & 0.9 & & \\ { }^{67} \mathrm{Ga} & -66879.7 & 1.3 & & \\ { }^{67} \mathrm{Ge} & -62658 & 5 & & \\ { }^{67} \mathrm{Ge} & -62640 & 5 & 18.2 & 0.05 \\ { }^{67} \mathrm{Ge}^{n} & -61906 & 5 & 751.70 & 0.06 \\ { }^{67} \mathrm{As} & -56650 & 100 & & \\ \ldots A \text {-group is continued on next page } \ldots\end{array}$

\begin{tabular}{|c|c|c|c|c|c|c|c|}
\hline $10 \#$ & $\mathrm{~ms}$ & $(>300 \mathrm{~ns})$ & $1 / 2^{-} \#$ & & $97 \mathrm{Be} 70$ & I & $\beta^{-}$? \\
\hline 45 & $\mathrm{~ms}$ & 3 & $5 / 2^{-} \#$ & 97 & 02So.A & TD & $\beta^{-}=100 ; \beta^{-} \mathrm{n}=?$ \\
\hline 394 & $\mathrm{~ms}$ & 9 & $1 / 2^{-} \#$ & 91 & 02So.A & TD & $\beta^{-}=100 ; \beta^{-} \mathrm{n} ?$ \\
\hline 64 & $\mu \mathrm{s}$ & 17 & $\left(5 / 2^{-}\right)$ & & 03Sa02 & ET & $\mathrm{IT}=100$ \\
\hline 425 & $\mathrm{~ms}$ & 20 & $7 / 2^{-} \#$ & 91 & 99We07 & $\mathrm{T}$ & $\beta^{-}=100$ \\
\hline 21 & $\mathrm{~s}$ & 1 & $1 / 2^{-}$ & 01 & 00Ri14 & $\mathrm{J}$ & $\beta^{-}=100$ \\
\hline 13.3 & $\mu \mathrm{s}$ & 0.2 & $9 / 2^{+}$ & 01 & 98Gr14 & E & $\mathrm{IT}=100$ \\
\hline 61.83 & $\mathrm{~h}$ & 0.12 & $3 / 2^{-}$ & 91 & & & $\beta^{-}=100$ \\
\hline STABLE & & & $5 / 2^{-}$ & 91 & & & $\mathrm{IS}=4.1013$ \\
\hline 3.2612 & $\mathrm{~d}$ & 0.0006 & $3 / 2^{-}$ & 96 & & & $\varepsilon=100$ \\
\hline 18.9 & $\mathrm{~m}$ & 0.3 & $1 / 2^{-}$ & 91 & & & $\beta^{+}=100$ \\
\hline 13.7 & $\mu \mathrm{s}$ & 0.9 & $5 / 2^{-}$ & 91 & & & $\mathrm{IT}=100$ \\
\hline 110.9 & ns & 1.4 & & 91 & & & $\mathrm{IT}=100$ \\
\hline 42.5 & $\mathrm{~s}$ & 1.2 & $\left(5 / 2^{-}\right)$ & 91 & & & $\beta^{+}=100$ \\
\hline
\end{tabular}




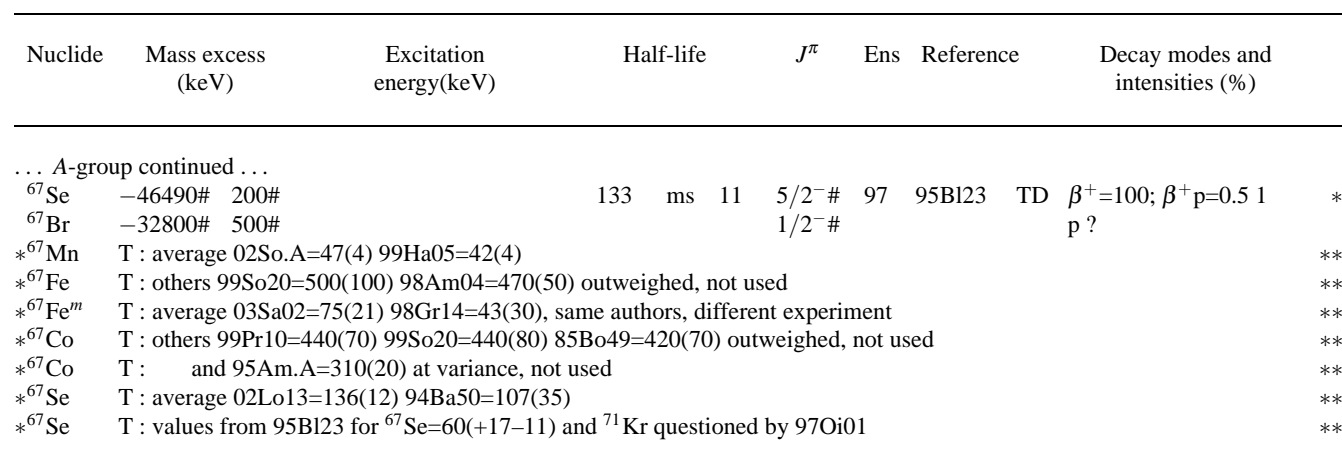

\begin{tabular}{|c|c|c|c|c|c|c|c|c|c|c|c|c|c|}
\hline${ }^{68} \mathrm{Mn}$ & $-28600 \#$ & $600 \#$ & & & & 28 & $\mathrm{~ms}$ & 4 & & 02 & 02So.A & $\mathrm{T}$ & $\beta^{-}=100 ; \beta^{-} \mathrm{n}=?$ \\
\hline${ }^{68} \mathrm{Fe}$ & -43130 & 700 & & & & 187 & $\mathrm{~ms}$ & 6 & $0^{+}$ & 02 & 02So.A & $\mathrm{T}$ & $\beta^{-}=100 ; \beta^{-} \mathrm{n} ?$ \\
\hline${ }^{68} \mathrm{Co}$ & -51350 & 320 & & & $*$ & 200 & $\mathrm{~ms}$ & 21 & $\left(7^{-}\right)$ & 02 & $00 \mathrm{Mu} 10$ & $\mathrm{~T}$ & $\beta^{-}=100$ \\
\hline${ }^{68} \mathrm{Co}^{m}$ & $-51200 \#$ & $350 \#$ & $150 \#$ & $150 \#$ & * & 1.6 & s & 0.3 & $\left(3^{+}\right)$ & 02 & $00 \mathrm{Mu} 10$ & JD & $\beta^{-}=? ;$ IT ? \\
\hline${ }^{68} \mathrm{Ni}$ & -63463.8 & 3.0 & & & & 29 & s & 2 & $0^{+}$ & 02 & & & $\beta^{-}=100$ \\
\hline${ }^{68} \mathrm{Ni}^{m}$ & -61694 & 3 & 1770.0 & 1.0 & & 276 & $\mathrm{~ns}$ & 65 & $0^{+}$ & 02 & & & IT $=100$ \\
\hline${ }^{68} \mathrm{Ni}^{n}$ & -60615 & 3 & 2849.1 & 0.3 & & 860 & $\mu \mathrm{s}$ & 50 & $5^{-}$ & 02 & & & $\mathrm{IT}=100$ \\
\hline${ }^{68} \mathrm{Cu}$ & -65567.0 & 1.6 & & & & 31.1 & $\mathrm{~s}$ & 1.5 & $1^{+}$ & 02 & & & $\beta^{-}=100$ \\
\hline${ }^{68} \mathrm{Cu}^{m}$ & -64845.4 & 1.7 & 721.6 & 0.7 & & 3.75 & $\mathrm{~m}$ & 0.05 & $\left(6^{-}\right)$ & 02 & & & $\mathrm{IT}=841 ; \beta^{-}=161$ \\
\hline${ }^{68} \mathrm{Zn}$ & -70007.2 & 1.0 & & & & StaBle & & & $0^{+}$ & 02 & & & $\mathrm{IS}=18.7551$ \\
\hline${ }^{68} \mathrm{Ga}$ & -67086.1 & 1.5 & & & & 67.71 & $\mathrm{~m}$ & 0.09 & $1^{+}$ & 02 & & & $\beta^{+}=100$ \\
\hline${ }^{68} \mathrm{Ga}^{m}$ & -65856.2 & 1.5 & 1229.87 & 0.04 & & 62.0 & $\mathrm{~ns}$ & 1.4 & $7^{-}$ & 02 & & & $\mathrm{IT}=100$ \\
\hline${ }^{68} \mathrm{Ge}$ & -66980 & 6 & & & & 270.95 & $\mathrm{~d}$ & 0.16 & $0^{+}$ & 02 & & & $\varepsilon=100$ \\
\hline${ }^{68} \mathrm{As}$ & -58900 & 40 & & & & 151.6 & s & 0.8 & $3^{+}$ & 02 & & & $\beta^{+}=100$ \\
\hline${ }^{68} \mathrm{As}^{m}$ & -58470 & 40 & 425.21 & 0.16 & & 111 & s & 20 & $1^{+}$ & 02 & & & $\mathrm{IT}=100$ \\
\hline${ }^{68} \mathrm{Se}$ & -54210 & 30 & & & & 35.5 & $\mathrm{~s}$ & 0.7 & $0^{+}$ & 02 & & & $\beta^{+}=100$ \\
\hline${ }^{68} \mathrm{Br}$ & $-38640 \#$ & $360 \#$ & & & & $<1.5$ & $\mu \mathrm{s}$ & & $3^{+} \#$ & 02 & $95 \mathrm{~B} 106$ & I & $\mathrm{p}$ ? \\
\hline$*^{68} \mathrm{Mn}$ & $\mathrm{T}$ : average & 02So. & $28(8)$ & $05=28$ & & & & & & & & & \\
\hline$*^{68} \mathrm{Fe}$ & $\mathrm{T}:$ others 9 & $9 \operatorname{So} 20=$ & $=155(50) 9$ & $\mathrm{Be} 33=10$ & & reighe & d, no & t used & & & & & \\
\hline$*^{68} \mathrm{Co}$ & $\mathrm{T}$ : average & $00 \mathrm{Mu} 1$ & $0=230(30$ & 99 So $20=$ & $O Y_{3}$ & ; not use & d 95 & Am.A & $310(30)$ & & & & \\
\hline$*^{68} \mathrm{Co}$ & $\mathrm{T}: 95 \mathrm{Am}$. & A super & edes $91 \mathrm{~B}$ & $3=180(1$ & & s & roup & & & & & & \\
\hline
\end{tabular}

\begin{tabular}{|c|c|c|c|c|c|c|c|c|c|c|c|c|c|c|}
\hline${ }^{69} \mathrm{Mn}$ & $-25300 \#$ & $800 \#$ & & & & 14 & $\mathrm{~ms}$ & 4 & $5 / 2^{-} \#$ & 00 & & & $\beta^{-}=100 ; \beta^{-} \mathrm{n}=24 \#$ & * \\
\hline${ }^{69} \mathrm{Fe}$ & $-38400 \#$ & $500 \#$ & & & & 109 & $\mathrm{~ms}$ & 9 & $1 / 2^{-} \#$ & 00 & 02So.A & $\mathrm{T}$ & $\beta^{-}=100 ; \beta^{-} \mathrm{n}=7 \#$ & \\
\hline${ }^{69} \mathrm{Co}$ & -50000 & 340 & & & & 227 & $\mathrm{~ms}$ & 13 & $7 / 2^{-} \#$ & 00 & 02So.A & $\mathrm{T}$ & $\beta^{-}=100 ; \beta^{-} \mathrm{n}=1 \#$ & $*$ \\
\hline${ }^{69} \mathrm{Ni}$ & -59979 & 4 & & & & 11.5 & $\mathrm{~s}$ & 0.3 & $9 / 2^{+}$ & 00 & $99 \operatorname{Pr} 10$ & $\mathrm{~T}$ & $\beta^{-}=100$ & $*$ \\
\hline${ }^{69} \mathrm{Ni}^{m}$ & -59658 & 4 & 321 & 2 & & 3.5 & $\mathrm{~s}$ & 0.4 & $\left(1 / 2^{-}\right)$ & 00 & 98Gr14 & $\mathrm{E}$ & $\beta^{-} \approx 100 ;$ IT ? & $*$ \\
\hline${ }^{69} \mathrm{Ni}^{n}$ & -57278 & 11 & 2701 & 10 & & 439 & ns & 3 & $\left(17 / 2^{-}\right)$ & 00 & & & $\mathrm{IT}=100$ & \\
\hline${ }^{69} \mathrm{Cu}$ & -65736.2 & 1.4 & & & & 2.85 & $\mathrm{~m}$ & 0.15 & $3 / 2^{-}$ & 00 & & & $\beta^{-}=100$ & \\
\hline${ }^{69} \mathrm{Cu}^{m}$ & -62994.4 & 1.7 & 2741.8 & 1.0 & & 360 & ns & 30 & $\left(13 / 2^{+}\right)$ & 00 & & & $\mathrm{IT}=100$ & \\
\hline${ }^{69} \mathrm{Zn}$ & -68418.0 & 1.0 & & & & 56.4 & $\mathrm{~m}$ & 0.9 & $1 / 2^{-}$ & 00 & & & $\beta^{-}=100$ & \\
\hline${ }^{69} \mathrm{Zn}^{m}$ & -67979.4 & 1.0 & 438.636 & 0.018 & & 13.76 & $\mathrm{~h}$ & 0.02 & $9 / 2^{+}$ & 00 & & & $\mathrm{IT} \approx 100 ; \beta^{-}=0.0333$ & \\
\hline${ }^{69} \mathrm{Ga}$ & -69327.8 & 1.2 & & & & STABLE & & & $3 / 2^{-}$ & 00 & & & $\mathrm{IS}=60.1089$ & \\
\hline${ }^{69} \mathrm{Ge}$ & -67100.6 & 1.3 & & & & 39.05 & $\mathrm{~h}$ & 0.10 & $5 / 2^{-}$ & 00 & & & $\beta^{+}=100$ & \\
\hline${ }^{69} \mathrm{Ge}^{m}$ & -67013.8 & 1.3 & 86.765 & 0.014 & & 5.1 & $\mu \mathrm{s}$ & 0.2 & $1 / 2^{-}$ & 00 & & & $\mathrm{IT}=100$ & \\
\hline${ }^{69} \mathrm{Ge}^{n}$ & -66702.7 & 1.3 & 397.944 & 0.018 & & 2.81 & $\mu \mathrm{s}$ & 0.05 & $9 / 2^{+}$ & 00 & & & $\mathrm{IT}=100$ & \\
\hline${ }^{69}$ As & -63090 & 30 & & & & 15.2 & $\mathrm{~m}$ & 0.2 & $5 / 2^{-}$ & 00 & & & $\beta^{+}=100$ & \\
\hline${ }^{69} \mathrm{Se}$ & -56300 & 30 & & & & 27.4 & $\mathrm{~s}$ & 0.2 & $\left(1 / 2^{-}\right)$ & 00 & $95 \mathrm{Po} 01$ & $\mathrm{~J}$ & $\beta^{+}=100 ; \beta^{+} \mathrm{p}=0.04510$ & \\
\hline${ }^{69} \mathrm{Se}^{m}$ & -56260 & 30 & 39.4 & 0.1 & & 2.0 & $\mu \mathrm{s}$ & 0.2 & $5 / 2^{-}$ & 00 & & & $\mathrm{IT}=100$ & \\
\hline${ }^{69} \mathrm{Se}^{n}$ & -55730 & 30 & 573.9 & 1.0 & & 955 & ns & 16 & $9 / 2^{+}$ & 00 & $00 \mathrm{Ch} 07$ & $\mathrm{~T}$ & $\mathrm{IT}=100$ & $*$ \\
\hline${ }^{69} \mathrm{Br}$ & $-46480 \#$ & $110 \#$ & & & $*$ & $<24$ & ns & & $1 / 2^{-} \#$ & 00 & 96Pf01 & I & $\mathrm{p}$ ? & $*$ \\
\hline${ }^{69} \mathrm{Br}^{m}$ & $-46440 \#$ & $150 \#$ & $40 \#$ & $100 \#$ & $*$ & & & & $5 / 2^{-} \#$ & & & & & \\
\hline${ }^{69} \mathrm{Br}^{n}$ & $-45910 \#$ & $150 \#$ & $570 \#$ & $100 \#$ & & & & & $9 / 2^{+} \#$ & & & & & \\
\hline
\end{tabular}




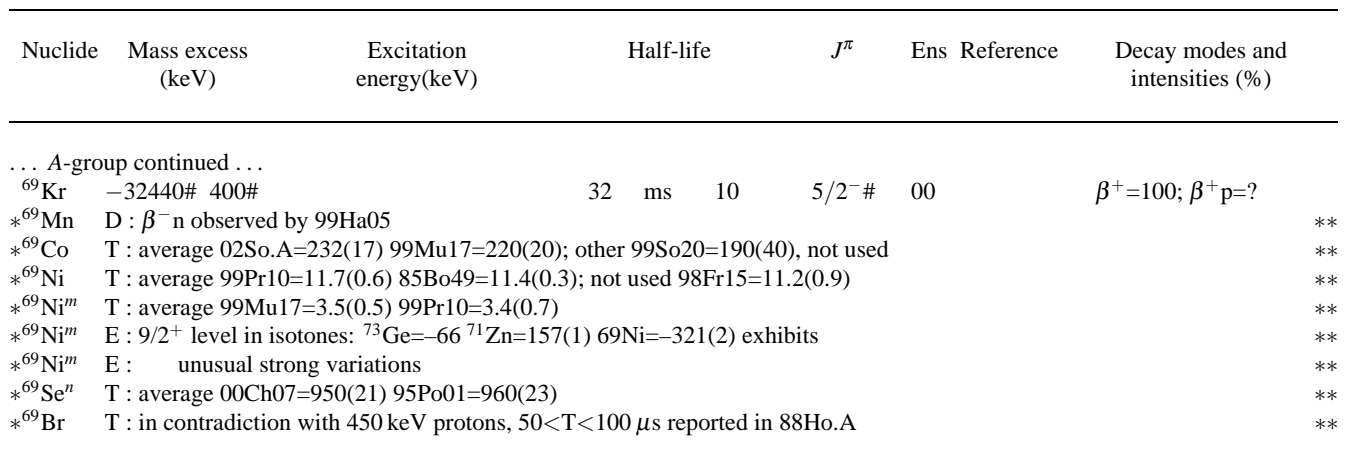

\begin{tabular}{|c|c|c|c|c|c|c|c|c|c|c|c|c|c|c|c|}
\hline${ }^{70} \mathrm{Fe}$ & $-35900 \#$ & $600 \#$ & & & & & 94 & $\mathrm{~ms}$ & 17 & $0^{+}$ & 97 & 02So.A & $\mathrm{TD}$ & $\beta^{-}=100$ & \\
\hline${ }^{70} \mathrm{Co}$ & -45640 & 840 & & & & * & 125 & $\mathrm{~ms}$ & 7 & $\left(6^{-}, 7^{-}\right)$ & 93 & $00 \mathrm{Mu} 10$ & TJD & $\beta^{-}=100 ; \beta^{-} \mathrm{n} ?$ & $*$ \\
\hline${ }^{70} \mathrm{Co}^{m}$ & $-45440 \#$ & $860 \#$ & $200 \#$ & $200 \#$ & & $*$ & 500 & $\mathrm{~ms}$ & 180 & $\left(3^{+}\right)$ & & $00 \mathrm{Mu} 10$ & TJD & $\beta^{-} \approx 100 ;$ IT $? ; \beta^{-} \mathrm{n}$ ? & \\
\hline${ }^{70} \mathrm{Ni}$ & -59150 & 350 & & & & & 6.0 & $\mathrm{~s}$ & 0.3 & $0^{+}$ & 03 & $98 \mathrm{Fr} 15$ & TD & $\beta^{-}=100$ & \\
\hline${ }^{70} \mathrm{Ni}^{m}$ & -56290 & 350 & 2860 & 2 & & & 232 & ns & 1 & $8^{+}$ & 03 & & & $\mathrm{IT}=100$ & \\
\hline${ }^{70} \mathrm{Cu}$ & -62976.1 & 1.6 & & & & $\&$ & 44.5 & $\mathrm{~s}$ & 0.2 & $\left(6^{-}\right)$ & 93 & $02 \mathrm{We} 03$ & $\mathrm{TJ}$ & $\beta^{-}=100$ & \\
\hline${ }^{70} \mathrm{Cu}^{m}$ & -62875.4 & 2.0 & 100.7 & 2.6 & MD & & 33 & $\mathrm{~s}$ & 2 & $\left(3^{-}\right)$ & & $02 \mathrm{We} 03$ & $\mathrm{TJ}$ & $\beta^{-} \approx 50 ; \mathrm{IT} \approx 50$ & \\
\hline${ }^{70} \mathrm{Cu}^{n}$ & -62734.1 & 2.1 & 242.0 & 2.7 & MD & $\&$ & 6.6 & $\mathrm{~s}$ & 0.2 & $1^{+}$ & 93 & $02 \mathrm{We} 03$ & TD & $\beta^{-} \approx 95 ; \mathrm{IT} \approx 5$ & * \\
\hline${ }^{70} \mathrm{Zn}$ & -69564.6 & 2.0 & & & & & STABLE & & & $0^{+}$ & 93 & & & $\mathrm{IS}=0.623 ; 2 \beta^{-} ?$ & $*$ \\
\hline${ }^{70} \mathrm{Ga}$ & -68910.1 & 1.2 & & & & & 21.14 & $\mathrm{~m}$ & 0.03 & $1^{+}$ & 93 & & & $\beta^{-} \approx 100 ; \varepsilon=0.416$ & \\
\hline${ }^{70} \mathrm{Ge}$ & -70563.1 & 1.0 & & & & & STABLE & & & $0^{+}$ & 93 & & & $\mathrm{IS}=20.8487$ & \\
\hline${ }^{70} \mathrm{As}$ & -64340 & 50 & & & & & 52.6 & $\mathrm{~m}$ & 0.3 & $4^{(+\#)}$ & 93 & & & $\beta^{+}=100$ & \\
\hline${ }^{70} \mathrm{As}{ }^{m}$ & -64310 & 50 & 32.06 & 0.03 & & & 96 & $\mu \mathrm{s}$ & 3 & $2^{(+)}$ & 93 & & & $\mathrm{IT}=100$ & \\
\hline${ }^{70} \mathrm{Se}$ & -62050 & 60 & & & & & 41.1 & $\mathrm{~m}$ & 0.3 & $0^{+}$ & 93 & & & $\beta^{+}=100$ & \\
\hline${ }^{70} \mathrm{Br}$ & $-51430 \#$ & $310 \#$ & & & & & 79.1 & $\mathrm{~ms}$ & 0.8 & $0^{+} \#$ & 93 & & & $\beta^{+}=100$ & \\
\hline${ }^{70} \mathrm{Br}^{m}$ & $-49140 \#$ & $310 \#$ & 2292.2 & 0.8 & & & 2.2 & $\mathrm{~s}$ & 0.2 & $\left(9^{+}\right)$ & 93 & 00Pi15 & $\mathrm{J}$ & $\beta^{+}=$?; IT ? & * \\
\hline${ }^{70} \mathrm{Kr}$ & $-41680 \#$ & $390 \#$ & & & & & 57 & $\mathrm{~ms}$ & 21 & $0^{+}$ & 97 & 00Oi02 & $\mathrm{TD}$ & $\beta^{+}$? & \\
\hline${ }^{70} \mathrm{Co}$ & $\mathrm{T}:$ averag & e 02 So & $A=121($ & $\mathrm{AmO}$ & J & 20 & others 0 & $0 \mathrm{Mu}$ & $0=120$ & 99So2 & $2(25$ & & & & F \\
\hline$*^{70} \mathrm{Cu}^{n}$ & $\mathrm{D}: \mathrm{IT}=\mathrm{fev}$ & $N$ perce & & $\mathrm{E}:$ post d & eadlin & e 03 & Va.2 10 & $1.1(0$ & ) and & $4(0.3)$ & & & & & $*$ \\
\hline$*^{70} \mathrm{Zn}$ & $\mathrm{T}:>500 \mathrm{~T}$ & Ty in E & NSDF is fo & $0 v-2 \beta^{-}$ & decay & alon & & & & & & & & & 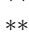 \\
\hline$*^{70} \mathrm{Br}^{m}$ & $\mathrm{E}$ : from 2 & 002Je0 & & & & & & & & & & & & & ** \\
\hline
\end{tabular}

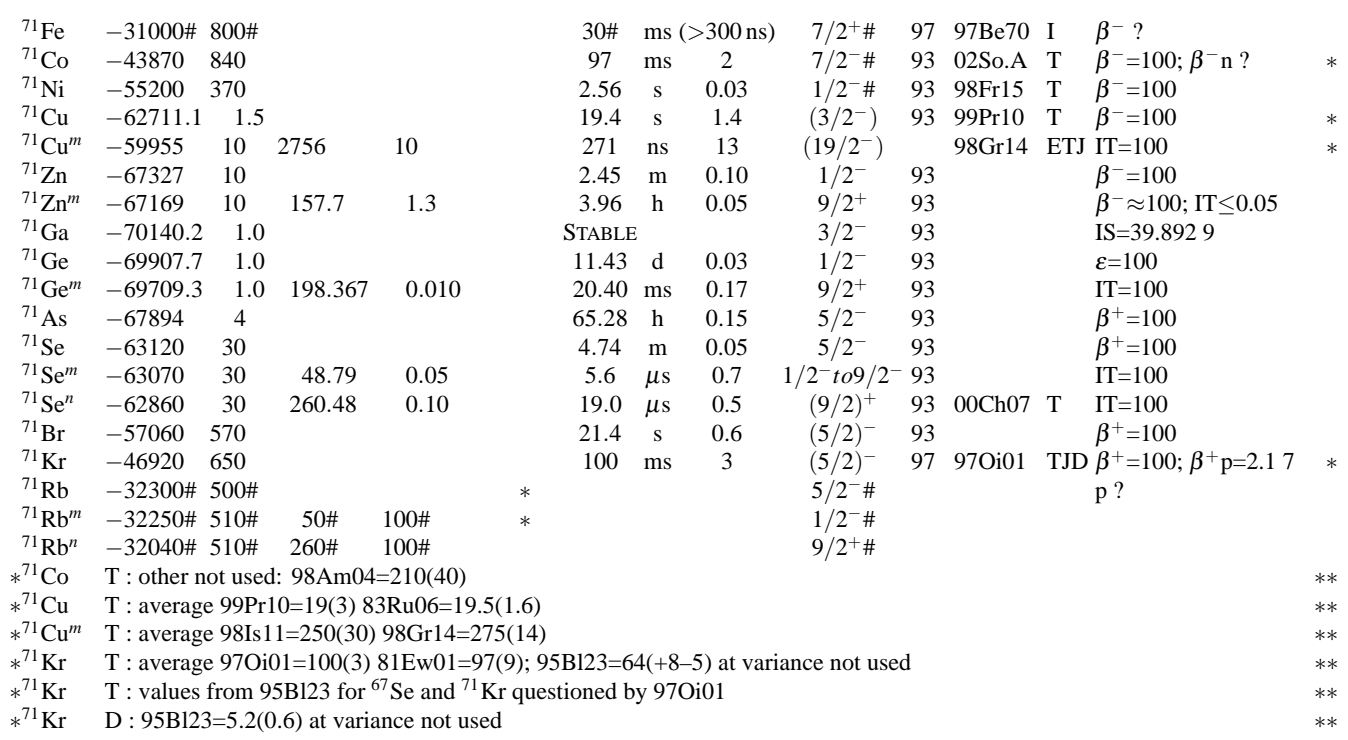




\begin{tabular}{|c|c|c|c|c|c|c|c|c|c|c|c|c|c|c|}
\hline Nuclide & \multicolumn{2}{|c|}{$\begin{array}{l}\text { Mass excess } \\
(\mathrm{keV})\end{array}$} & \multicolumn{3}{|c|}{$\begin{array}{c}\text { Excitation } \\
\text { energy(keV) }\end{array}$} & \multicolumn{3}{|c|}{ Half-life } & \multirow{2}{*}{$\begin{array}{c}J^{\pi} \\
0^{+}\end{array}$} & \multirow{2}{*}{$\begin{array}{c}\text { Ens } \\
97\end{array}$} & \multicolumn{2}{|c|}{ Reference } & \multicolumn{2}{|l|}{$\begin{array}{l}\text { Decay modes and } \\
\text { intensities }(\%)\end{array}$} \\
\hline${ }^{72} \mathrm{Fe}$ & $-28300 \#$ & $800 \#$ & & & & $10 \#$ & $\mathrm{~ms}$ & $(>300 \mathrm{~ns})$ & & & $97 \mathrm{Be} 70$ & I & $\beta^{-} ?$ & \\
\hline${ }^{72} \mathrm{Co}$ & $-39300 \#$ & $600 \#$ & & & & 90 & $\mathrm{~ms}$ & 20 & & & 98Am04 & TD & $\beta^{-}=100 ; \beta^{-} \mathrm{n} ?$ & \\
\hline${ }^{72} \mathrm{Ni}$ & -53940 & 440 & & & & 1.57 & $\mathrm{~s}$ & 0.05 & $0^{+}$ & & $98 \operatorname{Fr} 15$ & TD & $\beta^{-}=100 ; \beta^{-} \mathrm{n} ?$ & * \\
\hline${ }^{72} \mathrm{Cu}$ & -59783.0 & 1.4 & & & & 6.6 & $\mathrm{~s}$ & 0.1 & $\left(1^{+}\right)$ & 95 & & & $\beta^{-}=100$ & \\
\hline${ }^{72} \mathrm{Cu}^{m}$ & -59513 & 3 & 270 & 3 & & 1.76 & $\mu \mathrm{s}$ & 0.03 & $\left(4^{-}\right)$ & & $98 \mathrm{Gr} 14$ & ETJ & $\mathrm{IT}=100$ & \\
\hline${ }^{72} \mathrm{Zn}$ & -68131 & 6 & & & & 46.5 & $\mathrm{~h}$ & 0.1 & $0^{+}$ & 95 & & & $\beta^{-}=100$ & \\
\hline${ }^{72} \mathrm{Ga}$ & -68589.4 & 1.0 & & & & 14.10 & $\mathrm{~h}$ & 0.02 & $3^{-}$ & 95 & & & $\beta^{-}=100$ & \\
\hline${ }^{72} \mathrm{Ga}^{m}$ & -68469.7 & 1.0 & 119.66 & 0.05 & & 39.68 & $\mathrm{~ms}$ & 0.13 & $\left(0^{+}\right)$ & 95 & & & $\mathrm{IT}=100$ & \\
\hline${ }^{72} \mathrm{Ge}$ & -72585.9 & 1.6 & & & & STABLE & & & $0^{+}$ & 95 & & & $\mathrm{IS}=27.5434$ & \\
\hline${ }^{72} \mathrm{Ge}^{m}$ & -71894.5 & 1.6 & 691.43 & 0.04 & & 444.2 & $\mathrm{~ns}$ & 0.8 & $0^{+}$ & & & & & \\
\hline${ }^{72} \mathrm{As}$ & -68230 & 4 & & & & 26.0 & $\mathrm{~h}$ & 0.1 & $2^{-}$ & 95 & & & $\beta^{+}=100$ & \\
\hline${ }^{72} \mathrm{Se}$ & -67894 & 12 & & & & 8.40 & d & 0.08 & $0^{+}$ & 97 & & & $\varepsilon=100$ & \\
\hline${ }^{72} \mathrm{Br}$ & -59020 & 60 & & & & 78.6 & $\mathrm{~s}$ & 2.4 & $1^{+}$ & 95 & $03 \mathrm{Pi} 03$ & $\mathrm{~J}$ & $\beta^{+}=100$ & \\
\hline${ }^{72} \mathrm{Br}^{m}$ & -58920 & 60 & 100.92 & 0.03 & & 10.6 & $\mathrm{~s}$ & 0.3 & $1^{-}$ & 95 & & & $\mathrm{IT} \approx 100 ; \beta^{+}=?$ & \\
\hline${ }^{72} \mathrm{Kr}$ & -53941 & 8 & & & & 17.16 & $\mathrm{~s}$ & 0.18 & $0^{+}$ & 95 & $03 \mathrm{Pi} 03$ & $\mathrm{~T}$ & $\beta^{+}=100$ & * \\
\hline${ }^{72} \mathrm{Rb}$ & $-38120 \#$ & $500 \#$ & & & * & $<1.5$ & $\mu \mathrm{s}$ & & $3^{+} \#$ & 97 & $95 \mathrm{~B} 106$ & I & $\mathrm{p}$ ? & \\
\hline${ }^{72} \mathrm{Rb}^{m}$ & $-38020 \#$ & $510 \#$ & $100 \#$ & $100 \#$ & * & $1 \#$ & $\mu \mathrm{s}$ & & $1^{-} \#$ & & & & p? & \\
\hline$*^{72} \mathrm{Ni}$ & $\mathrm{T}:$ not use & ed $95 \mathrm{~A}$ & n. $A=1.30$ & 10) and 9 & $2 \mathrm{Be} \cdot \mathrm{A}=$ & $2.06(0.30$ & 0) (the & two of sal & Ime group & & & & & $* *$ \\
\hline$*^{72} \mathrm{Kr}$ & $\mathrm{T}:$ average & e $03 \mathrm{PiC}$ & $3=17.1(0.2$ & $73 \mathrm{Da} 22=$ & 17.4(0. & & & & & & & & & $* *$ \\
\hline${ }^{73} \mathrm{Co}$ & $-37040 \#$ & $700 \#$ & & & & $80 \#$ & $\mathrm{~ms}$ & $(>300 \mathrm{~ns})$ & $7 / 2^{-} \#$ & 02 & $97 \mathrm{Be} 70$ & I & $\beta^{-}$? & \\
\hline${ }^{73} \mathrm{Ni}$ & $-49860 \#$ & $300 \#$ & & & & 840 & $\mathrm{~ms}$ & 30 & $\left(9 / 2^{+}\right)$ & 02 & & & $\beta^{-}=100 ; \beta^{-} \mathrm{n} ?$ & \\
\hline${ }^{73} \mathrm{Cu}$ & -58987 & 4 & & & & 4.2 & $\mathrm{~s}$ & 0.3 & $\left(3 / 2^{-}\right)$ & 02 & $98 \mathrm{Fr} 15$ & $\mathrm{~J}$ & $\beta^{-}=100 ; \beta^{-} \mathrm{n} ?$ & \\
\hline${ }^{73} \mathrm{Zn}$ & -65410 & 40 & & & & 23.5 & $\mathrm{~s}$ & 1.0 & $(1 / 2)^{-}$ & 02 & & & $\beta^{-}=100$ & \\
\hline${ }^{73} \mathrm{Zn}^{m}$ & -65210 & 40 & 195.5 & 0.2 & & 13.0 & $\mathrm{~ms}$ & 0.2 & $\left(5 / 2^{+}\right)$ & 02 & & & $\mathrm{IT}=100$ & \\
\hline${ }^{73} \mathrm{Zn}^{n}$ & -65170 & 40 & 237.6 & 2.0 & $\mathrm{EU}$ & 5.8 & $\mathrm{~s}$ & 0.8 & $\left(7 / 2^{+}\right)$ & 02 & & & $\mathrm{IT}=? ; \beta^{-}=?$ & * \\
\hline${ }^{73} \mathrm{Ga}$ & -69699.3 & 1.7 & & & & 4.86 & $\mathrm{~h}$ & 0.03 & $3 / 2^{-}$ & 02 & & & $\beta^{-}=100$ & \\
\hline${ }^{73} \mathrm{Ge}$ & -71297.5 & 1.6 & & & & STABLE & & & $9 / 2^{+}$ & 02 & & & $\mathrm{IS}=7.735$ & \\
\hline${ }^{73} \mathrm{Ge}^{m}$ & -71284.2 & 1.6 & 13.2845 & 0.0015 & & 2.92 & $\mu \mathrm{s}$ & 0.03 & $5 / 2^{+}$ & 02 & & & $\mathrm{IT}=100$ & \\
\hline${ }^{73} \mathrm{Ge}^{n}$ & -71230.8 & 1.6 & 66.726 & 0.009 & & 499 & $\mathrm{~ms}$ & 11 & $1 / 2^{-}$ & 02 & & & $\mathrm{IT}=100$ & \\
\hline${ }^{73}$ As & -70957 & 4 & & & & 80.30 & $\mathrm{~d}$ & 0.06 & $3 / 2^{-}$ & 93 & & & $\varepsilon=100$ & \\
\hline${ }^{73} \mathrm{Se}$ & -68218 & 11 & & & & 7.15 & $\mathrm{~h}$ & 0.08 & $9 / 2^{+}$ & 03 & & & $\beta^{+}=100$ & \\
\hline${ }^{73} \mathrm{Se}^{m}$ & -68192 & 11 & 25.71 & 0.04 & & 39.8 & $\mathrm{~m}$ & 1.3 & $3 / 2^{-}$ & 03 & & & $\mathrm{IT}=72.63 ; \beta^{+}=27.43$ & \\
\hline${ }^{73} \mathrm{Br}$ & -63630 & 50 & & & & 3.4 & $\mathrm{~m}$ & 0.2 & $1 / 2^{-}$ & 02 & & & $\beta^{+}=100$ & \\
\hline${ }^{73} \mathrm{Kr}$ & -56552 & 7 & & & & 28.6 & $\mathrm{~s}$ & 0.6 & $3 / 2^{-}$ & 02 & $99 \mathrm{Mi} 17$ & $\mathrm{~T}$ & $\beta^{+}=100 ; \beta^{+} \mathrm{p}=0.253$ & * \\
\hline${ }^{73} \mathrm{Kr}^{m}$ & -56118 & 7 & 433.66 & 0.12 & & 107 & $\mathrm{~ns}$ & 10 & $\left(9 / 2^{+}\right)$ & 03 & & & $\mathrm{IT}=100$ & \\
\hline${ }^{73} \mathrm{Rb}$ & $-46050 \#$ & $150 \#$ & & & & $<30$ & ns & & $3 / 2^{-} \#$ & 03 & $96 \mathrm{Pf} 01$ & I & $\mathrm{p}$ ? & \\
\hline${ }^{73} \mathrm{Rb}^{m}$ & $-45620 \#$ & $180 \#$ & $430 \#$ & $100 \#$ & & & & & $9 / 2^{+} \#$ & & & & & \\
\hline${ }^{73} \mathrm{Sr}$ & $-31700 \#$ & $600 \#$ & & & & $>25$ & $\mathrm{~ms}$ & & $1 / 2^{-} \#$ & 03 & & & $\beta^{+}=100 ; \beta^{+} \mathrm{p}=?$ & \\
\hline$*^{73} \mathrm{Zn}^{n}$ & $\mathrm{E}:$ if 42.1 & $\operatorname{keV} \gamma$ & feeds ${ }^{73} \mathrm{Zn}^{n}$ & EU: see & liscussi & on in ENS & SDF'0 & & & & & & & ** \\
\hline$*^{73} \mathrm{Kr}$ & $\mathrm{T}:$ average & e 99Mi & $17=29.0(1$. & $81 \mathrm{Ha} 44$ & $=28.4(0$ & 7); 73Da & $22=2$ & $5.9(0.6$ & variar & & & & & $* *$ \\
\hline$*^{73} \mathrm{Kr}$ & $\mathrm{T}:$ & $t$ used & & & & & & & & & & & & $* *$ \\
\hline
\end{tabular}

\begin{tabular}{|c|c|c|c|c|c|c|c|c|c|c|c|c|}
\hline${ }^{74} \mathrm{Co}$ & $-32250 \#$ & $800 \#$ & & & $50 \#$ & $\mathrm{~ms}$ & $(>300 \mathrm{~ns})$ & & 03 & $97 \mathrm{Be} 70$ & I & $\beta^{-} ?$ \\
\hline${ }^{74} \mathrm{Ni}$ & $-48370 \#$ & $400 \#$ & & & 680 & $\mathrm{~ms}$ & 120 & $0^{+}$ & 03 & 98Fr15 & $\mathrm{T}$ & $\beta^{-}=100 ; \beta^{-} \mathrm{n} ?$ \\
\hline${ }^{74} \mathrm{Cu}$ & -56006 & 6 & & & 1.594 & $\mathrm{~s}$ & 0.010 & $1^{+} \#$ & 95 & & & $\beta^{-}=100$ \\
\hline${ }^{74} \mathrm{Zn}$ & -65710 & 50 & & & 95.6 & $\mathrm{~s}$ & 1.2 & $0^{+}$ & 95 & & & $\beta^{-}=100$ \\
\hline${ }^{74} \mathrm{Ga}$ & -68050 & 4 & & & 8.12 & $\mathrm{~m}$ & 0.12 & $\left(3^{-}\right)$ & 95 & & & $\beta^{-}=100$ \\
\hline${ }^{74} \mathrm{Ga}^{m}$ & -67990 & 4 & 59.571 & 0.014 & 9.5 & $\mathrm{~s}$ & 1.0 & $(0)$ & 95 & & & $\mathrm{IT}=? ; \beta^{-}=25 \#$ \\
\hline${ }^{74} \mathrm{Ge}$ & -73422.4 & 1.6 & & & StABle & & & $0^{+}$ & 95 & & & $\mathrm{IS}=36.2873$ \\
\hline${ }^{74} \mathrm{As}$ & -70860.0 & 2.3 & & & 17.77 & $\mathrm{~d}$ & 0.02 & $2^{-}$ & 95 & & & $\beta^{+}=662 ; \beta^{-}=342$ \\
\hline${ }^{74} \mathrm{Se}$ & -72212.7 & 1.7 & & & StABle & & & $0^{+}$ & 95 & & & $\mathrm{IS}=0.894 ; 2 \beta^{+} ?$ \\
\hline${ }^{74} \mathrm{Br}$ & -65306 & 15 & & & 25.4 & $\mathrm{~m}$ & 0.3 & $\left(0^{-}\right)$ & 95 & & & $\beta^{+}=100$ \\
\hline${ }^{74} \mathrm{Br}^{m}$ & -65292 & 15 & 13.58 & 0.21 & 46 & $\mathrm{~m}$ & 2 & $4^{(+\#)}$ & 95 & & & $\beta^{+}=100$ \\
\hline${ }^{74} \mathrm{Kr}$ & -62331.5 & 2.0 & & & 11.50 & $\mathrm{~m}$ & 0.11 & $0^{+}$ & 95 & & & $\beta^{+}=100$ \\
\hline${ }^{74} \mathrm{Kr}^{m}$ & -61824 & 10 & 508 & 10 & 29 & $\mathrm{~ns}$ & 6 & $0^{+}$ & & $00 \mathrm{Ch} 07$ & ETJ & $\mathrm{IT}=100$ \\
\hline${ }^{74} \mathrm{Rb}$ & -51917 & 4 & & & 64.76 & $\mathrm{~ms}$ & 0.03 & $\left(0^{+}\right)$ & 95 & 01Ba12 & $\mathrm{T}$ & $\beta^{+}=100$ \\
\hline${ }^{74} \mathrm{Sr}$ & $-40700 \#$ & $500 \#$ & & & $50 \#$ & $\mathrm{~ms}$ & $(>1.5 \mu \mathrm{s})$ & $0^{+}$ & 97 & $95 \mathrm{~B} 106$ & I & $\beta^{+} ?$ \\
\hline${ }^{4} \mathrm{Ni}$ & $\mathrm{T}$ : average & e $98 \mathrm{Erl}$ & (2) & $\mathrm{m}$ & & & & & & & & \\
\hline
\end{tabular}




\begin{tabular}{|c|c|c|c|c|c|c|c|c|c|c|c|c|c|}
\hline Nuclide & \multicolumn{2}{|c|}{$\begin{array}{l}\text { Mass excess } \\
(\mathrm{keV})\end{array}$} & \multicolumn{2}{|c|}{$\begin{array}{c}\text { Excitation } \\
\text { energy }(\mathrm{keV})\end{array}$} & \multicolumn{3}{|c|}{ Half-life } & $J^{\pi}$ & Ens & \multicolumn{2}{|c|}{ Reference } & \multicolumn{2}{|l|}{$\begin{array}{c}\text { Decay modes and } \\
\text { intensities }(\%)\end{array}$} \\
\hline${ }^{75} \mathrm{Co}$ & $-29500 \#$ & $800 \#$ & & & $40 \#$ & $\mathrm{~ms}($ & $(>300 \mathrm{~ns})$ & $7 / 2^{-} \#$ & 99 & 97Be70 & I & $\beta^{-} ?$ & \\
\hline${ }^{75} \mathrm{Ni}$ & $-43900 \#$ & $400 \#$ & & & 600 & $\mathrm{~ms}$ & 200 & $7 / 2^{+} \#$ & 99 & $85 \operatorname{Re} 01$ & $\mathrm{D}$ & $\beta^{-}=100 ; \beta^{-} \mathrm{n}=1.6 \#$ & * \\
\hline${ }^{75} \mathrm{Cu}$ & -54120 & 980 & & & 1.224 & $\mathrm{~s}$ & 0.003 & $3 / 2^{-} \#$ & 99 & & & $\beta^{-}=100 ; \beta^{-} \mathrm{n}=3.56$ & \\
\hline${ }^{75} \mathrm{Zn}$ & -62470 & 70 & & & 10.2 & $\mathrm{~s}$ & 0.2 & $7 / 2^{+} \#$ & 99 & & & $\beta^{-}=100$ & \\
\hline${ }^{75} \mathrm{Ga}$ & -68464.6 & 2.4 & & & 126 & $\mathrm{~s}$ & 2 & $(3 / 2)^{-}$ & 99 & & & $\beta^{-}=100$ & \\
\hline${ }^{75} \mathrm{Ge}$ & -71856.4 & 1.6 & & & 82.78 & $\mathrm{~m}$ & 0.04 & $1 / 2^{-}$ & 99 & & & $\beta^{-}=100$ & \\
\hline${ }^{75} \mathrm{Ge}^{m}$ & -71716.7 & 1.6 & 139.69 & 0.03 & 47.7 & $\mathrm{~s}$ & 0.5 & $7 / 2^{+}$ & 99 & & & $\mathrm{IT} \approx 100 ; \beta^{-}=0.0306$ & \\
\hline${ }^{75} \mathrm{As}$ & -73032.4 & 1.8 & & & StABLE & & & $3 / 2^{-}$ & 99 & & & $\mathrm{IS}=100$ & \\
\hline${ }^{75} \mathrm{As}^{m}$ & -72728.5 & 1.8 & 303.9241 & 0.0007 & 17.62 & $\mathrm{~ms}$ & 0.23 & $9 / 2^{+}$ & 99 & & & $\mathrm{IT}=100$ & \\
\hline${ }^{75} \mathrm{Se}$ & -72169.0 & 1.7 & & & 119.779 & $d$ & 0.004 & $5 / 2^{+}$ & 99 & & & $\varepsilon=100$ & \\
\hline${ }^{75} \mathrm{Br}$ & -69139 & 14 & & & 96.7 & $\mathrm{~m}$ & 1.3 & $3 / 2^{-}$ & 99 & & & $\beta^{+}=100$ & \\
\hline${ }^{75} \mathrm{Kr}$ & -64324 & 8 & & & 4.29 & $\mathrm{~m}$ & 0.17 & $5 / 2^{+}$ & 99 & & & $\beta^{+}=100$ & \\
\hline${ }^{75} \mathrm{Rb}$ & -57222 & 7 & & & 19.0 & $\mathrm{~s}$ & 1.2 & $\left(3 / 2^{-}\right)$ & 99 & & & $\beta^{+}=100$ & \\
\hline${ }^{75} \mathrm{Sr}$ & -46620 & 220 & & & 88 & $\mathrm{~ms}$ & 3 & $\left(3 / 2^{-}\right)$ & 99 & 03Hu01 & TJD & $\beta^{+}=100 ; \beta^{+} \mathrm{p}=5.29$ & \\
\hline$*^{75} \mathrm{Ni}$ & $\mathrm{D}: \beta^{-} \mathrm{n}=1$ & $1.6 \% \#$ & estimated & $85 \operatorname{Re} 01$ & & & & & & & & & $* *$ \\
\hline${ }^{76} \mathrm{Ni}$ & $-41610 \#$ & $900 \#$ & & & 470 & $\mathrm{~ms}$ & 390 & $0^{+}$ & 97 & 98Am04 & $\mathrm{T}$ & $\beta^{-}=100 ; \beta^{-} \mathrm{n} ?$ & \\
\hline${ }^{76} \mathrm{Cu}$ & -50976 & 7 & & & 641 & $\mathrm{~ms}$ & 6 & $(3,5)$ & 95 & 90Wi12 & $\mathrm{J}$ & $\beta^{-}=100 ; \beta^{-} \mathrm{n}=32$ & \\
\hline${ }^{76} \mathrm{Cu}^{m}$ & $-50980 \#$ & $200 \#$ & 0\# & $200 \#$ & 1.27 & $\mathrm{~s}$ & 0.30 & $(1,3)$ & 95 & 90Wi12 & $\mathrm{J}$ & $\beta^{-}=100$ & \\
\hline${ }^{76} \mathrm{Zn}$ & -62140 & 80 & & & 5.7 & $\mathrm{~s}$ & 0.3 & $0^{+}$ & 95 & & & $\beta^{-}=100$ & \\
\hline${ }^{76} \mathrm{Ga}$ & -66296.6 & 2.0 & & & 32.6 & $\mathrm{~s}$ & 0.6 & $\left(2^{+}, 3^{+}\right)$ & 95 & & & $\beta^{-}=100$ & \\
\hline${ }^{76} \mathrm{Ge}$ & -73213.0 & 1.7 & & & 1.58 & Zy & 0.17 & $0^{+}$ & 95 & $01 \mathrm{~K} 111$ & $\mathrm{~T}$ & $\mathrm{IS}=7.6138 ; 2 \beta^{-}=100$ & * \\
\hline${ }^{76} \mathrm{As}$ & -72289.5 & 1.8 & & & 1.0778 & $\mathrm{~d}$ & 0.0020 & $2^{-}$ & 95 & & & $\beta^{-} \approx 100 ; \varepsilon<0.02$ & \\
\hline${ }^{76} \mathrm{As}^{m}$ & -72245.1 & 1.8 & 44.425 & 0.001 & 1.84 & $\mu \mathrm{s}$ & 0.06 & $(1)^{+}$ & & & & & \\
\hline${ }^{76} \mathrm{Se}$ & -75252.1 & 1.7 & & & StABLE & & & $0^{+}$ & 95 & & & IS $=9.3729$ & \\
\hline${ }^{76} \mathrm{Br}$ & -70289 & 9 & & & 16.2 & $\mathrm{~h}$ & 0.2 & $1^{-}$ & 95 & & & $\beta^{+}=100$ & \\
\hline${ }^{76} \mathrm{Br}^{m}$ & -70186 & 9 & 102.58 & 0.03 & 1.31 & $\mathrm{~s}$ & 0.02 & $(4)^{+}$ & 95 & & & $\mathrm{IT}>99.4 ; \beta^{+}<0.6$ & \\
\hline${ }^{76} \mathrm{Kr}$ & -69014 & 4 & & & 14.8 & $\mathrm{~h}$ & 0.1 & $0^{+}$ & 95 & & & $\beta^{+}=100$ & \\
\hline${ }^{76} \mathrm{Rb}$ & -60479.8 & 1.9 & & & 36.5 & $\mathrm{~s}$ & 0.6 & $1^{(-)}$ & 95 & $78 \mathrm{Ha} 08$ & $\mathrm{D}$ & $\beta^{+}=100 ; \beta^{+} \alpha=3.8 \mathrm{e}-710$ & \\
\hline${ }^{76} \mathrm{Rb}^{m}$ & -60162.9 & 1.9 & 316.93 & 0.08 & 3.050 & $\mu \mathrm{s}$ & 0.007 & $\left(4^{+}\right)$ & 95 & $00 \mathrm{Ch} 07$ & $\mathrm{~T}$ & $\mathrm{IT}=100$ & \\
\hline${ }^{76} \mathrm{Sr}$ & -54240 & 40 & & & 8.9 & $\mathrm{~s}$ & 0.3 & $0^{+}$ & 95 & & & $\beta^{+}=100$ & \\
\hline${ }^{76} \mathrm{Y}$ & $-38700 \#$ & $500 \#$ & & & $500 \#$ & ns ( & $>170 \mathrm{~ns})$ & & & 00We.A & I & $\beta^{+} ? ; \mathrm{p}$ ? & * \\
\hline$*^{76} \mathrm{Ge}$ & $\mathrm{T}:$ from 01 & $1 \mathrm{~K} 111=$ & $=1.55(+0.1$ & $-0.15)$; oth & er results fror & m san & ne group: & & & & & & $* *$ \\
\hline$*^{76} \mathrm{Ge}$ & $\mathrm{T}: \quad 97 \mathrm{C}$ & Gu13=1 & $1.77(+0.13$ & $-0.11) 94 \mathrm{~B}$ & $15=1.42(0.1$ & & & & & & & & $* *$ \\
\hline$*^{76} \mathrm{Ge}$ & $\mathrm{T}:$ & her grou & ups $93 \mathrm{Br} 22$ & $=0.84(+0.1$ & $-0.08)(2 \sigma)$ & $90 \mathrm{Va}$ & $18=0.90(0$. & $.10)$ & & & & & $* *$ \\
\hline$*^{76} \mathrm{Ge}$ & $\mathrm{T}:$ & $\mathrm{d} 90 \mathrm{Mi} 2$ & $23=1.1(+0$ & $6-0.3)(2 \sigma)$ & & & & & & & & & $* *$ \\
\hline$*^{76} \mathrm{Ge}$ & TD : claim & $\mathrm{n}$ for $0 \mathrm{v}$ & $-\beta \beta 01 \mathrm{Kl}$ & $13=15 \mathrm{Yy} \mathrm{n}$ & t trusted. Se & ee alsc & $02 \mathrm{Aa}$. & and 02 & & & & & $* *$ \\
\hline$*^{76} \mathrm{Y}$ & $\mathrm{I}:$ also $01 \mathrm{~K}$ & $\mathrm{Ki} 13>2$ & $200 \mathrm{~ns}$, san & group & & & & & & & & & $* *$ \\
\hline
\end{tabular}

$\begin{array}{lllll}{ }^{77} \mathrm{Ni} & -36750 \# & 500 \# & & \\ { }^{77} \mathrm{Cu} & -48580 \# & 400 \# & & \\ { }^{77} \mathrm{Zn} & -58720 & 120 & & \\ { }^{77} \mathrm{Zn}^{m} & -57950 & 120 & 772.39 & 0.12 \\ { }^{77} \mathrm{Ga} & -65992.3 & 2.4 & & \\ { }^{77} \mathrm{Ge} & -71214.0 & 1.7 & & \\ { }^{77} \mathrm{Ge}^{m} & -71054.3 & 1.7 & 159.70 & 0.10 \\ { }^{77} \mathrm{As}^{m} & -73916.6 & 2.3 & & \\ { }^{77} \mathrm{As}^{m} & -73441.2 & 2.3 & 475.443 & 0.016 \\ { }^{77} \mathrm{Se} & -74599.6 & 1.7 & \\ { }^{77} \mathrm{Se} & -74437.7 & 1.7 & 161.9223 & 0.0007 \\ { }^{77} \mathrm{Br} & -73235 & 3 & & \\ { }^{77} \mathrm{Br} & -73129 & 3 & 105.86 & 0.08 \\ { }^{77} \mathrm{Kr} & -70169.4 & 2.0 & \\ { }^{77} \mathrm{Rb} & -64825 & 7 & \\ { }^{77} \mathrm{Sr} & -57804 & 9 & \\ { }^{77} \mathrm{Y} & -46910 \# & 60 \# \\ { }^{77} \mathrm{Y} & \mathrm{D}: \text { limit for } \mathrm{p} \text { is from 00We.A }\end{array}$

\begin{tabular}{|c|c|c|c|c|c|c|c|}
\hline 300\# & $\mathrm{ms}$ & $>300 \mathrm{~ns})$ & $9 / 2^{+} \#$ & 97 & $97 \mathrm{Be} 70$ & I & $\beta^{-} ?$ \\
\hline 469 & $\mathrm{~ms}$ & 8 & $3 / 2^{-} \#$ & 97 & & & $\beta^{-}=100$ \\
\hline 2.08 & $\mathrm{~s}$ & 0.05 & $7 / 2^{+} \#$ & 97 & & & $\beta^{-}=100$ \\
\hline 1.05 & $\mathrm{~s}$ & 0.10 & $1 / 2^{-} \#$ & 97 & & & $\mathrm{IT}>50 ; \beta^{-}<50$ \\
\hline 13.2 & $\mathrm{~s}$ & 0.2 & $\left(3 / 2^{-}\right)$ & 97 & & & $\beta^{-}=100$ \\
\hline 11.30 & $\mathrm{~h}$ & 0.01 & $7 / 2^{+}$ & 97 & & & $\beta^{-}=100$ \\
\hline 52.9 & $\mathrm{~s}$ & 0.6 & $1 / 2^{-}$ & 97 & & & $\beta^{-}=812 ; \mathrm{IT}=192$ \\
\hline 38.83 & $\mathrm{~h}$ & 0.05 & $3 / 2^{-}$ & 97 & & & $\beta^{-}=100$ \\
\hline 114.0 & $\mu \mathrm{s}$ & 2.5 & $9 / 2^{+}$ & 97 & & & $\mathrm{IT}=100$ \\
\hline STABLE & & & $1 / 2^{-}$ & 97 & & & $\mathrm{IS}=7.6316$ \\
\hline 17.36 & $\mathrm{~s}$ & 0.05 & $7 / 2^{+}$ & 97 & & & $\mathrm{IT}=100$ \\
\hline 57.036 & $\mathrm{~h}$ & 0.006 & $3 / 2^{-}$ & 97 & & & $\beta^{+}=100$ \\
\hline 4.28 & $\mathrm{~m}$ & 0.10 & $9 / 2^{+}$ & 97 & & & $\mathrm{IT}=100$ \\
\hline 74.4 & $\mathrm{~m}$ & 0.6 & $5 / 2^{+}$ & 97 & & & $\beta^{+}=100$ \\
\hline 3.77 & $\mathrm{~m}$ & 0.04 & $3 / 2^{-}$ & 97 & & & $\beta^{+}=100$ \\
\hline 9.0 & $\mathrm{~s}$ & 0.2 & $5 / 2^{+}$ & 97 & & & $\beta^{+}=100 ; \beta^{+} \mathrm{p}<0.25$ \\
\hline 63 & $\mathrm{~ms}$ & 17 & $5 / 2^{+} \#$ & 97 & $01 \mathrm{Ki} 13$ & $\mathrm{~T}$ & $\beta^{+}=? ; \beta^{+} \mathrm{p} ? ; \mathrm{p}<10$ \\
\hline
\end{tabular}




\begin{tabular}{|c|c|c|c|c|c|c|c|c|c|c|c|c|c|}
\hline Nuclide & \multicolumn{2}{|c|}{$\begin{array}{l}\text { Mass excess } \\
\quad(\mathrm{keV})\end{array}$} & \multicolumn{2}{|c|}{$\begin{array}{l}\text { Excitation } \\
\text { energy }(\mathrm{keV})\end{array}$} & \multicolumn{3}{|c|}{ Half-life } & \multirow{2}{*}{$\begin{array}{c}J^{\pi} \\
0^{+}\end{array}$} & \multirow{2}{*}{$\begin{array}{c}\text { Ens } \\
97\end{array}$} & \multicolumn{2}{|c|}{ Reference } & \multicolumn{2}{|l|}{$\begin{array}{l}\text { Decay modes and } \\
\text { intensities }(\%)\end{array}$} \\
\hline${ }^{78} \mathrm{Ni}$ & $-34300 \#$ & 1100\# & & & $200 \#$ & $\mathrm{~ms}$ & $(>300 \mathrm{~ns})$ & & & $97 \mathrm{Be} 70$ & I & $\beta^{-} ?$ & \\
\hline${ }^{78} \mathrm{Cu}$ & $-44750 \#$ & 400\# & & & 342 & $\mathrm{~ms}$ & 11 & & 97 & $91 \mathrm{Kr} 15$ & $\mathrm{~T}$ & $\beta^{-}=100$ & \\
\hline${ }^{78} \mathrm{Zn}$ & -57340 & 90 & & & 1.47 & $\mathrm{~s}$ & 0.15 & $0^{+}$ & 91 & & & $\beta^{-}=100$ & \\
\hline${ }^{78} \mathrm{Zn}^{m}$ & -54670 & 90 & 2673 & 1 & 319 & ns & 9 & $\left(8^{+}\right)$ & & 00Da07 & ET & $\mathrm{IT}=100$ & \\
\hline${ }^{78} \mathrm{Ga}$ & -63706.6 & 2.4 & & & 5.09 & $\mathrm{~s}$ & 0.05 & $\left(3^{+}\right)$ & 91 & & & $\beta^{-}=100$ & \\
\hline${ }^{78} \mathrm{Ge}$ & -71862 & 4 & & & 88 & $\mathrm{~m}$ & 1 & $0^{+}$ & 91 & & & $\beta^{-}=100$ & \\
\hline${ }^{78} \mathrm{As}$ & -72817 & 10 & & & 90.7 & $\mathrm{~m}$ & 0.2 & $2^{-}$ & 91 & & & $\beta^{-}=100$ & \\
\hline${ }^{78} \mathrm{Se}$ & -77026.1 & 1.7 & & & STABLE & & & $0^{+}$ & 91 & & & IS $=23.7728$ & \\
\hline${ }^{78} \mathrm{Br}$ & -73452 & 4 & & & 6.46 & $\mathrm{~m}$ & 0.04 & $1^{+}$ & 91 & & & $\beta^{+} \approx 100 ; \beta^{-}<0.01$ & * \\
\hline${ }^{78} \mathrm{Br}^{m}$ & -73271 & 4 & 180.82 & 0.13 & 119.2 & $\mu \mathrm{s}$ & & $4^{+}$ & & & & & \\
\hline${ }^{78} \mathrm{Kr}$ & -74179.7 & 1.1 & & & STABLE & & $(>110 \mathrm{Ey})$ & $0^{+}$ & 91 & $94 \mathrm{Sa} 31$ & $\mathrm{~T}$ & $\mathrm{IS}=0.351 ; 2 \beta^{+} ?$ & $*$ \\
\hline${ }^{78} \mathrm{Rb}$ & -66936 & 7 & & & 17.66 & $\mathrm{~m}$ & 0.08 & $0^{(+)}$ & 91 & & & $\beta^{+}=100$ & \\
\hline${ }^{78} \mathrm{Rb}^{m}$ & -66825 & 7 & 111.20 & 0.10 & 5.74 & $\mathrm{~m}$ & 0.05 & $4^{(-)}$ & 91 & 91Mc.A & $\mathrm{E}$ & $\beta^{+}=902 ; \mathrm{IT}=102$ & \\
\hline${ }^{78} \mathrm{Rb}^{x}$ & -66862 & 14 & 74 & 12 & $R=2.00 .5$ & & & spmix & & & & & \\
\hline${ }^{78} \mathrm{Sr}$ & -63174 & 7 & & & 159 & $\mathrm{~s}$ & 8 & $0^{+}$ & 91 & 92Gr09 & $\mathrm{T}$ & $\beta^{+}=100$ & \\
\hline${ }^{78} \mathrm{Y}$ & $-52530 \#$ & $400 \#$ & & & 54 & $\mathrm{~ms}$ & 5 & $\left(0^{+}\right)$ & 97 & $01 \mathrm{Ga} 24$ & TJD & $\beta^{+}=100 ; \beta^{+} \mathrm{p}$ ? & $*$ \\
\hline${ }^{78} \mathrm{Y}^{m}$ & $-52530 \#$ & $640 \#$ & $0 \#$ & $500 \#$ & 5.8 & $\mathrm{~s}$ & 0.5 & $5^{+} \#$ & & $01 \mathrm{Ki} 13$ & TD & $\beta^{+}=100 ; \beta^{+} \mathrm{p}$ ? & $*$ \\
\hline${ }^{78} \mathrm{Zr}$ & $-41700 \#$ & $500 \#$ & & & $50 \#$ & $\mathrm{~ms}$ & $(>170 \mathrm{~ns})$ & $0^{+}$ & & 00We.A & & $\beta^{+} ? ; \beta^{+} \mathrm{p} ?$ & $*$ \\
\hline$*^{78} \mathrm{Br}$ & $\mathrm{D}: \beta^{-}$bral & anch is $\mathrm{u}$ & ncertain. & ee ENSD & & & & & & & & & $* *$ \\
\hline$*^{78} \mathrm{Kr}$ & $\mathrm{T}$ : limit gi & iven here & is for the & $\mathrm{K}-\mathrm{e}^{+} \mathrm{dec}$ & y (theoretically & faste & & & & & & & $* *$ \\
\hline$*^{78} \mathrm{Y}$ & $\mathrm{T}:$ average & e $01 \mathrm{Ga} 2$ & $4=50(8) 0$ & $\mathrm{Ki} 13=55$ & $+9-6)$ & & & & & & & & $* *$ \\
\hline$*^{78} \mathrm{Y}^{m}$ & $\mathrm{~T}$ : average & e $01 \mathrm{Ki} 13$ & $=5.7(0.7)$ & 98Uu01= & $.8(0.6)$ & & & & & & & & $* *$ \\
\hline$*^{78} \mathrm{Zr}$ & $\mathrm{I}:$ also $01 \mathrm{~K}$ & $\mathrm{Ki} 13>2 \mathrm{C}$ & $0 \mathrm{~ns}$ sam & group & & & & & & & & & $* *$ \\
\hline
\end{tabular}

\begin{tabular}{|c|c|c|c|c|}
\hline${ }^{79} \mathrm{Cu}$ & $-42330 \#$ & $500 \#$ & & \\
\hline${ }^{79} \mathrm{Zn}$ & $-53420 \#$ & $260 \#$ & & \\
\hline${ }^{79} \mathrm{Ga}$ & -62510 & 100 & & \\
\hline${ }^{79} \mathrm{Ge}$ & -69490 & 90 & & \\
\hline${ }^{79} \mathrm{Ge}^{m}$ & -69300 & 90 & 185.95 & 0.0 \\
\hline${ }^{79} \mathrm{As}$ & -73637 & 6 & & \\
\hline${ }^{79} \mathrm{As}^{m}$ & -72864 & 6 & 772.81 & 0.0 \\
\hline${ }^{79} \mathrm{Se}$ & -75917.6 & 1.7 & & \\
\hline${ }^{79} \mathrm{Se}^{m}$ & -75821.8 & 1.7 & 95.77 & 0.0 \\
\hline${ }^{79} \mathrm{Br}$ & -76068.5 & 2.0 & & \\
\hline${ }^{79} \mathrm{Br}^{m}$ & -75860.9 & 2.0 & 207.61 & 0.0 \\
\hline${ }^{79} \mathrm{Kr}$ & -74443 & 4 & & \\
\hline${ }^{79} \mathrm{Kr}^{m}$ & -74313 & 4 & 129.77 & 0.0 \\
\hline${ }^{79} \mathrm{Kr}^{n}$ & -74296 & 4 & 147.06 & 0.0 \\
\hline${ }^{79} \mathrm{Rb}$ & -70803 & 6 & & \\
\hline${ }^{79} \mathrm{Sr}$ & -65477 & 8 & & \\
\hline${ }^{79} \mathrm{Y}$ & -58360 & 450 & & \\
\hline${ }^{79} \mathrm{Zr}$ & $-47360 \#$ & $400 \#$ & & \\
\hline$*^{79} \mathrm{As}^{m}$ & $\mathrm{~T}: 98 \mathrm{Hol}$ & $=0.87$ & 6) ou & hec \\
\hline
\end{tabular}

\begin{tabular}{|c|c|c|c|c|c|}
\hline 188 & $\mathrm{~ms}$ & 25 & $3 / 2^{-} \# 02$ & & $\beta^{-}=100 ; \beta^{-} \mathrm{n}=5517$ \\
\hline 995 & $\mathrm{~ms}$ & 19 & $\left(9 / 2^{+}\right) 02$ & & $\beta^{-}=100 ; \beta^{-} \mathrm{n}=1.34$ \\
\hline 2.847 & $\mathrm{~s}$ & 0.003 & $3 / 2^{-} \# 02$ & & $\beta^{-}=100 ; \beta^{-} \mathrm{n}=0.08919$ \\
\hline 18.98 & $\mathrm{~s}$ & 0.03 & $(1 / 2)^{-} 02$ & & $\beta^{-}=100$ \\
\hline 39.0 & s & 1.0 & $7 / 2^{+} \# 02$ & & $\beta^{-}=961 ; \mathrm{IT}=41$ \\
\hline 9.01 & $\mathrm{~m}$ & 0.15 & $3 / 2^{-} \quad 02$ & & $\beta^{-}=100$ \\
\hline 1.21 & $\mu \mathrm{s}$ & 0.01 & $(9 / 2)^{+} 02$ & $98 \mathrm{Gr} 14 \mathrm{~T}$ & $\mathrm{IT}=100$ \\
\hline 295 & ky & 38 & $7 / 2^{+} \quad 02$ & & $\beta^{-}=100$ \\
\hline 3.92 & $\mathrm{~m}$ & 0.01 & $1 / 2^{-} \quad 02$ & & $\mathrm{IT} \approx 100 ; \beta^{-}=0.05611$ \\
\hline STABLE & & & $3 / 2^{-} \quad 02$ & & $\mathrm{IS}=50.697$ \\
\hline 4.86 & s & 0.04 & $\left(9 / 2^{+}\right) 02$ & & $\mathrm{IT}=100$ \\
\hline 35.04 & $\mathrm{~h}$ & 0.10 & $1 / 2^{-} \quad 02$ & & $\beta^{+}=100$ \\
\hline 50 & $\mathrm{~s}$ & 3 & $7 / 2^{+} \quad 02$ & & $\mathrm{IT}=100$ \\
\hline 78.7 & ns & 1.0 & $\left(5 / 2^{-}\right) 02$ & & $\mathrm{IT}=100$ \\
\hline 22.9 & $\mathrm{~m}$ & 0.5 & $5 / 2^{+} \quad 02$ & & $\beta^{+}=100$ \\
\hline 2.25 & $\mathrm{~m}$ & 0.10 & $3 / 2^{(-)} 02$ & & $\beta^{+}=100$ \\
\hline 14.8 & s & 0.6 & $5 / 2^{+} \# 02$ & & $\beta^{+}=100 ; \beta^{+} \mathrm{p} ?$ \\
\hline 56 & $\mathrm{~ms}$ & 30 & $5 / 2^{+} \# 02$ & & $\beta^{+}=100 ; \beta^{+} \mathrm{p} ?$ \\
\hline
\end{tabular}

$\begin{array}{lllllr}{ }^{80} \mathrm{Cu} & -36450 \# & 600 \# & & & 100 \# \\ { }^{80} \mathrm{Zn} & -51840 & 170 & & & 5 \\ { }^{80} \mathrm{Ga} & -59140 & 120 & & & 1.697 \\ { }^{80} \mathrm{Ge} & -69515 & 28 & & & 29.5 \\ { }^{80} \mathrm{As} & -72159 & 23 & & & 15.2 \\ { }^{80} \mathrm{Se} & -77759.9 & 2.0 & & & \mathrm{ST} A \\ { }^{80} \mathrm{Br} & -75889.5 & 2.0 & & & 17.68 \\ { }^{80} \mathrm{Br} & -75803.7 & 2.0 & 85.843 & 0.004 & 4.4 \\ { }^{80} \mathrm{Kr} & -77892.5 & 1.5 & & & \mathrm{ST} \\ { }^{80} \mathrm{Rb} & -72173 & 7 & & & 3 \\ { }^{80} \mathrm{Rb} & -71679 & 7 & 494.4 & 0.5 & 1.6 \\ { }^{80} \mathrm{Sr} & -70308 & 7 & & & 106.3 \\ { }^{80} \mathrm{Y} & -61220 & 180 & & & 30.1 \\ { }^{80} \mathrm{Y}^{m} & -60990 & 180 & 228.5 & 0.1 & 4.8 \\ { }^{80} \mathrm{Y}^{n} & -60910 & 180 & 312.5 & 1.0 & 4.7\end{array}$

\begin{tabular}{|c|c|c|c|c|c|c|c|}
\hline $100 \#$ & $\mathrm{~ms}$ & $(>300 \mathrm{~ns})$ & & 97 & $97 \mathrm{Be} 70$ & I & $\beta^{-} ?$ \\
\hline 545 & $\mathrm{~ms}$ & 16 & $0^{+}$ & 92 & & & $\beta^{-}=100 ; \beta^{-} \mathrm{n}=1.05$ \\
\hline 1.697 & $\mathrm{~s}$ & 0.011 & (3) & 92 & 93Ru01 & $\mathrm{D}$ & $\beta^{-}=100 ; \beta^{-} \mathrm{n}=0.896$ \\
\hline 29.5 & $\mathrm{~s}$ & 0.4 & $0^{+}$ & 92 & & & $\beta^{-}=100$ \\
\hline 15.2 & $\mathrm{~s}$ & 0.2 & $1^{+}$ & 92 & & & $\beta^{-}=100$ \\
\hline STABLE & & & $0^{+}$ & 92 & & & $\mathrm{IS}=49.6141 ; 2 \beta^{-} ?$ \\
\hline 17.68 & $\mathrm{~m}$ & 0.02 & $1^{+}$ & 92 & & & $\beta^{-}=91.72 ; \beta^{+}=8.32$ \\
\hline 4.4205 & $\mathrm{~h}$ & 0.0008 & $5^{-}$ & 92 & & & $\mathrm{IT}=100$ \\
\hline STABLE & & & $0^{+}$ & 92 & & & $\mathrm{IS}=2.286$ \\
\hline 33.4 & $\mathrm{~s}$ & 0.7 & $1^{+}$ & 92 & 93Al03 & $\mathrm{T}$ & $\beta^{+}=100$ \\
\hline 1.6 & $\mu \mathrm{s}$ & 0.02 & $6^{+}$ & & 92Do10 & $\mathrm{E}$ & \\
\hline 106.3 & $\mathrm{~m}$ & 1.5 & $0^{+}$ & 99 & & & $\beta^{+}=100$ \\
\hline 30.1 & $\mathrm{~s}$ & 0.5 & $4^{-}$ & 92 & 98Do04 & TJ & $\beta^{+}=100$ \\
\hline 4.8 & $\mathrm{~s}$ & 0.3 & $\left(1^{-}\right)$ & & 98Do04 & ETJ & $\mathrm{IT}=812 ; \beta^{+}=192$ \\
\hline 4.7 & $\mu \mathrm{s}$ & 0.3 & $\left(2^{+}\right)$ & & $00 \mathrm{Ch} 07$ & ETJ & $\mathrm{IT}=100$ \\
\hline
\end{tabular}
.. A-group is continued on next page ... 


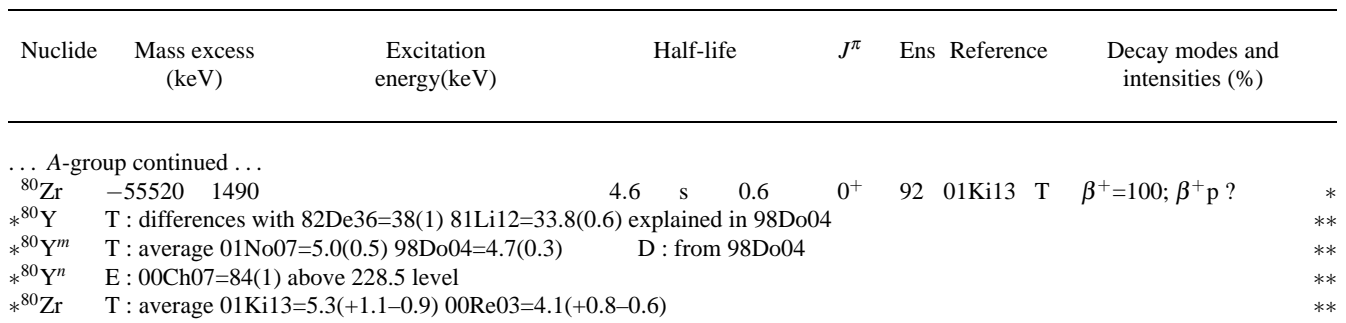

\begin{tabular}{|c|c|c|c|c|c|c|c|c|c|c|c|c|c|}
\hline${ }^{81} \mathrm{Zn}$ & $-46130 \#$ & $300 \#$ & & & & 290 & $\mathrm{~ms}$ & 50 & $5 / 2^{+} \#$ & 97 & & $\beta^{-}=100 ; \beta^{-} \mathrm{n}=7.530$ & \\
\hline${ }^{81} \mathrm{Ga}$ & -57980 & 190 & & & & 1.217 & $\mathrm{~s}$ & 0.005 & $\left(5 / 2^{-}\right)$ & 97 & & $\beta^{-}=100 ; \beta^{-} \mathrm{n}=11.97$ & \\
\hline${ }^{81} \mathrm{Ge}$ & -66300 & 120 & & & & 8 & $\mathrm{~s}$ & 2 & $9 / 2^{+} \#$ & 97 & & $\beta^{-}=100$ & * \\
\hline${ }^{81} \mathrm{Ge}^{m}$ & -65620 & 120 & 679.13 & 0.04 & & 8 & s & 2 & $\left(1 / 2^{+}\right)$ & 97 & & $\beta^{-} \approx 100 ; \mathrm{IT}<1$ & \\
\hline${ }^{81}$ As & -72533 & 6 & & & & 33.3 & s & 0.8 & $3 / 2^{-}$ & 97 & & $\beta^{-}=100$ & \\
\hline${ }^{81} \mathrm{Se}$ & -76389.5 & 2.0 & & & & 18.45 & $\mathrm{~m}$ & 0.12 & $1 / 2^{-}$ & 97 & & $\beta^{-}=100$ & \\
\hline${ }^{81} \mathrm{Se}^{m}$ & -76286.5 & 2.0 & 102.99 & 0.06 & & 57.28 & $\mathrm{~m}$ & 0.02 & $7 / 2^{+}$ & 97 & & $\mathrm{IT} \approx 100 ; \beta^{-}=0.05214$ & \\
\hline${ }^{81} \mathrm{Br}$ & -77974.8 & 2.0 & & & & StABLE & & & $3 / 2^{-}$ & 97 & & $\mathrm{IS}=49.317$ & \\
\hline${ }^{81} \mathrm{Br}^{m}$ & -77438.6 & 2.0 & 536.20 & 0.09 & & 34.6 & $\mu \mathrm{s}$ & & $9 / 2^{+}$ & & & & \\
\hline${ }^{81} \mathrm{Kr}$ & -77694.0 & 2.0 & & & & 229 & ky & 11 & $7 / 2^{+}$ & 97 & & $\varepsilon=100$ & \\
\hline${ }^{81} \mathrm{Kr}^{m}$ & -77503.4 & 2.0 & 190.62 & 0.04 & & 13.10 & $\mathrm{~s}$ & 0.03 & $1 / 2^{-}$ & 97 & & $\mathrm{IT} \approx 100 ; \varepsilon=0.00254$ & \\
\hline${ }^{81} \mathrm{Rb}$ & -75455 & 6 & & & & 4.576 & h & 0.005 & $3 / 2^{-}$ & 97 & & $\beta^{+}=100$ & \\
\hline${ }^{81} \mathrm{Rb}^{m}$ & -75369 & 6 & 86.31 & 0.07 & & 30.5 & $\mathrm{~m}$ & 0.3 & $9 / 2^{+}$ & 97 & & $\mathrm{IT}=97.66 ; \beta^{+}=2.46$ & \\
\hline${ }^{81} \mathrm{Sr}$ & -71528 & 6 & & & & 22.3 & $\mathrm{~m}$ & 0.4 & $1 / 2^{-}$ & 99 & & $\beta^{+}=100$ & \\
\hline${ }^{81} \mathrm{Y}$ & -66020 & 60 & & & & 70.4 & $\mathrm{~s}$ & 1.0 & $\left(5 / 2^{+}\right)$ & 98 & & $\beta^{+}=100$ & \\
\hline${ }^{81} \mathrm{Zr}$ & -58490 & 170 & & & & 5.5 & s & 0.4 & $3 / 2^{-} \#$ & 00 & & $\beta^{+}=100 ; \beta^{+} \mathrm{p}=0.122$ & \\
\hline${ }^{81} \mathrm{Nb}$ & $-47480 \#$ & $1500 \#$ & & & & $<44$ & ns & & $3 / 2^{-} \#$ & 97 & 00We.A I & $\mathrm{p} ? ; \beta^{+} ? ; \beta^{+} \mathrm{p} ?$ & * \\
\hline$*^{81} \mathrm{Ge}$ & \multicolumn{11}{|c|}{$\mathrm{T}$ : derived from $7.6(0.6)$, for mixture of ground-state and isomer with almost same half-life } & & $* *$ \\
\hline$*^{81} \mathrm{Nb}$ & $\mathrm{I}:$ also $99 \mathrm{~J}$ & $\mathrm{Ja} 02<80$ & $01 \mathrm{Ki} 13<2$ & $0 \mathrm{~ns}$ & $\mathrm{~T}$ : est & timated $\mathrm{h}$ & alf-li & fe for $\beta^{+}$: & $100 \# \mathrm{~m}$ & & & & ** \\
\hline${ }^{82} \mathrm{Zn}$ & $-42460 \#$ & $500 \#$ & & & & $100 \#$ & $\mathrm{~ms}($ & $>300 \mathrm{~ns})$ & $0^{+}$ & 03 & 97Be70 I & $\beta^{-} ?$ & \\
\hline${ }^{82} \mathrm{Ga}$ & $-53100 \#$ & $300 \#$ & & & & 599 & $\mathrm{~ms}$ & 2 & $(1,2,3)$ & 03 & 93Ru01 D & $\beta^{-}=100 ; \beta^{-} \mathrm{n}=21.313$ & $*$ \\
\hline${ }^{82} \mathrm{Ge}$ & -65620 & 240 & & & & 4.55 & $\mathrm{~s}$ & 0.05 & $0^{+}$ & 03 & & $\beta^{-}=100$ & \\
\hline${ }^{82} \mathrm{As}$ & -70320 & 200 & & & * & 19.1 & s & 0.5 & $\left(1^{+}\right)$ & 03 & & $\beta^{-}=100$ & \\
\hline${ }^{82} \mathrm{As}^{m}$ & -70075 & 25 & 250 & 200 & $\mathrm{BD} *$ & 13.6 & s & 0.4 & $\left(5^{-}\right)$ & 03 & & $\beta^{-}=100$ & \\
\hline${ }^{82} \mathrm{Se}$ & -77594.0 & 2.0 & & & & 97 & Ey & 5 & $0^{+}$ & 03 & 99Pi08 T & $\mathrm{IS}=8.7322 ; 2 \beta^{-}=100$ & $*$ \\
\hline${ }^{82} \mathrm{Br}$ & -77496.5 & 1.9 & & & & 35.282 & $\mathrm{~h}$ & 0.007 & $5^{-}$ & 03 & & $\beta^{-}=100$ & \\
\hline${ }^{82} \mathrm{Br}^{m}$ & -77450.6 & 1.9 & 45.9492 & 0.0010 & & 6.13 & $\mathrm{~m}$ & 0.05 & $2^{-}$ & 03 & & $\mathrm{IT}=97.63 ; \beta^{-}=2.43$ & \\
\hline${ }^{82} \mathrm{Kr}$ & -80589.5 & 1.8 & & & & STABLE & & & $0^{+}$ & 03 & & $\mathrm{IS}=11.5814$ & \\
\hline${ }^{82} \mathrm{Rb}$ & -76188.2 & 2.8 & & & & 1.273 & $\mathrm{~m}$ & 0.002 & $1^{+}$ & 03 & & $\beta^{+}=100$ & \\
\hline${ }^{82} \mathrm{Rb}^{m}$ & -76119.1 & 2.4 & 69.1 & 1.5 & MD & 6.472 & $\mathrm{~h}$ & 0.006 & $5^{-}$ & 03 & & $\beta^{+} \approx 100 ;$ IT $<0.33$ & \\
\hline${ }^{82} \mathrm{Sr}$ & -76008 & 6 & & & & 25.36 & d & 0.03 & $0^{+}$ & 03 & $87 \mathrm{Ho} 06 \mathrm{~T}$ & $\varepsilon=100$ & $*$ \\
\hline${ }^{82} \mathrm{Y}$ & -68190 & 100 & & & & 8.30 & s & 0.20 & $1^{+}$ & 03 & & $\beta^{+}=100$ & \\
\hline${ }^{82} \mathrm{Y}^{m}$ & -67790 & 100 & 402.63 & 0.14 & & 268 & ns & 25 & $4^{-}$ & 03 & & $\mathrm{IT}=100$ & \\
\hline${ }^{82} \mathrm{Zr}$ & $-64190 \#$ & $230 \#$ & & & & 32 & $\mathrm{~s}$ & 5 & $0^{+}$ & 03 & & $\beta^{+}=100$ & \\
\hline${ }^{82} \mathrm{Nb}$ & $-52970 \#$ & $300 \#$ & & & & 51 & $\mathrm{~ms}$ & 5 & $0^{+}$ & 03 & $01 \mathrm{Ga} 24 \mathrm{~T}$ & $\beta^{+}=100 ; \beta^{+} \mathrm{p} ?$ & $*$ \\
\hline$*^{82} \mathrm{Ga}$ & $\mathrm{D}$ : average & e $93 \mathrm{Ru} 01$ & $=31.1(4.4)$ & 86Wa17 & $19.8(1.7$ & $80 \mathrm{Lu} 0$ & $4=21$ & $4(2.2)$ & & & & & $* *$ \\
\hline$*^{82} \mathrm{Se}$ & $\mathrm{T}:$ average & e 99Pi08= & $=83(+9-7)$ & $8 \operatorname{Ar} 10=8$ & $3(12) 92$ & $\mathrm{E} 107=10$ & $8(+2$ & 5-6) 881 & $11=120($ & & & & $* *$ \\
\hline$*^{82} \mathrm{Sr}$ & $\mathrm{T}:$ average & e $87 \mathrm{Ho} 0 \mathrm{C}$ & $=25.36(0.0$ & 3) $87 \mathrm{Ju} 02$ & $=25.342$ & $(0.053)$ & & & & & & & ** \\
\hline$*^{82} \mathrm{Nb}$ & $\mathrm{T}$ : average & $\mathrm{e} 01 \mathrm{Ga} 24$ & $=52(6) 01 \mathrm{~K}$ & i1 $13=48(+$ & $8-6)$ & & & & & & & & $* *$ \\
\hline
\end{tabular}

\begin{tabular}{|c|c|c|c|c|c|c|c|c|c|c|}
\hline${ }^{83} \mathrm{Zn}$ & $-36300 \#$ & $500 \#$ & & & $80 \#$ & $\mathrm{~ms}$ & $300 \mathrm{~ns})$ & $5 / 2^{+} \#$ & $0197 \mathrm{Be} 70 \mathrm{I}$ & $\beta^{-} ?$ \\
\hline${ }^{83} \mathrm{Ga}$ & $-49390 \#$ & $300 \#$ & & & 308 & $\mathrm{~ms}$ & 1 & $3 / 2^{-} \#$ & 01 & $\beta^{-}=100 ; \beta^{-} \mathrm{n}=3717$ \\
\hline${ }^{83} \mathrm{Ge}$ & $-60900 \#$ & $200 \#$ & & & 1.85 & $\mathrm{~s}$ & 0.06 & $5 / 2^{+} \#$ & 01 & $\beta^{-}=100$ \\
\hline${ }^{83} \mathrm{As}$ & -69880 & 220 & & & 13.4 & $\mathrm{~s}$ & 0.3 & $3 / 2^{-} \#$ & 01 & $\beta^{-}=100$ \\
\hline${ }^{83} \mathrm{Se}$ & -75341 & 4 & & & 22.3 & $\mathrm{~m}$ & 0.3 & $9 / 2^{+}$ & 01 & $\beta^{-}=100$ \\
\hline${ }^{83} \mathrm{Se}^{m}$ & -75113 & 4 & 228.50 & 0.20 & 70.1 & $\mathrm{~s}$ & 0.4 & $1 / 2^{-}$ & 01 & $\beta^{-}=100$ \\
\hline${ }^{83} \mathrm{Br}$ & -79009 & 4 & & & 2.40 & $\mathrm{~h}$ & 0.02 & $3 / 2^{-}$ & 01 & $\beta^{-}=100$ \\
\hline${ }^{83} \mathrm{Br}^{m}$ & -75940 & 4 & 3068.8 & 0.6 & 700 & $\mathrm{~ns}$ & 100 & $\left(19 / 2^{-}\right)$ & 01 & $\mathrm{IT}=100$ \\
\hline${ }^{83} \mathrm{Kr}$ & -79981.7 & 2.8 & & & StABLE & & & $9 / 2^{+}$ & 01 & $\mathrm{IS}=11.496$ \\
\hline${ }^{83} \mathrm{Kr}^{m}$ & -79972.3 & 2.8 & 9.4053 & 0.0008 & 154.4 & $\mathrm{~ns}$ & 1.1 & $7 / 2^{+}$ & 01 & $\mathrm{IT}=100$ \\
\hline${ }^{83} \mathrm{Kr}^{n}$ & -79940.1 & 2.8 & 41.5569 & 0.0010 & 1.83 & $\mathrm{~h}$ & 0.02 & $1 / 2^{-}$ & 01 & $\mathrm{IT}=100$ \\
\hline
\end{tabular}




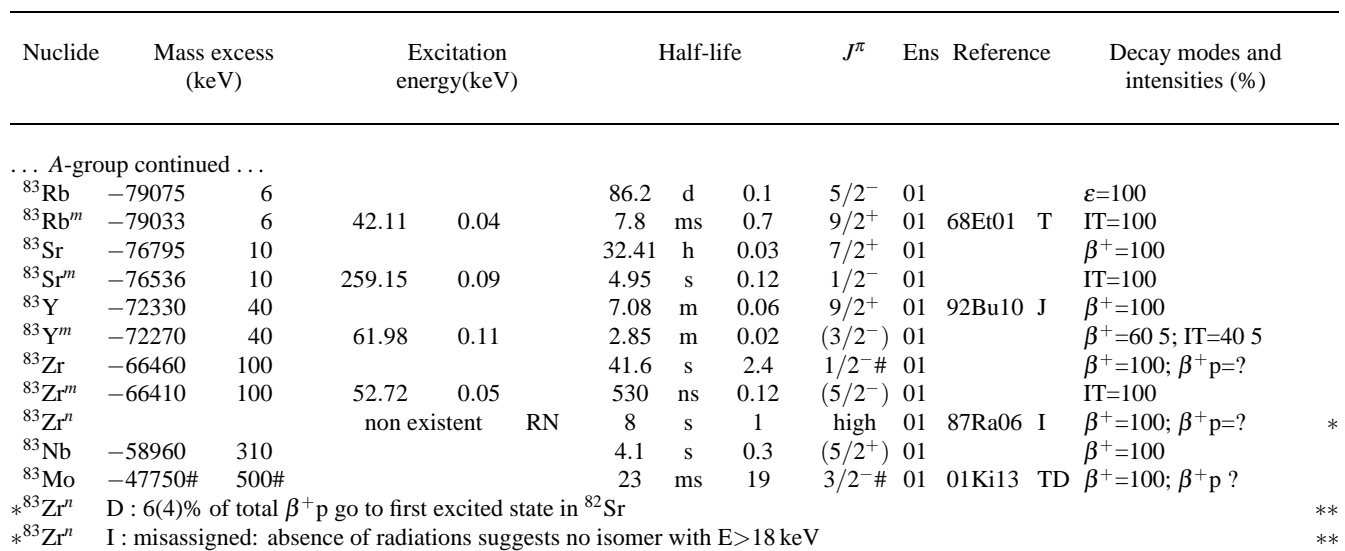

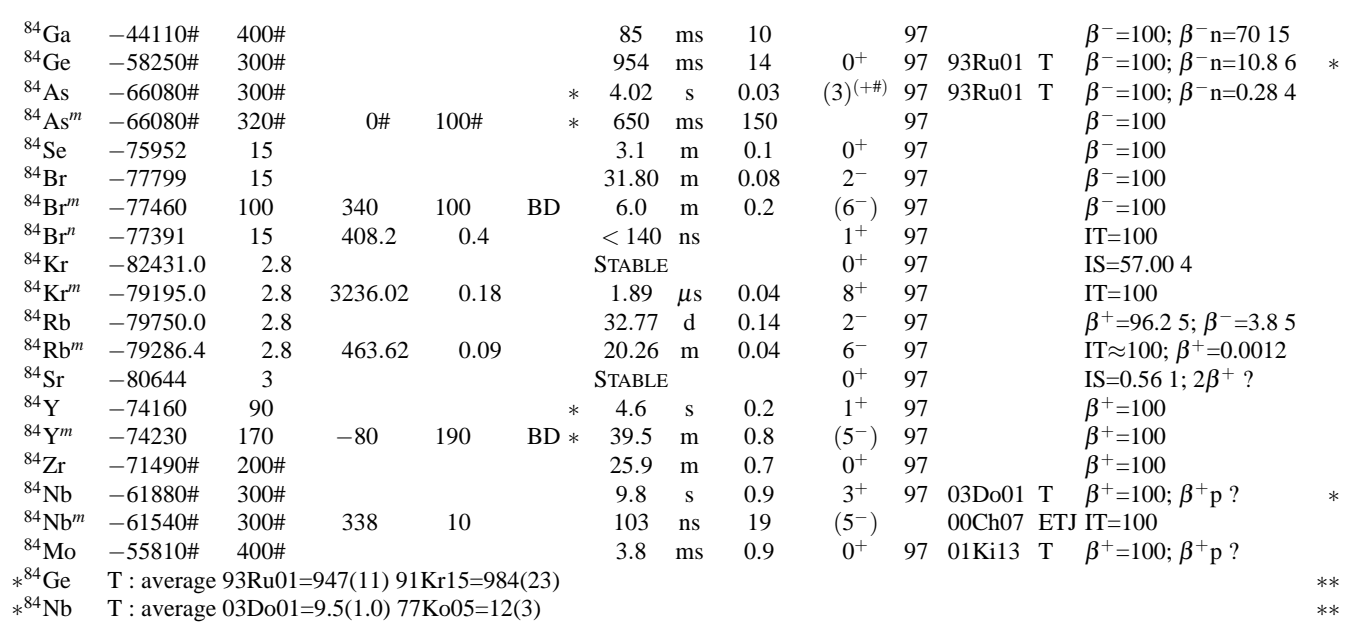

\begin{tabular}{|c|c|c|c|c|c|c|c|c|c|c|c|c|}
\hline${ }^{85} \mathrm{Ga}$ & $-40050 \#$ & $500 \#$ & & & $50 \#$ & $\mathrm{~ms}$ & $>300 \mathrm{~ns})$ & $3 / 2^{-} \#$ & 97 & $97 \mathrm{Be} 70 \mathrm{I}$ & I & $\beta^{-} ?$ \\
\hline${ }^{85} \mathrm{Ge}$ & $-53070 \#$ & $400 \#$ & & & 540 & $\mathrm{~ms}$ & 50 & $5 / 2^{+} \#$ & 97 & & & $\beta^{-}=100 ; \beta^{-} \mathrm{n}=143$ \\
\hline${ }^{85} \mathrm{As}$ & $-63320 \#$ & $200 \#$ & & & 2.021 & $\mathrm{~s}$ & 0.010 & $3 / 2^{-} \#$ & 97 & & & $\beta^{-}=100 ; \beta^{-} \mathrm{n}=59.424$ \\
\hline${ }^{85} \mathrm{Se}$ & -72428 & 30 & & & 31.7 & $\mathrm{~s}$ & 0.9 & $5 / 2^{+} \#$ & 97 & & & $\beta^{-}=100$ \\
\hline${ }^{85} \mathrm{Br}$ & -78610 & 19 & & & 2.90 & $\mathrm{~m}$ & 0.06 & $3 / 2^{-}$ & 91 & & & $\beta^{-}=100$ \\
\hline${ }^{85} \mathrm{Kr}$ & -81480.3 & 1.9 & & & 10.776 & $\mathrm{y}$ & 0.003 & $9 / 2^{+}$ & 91 & 02Un02 & $\mathrm{T}$ & $\beta^{-}=100$ \\
\hline${ }^{85} \mathrm{Kr}^{m}$ & -81175.4 & 1.9 & 304.871 & 0.020 & 4.480 & $\mathrm{~h}$ & 0.008 & $1 / 2^{-}$ & 91 & & & $\beta^{-}=78.64 ; \mathrm{IT}=21.44$ \\
\hline${ }^{85} \mathrm{Kr}^{n}$ & -79488.5 & 2.3 & 1991.8 & 1.3 & 1.6 & $\mu \mathrm{s}$ & 0.7 & $\left(17 / 2^{+}\right)$ & 91 & & & $\mathrm{IT}=100$ \\
\hline${ }^{85} \mathrm{Rb}$ & -82167.331 & 0.011 & & & STABLE & & & $5 / 2^{-}$ & 91 & & & IS $=72.172$ \\
\hline${ }^{85} \mathrm{Sr}$ & -81102.6 & 2.8 & & & 64.853 & $\mathrm{~d}$ & 0.008 & $9 / 2^{+}$ & 91 & 02Un02 & $\mathrm{T}$ & $\varepsilon=100$ \\
\hline${ }^{85} \mathrm{Sr}^{m}$ & -80863.9 & 2.8 & 238.66 & 0.06 & 67.63 & $\mathrm{~m}$ & 0.04 & $1 / 2^{-}$ & 91 & & & $\mathrm{IT}=86.64 ; \beta^{+}=13.44$ \\
\hline${ }^{85} \mathrm{Y}$ & -77842 & 19 & & & 2.68 & $\mathrm{~h}$ & 0.05 & $(1 / 2)^{-}$ & 94 & & & $\beta^{+}=100$ \\
\hline${ }^{85} \mathrm{Y}^{m}$ & -77822 & 19 & 19.8 & 0.5 & 4.86 & $\mathrm{~h}$ & 0.13 & $9 / 2^{+}$ & 94 & & & $\beta^{+} \approx 100 ;$ IT $<0.002$ \\
\hline${ }^{85} \mathrm{Zr}$ & -73150 & 100 & & & 7.86 & $\mathrm{~m}$ & 0.04 & $7 / 2^{+}$ & 94 & & & $\beta^{+}=100$ \\
\hline${ }^{85} \mathrm{Zr}^{m}$ & -72860 & 100 & 292.2 & 0.3 & 10.9 & $\mathrm{~s}$ & 0.3 & $\left(1 / 2^{-}\right)$ & 94 & & & $\mathrm{IT} \leq 92 ; \beta^{+}>8$ \\
\hline${ }^{85} \mathrm{Nb}$ & -67150 & 220 & & & 20.9 & $\mathrm{~s}$ & 0.7 & $\left(9 / 2^{+}\right)$ & 91 & & & $\beta^{+}=100$ \\
\hline${ }^{85} \mathrm{Nb}^{m}$ & -66390 & 220 & 759.0 & 1.0 & 12 & $\mathrm{~s}$ & 5 & $\left(1 / 2^{-}\right)$ & 91 & 980i.A & ETJ & $\beta^{+}=100$ \\
\hline${ }^{85} \mathrm{Mo}$ & $-59100 \#$ & $280 \#$ & & & 3.2 & $\mathrm{~s}$ & 0.2 & $1 / 2^{-} \#$ & 97 & $97 \mathrm{Hu} 15$ & TD & $\beta^{+}=100 ; \beta^{+} \mathrm{p}=?$ \\
\hline${ }^{85} \mathrm{Tc}$ & $-47670 \#$ & $400 \#$ & & & $<110$ & ns & & $1 / 2^{-} \#$ & & 00We.A I & & $\mathrm{p} ? ; \beta^{+} ? ; \beta^{+} \mathrm{p}$ ? \\
\hline${ }^{85} \mathrm{Tc}$ & $\mathrm{I}:$ also $99 \mathrm{Ja}$ & $2<100 \mathrm{~ns}$ & & $\mathrm{ma}$ & life for $\beta$ & $+\mathrm{de}$ & ay: $100 \#$ & $\mathrm{~ms}$ & & & & \\
\hline
\end{tabular}




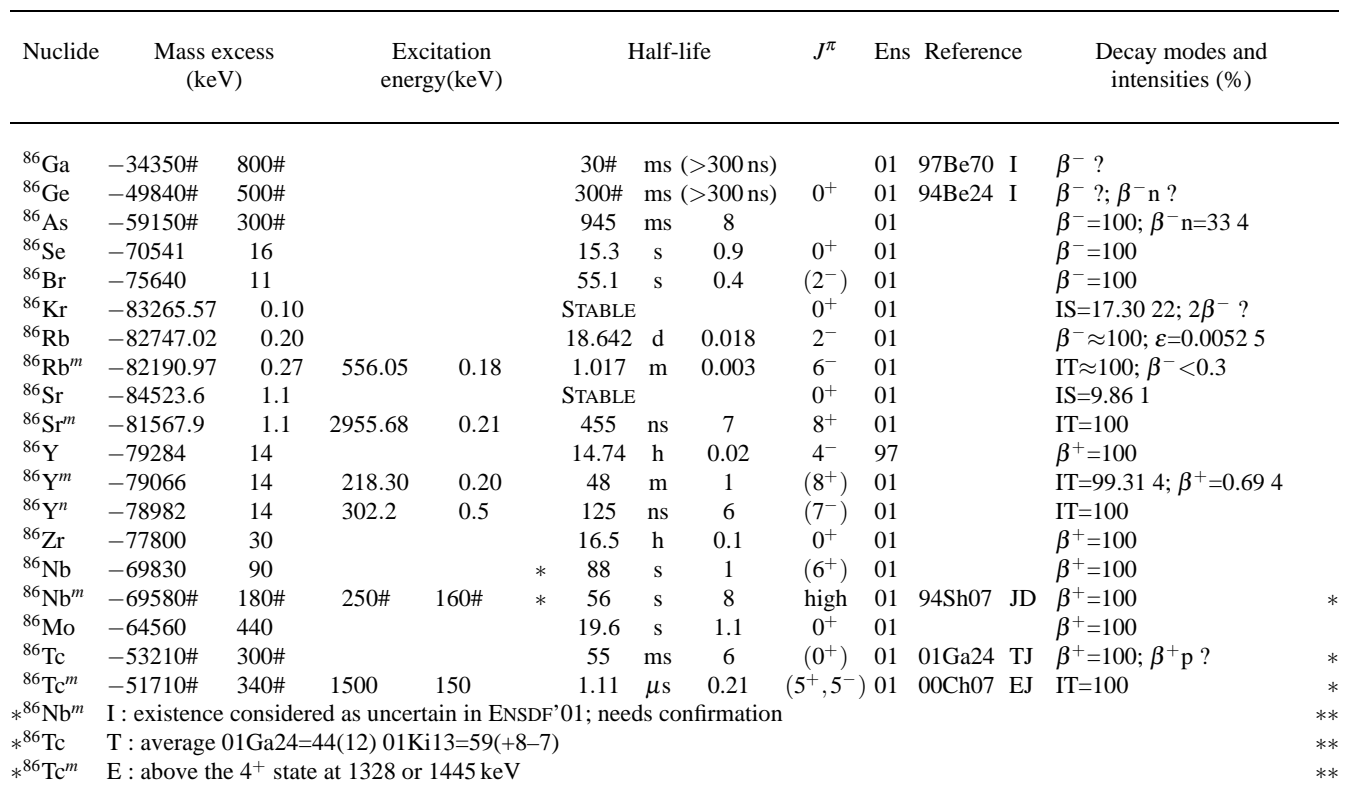

\begin{tabular}{|c|c|c|c|c|c|c|c|c|c|c|c|c|c|}
\hline${ }^{87} \mathrm{Ge}$ & $-44240 \#$ & $500 \#$ & & & $150 \#$ & $\mathrm{~ms}$ & $>300 \mathrm{~ns})$ & $5 / 2^{+} \#$ & 02 & 97Be70 & & $\beta^{-} ? ; \beta^{-} \mathrm{n} ?$ & \\
\hline${ }^{87} \mathrm{As}$ & $-55980 \#$ & $300 \#$ & & & 610 & $\mathrm{~ms}$ & 120 & $3 / 2^{-} \#$ & 02 & 93Ru01 & $\mathrm{T}$ & $\beta^{-}=100 ; \beta^{-} \mathrm{n}=15.422$ & $*$ \\
\hline${ }^{87} \mathrm{Se}$ & -66580 & 40 & & & 5.50 & $\mathrm{~s}$ & 0.12 & $5 / 2^{+} \#$ & 02 & & & $\beta^{-}=100 ; \beta^{-} \mathrm{n}=0.204$ & \\
\hline${ }^{87} \mathrm{Br}$ & -73857 & 18 & & & 55.65 & $\mathrm{~s}$ & 0.13 & $3 / 2^{-}$ & 02 & & & $\beta^{-}=100 ; \beta^{-} \mathrm{n}=2.604$ & \\
\hline${ }^{87} \mathrm{Kr}$ & -80709.43 & 0.27 & & & 76.3 & $\mathrm{~m}$ & 0.5 & $5 / 2^{+}$ & 02 & & & $\beta^{-}=100$ & \\
\hline${ }^{87} \mathrm{Rb}$ & -84597.795 & 0.012 & & & 49.23 & Gy & 0.22 & $3 / 2^{-}$ & 02 & $82 \mathrm{Mi14}$ & $\mathrm{T}$ & $\mathrm{IS}=27.832 ; \beta^{-}=100$ & . \\
\hline${ }^{87} \mathrm{Sr}$ & -84880.4 & 1.1 & & & StABLE & & & $9 / 2^{+}$ & 02 & & & $\mathrm{IS}=7.001$ & \\
\hline${ }^{87} \mathrm{Sr}^{m}$ & -84491.9 & 1.1 & 388.533 & 0.003 & 2.815 & $\mathrm{~h}$ & 0.012 & $1 / 2^{-}$ & 02 & & & $\mathrm{IT} \approx 100 ; \varepsilon=0.308$ & \\
\hline${ }^{87} \mathrm{Y}$ & -83018.7 & 1.6 & & & 79.8 & $\mathrm{~h}$ & 0.3 & $1 / 2^{-}$ & 02 & & & $\beta^{+}=100$ & \\
\hline${ }^{87} \mathrm{Y}^{m}$ & -82637.9 & 1.6 & 380.82 & 0.07 & 13.37 & $\mathrm{~h}$ & 0.03 & $9 / 2^{+}$ & 02 & & & $\mathrm{IT}=98.4310 ; \beta^{+}=1.5710$ & \\
\hline${ }^{87} \mathrm{Zr}$ & -79348 & 8 & & & 1.68 & $\mathrm{~h}$ & 0.01 & $(9 / 2)^{+}$ & 02 & & & $\beta^{+}=100$ & \\
\hline${ }^{87} \mathrm{Zr}^{m}$ & -79012 & 8 & 335.84 & 0.19 & 14.0 & s & 0.2 & $(1 / 2)^{-}$ & 02 & & & $\mathrm{IT}=100$ & \\
\hline${ }^{87} \mathrm{Nb}$ & -74180 & 60 & & & 3.75 & $\mathrm{~m}$ & 0.09 & $\left(1 / 2^{-}\right)$ & 02 & & & $\beta^{+}=100$ & \\
\hline${ }^{87} \mathrm{Nb}^{m}$ & -74180 & 60 & 3.84 & 0.14 & 2.6 & $\mathrm{~m}$ & 0.1 & $9 / 2^{+} \#$ & 02 & & & $\beta^{+}=100$ & \\
\hline${ }^{87} \mathrm{Mo}$ & -67690 & 220 & & & 14.05 & $\mathrm{~s}$ & 0.23 & $7 / 2^{+} \#$ & 02 & $97 \mathrm{Hu} 07$ & TD & $\beta^{+}=100 ; \beta^{+} \mathrm{p}=155$ & * \\
\hline${ }^{87} \mathrm{Tc}$ & $-59120 \#$ & $300 \#$ & & & 2.18 & s & 0.16 & $1 / 2^{-} \#$ & 02 & 00We.A & TD & $\beta^{+}=100 ; \beta^{+} \mathrm{p} ?$ & \\
\hline${ }^{87} \mathrm{Tc}^{m}$ & $-59100 \#$ & $310 \#$ & $20 \#$ & $60 \#$ & 2\# & s & & $9 / 2^{+} \#$ & & & & $\beta^{+}$?; IT? & \\
\hline${ }^{87} \mathrm{Ru}$ & $-47340 \#$ & $600 \#$ & & & $50 \#$ & $\mathrm{~ms}$ & $>1.5 \mu \mathrm{s})$ & $1 / 2^{-} \#$ & 02 & 95Ry03 & I & $\beta^{+} ?$ & \\
\hline${ }^{87} \mathrm{As}$ & \multicolumn{12}{|c|}{$\mathrm{T}:$ unweighed average $93 \mathrm{Ru} 01=485(40) 78 \mathrm{Cr} 03=730(60)($ Birge ratio $B=3.4)$} & ** \\
\hline${ }^{2}{ }^{87} \mathrm{Rb}$ & \multicolumn{12}{|c|}{$\mathrm{T}:$ average $82 \mathrm{Mi} 14=49.44(0.28) 74 \mathrm{Ne} 14=48.8(0.8) 77 \mathrm{Da} 22=48.9(0.4)$ obtained by } & $* *$ \\
\hline$*^{87} \mathrm{Rb}$ & \multicolumn{12}{|c|}{$\mathrm{T}$ : three methods, respectively: geochronology, decay counting, chemical } & ** \\
\hline${ }^{*}{ }^{87} \mathrm{Rb}$ & \multicolumn{12}{|c|}{$\mathrm{T}: \quad 77 \mathrm{Da} 22$ supersedes $66 \mathrm{Mc} 12=47.2(0.4)$ using the same material } & $* *$ \\
\hline$*^{87} \mathrm{Mo}$ & \multicolumn{12}{|c|}{$\mathrm{T}:$ average $97 \mathrm{Hu} 07=13.6(1.1) 91 \mathrm{Mi} 15=14.5(0.3) 83 \mathrm{Ha} 06=13.3(0.4)$} & ** \\
\hline$*^{87} \mathrm{Mo}$ & \multicolumn{12}{|c|}{ D : average $97 \mathrm{Hu} 07=15(6) \%$ (through 3 levels) $83 \mathrm{Ha} 06=15(8) \%$ first $2^{+}$state } & * \\
\hline
\end{tabular}




\begin{tabular}{|c|c|c|c|c|c|c|c|c|c|c|c|c|c|}
\hline Nuclide & \multicolumn{2}{|c|}{$\begin{array}{l}\text { Mass excess } \\
(\mathrm{keV})\end{array}$} & \multicolumn{3}{|c|}{$\begin{array}{l}\text { Excitation } \\
\text { energy }(\mathrm{keV})\end{array}$} & \multicolumn{3}{|c|}{ Half-life } & \multirow[t]{2}{*}{$J^{\pi}$} & \multirow{2}{*}{$\begin{array}{c}\text { Ens } \\
97\end{array}$} & Reference & \multicolumn{2}{|l|}{$\begin{array}{l}\text { Decay modes and } \\
\text { intensities }(\%)\end{array}$} \\
\hline${ }^{88} \mathrm{Ge}$ & $-40140 \#$ & $700 \#$ & & & & $80 \#$ & ms & $>300 \mathrm{~ns})$ & & & 97Be70 I & $\beta^{-} ?$ & \\
\hline${ }^{88} \mathrm{As}$ & $-51290 \#$ & $500 \#$ & & & & $300 \#$ & ms & $>300 \mathrm{~ns})$ & & 97 & 94Be24 I & $\beta^{-} ? ; \beta^{-} \mathrm{n} ?$ & \\
\hline${ }^{88} \mathrm{Se}$ & -63880 & 50 & & & & 1.53 & $\mathrm{~s}$ & 0.06 & $0^{+}$ & 97 & & $\beta^{-}=100 ; \beta^{-} \mathrm{n}=0.9910$ & \\
\hline${ }^{88} \mathrm{Br}$ & -70730 & 40 & & & & 16.36 & $\mathrm{~s}$ & 0.07 & $\left(2^{-}, 1^{+}\right)$ & 98 & 93Ru01 T & $\beta^{-}=100 ; \beta^{-} \mathrm{n}=6.5818$ & $*$ \\
\hline${ }^{88} \mathrm{Br}^{m}$ & -70460 & 40 & 272.7 & 0.3 & & 5.4 & $\mu \mathrm{s}$ & 0.7 & & 98 & & $\mathrm{IT}=100$ & \\
\hline${ }^{88} \mathrm{Kr}$ & -79692 & 13 & & & & 2.84 & $\mathrm{~h}$ & 0.03 & $0^{+}$ & 88 & & $\beta^{-}=100$ & \\
\hline${ }^{88} \mathrm{Rb}$ & -82609.00 & 0.16 & & & & 17.78 & $\mathrm{~m}$ & 0.11 & $2^{-}$ & 88 & & $\beta^{-}=100$ & \\
\hline${ }^{88} \mathrm{Sr}$ & -87921.7 & 1.1 & & & & STABLE & & & $0^{+}$ & 88 & & $\mathrm{IS}=82.581$ & \\
\hline${ }^{88} \mathrm{Y}$ & -84299.1 & 1.9 & & & & 106.65 & $\mathrm{~d}$ & 0.04 & $4^{-}$ & 88 & & $\beta^{+}=100$ & \\
\hline${ }^{88} \mathrm{Y}^{m}$ & -83624.6 & 1.9 & 674.55 & 0.0 & & 13.9 & $\mathrm{~ms}$ & 0.2 & $(8)^{+}$ & 88 & & $\mathrm{IT}=100$ & \\
\hline${ }^{88} \mathrm{Y}^{n}$ & -83906.2 & 1.9 & 392.86 & 0.0 & & 300 & $\mu \mathrm{s}$ & 3 & $1^{+}$ & 88 & & & \\
\hline${ }^{88} \mathrm{Zr}$ & -83623 & 10 & & & & 83.4 & $\mathrm{~d}$ & 0.3 & $0^{+}$ & 88 & & $\varepsilon=100$ & \\
\hline${ }^{88} \mathrm{Nb}$ & -76070 & 100 & & & * & 14.5 & $\mathrm{~m}$ & 0.1 & $\left(8^{+}\right)$ & 88 & & $\beta^{+}=100$ & \\
\hline${ }^{88} \mathrm{Nb}^{m}$ & -76030 & 100 & 40 & 140 & $\mathrm{BD} *$ & 7.8 & $\mathrm{~m}$ & 0.1 & $\left(4^{-}\right)$ & 88 & & $\beta^{+}=100$ & \\
\hline${ }^{88} \mathrm{Mo}$ & -72700 & 20 & & & & 8.0 & $\mathrm{~m}$ & 0.2 & $0^{+}$ & 97 & & $\beta^{+}=100$ & \\
\hline${ }^{88} \mathrm{Tc}$ & $-62710 \#$ & $200 \#$ & & & * & 5.8 & $\mathrm{~s}$ & 0.2 & $(2,3)$ & 97 & & $\beta^{+}=100$ & \\
\hline${ }^{88} \mathrm{Tc}^{m}$ & $-62710 \#$ & $360 \#$ & 0\# & $300 \#$ & * & 6.4 & s & 0.8 & $(6,7,8)$ & 97 & & $\beta^{+}=100$ & \\
\hline${ }^{88} \mathrm{Ru}$ & $-55650 \#$ & $400 \#$ & & & & 1.3 & $\mathrm{~s}$ & 0.3 & $0^{+}$ & 97 & $01 \mathrm{Ki} 13 \mathrm{TD}$ & $\beta^{+}=100 ; \beta^{+} \mathrm{p} ?$ & \\
\hline$*^{88} \mathrm{Br}$ & $\mathrm{T}$ : average & 93Ru01 & $=16.34(C$ & $0.08) 7$ & $\operatorname{Gr} 29=1$ & $6.5(0.2)$ & & $\mathbf{J}:$ syster & latics prefe & $\mathrm{s}\left(2^{-}\right)$ & & & T. \\
\hline
\end{tabular}

\begin{tabular}{|c|c|c|c|c|c|c|c|c|c|c|c|c|c|}
\hline${ }^{89} \mathrm{Ge}$ & $-33690 \#$ & 900\# & & & & $50 \#$ & $\mathrm{~ms}($ & $>300 \mathrm{~ns})$ & $3 / 2^{+} \#$ & 98 & $97 \mathrm{Be} 70$ & I & $\beta^{-} ?$ \\
\hline${ }^{89}$ As & $-47140 \#$ & $500 \#$ & & & & $200 \#$ & $\mathrm{~ms}($ & $>300 \mathrm{~ns})$ & $3 / 2^{-} \#$ & 98 & $94 \mathrm{Be} 24$ & I & $\beta^{-} ?$ \\
\hline${ }^{89} \mathrm{Se}$ & $-59200 \#$ & $300 \#$ & & & & 410 & $\mathrm{~ms}$ & 40 & $5 / 2^{+} \#$ & 98 & & & $\beta^{-}=100 ; \beta^{-} \mathrm{n}=7.825$ \\
\hline${ }^{89} \mathrm{Br}$ & -68570 & 60 & & & & 4.40 & $\mathrm{~s}$ & 0.03 & $\left(3 / 2^{-}, 5 / 2^{-}\right)$ & 98 & & & $\beta^{-}=100 ; \beta^{-} \mathrm{n}=13.84$ \\
\hline${ }^{89} \mathrm{Kr}$ & -76730 & 50 & & & & 3.15 & $\mathrm{~m}$ & 0.04 & $3 / 2^{(+\#)}$ & 98 & $95 \mathrm{Ke} 04$ & $\mathrm{~J}$ & $\beta^{-}=100$ \\
\hline${ }^{89} \mathrm{Rb}$ & -81713 & 5 & & & & 15.15 & $\mathrm{~m}$ & 0.12 & $3 / 2^{-}$ & 98 & & & $\beta^{-}=100$ \\
\hline${ }^{89} \mathrm{Sr}$ & -86209.1 & 1.1 & & & & 50.53 & $\mathrm{~d}$ & 0.07 & $5 / 2^{+}$ & 98 & & & $\beta^{-}=100$ \\
\hline${ }^{89} \mathrm{Y}$ & -87701.7 & 2.6 & & & & StABLE & & & $1 / 2^{-}$ & 98 & & & $\mathrm{IS}=100$ \\
\hline${ }^{89} \mathrm{Y}^{m}$ & -86792.7 & 2.6 & 908.97 & 0.03 & & 15.663 & $\mathrm{~s}$ & 0.005 & $9 / 2^{+}$ & 98 & 94It.A & $\mathrm{T}$ & $\mathrm{IT}=100$ \\
\hline${ }^{89} \mathrm{Zr}$ & -84869 & 4 & & & & 78.41 & $\mathrm{~h}$ & 0.12 & $9 / 2^{+}$ & 98 & & & $\beta^{+}=100$ \\
\hline${ }^{89} \mathrm{Zr}^{m}$ & -84281 & 4 & 587.82 & 0.10 & & 4.161 & $\mathrm{~m}$ & 0.017 & $1 / 2^{-}$ & 98 & & & $\mathrm{IT}=93.7712 ; \ldots$ \\
\hline${ }^{89} \mathrm{Nb}$ & -80650 & 27 & & & $*$ & 2.03 & $\mathrm{~h}$ & 0.07 & $\left(9 / 2^{+}\right)$ & 98 & & & $\beta^{+}=100$ \\
\hline${ }^{89} \mathrm{Nb}^{m}$ & $-80650 \#$ & $40 \#$ & 0\# & $30 \#$ & * & 1.10 & $\mathrm{~h}$ & 0.03 & $(1 / 2)^{-}$ & 98 & & & $\beta^{+}=100$ \\
\hline${ }^{89} \mathrm{Mo}$ & -75004 & 15 & & & & 2.11 & $\mathrm{~m}$ & 0.10 & $\left(9 / 2^{+}\right)$ & 98 & & & $\beta^{+}=100$ \\
\hline${ }^{89} \mathrm{Mo}^{m}$ & -74617 & 15 & 387.5 & 0.2 & & 190 & $\mathrm{~ms}$ & 15 & $\left(1 / 2^{-}\right)$ & 98 & & & $\mathrm{IT}=100$ \\
\hline${ }^{89} \mathrm{Tc}$ & $-67840 \#$ & $200 \#$ & & & & 12.8 & $\mathrm{~s}$ & 0.9 & $\left(9 / 2^{+}\right)$ & 98 & & & $\beta^{+}=100$ \\
\hline${ }^{89} \mathrm{Tc}^{m}$ & $-67780 \#$ & $200 \#$ & 62.6 & 0.5 & & 12.9 & s & 0.8 & $\left(1 / 2^{-}\right)$ & 98 & & & $\beta^{+} \approx 100 ;$ IT $<0.01$ \\
\hline${ }^{89} \mathrm{Ru}$ & $-59510 \#$ & $500 \#$ & & & & 1.38 & $\mathrm{~s}$ & 0.11 & $(7 / 2)^{(+\#)}$ & 98 & 00We.A & $\mathrm{T}$ & $\beta^{+}=100 ; \beta^{+} \mathrm{p}=?$ \\
\hline${ }^{89} \mathrm{Rh}$ & $-47660 \#$ & $450 \#$ & & & & $10 \#$ & $\mathrm{~ms}($ & $>1.5 \mu \mathrm{s})$ & $7 / 2^{+} \#$ & 98 & 95Ry03 & I & $\beta^{+} ?$ \\
\hline$*^{89} \mathrm{Br}$ & $\mathrm{T}:$ ENSDF & average: & 8 value & Also 93 & the & $=4.348(0$ & $.022)$ & & & & & & \\
\hline$*^{89} \mathrm{Zr}^{m}$ & $\mathrm{D}: \ldots ; \beta^{+}$ & $=6.231$ & & & & & & & & & & & \\
\hline${ }^{* 9} \mathrm{Ru}$ & $\mathrm{T}$ : average & 00We.t & $=1.45(0$ & 13) $99 \mathrm{I}$ & .2 & $(0.2)$; sa & ime $\mathrm{g}$ & roup $01 \mathrm{Ki}$ & $\mathrm{i} 13=1.5(0.2)$ & & & & \\
\hline${ }^{* 9}{ }^{89} \mathrm{~h}$ & I : unobs & $\mathrm{d}$ in & Ve.A, & tecti & & & & & & & & & \\
\hline
\end{tabular}




\begin{tabular}{|c|c|c|c|c|c|c|c|c|c|c|c|c|c|}
\hline Nuclide & \multicolumn{2}{|c|}{$\begin{array}{c}\text { Mass excess } \\
(\mathrm{keV})\end{array}$} & \multicolumn{3}{|c|}{$\begin{array}{c}\text { Excitation } \\
\text { energy }(\mathrm{keV})\end{array}$} & \multicolumn{3}{|c|}{ Half-life } & \multirow[t]{2}{*}{$J^{\pi}$} & \multirow[t]{2}{*}{ Ens } & Reference & \multicolumn{2}{|l|}{$\begin{array}{c}\text { Decay modes and } \\
\text { intensities (\%) }\end{array}$} \\
\hline${ }^{90} \mathrm{As}$ & $-41450 \#$ & $800 \#$ & & & & $80 \#$ & $\mathrm{~ms}$ & $>300 \mathrm{~ns})$ & & & $97 \mathrm{Be} 70 \mathrm{I}$ & $\beta^{-} ?$ & \\
\hline${ }^{90} \mathrm{Se}$ & $-55930 \#$ & $400 \#$ & & & & $300 \#$ & $\mathrm{~ms}$ & $>300 \mathrm{~ns})$ & $0^{+}$ & & $94 \mathrm{Be} 24 \mathrm{I}$ & $\beta^{-} ? ; \beta^{-} \mathrm{n} ?$ & \\
\hline${ }^{90} \mathrm{Br}$ & -64620 & 80 & & & & 1.910 & $\mathrm{~s}$ & 0.010 & & 98 & 93Ru01 T & $\beta^{-}=100 ; \beta^{-} \mathrm{n}=25.29$ & * \\
\hline${ }^{90} \mathrm{Kr}$ & -74970 & 19 & & & & 32.32 & $\mathrm{~s}$ & 0.09 & $0^{+}$ & 98 & & $\beta^{-}=100$ & \\
\hline${ }^{90} \mathrm{Rb}$ & -79362 & 7 & & & & 158 & $\mathrm{~s}$ & 5 & $0^{-}$ & 98 & & $\beta^{-}=100$ & \\
\hline${ }^{90} \mathrm{Rb}^{m}$ & -79255 & 7 & 106.90 & 0.03 & & 258 & $\mathrm{~s}$ & 4 & $3^{-}$ & 98 & & $\beta^{-}=97.44 ; \mathrm{IT}=2.64$ & \\
\hline${ }^{90} \mathrm{Rb}^{x}$ & -79291 & 14 & 71 & 12 & & $R=2 \quad 1$ & & & fsmix & & & & \\
\hline${ }^{90} \mathrm{Sr}$ & -85941.6 & 2.9 & & & & 28.79 & $\mathrm{y}$ & 0.06 & $0^{+}$ & 98 & & $\beta^{-}=100$ & \\
\hline${ }^{90} \mathrm{Y}$ & -86487.5 & 2.6 & & & & 64.00 & $\mathrm{~h}$ & 0.21 & $2^{-}$ & 98 & & $\beta^{-}=100$ & \\
\hline${ }^{90} \mathrm{Y}^{m}$ & -85805.8 & 2.6 & 681.67 & 0.10 & & 3.19 & $\mathrm{~h}$ & 0.06 & $7^{+}$ & 98 & & $\mathrm{IT} \approx 100 ; \beta^{-}=0.00182$ & \\
\hline${ }^{90} \mathrm{Zr}$ & -88767.3 & 2.4 & & & & STABLE & & & $0^{+}$ & 98 & & $\mathrm{IS}=51.4540$ & \\
\hline${ }^{90} \mathrm{Zr}^{m}$ & -86448.3 & 2.4 & 2319.000 & 0.010 & & 809.2 & $\mathrm{~ms}$ & 2.0 & $5^{-}$ & 98 & & $\mathrm{IT}=100$ & \\
\hline${ }^{90} \mathrm{Zr}^{n}$ & -85177.9 & 2.4 & 3589.419 & 0.016 & & 131 & $\mathrm{~ns}$ & 4 & $8^{+}$ & 98 & & $\mathrm{IT}=100$ & \\
\hline${ }^{90} \mathrm{Nb}$ & -82656 & 5 & & & & 14.60 & $\mathrm{~h}$ & 0.05 & $8^{+}$ & 98 & & $\beta^{+}=100$ & \\
\hline${ }^{90} \mathrm{Nb}^{m}$ & -82534 & 5 & 122.370 & 0.022 & & 63 & $\mu \mathrm{s}$ & 2 & $6^{+}$ & 98 & & $\mathrm{IT}=100$ & \\
\hline${ }^{90} \mathrm{Nb}^{n}$ & -82531 & 5 & 124.67 & 0.25 & & 18.81 & $\mathrm{~s}$ & 0.06 & $4^{-}$ & 98 & & $\mathrm{IT}=100$ & \\
\hline${ }^{90} \mathrm{Nb}^{p}$ & -82485 & 5 & 171.10 & 0.10 & & $<1$ & $\mu \mathrm{s}$ & & $7^{+}$ & 98 & & $\mathrm{IT}=100$ & \\
\hline${ }^{90} \mathrm{Nb}^{q}$ & -82274 & 5 & 382.01 & 0.25 & & 6.19 & $\mathrm{~ms}$ & 0.08 & $1^{+}$ & 98 & & $\mathrm{IT}=100$ & \\
\hline${ }^{90} \mathrm{Nb}^{r}$ & -80776 & 5 & 1880.21 & 0.20 & & 472 & ns & 13 & $\left(11^{-}\right)$ & 98 & & $\mathrm{IT}=100$ & \\
\hline${ }^{90} \mathrm{Mo}$ & -80167 & 6 & & & & 5.56 & $\mathrm{~h}$ & 0.09 & $0^{+}$ & 98 & & $\beta^{+}=100$ & \\
\hline${ }^{90} \mathrm{Mo}^{m}$ & -77292 & 6 & 2874.73 & 0.15 & & 1.12 & $\mu \mathrm{s}$ & 0.05 & $8^{+} \#$ & 98 & & $\mathrm{IT}=100$ & \\
\hline${ }^{90} \mathrm{Tc}$ & -71210 & 240 & & & $* \&$ & 8.7 & $\mathrm{~s}$ & 0.2 & $1^{+}$ & 98 & & $\beta^{+}=100$ & \\
\hline${ }^{90} \mathrm{Tc}^{m}$ & -70900 & 300 & 310 & 390 & $\mathrm{BD} * \&$ & 49.2 & $\mathrm{~s}$ & 0.4 & $\left(8^{+}\right)$ & 98 & 93Ru03 J & $\beta^{+}=100$ & * \\
\hline${ }^{90} \mathrm{Ru}$ & $-65310 \#$ & $300 \#$ & & & & 11 & $\mathrm{~s}$ & 3 & $0^{+}$ & 98 & & $\beta^{+}=100$ & \\
\hline${ }^{90} \mathrm{Rh}$ & $-53220 \#$ & $500 \#$ & & & * & 15 & $\mathrm{~ms}$ & 7 & $0^{+} \#$ & 98 & $01 \mathrm{Ki} 13 \mathrm{TD}$ & $\beta^{+}=100 ; \beta^{+} \mathrm{p} ?$ & \\
\hline${ }^{90} \mathrm{Rh}^{m}$ & $-53220 \#$ & $710 \#$ & 0\# & $500 \#$ & $*$ & 1.1 & $\mathrm{~s}$ & 0.3 & $9^{+} \#$ & & $01 \mathrm{Ki} 13 \mathrm{TD}$ & $\beta^{+}=100 ; \beta^{+} \mathrm{p}$ ? & \\
\hline${ }^{90} \mathrm{Br}$ & $\mathrm{T}$ : supers & edes 80 & $\mathrm{Al} 15=1.92$ & $2(0.02) \mathrm{fi}$ & m same g & group & & & & & & & $* *$ \\
\hline${ }^{90} \mathrm{Tc}^{m}$ & $\mathrm{E}$ : argum & ents are & given in 9 & $3 \mathrm{Ru} 03 \mathrm{fo}$ & r the $\left(8^{+}\right)$ & level to & be th & ground-s & tate & & & & $* *$ \\
\hline
\end{tabular}

\begin{tabular}{|c|c|c|c|c|}
\hline${ }^{91} \mathrm{As}$ & $-36860 \#$ & $900 \#$ & & \\
\hline${ }^{91} \mathrm{Se}$ & $-50340 \#$ & $500 \#$ & & \\
\hline${ }^{91} \mathrm{Br}$ & -61510 & 70 & & \\
\hline${ }^{91} \mathrm{Kr}$ & -71310 & 60 & & \\
\hline${ }^{91} \mathrm{Rb}$ & -77745 & 8 & & \\
\hline${ }^{91} \mathrm{Sr}$ & -83645 & 5 & & \\
\hline${ }^{91} \mathrm{Sr}^{x}$ & -83599 & 11 & 47 & 11 \\
\hline${ }^{91} \mathrm{Y}$ & -86345.0 & 2.9 & & \\
\hline${ }^{91} \mathrm{Y}^{m}$ & -85789.4 & 2.9 & 555.58 & 0.05 \\
\hline${ }^{91} \mathrm{Zr}$ & -87890.4 & 2.3 & & \\
\hline${ }^{91} \mathrm{Zr}^{m}$ & -84723.1 & 2.3 & 3167.3 & 0.4 \\
\hline${ }^{91} \mathrm{Nb}$ & -86632 & 4 & & \\
\hline${ }^{91} \mathrm{Nb}^{m}$ & -86527 & 4 & 104.60 & 0.05 \\
\hline${ }^{91} \mathrm{Nb}^{n}$ & -84598 & 4 & 2034.35 & 0.19 \\
\hline${ }^{91}$ Mo & -82204 & 11 & & \\
\hline${ }^{91} \mathrm{Mo}^{m}$ & -81551 & 11 & 653.01 & 0.09 \\
\hline${ }^{91} \mathrm{Tc}$ & -75980 & 200 & & \\
\hline${ }^{91} \mathrm{Tc}^{m}$ & -75840 & 200 & 139.3 & 0.3 \\
\hline${ }^{91} \mathrm{Ru}$ & $-68660 \#$ & $580 \#$ & & \\
\hline${ }^{91} \mathrm{Ru}^{m}$ & -68580 & 500 & $80 \#$ & $300 \#$ \\
\hline${ }^{91} \mathrm{Rh}$ & $-59100 \#$ & $400 \#$ & & \\
\hline${ }^{91} \mathrm{Pd}$ & $-47400 \#$ & $570 \#$ & & \\
\hline$*^{91} \mathrm{Nb}^{m}$ & $\mathrm{D}: \ldots ; \mathrm{e}^{+}$ & ${ }^{+}=0.002$ & 282 & \\
\hline
\end{tabular}

\begin{tabular}{|c|c|c|c|c|c|c|c|}
\hline $50 \#$ & $\mathrm{~ms}$ & $>300 \mathrm{~ns})$ & $3 / 2^{-} \#$ & 99 & $97 \mathrm{Be} 70$ & I & $\beta^{-}$? \\
\hline 270 & $\mathrm{~ms}$ & 50 & $1 / 2^{+} \#$ & 99 & & & $\beta^{-}=100 ; \beta^{-} \mathrm{n}=2110$ \\
\hline 541 & $\mathrm{~ms}$ & 5 & $3 / 2^{-} \#$ & 99 & & & $\beta^{-}=100 ; \beta^{-} \mathrm{n}=203$ \\
\hline 8.57 & $\mathrm{~s}$ & 0.04 & $5 / 2^{(+)}$ & 01 & & & $\beta^{-}=100$ \\
\hline 58.4 & s & 0.4 & $3 / 2^{(-)}$ & 99 & & & $\beta^{-}=100$ \\
\hline 9.63 & $\mathrm{~h}$ & 0.05 & $5 / 2^{+}$ & 01 & & & $\beta^{-}=100$ \\
\hline$R=6$ & & & $\operatorname{mix}$ & & & & \\
\hline 58.51 & $\mathrm{~d}$ & 0.06 & $1 / 2^{-}$ & 99 & & & $\beta^{-}=100$ \\
\hline 49.71 & $\mathrm{~m}$ & 0.04 & $9 / 2^{+}$ & 99 & & & $\mathrm{IT}>98.5 ; \beta^{-}<1.5$ \\
\hline STABLE & & & $5 / 2^{+}$ & 01 & & & $\mathrm{IS}=11.225$ \\
\hline 4.35 & $\mu \mathrm{s}$ & 0.14 & $\left(21 / 2^{+}\right)$ & 01 & & & $\mathrm{IT}=100$ \\
\hline 680 & $\mathrm{y}$ & 130 & $9 / 2^{+}$ & 99 & 91Hi.A & $\mathrm{D}$ & $\varepsilon \approx 100 ; \mathrm{e}^{+}=0.013825$ \\
\hline 60.86 & d & 0.22 & $1 / 2^{-}$ & 99 & 91Hi.A & $\mathrm{D}$ & $\mathrm{IT}=96.65 ; \varepsilon=3.45 ; \ldots$ \\
\hline 3.76 & $\mu \mathrm{s}$ & 0.12 & $\left(17 / 2^{-}\right)$ & 99 & & & $\mathrm{IT}=100$ \\
\hline 15.49 & $\mathrm{~m}$ & 0.01 & $9 / 2^{+}$ & 99 & & & $\beta^{+}=100$ \\
\hline 64.6 & $\mathrm{~s}$ & 0.6 & $1 / 2^{-}$ & 99 & & & $\mathrm{IT}=50.016 ; \beta^{+}=50.016$ \\
\hline 3.14 & $\mathrm{~m}$ & 0.02 & $(9 / 2)^{+}$ & 99 & & & $\beta^{+}=100$ \\
\hline 3.3 & $\mathrm{~m}$ & 0.1 & $(1 / 2)^{-}$ & 99 & & & $\beta^{+}>99 ; \mathrm{IT}<1$ \\
\hline 9 & $\mathrm{~s}$ & 1 & $\left(9 / 2^{+}\right)$ & 99 & & & $\beta^{+}=100$ \\
\hline 7.6 & s & 0.8 & $\left(1 / 2^{-}\right)$ & 99 & & & $\beta^{+} \approx 100 ; \beta^{+} \mathrm{p}=? ;$ IT ? \\
\hline 1.74 & $\mathrm{~s}$ & 0.14 & $7 / 2^{+} \#$ & 99 & 00We.A & $\mathrm{TD}$ & $\beta^{+}=100 ; \beta^{+} \mathrm{p}$ ? \\
\hline $10 \#$ & $\mathrm{~ms}$ & $>1.5 \mu \mathrm{s})$ & $7 / 2^{+} \#$ & 99 & 95Ry03 & I & $\beta^{+} ?$ \\
\hline
\end{tabular}




\begin{tabular}{|c|c|c|c|c|c|c|c|c|c|c|c|c|c|}
\hline Nuclide & \multicolumn{2}{|c|}{$\begin{array}{l}\text { Mass excess } \\
\quad(\mathrm{keV})\end{array}$} & \multicolumn{2}{|c|}{$\begin{array}{l}\text { Excitation } \\
\text { energy }(\mathrm{keV})\end{array}$} & \multicolumn{3}{|c|}{ Half-life } & \multirow[t]{2}{*}{$J^{\pi}$} & \multirow{2}{*}{$\begin{array}{c}\text { Ens } \\
01\end{array}$} & \multicolumn{2}{|c|}{ Reference } & \multicolumn{2}{|l|}{$\begin{array}{l}\text { Decay modes and } \\
\text { intensities }(\%)\end{array}$} \\
\hline${ }^{92} \mathrm{As}$ & $-30930 \#$ & 900\# & & & $30 \#$ & $\mathrm{~ms}$ & $(>300 \mathrm{~ns})$ & & & 97Be70 & & $\beta^{-} ?$ & \\
\hline${ }^{92} \mathrm{Se}$ & $-46650 \#$ & $600 \#$ & & & $100 \#$ & $\mathrm{~ms}$ & $(>300 \mathrm{~ns})$ & $0^{+}$ & 01 & $97 \mathrm{Be} 70$ & I & $\beta^{-} ?$ & \\
\hline${ }^{92} \mathrm{Br}$ & -56580 & 50 & & & 343 & $\mathrm{~ms}$ & 15 & $\left(2^{-}\right)$ & 01 & & & $\beta^{-}=100 ; \beta^{-} \mathrm{n}=33.125$ & \\
\hline${ }^{92} \mathrm{Kr}$ & -68785 & 12 & & & 1.840 & $\mathrm{~s}$ & 0.008 & $0^{+}$ & 01 & & & $\beta^{-}=100 ; \beta^{-} \mathrm{n}=0.033225$ & \\
\hline${ }^{92} \mathrm{Rb}$ & -74772 & 6 & & & 4.492 & s & 0.020 & $0^{-}$ & 01 & & & $\beta^{-}=100 ; \beta^{-} \mathrm{n}=0.01075$ & \\
\hline${ }^{92} \mathrm{Sr}$ & -82868 & 3 & & & 2.66 & $\mathrm{~h}$ & 0.04 & $0^{+}$ & 03 & & & $\beta^{-}=100$ & \\
\hline${ }^{92} \mathrm{Y}$ & -84813 & 9 & & & 3.54 & $\mathrm{~h}$ & 0.01 & $2^{-}$ & 01 & & & $\beta^{-}=100$ & \\
\hline${ }^{92} \mathrm{Zr}$ & -88453.9 & 2.3 & & & STABLE & & & $0^{+}$ & 01 & & & $\mathrm{IS}=17.158$ & \\
\hline${ }^{92} \mathrm{Nb}$ & -86448.3 & 2.8 & & & 34.7 & My & 2.4 & $(7)^{+}$ & 01 & & & $\beta^{+} \approx 100 ; \beta^{-}<0.05$ & \\
\hline${ }^{92} \mathrm{Nb}^{m}$ & -86312.8 & 2.8 & 135.5 & 0.4 & 10.15 & $\mathrm{~d}$ & 0.02 & $(2)^{+}$ & 01 & & & $\beta^{+}=100$ & \\
\hline${ }^{92} \mathrm{Nb}^{n}$ & -86222.6 & 2.8 & 225.7 & 0.4 & 5.9 & $\mu \mathrm{s}$ & 0.2 & $(2)^{-}$ & 01 & & & $\mathrm{IT}=100$ & \\
\hline${ }^{92} \mathrm{Nb}^{p}$ & -84245.0 & 2.8 & 2203.3 & 0.4 & 167 & ns & 4 & $\left(11^{-}\right)$ & 01 & & & $\mathrm{IT}=100$ & \\
\hline${ }^{92} \mathrm{Mo}$ & -86805 & 4 & & & STABLE & & (>190 Ey) & $0^{+}$ & 01 & 97Ba35 & $\mathrm{T}$ & $\mathrm{IS}=14.8435 ; 2 \beta^{+} ?$ & $*$ \\
\hline${ }^{92} \mathrm{Mo}^{m}$ & -84045 & 4 & 2760.46 & 0.16 & 190 & ns & 3 & $8^{+}$ & 01 & & & $\mathrm{IT}=100$ & \\
\hline${ }^{92} \mathrm{Tc}$ & -78935 & 26 & & & 4.25 & $\mathrm{~m}$ & 0.15 & $(8)^{+}$ & 01 & & & $\beta^{+}=100$ & \\
\hline${ }^{92} \mathrm{Tc}^{m}$ & -78665 & 26 & 270.15 & 0.11 & 1.03 & $\mu \mathrm{s}$ & 0.07 & $\left(4^{+}\right)$ & 01 & & & $\mathrm{IT}=100$ & \\
\hline${ }^{92} \mathrm{Ru}$ & $-74410 \#$ & $300 \#$ & & & 3.65 & $\mathrm{~m}$ & 0.05 & $0^{+}$ & 01 & & & $\beta^{+}=100$ & \\
\hline${ }^{92} \mathrm{Rh}$ & $-63360 \#$ & $400 \#$ & & & 4.3 & $\mathrm{~s}$ & 1.3 & $\left(6^{+}\right)$ & 01 & 01Xu05 & TJD & $\beta^{+}=100 ; \beta^{+} \mathrm{p}=?$ & $*$ \\
\hline${ }^{92} \mathrm{Pd}$ & $-55500 \#$ & $500 \#$ & & & 1.1 & s & 0.3 & $0^{+}$ & 01 & $01 \mathrm{Ki} 13$ & TD & $\beta^{+}=100 ; \beta^{+} \mathrm{p}$ ? & \\
\hline$*^{92} \mathrm{Mo}$ & $\mathrm{T}: T>190$ & Ey $(2 c$ & & & & & & & & & & & $* *$ \\
\hline$*{ }^{92} \mathrm{Rh}$ & $\mathrm{T}$ : unweig & hed av & erage $01 \mathrm{X}$ & $\mathrm{u} 05=3.0$ & $01 \mathrm{Ki} 1$ & $13-56$ & $6(05)(\operatorname{Bir}$ & $\therefore$ & & & & & $* *$ \\
\hline$*^{92} \mathrm{Rh}$ & $\mathrm{J}:$ from 97 & $\mathrm{Ka} 07$ & $01 \mathrm{Xu05}>$ & & & & & & & & & & $* *$ \\
\hline
\end{tabular}

\begin{tabular}{|c|c|c|c|c|c|c|c|c|c|c|c|c|}
\hline${ }^{93} \mathrm{Se}$ & $-40720 \#$ & $800 \#$ & & & $50 \#$ & $\mathrm{~ms}$ & $(>300 \mathrm{~ns})$ & $1 / 2^{+} \#$ & 97 & 97Be70 & I & $\beta^{-} ?$ \\
\hline${ }^{93} \mathrm{Br}$ & $-53050 \#$ & $300 \#$ & & & 102 & $\mathrm{~ms}$ & 10 & $3 / 2^{-} \#$ & 01 & & & $\beta^{-}=100 ; \beta^{-} \mathrm{n}=687$ \\
\hline${ }^{93} \mathrm{Kr}$ & -64020 & 100 & & & 1.286 & $\mathrm{~s}$ & 0.010 & $1 / 2^{+}$ & 01 & & & $\beta^{-}=100 ; \beta^{-} \mathrm{n}=1.9511$ \\
\hline${ }^{93} \mathrm{Rb}$ & -72618 & 8 & & & 5.84 & $\mathrm{~s}$ & 0.02 & $5 / 2^{-}$ & 97 & & & $\beta^{-}=100 ; \beta^{-} \mathrm{n}=1.397$ \\
\hline${ }^{93} \mathrm{Rb}^{m}$ & -72365 & 8 & 253.38 & 0.03 & 57 & $\mu \mathrm{s}$ & 15 & $\left(3 / 2^{-}, 5 / 2^{-}\right)$ & 97 & & & $\mathrm{IT}=100$ \\
\hline${ }^{93} \mathrm{Sr}$ & -80085 & 8 & & & 7.423 & $\mathrm{~m}$ & 0.024 & $5 / 2^{+}$ & 97 & & & $\beta^{-}=100$ \\
\hline${ }^{93} \mathrm{Y}$ & -84223 & 11 & & & 10.18 & $\mathrm{~h}$ & 0.08 & $1 / 2^{-}$ & 97 & & & $\beta^{-}=100$ \\
\hline${ }^{93} \mathrm{Y}^{m}$ & -83464 & 11 & 758.719 & 0.021 & 820 & $\mathrm{~ms}$ & 40 & $7 / 2^{+}$ & 97 & & & $\mathrm{IT}=100$ \\
\hline${ }^{93} \mathrm{Zr}$ & -87117.0 & 2.3 & & & 1.53 & My & 0.10 & $5 / 2^{+}$ & 97 & & & $\beta^{-}=100$ \\
\hline${ }^{93} \mathrm{Nb}$ & -87208.3 & 2.4 & & & STABLE & & & $9 / 2^{+}$ & 97 & & & $\mathrm{IS}=100$ \\
\hline${ }^{93} \mathrm{Nb}^{m}$ & -87177.5 & 2.4 & 30.77 & 0.02 & 16.13 & $\mathrm{y}$ & 0.14 & $1 / 2^{-}$ & 97 & & & $\mathrm{IT}=100$ \\
\hline${ }^{93} \mathrm{Mo}$ & -86803 & 4 & & & 4.0 & ky & 0.8 & $5 / 2^{+}$ & 97 & & & $\varepsilon=100$ \\
\hline${ }^{93} \mathrm{Mo}^{m}$ & -84378 & 4 & 2424.89 & 0.03 & 6.85 & $\mathrm{~h}$ & 0.07 & $21 / 2^{+}$ & 97 & & & $\mathrm{IT} \approx 100 ; \beta^{+}=0.121$ \\
\hline${ }^{93} \mathrm{Tc}$ & -83603 & 4 & & & 2.75 & $\mathrm{~h}$ & 0.05 & $9 / 2^{+}$ & 01 & & & $\beta^{+}=100$ \\
\hline${ }^{93} \mathrm{Tc}^{m}$ & -83211 & 4 & 391.84 & 0.08 & 43.5 & $\mathrm{~m}$ & 1.0 & $1 / 2^{-}$ & 01 & & & $\mathrm{IT}=76.611 ; \beta^{+}=23.411$ \\
\hline${ }^{93} \mathrm{Tc}^{n}$ & -81418 & 4 & 2185.16 & 0.15 & 10.2 & $\mu \mathrm{s}$ & 0.3 & $(17 / 2)^{-}$ & 01 & & & \\
\hline${ }^{93} \mathrm{Ru}$ & -77270 & 90 & & & 59.7 & $\begin{array}{c}\mu s \\
\mathrm{~s}\end{array}$ & 0.6 & $(9 / 2)^{+}$ & 97 & & & $\beta^{+}=100$ \\
\hline${ }^{93} \mathrm{Ru}^{m}$ & -76540 & 90 & 734.40 & 0.10 & 10.8 & $\mathrm{~s}$ & 0.3 & $(1 / 2)^{-}$ & 97 & 83Аy01 & $\mathrm{D}$ & $\beta^{+}=78.023$ \\
\hline${ }^{93} \mathrm{Ru}^{n}$ & -75190 & 90 & 2082.6 & 0.9 & 2.20 & $\mu \mathrm{s}$ & 0.17 & $(21 / 2)^{+}$ & 97 & & & $\mathrm{IT}=100$ \\
\hline${ }^{93} \mathrm{Rh}$ & -69170\# & $400 \#$ & & & 13.9 & $\begin{array}{c}\mu s \\
\mathrm{~s}\end{array}$ & 1.6 & $9 / 2^{+} \#$ & 01 & $01 \mathrm{Ki} 13$ & $\mathrm{TD}$ & $\beta^{+}=100 ; \beta^{+} \mathrm{p} ?$ \\
\hline${ }^{93} \mathrm{Pd}$ & $-59700 \#$ & $400 \#$ & & & 1.07 & $\mathrm{~s}$ & 0.12 & $\left(9 / 2^{+}\right)$ & 01 & $01 \mathrm{Ki13}$ & TJD & $\beta^{+}=100 ; \beta^{+} \mathrm{p}=$ ? \\
\hline${ }^{93} \mathrm{Ag}$ & $-46780 \#$ & $600 \#$ & & & $5 \#$ & $\mathrm{~ms}$ & $(>1.5 \mu \mathrm{s})$ & $9 / 2^{+} \#$ & 97 & 95Ry03 & I & $\mathrm{p} ? ; \beta^{+}$? \\
\hline$*^{93} \mathrm{Ru}^{m}$ & $\mathrm{D}: \ldots ; \mathrm{IT}$ & $\Gamma=22.0$ & $23 ; \beta^{+} \mathrm{p}=$ & .0275 & & & & & & & & \\
\hline$*^{93} \mathrm{Pd}$ & $\mathrm{T}:$ averag & $\mathrm{e} 01 \mathrm{Ki}$ & $13=1000(2$ & 00) $01 x$ & $=130$ & ) & $00 \mathrm{Sc} 31=$ & $00(200)$ & $\mathrm{D}:$ & $\beta^{+} \mathrm{p}=1.7 \#$ & & \\
\hline$*{ }^{93} \mathrm{Ag}$ & I : the few & events & reported is & n $94 \mathrm{He} 2$ & e not $t$ & usted & by NuBASt & & & & & \\
\hline$*{ }^{93} \mathrm{Ag}$ & $\mathrm{T}$ : estimat & ted hal & f-life is for & $\beta^{+}$deca & p-decay & woul & ld be much & shorter & & & & \\
\hline
\end{tabular}




\begin{tabular}{|c|c|c|c|c|c|c|c|c|c|c|c|c|c|c|}
\hline Nuclide & \multicolumn{2}{|c|}{$\begin{array}{l}\text { Mass excess } \\
(\mathrm{keV})\end{array}$} & \multicolumn{3}{|c|}{$\begin{array}{l}\text { Excitation } \\
\text { energy }(\mathrm{keV})\end{array}$} & \multicolumn{3}{|c|}{ Half-life } & \multirow{2}{*}{$\begin{array}{c}J^{\pi} \\
0^{+}\end{array}$} & \multirow{2}{*}{$\begin{array}{l}\text { Ens } \\
97\end{array}$} & \multicolumn{2}{|c|}{ Reference } & \multicolumn{2}{|l|}{$\begin{array}{l}\text { Decay modes and } \\
\text { intensities }(\%)\end{array}$} \\
\hline${ }^{94} \mathrm{Se}$ & $-36800 \#$ & $800 \#$ & & & & $20 \#$ & $\mathrm{~ms}$ & $(>300 \mathrm{~ns})$ & & & $97 \mathrm{Be} 70$ & I & $\beta^{-} ?$ & \\
\hline${ }^{94} \mathrm{Br}$ & $-47800 \#$ & $400 \#$ & & & & 70 & $\mathrm{~ms}$ & 20 & & 92 & & & $\beta^{-}=100 ; \beta^{-} \mathrm{n}=7015$ & \\
\hline${ }^{94} \mathrm{Kr}$ & $-61140 \#$ & $300 \#$ & & & & 210 & $\mathrm{~ms}$ & 4 & $0^{+}$ & 01 & 03Be05 & $\mathrm{TD}$ & $\beta^{-}=100 ; \beta^{-} \mathrm{n}=1.117$ & $*$ \\
\hline${ }^{94} \mathrm{Rb}$ & -68553 & 8 & & & & 2.702 & $\mathrm{~s}$ & 0.005 & $3^{(-)}$ & 92 & 93Ru01 & $\mathrm{D}$ & $\beta^{-}=100 ; \beta^{-} \mathrm{n}=10.0123$ & \\
\hline${ }^{94} \mathrm{Sr}$ & -78840 & 7 & & & & 75.3 & $\mathrm{~s}$ & 0.2 & $0^{+}$ & 92 & & & $\beta^{-}=100$ & \\
\hline${ }^{94} \mathrm{Y}$ & -82348 & 7 & & & & 18.7 & $\mathrm{~m}$ & 0.1 & $2^{-}$ & 92 & & & $\beta^{-}=100$ & \\
\hline${ }^{94} \mathrm{Zr}$ & -87266.8 & 2.4 & & & & STABLE & & $(>110 \mathrm{Py})$ & $0^{+}$ & 92 & 99Ar25 & $\mathrm{T}$ & $\mathrm{IS}=17.3828 ; 2 \beta^{-} ?$ & \\
\hline${ }^{94} \mathrm{Nb}$ & -86364.5 & 2.4 & & & & 20.3 & ky & 1.6 & $(6)^{+}$ & 92 & & & $\beta^{-}=100$ & \\
\hline${ }^{94} \mathrm{Nb}^{m}$ & -86323.6 & 2.4 & 40.902 & 0.012 & & 6.263 & $\mathrm{~m}$ & 0.004 & $3^{+}$ & 92 & & & $\mathrm{IT}=99.506 ; \beta^{-}=0.506$ & \\
\hline${ }^{94} \mathrm{Mo}$ & -88409.7 & 1.9 & & & & STABLE & & & $0^{+}$ & 97 & & & $\mathrm{IS}=9.2512$ & \\
\hline${ }^{94} \mathrm{Tc}$ & -84154 & 4 & & & & 293 & $\mathrm{~m}$ & 1 & $7^{+}$ & 92 & & & $\beta^{+}=100$ & \\
\hline${ }^{94} \mathrm{Tc}^{m}$ & -84079 & 4 & 75.5 & 1.9 & & 52.0 & $\mathrm{~m}$ & 1.0 & $(2)^{+}$ & 92 & & & $\beta^{+} \approx 100 ; \mathrm{IT}<0.1$ & \\
\hline${ }^{94} \mathrm{Ru}$ & -82568 & 13 & & & & 51.8 & $\mathrm{~m}$ & 0.6 & $0^{+}$ & 92 & & & $\beta^{+}=100$ & \\
\hline${ }^{94} \mathrm{Ru}^{m}$ & -79923 & 13 & 2644.55 & 0.25 & & 71 & $\mu \mathrm{s}$ & 4 & $\left(8^{+}\right)$ & 92 & & & $\mathrm{IT}=100$ & \\
\hline${ }^{94} \mathrm{Rh}$ & $-72940 \#$ & $450 \#$ & & & $*$ & 70.6 & $\mathrm{~s}$ & 0.6 & $\left(2^{+}, 4^{+}\right)$ & 92 & 96Jo06 & $\mathrm{J}$ & $\beta^{+}=100 ; \beta^{+} \mathrm{p}=1.85$ & \\
\hline${ }^{94} \mathrm{Rh}^{m}$ & -72640 & 400 & $300 \#$ & $200 \#$ & * & 25.8 & $\mathrm{~s}$ & 0.2 & $\left(8^{+}\right)$ & 92 & & & $\beta^{+}=100$ & \\
\hline${ }^{94} \mathrm{Pd}$ & $-66350 \#$ & $400 \#$ & & & & 9.0 & $\mathrm{~s}$ & 0.5 & $0^{+}$ & 02 & & & $\beta^{+}=100$ & \\
\hline${ }^{94} \mathrm{Pd}^{m}$ & $-61470 \#$ & $400 \#$ & 4884.4 & 0.5 & & 530 & $\mathrm{~ns}$ & 10 & $\left(14^{+}\right)$ & 02 & & & $\mathrm{IT}=100$ & \\
\hline${ }^{94} \mathrm{Ag}$ & $-53300 \#$ & $500 \#$ & & & & 37 & $\mathrm{~ms}$ & 18 & $0^{+} \#$ & 02 & & & $\beta^{+}=100 ; \beta^{+} \mathrm{p} ?$ & \\
\hline${ }^{94} \mathrm{Ag}^{m}$ & $-51950 \#$ & $640 \#$ & $1350 \#$ & $400 \#$ & & 422 & $\mathrm{~ms}$ & 16 & $\left(7^{+}\right)$ & 02 & 02La18 & $\mathrm{TJ}$ & $\beta^{+}=100 ; \beta^{+} \mathrm{p}=$ ? & * \\
\hline${ }^{94} \mathrm{Ag}^{n}$ & $-46800 \#$ & $500 \#$ & $6500 \#$ & $2000 \#$ & & 300 & $\mathrm{~ms}$ & 200 & $\left(21^{+}\right)$ & 02 & 02La18 & $\mathrm{TJ}$ & $\beta^{+}=100 ; \beta^{+} \mathrm{p}=?$ & \\
\hline$*{ }^{94} \mathrm{Kr}$ & $\mathrm{T}$ : averag & e $03 \mathrm{Be} 0$ & $5=212(5)$ & $72 \mathrm{Am01}=2$ & & 0 ); other & rs ou & tweighed no & ot used: & & & & & $* *$ \\
\hline$* *^{94} \mathrm{Kr}$ & $\mathrm{T}: \quad 03 \mathrm{I}$ & $\mathrm{Be} 05=2$ & $10(20) 75 \mathrm{~A}$ & $\mathrm{~s} 04=220$ & & nd $96 \mathrm{Me}$ & $\mathrm{e} 09=$ & $330(100)$ & & & & & & $* *$ \\
\hline$*^{94} \mathrm{Ag}^{m}$ & $\mathrm{~T}:$ averag & e $02 \mathrm{La} 1$ & $8=360(30)$ & $01 \mathrm{Ki} 13=4$ & & 0) $94 \mathrm{Sc} 3$ & $35=4$ & $20(50)$ & & & & & & $* *$ \\
\hline
\end{tabular}

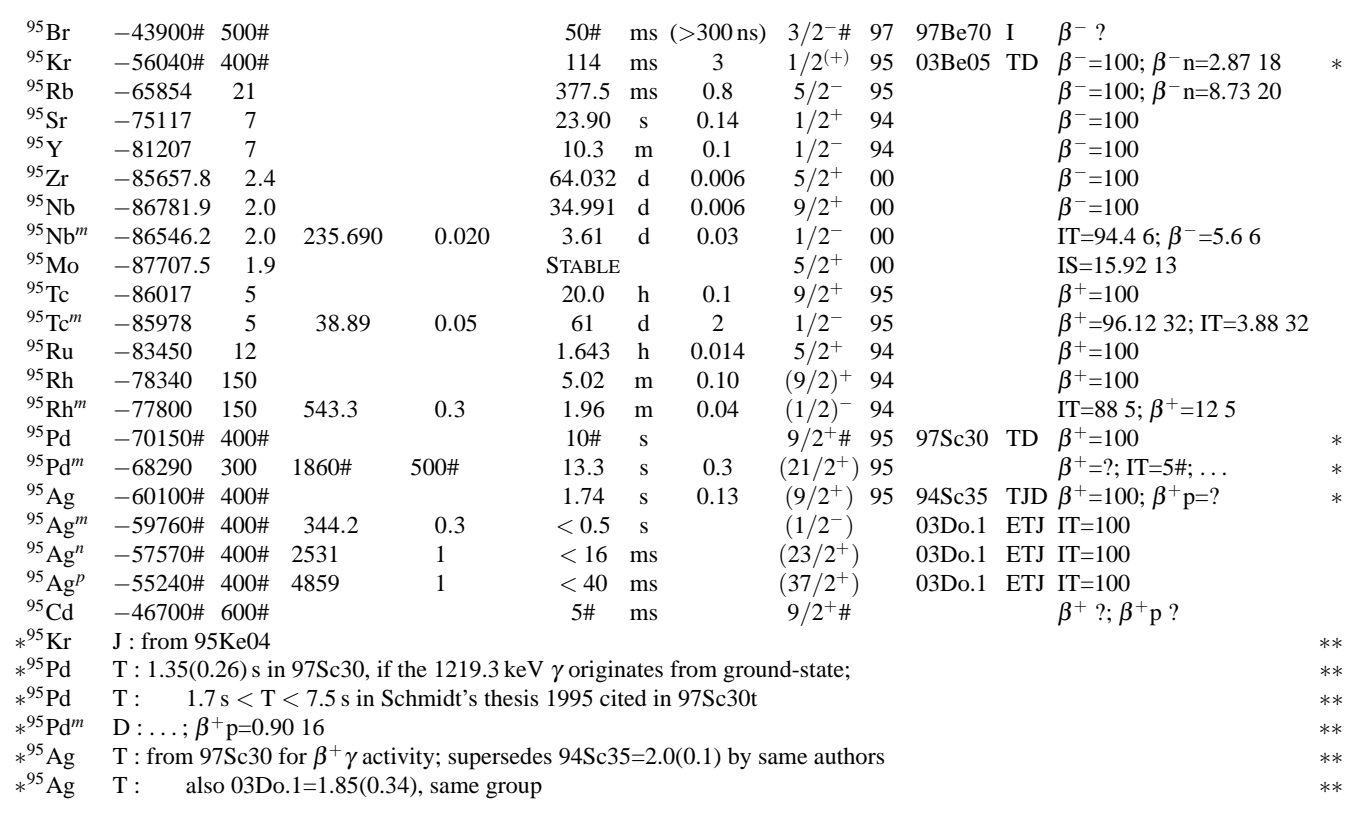




\begin{tabular}{|c|c|c|c|c|c|c|c|c|c|c|c|c|c|c|}
\hline Nuclide & \multicolumn{2}{|c|}{$\begin{array}{l}\text { Mass excess } \\
(\mathrm{keV})\end{array}$} & \multicolumn{3}{|c|}{$\begin{array}{l}\text { Excitation } \\
\text { energy }(\mathrm{keV})\end{array}$} & \multicolumn{3}{|c|}{ Half-life } & \multirow[t]{2}{*}{$J^{\pi}$} & \multirow{2}{*}{$\begin{array}{c}\text { Ens } \\
97\end{array}$} & \multicolumn{2}{|c|}{ Reference } & \multicolumn{2}{|l|}{$\begin{array}{l}\text { Decay modes and } \\
\text { intensities }(\%)\end{array}$} \\
\hline${ }^{96} \mathrm{Br}$ & $-38630 \#$ & $700 \#$ & & & & $20 \#$ & $\mathrm{~ms}$ & $(>300 \mathrm{~ns})$ & & & $97 \mathrm{Be} 70$ & I & $\beta^{-} ?$ & \\
\hline${ }^{96} \mathrm{Kr}$ & $-53030 \#$ & $500 \#$ & & & & 80 & $\mathrm{~ms}$ & 7 & $0^{+}$ & 97 & $03 \mathrm{Be} 05$ & TD & $\beta^{-}=100 ; \beta^{-} \mathrm{n}=3.74$ & \\
\hline${ }^{96} \mathrm{Rb}$ & -61225 & 29 & & & * & 203 & $\mathrm{~ms}$ & 3 & $2^{+}$ & 95 & 93Ru01 & $\mathrm{D}$ & $\beta^{-}=100 ; \beta^{-} \mathrm{n}=13.44$ & $*$ \\
\hline${ }^{96} \mathrm{Rb}^{m}$ & $-61230 \#$ & $200 \#$ & $0 \#$ & $200 \#$ & * & $200 \#$ & $\mathrm{~ms}$ & $(>1 \mathrm{~ms})$ & $1^{(-\#)}$ & & 81 Bo30 & JI & $\beta^{-}$?; IT ?; $\beta^{-} \mathrm{n} ?$ & $*$ \\
\hline${ }^{96} \mathrm{Sr}$ & -72939 & 27 & & & & 1.07 & $\mathrm{~s}$ & 0.01 & $0^{+}$ & 93 & & & $\beta^{-}=100$ & \\
\hline${ }^{96} \mathrm{Y}$ & -78347 & 23 & & & & 5.34 & $\mathrm{~s}$ & 0.05 & $0^{-}$ & 93 & & & $\beta^{-}=100$ & \\
\hline${ }^{96} \mathrm{Y}^{m}$ & -77206 & 21 & 1140 & 30 & $\mathrm{BD}$ & 9.6 & $\mathrm{~s}$ & 0.2 & $(8)^{+}$ & 93 & & & $\beta^{-}=100$ & \\
\hline${ }^{96} \mathrm{Zr}$ & -85442.8 & 2.8 & & & & 24 & Ey & 6 & $0^{+}$ & 98 & $99 \operatorname{Ar} 25$ & $\mathrm{~T}$ & $\mathrm{IS}=2.809 ; 2 \beta^{-}=100$ & * \\
\hline${ }^{96} \mathrm{Nb}$ & -85604 & 4 & & & & 23.35 & $\mathrm{~h}$ & 0.05 & $6^{+}$ & 93 & & & $\beta^{-}=100$ & \\
\hline${ }^{96} \mathrm{Mo}$ & -88790.5 & 1.9 & & & & STABLE & & & $0^{+}$ & 93 & & & $\mathrm{IS}=16.682$ & \\
\hline${ }^{96} \mathrm{Tc}$ & -85817 & 5 & & & & 4.28 & d & 0.07 & $7^{+}$ & 93 & & & $\beta^{+}=100$ & \\
\hline${ }^{96} \mathrm{Tc}^{m}$ & -85783 & 5 & 34.28 & 0.07 & & 51.5 & $\mathrm{~m}$ & 1.0 & $4^{+}$ & 93 & & & $\mathrm{IT}=98.05 ; \beta^{+}=2.05$ & \\
\hline${ }^{96} \mathrm{Ru}$ & -86072 & 8 & & & & StABLE & & (>67 Py) & $0^{+}$ & 01 & $85 \mathrm{No} 03$ & $\mathrm{~T}$ & $\mathrm{IS}=5.5414 ; 2 \beta^{+} ?$ & \\
\hline${ }^{96} \mathrm{Rh}$ & -79679 & 13 & & & & 9.90 & $\mathrm{~m}$ & 0.10 & $\left(6^{+}\right)$ & 93 & & & $\beta^{+}=100$ & \\
\hline${ }^{96} \mathrm{Rh}^{m}$ & -79627 & 13 & 52.0 & 0.1 & & 1.51 & $\mathrm{~m}$ & 0.02 & $\left(3^{+}\right)$ & 93 & & & $\mathrm{IT}=605 ; \beta^{+}=405$ & \\
\hline${ }^{96} \mathrm{Pd}$ & -76230 & 150 & & & & 122 & $\mathrm{~s}$ & 2 & $0^{+}$ & 93 & & & $\beta^{+}=100$ & \\
\hline${ }^{96} \mathrm{Pd}^{m}$ & -73700 & 150 & 2530.8 & 0.1 & & 1.81 & $\mu \mathrm{s}$ & 0.01 & $8^{+}$ & 93 & 98Gr.B & TD & $\mathrm{IT}=100$ & $*$ \\
\hline${ }^{96} \mathrm{Ag}$ & $-64570 \#$ & $400 \#$ & & & * & 4.45 & $\mathrm{~s}$ & 0.04 & $\left(8^{+}\right)$ & 93 & $03 \mathrm{Ba} 39$ & $\mathrm{TJ}$ & $\beta^{+}=100 ; \beta^{+} \mathrm{p}=9.717$ & $*$ \\
\hline${ }^{96} \mathrm{Ag}^{m}$ & $-64570 \#$ & $400 \#$ & $0 \#$ & $50 \#$ & $*$ & 6.9 & $\mathrm{~s}$ & 0.6 & $\left(2^{+}\right)$ & & 03Ba39 & TJD & $\beta^{+}=100 ; \beta^{+} \mathrm{p}=185$ & \\
\hline${ }^{96} \mathrm{Ag}^{n}$ & $-64570 \#$ & $400 \#$ & & & & 700 & ns & 200 & & & $97 \mathrm{Gr} 02$ & $\mathrm{~T}$ & IT ? & \\
\hline${ }^{96} \mathrm{Cd}$ & $-56100 \#$ & $500 \#$ & & & & $1 \#$ & s & & $0^{+}$ & & & & $\beta^{+} ?$ & \\
\hline$*{ }^{96} \mathrm{Rb}$ & $\mathrm{T}:$ ENSDF & average & of 8 valu & es. Ther & $\mathrm{e}$ is alss & $93 \mathrm{Ru} 01=$ & $=201($ & & & & & & & ** \\
\hline$*{ }^{96} \mathrm{Rb}^{m}$ & I : non-obs & servation & n by $81 \mathrm{Th}$ & 04 is no & in con & adiction & with & 81 Bo30 exp & eriment & & & & & $* *$ \\
\hline * ${ }^{96} \mathrm{Rb}^{m}$ & I : exis & stence o & f this ison & her is dis & cussed & ENSDF & & & & & & & & ** \\
\hline$*{ }^{96} \mathrm{Zr}$ & $\mathrm{T}:$ from 21 & $1(+8-4$ & statistics & +2 syste & matics) & other $93 \mathrm{~K}$ & $\mathrm{Ka} 12=$ & $=39(9)$ in $g$ & ochemic & & & & & $* *$ \\
\hline$*^{96} \mathrm{Zr}$ & $\mathrm{T}: \quad \exp$ & perimen & t, not usec & : obser & ation o & $2 \beta^{-}$dece & cay qu & lestionned b & $96 \mathrm{Ba} 3$ & & & & & ** \\
\hline$*{ }^{96} \mathrm{Pd}^{m}$ & $\mathrm{~T}$ : superse & edes $97 C$ & $\mathrm{Gr} 02=1.7($ & 0.1 ); oth & er $83 \mathrm{Gr}$ & $1=2.2(0$. & .3) ou & atweighed & & & & & & $* *$ \\
\hline$*{ }^{96} \mathrm{Ag}$ & $\mathrm{T}:$ average & e $03 \mathrm{Ba} 3$ & $39=4.40(0$ & .06) $97 \mathrm{~S}$ & $30=4.5$ & $(0.06)$ & & & & & & & & ** \\
\hline$*^{96} \mathrm{Ag}$ & $\mathrm{D}$ : averag & $\beta^{+} \mathrm{p} 9$ & $77 \mathrm{Sc} 30=1$ & $1.9(2.6)$ & $32 \mathrm{Ku} 15$ & $=8.0(2.3)$; & ; $96 \mathrm{H}$ & $\mathrm{He} 25=3.7(0$. & ) not use & & & & & $* *$ \\
\hline
\end{tabular}

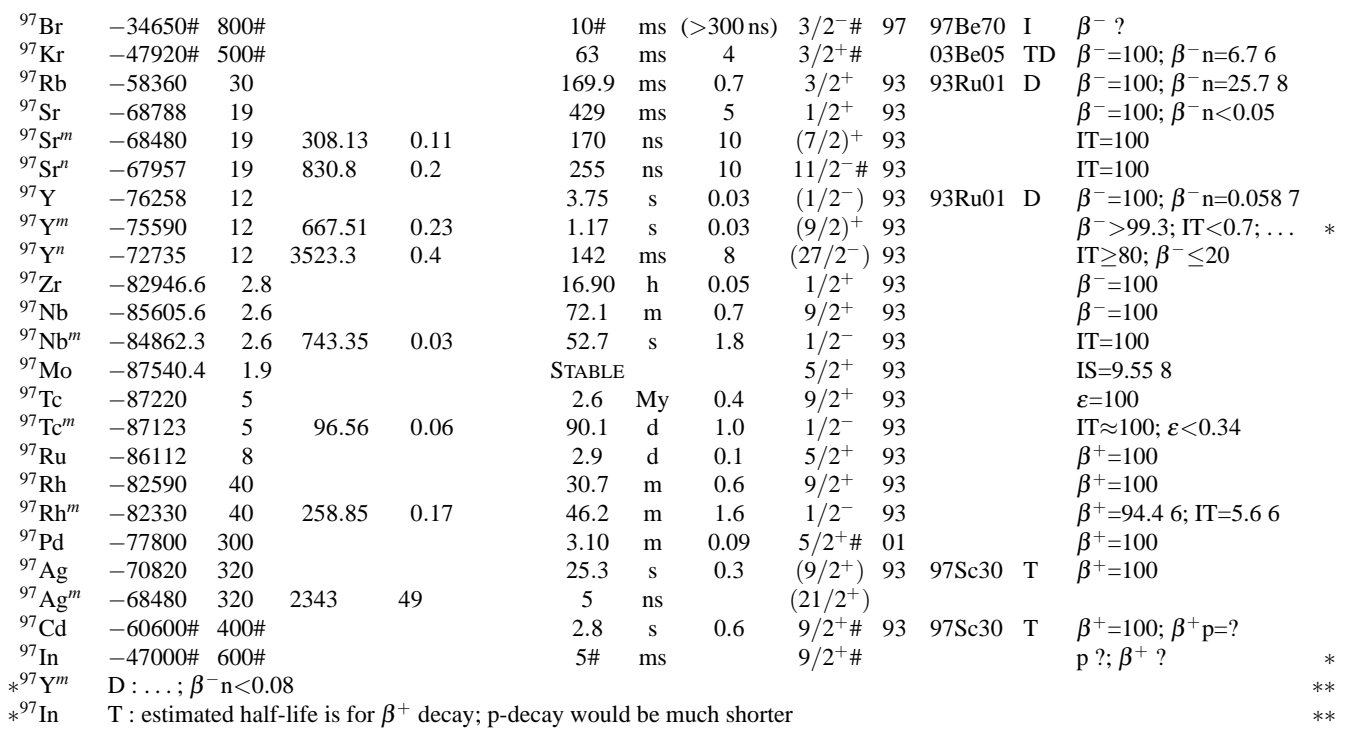




\begin{tabular}{|c|c|c|c|c|c|c|c|c|c|c|c|c|c|c|}
\hline Nuclide & \multicolumn{2}{|c|}{$\begin{array}{l}\text { Mass excess } \\
(\mathrm{keV})\end{array}$} & \multicolumn{3}{|c|}{$\begin{array}{l}\text { Excitation } \\
\text { energy }(\mathrm{keV})\end{array}$} & \multicolumn{3}{|c|}{ Half-life } & $J^{\pi}$ & \multicolumn{2}{|c|}{ Ens } & & \multicolumn{2}{|l|}{$\begin{array}{l}\text { Decay modes and } \\
\text { intensities }(\%)\end{array}$} \\
\hline${ }^{98} \mathrm{Kr}$ & $-44800 \#$ & $600 \#$ & & & & 46 & $\mathrm{~ms}$ & 8 & $0^{+}$ & 03 & & & $\beta^{-}=100 ; \beta^{-} \mathrm{n}=7.010$ & \\
\hline${ }^{98} \mathrm{Rb}$ & -54220 & 50 & & & & 114 & $\mathrm{~ms}$ & 5 & $(0,1)^{(-\#)}$ & 03 & & & $\beta^{-}=100 ; \beta^{-} \mathrm{n}=13.86$ & * \\
\hline${ }^{98} \mathrm{Rb}^{m}$ & -53940 & 120 & 290 & 130 & $\mathrm{BD}$ & 96 & $\mathrm{~ms}$ & 3 & $(3,4)^{(+\#)}$ & 03 & & & $\beta^{-}=100$ & \\
\hline${ }^{98} \mathrm{Sr}$ & -66646 & 26 & & & & 653 & $\mathrm{~ms}$ & 2 & $0^{+}$ & 03 & & & $\beta^{-}=100 ; \beta^{-} \mathrm{n}=0.255$ & \\
\hline${ }^{98} \mathrm{Y}$ & -72467 & 25 & & & & 548 & $\mathrm{~ms}$ & 2 & $(0)^{-}$ & 03 & & & $\beta^{-}=100 ; \beta^{-} \mathrm{n}=0.33124$ & \\
\hline${ }^{98} \mathrm{Y}^{m}$ & -72050 & 30 & 410 & 30 & $\mathrm{BD}$ & 2.0 & $\mathrm{~s}$ & 0.2 & $\left(5^{+}, 4^{-}\right)$ & 03 & & & $\beta^{-}=? ; \mathrm{IT}=10 \# ; \ldots$ & * \\
\hline${ }^{98} \mathrm{Y}^{n}$ & -71971 & 25 & 496.19 & 0.15 & & 7.6 & $\mu \mathrm{s}$ & 0.4 & $\left(2^{-}\right)$ & 03 & & & $\mathrm{IT}=100$ & \\
\hline${ }^{98} \mathrm{Y}^{p}$ & -72296 & 25 & 170.74 & 0.6 & & 620 & ns & 80 & $(2)^{-}$ & 03 & & & $\mathrm{IT}=100$ & \\
\hline${ }^{98} \mathrm{Zr}$ & -81287 & 20 & & & & 30.7 & $\mathrm{~s}$ & 0.4 & $0^{+}$ & 03 & & & $\beta^{-}=100$ & \\
\hline${ }^{98} \mathrm{Nb}$ & -83529 & 6 & & & & 2.86 & $\mathrm{~s}$ & 0.06 & $1^{+}$ & 03 & & & $\beta^{-}=100$ & \\
\hline${ }^{98} \mathrm{Nb}^{m}$ & -83445 & 7 & 84 & 4 & & 51.3 & $\mathrm{~m}$ & 0.4 & $\left(5^{+}\right)$ & 03 & & & $\beta^{-} \approx 100 ; \mathrm{IT}=0.1 \#$ & \\
\hline${ }^{98} \mathrm{Mo}$ & -88111.7 & $\begin{array}{ll}7 & 1.9\end{array}$ & & & & STABLE & & (>100 Ty) & $0^{+}$ & 03 & $52 \mathrm{Fr} 23$ & $\mathrm{~T}$ & $\mathrm{IS}=24.1331 ; 2 \beta^{-}$? & * \\
\hline${ }^{98} \mathrm{Tc}$ & -86428 & 4 & & & & 4.2 & My & 0.3 & $(6)^{+}$ & 03 & & & $\beta^{-}=100 ; \beta^{+}=0$ & \\
\hline${ }^{98} \mathrm{Tc}^{m}$ & -86337 & 4 & 90.76 & 0.16 & & 14.7 & $\mu \mathrm{s}$ & 3 & $(2)^{-}$ & 03 & & & $\mathrm{IT}=100$ & \\
\hline${ }^{98} \mathrm{Ru}$ & -88224 & 6 & & & & STABLE & & & $0^{+}$ & 03 & & & $\mathrm{IS}=1.873$ & \\
\hline${ }^{98} \mathrm{Rh}$ & -83175 & 12 & & & * & 8.72 & $\mathrm{~m}$ & 0.12 & $(2)^{+}$ & 03 & & & $\beta^{+}=100$ & \\
\hline${ }^{98} \mathrm{Rh}^{m}$ & $-83120 \#$ & $50 \#$ & $60 \#$ & $50 \#$ & $*$ & 3.6 & $\mathrm{~m}$ & 0.2 & $\left(5^{+}\right)$ & 03 & & & $\mathrm{IT}=895 ; \beta^{+}=115$ & \\
\hline${ }^{98} \mathrm{Pd}$ & -81300 & 21 & & & & 17.7 & $\mathrm{~m}$ & 0.3 & $0^{+}$ & 03 & & & $\beta^{+}=100$ & \\
\hline${ }^{98} \mathrm{Ag}$ & -73060 & 70 & & & & 47.5 & $\mathrm{~s}$ & 0.3 & $\left(5^{+}\right)$ & 03 & ABBW03. & $\mathrm{J}$ & $\beta^{+}=100 ; \beta^{+} \mathrm{p}=0.00125$ & $*$ \\
\hline${ }^{98} \mathrm{Ag}^{m}$ & -72890 & 70 & 167.83 & 0.15 & & 220 & ns & 20 & $\left(3^{+}\right)$ & 03 & 98Gr.B & ETD & $\mathrm{IT}=100$ & \\
\hline${ }^{98} \mathrm{Cd}$ & -67630 & 80 & & & & 9.2 & $\mathrm{~s}$ & 0.3 & $0^{+}$ & 03 & & & $\beta^{+}=100 ; \beta^{+} \mathrm{p}<0.025$ & \\
\hline${ }^{98} \mathrm{Cd}^{m}$ & -65200 & 80 & 2427.5 & 0.6 & & 190 & ns & 20 & $8^{+} \#$ & 98 & 98Gr.B & TD & $\mathrm{IT}=100$ & * \\
\hline${ }^{98} \mathrm{In}$ & $-53900 \#$ & $200 \#$ & & & * & 45 & $\mathrm{~ms}$ & 23 & $0^{+} \#$ & 03 & $01 \mathrm{Ki} 13$ & TD & $\beta^{+}=100 ; \beta^{+} \mathrm{p} ?$ & \\
\hline${ }^{98} \operatorname{In}^{m}$ & $-53900 \#$ & $540 \#$ & $0 \#$ & $500 \#$ & $*$ & 1.7 & $\mathrm{~s}$ & 0.8 & & 03 & $01 \mathrm{Ki} 13$ & TD & $\beta^{+}=100 ; \beta^{+} \mathrm{p}$ ? & \\
\hline$*{ }^{98} \mathrm{Rb}$ & $\mathrm{D}: \ldots ; \beta$ & $3^{-} 2 n=0$ & .0517 & & & & & & & & & & & $* *$ \\
\hline$*^{98} \mathrm{Y}^{m}$ & $\mathrm{D}: \ldots ; \beta$ & $3^{-} n=3$. & 410 & & & & & & & & & & & $* *$ \\
\hline$*^{98} \mathrm{Y}^{m}$ & $\mathrm{~J}: 94 \mathrm{St} 31$ & $=\left(5^{+}\right)$ & $95 \mathrm{Ha} \cdot \mathrm{B}=($ & & & & & & & & & & & ** \\
\hline$*^{98} \mathrm{Mo}$ & $\mathrm{T}$ : limit gi & given $h$ & ere is for & $\gamma-2 \beta^{-}$ & (1) & oretic & ly fast & ter, see tex & & & & & & $* *$ \\
\hline$*^{98} \mathrm{Ag}$ & $\mathrm{J}:\left(5^{+}\right)$wi & ith exp & erimental & asis pre & d to & $\left(6^{+}\right)$, se & disc & cussion in & ENSDF & & & & & $* *$ \\
\hline$*{ }^{98} \mathrm{Cd}^{m}$ & $\mathrm{~T}$ : superse & sedes 9 & $7 \mathrm{Gr} 02=20$ & $(+300-1$ & ); oth & e $97 \mathrm{Go}$ & $18=48$ & $80(160)$ ou & utweighed & & & & & $* *$ \\
\hline
\end{tabular}

\begin{tabular}{|c|c|c|c|c|c|c|c|c|c|c|c|c|}
\hline${ }^{99} \mathrm{Kr}$ & $-39500 \#$ & $600 \#$ & & & 40 & $\mathrm{~ms}$ & 11 & $3 / 2^{+} \#$ & 97 & 03Be05 & TD & $\beta^{-}=100 ; \beta^{-} \mathrm{n}=117$ \\
\hline${ }^{99} \mathrm{Rb}$ & -50880 & 130 & & & 50.3 & $\mathrm{~ms}$ & 0.7 & $\left(5 / 2^{+}\right)$ & 98 & & & $\beta^{-}=100 ; \beta^{-} \mathrm{n}=15.920$ \\
\hline${ }^{99} \mathrm{Sr}$ & -62190 & 80 & & & 269 & $\mathrm{~ms}$ & 1 & $3 / 2^{+}$ & 95 & & & $\beta^{-}=100 ; \beta^{-} \mathrm{n}=0.10019$ \\
\hline${ }^{99} \mathrm{Y}$ & -70201 & 24 & & & 1.470 & $\mathrm{~s}$ & 0.007 & $\left(5 / 2^{+}\right)$ & 95 & & & $\beta^{-}=100 ; \beta^{-} \mathrm{n}=1.94$ \\
\hline${ }^{99} \mathrm{Y}^{m}$ & -68059 & 24 & 2141.65 & 0.19 & 8.6 & $\mu \mathrm{s}$ & 0.8 & $\left(17 / 2^{+}\right)$ & 95 & & & IT $=100$ \\
\hline${ }^{99} \mathrm{Zr}$ & -77768 & 20 & & & 2.1 & $\mathrm{~s}$ & 0.1 & $1 / 2^{+}$ & 95 & $02 \mathrm{Ca} 37$ & $\mathrm{~J}$ & $\beta^{-}=100$ \\
\hline${ }^{99} \mathrm{Nb}$ & -82327 & 13 & & & 15.0 & $\mathrm{~s}$ & 0.2 & $9 / 2^{+}$ & 95 & & & $\beta^{-}=100$ \\
\hline${ }^{99} \mathrm{Nb}^{m}$ & -81962 & 13 & 365.29 & 0.14 & 2.6 & $\mathrm{~m}$ & 0.2 & $1 / 2^{-}$ & 95 & & & $\beta^{-}=? ; \mathrm{IT}<3.8$ \\
\hline${ }^{99} \mathrm{Mo}$ & -85965.8 & $8 \quad 1.9$ & & & 65.94 & $\mathrm{~h}$ & 0.01 & $1 / 2^{+}$ & 95 & & & $\beta^{-}=100$ \\
\hline${ }^{99} \mathrm{Mo}^{m}$ & -85868.0 & $0 \quad 1.9$ & 97.785 & 0.003 & 15.5 & $\mu \mathrm{s}$ & 0.2 & $5 / 2^{+}$ & 95 & & & $\mathrm{IT}=100$ \\
\hline${ }^{99} \mathrm{Tc}$ & -87323.1 & 12.0 & & & 211.1 & ky & 1.2 & $9 / 2^{+}$ & 01 & & & $\beta^{-}=100$ \\
\hline${ }^{99} \mathrm{Tc}^{m}$ & -87180.4 & $4 \quad 2.0$ & 142.6832 & 0.0011 & 6.015 & $\mathrm{~h}$ & 0.009 & $1 / 2^{-}$ & 01 & & & $\mathrm{IT} \approx 100 ; \beta^{-}=0.00376$ \\
\hline${ }^{99} \mathrm{Ru}$ & -87617.0 & $0 \quad 2.0$ & & & STABLE & & & $5 / 2^{+}$ & 95 & & & $\mathrm{IS}=12.7614$ \\
\hline${ }^{99} \mathrm{Rh}$ & -85574 & 7 & & & 16.1 & $\mathrm{~d}$ & 0.2 & $\left(1 / 2^{-}\right)$ & 95 & & & $\beta^{+}=100$ \\
\hline${ }^{99} \mathrm{Rh}^{m}$ & -85510 & 7 & 64.3 & 0.4 & 4.7 & $\mathrm{~h}$ & 0.1 & $9 / 2^{+}$ & 95 & & & $\beta^{+} \approx 100 ;$ IT $<0.16$ \\
\hline${ }^{99} \mathrm{Pd}$ & -82188 & 15 & & & 21.4 & $\mathrm{~m}$ & 0.2 & $(5 / 2)^{+}$ & 95 & & & $\beta^{+}=100$ \\
\hline${ }^{99} \mathrm{Ag}$ & -76760 & 150 & & & 124 & $\mathrm{~s}$ & 3 & $(9 / 2)^{+}$ & 95 & & & $\beta^{+}=100$ \\
\hline${ }^{99} \mathrm{Ag}^{m}$ & -76250 & 150 & 506.1 & 0.4 & 10.5 & $\mathrm{~s}$ & 0.5 & $\left(1 / 2^{-}\right)$ & 95 & & & $\mathrm{IT}=100$ \\
\hline${ }^{99} \mathrm{Cd}$ & $-69850 \#$ & $210 \#$ & & & 16 & $\mathrm{~s}$ & 3 & $\left(5 / 2^{+}\right)$ & 95 & & & $\beta^{+}=100 ; \beta^{+} \mathrm{p}=0.218 ; \ldots$ \\
\hline${ }^{99} \mathrm{In}$ & $-61270 \#$ & $400 \#$ & & & 3.1 & $\mathrm{~s}$ & 0.8 & $9 / 2^{+} \#$ & 97 & $01 \mathrm{Ki} 13$ & $\mathrm{TD}$ & $\beta^{+}=100 ; \beta^{+} \mathrm{p} ?$ \\
\hline${ }^{99} \mathrm{In}^{m}$ & $-60870 \#$ & $430 \#$ & $400 \#$ & $150 \#$ & 1\# & $\mathrm{s}$ & & $1 / 2^{-} \#$ & & & & $\beta^{+} ? ;$ IT ? \\
\hline${ }^{99} \mathrm{Sn}$ & $-47200 \#$ & $600 \#$ & & & $5 \#$ & $\mathrm{~ms}$ & & $9 / 2^{+} \#$ & & & & $\beta^{+} ? ; \beta^{+} \mathrm{p} ?$ \\
\hline${ }^{99} \mathrm{Sn}^{m}$ & $-46800 \#$ & $610 \#$ & $400 \#$ & $100 \#$ & & & & $1 / 2^{-} \#$ & & & & \\
\hline$*{ }^{99} \mathrm{Cd}$ & $\mathrm{D}: \ldots ; \beta$ & $3^{+} \alpha<10$ & & & & & & & & & & \\
\hline$*{ }^{99} \mathrm{Sn}$ & $\mathrm{I}$ : the $3 \mathrm{er}$ & vents re & eported in 95 & $5 \mathrm{Ry} 03$ are & usted by & NuB & & & & & & \\
\hline
\end{tabular}




\begin{tabular}{|c|c|c|c|c|c|c|c|c|c|c|c|c|c|c|}
\hline Nuclide & \multicolumn{2}{|c|}{$\begin{array}{l}\text { Mass excess } \\
\quad(\mathrm{keV})\end{array}$} & \multicolumn{3}{|c|}{$\begin{array}{l}\text { Excitation } \\
\text { energy }(\mathrm{keV})\end{array}$} & \multicolumn{3}{|c|}{ Half-life } & $J^{\pi}$ & Ens & \multicolumn{2}{|c|}{ Reference } & \multicolumn{2}{|l|}{$\begin{array}{l}\text { Decay modes and } \\
\text { intensities (\%) }\end{array}$} \\
\hline${ }^{100} \mathrm{Kr}$ & $-36200 \#$ & $500 \#$ & & & & $10 \#$ & $\mathrm{~ms}$ & $>300 \mathrm{~ns})$ & $0^{+}$ & 97 & $97 \mathrm{Be} 70$ & I & $\beta^{-?}$ ? & \\
\hline${ }^{100} \mathrm{Rb}$ & $-46700 \#$ & $300 \#$ & & & & 51 & $\mathrm{~ms}$ & 8 & $\left(3^{+}\right)$ & 97 & 93Ru01 & $\mathrm{D}$ & $\beta^{-}=100 ; \beta^{-} \mathrm{n}=5.612 ; \ldots$ & $*$ \\
\hline${ }^{100} \mathrm{Sr}$ & -60220 & 130 & & & & 202 & $\mathrm{~ms}$ & 3 & $0^{+}$ & 97 & & & $\beta^{-}=100 ; \beta^{-} \mathrm{n}=0.7813$ & \\
\hline${ }^{100} \mathrm{Y}$ & -67290 & 80 & & & * & 735 & $\mathrm{~ms}$ & 7 & $1^{-}, 2^{-}$ & 97 & & & $\beta^{-}=100 ; \beta^{-} \mathrm{n}=0.928$ & \\
\hline${ }^{100} \mathrm{Y}^{m}$ & $-67090 \#$ & $220 \#$ & $200 \#$ & $200 \#$ & * & 940 & $\mathrm{~ms}$ & 30 & $(3,4,5)^{+\#}$ & 97 & & & $\beta^{-}=100$ & \\
\hline${ }^{100} \mathrm{Zr}$ & -76600 & 40 & & & & 7.1 & $\mathrm{~s}$ & 0.4 & $0^{+}$ & 97 & & & $\beta^{-}=100$ & \\
\hline${ }^{100} \mathrm{Nb}$ & -79939 & 26 & & & & 1.5 & $\mathrm{~s}$ & 0.2 & $1^{+}$ & 97 & & & $\beta^{-}=100$ & \\
\hline${ }^{100} \mathrm{Nb}^{m}$ & -79471 & 28 & 470 & 40 & $\mathrm{BD}$ & 2.99 & $\mathrm{~s}$ & 0.11 & $\left(4^{+}, 5^{+}\right)$ & 97 & & & $\beta^{-}=100$ & \\
\hline${ }^{100} \mathrm{Mo}$ & -86184 & 6 & & & & 8.5 & Ey & 0.5 & $0^{+}$ & 97 & $97 \mathrm{Al} 02$ & $\mathrm{~T}$ & $\mathrm{IS}=9.6323 ; 2 \beta^{-}=100$ & * \\
\hline${ }^{100} \mathrm{Tc}$ & -86016.2 & 2.2 & & & & 15.8 & $\mathrm{~s}$ & 0.1 & $1^{+}$ & 97 & & & $\beta^{-} \approx 100 ; \varepsilon=0.00189$ & \\
\hline${ }^{100} \mathrm{Tc}^{m}$ & -85815.5 & 2.2 & 200.67 & 0.04 & & 8.32 & $\mu \mathrm{s}$ & 0.14 & $(4)^{+}$ & 97 & & & & \\
\hline${ }^{100} \mathrm{Tc}^{n}$ & -85772.2 & 2.2 & 243.96 & 0.04 & & 3.2 & $\mu \mathrm{s}$ & 0.2 & $(6)^{+}$ & 97 & & & & \\
\hline${ }^{100} \mathrm{Ru}$ & -89219.0 & 2.0 & & & & STABLE & & & $0^{+}$ & 97 & & & $\mathrm{IS}=12.607$ & \\
\hline${ }^{100} \mathrm{Rh}$ & -85584 & 18 & & & & 20.8 & $\mathrm{~h}$ & 0.1 & $1^{-}$ & 97 & & & $\beta^{+}=100$ & \\
\hline${ }^{100} \mathrm{Rh}^{m}$ & -85476 & 18 & 107.6 & 0.2 & & 4.6 & $\mathrm{~m}$ & 0.2 & $\left(5^{+}\right)$ & 97 & & & $\mathrm{IT} \approx 98.3 ; \beta^{+} \approx 1.7$ & \\
\hline${ }^{100} \mathrm{Pd}$ & -85226 & 11 & & & & 3.63 & $\mathrm{~d}$ & 0.09 & $0^{+}$ & 97 & & & $\varepsilon=100$ & \\
\hline${ }^{100} \mathrm{Ag}$ & -78150 & 80 & & & & 2.01 & $\mathrm{~m}$ & 0.09 & $(5)^{+}$ & 97 & & & $\beta^{+}=100$ & \\
\hline${ }^{100} \mathrm{Ag}^{m}$ & -78130 & 80 & 15.52 & 0.16 & & 2.24 & $\mathrm{~m}$ & 0.13 & $(2)^{+}$ & 97 & & & $\beta^{+}=$?; IT ? & \\
\hline${ }^{100} \mathrm{Cd}$ & -74250 & 100 & & & & 49.1 & $\mathrm{~s}$ & 0.5 & $0^{+}$ & 97 & & & $\beta^{+}=100$ & \\
\hline${ }^{100} \mathrm{Cd}^{m}$ & -71700 & 100 & 2548.6 & 0.5 & & 60 & ns & 3 & $(8)^{+}$ & 97 & & & $\mathrm{IT}=100$ & \\
\hline${ }^{100} \mathrm{In}$ & -64170 & 250 & & & & 5.9 & $\mathrm{~s}$ & 0.2 & $(6,7)^{+}$ & 97 & $02 \mathrm{P} 103$ & $\mathrm{TJ}$ & $\beta^{+}=100 ; \beta^{+} p>3.9$ & $*$ \\
\hline${ }^{100} \mathrm{Sn}$ & -56780 & 710 & & & & 1.1 & s & 0.4 & $0^{+}$ & 97 & & & $\beta^{+}=100 ; \beta^{+} \mathrm{p}<17$ & $*$ \\
\hline$*{ }^{100} \mathrm{Rb}$ & \multicolumn{12}{|c|}{$D: \ldots ; \beta^{-} 2 \mathrm{n}=0.155$} & & $* *$ \\
\hline$*{ }^{100} \mathrm{Rb}$ & \multicolumn{13}{|c|}{$\mathrm{T}:$ ENSDF average of 3 values. See also 53(2) of 85Pf.A $\quad \mathrm{J}:$ from $95 \mathrm{Pf} 04$} & $* *$ \\
\hline$*^{100} \mathrm{Rb}$ & \multicolumn{13}{|c|}{$\mathrm{D}: \beta^{-} 2 \mathrm{n}$ intensity is derived from $\beta^{-} 2 \mathrm{n} / \beta^{-} \mathrm{n}=0.027(7)$, in $81 \mathrm{Jo} . \mathrm{A}$} & $* *$ \\
\hline$*^{100} \mathrm{Mo}$ & \multicolumn{13}{|c|}{$\mathrm{T}:$ average $97 \mathrm{Al} 02=7.6(+2.2-1.4) 97 \mathrm{De} 40=6.82(+0.38-0.53$ statistics +0.68 systematics $)$} & $* *$ \\
\hline$*^{100} \mathrm{Mo}$ & \multicolumn{13}{|c|}{$\mathrm{T}: \quad 95 \mathrm{Da} 37=9.5(0.9) 91 \mathrm{Ej} 02=11.5(+3-2)$ and $91 \mathrm{E} 104=11.6(+3.4-0.8)$} & $* *$ \\
\hline$*^{100} \mathrm{In}$ & \multicolumn{13}{|c|}{$\mathrm{T}:$ others: $95 \mathrm{Sz} 01=6.1(0.9) 95 \mathrm{Fa} . \mathrm{A}=6.3(+1.0-.9) ; 95 \mathrm{Fa} . \mathrm{A}$ supersedes $95 \mathrm{Sc} 33=7.8(.8)$} & $* *$ \\
\hline$*^{100} \mathrm{Sn}$ & \multicolumn{13}{|c|}{$\mathrm{D}$ : from $97 \mathrm{Su} 06 \beta^{+} \mathrm{p} / \beta^{+}<20 \%$} & $* *$ \\
\hline
\end{tabular}

\begin{tabular}{|c|c|c|c|c|c|c|c|c|c|c|c|}
\hline${ }^{101} \mathrm{Rb}$ & -43600 & 170 & & & 32 & $\mathrm{~ms}$ & 4 & $3 / 2^{+} \#$ & 98 & & $\beta^{-}=100 ; \beta^{-} \mathrm{n}=284$ \\
\hline${ }^{101} \mathrm{Sr}$ & -55410 & 120 & & & 118 & $\mathrm{~ms}$ & 3 & $\left(5 / 2^{-}\right)$ & 98 & & $\beta^{-}=100 ; \beta^{-} \mathrm{n}=2.3714$ \\
\hline${ }^{101} \mathrm{Y}$ & -64910 & 100 & & & 426 & $\mathrm{~ms}$ & 20 & $\left(5 / 2^{+}\right)$ & 98 & $96 \mathrm{Me} 09 \mathrm{~T}$ & $\beta^{-}=100 ; \beta^{-} \mathrm{n}=1.9418$ \\
\hline${ }^{101} \mathrm{Zr}$ & -73460 & 30 & & & 2.3 & $\mathrm{~s}$ & 0.1 & $3 / 2^{+}$ & 98 & $02 \mathrm{Ca} 37 \mathrm{~J}$ & $\beta^{-}=100$ \\
\hline${ }^{101} \mathrm{Nb}$ & -78942 & 19 & & & 7.1 & $\mathrm{~s}$ & 0.3 & $(5 / 2 \#)^{+}$ & 98 & & $\beta^{-}=100$ \\
\hline${ }^{101}$ Mo & -83511 & 6 & & & 14.61 & $\mathrm{~m}$ & 0.03 & $1 / 2^{+}$ & 98 & & $\beta^{-}=100$ \\
\hline${ }^{101} \mathrm{Tc}$ & -86336 & 24 & & & 14.22 & $\mathrm{~m}$ & 0.01 & $9 / 2^{+}$ & 98 & & $\beta^{-}=100$ \\
\hline${ }^{101} \mathrm{Tc}^{m}$ & -86128 & 24 & 207.53 & 0.04 & 636 & $\mu \mathrm{s}$ & 8 & $1 / 2^{-}$ & 98 & & $\mathrm{IT}=100$ \\
\hline${ }^{101} \mathrm{Ru}$ & -87949.7 & 2.0 & & & STABLE & & & $5 / 2^{+}$ & 98 & & $\mathrm{IS}=17.062$ \\
\hline${ }^{101} \mathrm{Ru}^{m}$ & -87422.2 & 2.0 & 527.5 & 0.4 & 17.5 & $\mu \mathrm{s}$ & 0.4 & $11 / 2^{-}$ & 98 & & $\mathrm{IT}=100$ \\
\hline${ }^{101} \mathrm{Rh}$ & -87408 & 17 & & & 3.3 & $\mathrm{y}$ & 0.3 & $1 / 2^{-}$ & 98 & & $\varepsilon=100$ \\
\hline${ }^{101} \mathrm{Rh}^{m}$ & -87251 & 17 & 157.32 & 0.04 & 4.34 & $\mathrm{~d}$ & 0.01 & $9 / 2^{+}$ & 98 & & $\varepsilon=93.62 ; \mathrm{IT}=6.42$ \\
\hline${ }^{101} \mathrm{Pd}$ & -85428 & 18 & & & 8.47 & $\mathrm{~h}$ & 0.06 & $5 / 2^{+}$ & 98 & & $\beta^{+}=100$ \\
\hline${ }^{101} \mathrm{Ag}$ & -81220 & 100 & & & 11.1 & $\mathrm{~m}$ & 0.3 & $9 / 2^{+}$ & 98 & & $\beta^{+}=100$ \\
\hline${ }^{101} \mathrm{Ag}^{m}$ & -80950 & 100 & 274.1 & 0.3 & 3.10 & $\mathrm{~s}$ & 0.10 & $1 / 2^{-}$ & 98 & & $\mathrm{IT}=100$ \\
\hline${ }^{101} \mathrm{Cd}$ & -75750 & 150 & & & 1.36 & $\mathrm{~m}$ & 0.05 & $\left(5 / 2^{+}\right)$ & 98 & & $\beta^{+}=100$ \\
\hline${ }^{101} \mathrm{In}$ & $-68610 \#$ & $300 \#$ & & & 15.1 & $\mathrm{~s}$ & 1.1 & $9 / 2^{+} \#$ & 98 & & $\beta^{+}=100 ; \beta^{+} \mathrm{p}=?$ \\
\hline${ }^{101} \operatorname{In}^{m}$ & $-68060 \#$ & $320 \#$ & $550 \#$ & $100 \#$ & $10 \#$ & $\mathrm{~s}$ & & $1 / 2^{-} \#$ & & & $\beta^{+}=95 \# ; \mathrm{IT}=5 \#$ \\
\hline${ }^{101} \mathrm{Sn}$ & $-59560 \#$ & $300 \#$ & & & 3 & $\mathrm{~s}$ & 1 & $5 / 2^{+} \#$ & 98 & & $\beta^{+}=100 ; \beta^{+} \mathrm{p}=$ ? \\
\hline${ }^{01} \mathrm{Y}$ & $\mathrm{T}$ : averag & $\mathrm{e} 96 \mathrm{Me}$ & -400 & $36 \mathrm{~V}-2$ & 20) an & $83 \mathrm{~V}$ & $=5$ & & & & \\
\hline${ }^{01} \mathrm{Y}$ & $\mathrm{T}:$ & $\mathrm{Ru} 01=$ & (9) at & iance & & & & & & & \\
\hline
\end{tabular}




\begin{tabular}{|c|c|c|c|c|c|c|c|c|c|c|c|c|c|c|}
\hline Nuclide & \multicolumn{2}{|c|}{$\begin{array}{c}\text { Mass excess } \\
(\mathrm{keV})\end{array}$} & \multicolumn{3}{|c|}{$\begin{array}{l}\text { Excitation } \\
\text { energy }(\mathrm{keV})\end{array}$} & \multicolumn{3}{|c|}{ Half-life } & \multirow[t]{2}{*}{$J^{\pi}$} & Ens & \multicolumn{2}{|c|}{ Reference } & \multicolumn{2}{|l|}{$\begin{array}{c}\text { Decay modes and } \\
\text { intensities }(\%)\end{array}$} \\
\hline${ }^{102} \mathrm{Rb}$ & $-38310 \#$ & $500 \#$ & & & & 37 & $\mathrm{~ms}$ & 5 & & 98 & & & $\beta^{-}=100 ; \beta^{-} \mathrm{n}=188$ & \\
\hline${ }^{102} \mathrm{Sr}$ & -53080 & 110 & & & & 69 & $\mathrm{~ms}$ & 6 & $0^{+}$ & 98 & 93Ru01 & $\mathrm{D}$ & $\beta^{-}=100 ; \beta^{-} \mathrm{n}=5.515$ & \\
\hline${ }^{102} \mathrm{Y}$ & -61890 & 90 & & & $* \&$ & 300 & $\mathrm{~ms}$ & 10 & low & 98 & & & $\beta^{-}=100 ; \beta^{-} \mathrm{n}=4.912$ & \\
\hline${ }^{102} \mathrm{Y}^{m}$ & $-61690 \#$ & $220 \#$ & $200 \#$ & $200 \#$ & $* \&$ & 360 & $\mathrm{~ms}$ & 40 & high & 98 & & & $\beta^{-}=100 ; \beta^{-} \mathrm{n}=4.912$ & \\
\hline${ }^{102} \mathrm{Zr}$ & -71740 & 50 & & & & 2.9 & $\mathrm{~s}$ & 0.2 & $0^{+}$ & 98 & & & $\beta^{-}=100$ & \\
\hline${ }^{102} \mathrm{Nb}$ & -76350 & 40 & & & & 1.3 & $\mathrm{~s}$ & 0.2 & $1^{+}$ & 98 & & & $\beta^{-}=100$ & \\
\hline${ }^{102} \mathrm{Nb}^{m}$ & -76220 & 50 & 130 & 50 & BD & 4.3 & $\mathrm{~s}$ & 0.4 & high & 98 & & & $\beta^{-}=100$ & \\
\hline${ }^{102} \mathrm{Mo}$ & -83557 & 21 & & & & 11.3 & $\mathrm{~m}$ & 0.2 & $0^{+}$ & 01 & & & $\beta^{-}=100$ & \\
\hline${ }^{102} \mathrm{Tc}$ & -84566 & 9 & & & * & 5.28 & $\mathrm{~s}$ & 0.15 & $1^{+}$ & 98 & & & $\beta^{-}=100$ & \\
\hline${ }^{102} \mathrm{Tc}^{m}$ & -84546 & 13 & 20 & 10 & $*$ & 4.35 & $\mathrm{~m}$ & 0.07 & $(4,5)$ & 98 & & & $\beta^{-}=982 ; \mathrm{IT}=22$ & \\
\hline${ }^{102} \mathrm{Ru}$ & -89098.0 & 2.0 & & & & STABLE & & & $0^{+}$ & 98 & & & $\mathrm{IS}=31.5514$ & \\
\hline${ }^{102} \mathrm{Rh}$ & -86775 & 5 & & & & 207.0 & $\mathrm{~d}$ & 1.5 & $\left(1^{-}, 2^{-}\right)$ & 98 & $98 \operatorname{Sh} 21$ & $\mathrm{~T}$ & $\beta^{+}=785 ; \beta^{-}=225$ & * \\
\hline${ }^{102} \mathrm{Rh}^{m}$ & -86634 & 5 & 140.75 & 0.08 & & 3.742 & $\mathrm{y}$ & 0.010 & $6^{+}$ & 98 & $98 \mathrm{Sh} 21$ & $\mathrm{~T}$ & $\beta^{+} \approx 100 ; \mathrm{IT}=0.23324$ & $*$ \\
\hline${ }^{102} \mathrm{Pd}$ & -87925.1 & 3.0 & & & & STABLE & & & $0^{+}$ & 98 & & & $\mathrm{IS}=1.021 ; 2 \beta^{+} ?$ & \\
\hline${ }^{102} \mathrm{Ag}$ & -82265 & 28 & & & & 12.9 & $\mathrm{~m}$ & 0.3 & $5^{+}$ & 98 & & & $\beta^{+}=100$ & \\
\hline${ }^{102} \mathrm{Ag}^{m}$ & -82256 & 28 & 9.3 & 0.4 & & 7.7 & $\mathrm{~m}$ & 0.5 & $2^{+}$ & 98 & & & $\beta^{+}=515 ; \mathrm{IT}=495$ & \\
\hline${ }^{102} \mathrm{Cd}$ & -79678 & 29 & & & & 5.5 & $\mathrm{~m}$ & 0.5 & $0^{+}$ & 98 & & & $\beta^{+}=100$ & \\
\hline${ }^{102} \mathrm{In}$ & -70710 & 110 & & & & 23.3 & $\mathrm{~s}$ & 0.1 & $\left(6^{+}\right)$ & 98 & $03 \mathrm{Gi} 06$ & $\mathrm{~T}$ & $\beta^{+}=100 ; \beta^{+} \mathrm{p}=0.009313$ & * \\
\hline${ }^{102} \mathrm{Sn}$ & -64930 & 130 & & & & 4.6 & $\mathrm{~s}$ & 1.4 & $0^{+}$ & 98 & 95Fa.A & $\mathrm{T}$ & $\beta^{+}=100 ; \beta^{+} \mathrm{p} ?$ & $*$ \\
\hline${ }^{102} \mathrm{Sn}^{m}$ & -62910 & 130 & 2017 & 2 & & 720 & ns & 220 & $\left(6^{+}\right)$ & 98 & $98 \operatorname{Li} 50$ & EJT & $\mathrm{IT}=100$ & * \\
\hline$*^{102} \mathrm{Rh}$ & $\mathrm{T}$ : averag & $98 \mathrm{Sh}$ & $21=207.3$ & $(1.7) 61 \mathrm{H}$ & $06=206(3)$ & & & & & & & & & ** \\
\hline$*^{102} \mathrm{Rh}^{m}$ & $\mathrm{~J}:$ from 9 & $9 \mathrm{Gi} 14$ & & & & & & & & & & & & $* *$ \\
\hline$*^{102} \mathrm{In}$ & $\mathrm{J}:$ from 9 & $5 \mathrm{Sz} 01$ & & & & & & & & & & & & ** \\
\hline$*^{102} \mathrm{Sn}$ & $\mathrm{T}: 95 \mathrm{Fa} . \mathrm{A}$ & A, super & rsedes 95 & $\operatorname{Sc} 28=4$ & 7), prelir & minary $f$ & fron & ame $g$ & & & & & & $* *$ \\
\hline$*^{102} \mathrm{Sn}^{m}$ & $\mathrm{~T}$ : averag & $98 \mathrm{Li}$ & $50=620(+$ & $430-190)$ & $97 \mathrm{Gr} 02=3$ & $00(+50 c$ & $0-20$ & $96 \mathrm{Li} 5$ & $=1000(5$ & & & & & $* *$ \\
\hline
\end{tabular}

\begin{tabular}{|c|c|c|c|c|c|c|c|c|c|c|c|c|}
\hline${ }^{103} \mathrm{Sr}$ & $-47550 \#$ & $500 \#$ & & & $50 \#$ & $\mathrm{~ms}$ & $>300 \mathrm{~ns})$ & & 01 & $97 \mathrm{Be} 70$ & I & $\beta^{-} ?$ \\
\hline${ }^{103} \mathrm{Y}$ & $-58940 \#$ & $300 \#$ & & & 224 & $\mathrm{~ms}$ & 19 & $5 / 2^{+} \#$ & 01 & $96 \mathrm{Me} 09$ & $\mathrm{~T}$ & $\beta^{-}=100 ; \beta^{-} \mathrm{n}=83$ \\
\hline${ }^{103} \mathrm{Zr}$ & -68370 & 110 & & & 1.3 & $\mathrm{~s}$ & 0.1 & $\left(5 / 2^{-}\right)$ & 01 & & & $\beta^{-}=100$ \\
\hline${ }^{103} \mathrm{Nb}$ & -75320 & 70 & & & 1.5 & $\mathrm{~s}$ & 0.2 & $\left(5 / 2^{+}\right)$ & 01 & & & $\beta^{-}=100$ \\
\hline${ }^{103}$ Mo & -80850 & 60 & & & 67.5 & $\mathrm{~s}$ & 1.5 & $\left(3 / 2^{+}\right)$ & 01 & & & $\beta^{-}=100$ \\
\hline${ }^{103} \mathrm{Tc}$ & -84597 & 10 & & & 54.2 & $\mathrm{~s}$ & 0.8 & $5 / 2^{+}$ & 01 & & & $\beta^{-}=100$ \\
\hline${ }^{103} \mathrm{Ru}$ & -87258.8 & 2.0 & & & 39.26 & d & 0.02 & $3 / 2^{+}$ & 01 & & & $\beta^{-}=100$ \\
\hline${ }^{103} \mathrm{Ru}^{m}$ & -87020.6 & 2.1 & 238.2 & 0.7 & 1.69 & $\mathrm{~ms}$ & 0.07 & $11 / 2^{-}$ & 01 & & & $\mathrm{IT}=100$ \\
\hline${ }^{103} \mathrm{Rh}$ & -88022.2 & 2.8 & & & STABLE & & & $1 / 2^{-}$ & 01 & & & $\mathrm{IS}=100$. \\
\hline${ }^{103} \mathrm{Rh}^{m}$ & -87982.4 & 2.8 & 39.756 & 0.006 & 56.114 & $\mathrm{~m}$ & 0.009 & $7 / 2^{+}$ & 01 & & & $\mathrm{IT}=100$ \\
\hline${ }^{103} \mathrm{Pd}$ & -87479.1 & 2.9 & & & 16.991 & $\mathrm{~d}$ & 0.019 & $5 / 2^{+}$ & 01 & & & $\varepsilon=100$ \\
\hline${ }^{103} \mathrm{Pd}^{m}$ & -86694.3 & 2.9 & 784.79 & 0.10 & 25 & $\mathrm{~ns}$ & 2 & $11 / 2^{-}$ & 01 & & & $\mathrm{IT}=100$ \\
\hline${ }^{103} \mathrm{Ag}$ & -84791 & 17 & & & 65.7 & $\mathrm{~m}$ & 0.7 & $7 / 2^{+}$ & 01 & & & $\beta^{+}=100$ \\
\hline${ }^{103} \mathrm{Ag}^{m}$ & -84657 & 17 & 134.45 & 0.04 & 5.7 & $\mathrm{~s}$ & 0.3 & $1 / 2^{-}$ & 01 & & & $\mathrm{IT}=100$ \\
\hline${ }^{103} \mathrm{Cd}$ & -80649 & 15 & & & 7.3 & $\mathrm{~m}$ & 0.1 & $5 / 2^{+}$ & 01 & & & $\beta^{+}=100$ \\
\hline${ }^{103} \mathrm{In}$ & -74599 & 25 & & & 60 & $\mathrm{~s}$ & 1 & $9 / 2^{+} \#$ & 01 & $97 \mathrm{Sz} 04$ & $\mathrm{~T}$ & $\beta^{+}=100$ \\
\hline${ }^{103} \mathrm{In}^{m}$ & -73967 & 25 & 631.7 & 0.1 & 34 & $\mathrm{~s}$ & 2 & $1 / 2^{-} \#$ & 01 & 97Sz04 & ETD & $\beta^{+}=67 ; \mathrm{IT}=33$ \\
\hline${ }^{103} \mathrm{Sn}$ & $-66970 \#$ & $300 \#$ & & & 7 & $\mathrm{~s}$ & 3 & $5 / 2^{+} \#$ & 01 & & & $\beta^{+}=100 ; \beta^{+} \mathrm{p}=?$ \\
\hline${ }^{103} \mathrm{Sb}$ & $-56180 \#$ & $300 \#$ & & & $100 \#$ & $\mathrm{~ms}$ & $>1.5 \mu \mathrm{s})$ & $5 / 2^{+} \#$ & 01 & 95Ry03 & I & $\beta^{+} ?$ \\
\hline${ }^{103} \mathrm{Y}$ & $\mathrm{T}$ : averag & $96 \mathrm{M}$ & 2300 & $5 \mathrm{Lh} 04=190(50)$ & & & & & & & & \\
\hline
\end{tabular}




\begin{tabular}{|c|c|c|c|c|c|c|c|c|c|c|c|c|c|}
\hline Nuclide & \multicolumn{2}{|c|}{$\begin{array}{l}\text { Mass excess } \\
(\mathrm{keV})\end{array}$} & \multicolumn{3}{|c|}{$\begin{array}{l}\text { Excitation } \\
\text { energy }(\mathrm{keV})\end{array}$} & \multicolumn{3}{|c|}{ Half-life } & \multirow[t]{2}{*}{$J^{\pi}$} & \multirow[t]{2}{*}{ Ens } & Reference & \multicolumn{2}{|l|}{$\begin{array}{l}\text { Decay modes and } \\
\text { intensities }(\%)\end{array}$} \\
\hline${ }^{104} \mathrm{Sr}$ & $-44400 \#$ & $700 \#$ & & & & 30\# & $\mathrm{ms}$ & $(>300 \mathrm{~ns})$ & & & 97Be70 I & $\beta^{-} ?$ & \\
\hline${ }^{104} \mathrm{Y}$ & $-54910 \#$ & $400 \#$ & & & & 180 & $\mathrm{~ms}$ & 60 & & 00 & 99Wa09 D & $\beta^{-}=100 ; \beta^{-} \mathrm{n}=?$ & \\
\hline${ }^{104} \mathrm{Zr}$ & $-66340 \#$ & $400 \#$ & & & & 1.2 & $\mathrm{~s}$ & 0.3 & $0^{+}$ & 00 & & $\beta^{-}=100$ & \\
\hline${ }^{104} \mathrm{Nb}$ & -72220 & 100 & & & * & 4.9 & $\mathrm{~s}$ & 0.3 & $\left(1^{+}\right)$ & 00 & & $\beta^{-}=100 ; \beta^{-} \mathrm{n}=0.063$ & * \\
\hline${ }^{104} \mathrm{Nb}^{m}$ & -72010 & 100 & 220 & 120 & $\mathrm{BD} *$ & 940 & $\mathrm{~ms}$ & 40 & high & 00 & & $\beta^{-}=100 ; \beta^{-} \mathrm{n}=0.053$ & \\
\hline${ }^{104} \mathrm{Mo}$ & -80330 & 50 & & & & 60 & $\mathrm{~s}$ & 2 & $0^{+}$ & 00 & & $\beta^{-}=100$ & \\
\hline${ }^{104} \mathrm{Tc}$ & -82490 & 50 & & & & 18.3 & $\mathrm{~m}$ & 0.3 & $3^{+} \#$ & 00 & & $\beta^{-}=100$ & \\
\hline${ }^{104} \mathrm{Tc}^{m}$ & -82420 & 50 & 69.7 & 0.2 & & 3.5 & $\mu \mathrm{s}$ & 0.3 & $2^{(+)}$ & 00 & & $\mathrm{IT}=100$ & \\
\hline${ }^{104} \mathrm{Ru}$ & -88089 & 3 & & & & STABLE & & & $0^{+}$ & 00 & & $\mathrm{IS}=18.6227 ; 2 \beta^{-} ?$ & \\
\hline${ }^{104} \mathrm{Rh}$ & -86949.8 & 2.8 & & & & 42.3 & $\mathrm{~s}$ & 0.4 & $1^{+}$ & 00 & & $\beta^{-} \approx 100 ; \beta^{+}=0.4510$ & \\
\hline${ }^{104} \mathrm{Rh}^{m}$ & -86820.8 & 2.8 & 128.967 & 0.004 & & 4.34 & $\mathrm{~m}$ & 0.03 & $5^{+}$ & 00 & & $\mathrm{IT} \approx 100 ; \beta^{-}=0.131$ & \\
\hline${ }^{104} \mathrm{Pd}$ & -89390 & 4 & & & & STABLE & & & $0^{+}$ & 00 & & $\mathrm{IS}=11.148$ & \\
\hline${ }^{104} \mathrm{Ag}$ & -85111 & 6 & & & & 69.2 & $\mathrm{~m}$ & 1.0 & $5^{+}$ & 00 & & $\beta^{+}=100$ & \\
\hline${ }^{104} \mathrm{Ag}^{m}$ & -85104 & 6 & 6.9 & 0.4 & & 33.5 & $\mathrm{~m}$ & 2.0 & $2^{+}$ & 00 & & $\beta^{+} \approx 100 ;$ IT $<0.07$ & \\
\hline${ }^{104} \mathrm{Cd}$ & -83975 & 9 & & & & 57.7 & $\mathrm{~m}$ & 1.0 & $0^{+}$ & 00 & & $\beta^{+}=100$ & \\
\hline${ }^{104}$ In & -76110 & 80 & & & & 1.80 & $\mathrm{~m}$ & 0.03 & $5,6^{(+)}$ & 00 & & $\beta^{+}=100$ & \\
\hline${ }^{104} \mathrm{In}^{m}$ & -76020 & 80 & 93.48 & 0.10 & & 15.7 & s & 0.5 & $\left(3^{+}\right)$ & 00 & & $\mathrm{IT}=80 ; \beta^{+}=20$ & \\
\hline${ }^{104} \mathrm{Sn}$ & -71590 & 100 & & & & 20.8 & $\mathrm{~s}$ & 0.5 & $0^{+}$ & 00 & & $\beta^{+}=100$ & \\
\hline${ }^{104} \mathrm{Sb}$ & $-59180 \#$ & $360 \#$ & & & & 470 & $\mathrm{~ms}$ & 130 & & 00 & 95Fa.A D & $\beta^{+}=? ; \beta^{+} \mathrm{p}<7 ; \mathrm{p}<7 ; \alpha ?$ & * \\
\hline$*^{104} \mathrm{Nb}$ & $\mathrm{D}: \beta^{-} \mathrm{n}=0$ & $.71 \% \mathrm{o}$ & f 83En03, & at var & , no & & & & & & & & * \\
\hline$*^{104} \mathrm{Sb}$ & D : $95 \mathrm{Fa} . \mathrm{A}$ & super: & edes $95 \mathrm{Sc}$ & $28 p<1$ & & & & & & & & & $* *$ \\
\hline
\end{tabular}

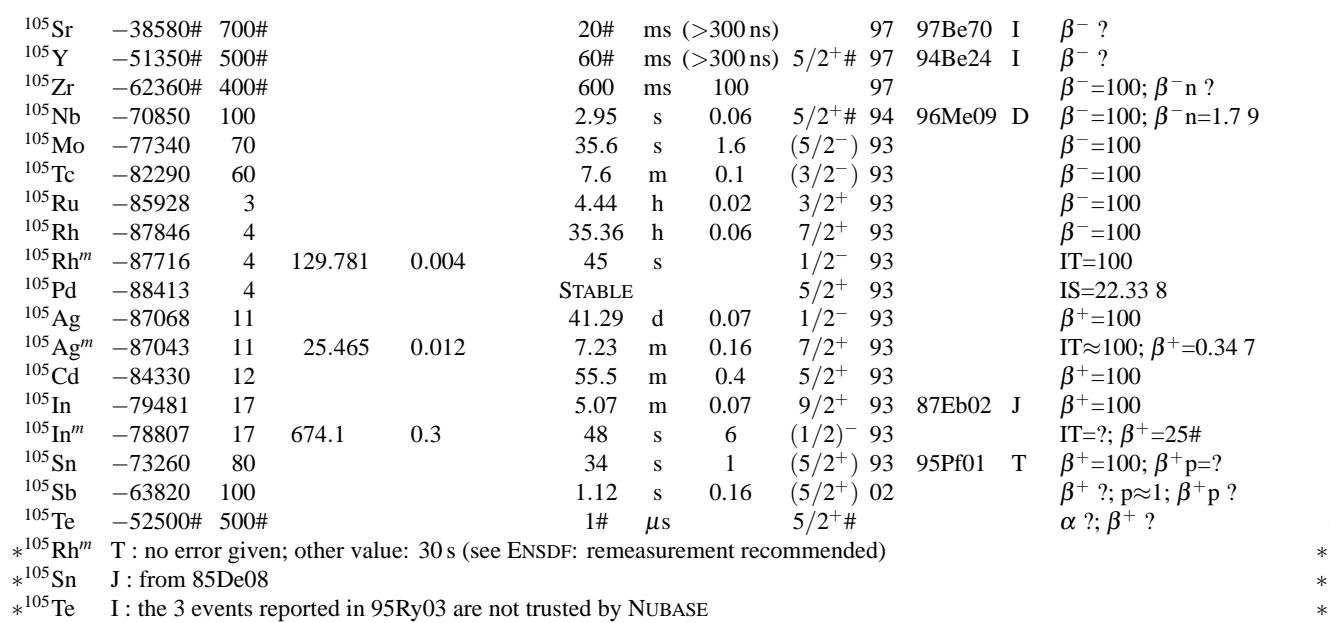

\begin{tabular}{|c|c|c|c|c|c|c|c|c|c|c|c|c|c|}
\hline${ }^{106} \mathrm{Y}$ & $-46770 \#$ & $700 \#$ & & & & $50 \#$ & $\mathrm{~ms}$ & $(>300 \mathrm{~ns})$ & & 97 & $97 \mathrm{Be} 70$ & I & $\beta^{-} ?$ \\
\hline${ }^{106} \mathrm{Zr}$ & $-59700 \#$ & $500 \#$ & & & & $200 \#$ & $\mathrm{~ms}$ & $(>300 \mathrm{~ns})$ & $0^{+}$ & 97 & $94 \mathrm{Be} 24$ & I & $\beta^{-} ?$ \\
\hline${ }^{106} \mathrm{Nb}$ & $-67100 \#$ & $200 \#$ & & & & 920 & $\mathrm{~ms}$ & 40 & $2^{+} \#$ & 94 & $96 \mathrm{Me} 09$ & $\mathrm{TD}$ & $\beta^{-}=100 ; \beta^{-} \mathrm{n}=4.53$ \\
\hline${ }^{106} \mathrm{Mo}$ & -76255 & 18 & & & & 8.73 & $\mathrm{~s}$ & 0.12 & $0^{+}$ & 94 & 95Jo02 & $\mathrm{T}$ & $\beta^{-}=100$ \\
\hline${ }^{106} \mathrm{Tc}$ & -79775 & 13 & & & & 35.6 & $\mathrm{~s}$ & 0.6 & $(1,2)$ & 94 & & & $\beta^{-}=100$ \\
\hline${ }^{106} \mathrm{Ru}$ & -86322 & 8 & & & & 373.59 & $\mathrm{~d}$ & 0.15 & $0^{+}$ & 94 & & & $\beta^{-}=100$ \\
\hline${ }^{106} \mathrm{Rh}$ & -86361 & 8 & & & & 29.80 & $\mathrm{~s}$ & 0.08 & $1^{+}$ & 94 & & & $\beta^{-}=100$ \\
\hline${ }^{106} \mathrm{Rh}^{m}$ & -86225 & 11 & 136 & 12 & $\mathrm{BD}$ & 131 & $\mathrm{~m}$ & 2 & $(6)^{+}$ & 94 & & & $\beta^{-}=100$ \\
\hline${ }^{106} \mathrm{Pd}$ & -89902 & 4 & & & & STABLE & & & $0^{+}$ & 94 & & & $\mathrm{IS}=27.333$ \\
\hline${ }^{106} \mathrm{Ag}$ & -86937 & 5 & & & & 23.96 & $\mathrm{~m}$ & 0.04 & $1^{+}$ & 94 & & & $\beta^{+}=? ; \beta^{-} \approx 0.5$ \\
\hline${ }^{106} \mathrm{Ag}^{m}$ & -86847 & 5 & 89.66 & 0.07 & & 8.28 & $\mathrm{~d}$ & 0.02 & $6^{+}$ & 94 & & & $\beta^{+}=100 ; \mathrm{IT}<4.2 \mathrm{e}-6$ \\
\hline
\end{tabular}




\begin{tabular}{|c|c|c|c|c|c|c|c|c|c|c|c|c|}
\hline Nuclide & $\begin{array}{c}\text { Mass excess } \\
(\mathrm{keV})\end{array}$ & & $\begin{array}{l}\text { xcitation } \\
\text { ergy }(\mathrm{keV})\end{array}$ & & Half- & -life & $J^{\pi}$ & Ens & Referenc & & $\begin{array}{l}\text { Decay modes and } \\
\text { intensities }(\%)\end{array}$ & \\
\hline \multicolumn{13}{|c|}{$\ldots A$-group continued $\ldots$} \\
\hline${ }^{106} \mathrm{Cd}$ & $-87132 \quad 6$ & & & STABLE & & $(>410$ Ey) & $0^{+}$ & 94 & $02 \operatorname{Tr} 04$ & $\mathrm{~T}$ & $\mathrm{IS}=1.256 ; 2 \beta^{+} ?$ & \\
\hline${ }^{106} \mathrm{In}$ & $-80606 \quad 12$ & & & 6.2 & $\mathrm{~m}$ & 0.1 & $7^{+}$ & 94 & & & $\beta^{+}=100$ & \\
\hline${ }^{106} \mathrm{In}^{m}$ & $-80577 \quad 12$ & 28.6 & 0.3 & 5.2 & $\mathrm{~m}$ & 0.1 & $\left(3^{+}\right)$ & 94 & & & $\beta^{+}=100$ & \\
\hline${ }^{106} \mathrm{Sn}$ & $-77430 \quad 50$ & & & 1.92 & $\mathrm{~m}$ & 0.08 & $0^{+}$ & 94 & & & $\beta^{+}=100$ & \\
\hline${ }^{106} \mathrm{Sb}$ & $-66330 \# 310 \#$ & & & 600 & $\mathrm{~ms}$ & 200 & $\left(4^{+}\right)$ & 97 & $94 \mathrm{Se} 01$ & $\mathrm{~J}$ & $\beta^{+}=100$ & $*$ \\
\hline${ }^{106} \mathrm{Sb}^{m}$ & $-65330 \#$ 590\# & $1000 \#$ & $500 \#$ & 220 & ns & 20 & & & 98Li50 & $\mathrm{T}$ & $\mathrm{IT}=100$ & \\
\hline${ }^{106} \mathrm{Te}$ & $-58210 \quad 130$ & & & 70 & $\mu \mathrm{s}$ & 20 & $0^{+}$ & 94 & 94Pa11 & $\mathrm{T}$ & $\alpha=100$ & $*$ \\
\hline$*^{106} \mathrm{Zr}$ & \multicolumn{11}{|c|}{$\mathrm{I}:$ and $T>240 \mathrm{~ns}$ in $97 \mathrm{So} 07$} & $* *$ \\
\hline$*^{106} \mathrm{Nb}$ & \multicolumn{11}{|c|}{$\mathrm{T}:$ average $96 \mathrm{Me} 09=900(20) 83 \mathrm{Sh} 06=1020(50)$} & $* *$ \\
\hline$*^{106} \mathrm{Sb}$ & \multicolumn{11}{|c|}{$\mathrm{T}$ : from 95Le.C, Fig. 4, preliminary } & $* *$ \\
\hline$*^{106} \mathrm{Te}$ & \multicolumn{11}{|c|}{$\mathrm{T}:$ average $94 \mathrm{~Pa} 11=60(+40-20) 81 \mathrm{Sc} 17=60(+30-10)$} & $* *$ \\
\hline
\end{tabular}

\begin{tabular}{|c|c|c|c|c|c|c|c|c|c|c|c|}
\hline${ }^{107} \mathrm{Y}$ & $-42720 \#$ & $500 \#$ & & & $30 \#$ & $\mathrm{~ms}$ & $(>300 \mathrm{~ns})$ & $5 / 2^{+} \#$ & 00 & $97 \mathrm{Be} 70 \mathrm{I}$ & $\beta^{-}$? \\
\hline${ }^{107} \mathrm{Zr}$ & $-55190 \#$ & $300 \#$ & & & $150 \#$ & $\mathrm{~ms}$ & $(>300 \mathrm{~ns})$ & & 00 & $94 \mathrm{Be} 24 \mathrm{I}$ & $\beta^{-}$? \\
\hline${ }^{107} \mathrm{Nb}$ & $-64920 \#$ & $400 \#$ & & & 300 & $\mathrm{~ms}$ & 9 & $5 / 2^{+} \#$ & 00 & $96 \mathrm{Me} 09 \mathrm{TD}$ & $\beta^{-}=100 ; \beta^{-} \mathrm{n}=6.015$ \\
\hline${ }^{107} \mathrm{Mo}$ & -72940 & 160 & & & 3.5 & $\mathrm{~s}$ & 0.5 & $\left(7 / 2^{-}\right)$ & 00 & & $\beta^{-}=100$ \\
\hline${ }^{107} \mathrm{Mo}^{m}$ & -72870 & 160 & 66.3 & 0.2 & 470 & ns & 30 & $\left(5 / 2^{-}\right)$ & 00 & & $\mathrm{IT}=100$ \\
\hline${ }^{107} \mathrm{Tc}$ & -79100 & 150 & & & 21.2 & $\mathrm{~s}$ & 0.2 & $\left(3 / 2^{-}\right)$ & 00 & & $\beta^{-}=100$ \\
\hline${ }^{107} \mathrm{Tc}^{m}$ & -79030 & 150 & 65.7 & 1.0 & 184 & $\mathrm{~ns}$ & 3 & $\left(5 / 2^{-}\right)$ & 00 & & $\mathrm{IT}=100$ \\
\hline${ }^{107} \mathrm{Ru}$ & -83920 & 120 & & & 3.75 & $\mathrm{~m}$ & 0.05 & $(5 / 2)^{+}$ & 00 & & $\beta^{-}=100$ \\
\hline${ }^{107} \mathrm{Rh}$ & -86863 & 12 & & & 21.7 & $\mathrm{~m}$ & 0.4 & $7 / 2^{+}$ & 00 & & $\beta^{-}=100$ \\
\hline${ }^{107} \mathrm{Rh}^{m}$ & -86595 & 12 & 268.36 & 0.04 & $>10$ & $\mu \mathrm{s}$ & & $1 / 2^{-}$ & 00 & & $\mathrm{IT}=100$ \\
\hline${ }^{107} \mathrm{Pd}$ & -88368 & 4 & & & 6.5 & My & 0.3 & $5 / 2^{+}$ & 00 & & $\beta^{-}=100$ \\
\hline${ }^{107} \mathrm{Pd}^{m}$ & -88153 & 4 & 214.6 & 0.3 & 21.3 & $\mathrm{~s}$ & 0.5 & $11 / 2^{-}$ & 00 & & $\mathrm{IT}=100$ \\
\hline${ }^{107} \mathrm{Ag}$ & -88402 & 4 & & & STABLE & & & $1 / 2^{-}$ & 00 & & $\mathrm{IS}=51.8398$ \\
\hline${ }^{107} \mathrm{Ag}^{m}$ & -88309 & 4 & 93.125 & 0.019 & 44.3 & s & 0.2 & $7 / 2^{+}$ & 00 & & $\mathrm{IT}=100$ \\
\hline${ }^{107} \mathrm{Cd}$ & -86985 & 6 & & & 6.50 & $\mathrm{~h}$ & 0.02 & $5 / 2^{+}$ & 00 & & $\beta^{+}=100$ \\
\hline${ }^{107} \mathrm{In}$ & -83560 & 11 & & & 32.4 & $\mathrm{~m}$ & 0.3 & $9 / 2^{+}$ & 00 & & $\beta^{+}=100$ \\
\hline${ }^{107} \mathrm{In}^{m}$ & -82882 & 11 & 678.5 & 0.3 & 50.4 & $\mathrm{~s}$ & 0.6 & $1 / 2^{-}$ & 00 & & $\mathrm{IT}=100$ \\
\hline${ }^{107} \mathrm{Sn}$ & -78580 & 80 & & & 2.90 & $\mathrm{~m}$ & 0.05 & $\left(5 / 2^{+}\right)$ & 00 & & $\beta^{+}=100$ \\
\hline${ }^{107} \mathrm{Sb}$ & $-70650 \#$ & $300 \#$ & & & 4.6 & $\mathrm{~s}$ & 0.8 & $5 / 2^{+} \#$ & 00 & & $\beta^{+}=100$ \\
\hline${ }^{107} \mathrm{Te}$ & $-60540 \#$ & $300 \#$ & & & 3.1 & $\mathrm{~ms}$ & 0.1 & $5 / 2^{+} \#$ & 00 & & $\alpha=7030 ; \beta^{+}=3030$ \\
\hline$*^{107} \mathrm{Zr}$ & $\mathrm{I}:$ and $T$ & $>240 \mathrm{n}$ & in $97 \mathrm{So} 07$ & & & & & & & & \\
\hline$*^{107} \mathrm{Nb}$ & $\mathrm{T}:$ averas & ge $96 \mathrm{M}$ & $09=300(30$ & He & & & & & & & \\
\hline
\end{tabular}

\begin{tabular}{|c|c|c|c|c|c|c|c|c|c|c|c|c|c|c|}
\hline${ }^{108} \mathrm{Y}$ & $-37740 \#$ & $800 \#$ & & & & $20 \#$ & $\mathrm{~ms}$ & $(>300 \mathrm{~ns})$ & & 00 & 95Cz.A & I & $\beta^{-} ? ; \beta^{-} \mathrm{n} ?$ & \\
\hline${ }^{108} \mathrm{Zr}$ & $-52200 \#$ & $600 \#$ & & & & $80 \#$ & $\mathrm{~ms}$ & $(>300 \mathrm{~ns})$ & $0^{+}$ & 00 & $97 \mathrm{Be} 70$ & I & $\beta^{-} ? ; \beta^{-} \mathrm{n} ?$ & \\
\hline${ }^{108} \mathrm{Nb}$ & $-60700 \#$ & $300 \#$ & & & & 193 & $\mathrm{~ms}$ & 17 & $\left(2^{+}\right)$ & 00 & & & $\beta^{-}=100 ; \beta^{-} \mathrm{n}=6.25$ & \\
\hline${ }^{108} \mathrm{Mo}$ & $-71300 \#$ & $200 \#$ & & & & 1.09 & $\mathrm{~s}$ & 0.02 & $0^{+}$ & 00 & & & $\beta^{-}=100$ & \\
\hline${ }^{108} \mathrm{Tc}$ & -75950 & 130 & & & & 5.17 & $\mathrm{~s}$ & 0.07 & $(2)^{+}$ & 00 & & & $\beta^{-}=100$ & \\
\hline${ }^{108} \mathrm{Ru}$ & -83670 & 120 & & & & 4.55 & $\mathrm{~m}$ & 0.05 & $0^{+}$ & 00 & & & $\beta^{-}=100$ & \\
\hline${ }^{108} \mathrm{Rh}$ & -85020 & 110 & & & * & 16.8 & $\mathrm{~s}$ & 0.5 & $1^{+}$ & 00 & & & $\beta^{-}=100$ & \\
\hline${ }^{108} \mathrm{Rh}^{m}$ & -85080 & 40 & -60 & 110 & $\mathrm{BD} *$ & 6.0 & $\mathrm{~m}$ & 0.3 & $(5)^{(+\#)}$ & 00 & & & $\beta^{-}=100$ & \\
\hline${ }^{108} \mathrm{Pd}$ & -89524 & 3 & & & & STABLE & & & $0^{+}$ & 00 & & & $\mathrm{IS}=26.469$ & \\
\hline${ }^{108} \mathrm{Ag}$ & -87602 & 4 & & & & 2.37 & $\mathrm{~m}$ & 0.01 & $1^{+}$ & 00 & & & $\beta^{-}=97.1520 ; \beta^{+}=2.8520$ & \\
\hline${ }^{108} \mathrm{Ag}^{m}$ & -87493 & 4 & 109.440 & 0.007 & & 418 & $\mathrm{y}$ & 21 & $6^{+}$ & 00 & & & $\beta^{+}=91.39 ; \mathrm{IT}=8.79$ & $*$ \\
\hline${ }^{108} \mathrm{Cd}$ & -89252 & 6 & & & & STABLE & & (>410 Py) & $0^{+}$ & 02 & $95 \mathrm{Ge} 14$ & $\mathrm{~T}$ & $\mathrm{IS}=0.893 ; 2 \beta^{+} ?$ & \\
\hline${ }^{108} \mathrm{In}$ & -84116 & 10 & & & & 58.0 & $\mathrm{~m}$ & 1.2 & $7^{+}$ & 00 & & & $\beta^{+}=100$ & \\
\hline${ }^{108} \operatorname{In}^{m}$ & -84086 & 10 & 29.75 & 0.05 & & 39.6 & $\mathrm{~m}$ & 0.7 & $2^{+}$ & 00 & & & $\beta^{+}=100$ & \\
\hline${ }^{108} \mathrm{Sn}$ & -82041 & 20 & & & & 10.30 & $\mathrm{~m}$ & 0.08 & $0^{+}$ & 00 & & & $\beta^{+}=100$ & \\
\hline${ }^{108} \mathrm{Sb}$ & $-72510 \#$ & $210 \#$ & & & & 7.4 & $\mathrm{~s}$ & 0.3 & $\left(4^{+}\right)$ & 00 & & & $\beta^{+}=100 ; \beta^{+} \mathrm{p} ?$ & \\
\hline${ }^{108} \mathrm{Te}$ & -65720 & 100 & & & & 2.1 & $\mathrm{~s}$ & 0.1 & $0^{+}$ & 00 & $85 \mathrm{Ti} 02$ & $\mathrm{D}$ & $\beta^{+}=514 ; \alpha=494$ & * \\
\hline${ }^{108} \mathrm{I}$ & $-52650 \#$ & $360 \#$ & & & & 36 & $\mathrm{~ms}$ & 6 & $1^{+} \#$ & 00 & $94 \mathrm{~Pa} 12$ & $\mathrm{D}$ & $\alpha=? ; \beta^{+}=9 \# ; \mathrm{p}<1$ & $*$ \\
\hline${ }^{108} \mathrm{Ag}^{m}$ & $\mathrm{~T}:$ discre & pant re & lts: 418 & 7) $310(13$ & 30) 127( & (21), see $\mathrm{E}$ & ENSDI & & & & & & & $* *$ \\
\hline${ }^{108} \mathrm{Te}$ & $\mathrm{D}: \ldots ; \beta$ & $3^{+} p=2$ & $410 ; \beta^{+}$ & $<0.065$ & & & & & & & & & & $* *$ \\
\hline${ }^{108} I$ & $\mathrm{D}: \beta^{+}=9$ & $\% \%$ es & timated by & $94 \mathrm{~Pa} 12 \mathrm{u}$ & asing $t$ & eoretical & $\beta^{+}$ha & nalf-life $\approx$ & $00 \mathrm{~ms}$ & & & & & $* *$ \\
\hline
\end{tabular}




\begin{tabular}{|c|c|c|c|c|c|c|c|c|c|c|c|c|}
\hline \multirow{2}{*}{$\begin{array}{l}\text { Nuclide } \\
{ }^{109} \mathrm{Zr}\end{array}$} & \multicolumn{2}{|c|}{$\begin{array}{l}\text { Mass excess } \\
(\mathrm{keV})\end{array}$} & \multicolumn{2}{|c|}{$\begin{array}{l}\text { Excitation } \\
\text { energy }(\mathrm{keV})\end{array}$} & \multicolumn{3}{|c|}{ Half-life } & \multirow[t]{2}{*}{$J^{\pi}$} & \multirow{2}{*}{$\begin{array}{c}\text { Ens } \\
99\end{array}$} & Reference & \multicolumn{2}{|l|}{$\begin{array}{l}\text { Decay modes and } \\
\text { intensities }(\%)\end{array}$} \\
\hline & $-47280 \#$ & $500 \#$ & & & $60 \#$ & $\mathrm{~ms}$ & $>300 \mathrm{~ns})$ & & & $97 \mathrm{Be} 70 \mathrm{I}$ & $\beta^{-} ?$ & \\
\hline${ }^{109} \mathrm{Nb}$ & $-58100 \#$ & $500 \#$ & & & 190 & $\mathrm{~ms}$ & 30 & $5 / 2^{+} \#$ & 99 & & $\beta^{-}=100 ; \beta^{-} \mathrm{n}=315$ & \\
\hline${ }^{109} \mathrm{Mo}$ & $-67250 \#$ & $300 \#$ & & & 530 & $\mathrm{~ms}$ & 60 & $7 / 2^{-} \#$ & 99 & & $\beta^{-}=100$ & \\
\hline${ }^{109} \mathrm{Tc}$ & -74540 & 100 & & & 860 & $\mathrm{~ms}$ & 40 & $3 / 2^{-} \#$ & 99 & & $\beta^{-}=100 ; \beta^{-} \mathrm{n}=0.082$ & \\
\hline${ }^{109} \mathrm{Ru}$ & -80850 & 70 & & & 34.5 & $\mathrm{~s}$ & 1.0 & $5 / 2^{+} \#$ & 99 & & $\beta^{-}=100$ & \\
\hline${ }^{109} \mathrm{Rh}$ & -85011 & 12 & & & 80 & s & 2 & $7 / 2^{+}$ & 99 & & $\beta^{-}=100$ & \\
\hline${ }^{109} \mathrm{Pd}$ & -87607 & 3 & & & 13.7012 & $\mathrm{~h}$ & 0.0024 & $5 / 2^{+}$ & 99 & & $\beta^{-}=100$ & \\
\hline${ }^{109} \mathrm{Pd}^{m}$ & -87418 & 3 & 188.990 & 0.010 & 4.696 & $\mathrm{~m}$ & 0.003 & $11 / 2^{-}$ & 99 & & $\mathrm{IT}=100$ & \\
\hline${ }^{109} \mathrm{Ag}$ & -88722.7 & 2.9 & & & STABLE & & & $1 / 2^{-}$ & 99 & & $\mathrm{IS}=48.1618$ & \\
\hline${ }^{109} \mathrm{Ag}^{m}$ & -88634.7 & 2.9 & 88.0341 & 0.0011 & 39.6 & $\mathrm{~s}$ & 0.2 & $7 / 2^{+}$ & 99 & & $\mathrm{IT}=100$ & \\
\hline${ }^{109} \mathrm{Cd}$ & -88508 & 4 & & & 461.4 & $\mathrm{~d}$ & 1.2 & $5 / 2^{+}$ & 99 & & $\varepsilon=100$ & \\
\hline${ }^{109} \mathrm{Cd}^{m}$ & -88448 & 4 & 59.6 & 0.4 & 12 & $\mu \mathrm{s}$ & 2 & $1 / 2^{+}$ & 99 & & $\mathrm{IT}=100$ & \\
\hline${ }^{109} \mathrm{Cd}^{n}$ & -88045 & 4 & 463.0 & 0.5 & 10.9 & $\mu \mathrm{s}$ & 0.5 & $11 / 2^{-}$ & 99 & & $\mathrm{IT}=100$ & \\
\hline${ }^{109} \mathrm{In}$ & -86489 & 6 & & & 4.2 & $\mathrm{~h}$ & 0.1 & $9 / 2^{+}$ & 99 & & $\beta^{+}=100$ & \\
\hline${ }^{109} \mathrm{In}^{m}$ & -85839 & 6 & 650.1 & 0.3 & 1.34 & $\mathrm{~m}$ & 0.07 & $1 / 2^{-}$ & 99 & & $\mathrm{IT}=100$ & \\
\hline${ }^{109} \mathrm{In}^{n}$ & -84387 & 6 & 2101.8 & 0.2 & 209 & $\mathrm{~ms}$ & 6 & $\left(19 / 2^{+}\right)$ & 99 & & $\mathrm{IT}=100$ & \\
\hline${ }^{109} \mathrm{Sn}$ & -82639 & 10 & & & 18.0 & $\mathrm{~m}$ & 0.2 & $5 / 2^{(+)}$ & 99 & & $\beta^{+}=100$ & \\
\hline${ }^{109} \mathrm{Sb}$ & -76259 & 19 & & & 17.0 & $\mathrm{~s}$ & 0.7 & $5 / 2^{+} \#$ & 99 & & $\beta^{+}=100$ & \\
\hline${ }^{109} \mathrm{Te}$ & -67610 & 60 & & & 4.6 & s & 0.3 & $\left(5 / 2^{+}\right)$ & 99 & & $\beta^{+}=? ; \alpha=3.913 ; \ldots$ & * \\
\hline${ }^{109} \mathrm{I}$ & -57610 & 100 & & & 103 & $\mu \mathrm{s}$ & 5 & $\left(5 / 2^{+}\right)$ & 02 & $87 \mathrm{Gi02} \mathrm{J}$ & $\mathrm{p}=100$ & \\
\hline${ }^{109} \mathrm{Te}$ & $\mathrm{D}: \ldots ; \beta^{+}$ & $p=9.4$ & $\beta+$ & & & & & & & & & ** \\
\hline
\end{tabular}

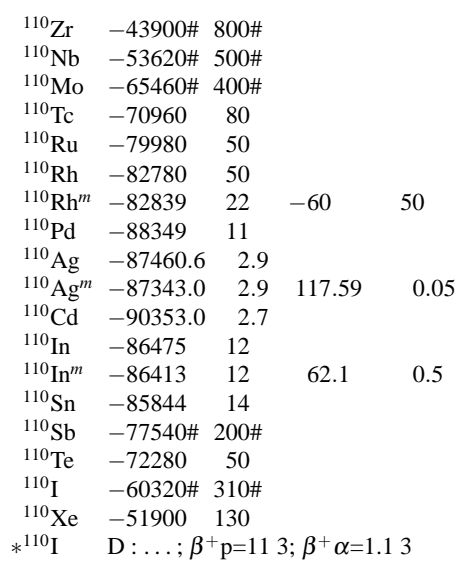

$\begin{array}{cccc}30 \# & \mathrm{~ms} & (>300 \mathrm{~ns}) \\ 170 & \mathrm{~ms} & 20 \\ 300 & \mathrm{~ms} & 40 \\ 920 & \mathrm{~ms} & 30 \\ 11.6 & \mathrm{~s} & 0.6 \\ 28.5 & \mathrm{~s} & 1.5 \\ \text { * } & 2.2 & \mathrm{~s} & 0.2 \\ \text { BD } * & & (>600 \mathrm{Py}) \\ & \text { STABLE } & & \\ 24.6 & \mathrm{~s} & 0.2 \\ 249.950 & \mathrm{~d} & 0.024 \\ \text { STABLE } & & \\ 4.9 & \mathrm{~h} & 0.1 \\ 69.1 & \mathrm{~m} & 0.5 \\ 4.11 & \mathrm{~h} & 0.10 \\ 23.0 & \mathrm{~s} & 0.4 \\ 18.6 & \mathrm{~s} & 0.8 \\ 650 & \mathrm{~ms} & 20 \\ 310 & \mathrm{~ms} & 190\end{array}$

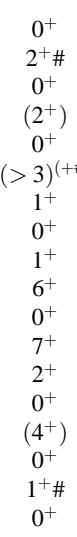

00 97Be70 I $\beta^{-}$?

$00 \quad \beta^{-}=100 ; \beta^{-} \mathrm{n}=408$

$\beta^{-}=100 ; \beta^{-} \mathrm{n}$ ?

$0096 \mathrm{Me} 09 \mathrm{D} \quad \beta^{-}=100 ; \beta^{-} \mathrm{n}=0.042$

$0^{+} \quad 00 \quad \beta^{-}=100$

$\beta^{-}=100$

$\beta^{-}=100$
$\beta^{-}=100$

$\begin{array}{llll}00 & & \beta^{-}=100 \\ 00 & 52 \mathrm{Wi26} \quad \mathrm{T} & \mathrm{IS}=11.729 ; 2 \beta^{-}\end{array}$

$00 \quad \beta^{-} \approx 100 ; \varepsilon=0.306$

$\begin{array}{llll}00 & 02 \mathrm{Un} 02 \mathrm{~T} & \beta^{-} \approx 100 ; \varepsilon=0.306 \\ \beta^{-}=98.646 ; \mathrm{IT}=1.366\end{array}$

$00 \quad$ IS $=12.4918$

$00 \quad \beta^{+}=100$

$\begin{array}{ll}00 & \beta^{+}=100 \\ 00 & \varepsilon=100\end{array}$

$\begin{array}{llll}00 & & & \varepsilon=100 \\ 00 & 97 \mathrm{La} 13 & \mathrm{~J} & \beta^{+}=100\end{array}$

$00 \quad \beta^{+} \approx 100 ; \alpha=0.003 \#$

\# $00 \quad \beta^{+}=834 ; \alpha=174$

00 02Ma19 TD $\alpha=6435 ; \beta^{+}$? 


\begin{tabular}{|c|c|c|c|c|c|c|c|c|c|c|c|c|c|}
\hline Nuclide & \multicolumn{2}{|c|}{$\begin{array}{l}\text { Mass excess } \\
\quad(\mathrm{keV})\end{array}$} & \multicolumn{3}{|c|}{$\begin{array}{l}\text { Excitation } \\
\text { energy }(\mathrm{keV})\end{array}$} & \multicolumn{3}{|c|}{ Half-life } & \multirow[t]{2}{*}{$J^{\pi}$} & \multirow{2}{*}{$\begin{array}{c}\text { Ens } \\
97\end{array}$} & Reference & \multicolumn{2}{|l|}{$\begin{array}{l}\text { Decay modes and } \\
\text { intensities }(\%)\end{array}$} \\
\hline${ }^{111} \mathrm{Nb}$ & $-50630 \#$ & $500 \#$ & & & & $80 \#$ & ms & $(>300 \mathrm{~ns})$ & & & $97 \mathrm{Be} 70 \mathrm{I}$ & $\beta^{-} ?$ & \\
\hline${ }^{111} \mathrm{Mo}$ & $-61100 \#$ & $400 \#$ & & & & $200 \#$ & ms & $(>300 \mathrm{~ns})$ & & 97 & $94 \mathrm{Be} 24 \mathrm{I}$ & $\beta^{-} ?$ & $*$ \\
\hline${ }^{111} \mathrm{Tc}$ & -69220 & 110 & & & & 290 & $\mathrm{~ms}$ & 20 & $3 / 2^{-} \#$ & 96 & $96 \mathrm{Me} 09 \mathrm{TD}$ & $\beta^{-}=100 ; \beta^{-} \mathrm{n}=0.8520$ & $*$ \\
\hline${ }^{111} \mathrm{Ru}$ & -76670 & 70 & & & & 2.12 & $\mathrm{~s}$ & 0.07 & $\left(5 / 2^{+}\right)$ & 96 & $98 \mathrm{Lh02} \mathrm{J}$ & $\beta^{-}=100$ & \\
\hline${ }^{111} \mathrm{Rh}$ & -82357 & 30 & & & & 11 & $\mathrm{~s}$ & 1 & $\left(7 / 2^{+}\right)$ & 96 & & $\beta^{-}=100$ & \\
\hline${ }^{111} \mathrm{Pd}$ & -86004 & 11 & & & & 23.4 & $\mathrm{~m}$ & 0.2 & $5 / 2^{+}$ & 96 & & $\beta^{-}=100$ & \\
\hline${ }^{111} \mathrm{Pd}^{m}$ & -85832 & 11 & 172.18 & 0.08 & & 5.5 & $\mathrm{~h}$ & 0.1 & $11 / 2^{-}$ & 96 & & $\mathrm{IT}=733 ; \beta^{-}=273$ & \\
\hline${ }^{111} \mathrm{Ag}$ & -88221 & 3 & & & & 7.45 & d & 0.01 & $1 / 2^{-}$ & 96 & & $\beta^{-}=100$ & \\
\hline${ }^{111} \mathrm{Ag}^{m}$ & -88161 & 3 & 59.82 & 0.04 & & 64.8 & $\mathrm{~s}$ & 0.8 & $7 / 2^{+}$ & 96 & & $\mathrm{IT}=99.32 ; \beta^{-}=0.72$ & \\
\hline${ }^{111} \mathrm{Cd}$ & -89257.5 & 2.7 & & & & STABLE & & & $1 / 2^{+}$ & 00 & & $\mathrm{IS}=12.8012$ & \\
\hline${ }^{111} \mathrm{Cd}^{m}$ & -88861.3 & 2.7 & 396.214 & 0.021 & & 48.50 & $\mathrm{~m}$ & 0.09 & $11 / 2^{-}$ & 00 & & $\mathrm{IT}=100$ & \\
\hline${ }^{111}$ In & -88396 & 5 & & & & 2.8047 & $\mathrm{~d}$ & 0.0004 & $9 / 2^{+}$ & 00 & & $\varepsilon=100$ & \\
\hline${ }^{111} \operatorname{In}^{m}$ & -87859 & 5 & 536.95 & 0.06 & & 7.7 & $\mathrm{~m}$ & 0.2 & $1 / 2^{-}$ & 00 & & $\mathrm{IT}=100$ & \\
\hline${ }^{111} \mathrm{Sn}$ & -85945 & 7 & & & & 35.3 & $\mathrm{~m}$ & 0.6 & $7 / 2^{+}$ & 96 & & $\beta^{+}=100$ & \\
\hline${ }^{111} \mathrm{Sn}^{m}$ & -85690 & 7 & 254.72 & 0.08 & & 12.5 & $\mu \mathrm{s}$ & 1.0 & $1 / 2^{+}$ & & & & \\
\hline${ }^{111} \mathrm{Sb}$ & -80888 & 28 & & & & 75 & $\mathrm{~s}$ & 1 & $\left(5 / 2^{+}\right)$ & 96 & & $\beta^{+}=100$ & \\
\hline${ }^{111} \mathrm{Te}$ & -73480 & 70 & & & & 19.3 & $\mathrm{~s}$ & 0.4 & $5 / 2^{+} \#$ & 97 & & $\beta^{+}=100 ; \beta^{+} \mathrm{p}=$ ? & \\
\hline${ }^{111} \mathrm{I}$ & $-64950 \#$ & $300 \#$ & & & & 2.5 & $\mathrm{~s}$ & 0.2 & $5 / 2^{+} \#$ & 96 & & $\beta^{+} \approx 100 ; \alpha=0.088$ & \\
\hline${ }^{111} \mathrm{I}^{m}$ & $-63550 \#$ & $300 \#$ & 1398 & 1 & & 21 & ns & 2 & $\left(11 / 2^{-}\right)$ & & & & \\
\hline${ }^{111} \mathrm{Xe}$ & $-54400 \#$ & $300 \#$ & & & & 740 & $\mathrm{~ms}$ & 200 & $5 / 2^{+} \#$ & 96 & $94 \mathrm{~Pa} 11 \quad \mathrm{D}$ & $\beta^{+} ? ; \alpha=107$ & \\
\hline${ }^{111} \mathrm{Xe}^{m}$ & & & non ex & stent & $\mathrm{RN}$ & 900 & $\mathrm{~ms}$ & 200 & & & 90Tu.A T & & $*$ \\
\hline$*^{111} \mathrm{Mo}$ & $\mathrm{I}:$ and $T>$ & $240 \mathrm{~ns}$ & in $97 \mathrm{So} 07$ & & & & & & & & & & $* *$ \\
\hline$*^{111} \mathrm{Tc}$ & $\mathrm{T}$ : superse & edes 88 & $\mathrm{Pe} 13=300$ & 0) fro & 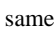 & oup & & & & & & & $* *$ \\
\hline$*^{111} \mathrm{Xe}^{m}$ & $\mathrm{I}$ : from as & signing & $\alpha$ decay to & isomer & in old & r version & of EN & NSDF & & & & & $* *$ \\
\hline
\end{tabular}

\begin{tabular}{|c|c|c|c|c|c|c|c|c|c|c|c|c|c|c|}
\hline${ }^{112} \mathrm{Nb}$ & $-45800 \#$ & $700 \#$ & & & & $60 \#$ & $\mathrm{~ms}$ & $(>300 \mathrm{~ns})$ & $2^{+} \#$ & 97 & $97 \mathrm{Be} 70$ & I & $\beta^{-} ?$ & \\
\hline${ }^{112} \mathrm{Mo}$ & $-58830 \#$ & $600 \#$ & & & & $150 \#$ & $\mathrm{~ms}$ & $(>300 \mathrm{~ns})$ & $0^{+}$ & 97 & $94 \mathrm{Be} 24$ & I & $\beta^{-}$? & \\
\hline${ }^{112} \mathrm{Tc}$ & -66000 & 120 & & & & 290 & $\mathrm{~ms}$ & 20 & $2^{+} \#$ & 97 & 99Wa09 & TD & $\beta^{-}=100 ; \beta^{-} \mathrm{n}=1.52$ & \\
\hline${ }^{112} \mathrm{Ru}$ & -75480 & 70 & & & & 1.75 & $\mathrm{~s}$ & 0.07 & $0^{+}$ & 97 & & & $\beta^{-}=100$ & \\
\hline${ }^{112} \mathrm{Rh}$ & -79740 & 50 & & & & 3.4 & s & 0.4 & $1^{+}$ & 97 & 99Lh01 & $\mathrm{T}$ & $\beta^{-}=100$ & $*$ \\
\hline${ }^{112} \mathrm{Rh}^{m}$ & -79410 & 60 & 330 & 70 & $\mathrm{BD}$ & 6.73 & s & 0.15 & $>3$ & 97 & 99Lh01 & $\mathrm{T}$ & $\beta^{-}=100$ & * \\
\hline${ }^{112} \mathrm{Pd}$ & -86336 & 18 & & & & 21.03 & $\mathrm{~h}$ & 0.05 & $0^{+}$ & 97 & & & $\beta^{-}=100$ & \\
\hline${ }^{112} \mathrm{Ag}$ & -86624 & 17 & & & & 3.130 & $\mathrm{~h}$ & 0.009 & $2^{(-)}$ & 97 & & & $\beta^{-}=100$ & \\
\hline${ }^{112} \mathrm{Cd}$ & -90580.5 & 2.7 & & & & STABLE & & & $0^{+}$ & 97 & & & IS $=24.1321$ & \\
\hline${ }^{112} \mathrm{In}$ & -87996 & 5 & & & & 14.97 & $\mathrm{~m}$ & 0.10 & $1^{+}$ & 97 & & & $\beta^{+}=563 ; \beta^{-}=443$ & \\
\hline${ }^{112} \mathrm{In}^{m}$ & -87839 & 5 & 156.59 & 0.05 & & 20.56 & $\mathrm{~m}$ & 0.06 & $4^{+}$ & 97 & & & $\mathrm{IT}=100$ & \\
\hline${ }^{112} \operatorname{In}^{n}$ & -87645 & 5 & 350.76 & 0.09 & & 690 & $\mathrm{~ns}$ & 50 & $7^{+}$ & 97 & & & $\mathrm{IT}=100$ & \\
\hline${ }^{112} \operatorname{In}^{p}$ & -87382 & 5 & 613.69 & 0.14 & & 2.81 & $\mu \mathrm{s}$ & 0.03 & $8^{-}$ & 97 & 87Eb02 & $\mathrm{J}$ & $\mathrm{IT}=100$ & \\
\hline${ }^{112} \mathrm{Sn}$ & -88661 & 4 & & & & STABLE & & & $0^{+}$ & 97 & & & $\mathrm{IS}=0.971 ; 2 \beta^{+} ?$ & \\
\hline${ }^{112} \mathrm{Sb}$ & -81601 & 18 & & & & 51.4 & $\mathrm{~s}$ & 1.0 & $3^{+}$ & 97 & & & $\beta^{+}=100$ & \\
\hline${ }^{112} \mathrm{Te}$ & -77300 & 170 & & & & 2.0 & $\mathrm{~m}$ & 0.2 & $0^{+}$ & 97 & & & $\beta^{+}=100$ & \\
\hline${ }^{112} \mathrm{I}$ & $-67100 \#$ & $210 \#$ & & & & 3.42 & $\mathrm{~s}$ & 0.11 & $1^{+} \#$ & 97 & 78Ro19 & $\mathrm{D}$ & $\beta^{+} \approx 100 ; \alpha=0.0012 ; \ldots$ & $*$ \\
\hline${ }^{112} \mathrm{Xe}$ & -59970 & 100 & & & & 2.7 & $\mathrm{~s}$ & $\begin{array}{c}0.11 \\
0.8\end{array}$ & $0^{+}$ & 97 & $94 \mathrm{~Pa} 11$ & $\mathrm{D}$ & $\beta^{+} \approx 100 ; \alpha=0.98$ & $*$ \\
\hline${ }^{112} \mathrm{Cs}$ & $-46290 \#$ & $300 \#$ & & & & 500 & $\mu \mathrm{s}$ & 100 & $1^{+} \#$ & 02 & & & $\mathrm{p}=100$ & \\
\hline${ }^{112} \mathrm{Rh}$ & $\mathrm{T}$ : superse & edes $91 \mathrm{~J}$ & $11=2.1(C$ & .3) and & $88 \mathrm{Ay} 0$ & $=3.8(0.6)$ & of $s$ & ame group & & & & & & $* *$ \\
\hline${ }^{112} \mathrm{Rh}^{m}$ & $\mathrm{~T}$ : superse & edes 88 & $y 02=6.8($ & & & & & & & & & & & $* *$ \\
\hline$*^{112} \mathrm{I}$ & $\mathrm{D}: \ldots ; \beta$ & ${ }^{+} \mathrm{p}=0.88$ & $10 ; \beta^{+} \alpha$ & $=0.104$ & & & & & & & & & & $* *$ \\
\hline$*^{112} \mathrm{I}$ & $\mathrm{D}: \beta^{+} \mathrm{p}$ an & nd $\beta^{+} \alpha$ & are derive & from & $3^{+} \mathrm{p} /$ & $35(80$ & & $3+\alpha$ & in & & & & & $* *$ \\
\hline$*^{112} \mathrm{Xe}$ & $\mathrm{D}: \alpha$ inten & nsity is & stimated & from 94 & a $11=0$ & $(+1.1-0$ & $.5) \%$ & and $78 \mathrm{Ro}$ & $=0.8$ & & & & & $<*$ \\
\hline
\end{tabular}




\begin{tabular}{|c|c|c|c|c|c|c|c|c|c|c|c|c|c|c|}
\hline Nuclide & \multicolumn{2}{|c|}{$\begin{array}{c}\text { Mass excess } \\
(\mathrm{keV})\end{array}$} & \multicolumn{3}{|c|}{$\begin{array}{l}\text { Excitation } \\
\text { energy }(\mathrm{keV})\end{array}$} & \multicolumn{3}{|c|}{ Half-life } & $J^{\pi}$ & Ens & \multicolumn{2}{|c|}{ Reference } & \multicolumn{2}{|l|}{$\begin{array}{l}\text { Decay modes and } \\
\text { intensities }(\%)\end{array}$} \\
\hline${ }^{113} \mathrm{Nb}$ & $-42200 \#$ & $800 \#$ & & & & $30 \#$ & ms ( & $>300 \mathrm{~ns})$ & $5 / 2^{+} \#$ & 98 & 97Be70 & I & $\beta^{-} ?$ & \\
\hline${ }^{113} \mathrm{Mo}$ & $-54140 \#$ & $600 \#$ & & & & $100 \#$ & ms ( & $>300 \mathrm{~ns})$ & & 98 & $94 \mathrm{Be} 24$ & I & $\beta^{-} ?$ & \\
\hline${ }^{113} \mathrm{Tc}$ & $-63720 \#$ & $300 \#$ & & & & 170 & $\mathrm{~ms}$ & 20 & $3 / 2^{-} \#$ & 98 & 99Wa09 & TD & $\beta^{-}=100 ; \beta^{-} \mathrm{n}=2.13$ & $*$ \\
\hline${ }^{113} \mathrm{Ru}$ & -72200 & 70 & & & & 800 & $\mathrm{~ms}$ & 50 & $\left(5 / 2^{+}\right)$ & 98 & $98 \mathrm{Ku} 17$ & $\mathrm{~J}$ & $\beta^{-}=100$ & \\
\hline${ }^{113} \mathrm{Ru}^{m}$ & -72070 & 70 & 130 & 18 & & 510 & $\mathrm{~ms}$ & 30 & $\left(11 / 2^{-}\right)$ & & $98 \mathrm{Ku} 17$ & ETJ & $\mathrm{IT}=? ; \beta^{-}=$? & * \\
\hline${ }^{113} \mathrm{Rh}$ & -78680 & 50 & & & & 2.80 & $\mathrm{~s}$ & 0.12 & $\left(7 / 2^{+}\right)$ & 98 & 93Pe11 & $\mathrm{J}$ & $\beta^{-}=100$ & \\
\hline${ }^{113} \mathrm{Pd}$ & -83690 & 40 & & & & 93 & $\mathrm{~s}$ & 5 & $\left(5 / 2^{+}\right)$ & 98 & & & $\beta^{-}=100$ & \\
\hline${ }^{113} \mathrm{Pd}^{m}$ & -83610 & 40 & 81.1 & 0.3 & \multirow{3}{*}{$\mathrm{RN}$} & 300 & $\mathrm{~ms}$ & 100 & $\left(9 / 2^{-}\right)$ & 98 & & & $\mathrm{IT}=100$ & \\
\hline${ }^{113} \mathrm{Pd}^{n}$ & & & \multicolumn{2}{|c|}{ non existent } & & $>100$ & $\mathrm{~s}$ & & & 98 & $81 \mathrm{Me} 17$ & I & & * \\
\hline${ }^{113} \mathrm{Ag}$ & -87033 & 17 & & & & 5.37 & $\mathrm{~h}$ & 0.05 & $1 / 2^{-}$ & 98 & & & $\beta^{-}=100$ & \\
\hline${ }^{113} \mathrm{Ag}^{m}$ & -86990 & 17 & 43.50 & 0.10 & & 68.7 & $\mathrm{~s}$ & 1.6 & $7 / 2^{+}$ & 98 & & & $\mathrm{IT}=647 ; \beta^{-}=367$ & \\
\hline${ }^{113} \mathrm{Cd}$ & -89049.3 & 2.7 & & & & 7.7 & Py & 0.3 & $1 / 2^{+}$ & 98 & & & $\mathrm{IS}=12.2212 ; \beta^{-}=100$ & \\
\hline${ }^{113} \mathrm{Cd}^{m}$ & -88785.8 & 2.7 & 263.54 & 0.03 & & 14.1 & $\mathrm{y}$ & 0.5 & $11 / 2^{-}$ & 98 & & & $\beta^{-} \approx 100 ; \mathrm{IT}=0.14$ & \\
\hline${ }^{113} \mathrm{In}$ & -89370 & 3 & & & & STABLE & & & $9 / 2^{+}$ & 99 & & & $\mathrm{IS}=4.295$ & \\
\hline${ }^{113} \operatorname{In}^{m}$ & -88978 & 3 & 391.699 & 0.003 & & 1.6579 & $\mathrm{~h}$ & 0.0004 & $1 / 2^{-}$ & 99 & & & $\mathrm{IT}=100$ & \\
\hline${ }^{113} \mathrm{Sn}$ & -88333 & 4 & & & & 115.09 & $\mathrm{~d}$ & 0.03 & $1 / 2^{+}$ & 00 & & & $\beta^{+}=100$ & \\
\hline${ }^{113} \mathrm{Sn}^{m}$ & -88256 & 4 & 77.386 & 0.019 & & 21.4 & $\mathrm{~m}$ & 0.4 & $7 / 2^{+}$ & 00 & & & $\mathrm{IT}=91.123 ; \beta^{+}=8.923$ & \\
\hline${ }^{113} \mathrm{Sb}$ & -84420 & 18 & & & & 6.67 & $\mathrm{~m}$ & 0.07 & $5 / 2^{+}$ & 98 & & & $\beta^{+}=100$ & \\
\hline${ }^{113} \mathrm{Te}$ & -78347 & 28 & & & & 1.7 & $\mathrm{~m}$ & 0.2 & $\left(7 / 2^{+}\right)$ & 98 & & & $\beta^{+}=100$ & \\
\hline${ }^{113} \mathrm{I}$ & -71130 & 50 & & & & 6.6 & $\mathrm{~s}$ & 0.2 & $5 / 2^{+} \#$ & 98 & & & $\beta^{+}=100 ; \alpha=3.31 \mathrm{e}-7 ; \ldots$ & * \\
\hline${ }^{113} \mathrm{Xe}$ & -62090 & 80 & & & & 2.74 & $\mathrm{~s}$ & 0.08 & $5 / 2^{+} \#$ & 98 & $85 \mathrm{Ti0} 2$ & $\mathrm{D}$ & $\beta^{+} \approx 100 ; \alpha=0.0115 ; \ldots$ & * \\
\hline${ }^{113} \mathrm{Cs}$ & -51700 & 100 & & & & 16.7 & $\mu \mathrm{s}$ & 0.7 & $5 / 2^{+} \#$ & 02 & & & $\mathrm{p}=100 ; \alpha=0$ & \\
\hline$*^{113} \mathrm{Tc}$ & $\mathrm{T}: 98 \mathrm{Ku} 1$ & $17=110$ & 30) and 92 & $A y 02=13$ & $0(50)$ & are from & same & authors & & & & & & $* *$ \\
\hline${ }^{113} \mathrm{Ru}^{m}$ & E : above $t$ & the $99 \mathrm{k}$ & eV level a & d below & $160 \mathrm{k}$ & & & & & & & & & $* *$ \\
\hline$*{ }^{113} \mathrm{Pd}^{n}$ & I : existenc & ce is no & possible & ince disce & covery & of ${ }^{113} \mathrm{Pd}^{m}$ & ${ }^{m}$ by & 3Pe11 & & & & & & ** \\
\hline$*^{113} \mathrm{I}$ & $\mathrm{D}: \ldots ; \beta^{-}$ & ${ }^{+} \alpha ?$ & & & & & & & & & & & & $* *$ \\
\hline$*{ }^{113} \mathrm{Xe}$ & $\mathrm{D}: \ldots ; \beta^{-}$ & $+p=74$ & $\beta^{+} \alpha \approx 0$ & & & & & & & & & & & $* *$ \\
\hline$*^{113} \mathrm{Xe}$ & $\mathrm{D}: \alpha=0.00$ & $024-0.0$ & $04 \%$ fron & estimate & ed lin & $t$ for the $r$ & redu & ed width, & , see & & & & & $* *$ \\
\hline$*^{113} \mathrm{Xe}$ & $\mathrm{D}: \beta^{+}$ & $+\mathrm{p}$ and & $3^{+} \alpha$ deriv & from $\beta$ & $3^{+} \mathrm{p} / \alpha$ & $=605(35)$ & and & $\beta^{+} \mathrm{p} / \beta^{+} \alpha$ & $\alpha=500-15$ & 00 in & $85 \mathrm{Ti02}$ & & & $* *$ \\
\hline
\end{tabular}

\begin{tabular}{|c|c|c|c|c|c|c|c|c|c|c|c|c|c|c|}
\hline${ }^{114} \mathrm{Mo}$ & $-51310 \#$ & $700 \#$ & & & & $80 \#$ & $\mathrm{~ms}($ & $(>300 \mathrm{~ns})$ & $0^{+}$ & 03 & $97 \mathrm{Be} 70$ & I & $\beta^{-} ?$ & \\
\hline${ }^{114} \mathrm{Tc}$ & $-59730 \#$ & $600 \#$ & & & & 150 & $\mathrm{~ms}$ & 30 & $2^{+} \#$ & 03 & & & $\beta^{-}=100 ; \beta^{-} \mathrm{n}=?$ & \\
\hline${ }^{114} \mathrm{Ru}$ & $-70530 \#$ & $230 \#$ & & & & 530 & $\mathrm{~ms}$ & 60 & $0^{+}$ & 03 & & & $\beta^{-}=100 ; \beta^{-} \mathrm{n} ?$ & \\
\hline${ }^{114} \mathrm{Rh}$ & -75630 & 110 & & & * & 1.85 & s & 0.05 & $1^{+}$ & 03 & & & $\beta^{-}=100 ; \beta^{-} \mathrm{n} ?$ & \\
\hline${ }^{114} \mathrm{Rh}^{m}$ & $-75430 \#$ & $190 \#$ & $200 \#$ & $150 \#$ & $*$ & 1.85 & s & 0.05 & $(4,5)$ & 03 & & & $\beta^{-}=100$ & \\
\hline${ }^{114} \mathrm{Pd}$ & -83497 & 24 & & & & 2.42 & $\mathrm{~m}$ & 0.06 & $0^{+}$ & 03 & & & $\beta^{-}=100$ & \\
\hline${ }^{114} \mathrm{Ag}$ & -84949 & 25 & & & & 4.6 & $\mathrm{~s}$ & 0.1 & $1^{+}$ & 03 & & & $\beta^{-}=100$ & \\
\hline${ }^{114} \mathrm{Ag}^{m}$ & -84750 & 25 & 199 & 5 & & 1.50 & $\mathrm{~ms}$ & 0.05 & $\left(<7^{+}\right)$ & 03 & & & $\mathrm{IT}=100$ & \\
\hline${ }^{114} \mathrm{Cd}$ & -90020.9 & 2.7 & & & & STABLE & & (>92 Py) & $0^{+}$ & 03 & $95 \mathrm{Ge} 14$ & $\mathrm{~T}$ & $\mathrm{IS}=28.7342 ; 2 \beta^{-} ?$ & \\
\hline${ }^{114} \mathrm{In}$ & -88572 & 3 & & & & 71.9 & s & 0.1 & $1^{+}$ & 03 & & & $\beta^{-}=99.5015 ; \beta^{+}=0.5015$ & \\
\hline${ }^{114} \operatorname{In}^{m}$ & -88382 & 3 & 190.29 & 0.03 & & 49.51 & $\mathrm{~d}$ & 0.01 & $5^{+}$ & 03 & & & $\mathrm{IT}=96.7524 ; \beta^{+}=3.2524$ & \\
\hline${ }^{114} \operatorname{In}^{n}$ & -88070 & 3 & 501.94 & 0.03 & & 43.1 & $\mathrm{~ms}$ & 0.6 & $\left(8^{-}\right)$ & 03 & & & $\mathrm{IT}=100$ & \\
\hline${ }^{114} \operatorname{In}^{p}$ & -87930 & 3 & 641.72 & 0.03 & & 4.3 & $\mu \mathrm{s}$ & 0.4 & $\left(7^{+}\right)$ & 03 & & & $\mathrm{IT}=100$ & \\
\hline${ }^{114} \mathrm{Sn}$ & -90561 & 3 & & & & STABLE & & & $0^{+}$ & 03 & & & $\mathrm{IS}=0.661$ & \\
\hline${ }^{114} \mathrm{Sn}^{m}$ & -87474 & 3 & 3087.37 & 0.07 & & 733 & ns & 14 & $7^{-}$ & 03 & & & $\mathrm{IT}=100$ & \\
\hline${ }^{114} \mathrm{Sb}$ & -84515 & 28 & & & & 3.49 & $\mathrm{~m}$ & 0.03 & $\left(3^{+}\right)$ & 03 & & & $\beta^{+}=100$ & \\
\hline${ }^{114} \mathrm{Sb}^{m}$ & -84020 & 28 & 495.5 & 0.07 & & 219 & $\mu \mathrm{s}$ & 12 & $\left(8^{-}\right)$ & 03 & & & $\mathrm{IT}=100$ & \\
\hline${ }^{114} \mathrm{Te}$ & -81889 & 28 & & & & 15.2 & $\mathrm{~m}$ & 0.7 & $0^{+}$ & 03 & & & $\beta^{+}=100$ & \\
\hline${ }^{114} \mathrm{I}$ & $-72800 \#$ & $300 \#$ & & & & 2.1 & s & 0.2 & $1^{+}$ & 03 & & & $\beta^{+}=100 ; \beta^{+} \mathrm{p} ?$ & \\
\hline${ }^{114} I^{m}$ & $-72530 \#$ & $300 \#$ & 265.9 & 0.5 & & 6.2 & $\mathrm{~s}$ & 0.5 & (7) & 03 & ABBW96 & $\mathrm{D}$ & $\beta^{+}=912 ; \mathrm{IT}=92$ & * \\
\hline${ }^{114} \mathrm{Xe}$ & -67086 & 11 & & & & 10.0 & s & 0.4 & $0^{+}$ & 03 & & & $\beta^{+}=100$ & \\
\hline${ }^{114} \mathrm{Cs}$ & $-54540 \#$ & $310 \#$ & & & & 570 & $\mathrm{~ms}$ & 20 & $\left(1^{+}\right)$ & 03 & & & $\beta^{+} \approx 100 ; \alpha=0.0186 ;$. & * \\
\hline${ }^{114} \mathrm{Ba}$ & -45950 & 140 & & & & 530 & $\mathrm{~ms}$ & 230 & $0^{+}$ & 03 & $02 \mathrm{Ma} 19$ & $\mathrm{D}$ & $\beta^{+} \approx 100 ; \beta^{+} \mathrm{p}=2010 ; \ldots$ & * \\
\hline$*^{114} \mathrm{I}^{m}$ & \multicolumn{13}{|c|}{ D : evaluated for NUBASE by J. Blachot, based on ${ }^{114}$ I IT decay } & $* *$ \\
\hline$*^{114} \mathrm{Cs}$ & \multicolumn{13}{|c|}{$\mathrm{D}: \ldots ; \beta^{+} \mathrm{p}=8.713 ; \beta^{+} \alpha=0.193$} & $* *$ \\
\hline$*^{114} \mathrm{Ba}$ & \multicolumn{13}{|c|}{$\mathrm{D}: \ldots ; \alpha=0.93 ;{ }^{12} \mathrm{C}<0.038$} & ** \\
\hline${ }^{114} \mathrm{Ba}$ & \multicolumn{13}{|c|}{$\mathrm{D}:{ }^{12} \mathrm{C}$ intensity is from $95 \mathrm{Gu} 10$} & $* *$ \\
\hline
\end{tabular}




\begin{tabular}{|c|c|c|c|c|c|c|c|c|c|c|c|c|c|}
\hline Nuclide & \multicolumn{2}{|c|}{$\begin{array}{c}\text { Mass excess } \\
(\mathrm{keV})\end{array}$} & \multicolumn{3}{|c|}{$\begin{array}{c}\text { Excitation } \\
\text { energy }(\mathrm{keV})\end{array}$} & \multicolumn{3}{|c|}{ Half-life } & $J^{\pi}$ & Ens & Reference & \multicolumn{2}{|l|}{$\begin{array}{c}\text { Decay modes and } \\
\text { intensities }(\%)\end{array}$} \\
\hline${ }^{115} \mathrm{Mo}$ & $-46310 \#$ & $800 \#$ & & & & $60 \#$ & $\mathrm{~ms}$ & $>300 \mathrm{~ns})$ & & 99 & & $\beta^{-} ? ; \beta^{-} \mathrm{n} ?$ & \\
\hline${ }^{115} \mathrm{Tc}$ & $-57110 \#$ & $700 \#$ & & & & $100 \#$ & $\mathrm{~ms}$ & $>300 \mathrm{~ns})$ & $3 / 2^{-} \#$ & 99 & & $\beta^{-} ? ; \beta^{-} \mathrm{n} ?$ & \\
\hline${ }^{115} \mathrm{Ru}$ & -66430 & 130 & & & & 740 & $\mathrm{~ms}$ & 80 & & 99 & & $\beta^{-}=100 ; \beta^{-} \mathrm{n} ?$ & \\
\hline${ }^{115} \mathrm{Rh}$ & -74210 & 80 & & & & 990 & $\mathrm{~ms}$ & 50 & $7 / 2^{+} \#$ & 99 & & $\beta^{-}=100$ & \\
\hline${ }^{115} \mathrm{Pd}$ & -80400 & 60 & & & & 25 & $\mathrm{~s}$ & 2 & $5 / 2^{+} \#$ & 99 & & $\beta^{-}=100$ & \\
\hline${ }^{115} \mathrm{Pd}^{m}$ & -80310 & 60 & 89.18 & 0.25 & & 50 & $\mathrm{~s}$ & 3 & $11 / 2^{-} \#$ & 99 & & $\beta^{-}=92.020 ; \mathrm{IT}=8.020$ & * \\
\hline${ }^{115} \mathrm{Ag}$ & -84990 & 30 & & & & 20.0 & $\mathrm{~m}$ & 0.5 & $1 / 2^{-}$ & 99 & & $\beta^{-}=100$ & \\
\hline${ }^{115} \mathrm{Ag}^{m}$ & -84950 & 30 & 41.16 & 0.10 & & 18.0 & $\mathrm{~s}$ & 0.7 & $7 / 2^{+}$ & 99 & & $\beta^{-}=79.03 ; \mathrm{IT}=21.03$ & \\
\hline${ }^{115} \mathrm{Cd}$ & -88090.5 & 2.7 & & & & 53.46 & $\mathrm{~h}$ & 0.10 & $1 / 2^{+}$ & 99 & & $\beta^{-}=100$ & \\
\hline${ }^{115} \mathrm{Cd}^{m}$ & -87909.5 & 2.7 & 181.0 & 0.5 & & 44.56 & d & 0.24 & $(11 / 2)^{-}$ & 99 & & $\beta^{-} \approx 100 ;$ IT $<0.003$ & \\
\hline${ }^{115} \mathrm{In}$ & -89537 & 4 & & & & 441 & Ty & 25 & $9 / 2^{+}$ & 99 & & $\mathrm{IS}=95.715 ; \beta^{-}=100$ & \\
\hline${ }^{115} \mathrm{In}^{m}$ & -89201 & 4 & 336.244 & 0.017 & & 4.486 & $\mathrm{~h}$ & 0.004 & $1 / 2^{-}$ & 99 & & $\mathrm{IT}=95.07 ; \beta^{-}=5.07$ & \\
\hline${ }^{115} \mathrm{Sn}$ & -90036.0 & 2.9 & & & & STABLE & & & $1 / 2^{+}$ & 99 & & IS $=0.341$ & \\
\hline${ }^{115} \mathrm{Sn}^{m}$ & -89423.2 & 2.9 & 612.81 & 0.04 & & 3.26 & $\mu \mathrm{s}$ & 0.08 & $7 / 2^{+}$ & 99 & & $\mathrm{IT}=100$ & \\
\hline${ }^{115} \mathrm{Sn}^{n}$ & -89322.4 & 2.9 & 713.64 & 0.12 & & 159 & $\mu \mathrm{s}$ & 1 & $11 / 2^{-}$ & 99 & & $\mathrm{IT}=100$ & \\
\hline${ }^{115} \mathrm{Sb}$ & -87003 & 16 & & & & 32.1 & $\mathrm{~m}$ & 0.3 & $5 / 2^{+}$ & 99 & & $\beta^{+}=100$ & \\
\hline${ }^{115} \mathrm{Te}$ & -82063 & 28 & & & $*$ & 5.8 & $\mathrm{~m}$ & 0.2 & $7 / 2^{+}$ & 99 & & $\beta^{+}=100$ & \\
\hline${ }^{115} \mathrm{Te}^{m}$ & -82053 & 29 & 10 & 7 & * & 6.7 & $\mathrm{~m}$ & 0.4 & $(1 / 2)^{+}$ & 99 & ABBW E & $\beta^{+} \approx 100 ;$ IT $<0.06$ & * \\
\hline${ }^{115} \mathrm{Te}^{n}$ & -81783 & 28 & 280.05 & 0.20 & & 7.5 & $\mu \mathrm{s}$ & 0.2 & $11 / 2^{-}$ & 99 & & $\mathrm{IT}=100$ & \\
\hline${ }^{115} \mathrm{I}$ & -76338 & 29 & & & & 1.3 & $\mathrm{~m}$ & 0.2 & $5 / 2^{+} \#$ & 99 & & $\beta^{+}=100$ & \\
\hline${ }^{115} \mathrm{Xe}$ & -68657 & 12 & & & & 18 & $\mathrm{~s}$ & 4 & $\left(5 / 2^{+}\right)$ & 99 & & $\beta^{+}=100 ; \beta^{+} \mathrm{p}=0.346$ & * \\
\hline${ }^{115} \mathrm{Cs}$ & $-59700 \#$ & $300 \#$ & & & & 1.4 & $\mathrm{~s}$ & 0.8 & $9 / 2^{+} \#$ & 99 & & $\beta^{+}=100 ; \beta^{+} \mathrm{p} \approx 0.07$ & \\
\hline${ }^{115} \mathrm{Ba}$ & $-49030 \#$ & $600 \#$ & & & & 450 & $\mathrm{~ms}$ & 50 & $5 / 2^{+} \#$ & 99 & 97Ja12 D & $\beta^{+}=100 ; \beta^{+} \mathrm{p}>15$ & \\
\hline$*^{115} \mathrm{Pd}^{m}$ & $\mathrm{~J}: \mathrm{E} 3 \mathrm{tran}$ & sition $\mathrm{t}$ & o ground- & state & & & & & & & & & $* *$ \\
\hline$*^{115} \mathrm{Te}^{m}$ & $\mathrm{E}:$ less tha & an $20 \mathrm{k}$ & $\mathrm{eV}$, from $\mathrm{E}$ & NSDF & & & & & & & & & $* *$ \\
\hline$*^{115} \mathrm{Xe}$ & $\mathrm{D}: \ldots ; \beta$ & $\alpha=0$ & 00031 & & & & & & & & & & $* *$ \\
\hline${ }^{116} \mathrm{Tc}$ & $-52750 \#$ & $700 \#$ & & & & $90 \#$ & $\mathrm{~ms}$ & $>300 \mathrm{~ns})$ & $2^{+} \#$ & 01 & $97 \mathrm{Be} 70 \mathrm{I}$ & $\beta^{-} ?$ & \\
\hline${ }^{116} \mathrm{Ru}$ & $-64450 \#$ & $700 \#$ & & & & $400 \#$ & $\mathrm{~ms}$ & $>300 \mathrm{~ns})$ & $0^{+}$ & 01 & $94 \mathrm{Be} 24 \mathrm{I}$ & $\beta^{-} ?$ & $*$ \\
\hline${ }^{116} \mathrm{Rh}$ & -70740 & 140 & & & $*$ & 680 & $\mathrm{~ms}$ & 60 & $1^{+}$ & 01 & & $\beta^{-}=100 ; \beta^{-} \mathrm{n} ?$ & \\
\hline${ }^{116} \mathrm{Rh}^{m}$ & $-70540 \#$ & $210 \#$ & $200 \#$ & $150 \#$ & $*$ & 570 & $\mathrm{~ms}$ & 50 & $\left(6^{-}\right)$ & 01 & & $\beta^{-}=100$ & \\
\hline${ }^{116} \mathrm{Pd}$ & -79960 & 60 & & & & 11.8 & $\mathrm{~s}$ & 0.4 & $0^{+}$ & 01 & & $\beta^{-}=100$ & \\
\hline${ }^{116} \mathrm{Ag}$ & -82570 & 50 & & & & 2.68 & $\mathrm{~m}$ & 0.10 & $(2)^{-}$ & 01 & & $\beta^{-}=100$ & \\
\hline${ }^{116} \mathrm{Ag}^{m}$ & -82490 & 50 & 81.90 & 0.20 & & 8.6 & $\mathrm{~s}$ & 0.3 & $\left(5^{+}\right)$ & 01 & & $\beta^{-}=94.015 ; \mathrm{IT}=6.015$ & \\
\hline${ }^{116} \mathrm{Cd}$ & -88719 & 3 & & & & 30 & Ey & 4 & $0^{+}$ & 01 & 03Da09 T & $\mathrm{IS}=7.4918 ; 2 \beta^{-}=100$ & $*$ \\
\hline${ }^{116} \mathrm{In}$ & -88250 & 4 & & & & 14.10 & $\mathrm{~s}$ & 0.03 & $1^{+}$ & 01 & 98Bh04 D & $\beta^{-} \approx 100 ; \varepsilon=0.236$ & \\
\hline${ }^{116} \mathrm{In}^{m}$ & -88123 & 4 & 127.267 & 0.006 & & 54.29 & $\mathrm{~m}$ & 0.17 & $5^{+}$ & 01 & & $\beta^{-}=100$ & \\
\hline${ }^{116} \mathrm{In}^{n}$ & -87960 & 4 & 289.660 & 0.006 & & 2.18 & $\mathrm{~s}$ & 0.04 & $8^{-}$ & 01 & & $\mathrm{IT}=100$ & \\
\hline${ }^{116} \mathrm{Sn}$ & -91528.1 & 2.9 & & & & STABLE & & & $0^{+}$ & 01 & & $\mathrm{IS}=14.549$ & \\
\hline${ }^{116} \mathrm{Sb}$ & -86821 & 6 & & & & 15.8 & $\mathrm{~m}$ & 0.8 & $3^{+}$ & 01 & & $\beta^{+}=100$ & \\
\hline${ }^{116} \mathrm{Sb}^{m}$ & -86440 & 40 & 380 & 40 & $\mathrm{BD}$ & 60.3 & $\mathrm{~m}$ & 0.6 & $8^{-}$ & 01 & & $\beta^{+}=100$ & \\
\hline${ }^{116} \mathrm{Te}$ & -85269 & 28 & & & & 2.49 & $\mathrm{~h}$ & 0.04 & $0^{+}$ & 01 & & $\beta^{+}=100$ & \\
\hline${ }^{116} \mathrm{I}$ & -77490 & 100 & & & & 2.91 & $\mathrm{~s}$ & 0.15 & $1^{+}$ & 01 & & $\beta^{+}=100$ & \\
\hline${ }^{116} I^{m}$ & $-77090 \#$ & $110 \#$ & $400 \#$ & $50 \#$ & & 3.27 & $\mu \mathrm{s}$ & 0.16 & $\left(7^{-}\right)$ & 01 & & $\mathrm{IT}=100$ & \\
\hline${ }^{116} \mathrm{Xe}$ & -73047 & 13 & & & & 59 & $\mathrm{~s}$ & 2 & $0^{+}$ & 01 & & $\beta^{+}=100$ & \\
\hline${ }^{116} \mathrm{Cs}$ & $-62070 \#$ & $100 \#$ & & & $*$ & 700 & $\mathrm{~ms}$ & 40 & $\left(1^{+}\right)$ & 01 & & $\beta^{+}=100 ; \beta^{+} \mathrm{p}=0.287 ; \ldots$ & * \\
\hline${ }^{116} \mathrm{Cs}^{m}$ & $-61970 \#$ & $120 \#$ & $100 \#$ & $60 \#$ & * & 3.85 & $\mathrm{~s}$ & 0.13 & $4^{+}, 5,6$ & 01 & & $\beta^{+}=100 ; \beta^{+} \mathrm{p}=0.5115 ; \ldots$ & * \\
\hline${ }^{116} \mathrm{Ba}$ & $-54600 \#$ & $400 \#$ & & & & 1.3 & $\mathrm{~s}$ & 0.2 & $0^{+}$ & 01 & & $\beta^{+}=100 ; \beta^{+} \mathrm{p}=31$ & \\
\hline$*{ }^{116} \mathrm{Ru}$ & $\mathrm{I}:$ and $T>$ & $240 \mathrm{~ns}$ & in $97 \mathrm{So} 0$ & & & & & & & & & & ** \\
\hline$*^{116} \mathrm{Cd}$ & $\mathrm{T}:$ from 2 & $29(1 \mathrm{stat}$ & tistics +4 & 3 systema & tics); s & supersede & $00 D$ & $a 27=26(1$ & statistics & $5+1$ & -4 systematics & & $* *$ \\
\hline$*^{116} \mathrm{Cs}$ & $\mathrm{D}: \ldots ; \beta$ & $+\alpha=0$ & 04925 & & & & & & & & & & $* *$ \\
\hline$*^{116} \mathrm{Cs}^{m}$ & $\mathrm{D}: \ldots ; \beta$ & ${ }^{+} \alpha=0$ & 0082 & & & & & & & & & & $* *$ \\
\hline
\end{tabular}




\begin{tabular}{|c|c|c|c|c|c|c|c|c|c|c|c|c|c|}
\hline Nuclide & \multicolumn{2}{|c|}{$\begin{array}{c}\text { Mass excess } \\
(\mathrm{keV})\end{array}$} & \multicolumn{3}{|c|}{$\begin{array}{l}\text { Excitation } \\
\text { energy }(\mathrm{keV})\end{array}$} & \multicolumn{3}{|c|}{ Half-life } & $J^{\pi}$ & Ens & Reference & \multicolumn{2}{|l|}{$\begin{array}{l}\text { Decay modes and } \\
\text { intensities }(\%)\end{array}$} \\
\hline${ }^{117} \mathrm{Tc}$ & $-49850 \#$ & $700 \#$ & & & & $40 \#$ & $\mathrm{~ms}($ & $>300 \mathrm{~ns})$ & $3 / 2^{-} \#$ & 02 & 97Be70 I & $\beta^{-} ?$ & \\
\hline${ }^{117} \mathrm{Ru}$ & $-60010 \#$ & $700 \#$ & & & & $300 \#$ & $\mathrm{~ms}($ & $>300 \mathrm{~ns})$ & & 02 & $94 \mathrm{Be} 24 \mathrm{I}$ & $\beta^{-} ?$ & $*$ \\
\hline${ }^{117} \mathrm{Rh}$ & $-68950 \#$ & $500 \#$ & & & & 440 & $\mathrm{~ms}$ & 40 & $7 / 2^{+} \#$ & 02 & & $\beta^{-}=100$ & \\
\hline${ }^{117} \mathrm{Pd}$ & -76530 & 60 & & & & 4.3 & $\mathrm{~s}$ & 0.3 & $\left(5 / 2^{+}\right)$ & 02 & & $\beta^{-}=100$ & \\
\hline${ }^{117} \mathrm{Pd}^{m}$ & -76330 & 60 & 203.2 & 0.3 & & 19.1 & $\mathrm{~ms}$ & 0.7 & $11 / 2^{-} \#$ & 02 & & $\mathrm{IT}=100$ & \\
\hline${ }^{117} \mathrm{Ag}$ & -82270 & 50 & & & & 73.6 & $\mathrm{~s}$ & 1.4 & $1 / 2^{-} \#$ & 02 & & $\beta^{-}=100$ & \\
\hline${ }^{117} \mathrm{Ag}^{m}$ & -82240 & 50 & 28.6 & 0.2 & & 5.34 & s & 0.05 & $\left(7 / 2^{+}\right)$ & 02 & & $\beta^{-}=94.015 ; \mathrm{IT}=6.015$ & \\
\hline${ }^{117} \mathrm{Cd}$ & -86425 & 3 & & & & 2.49 & $\mathrm{~h}$ & 0.04 & $1 / 2^{+}$ & 02 & & $\beta^{-}=100$ & \\
\hline${ }^{117} \mathrm{Cd}^{m}$ & -86289 & 3 & 136.4 & 0.2 & & 3.36 & $\mathrm{~h}$ & 0.05 & $(11 / 2)^{-}$ & 02 & & $\beta^{-} \approx 100 ; \mathrm{IT} \approx 0$ & \\
\hline${ }^{117} \mathrm{In}$ & -88945 & 6 & & & & 43.2 & $\mathrm{~m}$ & 0.3 & $9 / 2^{+}$ & 02 & & $\beta^{-}=100$ & \\
\hline${ }^{117} \mathrm{In}^{m}$ & -88630 & 6 & 315.302 & 0.012 & & 116.2 & $\mathrm{~m}$ & 0.3 & $1 / 2^{-}$ & 02 & & $\beta^{-}=52.915 ; \mathrm{IT}=47.115$ & \\
\hline${ }^{117} \mathrm{Sn}$ & -90400.0 & 2.9 & & & & STABLE & & & $1 / 2^{+}$ & 02 & & $\mathrm{IS}=7.687$ & \\
\hline${ }^{117} \mathrm{Sn}^{m}$ & -90085.4 & 2.9 & 314.58 & 0.04 & & 13.76 & $\mathrm{~d}$ & 0.04 & $11 / 2^{-}$ & 02 & & $\mathrm{IT}=100$ & \\
\hline${ }^{117} \mathrm{Sb}$ & -88645 & 9 & & & & 2.80 & $\mathrm{~h}$ & 0.01 & $5 / 2^{+}$ & 02 & & $\beta^{+}=100$ & \\
\hline${ }^{117} \mathrm{Te}$ & -85097 & 13 & & & & 62 & $\mathrm{~m}$ & 2 & $1 / 2^{+}$ & 02 & & $\beta^{+}=100 ; \mathrm{e}^{+}=251$ & \\
\hline${ }^{117} \mathrm{Te}^{m}$ & -84801 & 13 & 296.1 & 0.5 & & 103 & $\mathrm{~ms}$ & 3 & $\left(11 / 2^{-}\right)$ & 02 & 99Мo30 J & IT ? & \\
\hline${ }^{117} \mathrm{Te}^{n}$ & -84823 & 13 & 274.4 & 0.1 & & 19.9 & ns & 0.4 & $5 / 2^{+}$ & 02 & & $\mathrm{IT}=100$ & \\
\hline${ }^{117} \mathrm{I}$ & -80435 & 28 & & & & 2.22 & $\mathrm{~m}$ & 0.04 & $(5 / 2)^{+}$ & 02 & & $\beta^{+}=100 ; \mathrm{e}^{+} \approx 77$ & \\
\hline${ }^{117} \mathrm{Xe}$ & -74185 & 10 & & & & 61 & $\mathrm{~s}$ & 2 & $5 / 2^{(+)}$ & 02 & & $\beta^{+}=100 ; \beta^{+} \mathrm{p}=0.00296$ & \\
\hline${ }^{117} \mathrm{Cs}$ & -66440 & 60 & & & $*$ & 8.4 & s & 0.6 & $9 / 2^{+} \#$ & 02 & & $\beta^{+}=100$ & \\
\hline${ }^{117} \mathrm{Cs}^{m}$ & $-66290 \#$ & $100 \#$ & $150 \#$ & $80 \#$ & $*$ & 6.5 & s & 0.4 & $3 / 2^{+} \#$ & 02 & & $\beta^{+}=100$ & \\
\hline${ }^{117} \mathrm{Cs}^{x}$ & -66390 & 80 & 50 & 50 & & $R=$ ? & & & spmix & & & & \\
\hline${ }^{117} \mathrm{Ba}$ & $-57290 \#$ & $300 \#$ & & & & 1.75 & $\mathrm{~s}$ & 0.07 & $(3 / 2)^{(+\#)}$ & 02 & 97Ja12 D & $\beta^{+}=100 ; \beta^{+} \mathrm{p}=133 ;$ & $*$ \\
\hline${ }^{117} \mathrm{La}$ & $-46510 \#$ & $400 \#$ & & & & 23.5 & $\mathrm{~ms}$ & 2.6 & $\left(3 / 2^{+}, 3 / 2^{-}\right)$ & 02 & & $\mathrm{p}=? ; \beta^{+}=6 \#$ & \\
\hline${ }^{117} \mathrm{La}^{m}$ & $-46370 \#$ & $400 \#$ & 138 & 15 & $\mathrm{p}$ & 10 & $\mathrm{~ms}$ & 5 & $\left(9 / 2^{+}\right)$ & 02 & & $\mathrm{p}=? ; \beta^{+}=3 \#$ & \\
\hline${ }^{117} \mathrm{Ru}$ & $\mathrm{I}:$ and $T>$ & $>240 \mathrm{n}$ & in $97 \mathrm{So} 0$ & & & & & & & & & & $* *$ \\
\hline${ }^{117} \mathrm{Ba}$ & $\mathrm{D}: \ldots ; \beta$ & $3^{+} \alpha=0$ & 0248 & & & & & & & & & & $* *$ \\
\hline${ }^{117} \mathrm{Ba}$ & $\mathrm{D}: \beta^{+} \mathrm{p}$ fr & from 97 & Ja12. $\beta^{+}$ & $\mathrm{p} / \beta^{+} \alpha$ & $0-12$ & 200 from & 11 & 2 yielc & $\beta^{+} \alpha=0.011$ & 0.037 & & & $* *$ \\
\hline
\end{tabular}

\begin{tabular}{|c|c|c|c|c|c|c|c|c|c|c|c|c|c|c|}
\hline${ }^{118} \mathrm{Tc}$ & $-45200 \#$ & $900 \#$ & & & & $30 \#$ & $\mathrm{~ms}$ & $>300 \mathrm{~ns})$ & $2^{+} \#$ & 97 & 95Cz.A & I & $\beta^{-} ?$ & \\
\hline${ }^{118} \mathrm{Ru}$ & $-57920 \#$ & $800 \#$ & & & & $200 \#$ & $\mathrm{~ms}$ & $>300 \mathrm{~ns})$ & $0^{+}$ & & $94 \mathrm{Be} 24$ & I & $\beta^{-}$? & \\
\hline${ }^{118} \mathrm{Rh}$ & $-65140 \#$ & $500 \#$ & & & & 310 & $\mathrm{~ms}$ & 30 & $\left(4^{-} 10\right)^{(+\#)}$ & 97 & 00Jo18 & TJD & $\beta^{-}=100$ & \\
\hline${ }^{118} \mathrm{Pd}$ & -75470 & 210 & & & & 1.9 & $\mathrm{~s}$ & 0.1 & $0^{+}$ & 95 & & & $\beta^{-}=100$ & \\
\hline${ }^{118} \mathrm{Ag}$ & -79570 & 60 & & & & 3.76 & $\mathrm{~s}$ & 0.15 & $1^{-}$ & 95 & $93 \mathrm{Ja} 03$ & $\mathrm{~J}$ & $\beta^{-}=100$ & \\
\hline${ }^{118} \mathrm{Ag}^{m}$ & -79440 & 60 & 127.49 & 0.05 & & 2.0 & s & 0.2 & $4^{(+)}$ & 95 & 95Ap.A & E & $\beta^{-}=59 ; \mathrm{IT}=41$ & \\
\hline${ }^{118} \mathrm{Cd}$ & -86709 & 20 & & & & 50.3 & $\mathrm{~m}$ & 0.2 & $0^{+}$ & 95 & & & $\beta^{-}=100$ & \\
\hline${ }^{118} \mathrm{In}$ & -87230 & 8 & & & $*$ & 5.0 & $\mathrm{~s}$ & 0.5 & $1^{+}$ & 95 & & & $\beta^{-}=100$ & \\
\hline${ }^{118} \operatorname{In}^{m}$ & $-87130 \#$ & $50 \#$ & $100 \#$ & $50 \#$ & * & 4.364 & $\mathrm{~m}$ & 0.007 & $5^{+}$ & 95 & 94It.A & $\mathrm{T}$ & $\beta^{-}=100$ & \\
\hline${ }^{118} \operatorname{In}^{n}$ & $-86990 \#$ & $50 \#$ & $240 \#$ & $50 \#$ & & 8.5 & $\mathrm{~s}$ & 0.3 & $8^{-}$ & 95 & & & $\mathrm{IT}=98.63 ; \beta^{-}=1.43$ & * \\
\hline${ }^{118} \mathrm{Sn}$ & -91656.1 & 2.9 & & & & STABLE & & & $0^{+}$ & 95 & & & $\mathrm{IS}=24.229$ & \\
\hline${ }^{118} \mathrm{Sb}$ & -87999 & 4 & & & & 3.6 & $\mathrm{~m}$ & 0.1 & $1^{+}$ & 95 & & & $\beta^{+}=100$ & \\
\hline${ }^{118} \mathrm{Sb}^{m}$ & -87749 & 6 & 250 & 6 & BD & 5.00 & $\mathrm{~h}$ & 0.02 & $8^{-}$ & 95 & & & $\beta^{+}=100$ & \\
\hline${ }^{118} \mathrm{Sb}^{n}$ & -87948 & 4 & 50.814 & 0.021 & & 20.6 & $\mu \mathrm{s}$ & 0.6 & $(3)^{+}$ & & & & & \\
\hline${ }^{118} \mathrm{Te}$ & -87721 & 15 & & & & 6.00 & $\mathrm{~d}$ & 0.02 & $0^{+}$ & 95 & & & $\varepsilon=100$ & \\
\hline${ }^{118} \mathbf{I}$ & -80971 & 20 & & & & 13.7 & $\mathrm{~m}$ & 0.5 & $2^{-}$ & 95 & & & $\beta^{+}=100$ & \\
\hline${ }^{118} \mathrm{I}^{m}$ & -80781 & 20 & 190.1 & 1.0 & & 8.5 & $\mathrm{~m}$ & 0.5 & $\left(7^{-}\right)$ & 95 & $94 \mathrm{Ka} 39$ & E & $\beta^{+} \approx 100 ; \mathrm{IT}=$ ? & \\
\hline${ }^{118} \mathrm{Xe}$ & -78079 & 10 & & & & 3.8 & $\mathrm{~m}$ & 0.9 & $0^{+}$ & 95 & & & $\beta^{+}=100$ & \\
\hline${ }^{118} \mathrm{Cs}$ & -68409 & 13 & & & * & 14 & $\mathrm{~s}$ & 2 & 2 & 95 & & & $\beta^{+}=100 ; \beta^{+} \mathrm{p}=0.02114 ; \ldots$ & * \\
\hline${ }^{118} \mathrm{Cs}^{m}$ & $-68310 \#$ & $60 \#$ & $100 \#$ & $60 \#$ & $*$ & 17 & $\mathrm{~s}$ & 3 & $\left(7^{-}\right)$ & 95 & $93 \mathrm{Be} 46$ & $\mathbf{J}$ & $\beta^{+}=100 ; \beta^{+} \mathrm{p}=0.02114 ; \ldots$ & $*$ \\
\hline${ }^{118} \mathrm{Cs}^{x}$ & -68404 & 12 & 5 & 4 & & $R<0.1$ & & & spmix & & & & & \\
\hline${ }^{118} \mathrm{Ba}$ & $-62370 \#$ & $200 \#$ & & & & 5.2 & $\mathrm{~s}$ & 0.2 & $0^{+}$ & 97 & 97Ja12 & TD & $\beta^{+}=100 ; \beta^{+} \mathrm{p} ?$ & \\
\hline${ }^{118} \mathrm{La}$ & $-49620 \#$ & $300 \#$ & & & & $200 \#$ & $\mathrm{~ms}$ & & & & & & $\beta^{+} ?$ & \\
\hline${ }^{118} \operatorname{In}^{n}$ & \multicolumn{13}{|c|}{$\mathrm{E}: 138.2(0.5) \mathrm{keV}$ above ${ }^{118} \mathrm{In}^{m}$, from ENSDF } & $* *$ \\
\hline${ }^{118} \mathrm{Cs}$ & \multicolumn{14}{|c|}{$\mathrm{D}: \ldots ; \beta^{+} \alpha=0.00125$} \\
\hline${ }^{118} \mathrm{Cs}$ & \multicolumn{14}{|c|}{$\mathrm{D}:$ derived from $\beta^{+} \mathrm{p}=0.042(6) \%, \beta^{+} \alpha=0.0024(4) \%$ for mixture of ground-state and isomer. } \\
\hline${ }^{118} \mathrm{Cs}$ & \multicolumn{14}{|c|}{ D : $\quad$ Replaced by uniform distributions from zero to values for each isomer } \\
\hline${ }^{118} \mathrm{Cs}^{m}$ & \multicolumn{13}{|c|}{$\mathrm{D}: \ldots ; \beta^{+} \alpha=0.00125$} & ** \\
\hline
\end{tabular}




\begin{tabular}{|c|c|c|c|c|c|c|c|c|c|c|c|c|c|}
\hline \multirow{2}{*}{$\begin{array}{l}\text { Nuclide } \\
{ }^{119} \mathrm{Ru}\end{array}$} & \multicolumn{2}{|c|}{$\begin{array}{l}\text { Mass excess } \\
(\mathrm{keV})\end{array}$} & \multicolumn{3}{|c|}{$\begin{array}{l}\text { Excitation } \\
\text { energy }(\mathrm{keV})\end{array}$} & \multicolumn{3}{|c|}{ Half-life } & \multirow[t]{2}{*}{$J^{\pi}$} & \multicolumn{2}{|c|}{ Ens $\mathrm{P}$} & \multicolumn{2}{|l|}{$\begin{array}{l}\text { Decay modes and } \\
\text { intensities }(\%)\end{array}$} \\
\hline & $-53240 \#$ & 700\# & & & & 170\# & $\mathrm{ms}(7$ & $>300 \mathrm{~ns})$ & & & 97Be70 I & $\beta^{-} ?$ & \\
\hline${ }^{119} \mathrm{Rh}$ & $-63240 \#$ & $600 \#$ & & & & $300 \#$ & $\mathrm{~ms}(>$ & $>300 \mathrm{~ns})$ & $7 / 2^{+} \#$ & & $94 \mathrm{Be} 24 \mathrm{I}$ & $\beta^{-} ?$ & \\
\hline${ }^{119} \mathrm{Pd}$ & $-71620 \#$ & $300 \#$ & & & & 920 & $\mathrm{~ms}$ & 130 & & 00 & & $\beta^{-}=100$ & \\
\hline${ }^{119} \mathrm{Ag}$ & -78560 & 90 & & & $* \&$ & 6.0 & $\mathrm{~s}$ & 0.5 & $1 / 2^{-} \#$ & 00 & & $\beta^{-}=100$ & \\
\hline${ }^{119} \mathrm{Ag}^{m}$ & $-78540 \#$ & $90 \#$ & $20 \#$ & $20 \#$ & $* \&$ & 2.1 & s & 0.1 & $7 / 2^{+} \#$ & 00 & & $\beta^{-}=100$ & $*$ \\
\hline${ }^{119} \mathrm{Cd}$ & -83910 & 80 & & & & 2.69 & $\mathrm{~m}$ & 0.02 & $\left(3 / 2^{+}\right)$ & 00 & & $\beta^{-}=100$ & \\
\hline${ }^{119} \mathrm{Cd}^{m}$ & -83760 & 80 & 146.54 & 0.11 & & 2.20 & $\mathrm{~m}$ & 0.02 & $11 / 2^{-} \#$ & 00 & & $\beta^{-}=100$ & \\
\hline${ }^{119} \mathrm{In}$ & -87704 & 8 & & & & 2.4 & $\mathrm{~m}$ & 0.1 & $9 / 2^{+}$ & 00 & & $\beta^{-}=100$ & \\
\hline${ }^{119} \operatorname{In}^{m}$ & -87393 & 8 & 311.37 & 0.03 & & 18.0 & $\mathrm{~m}$ & 0.3 & $1 / 2^{-}$ & 00 & & $\beta^{-}=94.415 ; \mathrm{IT}=5.615$ & \\
\hline${ }^{119} \mathrm{Sn}$ & -90068.4 & +2.9 & & & & STABLE & & & $1 / 2^{+}$ & 00 & & $\mathrm{IS}=8.594$ & \\
\hline${ }^{119} \mathrm{Sn}^{m}$ & -89978.9 & 2.9 & 89.531 & 0.013 & & 293.1 & d & 0.7 & $11 / 2^{-}$ & 00 & & $\mathrm{IT}=100$ & \\
\hline${ }^{119} \mathrm{Sb}$ & -89477 & 8 & & & & 38.19 & $\mathrm{~h}$ & 0.22 & $5 / 2^{+}$ & 00 & & $\varepsilon=100$ & \\
\hline${ }^{119} \mathrm{Sb}^{m}$ & -86625 & 11 & 2852 & 7 & & 850 & $\mathrm{~ms}$ & 90 & $27 / 2^{+} \#$ & 00 & ABBW E & $\mathrm{IT}=100$ & * \\
\hline${ }^{119} \mathrm{Te}$ & -87184 & 8 & & & & 16.05 & $\mathrm{~h}$ & 0.05 & $1 / 2^{+}$ & 00 & & $\beta^{+}=100$ & \\
\hline${ }^{119} \mathrm{Te}^{m}$ & -86923 & 8 & 260.96 & 0.05 & & 4.70 & d & 0.04 & $11 / 2^{-}$ & 00 & & $\varepsilon=99.594 ; \mathrm{e}^{+}=0.414 ; \ldots$ & * \\
\hline${ }^{119} \mathrm{I}$ & -83766 & 28 & & & & 19.1 & $\mathrm{~m}$ & 0.4 & $5 / 2^{+}$ & 00 & & $\beta^{+}=100$ & \\
\hline${ }^{119} \mathrm{Xe}$ & -78794 & 10 & & & & 5.8 & $\mathrm{~m}$ & 0.3 & $5 / 2^{(+)}$ & 00 & 90Ne.A J & $\mathrm{e}^{+}=795 ; \varepsilon=215$ & \\
\hline${ }^{119} \mathrm{Cs}$ & -72305 & 14 & & & $*$ & 43.0 & $\mathrm{~s}$ & 0.2 & $9 / 2^{+}$ & 00 & & $\beta^{+}=100 ; \beta^{+} \alpha<2 \mathrm{e}-6$ & \\
\hline${ }^{119} \mathrm{Cs}^{m}$ & $-72260 \#$ & $30 \#$ & $50 \#$ & $30 \#$ & $*$ & 30.4 & s & 0.1 & $3 / 2^{(+)}$ & 00 & & $\beta^{+}=100$ & \\
\hline${ }^{119} \mathrm{Cs}^{x}$ & -72289 & 9 & 16 & 11 & & $R=.5 \quad .25$ & & & spmix & & & & \\
\hline${ }^{119} \mathrm{Ba}$ & -64590 & 200 & & & & 5.4 & $\mathrm{~s}$ & 0.3 & $\left(5 / 2^{+}\right)$ & 00 & & $\beta^{+}=100 ; \beta^{+} \mathrm{p}<25$ & \\
\hline${ }^{119} \mathrm{La}$ & $-54970 \#$ & $400 \#$ & & & & $1 \#$ & s & & $11 / 2^{-} \#$ & & & $\beta^{+}$? & \\
\hline${ }^{119} \mathrm{Ce}$ & $-44000 \#$ & $600 \#$ & & & & $200 \#$ & $\mathrm{~ms}$ & & $5 / 2^{+} \#$ & & & $\beta^{+} ?$ & \\
\hline$*^{119} \mathrm{Ag}^{m}$ & E : estimat & ted fror & $\mathrm{m} 7 / 2^{+}$le & 1 in isot & opes & ${ }^{113} \mathrm{Ag}=43$ & ${ }^{115} \mathrm{Aq}$ & 41 & $y=28$ & & & & ** \\
\hline$*^{119} \mathrm{Sb}^{m}$ & E : estimat & ted less & $\mathrm{s}$ than $20 \mathrm{ke}$ & $\mathrm{V}$ above & 284 & 1.7 level & & & & & & & ** \\
\hline$*^{119} \mathrm{Te}^{m}$ & $\mathrm{D}: \ldots ; \mathrm{IT}$ & $\Gamma<0.00$ & & & & & & & & & & & ** \\
\hline
\end{tabular}

\begin{tabular}{|c|c|c|c|c|c|c|c|c|c|c|c|c|c|}
\hline${ }^{120} \mathrm{Ru}$ & $-50940 \#$ & $800 \#$ & & & & $80 \#$ & $\mathrm{~ms}($ & $>300 \mathrm{~ns})$ & $0^{+}$ & 02 & 95Cz.A I & $\beta^{-} ?$ & \\
\hline${ }^{120} \mathrm{Rh}$ & $-59230 \#$ & $600 \#$ & & & & 200\# & \multicolumn{4}{|c|}{$\mathrm{ms}(>300 \mathrm{~ns})$} & $94 \mathrm{Be} 24 \mathrm{I}$ & $\beta^{-} ?$ & \\
\hline${ }^{120} \mathrm{Pd}$ & -70150 & 120 & & & & 500 & $\mathrm{~ms}$ & 100 & $0^{+}$ & 02 & & $\beta^{-}=100$ & \\
\hline${ }^{120} \mathrm{Ag}$ & -75650 & 70 & & & & 1.23 & $\mathrm{~s}$ & 0.04 & $3^{(+\#)}$ & 02 & 93Ru01 D & $\beta^{-}=100 ; \beta^{-} \mathrm{n}<0.003$ & \\
\hline${ }^{120} \mathrm{Ag}^{m}$ & -75450 & 70 & 203.0 & 1.0 & & 371 & $\mathrm{~ms}$ & 24 & $6^{(-)}$ & 02 & 03Wa13 T & $\beta^{-} \approx 63 ; \mathrm{IT} \approx 37$ & * \\
\hline${ }^{120} \mathrm{Cd}$ & -83974 & 19 & & & & 50.80 & $\mathrm{~s}$ & 0.21 & $0^{+}$ & 02 & & $\beta^{-}=100$ & \\
\hline${ }^{120} \mathrm{In}$ & -85740 & 40 & & & $*$ & 3.08 & $\mathrm{~s}$ & 0.08 & $1^{+}$ & 02 & & $\beta^{-}=100$ & \\
\hline${ }^{120} \mathrm{In}^{m}$ & $-85690 \#$ & $50 \#$ & $50 \#$ & $60 \#$ & $* \&$ & 46.2 & $\mathrm{~s}$ & 0.8 & $5^{+}$ & 02 & 87Eb02 J & $\beta^{-}=100$ & \\
\hline${ }^{120} \mathrm{In}^{n}$ & $-85440 \#$ & $200 \#$ & $300 \#$ & $200 \#$ & $* \&$ & 47.3 & $\mathrm{~s}$ & 0.5 & $8^{(-)}$ & 02 & 79 Fo10 J & $\beta^{-}=100$ & \\
\hline${ }^{120} \mathrm{Sn}$ & -91105.1 & 2.5 & & & & STABLE & & & $0^{+}$ & 02 & & $\mathrm{IS}=32.589$ & \\
\hline${ }^{120} \mathrm{Sn}^{m}$ & -88623.5 & 2.5 & 2481.63 & 0.06 & & 11.8 & $\mu \mathrm{s}$ & 0.5 & $\left(7^{-}\right)$ & 02 & & $\mathrm{IT}=100$ & \\
\hline${ }^{120} \mathrm{Sn}^{n}$ & -88202.9 & 2.5 & 2902.22 & 0.22 & & 6.26 & $\mu \mathrm{s}$ & 0.11 & $10^{+} \#$ & 02 & & $\mathrm{IT}=100$ & \\
\hline${ }^{120} \mathrm{Sb}$ & -88424 & 8 & & & $*$ & 15.89 & $\mathrm{~m}$ & 0.04 & $1^{+}$ & 02 & & $\beta^{+}=100$ & \\
\hline${ }^{120} \mathrm{Sb}^{m}$ & $-88420 \#$ & $100 \#$ & 0\# & $100 \#$ & $*$ & 5.76 & $\mathrm{~d}$ & 0.02 & $8^{-}$ & 02 & & $\beta^{+}=100$ & \\
\hline${ }^{120} \mathrm{Sb}^{n}$ & -88346 & 8 & 78.16 & 0.05 & & 246 & ns & 2 & $\left(3^{+}\right)$ & 02 & & $\mathrm{IT}=100$ & \\
\hline${ }^{120} \mathrm{Sb}^{p}$ & -86096 & 8 & 2328.3 & 0.6 & & 400 & ns & 8 & $(6)$ & 02 & & $\mathrm{IT}=100$ & \\
\hline${ }^{120} \mathrm{Te}$ & -89405 & 10 & & & & StABlE & & & $0^{+}$ & 02 & & $\mathrm{IS}=0.091 ; 2 \beta^{+} ?$ & \\
\hline${ }^{120} \mathrm{I}$ & -83790 & 18 & & & & 81.6 & $\mathrm{~m}$ & 0.2 & $2^{-}$ & 02 & & $\beta^{+}=100$ & \\
\hline${ }^{120} \mathrm{I}^{m}$ & -83717 & 18 & 72.61 & 0.09 & & 228 & ns & 15 & $\left(1^{+}, 2^{+}, 3^{+}\right)$ & 02 & & $\mathrm{IT}=100$ & \\
\hline${ }^{120} \mathrm{I}^{n}$ & -83470 & 23 & 320 & 15 & & 53 & $\mathrm{~m}$ & 4 & $\left(7^{-}\right)$ & 02 & & $\beta^{+}=100$ & \\
\hline${ }^{120} \mathrm{Xe}$ & -82172 & 12 & & & & 40 & $\mathrm{~m}$ & 1 & $0^{+}$ & 02 & & $\beta^{+}=100$ & \\
\hline${ }^{120} \mathrm{Cs}$ & -73889 & 10 & & & * & 61.2 & $\mathrm{~s}$ & 1.8 & $2^{(-\#)}$ & 02 & & $\beta^{+}=100 ; \beta^{+} \alpha<2.0 \mathrm{e}-54 ; \ldots$ & $*$ \\
\hline${ }^{120} \mathrm{Cs}^{m}$ & $-73790 \#$ & $60 \#$ & $100 \#$ & $60 \#$ & $*$ & 57 & $\mathrm{~s}$ & 6 & $\left(7^{-}\right)$ & 02 & 75Ho09 D & $\beta^{+}=100 ; \beta^{+} \alpha<2.0 \mathrm{e}-54 ; \ldots$ & $*$ \\
\hline${ }^{120} \mathrm{Cs}^{x}$ & -73884 & 9 & 5 & 4 & & $R<0.1$ & & & spmix & & & & \\
\hline${ }^{120} \mathrm{Ba}$ & -68890 & 300 & & & & 24 & $\mathrm{~s}$ & 2 & $0^{+}$ & 02 & 92Xu04 T & $\beta^{+}=100$ & \\
\hline${ }^{120} \mathrm{La}$ & $-57690 \#$ & $500 \#$ & & & & 2.8 & $\mathrm{~s}$ & 0.2 & & 02 & & $\beta^{+}=100 ; \beta^{+} \mathrm{p}=$ ? & \\
\hline${ }^{120} \mathrm{Ce}$ & $-49710 \#$ & $700 \#$ & & & & $250 \#$ & $\mathrm{~ms}$ & & $0^{+}$ & & & $\beta^{+} ?$ & \\
\hline${ }^{120} \mathrm{Ag}^{m}$ & $\mathrm{~T}:$ averag & $03 \mathrm{~W}$ & $=400$ & $71 \mathrm{Fo}$ & $2=32$ & $(40)$ & & & & & & & ** \\
\hline${ }^{120} \mathrm{Cs}$ & $\mathrm{D}: \ldots ; \beta$ & ${ }^{+} \mathrm{p}<7 \mathrm{e}$ & -63 & & & & & & & & & & ** \\
\hline${ }^{120} \mathrm{Cs}$ & $\mathrm{D}$ : isomer & rs not d & listinguis & ed by 7 & Ho09 & $\beta^{+} \alpha$ & d $\beta^{+}$ & Valu & replaced & & & & ** \\
\hline${ }^{120} \mathrm{Cs}$ & D : by & upper & limits for & both (c & 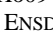 & evalu & on 0 & $\left.{ }^{8} \mathrm{Cs}\right)$ & & & & & $* *$ \\
\hline${ }^{120} \mathrm{Cs}^{m}$ & $\mathrm{D}: \ldots ; \beta$ & $+\mathrm{p}<7 \mathrm{e}$ & -63 & & & & & & & & & & ** \\
\hline
\end{tabular}




\begin{tabular}{|c|c|c|c|c|c|c|c|c|c|c|c|c|}
\hline \multirow{2}{*}{$\begin{array}{l}\text { Nuclide } \\
{ }^{121} \mathrm{Rh}\end{array}$} & \multicolumn{2}{|c|}{$\begin{array}{l}\text { Mass excess } \\
\quad(\mathrm{keV})\end{array}$} & \multicolumn{2}{|c|}{$\begin{array}{l}\text { Excitation } \\
\text { energy }(\mathrm{keV})\end{array}$} & \multicolumn{3}{|c|}{ Half-life } & \multirow{2}{*}{$\begin{array}{c}J^{\pi} \\
7 / 2^{+} \#\end{array}$} & \multirow[t]{2}{*}{ Ens } & Reference & \multicolumn{2}{|l|}{$\begin{array}{l}\text { Decay modes and } \\
\text { intensities }(\%)\end{array}$} \\
\hline & $-57080 \#$ & $900 \#$ & & & $100 \#$ & $\mathrm{~ms}($ & $>300 \mathrm{~ns})$ & & & $94 \mathrm{Be} 24 \mathrm{I}$ & $\beta^{-?}$ & \\
\hline${ }^{121} \mathrm{Pd}$ & $-66260 \#$ & $500 \#$ & & & $400 \#$ & $\mathrm{~ms}$ & $>300 \mathrm{~ns})$ & & 00 & $94 \mathrm{Be} 24 \mathrm{I}$ & $\beta^{-} ?$ & * \\
\hline${ }^{121} \mathrm{Ag}$ & -74660 & 150 & & & 790 & $\mathrm{~ms}$ & 20 & $7 / 2^{+} \#$ & 00 & & $\beta^{-}=100 ; \beta^{-} \mathrm{n}=0.08013$ & \\
\hline${ }^{121} \mathrm{Cd}$ & -81060 & 80 & & & 13.5 & $\mathrm{~s}$ & 0.3 & $\left(3 / 2^{+}\right)$ & 00 & & $\beta^{-}=100$ & \\
\hline${ }^{121} \mathrm{Cd}^{m}$ & -80850 & 80 & 214.86 & 0.15 & 8.3 & $\mathrm{~s}$ & 0.8 & $\left(11 / 2^{-}\right)$ & 00 & & $\beta^{-}=100$ & \\
\hline${ }^{121} \mathrm{In}$ & -85841 & 27 & & & 23.1 & $\mathrm{~s}$ & 0.6 & $9 / 2^{+}$ & 00 & & $\beta^{-}=100$ & \\
\hline${ }^{121} \mathrm{In}^{m}$ & -85528 & 27 & 312.98 & 0.08 & 3.88 & $\mathrm{~m}$ & 0.10 & $1 / 2^{-}$ & 00 & & $\beta^{-}=98.82 ; \mathrm{IT}=1.22$ & \\
\hline${ }^{121} \mathrm{Sn}$ & -89204.1 & 2.5 & & & 27.03 & $\mathrm{~h}$ & 0.04 & $3 / 2^{+}$ & 00 & & $\beta^{-}=100$ & \\
\hline${ }^{121} \mathrm{Sn}^{m}$ & -89197.8 & 2.5 & 6.30 & 0.06 & 43.9 & $\mathrm{y}$ & 0.5 & $11 / 2^{-}$ & 00 & $02 \operatorname{Re} 18 \mathrm{~T}$ & $\mathrm{IT}=77.620 ; \beta^{-}=22.420$ & \\
\hline${ }^{121} \mathrm{Sn}^{n}$ & -87205.3 & 2.7 & 1998.8 & 0.9 & 5.3 & $\mu \mathrm{s}$ & 0.5 & $19 / 2^{+} \#$ & 00 & & $\mathrm{IT}=100$ & \\
\hline${ }^{121} \mathrm{Sb}$ & -89595.1 & 2.2 & & & STABLE & & & $5 / 2^{+}$ & 00 & & $\mathrm{IS}=57.215$ & \\
\hline${ }^{121} \mathrm{Te}$ & -88551 & 26 & & & 19.16 & $\mathrm{~d}$ & 0.05 & $1 / 2^{+}$ & 00 & & $\beta^{+}=100$ & \\
\hline${ }^{121} \mathrm{Te}^{m}$ & -88257 & 26 & 293.991 & 0.022 & 154 & $\mathrm{~d}$ & 7 & $11 / 2^{-}$ & 00 & & $\mathrm{IT}=88.611 ; \beta^{+}=11.411$ & \\
\hline${ }^{121} \mathrm{I}$ & -86287 & 10 & & & 2.12 & $\mathrm{~h}$ & 0.01 & $5 / 2^{+}$ & 00 & & $\beta^{+}=100$ & \\
\hline${ }^{121} \mathrm{I}^{m}$ & -83910 & 10 & 2376.9 & 0.4 & 9.0 & $\mu \mathrm{s}$ & 1.5 & & 00 & & $\mathrm{IT}=100$ & \\
\hline${ }^{121} \mathrm{Xe}$ & -82473 & 11 & & & 40.1 & $\mathrm{~m}$ & 2.0 & $\left(5 / 2^{+}\right)$ & 00 & & $\beta^{+}=100$ & \\
\hline${ }^{121} \mathrm{Cs}$ & -77100 & 14 & & & 155 & s & 4 & $3 / 2^{(+)}$ & 00 & & $\beta^{+}=100$ & \\
\hline${ }^{121} \mathrm{Cs}^{m}$ & -77032 & 14 & 68.5 & 0.3 & 122 & $\mathrm{~s}$ & 3 & $9 / 2^{(+)}$ & 00 & & $\beta^{+}=83 ; \mathrm{IT}=17$ & \\
\hline${ }^{121} \mathrm{Ba}$ & -70740 & 140 & & & 29.7 & $\mathrm{~s}$ & 1.5 & $5 / 2^{(+)}$ & 00 & & $\beta^{+}=100 ; \beta^{+} \mathrm{p}=0.021$ & \\
\hline${ }^{121} \mathrm{La}$ & $-62400 \#$ & $500 \#$ & & & 5.3 & $\mathrm{~s}$ & 0.2 & $11 / 2^{-} \#$ & 00 & & $\beta^{+}=100 ; \beta^{+} \mathrm{p}$ ? & \\
\hline${ }^{121} \mathrm{Ce}$ & $-52700 \#$ & $500 \#$ & & & 1.1 & $\mathrm{~s}$ & 0.1 & $(5 / 2)^{(+\#)}$ & 00 & $99 \mathrm{Li} 46 \mathrm{~J}$ & $\beta^{+}=100 ; \beta^{+} \mathrm{p} \approx 1$ & \\
\hline${ }^{121} \mathrm{Pr}$ & $-41580 \#$ & $700 \#$ & & & 600 & $\mathrm{~ms}$ & 300 & $\left(3 / 2^{-}\right)$ & 00 & 90Bo39 TJD & $\mathrm{p}=? ; \beta^{+} ? ; \beta^{+} \mathrm{p} ?$ & $*$ \\
\hline$*^{121} \mathrm{Pd}$ & $\mathrm{I}:$ and $T>$ & $240 \mathrm{~ns}$ & in $97 \mathrm{So} 07$ & & & & & & & & & $* *$ \\
\hline$*^{121} \mathrm{Pr}$ & $\mathrm{T}: T=1.4$ & $(0.8) \mathrm{si}$ & n ENSDF: $\mathrm{n}$ & trusted to & $\mathrm{g}$ to this & nucli & & & & & & ** \\
\hline
\end{tabular}

\begin{tabular}{|c|c|c|c|c|c|c|c|c|c|c|c|c|c|}
\hline${ }^{122} \mathrm{Rh}$ & $-52900 \#$ & $700 \#$ & & & & $50 \#$ & $\mathrm{~ms}$ & $>300 \mathrm{~ns})$ & & & 97Be70 I & $\beta^{-?} ?$ & \\
\hline${ }^{122} \mathrm{Pd}$ & $-64690 \#$ & $400 \#$ & & & & $300 \#$ & $\mathrm{~ms}$ & $>300 \mathrm{~ns})$ & $0^{+}$ & 98 & $94 \mathrm{Be} 24 \mathrm{I}$ & $\beta^{-} ?$ & * \\
\hline${ }^{122} \mathrm{Ag}$ & $-71230 \#$ & $210 \#$ & & & * & 520 & $\mathrm{~ms}$ & 14 & $\left(3^{+}\right)$ & 94 & $95 \mathrm{Fe} 12 \mathrm{~T}$ & $\beta^{-}=100 ; \beta^{-} \mathrm{n}=0.18610$ & $*$ \\
\hline${ }^{122} \mathrm{Ag}^{m}$ & $-71150 \#$ & $220 \#$ & $80 \#$ & $50 \#$ & * & 1.5 & $\mathrm{~s}$ & 0.5 & $8^{-} \#$ & 94 & & $\beta^{-}=100 ; \beta^{-} \mathrm{n} ?$ & \\
\hline${ }^{122} \mathrm{Cd}$ & -80730 & 40 & & & & 5.24 & $\mathrm{~s}$ & 0.03 & $0^{+}$ & 94 & & $\beta^{-}=100$ & \\
\hline${ }^{122}$ In & -83580 & 50 & & & * & 1.5 & $\mathrm{~s}$ & 0.3 & $1^{+}$ & 94 & & $\beta^{-}=100$ & \\
\hline${ }^{122} \operatorname{In}^{m}$ & $-83540 \#$ & 80\# & $40 \#$ & $60 \#$ & * & 10.3 & $\mathrm{~s}$ & 0.6 & $5^{+}$ & 94 & & $\beta^{-}=100$ & \\
\hline${ }^{122} \operatorname{In}^{n}$ & -83290 & 130 & 290 & 140 & $\mathrm{BD}$ & 10.8 & $\mathrm{~s}$ & 0.4 & $8^{-}$ & 94 & & $\beta^{-}=100$ & \\
\hline${ }^{122} \mathrm{Sn}$ & -89945.9 & 2.7 & & & & StABLE & & & $0^{+}$ & 94 & & $\mathrm{IS}=4.633 ; 2 \beta^{-} ?$ & \\
\hline${ }^{122} \mathrm{Sb}$ & -88330.2 & 2.2 & & & & 2.7238 & $\mathrm{~d}$ & 0.0002 & $2^{-}$ & 94 & & $\beta^{-}=97.5912 ; \ldots$ & $*$ \\
\hline${ }^{122} \mathrm{Sb}^{m}$ & -88166.6 & 2.2 & 163.5591 & 0.0017 & & 4.191 & $\mathrm{~m}$ & 0.003 & $(8)^{-}$ & 94 & & $\mathrm{IT}=100$ & \\
\hline${ }^{122} \mathrm{Sb}^{n}$ & -88192.7 & 2.2 & 137.472 & 0.001 & & 530 & $\mu \mathrm{s}$ & & $5^{+}$ & & & & \\
\hline${ }^{122} \mathrm{Te}$ & -90314.0 & 1.5 & & & & STABLE & & & $0^{+}$ & 94 & & $\mathrm{IS}=2.5512$ & \\
\hline${ }^{122} \mathrm{I}$ & -86080 & 5 & & & & 3.63 & $\mathrm{~m}$ & 0.06 & $1^{+}$ & 94 & & $\beta^{+}=100$ & \\
\hline${ }^{122} \mathrm{Xe}$ & -85355 & 11 & & & & 20.1 & $\mathrm{~h}$ & 0.1 & $0^{+}$ & 94 & & $\varepsilon=100$ & \\
\hline${ }^{122} \mathrm{Cs}$ & -78140 & 30 & & & & 21.18 & $\mathrm{~s}$ & 0.19 & $1^{+}$ & 96 & $93 \mathrm{Al} 03 \mathrm{~T}$ & $\beta^{+}=100 ; \beta^{+} \alpha<2 \mathrm{e}-7$ & * \\
\hline${ }^{122} \mathrm{Cs}^{m}$ & -78005 & 9 & 140 & 30 & MD & 3.70 & $\mathrm{~m}$ & 0.11 & $8^{-}$ & 96 & & $\beta^{+}=100$ & \\
\hline${ }^{122} \mathrm{Cs}^{n}$ & -78010 & 30 & 127.0 & 0.5 & & 360 & $\mathrm{~ms}$ & 20 & $(5)^{-}$ & 96 & & $\mathrm{IT}=100$ & \\
\hline${ }^{122} \mathrm{Ba}$ & -74609 & 28 & & & & 1.95 & $\mathrm{~m}$ & 0.15 & $0^{+}$ & 94 & & $\beta^{+}=100$ & \\
\hline${ }^{122} \mathrm{La}$ & $-64540 \#$ & $300 \#$ & & & & 8.7 & $\mathrm{~s}$ & 0.7 & & 94 & & $\beta^{+}=100 ; \beta^{+} \mathrm{p}=?$ & \\
\hline${ }^{122} \mathrm{Ce}$ & $-57840 \#$ & $400 \#$ & & & & $2 \#$ & $\mathrm{~s}$ & & $0^{+}$ & 94 & & $\beta^{+} ? ; \beta^{+} \mathrm{p} ?$ & $*$ \\
\hline${ }^{122} \mathrm{Pr}$ & $-44890 \#$ & $500 \#$ & & & & $500 \#$ & $\mathrm{~ms}$ & & & & & $\beta^{+} ?$ & \\
\hline${ }^{122} \mathrm{Pd}$ & $\mathrm{I}:$ and $T>$ & $240 \mathrm{~ns}$ & in $97 \mathrm{So} 07$ & & & & & & & & & & $* *$ \\
\hline${ }^{122} \mathrm{Ag}$ & $\mathrm{D}: \beta^{-} \mathrm{n}$ in & tensity & is from $93 \mathrm{I}$ & Ru01 & & & & & & & & & $* *$ \\
\hline${ }^{122} \mathrm{Sb}$ & $\mathrm{D}: \ldots ; \beta$ & $+=2.41$ & & & & & & & & & & & $* *$ \\
\hline${ }^{122} \mathrm{Cs}$ & $\mathrm{T}$ : averag & e $93 \mathrm{All}$ & $3=21.2(0.2$ & ) $69 \mathrm{Ch} 18=$ & $21.0(0$ & & & & & & & & $* *$ \\
\hline${ }^{122} \mathrm{Cs}$ & $\mathrm{D}: \beta^{+} \alpha$ ir & ntensity & upper limi & $\mathrm{t}$ is from 75 & $5 \mathrm{Ho} 09$ & & & & & & & & ** \\
\hline${ }^{122} \mathrm{Ce}$ & $\mathrm{I}: T=8.7(0$ & $0.7) \mathrm{s}$ in & NDS 71 (1 & 994) was $n$ & nisprin & t for ${ }^{122} \mathrm{~L}$ & $\mathrm{a} ; \mathrm{co}$ & rrected in & SDF & & & & ** \\
\hline
\end{tabular}




\begin{tabular}{|c|c|c|c|c|c|c|c|c|c|c|c|c|}
\hline Nuclide & \multicolumn{2}{|c|}{$\begin{array}{l}\text { Mass excess } \\
\quad(\mathrm{keV})\end{array}$} & \multicolumn{2}{|c|}{$\begin{array}{l}\text { Excitation } \\
\text { energy }(\mathrm{keV})\end{array}$} & \multicolumn{3}{|c|}{ Half-life } & \multirow[t]{2}{*}{$J^{\pi}$} & \multirow[t]{2}{*}{ Ens } & Reference & \multicolumn{2}{|l|}{$\begin{array}{l}\text { Decay modes and } \\
\text { intensities }(\%)\end{array}$} \\
\hline${ }^{123} \mathrm{Pd}$ & $-60610 \#$ & $600 \#$ & & & $200 \#$ & $\mathrm{~ms}($ & $(>300 \mathrm{~ns})$ & & & $94 \mathrm{Be} 24 \mathrm{I}$ & $\beta^{-} ?$ & \\
\hline${ }^{123} \mathrm{Ag}$ & $-69960 \#$ & $210 \#$ & & & 296 & $\mathrm{~ms}$ & 6 & $\left(7 / 2^{+}\right)$ & 94 & $95 \mathrm{Fe} 12 \mathrm{~T}$ & $\beta^{-}=100 ; \beta^{-} \mathrm{n}=0.555$ & * \\
\hline${ }^{123} \mathrm{Cd}$ & -77310 & 40 & & & 2.10 & $\mathrm{~s}$ & 0.02 & $(3 / 2)^{+}$ & 94 & & $\beta^{-}=100$ & \\
\hline${ }^{123} \mathrm{Cd}^{m}$ & -76990 & 40 & 316.52 & 0.23 & 1.82 & $\mathrm{~s}$ & 0.03 & $\left(11 / 2^{-}\right)$ & 94 & & $\beta^{-}=? ; \mathrm{IT}=$ ? & \\
\hline${ }^{123} \mathrm{In}$ & -83426 & 24 & & & 5.98 & $\mathrm{~s}$ & 0.06 & $9 / 2^{+}$ & 94 & & $\beta^{-}=100$ & \\
\hline${ }^{123} \operatorname{In}^{m}$ & -83099 & 24 & 327.21 & 0.04 & 47.8 & $\mathrm{~s}$ & 0.5 & $1 / 2^{-}$ & 94 & & $\beta^{-}=100$ & \\
\hline${ }^{123} \mathrm{Sn}$ & -87820.5 & 2.7 & & & 129.2 & d & 0.4 & $11 / 2^{-}$ & 94 & & $\beta^{-}=100$ & \\
\hline${ }^{123} \mathrm{Sn}^{m}$ & -87795.9 & 2.7 & 24.6 & 0.4 & 40.06 & $\mathrm{~m}$ & 0.01 & $3 / 2^{+}$ & 94 & & $\beta^{-}=100$ & \\
\hline${ }^{123} \mathrm{Sb}$ & -89224.1 & 2.1 & & & STABLE & & & $7 / 2^{+}$ & 94 & & $\mathrm{IS}=42.795$ & \\
\hline${ }^{123} \mathrm{Te}$ & -89171.9 & 1.5 & & & $>600$ & Ty & & $1 / 2^{+}$ & 94 & $96 \mathrm{Al} 30 \mathrm{~T}$ & $\mathrm{IS}=0.893 ; \varepsilon=100$ & * \\
\hline${ }^{123} \mathrm{Te}^{m}$ & -88924.3 & 1.5 & 247.55 & 0.04 & 119.25 & $\mathrm{~d}$ & 0.15 & $11 / 2^{-}$ & 94 & & $\mathrm{IT}=100$ & \\
\hline${ }^{123} \mathrm{I}$ & -87943 & 4 & & & 13.2235 & $\mathrm{~h}$ & 0.0019 & $5 / 2^{+}$ & 94 & 02Un02 T & $\beta^{+}=100$ & \\
\hline${ }^{123} \mathrm{Xe}$ & -85249 & 10 & & & 2.08 & $\mathrm{~h}$ & 0.02 & $1 / 2^{+}$ & 94 & 90Ne.A J & $\beta^{+}=100$ & \\
\hline${ }^{123} \mathrm{Xe}^{m}$ & -85064 & 10 & 185.18 & 0.22 & 5.49 & $\mu \mathrm{s}$ & 0.26 & $7 / 2^{(-)}$ & & & & \\
\hline${ }^{123} \mathrm{Cs}$ & -81044 & 12 & & & 5.87 & $\mathrm{~m}$ & 0.04 & $1 / 2^{+}$ & 94 & $93 \mathrm{Al} 03 \mathrm{~T}$ & $\beta^{+}=100$ & $*$ \\
\hline${ }^{123} \mathrm{Cs}^{m}$ & -80887 & 12 & 156.74 & 0.21 & 1.64 & $\mathrm{~s}$ & 0.12 & $(11 / 2)^{-}$ & 94 & & $\mathrm{IT}=100$ & \\
\hline${ }^{123} \mathrm{Cs}^{x}$ & -81037 & 13 & 7 & 4 & $R<0.1$ & & & spmix & & & & \\
\hline${ }^{123} \mathrm{Ba}$ & -75655 & 12 & & & 2.7 & $\mathrm{~m}$ & 0.4 & $5 / 2^{+}$ & 94 & & $\beta^{+}=100$ & \\
\hline${ }^{123} \mathrm{La}$ & $-68710 \#$ & $200 \#$ & & & 17 & $\mathrm{~s}$ & 3 & $11 / 2^{-} \#$ & 94 & & $\beta^{+}=100$ & \\
\hline${ }^{123} \mathrm{Ce}$ & $-60180 \#$ & $300 \#$ & & & 3.8 & $\mathrm{~s}$ & 0.2 & $(5 / 2)^{(+\#)}$ & 94 & & $\beta^{+}=100 ; \beta^{+} \mathrm{p}=$ ? & \\
\hline${ }^{123} \mathrm{Pr}$ & $-50340 \#$ & $600 \#$ & & & $800 \#$ & $\mathrm{~ms}$ & & $3 / 2^{+} \#$ & & & $\beta^{+}$? & \\
\hline$*^{123} \mathrm{Ag}$ & $\mathrm{T}$ : averag & $95 \mathrm{Fe}$ & $2=293(7)$ & $\mathrm{Ma} 42=3 \mathrm{C}$ & $83 \operatorname{Re} 05=3 \mathrm{C}$ & $00(10$ & & D : from 93 & $3 \mathrm{Ru} 0$ & & & $* *$ \\
\hline$*^{123} \mathrm{Te}$ & $\mathrm{T}:$ and $T=$ & $=24(9) \mathrm{I}$ & $\mathrm{y}$ for $\varepsilon(\mathrm{K}$ & Ime auth & & & & & & & & $* *$ \\
\hline$*^{123} \mathrm{Te}$ & $\mathrm{I}:$ this nuc & clide is & ot consid & 'stable & $\mathrm{K} \varepsilon$ has be & een ob & bserved & & & & & $* *$ \\
\hline$*^{123} \mathrm{Cs}$ & $\mathrm{T}$ : averag & e $93 \mathrm{AlC}$ & $3=5.87(0$ & $68 \mathrm{Ch} 18$ & $0.05)$ & & & & & & & $* *$ \\
\hline
\end{tabular}

\begin{tabular}{|c|c|c|c|c|c|c|c|c|c|c|c|c|c|c|}
\hline${ }^{124} \mathrm{Pd}$ & $-58800 \#$ & $500 \#$ & & & & $100 \#$ & $\mathrm{~ms}$ & $(>300 \mathrm{~ns})$ & $0^{+}$ & & $97 \mathrm{Be} 70$ & I & $\beta^{-}$? & \\
\hline${ }^{124} \mathrm{Ag}$ & $-66470 \#$ & $200 \#$ & & & * & 172 & $\mathrm{~ms}$ & 5 & $3^{+} \#$ & 97 & & & $\beta^{-}=100 ; \beta^{-} \mathrm{n}>0.1$ & \\
\hline${ }^{124} \mathrm{Ag}^{m}$ & $-66470 \#$ & $220 \#$ & $0 \#$ & $100 \#$ & $*$ & $200 \#$ & $\mathrm{~ms}$ & & $8^{-} \#$ & & 95Kr.A & I & $\beta^{-}$?; IT ? & $*$ \\
\hline${ }^{124} \mathrm{Cd}$ & -76710 & 60 & & & & 1.25 & $\mathrm{~s}$ & 0.02 & $0^{+}$ & 97 & & & $\beta^{-}=100$ & \\
\hline${ }^{124} \mathrm{In}$ & -80880 & 50 & & & $*$ & 3.11 & $\mathrm{~s}$ & 0.10 & $3^{+}$ & 97 & & & $\beta^{-}=100$ & \\
\hline${ }^{124} \mathrm{In}^{m}$ & -80900 & 50 & -20 & 70 & $\mathrm{BD} *$ & 3.7 & $\mathrm{~s}$ & 0.2 & $(8)^{(-\#)}$ & 97 & & & $\beta^{-} \approx 100$; IT ? & \\
\hline${ }^{124} \mathrm{Sn}$ & -88236.8 & 1.4 & & & & STABLE & & $(>100 \mathrm{Py})$ & $0^{+}$ & 97 & $52 \mathrm{Ka} 41$ & $\mathrm{~T}$ & $\mathrm{IS}=5.795 ; 2 \beta^{-} ?$ & \\
\hline${ }^{124} \mathrm{Sn}^{m}$ & -85911.8 & 1.4 & 2325.01 & 0.04 & & 3.1 & $\mu \mathrm{s}$ & 0.5 & $7^{-}$ & 97 & & & $\mathrm{IT}=100$ & \\
\hline${ }^{124} \mathrm{Sn}^{n}$ & -85580.2 & 1.5 & 2656.6 & 0.5 & & 45 & $\mu \mathrm{s}$ & 5 & $10^{+} \#$ & 97 & & & $\mathrm{IT}=100$ & \\
\hline${ }^{124} \mathrm{Sb}$ & -87620.3 & 2.1 & & & & 60.20 & $\mathrm{~d}$ & 0.03 & $3^{-}$ & 98 & & & $\beta^{-}=100$ & \\
\hline${ }^{124} \mathrm{Sb}^{m}$ & -87609.4 & 2.1 & 10.8627 & 0.0008 & & 93 & $\mathrm{~s}$ & 5 & $5^{+}$ & 97 & & & $\mathrm{IT}=755 ; \beta^{-}=255$ & \\
\hline${ }^{124} \mathrm{Sb}^{n}$ & -87583.5 & 2.1 & 36.8440 & 0.0014 & & 20.2 & $\mathrm{~m}$ & 0.2 & $(8)^{-}$ & 97 & & & $\mathrm{IT}=100$ & \\
\hline${ }^{124} \mathrm{Sb}^{p}$ & -87579.5 & 2.1 & 40.8038 & 0.0007 & & 3.2 & $\mu \mathrm{s}$ & 0.3 & $\left(3^{+}, 4^{+}\right)$ & 97 & & & $\mathrm{IT}=100$ & \\
\hline${ }^{124} \mathrm{Te}$ & -90524.5 & 1.5 & & & & STABLE & & & $0^{+}$ & 97 & & & $\mathrm{IS}=4.7414$ & \\
\hline${ }^{124} \mathrm{I}$ & -87365.0 & 2.4 & & & & 4.1760 & $\mathrm{~d}$ & 0.0003 & $2^{-}$ & 97 & & & $\beta^{+}=100$ & \\
\hline${ }^{124} \mathrm{Xe}$ & -87660.1 & 1.8 & & & & STABLE & & $(>48$ Py $)$ & $0^{+}$ & 97 & 89Ba22 & $\mathrm{T}$ & $\mathrm{IS}=0.091 ; 2 \beta^{+} ?$ & \\
\hline${ }^{124} \mathrm{Cs}$ & -81731 & 8 & & & & 30.9 & $\mathrm{~s}$ & 0.4 & $1^{+}$ & 97 & $93 \mathrm{Al} 03$ & $\mathrm{~T}$ & $\beta^{+}=100$ & $*$ \\
\hline${ }^{124} \mathrm{Cs}^{m}$ & -81268 & 8 & 462.55 & 0.17 & & 6.3 & $\mathrm{~s}$ & 0.2 & $(7)^{+}$ & 97 & & & $\mathrm{IT}=100$ & \\
\hline${ }^{124} \mathrm{Cs}^{x}$ & -81701 & 22 & 30 & 20 & & $R=$ ? & & & spmix & & & & & \\
\hline${ }^{124} \mathrm{Ba}$ & -79090 & 12 & & & & 11.0 & $\mathrm{~m}$ & 0.5 & $0^{+}$ & 97 & & & $\beta^{+}=100$ & \\
\hline${ }^{124} \mathrm{La}$ & -70260 & 60 & & & * & 29.21 & $\mathrm{~s}$ & 0.17 & $\left(7^{-}, 8^{-}\right)$ & 97 & 97As05 & $\mathrm{T}$ & $\beta^{+}=100$ & $*$ \\
\hline${ }^{124} \mathrm{La}^{m}$ & $-70160 \#$ & $120 \#$ & $100 \#$ & $100 \#$ & * & 21 & $\mathrm{~s}$ & 4 & low ${ }^{(+\#)}$ & 97 & $97 \mathrm{As} 05$ & $\mathrm{~T}$ & $\beta^{+}=100$ & \\
\hline${ }^{124} \mathrm{Ce}$ & $-64820 \#$ & $300 \#$ & & & & 9.1 & $\mathrm{~s}$ & 1.2 & $0^{+}$ & 98 & $97 \mathrm{As} 05$ & $\mathrm{~T}$ & $\beta^{+}=100$ & $*$ \\
\hline${ }^{124} \mathrm{Pr}$ & $-53130 \#$ & $600 \#$ & & & & 1.2 & $\mathrm{~s}$ & 0.2 & & 97 & & & $\beta^{+}=100 ; \beta^{+} \mathrm{p}=?$ & \\
\hline${ }^{124} \mathrm{Nd}$ & $-44500 \#$ & $600 \#$ & & & & $500 \#$ & $\mathrm{~ms}$ & & $0^{+}$ & & & & $\beta^{+}$? & \\
\hline${ }^{124} \mathrm{Ag}^{m}$ & I : "There & is some & e evidence fo & or a low-spi & in and & high-spi & in iso & omer in ${ }^{1}$ & & & & & & ** \\
\hline${ }^{124} \mathrm{Cs}$ & $\mathrm{T}$ : average & e $93 \mathrm{All}$ & $03=30.9(0.5)$ & $78 \mathrm{Ek} 05=3$ & $30.8(0.5$ & & & & & & & & & ** \\
\hline${ }^{124} \mathrm{La}$ & $\mathrm{J}:$ for ${ }^{124} \mathrm{~L}$ & a and 12 & ${ }^{124} \mathrm{La}^{m}$ are $\mathrm{fr}$ & rom 92Id01 & & & & & & & & & & ** \\
\hline${ }^{124} \mathrm{Ce}$ & $\mathrm{T}$ : averag & e $97 \mathrm{As} 0$ & $05=10.8(1.5$ & $78 \mathrm{Bo} 32=6$ & $6(2)$ & & & & & & & & & ** \\
\hline
\end{tabular}




\begin{tabular}{|c|c|c|c|c|c|c|c|c|c|c|c|c|c|}
\hline Nuclide & \multicolumn{2}{|c|}{$\begin{array}{c}\text { Mass excess } \\
(\mathrm{keV})\end{array}$} & \multicolumn{3}{|c|}{$\begin{array}{l}\text { Excitation } \\
\text { energy }(\mathrm{keV})\end{array}$} & \multicolumn{3}{|c|}{ Half-life } & $J^{\pi}$ & \multirow[t]{2}{*}{ Ens } & Reference & \multicolumn{2}{|l|}{$\begin{array}{l}\text { Decay modes and } \\
\text { intensities }(\%)\end{array}$} \\
\hline${ }^{125} \mathrm{Ag}$ & $-64800 \#$ & $300 \#$ & & & & 166 & $\mathrm{~ms}$ & 7 & $7 / 2^{+} \#$ & & & $\beta^{-}=100 ; \beta^{-} \mathrm{n}=?$ & \\
\hline${ }^{125} \mathrm{Cd}$ & -73360 & 70 & & & * & 650 & $\mathrm{~ms}$ & 20 & $3 / 2^{+} \#$ & 99 & & $\beta^{-}=100$ & \\
\hline${ }^{125} \mathrm{Cd}^{m}$ & -73310 & 50 & 50 & 70 & $\mathrm{BD} *$ & 570 & $\mathrm{~ms}$ & 90 & $11 / 2^{-} \#$ & 99 & $89 \mathrm{Hu} 03 \mathrm{~T}$ & $\beta^{-}=100$ & $*$ \\
\hline${ }^{125} \mathrm{In}$ & -80480 & 30 & & & & 2.36 & $\mathrm{~s}$ & 0.04 & $9 / 2^{+}$ & 99 & & $\beta^{-}=100$ & \\
\hline${ }^{125} \operatorname{In}^{m}$ & -80120 & 30 & 360.12 & 0.09 & & 12.2 & $\mathrm{~s}$ & 0.2 & $1 / 2^{(-)}$ & 99 & & $\beta^{-}=100$ & \\
\hline${ }^{125} \mathrm{Sn}$ & -85898.5 & 1.5 & & & & 9.64 & $\mathrm{~d}$ & 0.03 & $11 / 2^{-}$ & 99 & & $\beta^{-}=100$ & \\
\hline${ }^{125} \mathrm{Sn}^{m}$ & -85871.0 & 1.5 & 27.50 & 0.14 & & 9.52 & $\mathrm{~m}$ & 0.05 & $3 / 2^{+}$ & 99 & & $\beta^{-}=100$ & \\
\hline${ }^{125} \mathrm{Sb}$ & -88255.5 & 2.6 & & & & 2.75856 & $6 y$ & 0.00025 & $7 / 2^{+}$ & 99 & & $\beta^{-}=100$ & \\
\hline${ }^{125} \mathrm{Te}$ & -89022.2 & 1.5 & & & & STABLE & & & $1 / 2^{+}$ & 99 & & $\mathrm{IS}=7.0715$ & \\
\hline${ }^{125} \mathrm{Te}^{m}$ & -88877.4 & 1.5 & 144.772 & 0.009 & & 57.40 & $\mathrm{~d}$ & 0.15 & $11 / 2^{-}$ & 99 & & $\mathrm{IT}=100$ & \\
\hline${ }^{125} \mathrm{I}$ & -88836.4 & 1.5 & & & & 59.400 & $\mathrm{~d}$ & 0.010 & $5 / 2^{+}$ & 99 & & $\varepsilon=100$ & \\
\hline${ }^{125} \mathrm{Xe}$ & -87192.1 & 1.9 & & & & 16.9 & $\mathrm{~h}$ & 0.2 & $1 / 2^{(+)}$ & 99 & & $\beta^{+}=100$ & \\
\hline${ }^{125} \mathrm{Xe}^{m}$ & -86939.5 & 1.9 & 252.60 & 0.14 & & 56.9 & $\mathrm{~s}$ & 0.9 & $9 / 2^{(-)}$ & 99 & & $\mathrm{IT}=100$ & \\
\hline${ }^{125} \mathrm{Cs}$ & -84088 & 8 & & & & 45 & $\mathrm{~m}$ & 1 & $1 / 2^{(+)}$ & 99 & & $\beta^{+}=100$ & \\
\hline${ }^{125} \mathrm{Cs}^{m}$ & -83821 & 8 & 266.6 & 1.1 & & 900 & $\mathrm{~ms}$ & 30 & $\left(11 / 2^{-}\right)$ & 99 & 98Su16 TJ & $\mathrm{IT}=100$ & \\
\hline${ }^{125} \mathrm{Ba}$ & -79668 & 11 & & & & 3.5 & $\mathrm{~m}$ & 0.4 & $1 / 2^{(+\#)}$ & 99 & & $\beta^{+}=100$ & \\
\hline${ }^{125} \mathrm{La}$ & -73759 & 26 & & & & 64.8 & $\mathrm{~s}$ & 1.2 & $\left(11 / 2^{-}\right)$ & 99 & & $\beta^{+}=100$ & * \\
\hline${ }^{125} \mathrm{La}^{m}$ & -73652 & 26 & 107.0 & 0.1 & & 390 & $\mathrm{~ms}$ & 40 & $\left(3 / 2^{+}\right)$ & 99 & 99Ca21 ETJ & $\mathrm{IT}=100$ & $*$ \\
\hline${ }^{125} \mathrm{Ce}$ & $-66660 \#$ & $200 \#$ & & & & 9.3 & $\mathrm{~s}$ & 0.3 & $\left(7 / 2^{-}\right)$ & 99 & $02 \mathrm{Pe} 15 \mathrm{~J}$ & $\beta^{+}=100 ; \beta^{+} \mathrm{p}=?$ & * \\
\hline${ }^{125} \mathrm{Pr}$ & $-57910 \#$ & $400 \#$ & & & & 3.3 & $\mathrm{~s}$ & 0.7 & $3 / 2^{+} \#$ & 02 & & $\beta^{+}=100 ; \beta^{+} \mathrm{p} ?$ & \\
\hline${ }^{125} \mathrm{Nd}$ & $-47620 \#$ & $400 \#$ & & & & & $\mathrm{~ms}$ & 150 & $5 / 2^{(+\#)}$ & 02 & & $\beta^{+}=100$ & \\
\hline$*^{125} \mathrm{Cd}^{m}$ & $\mathrm{~T}$ : unweig & shed av & rage $89 \mathrm{Hu}$ & $03=480$ & (30) $86 \mathrm{~N}$ & Ma $42=66$ & $60(30)$ & ) (Birge ra & o $B=4.24$ ) & & & & $* *$ \\
\hline$*^{125} \mathrm{La}$ & J : ENSDF' & 99 say & ground-sta & te spin $u$ & inknown & ; $; a(11 / 2$ & $\left.2^{-}\right) \mathrm{le}$ & evel lies at & $-9 \mathrm{keV}$ abo & ve gr & round-state & & ** \\
\hline$*{ }^{125} \mathrm{La}^{m}$ & $\mathrm{~J}: 3 / 2^{+} \#$ & from sy & tematics; & ow spin & and eve & n-parity & from & $99 \mathrm{Ca} 21$ & & & & & $* *$ \\
\hline$*{ }^{125} \mathrm{Ce}$ & $\mathrm{T}$ : averag & e $99 \mathrm{Ca}$ & $1=9.6(0.4)$ & $86 \mathrm{Wi15}$ & $=9.2(1$. & 0) $83 \mathrm{Ni} 0$ & $05=8$. & $9(0.5)$ & & & & & $* *$ \\
\hline
\end{tabular}

\begin{tabular}{|c|c|c|c|c|c|c|c|c|c|c|c|c|c|}
\hline${ }^{126} \mathrm{Ag}$ & $-61010 \#$ & $300 \#$ & & & & 107 & $\mathrm{~ms}$ & 12 & $3^{+} \#$ & 03 & & & $\beta^{-}=100 ; \beta^{-} \mathrm{n}=?$ \\
\hline${ }^{126} \mathrm{Cd}$ & -72330 & 50 & & & & 515 & $\mathrm{~ms}$ & 17 & $0^{+}$ & 03 & & & $\beta^{-}=100$ \\
\hline${ }^{126} \mathrm{In}$ & -77810 & 40 & & & $*$ & 1.53 & $\mathrm{~s}$ & 0.01 & $3^{(+\#)}$ & 03 & & & $\beta^{-}=100$ \\
\hline${ }^{126} \mathrm{In}^{m}$ & -77710 & 50 & 100 & 60 & $\mathrm{BD} *$ & 1.64 & $\mathrm{~s}$ & 0.05 & $8^{(-\#)}$ & 03 & 79Fo10 J & J & $\beta^{-}=100$ \\
\hline${ }^{126} \mathrm{Sn}$ & -86020 & 11 & & & & 230 & ky & 14 & $0^{+}$ & 03 & & & $\beta^{-}=100$ \\
\hline${ }^{126} \mathrm{Sn}^{m}$ & -83801 & 11 & 2218.99 & 0.08 & & 6.6 & $\mu \mathrm{s}$ & 1.4 & $7^{-}$ & 03 & & & $\mathrm{IT}=100$ \\
\hline${ }^{126} \mathrm{Sn}^{n}$ & -83456 & 11 & 2564.5 & 0.5 & & 7.7 & $\mu \mathrm{s}$ & 0.5 & $10^{+} \#$ & 03 & & & $\mathrm{IT}=100$ \\
\hline${ }^{126} \mathrm{Sb}$ & -86400 & 30 & & & & 12.35 & $\mathrm{~d}$ & 0.06 & $\left(8^{-}\right)$ & 03 & & & $\beta^{-}=100$ \\
\hline${ }^{126} \mathrm{Sb}^{m}$ & -86380 & 30 & 17.7 & 0.3 & & 19.15 & $\mathrm{~m}$ & 0.08 & $\left(5^{+}\right)$ & 03 & & & $\beta^{-}=864 ; \mathrm{IT}=144$ \\
\hline${ }^{126} \mathrm{Sb}^{n}$ & -86360 & 30 & 40.4 & 0.3 & & 11 & $\mathrm{~s}$ & & $\left(3^{-}\right)$ & 03 & & & $\mathrm{IT}=100$ \\
\hline${ }^{126} \mathrm{Sb}^{p}$ & -86300 & 30 & 104.6 & 0.3 & & 553 & ns & 5 & $\left(3^{+}\right)$ & 03 & & & $\mathrm{IT}=100$ \\
\hline${ }^{126} \mathrm{Te}$ & -90064.6 & 1.5 & & & & STABLE & & & $0^{+}$ & 03 & & & $\mathrm{IS}=18.8425$ \\
\hline${ }^{126} \mathrm{I}$ & -87911 & 4 & & & & 12.93 & d & 0.05 & $2^{-}$ & 03 & & & $\beta^{+}=52.75 ; \beta^{-}=47.35$ \\
\hline${ }^{126} \mathrm{Xe}$ & -89169 & 6 & & & & Stable & & & $0^{+}$ & 03 & & & $\mathrm{IS}=0.091 ; 2 \beta^{+} ?$ \\
\hline${ }^{126} \mathrm{Cs}$ & -84345 & 12 & & & & 1.64 & $\mathrm{~m}$ & 0.02 & $1^{+}$ & 03 & & & $\beta^{+}=100$ \\
\hline${ }^{126} \mathrm{Cs}^{m}$ & -84072 & 12 & 273.0 & 0.7 & & $>1$ & $\mu \mathrm{s}$ & & & 03 & & & $\mathrm{IT}=100$ \\
\hline${ }^{126} \mathrm{Cs}^{n}$ & -83749 & 12 & 596.1 & 1.1 & & 171 & $\mu \mathrm{s}$ & 14 & & 03 & & & $\mathrm{IT}=100$ \\
\hline${ }^{126} \mathrm{Ba}$ & -82670 & 12 & & & & 100 & $\mathrm{~m}$ & 2 & $0^{+}$ & 03 & & & $\beta^{+}=100$ \\
\hline${ }^{126} \mathrm{La}$ & -74970 & 90 & & & $*$ & 54 & $\mathrm{~s}$ & 2 & $(5)^{(+\#)}$ & 03 & & & $\beta^{+}=100$ \\
\hline${ }^{126} \mathrm{La}^{m}$ & -74760 & 400 & 210 & 410 & $\mathrm{BD} *$ & 20 & $\mathrm{~s}$ & 20 & $\left(0^{-}, 1^{-}, 2^{-}\right)$ & 03 & & & $\beta^{+}=100$ \\
\hline${ }^{126} \mathrm{Ce}$ & -70821 & 28 & & & & 51.0 & $\mathrm{~s}$ & 0.3 & $0^{+}$ & 03 & & & $\beta^{+}=100$ \\
\hline${ }^{126} \mathrm{Pr}$ & $-60260 \#$ & $200 \#$ & & & & 3.12 & $\mathrm{~s}$ & 0.18 & $(4,5,6)$ & 03 & $88 \mathrm{Ba} 42 \mathrm{~T}$ & $\mathrm{~T}$ & $\beta^{+}=100 ; \beta^{+} \mathrm{p}=$ ? \\
\hline${ }^{126} \mathrm{Nd}$ & $-52890 \#$ & $400 \#$ & & & & $1 \#$ & s & $(>200 \mathrm{~ns})$ & $0^{+}$ & 03 & 00So11 I & & $\beta^{+}$? \\
\hline${ }^{126} \mathrm{Pm}$ & $-39570 \#$ & $500 \#$ & & & & $500 \#$ & $\mathrm{~ms}$ & & & & & & $\beta^{+}$? \\
\hline${ }^{126} \mathrm{La}^{m}$ & $\mathrm{~T}: 97 \mathrm{As} 0$ & 5: "by & far short & han $50 \mathrm{~s}^{\prime}$ & & & & & & & & & \\
\hline${ }^{126} \mathrm{Pr}$ & $\mathrm{T}$ : averag & e $95 \mathrm{Os}$ & $03=3.14(C$ & .22) $88 \mathrm{Ba}$ & $42=3.0$ & $0.4)$ and & $83 N$ & $\mathrm{i} 05=3.2(0$. & & & & & \\
\hline
\end{tabular}




\begin{tabular}{|c|c|c|c|c|c|c|c|c|c|c|c|c|c|c|}
\hline Nuclide & \multicolumn{2}{|c|}{$\begin{array}{l}\text { Mass excess } \\
\quad(\mathrm{keV})\end{array}$} & \multicolumn{3}{|c|}{$\begin{array}{l}\text { Excitation } \\
\text { energy }(\mathrm{keV})\end{array}$} & \multicolumn{3}{|c|}{ Half-life } & $J^{\pi}$ & \multirow[t]{2}{*}{ Ens } & \multicolumn{2}{|c|}{ Reference } & \multicolumn{2}{|l|}{$\begin{array}{l}\text { Decay modes and } \\
\text { intensities }(\%)\end{array}$} \\
\hline${ }^{127} \mathrm{Ag}$ & $-58900 \#$ & $300 \#$ & & & & 79 & $\mathrm{~ms}$ & 3 & $7 / 2^{+} \#$ & & 96Wo.A & $\mathrm{TD}$ & $\beta^{-}=100 ; \beta^{-} \mathrm{n}=?$ & * \\
\hline${ }^{127} \mathrm{Cd}$ & -68520 & 70 & & & & 370 & $\mathrm{~ms}$ & 70 & $\left(3 / 2^{+}\right)$ & 96 & & & $\beta^{-}=100$ & \\
\hline${ }^{127} \mathrm{In}$ & -76990 & 40 & & & & 1.09 & $\mathrm{~s}$ & 0.01 & $9 / 2^{(+)}$ & 96 & 87Eb02 & $\mathrm{J}$ & $\beta^{-}=100 ; \beta^{-} \mathrm{n} \leq 0.03$ & \\
\hline${ }^{127} \operatorname{In}^{m}$ & -76520 & 70 & 460 & 70 & $\mathrm{BD}$ & 3.67 & s & 0.04 & $\left(1 / 2^{-}\right)$ & 96 & & & $\beta^{-}=100 ; \beta^{-} \mathrm{n}=0.694$ & \\
\hline${ }^{127} \mathrm{Sn}$ & -83499 & 25 & & & & 2.10 & $\mathrm{~h}$ & 0.04 & $\left(11 / 2^{-}\right)$ & 96 & & & $\beta^{-}=100$ & \\
\hline${ }^{127} \mathrm{Sn}^{m}$ & -83494 & 25 & 4.7 & 0.3 & & 4.13 & $\mathrm{~m}$ & 0.03 & $\left(3 / 2^{+}\right)$ & 96 & & & $\beta^{-}=100$ & \\
\hline${ }^{127} \mathrm{Sb}$ & -86700 & 5 & & & & 3.85 & $\mathrm{~d}$ & 0.05 & $7 / 2^{+}$ & 96 & & & $\beta^{-}=100$ & \\
\hline${ }^{127} \mathrm{Te}$ & -88281.1 & 1.5 & & & & 9.35 & $\mathrm{~h}$ & 0.07 & $3 / 2^{+}$ & 96 & & & $\beta^{-}=100$ & \\
\hline${ }^{127} \mathrm{Te}^{m}$ & -88192.8 & 1.5 & 88.26 & 0.08 & & 109 & d & 2 & $11 / 2^{-}$ & 96 & & & $\mathrm{IT}=97.62 ; \beta^{-}=2.42$ & \\
\hline${ }^{127} \mathrm{I}$ & -88983 & 4 & & & & STABLE & & & $5 / 2^{+}$ & 96 & & & $\mathrm{IS}=100$. & \\
\hline${ }^{127} \mathrm{Xe}$ & -88321 & 4 & & & & 36.345 & $\mathrm{~d}$ & 0.003 & $1 / 2^{+}$ & 96 & 02Un02 & $\mathrm{T}$ & $\varepsilon=100$ & \\
\hline${ }^{127} \mathrm{Xe}^{m}$ & -88024 & 4 & 297.10 & 0.08 & & 69.2 & s & 0.9 & $9 / 2^{-}$ & 96 & & & $\mathrm{IT}=100$ & \\
\hline${ }^{127} \mathrm{Cs}$ & -86240 & 6 & & & & 6.25 & $\mathrm{~h}$ & 0.10 & $1 / 2^{+}$ & 96 & & & $\beta^{+}=100$ & \\
\hline${ }^{127} \mathrm{Cs}^{m}$ & -85788 & 6 & 452.23 & 0.21 & & 55 & $\mu \mathrm{s}$ & 3 & $(11 / 2)^{-}$ & 96 & & & $\mathrm{IT}=100$ & \\
\hline${ }^{127} \mathrm{Ba}$ & -82816 & 11 & & & & 12.7 & $\mathrm{~m}$ & 0.4 & $1 / 2^{+}$ & 96 & & & $\beta^{+}=100$ & \\
\hline${ }^{127} \mathrm{Ba}^{m}$ & -82736 & 11 & 80.33 & 0.12 & & 1.9 & $\mathrm{~s}$ & 0.2 & $7 / 2^{-}$ & 96 & & & $\mathrm{IT}=100$ & \\
\hline${ }^{127} \mathrm{La}$ & -77896 & 26 & & & & 5.1 & $\mathrm{~m}$ & 0.1 & $\left(11 / 2^{-}\right)$ & 96 & & & $\beta^{+}=100$ & \\
\hline${ }^{127} \mathrm{La}^{m}$ & -77881 & 26 & 14.8 & 1.2 & & 3.7 & $\mathrm{~m}$ & 0.4 & $\left(3 / 2^{+}\right)$ & 96 & & & $\beta^{+} \approx 100 ;$ IT ? & \\
\hline${ }^{127} \mathrm{Ce}$ & -71980 & 60 & & & $*$ & 29 & $\mathrm{~s}$ & 2 & $5 / 2^{+} \#$ & 98 & $96 \mathrm{Ge} 07$ & $\mathrm{~T}$ & $\beta^{+}=100$ & \\
\hline${ }^{127} \mathrm{Ce}^{m}$ & $-71980 \#$ & $120 \#$ & $0 \#$ & $100 \#$ & $*$ & 34 & $\mathrm{~s}$ & 2 & $\left(1 / 2^{+}\right)$ & & $96 \mathrm{Ge} 07$ & TJD & $\beta^{+}=100$ & \\
\hline${ }^{127} \mathrm{Pr}$ & $-64430 \#$ & $200 \#$ & & & & 4.2 & s & 0.3 & $3 / 2^{+} \#$ & 98 & & & $\beta^{+}=100$ & \\
\hline${ }^{127} \operatorname{Pr}^{m}$ & -63830\# & $280 \#$ & $600 \#$ & $200 \#$ & & $50 \#$ & $\mathrm{~ms}$ & & $11 / 2^{-}$ & & $98 \mathrm{Mo} 30$ & J & $\beta^{+}$?; IT ? & \\
\hline${ }^{127} \mathrm{Nd}$ & $-55420 \#$ & $400 \#$ & & & & 1.8 & $\mathrm{~s}$ & 0.4 & $5 / 2^{+} \#$ & 96 & & & $\beta^{+}=100 ; \beta^{+} \mathrm{p}=?$ & \\
\hline${ }^{127} \mathrm{Pm}$ & $-45060 \#$ & $600 \#$ & & & & $1 \#$ & s & & $5 / 2^{+} \#$ & & & & $\beta^{+}$?; p ? & \\
\hline${ }^{127} \mathrm{Ag}$ & $\mathrm{T}$ : superse & edes $95 \mathrm{~F}$ & $12=109$ & 5) from sa & ame gros & & & & & & & & & 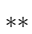 \\
\hline
\end{tabular}

\begin{tabular}{|c|c|c|c|c|c|c|c|c|c|c|c|c|c|c|}
\hline${ }^{128} \mathrm{Ag}$ & $-54800 \#$ & $300 \#$ & & & & 58 & $\mathrm{~ms}$ & 5 & & 01 & & & $\beta^{-}=100 ; \beta^{-} \mathrm{n}=?$ & \\
\hline${ }^{128} \mathrm{Cd}$ & -67290 & 290 & & & & 280 & $\mathrm{~ms}$ & 40 & $0^{+}$ & 01 & & & $\beta^{-}=100$ & \\
\hline${ }^{128} \mathrm{In}$ & -74360 & 50 & & & & 840 & $\mathrm{~ms}$ & 60 & $(3)^{+}$ & 01 & 93Ru01 & $\mathrm{D}$ & $\beta^{-}=100 ; \beta^{-} \mathrm{n}=0.0383$ & \\
\hline${ }^{128} \operatorname{In}^{m}$ & -74110 & 50 & 247.87 & 0.10 & & 10 & $\mathrm{~ms}$ & 7 & $(1)^{-}$ & 01 & & & $\mathrm{IT}=100$ & * \\
\hline${ }^{128} \operatorname{In}^{n}$ & -74040 & 50 & 320 & 60 & $\mathrm{BD}$ & 720 & $\mathrm{~ms}$ & 100 & $\left(8^{-}\right)$ & 01 & & & $\beta^{-}=100$ & \\
\hline${ }^{128} \mathrm{Sn}$ & -83335 & 27 & & & & 59.07 & $\mathrm{~m}$ & 0.14 & $0^{+}$ & 01 & & & $\beta^{-}=100$ & \\
\hline${ }^{128} \mathrm{Sn}^{m}$ & -81244 & 27 & 2091.50 & 0.11 & & 6.5 & $\mathrm{~s}$ & 0.5 & $\left(7^{-}\right)$ & 01 & & & $\mathrm{IT}=100$ & \\
\hline${ }^{128} \mathrm{Sb}$ & -84609 & 25 & & & * & 9.01 & $\mathrm{~h}$ & 0.04 & $8^{-}$ & 01 & & & $\beta^{-}=100$ & \\
\hline${ }^{128} \mathrm{Sb}^{m}$ & -84599 & 24 & 10 & 7 & $*$ & 10.4 & $\mathrm{~m}$ & 0.2 & $5^{+}$ & 01 & & & $\beta^{-}=96.410 ; \mathrm{IT}=3.610$ & * \\
\hline${ }^{128} \mathrm{Te}$ & -88992.1 & 1.7 & & & & 2.2 & Yy & 0.3 & $0^{+}$ & 01 & $96 \mathrm{Ta} 04$ & $\mathrm{~T}$ & $\mathrm{IS}=31.748 ; 2 \beta^{-}=100$ & * \\
\hline${ }^{128} \mathrm{Te}^{m}$ & -86201.4 & 1.7 & 2790.7 & 0.4 & & 370 & $\mathrm{~ns}$ & 30 & $10^{+}$ & 01 & & & $\mathrm{IT}=100$ & \\
\hline${ }^{128} \mathrm{I}$ & -87738 & 4 & & & & 24.99 & $\mathrm{~m}$ & 0.02 & $1^{+}$ & 01 & & & $\beta^{-}=93.18 ; \beta^{+}=6.98$ & \\
\hline${ }^{128} \mathrm{I}^{m}$ & -87600 & 4 & 137.850 & 0.004 & & 845 & ns & 20 & $4^{-}$ & 01 & & & $\mathrm{IT}=100$ & \\
\hline${ }^{128} \mathrm{I}^{n}$ & -87571 & 4 & 167.367 & 0.005 & & 175 & ns & 15 & $(6)^{-}$ & 01 & & & $\mathrm{IT}=100$ & \\
\hline${ }^{128} \mathrm{Xe}$ & -89860.0 & 1.4 & & & & STABLE & & & $0^{+}$ & 01 & & & $\mathrm{IS}=1.923$ & \\
\hline${ }^{128} \mathrm{Xe}^{m}$ & -87072.7 & 1.5 & 2787.3 & 0.4 & & 83 & ns & 2 & $8^{-}$ & 01 & & & $\mathrm{IT}=100$ & \\
\hline${ }^{128} \mathrm{Cs}$ & -85931 & 5 & & & & 3.640 & $\mathrm{~m}$ & 0.014 & $1^{+}$ & 01 & 93Al03 & $\mathrm{T}$ & $\beta^{+}=100$ & * \\
\hline${ }^{128} \mathrm{Ba}$ & -85402 & 10 & & & & 2.43 & $\mathrm{~d}$ & 0.05 & $0^{+}$ & 01 & & & $\varepsilon=100$ & \\
\hline${ }^{128} \mathrm{La}$ & -78630 & 50 & & & * & 5.18 & $\mathrm{~m}$ & 0.14 & $\left(5^{+}\right)$ & 01 & & & $\beta^{+}=100$ & \\
\hline${ }^{128} \mathrm{La}^{m}$ & $-78530 \#$ & $110 \#$ & $100 \#$ & $100 \#$ & * & $<1.4$ & $\mathrm{~m}$ & & $\left(1^{+}, 2^{-}\right)$ & 01 & & & $\beta^{+}=100$ & \\
\hline${ }^{128} \mathrm{Ce}$ & -75534 & 28 & & & & 3.93 & $\mathrm{~m}$ & 0.02 & $0^{+}$ & 01 & & & $\beta^{+}=100$ & \\
\hline${ }^{128} \mathrm{Pr}$ & -66331 & 30 & & & & 2.84 & $\mathrm{~s}$ & 0.09 & $\left(3^{+}\right)$ & 01 & 99Xi03 & $\mathrm{J}$ & $\beta^{+}=100 ; \beta^{+} \mathrm{p}=$ ? & * \\
\hline${ }^{128} \mathrm{Nd}$ & $-60180 \#$ & $200 \#$ & & & & $5 \#$ & $\mathrm{~s}$ & & $0^{+}$ & 01 & & & $\beta^{+} ? ; \beta^{+} \mathrm{p} ?$ & * \\
\hline${ }^{128} \mathrm{Pm}$ & $-48050 \#$ & $400 \#$ & & & & 1.0 & $\mathrm{~s}$ & 0.3 & $6^{+} \#$ & 01 & 93Li40 & $\mathrm{D}$ & $\beta^{+} \approx 100 ; \beta^{+} \mathrm{p} ? ; \mathrm{p}=0$ & * \\
\hline${ }^{128} \mathrm{Sm}$ & $-39050 \#$ & $500 \#$ & & & & $500 \#$ & $\mathrm{~ms}$ & & $0^{+}$ & & & & $\beta^{+} ? ; \mathrm{p} ?$ & \\
\hline$*^{128} \operatorname{In}^{m}$ & $\mathrm{~T}: 10 \mu \mathrm{s}$ & $<$ half-li & ife $<20 \mathrm{~ms}$ & , cf. ENSI & & & & & & & & & & k* \\
\hline$*^{128} \mathrm{Sb}^{m}$ & $\mathrm{E}$ : less tha & an $20 \mathrm{ke}$ & $\mathrm{V}$ above $\mathrm{gr}$ & ound stat & , cf. EN & & & & & & & & & $* *$ \\
\hline${ }^{128} \mathrm{Te}$ & $\mathrm{T}:$ see also & o $92 \mathrm{Be} 3$ & $30=7.7(0.4)$ & not used & for cons & stency v & n & ${ }^{0} \mathrm{Te}$ (se & below) & & & & & $* *$ \\
\hline${ }^{128} \mathrm{Cs}$ & $\mathrm{T}:$ average & e $93 \mathrm{Al} 0$ & $3=3.66(0.0$ & 2) $76 \mathrm{He} 0$ & $4=3.62(0$ & & & & & & & & & * \\
\hline${ }^{2}{ }^{128} \mathrm{Pr}$ & $\mathrm{D}:$ from 8 & $35 \mathrm{Wi} 07$ & & & & & & & & & & & & $k x$ \\
\hline$*^{128} \mathrm{Nd}$ & $\mathrm{T}: 83 \mathrm{Ni05}$ & 5 gave 4 & 4(2) s. Pr & d, by 8. & $\mathrm{Ni07,t}$ & e due $t$ & & r, not to & ${ }^{28} \mathrm{Nd}$ & & & & & " \\
\hline$*^{128} \mathrm{Pm}$ & $D: p=0$ fro & om $93 \mathrm{Li}$ & & $\mathrm{J}$ : as calc & lated by & $02 X u 11$ & & & & & & & & 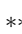 \\
\hline
\end{tabular}




\begin{tabular}{|c|c|c|c|c|c|c|c|c|c|c|c|c|c|c|}
\hline Nuclide & \multicolumn{2}{|c|}{$\begin{array}{l}\text { Mass excess } \\
(\mathrm{keV})\end{array}$} & \multicolumn{3}{|c|}{$\begin{array}{l}\text { Excitation } \\
\text { energy }(\mathrm{keV})\end{array}$} & \multicolumn{3}{|c|}{ Half-life } & $J^{\pi}$ & Ens & \multicolumn{2}{|c|}{ Reference } & \multicolumn{2}{|l|}{$\begin{array}{l}\text { Decay modes and } \\
\text { intensities }(\%)\end{array}$} \\
\hline${ }^{129} \mathrm{Ag}$ & $-52450 \#$ & $400 \#$ & & & $*$ & 44 & $\mathrm{~ms}$ & 7 & $7 / 2^{+} \#$ & 03 & & & $\beta^{-}=100 ; \beta^{-} \mathrm{n}=?$ & \\
\hline${ }^{129} \mathrm{Ag}^{m}$ & $-52450 \#$ & $450 \#$ & $0 \#$ & $200 \#$ & $\mathrm{EU} *$ & 160 & $\mathrm{~ms}$ & & $1 / 2^{-} \#$ & 03 & & & $\beta^{-} ? ; \beta^{-} \mathrm{n} ?$ & * \\
\hline${ }^{129} \mathrm{Cd}$ & $-63200 \#$ & $300 \#$ & & & * & 242 & $\mathrm{~ms}$ & 8 & $3 / 2^{+} \#$ & 96 & 03Pf.A & TD & $\beta^{-}=100 ; \beta^{-} \mathrm{n}=?$ & \\
\hline${ }^{129} \mathrm{Cd}^{m}$ & $-63200 \#$ & $360 \#$ & $0 \#$ & $200 \#$ & * & 104 & $\mathrm{~ms}$ & 6 & $11 / 2^{-} \#$ & & 03Pf.A & TD & $\beta^{-}=100 ; \beta^{-} \mathrm{n}=$ ? & \\
\hline${ }^{129} \mathrm{In}$ & -72940 & 40 & & & & 611 & $\mathrm{~ms}$ & 4 & $9 / 2^{+} \#$ & 96 & $93 \mathrm{Ru} 01$ & $\mathrm{~T}$ & $\beta^{-}=100 ; \beta^{-} \mathrm{n}=0.255$ & $*$ \\
\hline${ }^{129} \mathrm{In}^{m}$ & -72560 & 70 & 380 & 70 & $\mathrm{BD}$ & 1.23 & $\mathrm{~s}$ & 0.03 & $1 / 2^{-} \#$ & 96 & & & $\beta^{-} \approx 100 ;$ IT $<0.3 ; \ldots$ & $*$ \\
\hline${ }^{129} \mathrm{In}^{n}$ & -71250 & 40 & 1688.0 & 0.5 & & 8.5 & $\mu \mathrm{s}$ & 0.5 & $17 / 2^{-}$ & & 03Ge04 & ETJ & $\mathrm{IT}=100$ & \\
\hline${ }^{129} \mathrm{Sn}$ & -80594 & 29 & & & & 2.23 & $\mathrm{~m}$ & 0.04 & $3 / 2^{+} \#$ & 96 & & & $\beta^{-}=100$ & \\
\hline${ }^{129} \mathrm{Sn}^{m}$ & -80559 & 29 & 35.2 & 0.3 & & 6.9 & $\mathrm{~m}$ & 0.1 & $11 / 2^{-} \#$ & 96 & & & $\beta^{-} \approx 100 ; \mathrm{IT} \approx 0.002$ & \\
\hline${ }^{129} \mathrm{Sb}$ & -84628 & 21 & & & & 4.40 & $\mathrm{~h}$ & 0.01 & $7 / 2^{+}$ & 96 & & & $\beta^{-}=100$ & \\
\hline${ }^{129} \mathrm{Sb}^{m}$ & -82777 & 21 & 1851.05 & 0.10 & & 17.7 & $\mathrm{~m}$ & 0.1 & $\left(19 / 2^{-}\right)$ & 96 & & & $\beta^{-}=85 ; \mathrm{IT}=15$ & \\
\hline${ }^{129} \mathrm{Sb}^{n}$ & -82767 & 21 & 1860.90 & 0.10 & & $>2$ & $\mu \mathrm{s}$ & & $\left(15 / 2^{-}\right)$ & 96 & & & $\mathrm{IT}=100$ & \\
\hline${ }^{129} \mathrm{Sb}^{p}$ & -82489 & 21 & 2138.9 & 0.5 & & 1.1 & $\mu \mathrm{s}$ & 0.1 & $\left(23 / 2^{+}\right)$ & & 03Ge04 & ETJ & $\mathrm{IT}=100$ & \\
\hline${ }^{129} \mathrm{Te}$ & -87003.2 & 1.8 & & & & 69.6 & $\mathrm{~m}$ & 0.3 & $3 / 2^{+}$ & 96 & & & $\beta^{-}=100$ & \\
\hline${ }^{129} \mathrm{Te}^{m}$ & -86897.7 & 1.8 & 105.50 & 0.05 & & 33.6 & $\mathrm{~d}$ & 0.1 & $11 / 2^{-}$ & 96 & & & $\mathrm{IT}=6317 ; \beta^{-}=3717$ & \\
\hline${ }^{129} \mathrm{I}$ & -88503 & 3 & & & & 15.7 & My & 0.4 & $7 / 2^{+}$ & 96 & & & $\beta^{-}=100$ & \\
\hline${ }^{129} \mathrm{Xe}$ & -88697.4 & 0.7 & & & & STABLE & & & $1 / 2^{+}$ & 96 & & & $\mathrm{IS}=26.4424$ & \\
\hline${ }^{129} \mathrm{Xe}^{m}$ & -88461.3 & 0.7 & 236.14 & 0.05 & & 8.88 & $\mathrm{~d}$ & 0.02 & $11 / 2^{-}$ & 96 & & & $\mathrm{IT}=100$ & \\
\hline${ }^{129} \mathrm{Cs}$ & -87500 & 5 & & & & 32.06 & $\mathrm{~h}$ & 0.06 & $1 / 2^{+}$ & 96 & & & $\beta^{+}=100$ & \\
\hline${ }^{129} \mathrm{Ba}$ & -85065 & 11 & & & & 2.23 & $\mathrm{~h}$ & 0.11 & $1 / 2^{+}$ & 96 & & & $\beta^{+}=100$ & \\
\hline${ }^{129} \mathrm{Ba}^{m}$ & -85057 & 11 & 8.42 & 0.06 & & 2.16 & $\mathrm{~h}$ & 0.02 & $7 / 2^{+} \#$ & 96 & & & $\beta^{+} \approx 100 ; \mathrm{IT}=?$ & \\
\hline${ }^{129} \mathrm{La}$ & -81326 & 21 & & & & 11.6 & $\mathrm{~m}$ & 0.2 & $3 / 2^{+}$ & 96 & & & $\beta^{+}=100$ & \\
\hline${ }^{129} \mathrm{La}^{m}$ & -81154 & 21 & 172.1 & 0.4 & & 560 & $\mathrm{~ms}$ & 50 & $11 / 2^{-}$ & 96 & & & $\mathrm{IT}=100$ & \\
\hline${ }^{129} \mathrm{Ce}$ & -76287 & 28 & & & & 3.5 & $\mathrm{~m}$ & 0.3 & $\left(5 / 2^{+}\right)$ & 97 & $93 \mathrm{Al} 03$ & $\mathrm{~T}$ & $\beta^{+}=100$ & $*$ \\
\hline${ }^{129} \mathrm{Ce}^{m}$ & -76179 & 28 & 107.6 & 0.1 & & 62 & ns & 5 & $\left(7 / 2^{-}\right)$ & 96 & & & $\mathrm{IT}=100$ & \\
\hline${ }^{129} \mathrm{Pr}$ & -69774 & 30 & & & $\&$ & 30 & $\mathrm{~s}$ & 4 & $\left(3 / 2^{+}\right)$ & 96 & $96 \mathrm{Gi0} 8$ & J & $\beta^{+}=100$ & \\
\hline${ }^{129} \operatorname{Pr}^{m}$ & -69390 & 30 & 382.7 & 0.5 & \& & $1 \#$ & $\mathrm{~ms}$ & & $\left(11 / 2^{-}\right)$ & & $97 \mathrm{Gi07}$ & EJD & $\mathrm{IT}=100$ & \\
\hline${ }^{129} \mathrm{Nd}$ & $-62240 \#$ & $200 \#$ & & & & 4.9 & $\mathrm{~s}$ & 0.2 & $5 / 2^{+} \#$ & 96 & & & $\beta^{+}=100 ; \beta^{+} \mathrm{p}=?$ & \\
\hline${ }^{129} \mathrm{Pm}$ & $-52950 \#$ & $400 \#$ & & & & $3 \#$ & s ( & $(>200 \mathrm{~ns})$ & $5 / 2^{+} \#$ & & 00So11 & I & $\beta^{+}$? & \\
\hline${ }^{129} \mathrm{Sm}$ & $-42250 \#$ & $500 \#$ & & & & 550 & $\mathrm{~ms}$ & 100 & $5 / 2^{+} \#$ & & $99 \mathrm{Xu} 05$ & TD & $\beta^{+}=100$ & \\
\hline${ }^{129} \mathrm{Ag}$ & I : the eval & luators & are not c & nvinced & the identi & ication & rgume & & & & & & & $* *$ \\
\hline${ }^{129} \mathrm{In}$ & $\mathrm{T}$ : averag & e $93 R u$ & $01=611$ & $86 \mathrm{Wa} 17=$ & $10(10)$ & & & & & & & & & $* *$ \\
\hline${ }^{* 129} \operatorname{In}^{m}$ & $\mathrm{D}: \ldots ; \beta$ & $\mathrm{n}=2.5$ & & & & & & & & & & & & $* *$ \\
\hline${ }^{129} \mathrm{Ce}$ & $\mathrm{J}:$ from 96 & $6 \mathrm{Gi} 08$ & $\left(5 / 2^{+}\right.$in $\mathrm{E}$ & SDF was & om theor: & & & & & & & & & $* *$ \\
\hline
\end{tabular}

\begin{tabular}{|c|c|c|c|c|c|c|c|c|c|c|c|c|c|c|}
\hline${ }^{130} \mathrm{Ag}$ & $-46160 \#$ & $330 \#$ & & & & 50 & $\mathrm{~ms}$ & & $0^{+}$ & 01 & & & $\beta^{-}=100 ; \beta^{-} \mathrm{n} ?$ & \\
\hline${ }^{130} \mathrm{Cd}$ & -61570 & 280 & & & & 162 & $\mathrm{~ms}$ & 7 & $0^{+}$ & 01 & 01Нa39 & TD & $\beta^{-}=100 ; \beta^{-} \mathrm{n}=3.510$ & \\
\hline${ }^{130} \mathrm{In}$ & -69890 & 40 & & & * & 290 & $\mathrm{~ms}$ & 20 & $\left(1^{-}\right)$ & 01 & & & $\beta^{-}=100 ; \beta^{-} \mathrm{n}=0.9313$ & \\
\hline${ }^{130} \operatorname{In}^{m}$ & -69840 & 40 & 50 & 50 & $\mathrm{BD} *$ & 538 & $\mathrm{~ms}$ & 5 & $10^{-} \#$ & 01 & 93Ru01 & $\mathrm{T}$ & $\beta^{-}=100 ; \beta^{-} \mathrm{n}=1.6515$ & $*$ \\
\hline${ }^{130} \operatorname{In}^{n}$ & -69490 & 50 & 400 & 60 & $\mathrm{BD}$ & 540 & $\mathrm{~ms}$ & 10 & $\left(5^{+}\right)$ & 01 & & & $\beta^{-}=100 ; \beta^{-} \mathrm{n}=1.6515$ & \\
\hline${ }^{130} \mathrm{Sn}$ & -80139 & 11 & & & & 3.72 & $\mathrm{~m}$ & 0.07 & $0^{+}$ & 01 & & & $\beta^{-}=100$ & \\
\hline${ }^{130} \mathrm{Sn}^{m}$ & -78192 & 11 & 1946.88 & 0.10 & & 1.7 & $\mathrm{~m}$ & 0.1 & $7^{-} \#$ & 01 & & & $\beta^{-}=100$ & \\
\hline${ }^{130} \mathrm{Sb}$ & -82292 & 17 & & & & 39.5 & $\mathrm{~m}$ & 0.8 & $8^{-} \#$ & 01 & & & $\beta^{-}=100$ & \\
\hline${ }^{130} \mathrm{Sb}^{m}$ & -82287 & 17 & 4.80 & 0.20 & & 6.3 & $\mathrm{~m}$ & 0.2 & $(4,5)^{+}$ & 01 & & & $\beta^{-}=100$ & \\
\hline${ }^{130} \mathrm{Te}$ & -87351.4 & 1.9 & & & & 790 & Ey & 100 & $0^{+}$ & 01 & $96 \mathrm{Ta} 04$ & TD & $\mathrm{IS}=34.0862 ; 2 \beta^{-}=100$ & * \\
\hline${ }^{130} \mathrm{Te}^{m}$ & -85205.0 & 1.9 & 2146.41 & 0.04 & & 115 & ns & 8 & $(7)^{-}$ & 01 & & & $\mathrm{IT}=100$ & \\
\hline${ }^{130} \mathrm{Te}^{n}$ & -84690 & 7 & 2661 & 7 & & 1.90 & $\mu \mathrm{s}$ & 0.08 & $\left(10^{+}\right)$ & 01 & & & $\mathrm{IT}=100$ & * \\
\hline${ }^{130} \mathrm{Te}^{p}$ & -82976.0 & 2.6 & 4375.4 & 1.8 & & 261 & ns & 33 & & 01 & & & $\mathrm{IT}=100$ & \\
\hline${ }^{130} \mathrm{I}$ & -86932 & 3 & & & & 12.36 & $\mathrm{~h}$ & 0.01 & $5^{+}$ & 01 & & & $\beta^{-}=100$ & \\
\hline${ }^{130} \mathrm{I}^{m}$ & -86892 & 3 & 39.9525 & 0.0013 & & 8.84 & $\mathrm{~m}$ & 0.06 & $2^{+}$ & 01 & & & $\mathrm{IT}=842 ; \beta^{-}=162$ & \\
\hline${ }^{130} \mathrm{Xe}$ & -89881.7 & 0.7 & & & & Stable & & & $0^{+}$ & 01 & & & $\mathrm{IS}=4.082$ & \\
\hline${ }^{130} \mathrm{Cs}$ & -86900 & 8 & & & & 29.21 & $\mathrm{~m}$ & 0.04 & $1^{+}$ & 01 & & & $\beta^{+}=98.4 ; \beta^{-}=1.6$ & \\
\hline${ }^{130} \mathrm{Cs}^{m}$ & -86737 & 8 & 163.25 & 0.11 & & 3.46 & $\mathrm{~m}$ & 0.06 & $5^{-}$ & 01 & & & $\mathrm{IT} \approx 100 ; \beta^{+}=0.162$ & \\
\hline${ }^{130} \mathrm{Cs}^{x}$ & -86873 & 17 & 27 & 15 & & $R=.2 \quad .1$ & & & fsmix & & & & & \\
\hline${ }^{130} \mathrm{Ba}$ & -87261.6 & 2.8 & & & & STABLE & & $(>4.0 \mathrm{Zy})$ & $0^{+}$ & 01 & $96 \mathrm{Ba} 24$ & $\mathrm{~T}$ & $\mathrm{IS}=0.1061 ; 2 \beta^{+} ?$ & \\
\hline${ }^{130} \mathrm{Ba}^{m}$ & -84786.5 & 2.8 & 2475.12 & 0.18 & & 9.54 & $\mathrm{~ms}$ & 0.14 & $8^{-}$ & 01 & $02 \mathrm{Mo} 31$ & $\mathrm{~T}$ & $\mathrm{IT}=100$ & * \\
\hline
\end{tabular}




\begin{tabular}{|c|c|c|c|c|c|c|c|c|c|c|c|c|c|}
\hline Nuclide & \multicolumn{2}{|c|}{$\begin{array}{l}\text { Mass excess } \\
\quad(\mathrm{keV})\end{array}$} & \multicolumn{2}{|c|}{$\begin{array}{l}\text { Excitation } \\
\text { energy }(\mathrm{keV})\end{array}$} & \multicolumn{3}{|c|}{ Half-life } & \multirow[t]{2}{*}{$J^{\pi}$} & \multirow[t]{2}{*}{ Ens } & \multicolumn{2}{|c|}{ Reference } & \multicolumn{2}{|l|}{$\begin{array}{l}\text { Decay modes and } \\
\text { intensities }(\%)\end{array}$} \\
\hline \multicolumn{12}{|c|}{$A$-group continued } & & \\
\hline${ }^{130} \mathrm{La}$ & -81628 & 26 & & & 8.7 & $\mathrm{~m}$ & 0.1 & $3^{(+)}$ & 01 & & & $\beta^{+}=100$ & \\
\hline${ }^{130} \mathrm{Ce}$ & -79423 & 28 & & & 22.9 & $\mathrm{~m}$ & 0.5 & $0^{+}$ & 01 & & & $\beta^{+}=100$ & \\
\hline${ }^{130} \mathrm{Ce}^{m}$ & -76969 & 28 & 2453.6 & 0.3 & 100 & $\mathrm{~ns}$ & 8 & $\left(7^{-}\right)$ & 01 & & & $\mathrm{IT}=100$ & \\
\hline${ }^{130} \mathrm{Pr}$ & -71180 & 60 & & & 40.0 & $\mathrm{~s}$ & 0.4 & $(6,7)^{(+\#)}$ & 01 & $88 \mathrm{Ba} 42$ & $\mathrm{~J}$ & $\beta^{+}=100$ & \\
\hline${ }^{130} \mathrm{Pr}^{m}$ & $-71080 \#$ & $120 \#$ & $100 \#$ & $100 \#$ & $10 \#$ & s & & $2^{+} \#$ & 01 & $88 \mathrm{Ba} 42$ & $\mathrm{~J}$ & $\beta^{+}$? & $*$ \\
\hline${ }^{130} \mathrm{Nd}$ & -66596 & 28 & & & 21 & s & 3 & $0^{+}$ & 01 & $01 \mathrm{Gi} 17$ & $\mathrm{~T}$ & $\beta^{+}=100$ & $*$ \\
\hline${ }^{130} \mathrm{Pm}$ & $-55470 \#$ & $300 \#$ & & & 2.6 & s & 0.2 & $\left(5^{+}, 6^{+}, 4^{+}\right)$ & 01 & $99 \mathrm{Xi03}$ & $\mathrm{J}$ & $\beta^{+}=100 ; \beta^{+} \mathrm{p}=$ ? & \\
\hline${ }^{130} \mathrm{Sm}$ & $-47580 \#$ & $400 \#$ & & & $1 \#$ & s & & $0^{+}$ & 01 & & & $\beta^{+}$? & \\
\hline${ }^{130} \mathrm{Eu}$ & $-33940 \#$ & $500 \#$ & & & 1.1 & $\mathrm{~ms}$ & 0.5 & $2^{+} \#$ & & $02 \mathrm{Ma} 61$ & TD & $\mathrm{p}=? ; \beta^{+}=1 \#$ & \\
\hline$*^{130} \mathrm{In}^{m}$ & \multicolumn{12}{|c|}{$\mathrm{T}:$ average $93 \mathrm{Ru} 01=542(9) 85 \mathrm{Re} \cdot \mathrm{A}=532(6)$ and $86 \mathrm{Wa1}=550(10)$} & $* *$ \\
\hline$*^{130} \mathrm{In}^{m}$ & \multicolumn{12}{|c|}{$\mathrm{T}: \quad 76 \mathrm{Lu02}=580(10)$ at variance, not used } & $* *$ \\
\hline$*{ }^{130} \mathrm{Te}$ & \multicolumn{12}{|c|}{$\mathrm{T}$ : see also numerous (not used) results in $95 \mathrm{Tr} 07$} & $* *$ \\
\hline$*{ }^{130} \mathrm{Te}$ & \multicolumn{12}{|c|}{$\mathrm{T}$ : treated by ENSDF'01 as a lower limit (not accepted by NUBASE) } & $* *$ \\
\hline$*^{130} \mathrm{Te}^{n}$ & \multicolumn{12}{|c|}{$\mathrm{E}:$ less than $25 \mathrm{keV}$ above $2648.57(0.22)\left(8^{+}\right)$level, see ENSDF'01 } & $* *$ \\
\hline$*{ }^{130} \mathrm{Ba}^{m}$ & \multicolumn{12}{|c|}{$\mathrm{T}:$ others $66 \mathrm{Br} 14=8.8(0.2) 69 \mathrm{Wa} . \mathrm{A}=13.5(1.0)$ not used } & $* *$ \\
\hline$*{ }^{130} \operatorname{Pr}^{m}$ & \multicolumn{12}{|c|}{$\mathrm{J}: 88 \mathrm{Ba} 42:$ there is also a low-spin component in ${ }^{130} \mathrm{Pr}$ activity } & $* *$ \\
\hline$*^{130} \mathrm{Pr}^{m}$ & \multicolumn{12}{|c|}{$\mathrm{J}$ : see also the discussion in $01 \mathrm{Gi} 17$ on three isomeric states in ${ }^{130} \mathrm{Pr}$} & $* *$ \\
\hline$*{ }^{130} \mathrm{Nd}$ & \multicolumn{12}{|c|}{$\mathrm{T}:$ other conflicting data, not used: $00 \mathrm{Xu} 08=13(3) 77 \mathrm{Bo} 02=28(3)$} & $* *$ \\
\hline
\end{tabular}

\begin{tabular}{|c|c|c|c|c|c|c|c|c|c|c|c|c|c|c|}
\hline${ }^{131} \mathrm{Cd}$ & $-55270 \#$ & $300 \#$ & & & & 68 & $\mathrm{~ms}$ & 3 & $7 / 2^{-} \#$ & & 00Ha55 & $\mathrm{TD}$ & $\beta^{-}=100 ; \beta^{-} \mathrm{n}=3.510$ & \\
\hline${ }^{131}$ In & -68137 & 28 & & & & 280 & $\mathrm{~ms}$ & 30 & $\left(9 / 2^{+}\right)$ & 94 & 93Ru01 & $\mathrm{D}$ & $\beta^{-}=100 ; \beta^{-} \mathrm{n}=2.23$ & \\
\hline${ }^{131} \mathrm{In}^{m}$ & -67790 & 40 & 350 & 40 & $\mathrm{BD}$ & 350 & $\mathrm{~ms}$ & 50 & $\left(1 / 2^{-}\right)$ & 94 & & & $\beta^{-} \approx 100 ; \ldots$ & $*$ \\
\hline${ }^{131} \operatorname{In}^{n}$ & -64040 & 70 & 4100 & 70 & $\mathrm{BD}$ & 320 & $\mathrm{~ms}$ & 60 & $\left(19 . .23 / 2^{+}\right)$ & 94 & & & $\beta^{-}>99 ; \ldots$ & $*$ \\
\hline${ }^{131} \mathrm{Sn}$ & -77314 & 21 & & & & 56.0 & $\mathrm{~s}$ & 0.5 & $\left(3 / 2^{+}\right)$ & 94 & & & $\beta^{-}=100$ & \\
\hline${ }^{131} \mathrm{Sn}^{m}$ & $-77230 \#$ & $40 \#$ & $80 \#$ & $30 \#$ & & 58.4 & $\mathrm{~s}$ & 0.5 & $\left(11 / 2^{-}\right)$ & 94 & 01Si.A & $\mathrm{E}$ & $\beta^{-}=100 ; \mathrm{IT}<0.0004 \#$ & * \\
\hline${ }^{131} \mathrm{Sb}$ & -81988 & 21 & & & & 23.03 & $\mathrm{~m}$ & 0.04 & $\left(7 / 2^{+}\right)$ & 94 & & & $\beta^{-}=100$ & \\
\hline${ }^{131} \mathrm{Te}$ & -85209.5 & 1.9 & & & & 25.0 & $\mathrm{~m}$ & 0.1 & $3 / 2^{+}$ & 94 & & & $\beta^{-}=100$ & \\
\hline${ }^{131} \mathrm{Te}^{m}$ & -85027.3 & 1.9 & 182.250 & 0.020 & & 30 & $\mathrm{~h}$ & 2 & $11 / 2^{-}$ & 94 & & & $\beta^{-}=77.816 ; \mathrm{IT}=22.216$ & \\
\hline${ }^{131} \mathrm{I}$ & -87444.4 & 1.1 & & & & 8.02070 & $d$ & 0.00011 & $7 / 2^{+}$ & 94 & & & $\beta^{-}=100$ & \\
\hline${ }^{131} \mathrm{Xe}$ & -88415.2 & 1.0 & & & & StABLE & & & $3 / 2^{+}$ & 94 & & & $\mathrm{IS}=21.183$ & \\
\hline${ }^{131} \mathrm{Xe}^{m}$ & -88251.3 & 1.0 & 163.930 & 0.008 & & 11.84 & $\mathrm{~d}$ & 0.07 & $11 / 2^{-}$ & 94 & & & $\mathrm{IT}=100$ & \\
\hline${ }^{131} \mathrm{Cs}$ & -88060 & 5 & & & & 9.689 & $\mathrm{~d}$ & 0.016 & $5 / 2^{+}$ & 94 & & & $\varepsilon=100$ & \\
\hline${ }^{131} \mathrm{Ba}$ & -86683.8 & 2.8 & & & & 11.50 & $\mathrm{~d}$ & 0.06 & $1 / 2^{+}$ & 94 & & & $\beta^{+}=100$ & \\
\hline${ }^{131} \mathrm{Ba}^{m}$ & -86496.7 & 2.8 & 187.14 & 0.12 & & 14.6 & $\mathrm{~m}$ & 0.2 & $9 / 2^{-}$ & 94 & & & $\mathrm{IT}=100$ & \\
\hline${ }^{131} \mathrm{La}$ & -83769 & 28 & & & & 59 & $\mathrm{~m}$ & 2 & $3 / 2^{+}$ & 94 & & & $\beta^{+}=100$ & \\
\hline${ }^{131} \mathrm{La}^{m}$ & -83464 & 28 & 304.52 & 0.24 & & 170 & $\mu \mathrm{s}$ & 10 & $11 / 2^{-}$ & 94 & & & $\mathrm{IT}=100$ & \\
\hline${ }^{131} \mathrm{Ce}$ & -79720 & 30 & & & & 10.2 & $\mathrm{~m}$ & 0.3 & $\left(7 / 2^{+}\right)$ & 99 & & & $\beta^{+}=100$ & \\
\hline${ }^{131} \mathrm{Ce}^{m}$ & -79660 & 30 & 61.8 & 0.1 & & 5.0 & $\mathrm{~m}$ & 1.0 & $\left(1 / 2^{+}\right)$ & 99 & 96Gi08 & $\mathrm{E}$ & $\beta^{+}=100$ & \\
\hline${ }^{131} \mathrm{Ce}^{n}$ & -79560 & 30 & 162.00 & 0.09 & & 70 & ns & 5 & $\left(9 / 2^{-}\right)$ & & & & & \\
\hline${ }^{131} \mathrm{Pr}$ & -74280 & 50 & & & & 1.50 & $\mathrm{~m}$ & 0.03 & $\left(3 / 2^{+}\right)$ & 94 & 96Gi08 & $\mathrm{T}$ & $\beta^{+}=100$ & * \\
\hline${ }^{131} \mathrm{Pr}^{m}$ & -74130 & 50 & 152.4 & 0.2 & & 5.7 & $\mathrm{~s}$ & 0.2 & $\left(11 / 2^{-}\right)$ & 94 & $96 \mathrm{Ge} 12$ & ED & $\mathrm{IT}=96.412 ; \beta^{+}=3.612$ & \\
\hline${ }^{131} \mathrm{Nd}$ & -67769 & 28 & & & & 33 & $\mathrm{~s}$ & 3 & $(5 / 2)^{(+\#)}$ & 94 & $96 \mathrm{Ge} 12$ & $\mathrm{~T}$ & $\beta^{+}=100 ; \beta^{+} \mathrm{p}=?$ & \\
\hline${ }^{131} \mathrm{Nd}^{m}$ & -67412 & 28 & 357 & 3 & & 50 & $\mathrm{~ns}$ & & $\left(7 / 2^{-}\right)$ & 94 & $96 \mathrm{Ge} 12$ & $\mathrm{~J}$ & $\mathrm{IT}=100$ & \\
\hline${ }^{131} \mathrm{Pm}$ & $-59740 \#$ & $200 \#$ & & & & 6.3 & $\mathrm{~s}$ & 0.8 & $5 / 2^{+} \#$ & 94 & $99 \mathrm{Ga} 41$ & $\mathrm{~T}$ & $\beta^{+}=100 ; \beta^{+} \mathrm{p} ?$ & \\
\hline${ }^{131} \mathrm{Sm}$ & $-50200 \#$ & $300 \#$ & & & & 1.2 & $\mathrm{~s}$ & 0.2 & $5 / 2^{+} \#$ & 94 & & & $\beta^{+}=100 ; \beta^{+} \mathrm{p}=?$ & \\
\hline${ }^{131} \mathrm{Eu}$ & $-39350 \#$ & $400 \#$ & & & & 17.8 & $\mathrm{~ms}$ & 1.9 & $3 / 2^{+}$ & 02 & & & $\mathrm{p}=? ; \beta^{+}=12 \#$ & \\
\hline${ }^{131} \mathrm{In}^{m}$ & $\mathrm{D}: \ldots ; \beta^{-}$ & $-\mathrm{n} \leq 2$ & $4 ; \mathrm{IT} \leq 0$. & & & & & & & & & & & 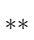 \\
\hline$*^{131} \operatorname{In}^{n}$ & $\mathrm{D}: \ldots ; \beta^{-}$ & $-\mathrm{n}=0.02$ & $285 ;$ IT $<1$ & & & & & & & & & & & k* \\
\hline${ }^{131} \mathrm{Sn}^{m}$ & E : ENSDF' & $' 94=24$ & $1.8(0.8) \mathrm{qu}$ & 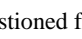 & & etic & at & n & erations & & & & & $*$ \\
\hline$*^{131} \mathrm{Pr}$ & $\mathrm{T}$ : average & e $96 \mathrm{Gi}$ & $8=1.57(0.0$ & $93 \mathrm{~A}$ & $3=1$. & 0.02 ) an & nd 83 & $3 \mathrm{Ga} \cdot \mathrm{A}=1$. & $.58(0.05)$ & & & & & $* *$ \\
\hline
\end{tabular}




\begin{tabular}{|c|c|c|c|c|c|c|c|c|c|c|c|c|}
\hline \multirow{2}{*}{$\begin{array}{l}\text { Nuclide } \\
{ }^{132} \mathrm{Cd}\end{array}$} & \multicolumn{2}{|c|}{$\begin{array}{l}\text { Mass excess } \\
\quad(\mathrm{keV})\end{array}$} & \multicolumn{3}{|c|}{$\begin{array}{c}\text { Excitation } \\
\text { energy(keV) }\end{array}$} & \multicolumn{3}{|c|}{ Half-life } & \multirow{2}{*}{$\frac{J^{\pi}}{0^{+}}$} & \multirow[t]{2}{*}{ Ens } & Reference & $\begin{array}{l}\text { Decay modes and } \\
\text { intensities }(\%)\end{array}$ \\
\hline & $-50720 \#$ & $500 \#$ & & & & 97 & $\mathrm{~ms}$ & 10 & & & 00Ha55 TD & $\beta^{-}=100 ; \beta^{-} \mathrm{n}=6015$ \\
\hline${ }^{132} \mathrm{In}$ & -62420 & 60 & & & & 206 & $\mathrm{~ms}$ & 4 & $\left(7^{-}\right)$ & 02 & & $\beta^{-}=100 ; \beta^{-} \mathrm{n}=6.211$ \\
\hline${ }^{132} \mathrm{Sn}$ & -76554 & 14 & & & & 39.7 & $\mathrm{~s}$ & 0.5 & $0^{+}$ & 92 & & $\beta^{-}=100$ \\
\hline${ }^{132} \mathrm{Sb}$ & -79674 & 14 & & & & 2.79 & $\mathrm{~m}$ & 0.05 & $\left(4^{+}\right)$ & 92 & & $\beta^{-}=100$ \\
\hline${ }^{132} \mathrm{Sb}^{m}$ & -79470 & 30 & 200 & 30 & & 4.15 & $\mathrm{~m}$ & 0.05 & $\left(8^{-}\right)$ & 92 & 89St06 E & $\beta^{-}=100$ \\
\hline${ }^{132} \mathrm{Te}$ & -85182 & 7 & & & & 3.204 & $\mathrm{~d}$ & 0.013 & $0^{+}$ & 92 & & $\beta^{-}=100$ \\
\hline${ }^{132} \mathrm{I}$ & -85700 & 6 & & & & 2.295 & $\mathrm{~h}$ & 0.013 & $4^{+}$ & 92 & & $\beta^{-}=100$ \\
\hline${ }^{132} \mathrm{I}^{m}$ & -85595 & 10 & 104 & 12 & $\mathrm{BD}$ & 1.387 & $\mathrm{~h}$ & 0.015 & $\left(8^{-}\right)$ & 92 & & $\mathrm{IT}=862 ; \beta^{-}=142$ \\
\hline${ }^{132} \mathrm{Xe}$ & -89280.5 & 1.0 & & & & STABLE & & & $0^{+}$ & 92 & & $\mathrm{IS}=26.896$ \\
\hline${ }^{132} \mathrm{Xe}^{m}$ & -86528.2 & 1.0 & 2752.27 & 0.17 & & 8.39 & $\mathrm{~ms}$ & 0.11 & $\left(10^{+}\right)$ & 92 & & $\mathrm{IT}=100$ \\
\hline${ }^{132} \mathrm{Cs}$ & -87155.9 & 1.9 & & & & 6.479 & $\mathrm{~d}$ & 0.007 & $2^{+}$ & 92 & & $\beta^{+}=98.139 ; \beta^{-}=1.879$ \\
\hline${ }^{132} \mathrm{Ba}$ & -88434.8 & 1.1 & & & & STABLE & & (>300 Ey) & $0^{+}$ & 94 & $96 \mathrm{Ba} 24 \mathrm{~T}$ & $\mathrm{IS}=0.1011 ; 2 \beta^{+} ?$ \\
\hline${ }^{132} \mathrm{La}$ & -83740 & 40 & & & & 4.8 & $\mathrm{~h}$ & 0.2 & $2^{-}$ & 94 & & $\beta^{+}=100$ \\
\hline${ }^{132} \mathrm{La}^{m}$ & -83550 & 40 & 188.18 & 0.11 & & 24.3 & $\mathrm{~m}$ & 0.5 & $6^{-}$ & 94 & & $\mathrm{IT}=76 ; \beta^{+}=24$ \\
\hline${ }^{132} \mathrm{Ce}$ & -82474 & 21 & & & & 3.51 & $\mathrm{~h}$ & 0.11 & $0^{+}$ & 99 & & $\beta^{+}=100$ \\
\hline${ }^{132} \mathrm{Ce}^{m}$ & -80133 & 21 & 2340.8 & 0.5 & & 9.4 & $\mathrm{~ms}$ & 0.3 & $\left(8^{-}\right)$ & 99 & 01Mo05 TJ & $\mathrm{IT}=100$ \\
\hline${ }^{132} \mathrm{Pr}$ & -75210 & 60 & & & * & 1.49 & $\mathrm{~m}$ & 0.11 & $\left(2^{+}\right)$ & 01 & $94 \mathrm{Bu} 18 \mathrm{TJ}$ & $\beta^{+}=100$ \\
\hline${ }^{132} \mathrm{Pr}^{m}$ & $-75210 \#$ & $120 \#$ & $0 \#$ & $100 \#$ & $*$ & $20 \#$ & $\mathrm{~s}$ & & $\left(5^{+}\right)$ & & $90 \mathrm{Ko} 25 \mathrm{~J}$ & $\beta^{+}$? \\
\hline${ }^{132} \mathrm{Nd}$ & -71426 & 24 & & & & 1.56 & $\mathrm{~m}$ & 0.10 & $0^{+}$ & 97 & $95 \mathrm{Bu} 11 \mathrm{~T}$ & $\beta^{+}=100$ \\
\hline${ }^{132} \mathrm{Pm}$ & $-61710 \#$ & $200 \#$ & & & & 6.3 & $\mathrm{~s}$ & 0.7 & $\left(3^{+}\right)$ & 92 & & $\beta^{+}=100 ; \beta^{+} \mathrm{p} \approx 5 \mathrm{e}-5$ \\
\hline${ }^{132} \mathrm{Sm}$ & $-55250 \#$ & $300 \#$ & & & & 4.0 & $\mathrm{~s}$ & 0.3 & $0^{+}$ & 92 & & $\beta^{+}=100 ; \beta^{+} \mathrm{p}$ ? \\
\hline${ }^{132} \mathrm{Eu}$ & $-42500 \#$ & $400 \#$ & & & & $100 \#$ & $\mathrm{~ms}$ & & & & $93 \mathrm{Li} 40 \quad \mathrm{D}$ & $\beta^{+} ? ; \mathrm{p}=0$ \\
\hline${ }^{132} \mathrm{Pr}$ & $\mathrm{T}:$ average & $94 \mathrm{Bu} 18$ & $47(0.12$ & $74 \mathrm{Ar} 2$ & $6(0.3$ & & & & & & & \\
\hline${ }^{132} \mathrm{Nd}$ & $\mathrm{T}:$ average & $95 \mathrm{Bu} 11$ & $47(0.12)$ & 77Bo02 & $1.75(0$ & & & & & & & \\
\hline
\end{tabular}

\begin{tabular}{|c|c|c|c|c|c|c|c|c|c|c|c|c|c|}
\hline${ }^{133} \mathrm{In}$ & $-57930 \#$ & $300 \#$ & & & & 165 & $\mathrm{~ms}$ & 3 & $\left(9 / 2^{+}\right) 02$ & $96 \mathrm{Ho} 16$ & & $\beta^{-}=100 ; \beta^{-} \mathrm{n}=8510$ & * \\
\hline${ }^{133} \mathrm{In}^{m}$ & $-57600 \#$ & $300 \#$ & $330 \#$ & $40 \#$ & & $180 \#$ & $\mathrm{~ms}$ & & $\left(1 / 2^{-}\right)$ & 96Ho16 & $\mathrm{J}$ & IT ? & \\
\hline${ }^{133} \mathrm{Sn}$ & -70950 & 40 & & & & 1.45 & $\mathrm{~s}$ & 0.03 & $7 / 2^{-} \# 98$ & $93 \mathrm{Ru} 01$ & $\mathrm{D}$ & $\beta^{-}=100 ; \beta^{-} \mathrm{n}=0.029424$ & \\
\hline${ }^{133} \mathrm{Sb}$ & -78943 & 25 & & & & 2.5 & $\mathrm{~m}$ & 0.1 & $\left(7 / 2^{+}\right) 95$ & & & $\beta^{-}=100$ & \\
\hline${ }^{133} \mathrm{Te}$ & -82945 & 24 & & & & 12.5 & $\mathrm{~m}$ & 0.3 & $\left(3 / 2^{+}\right) 95$ & & & $\beta^{-}=100$ & \\
\hline${ }^{133} \mathrm{Te}^{m}$ & -82611 & 24 & 334.26 & 0.04 & & 55.4 & $\mathrm{~m}$ & 0.4 & $\left(11 / 2^{-}\right) 95$ & & & $\beta^{-}=82.530 ; \mathrm{IT}=17.530$ & \\
\hline${ }^{133} \mathrm{I}$ & -85887 & 5 & & & & 20.8 & $\mathrm{~h}$ & 0.1 & $7 / 2^{+} \quad 95$ & & & $\beta^{-}=100$ & \\
\hline${ }^{133} \mathrm{I}^{m}$ & -84253 & 5 & 1634.174 & 0.017 & & 9 & $\mathrm{~s}$ & 2 & $\left(19 / 2^{-}\right) 95$ & & & $\mathrm{IT}=100$ & \\
\hline${ }^{133} \mathrm{Xe}$ & -87643.6 & 2.4 & & & & 5.2475 & d & 0.0005 & $3 / 2^{+} \quad 95$ & 02Un02 & $\mathrm{T}$ & $\beta^{-}=100$ & \\
\hline${ }^{133} \mathrm{Xe}^{m}$ & -87410.4 & 2.4 & 233.221 & 0.018 & & 2.19 & d & 0.01 & $11 / 2^{-} 95$ & & & $\mathrm{IT}=100$ & \\
\hline${ }^{133} \mathrm{Cs}$ & -88070.958 & 0.022 & & & & STABLE & & & $7 / 2^{+} \quad 95$ & & & $\mathrm{IS}=100$. & \\
\hline${ }^{133} \mathrm{Ba}$ & -87553.5 & 1.0 & & & & 10.51 & $\mathrm{y}$ & 0.05 & $1 / 2^{+} \quad 95$ & & & $\varepsilon=100$ & \\
\hline${ }^{133} \mathrm{Ba}^{m}$ & -87265.3 & 1.0 & 288.247 & 0.009 & & 38.9 & $\mathrm{~h}$ & 0.1 & $11 / 2^{-} 95$ & & & $\mathrm{IT} \approx 100 ; \varepsilon=0.009611$ & \\
\hline${ }^{133} \mathrm{La}$ & -85494 & 28 & & & & 3.912 & $\mathrm{~h}$ & 0.008 & $5 / 2^{+} \quad 95$ & & & $\beta^{+}=100$ & \\
\hline${ }^{133} \mathrm{La}^{m}$ & -84958 & 28 & 535.60 & 0.02 & & 62 & $\mathrm{~ns}$ & 3 & $11 / 2^{-}$ & & & & \\
\hline${ }^{133} \mathrm{Ce}$ & -82423 & 16 & & & & 97 & $\mathrm{~m}$ & 4 & $1 / 2^{+} \quad 97$ & & & $\beta^{+}=100$ & \\
\hline${ }^{133} \mathrm{Ce}^{m}$ & -82386 & 16 & 37.1 & 0.8 & & 4.9 & $\mathrm{~h}$ & 0.4 & $9 / 2^{-} \quad 97$ & & & $\beta^{+}=100$ & \\
\hline${ }^{133} \mathrm{Pr}$ & -77938 & 12 & & & & 6.5 & $\mathrm{~m}$ & 0.3 & $\left(3 / 2^{+}\right) 97$ & & & $\beta^{+}=100$ & \\
\hline${ }^{133} \operatorname{Pr}^{m}$ & -77746 & 12 & 192.05 & 0.14 & & 1.1 & $\mu \mathrm{s}$ & 0.2 & $\left(11 / 2^{-}\right) 97$ & 01Xu04 & $\mathrm{T}$ & $\mathrm{IT}=100$ & \\
\hline${ }^{133} \mathrm{Nd}$ & -72330 & 50 & & & & 70 & $\mathrm{~s}$ & 10 & $\left(7 / 2^{+}\right) 97$ & & & $\beta^{+}=100$ & \\
\hline${ }^{133} \mathrm{Nd}^{m}$ & -72200 & 50 & 127.97 & 0.11 & & 70 & $\mathrm{~s}$ & & $(1 / 2)^{+} 97$ & $95 \mathrm{Br} 24$ & $\mathrm{D}$ & $\beta^{+} \approx 100 ; \mathrm{IT}=$ ? & \\
\hline${ }^{133} \mathrm{Nd}^{n}$ & -72150 & 50 & 176.10 & 0.10 & & 300 & $\mathrm{~ns}$ & & $\left(9 / 2^{-}\right) 97$ & & & $\mathrm{IT}=100$ & \\
\hline${ }^{133} \mathrm{Pm}$ & -65410 & 50 & & & $\&$ & 15 & $\mathrm{~s}$ & 3 & $\left(3 / 2^{+}\right) 95$ & $96 \mathrm{Ga} 17$ & $\mathrm{~J}$ & $\beta^{+}=100$ & \\
\hline${ }^{133} \mathrm{Pm}^{m}$ & -65280 & 50 & 130.4 & 1.0 & $\&$ & $10 \#$ & $\mathrm{~s}$ & & $\left(11 / 2^{-}\right)$ & 96Ga17 & EJ & $\beta^{+}$?; IT ? & * \\
\hline${ }^{133} \mathrm{Sm}$ & $-57130 \#$ & $200 \#$ & & & & 2.90 & $\mathrm{~s}$ & 0.17 & $\left(5 / 2^{+}\right) 01$ & 01Xu04 & $\mathrm{T}$ & $\beta^{+}=100 ; \beta^{+} \mathrm{p}=?$ & * \\
\hline${ }^{133} \mathrm{Eu}$ & $-47280 \#$ & $300 \#$ & & & & $200 \#$ & $\mathrm{~ms}$ & & $11 / 2^{-} \#$ & & & $\beta^{+} ?$ & \\
\hline${ }^{133} \mathrm{In}$ & \multirow{3}{*}{\multicolumn{12}{|c|}{$\begin{array}{l}\mathrm{D}: \beta^{-} \mathrm{n} \text { intensity is from 93Ru01 } \\
\mathrm{E}: \text { combining } \gamma_{\mathrm{s}} \text { from Table 1: } 21\end{array}$}} & * \\
\hline${ }^{133} \mathrm{Pm}^{m}$ & & & & & & & & & & & & & Fit \\
\hline${ }^{133} \mathrm{Sm}$ & $\mathrm{T}:$ average 0 & $01 \times u 04=$ & $3.1(0.5) 8$ & $07=2$ & & $7 \mathrm{Bo} 02=$ & 3.2( & & & & & & k* \\
\hline
\end{tabular}




\begin{tabular}{|c|c|c|c|c|c|c|c|c|c|c|c|c|c|}
\hline \multirow{2}{*}{$\begin{array}{l}\text { Nuclide } \\
{ }^{134} \text { In }\end{array}$} & \multicolumn{2}{|c|}{$\begin{array}{l}\text { Mass excess } \\
\quad(\mathrm{keV})\end{array}$} & \multicolumn{3}{|c|}{$\begin{array}{c}\text { Excitation } \\
\text { energy }(\mathrm{keV})\end{array}$} & \multicolumn{3}{|c|}{ Half-life } & \multirow{2}{*}{$\begin{array}{c}J^{\pi} \\
\text { high }\end{array}$} & \multicolumn{2}{|c|}{ Ens Reference } & \multicolumn{2}{|l|}{$\begin{array}{l}\text { Decay modes and } \\
\text { intensities }(\%)\end{array}$} \\
\hline & $-52020 \#$ & $400 \#$ & & & & 140 & $\mathrm{~ms}$ & 4 & & 02 & $96 \mathrm{Ho} 16 \mathrm{~J}$ & $\beta^{-}=100 ; \beta^{-} \mathrm{n}=65 ; \ldots$ & * \\
\hline${ }^{134} \mathrm{Sn}$ & -66800 & 100 & & & & 1.12 & $\mathrm{~s}$ & 0.08 & $0^{+}$ & 94 & & $\beta^{-}=100 ; \beta^{-} \mathrm{n}=1713$ & \\
\hline${ }^{134} \mathrm{Sb}$ & -74170 & 40 & & & $*$ & 780 & $\mathrm{~ms}$ & 60 & $\left(0^{-}\right)$ & 95 & & $\beta^{-}=100$ & \\
\hline${ }^{134} \mathrm{Sb}^{m}$ & -74090 & 100 & 80 & 110 & $\mathrm{BD} *$ & 10.22 & $\mathrm{~s}$ & 0.09 & $\left(7^{-}\right)$ & 95 & & $\beta^{-}=100 ; \beta^{-} \mathrm{n}=0.0918$ & \\
\hline${ }^{134} \mathrm{Te}$ & -82559 & 11 & & & & 41.8 & $\mathrm{~m}$ & 0.8 & $0^{+}$ & 98 & & $\beta^{-}=100$ & \\
\hline${ }^{134} \mathrm{Te}^{m}$ & -80868 & 11 & 1691.24 & 0.17 & & 164 & $\mathrm{~ns}$ & 1 & $6^{+}$ & 98 & & $\mathrm{IT}=100$ & \\
\hline${ }^{134} \mathbf{I}$ & -84072 & 8 & & & & 52.5 & $\mathrm{~m}$ & 0.2 & $(4)^{+}$ & 94 & & $\beta^{-}=100$ & \\
\hline${ }^{134} \mathrm{I}^{m}$ & -83756 & 8 & 316.49 & 0.22 & & 3.60 & $\mathrm{~m}$ & 0.10 & $(8)^{-}$ & 94 & & $\mathrm{IT}=97.710 ; \beta^{-}=2.310$ & \\
\hline${ }^{134} \mathrm{Xe}$ & -88124.5 & 0.8 & & & & STABLE & & (>11 Py) & $0^{+}$ & 94 & $89 \mathrm{Ba} 22 \mathrm{~T}$ & $\mathrm{IS}=10.4410 ; 2 \beta^{-} ?$ & \\
\hline${ }^{134} \mathrm{Xe}^{m}$ & -86159.0 & 0.9 & 1965.5 & 0.5 & & 290 & $\mathrm{~ms}$ & 17 & $7^{-}$ & 94 & & $\mathrm{IT}=100$ & \\
\hline${ }^{134} \mathrm{Cs}$ & -86891.181 & 10.026 & & & & 2.0648 & $y$ & 0.0010 & $4^{+}$ & 94 & & $\beta^{-}=100 ; \varepsilon=0.00031$ & \\
\hline${ }^{134} \mathrm{Cs}^{m}$ & -86752.437 & $7 \quad 0.026$ & 138.7441 & 0.0026 & & 2.903 & $\mathrm{~h}$ & 0.008 & $8^{-}$ & 94 & & $\mathrm{IT}=100$ & \\
\hline${ }^{134} \mathrm{Ba}$ & -88949.9 & 0.4 & & & & STABLE & & & $0^{+}$ & 95 & & $\mathrm{IS}=2.41718$ & \\
\hline${ }^{134} \mathrm{La}$ & -85219 & 20 & & & & 6.45 & $\mathrm{~m}$ & 0.16 & $1^{+}$ & 94 & & $\beta^{+}=100$ & \\
\hline${ }^{134} \mathrm{Ce}$ & -84836 & 20 & & & & 3.16 & $\mathrm{~d}$ & 0.04 & $0^{+}$ & 94 & & $\varepsilon=100$ & \\
\hline${ }^{134} \mathrm{Pr}$ & -78510 & 40 & & & $\&$ & 11 & $\mathrm{~m}$ & & $\left(5^{-}\right)$ & 94 & & $\beta^{+}=100$ & \\
\hline${ }^{134} \mathrm{Pr}^{m}$ & $-78510 \#$ & $110 \#$ & $0 \#$ & $100 \#$ & $\&$ & 17 & $\mathrm{~m}$ & 2 & $2^{-}$ & 94 & & $\beta^{+}=100$ & \\
\hline${ }^{134} \mathrm{Nd}$ & -75646 & 12 & & & & 8.5 & $\mathrm{~m}$ & 1.5 & $0^{+}$ & 99 & & $\beta^{+}=100$ & \\
\hline${ }^{134} \mathrm{Nd}^{m}$ & -73353 & 12 & 2293.1 & 0.4 & & 410 & $\mu \mathrm{s}$ & 30 & $(8)^{-}$ & 99 & & $\mathrm{IT}=100$ & \\
\hline${ }^{134} \mathrm{Pm}$ & -66740 & 60 & & & $*$ & 22 & $\mathrm{~s}$ & 1 & $\left(5^{+}\right)$ & 94 & & $\beta^{+}=100$ & \\
\hline${ }^{134} \mathrm{Pm}^{m}$ & $-66740 \#$ & $120 \#$ & $0 \#$ & $100 \#$ & $*$ & 5 & $\mathrm{~s}$ & & $\left(2^{+}\right)$ & 94 & & $\beta^{+}=100$ & \\
\hline${ }^{134} \mathrm{Sm}$ & $-61510 \#$ & $200 \#$ & & & & 10 & $\mathrm{~s}$ & 1 & $0^{+}$ & 94 & & $\beta^{+}=100$ & \\
\hline${ }^{134} \mathrm{Eu}$ & $-49830 \#$ & $200 \#$ & & & & 500 & $\mathrm{~ms}$ & 200 & & 94 & & $\beta^{+}=100 ; \beta^{+} \mathrm{p}=?$ & \\
\hline${ }^{134} \mathrm{Gd}$ & $-41570 \#$ & $400 \#$ & & & & $400 \#$ & $\mathrm{~ms}$ & & $0^{+}$ & & & $\beta^{+}$? & \\
\hline$*{ }^{134} \mathrm{In} \quad \mathrm{I}$ & $\mathrm{D}: \ldots ; \beta^{-2}$ & $2 n<4$ & & & & & & & & & & & $* *$ \\
\hline$*^{134}$ In I & $\mathrm{D}: \beta^{-} 2 \mathrm{n}$ in & tensity lin & mits is from & 95Jo.A & & & & & & & & & $* *$ \\
\hline${ }^{135} \mathrm{In}$ & $-47200 \#$ & $500 \#$ & & & & 92 & $\mathrm{~ms}$ & 10 & $9 / 2^{+} \#$ & 02 & & $\beta^{-} ? ; \beta^{-} \mathrm{n} ?$ & \\
\hline${ }^{135} \mathrm{Sn}$ & $-60800 \#$ & $400 \#$ & & & & 530 & $\mathrm{~ms}$ & 20 & $\left(7 / 2^{-}\right)$ & 02 & & $\beta^{-}=100 ; \beta^{-} \mathrm{n}=213$ & \\
\hline${ }^{135} \mathrm{Sb}$ & -69710 & 100 & & & & 1.68 & $\mathrm{~s}$ & 0.02 & $\left(7 / 2^{+}\right)$ & 02 & 02Sh08 J & $\beta^{-}=100 ; \beta^{-} \mathrm{n}=223$ & \\
\hline${ }^{135} \mathrm{Te}$ & -77830 & 90 & & & & 19.0 & s & 0.2 & $\left(7 / 2^{-}\right)$ & 98 & & $\beta^{-}=100$ & \\
\hline${ }^{135} \mathrm{Te}^{m}$ & -76280 & 90 & 1554.88 & 0.17 & & 510 & $\mathrm{~ns}$ & 20 & $\left(19 / 2^{-}\right)$ & 98 & & $\mathrm{IT}=100$ & \\
\hline${ }^{135} \mathrm{I}$ & -83790 & 7 & & & & 6.57 & $\mathrm{~h}$ & 0.02 & $7 / 2^{+}$ & 98 & & $\beta^{-}=100$ & \\
\hline${ }^{135} \mathrm{Xe}$ & -86417 & 5 & & & & 9.14 & $\mathrm{~h}$ & 0.02 & $3 / 2^{+}$ & 98 & & $\beta^{-}=100$ & \\
\hline${ }^{135} \mathrm{Xe}^{m}$ & -85890 & 5 & 526.551 & 0.013 & & 15.29 & $\mathrm{~m}$ & 0.05 & $11 / 2^{-}$ & 98 & & $\mathrm{IT} \approx 100 ; \beta^{-}=0.3017$ & * \\
\hline${ }^{135} \mathrm{Cs}$ & -87581.9 & 1.0 & & & & 2.31 & My & 0.3 & $7 / 2^{+}$ & 98 & & $\beta^{-}=100$ & \\
\hline${ }^{135} \mathrm{Cs}^{m}$ & -85949.0 & 1.8 & 1632.9 & 1.5 & & 53 & $\mathrm{~m}$ & 2 & $19 / 2^{-}$ & 98 & & $\mathrm{IT}=100$ & \\
\hline${ }^{135} \mathrm{Ba}$ & -87850.5 & 0.4 & & & & STABLE & & & $3 / 2^{+}$ & 98 & & $\mathrm{IS}=6.59212$ & \\
\hline${ }^{135} \mathrm{Ba}^{m}$ & -87582.3 & 0.4 & 268.22 & 0.02 & & 28.7 & $\mathrm{~h}$ & 0.2 & $11 / 2^{-}$ & 98 & & $\mathrm{IT}=100$ & \\
\hline${ }^{135} \mathrm{La}$ & -86651 & 10 & & & & 19.5 & $\mathrm{~h}$ & 0.2 & $5 / 2^{+}$ & 98 & & $\beta^{+}=100$ & \\
\hline${ }^{135} \mathrm{Ce}$ & -84625 & 11 & & & & 17.7 & $\mathrm{~h}$ & 0.3 & $1 / 2^{(+)}$ & 98 & & $\beta^{+}=100$ & \\
\hline${ }^{135} \mathrm{Ce}^{m}$ & -84179 & 11 & 445.8 & 0.2 & & 20 & $\mathrm{~s}$ & 1 & $\left(11 / 2^{-}\right)$ & 98 & & $\mathrm{IT}=100$ & \\
\hline${ }^{135} \mathrm{Pr}$ & -80936 & 12 & & & & 24 & $\mathrm{~m}$ & 2 & $3 / 2^{(+)}$ & 98 & & $\beta^{+}=100$ & \\
\hline${ }^{135} \mathrm{Pr}^{m}$ & -80578 & 12 & 358.06 & 0.06 & & 105 & $\mu \mathrm{s}$ & 10 & $\left(11 / 2^{-}\right)$ & 98 & & $\mathrm{IT}=100$ & \\
\hline${ }^{135} \mathrm{Nd}$ & -76214 & 19 & & & & 12.4 & $\mathrm{~m}$ & 0.6 & $9 / 2^{(-)}$ & 98 & & $\beta^{+}=100$ & \\
\hline${ }^{135} \mathrm{Nd}^{m}$ & -76149 & 19 & 65.0 & 0.2 & & 5.5 & $\mathrm{~m}$ & 0.5 & $\left(1 / 2^{+}\right)$ & 98 & & $\beta^{+}>99.97 ;$ IT $<0.03$ & \\
\hline${ }^{135} \mathrm{Pm}$ & -69980 & 60 & & & $* \&$ & 49 & $\mathrm{~s}$ & 3 & $\left(5 / 2^{+}, 3 / 2^{+}\right)$ & 98 & & $\beta^{+}=100$ & \\
\hline${ }^{135} \mathrm{Pm}^{m}$ & $-69930 \#$ & $120 \#$ & $50 \#$ & $100 \#$ & $* \&$ & 40 & s & 3 & $\left(11 / 2^{-}\right)$ & 98 & 89Ko07 TJ & $\beta^{+}=100$ & \\
\hline${ }^{135} \mathrm{Sm}$ & -62860 & 150 & & & $*$ & 10.3 & s & 0.5 & $\left(7 / 2^{+}\right)$ & 98 & 77Bo02 J & $\beta^{+}=100 ; \beta^{+} \mathrm{p}=0.021$ & \\
\hline${ }^{135} \mathrm{Sm}^{m}$ & $-62860 \#$ & $340 \#$ & $0 \#$ & $300 \#$ & $*$ & 2.4 & s & 0.9 & $\left(3 / 2^{+}, 5 / 2^{+}\right)$ & 98 & 89Vi04 TJD & $\beta^{+}=100$ & $*$ \\
\hline${ }^{135} \mathrm{Eu}$ & $-54190 \#$ & $300 \#$ & & & & 1.5 & s & 0.2 & $11 / 2^{-} \#$ & 98 & & $\beta^{+}=100 ; \beta^{+} \mathrm{p} ?$ & \\
\hline${ }^{135} \mathrm{Gd}$ & $-44180 \#$ & $500 \#$ & & & & 1.1 & $\mathrm{~s}$ & 0.2 & $3 / 2^{-}$ & 98 & $98 \mathrm{St} 28 \mathrm{~J}$ & $\beta^{+}=100 ; \beta^{+} \mathrm{p} \approx 2$ & \\
\hline${ }^{135} \mathrm{Xe}^{m} \mathrm{I}$ & $\mathrm{D}: \beta^{-}$ran & ing 0.004 & & & & & & & & & & & \\
\hline
\end{tabular}




\begin{tabular}{|c|c|c|c|c|c|c|c|c|c|c|c|c|}
\hline Nuclide & \multicolumn{2}{|c|}{$\begin{array}{c}\text { Mass excess } \\
(\mathrm{keV})\end{array}$} & \multicolumn{3}{|c|}{$\begin{array}{l}\text { Excitation } \\
\text { energy }(\mathrm{keV})\end{array}$} & \multicolumn{3}{|c|}{ Half-life } & \multirow[t]{2}{*}{$J^{\pi}$} & \multirow{2}{*}{$\begin{array}{c}\text { Ens } \\
02\end{array}$} & Reference & $\begin{array}{l}\text { Decay modes and } \\
\text { intensities }(\%)\end{array}$ \\
\hline${ }^{136} \mathrm{Sn}$ & $-56500 \#$ & $500 \#$ & & & & 250 & $\mathrm{~ms}$ & 30 & & & & $\beta^{-}=100 ; \beta^{-} \mathrm{n}=305$ \\
\hline${ }^{136} \mathrm{Sb}$ & $-64880 \#$ & $300 \#$ & & & & 923 & $\mathrm{~ms}$ & 14 & $1^{-} \#$ & 02 & & $\beta^{-}=100 ; \beta^{-} \mathrm{n}=16.332 ; \ldots$ \\
\hline${ }^{136} \mathrm{Sb}^{m}$ & $-64710 \#$ & $300 \#$ & 173 & 3 & & 570 & ns & 50 & $6^{-} \#$ & 02 & $01 \mathrm{Mi} 22 \mathrm{E}$ & $\mathrm{IT}=100$ \\
\hline${ }^{136} \mathrm{Te}$ & -74430 & 50 & & & & 17.63 & $\mathrm{~s}$ & 0.08 & $0^{+}$ & 02 & & $\beta^{-}=100 ; \beta^{-} \mathrm{n}=1.315$ \\
\hline${ }^{136} \mathrm{I}$ & -79500 & 50 & & & & 83.4 & s & 1.0 & $\left(1^{-}\right)$ & 02 & & $\beta^{-}=100$ \\
\hline${ }^{136} \mathrm{I}^{m}$ & -78850 & 110 & 650 & 120 & $\mathrm{BD}$ & 46.9 & s & 1.0 & $\left(6^{-}\right)$ & 02 & & $\beta^{-}=100 ; \mathrm{IT}=0$ \\
\hline${ }^{136} \mathrm{Xe}$ & -86425 & 7 & & & & STABLE & & $(>10 \mathrm{Zy})$ & $0^{+}$ & 02 & 02Be74 T & $\mathrm{IS}=8.8716 ; 2 \beta^{-} ?$ \\
\hline${ }^{136} \mathrm{Xe}^{m}$ & -84533 & 7 & 1891.703 & 0.014 & & 2.95 & $\mu \mathrm{s}$ & 0.09 & $6^{+}$ & 02 & & $\mathrm{IT}=100$ \\
\hline${ }^{136} \mathrm{Cs}$ & -86338.7 & 1.9 & & & $*$ & 13.16 & $\mathrm{~d}$ & 0.03 & $5^{+}$ & 02 & & $\beta^{-}=100$ \\
\hline${ }^{136} \mathrm{Cs}^{m}$ & -85821 & 5 & 518 & 5 & * & 19 & $\mathrm{~s}$ & 2 & $8^{-}$ & 02 & 83We07 E & $\mathrm{IT}=? ; \beta^{-} ?$ \\
\hline${ }^{136} \mathrm{Ba}$ & -88886.9 & 0.4 & & & & StaBle & & & $0^{+}$ & 02 & & IS $=7.85424$ \\
\hline${ }^{136} \mathrm{Ba}^{m}$ & -86856.4 & 0.4 & 2030.466 & 0.018 & & 308.4 & $\mathrm{~ms}$ & 1.9 & $7^{-}$ & 02 & & $\mathrm{IT}=100$ \\
\hline${ }^{136} \mathrm{La}$ & -86040 & 50 & & & & 9.87 & $\mathrm{~m}$ & 0.03 & $1^{+}$ & 02 & & $\beta^{+}=100$ \\
\hline${ }^{136} \mathrm{La}^{m}$ & -85790 & 50 & 255 & 9 & & 114 & $\mathrm{~ms}$ & 3 & $(8)^{(-\#)}$ & 02 & ABBW E & $\mathrm{IT}=100$ \\
\hline${ }^{136} \mathrm{Ce}$ & -86468 & 13 & & & & STABLE & & (>38 Py) & $0^{+}$ & 02 & $01 \mathrm{Da} 22 \mathrm{~T}$ & $\mathrm{IS}=0.1852 ; 2 \beta^{+} ?$ \\
\hline${ }^{136} \mathrm{Ce}^{m}$ & -83373 & 13 & 3095.5 & 0.4 & & 2.2 & $\mu \mathrm{s}$ & 0.2 & $10^{+}$ & 02 & & $\mathrm{IT}=100$ \\
\hline${ }^{136} \mathrm{Pr}$ & -81327 & 12 & & & & 13.1 & $\mathrm{~m}$ & 0.1 & $2^{+}$ & 02 & & $\beta^{+}=100$ \\
\hline${ }^{136} \operatorname{Pr}^{m}$ & -80732 & 12 & 594.62 & 0.22 & & 91.7 & ns & 0.9 & $(6)^{+}$ & 02 & & $\mathrm{IT}=100$ \\
\hline${ }^{136} \mathrm{Nd}$ & -79199 & 12 & & & & 50.7 & $\mathrm{~m}$ & 0.3 & $0^{+}$ & 02 & & $\beta^{+}=100$ \\
\hline${ }^{136} \mathrm{Pm}$ & -71200 & 80 & & & $* \&$ & 107 & $\mathrm{~s}$ & 6 & $\left(5^{-}\right)$ & 02 & & $\beta^{+}=100$ \\
\hline${ }^{136} \mathrm{Pm}^{m}$ & -71070 & 90 & 130 & 120 & $\mathrm{BD} * \&$ & 47 & $\mathrm{~s}$ & 2 & $\left(2^{+}\right)$ & 02 & & $\beta^{+}=100$ \\
\hline${ }^{136} \mathrm{Sm}$ & -66811 & 12 & & & & 47 & s & 2 & $0^{+}$ & 02 & & $\beta^{+}=100$ \\
\hline${ }^{136} \mathrm{Sm}^{m}$ & -64546 & 12 & 2264.7 & 1.1 & & 15 & $\mu \mathrm{s}$ & 1 & $\left(8^{-}\right)$ & 02 & & $\mathrm{IT}=100$ \\
\hline${ }^{136} \mathrm{Eu}$ & $-56260 \#$ & $200 \#$ & & & * & 3.3 & $\mathrm{~s}$ & 0.3 & $\left(7^{+}\right)$ & 02 & 89Vi04 D & $\beta^{+}=100 ; \beta^{+} \mathrm{p}=0.093$ \\
\hline${ }^{136} \mathrm{Eu}^{m}$ & $-56260 \#$ & $540 \#$ & 0\# & $500 \#$ & $*$ & 3.8 & s & 0.3 & $\left(3^{+}\right)$ & 02 & 89Vi04 D & $\beta^{+}=100 ; \beta^{+} \mathrm{p}=0.093$ \\
\hline${ }^{136} \mathrm{Gd}$ & $-49050 \#$ & $400 \#$ & & & & $1 \#$ & s & $(>200 \mathrm{~ns})$ & $0^{+}$ & 02 & 00So11 I & $\beta^{+}$? \\
\hline${ }^{136} \mathrm{~Tb}$ & $-35970 \#$ & $600 \#$ & & & & $200 \#$ & $\mathrm{~ms}$ & & & 02 & & $\beta^{+} ?$ \\
\hline${ }^{136} \mathrm{Sb}$ & $\mathrm{D}: \ldots ; \beta$ & $-2 \mathrm{n}=0$. & & & & & & & & & & \\
\hline${ }^{136} \mathrm{La}^{m}$ & E : approx & $10-40$ & & 230 & f & ISD & & 3 & $25(9$ & & & \\
\hline
\end{tabular}

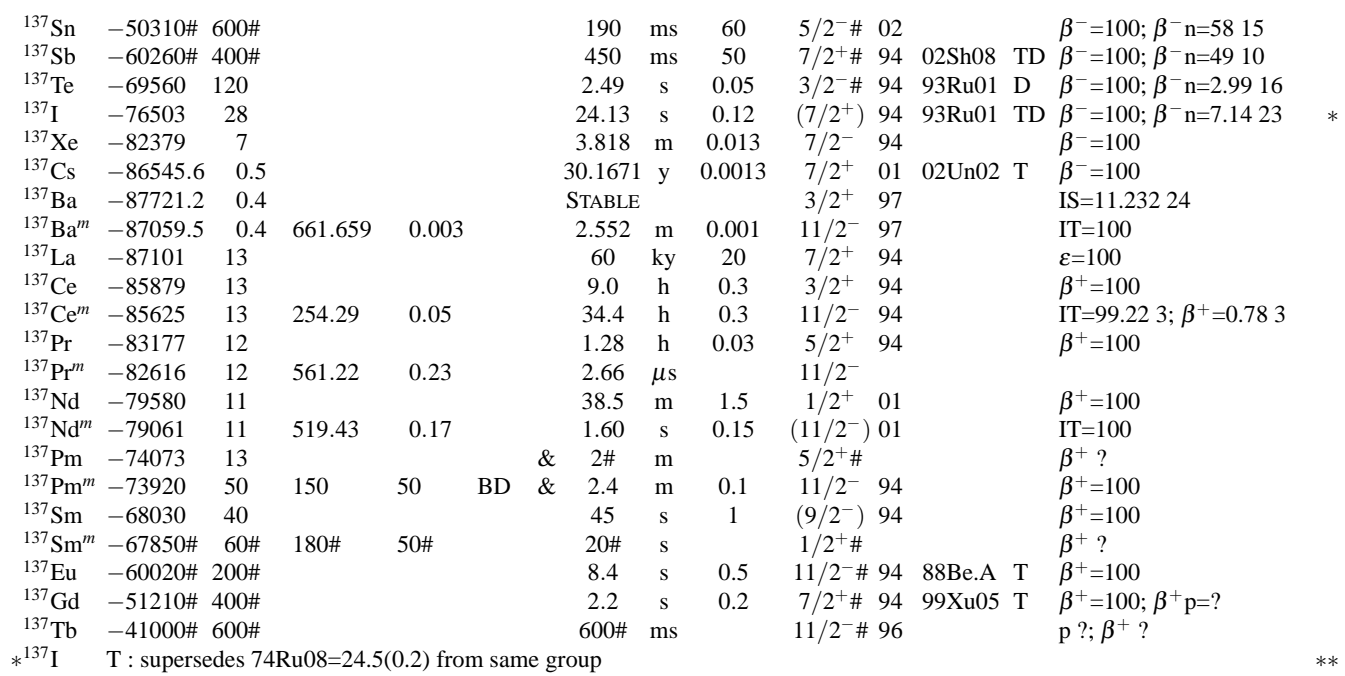




\begin{tabular}{|c|c|c|c|c|c|c|c|c|c|c|c|c|c|c|}
\hline \multirow{2}{*}{$\begin{array}{l}\text { Nuclide } \\
{ }^{138} \mathrm{Sb}\end{array}$} & \multicolumn{2}{|c|}{$\begin{array}{l}\text { Mass excess } \\
\quad(\mathrm{keV})\end{array}$} & \multicolumn{3}{|c|}{$\begin{array}{l}\text { Excitation } \\
\text { energy }(\mathrm{keV})\end{array}$} & \multicolumn{3}{|c|}{ Half-life } & \multirow{2}{*}{$\begin{array}{c}J^{\pi} \\
2^{-} \#\end{array}$} & \multirow{2}{*}{$\begin{array}{c}\text { Ens } \\
03\end{array}$} & \multicolumn{2}{|c|}{ Reference } & \multicolumn{2}{|l|}{$\begin{array}{l}\text { Decay modes and } \\
\text { intensities }(\%)\end{array}$} \\
\hline & $-55150 \#$ & $300 \#$ & & & & $500 \#$ & $\mathrm{~ms}$ & $(>300 \mathrm{~ns})$ & & & $94 \mathrm{Be} 24$ & I & $\beta^{-} ? ; \beta^{-} \mathrm{n} ?$ & \\
\hline${ }^{138} \mathrm{Te}$ & $-65930 \#$ & $210 \#$ & & & & 1.4 & $\mathrm{~s}$ & 0.4 & $0^{+}$ & 03 & & & $\beta^{-}=100 ; \beta^{-} \mathrm{n}=6.321$ & \\
\hline${ }^{138} \mathrm{I}$ & -72330 & 80 & & & & 6.23 & $\mathrm{~s}$ & 0.03 & $\left(2^{-}\right)$ & 03 & 93Ru01 & $\mathrm{D}$ & $\beta^{-}=100 ; \beta^{-} \mathrm{n}=5.4618$ & \\
\hline${ }^{138} \mathrm{Xe}$ & -80150 & 40 & & & & 14.08 & $\mathrm{~m}$ & 0.08 & $0^{+}$ & 03 & & & $\beta^{-}=100$ & \\
\hline${ }^{138} \mathrm{Cs}$ & -82887 & 9 & & & & 33.41 & $\mathrm{~m}$ & 0.18 & $3^{-}$ & 03 & & & $\beta^{-}=100$ & \\
\hline${ }^{138} \mathrm{Cs}^{m}$ & -82807 & 9 & 79.9 & 0.3 & & 2.91 & $\mathrm{~m}$ & 0.08 & $6^{-}$ & 03 & & & $\mathrm{IT}=812 ; \beta^{-}=192$ & \\
\hline${ }^{138} \mathrm{Cs}^{x}$ & -82847 & 25 & 40 & 23 & & $R=?$ & & & fsmix & & & & & \\
\hline${ }^{138} \mathrm{Ba}$ & -88261.6 & 0.4 & & & & STABLE & & & $0^{+}$ & 03 & & & IS $=71.69842$ & \\
\hline${ }^{138} \mathrm{Ba}^{m}$ & -86171.1 & 0.4 & 2090.54 & 0.0 & & 800 & ns & 100 & $6^{+}$ & 03 & & & $\mathrm{IT}=100$ & \\
\hline${ }^{138} \mathrm{La}$ & -86525 & 4 & & & & 102 & Gy & 1 & $5^{+}$ & 03 & & & $\mathrm{IS}=0.0901 ; \ldots$ & $*$ \\
\hline${ }^{138} \mathrm{La}^{m}$ & -86452 & 4 & 72.57 & 0.0 & & 116 & $\mathrm{~ns}$ & 5 & $(3)^{+}$ & 03 & & & $\mathrm{IT}=100$ & \\
\hline${ }^{138} \mathrm{Ce}$ & -87569 & 10 & & & & STABLE & & (>150 Ty) & $0^{+}$ & 03 & $01 \mathrm{Da} 22$ & $\mathrm{~T}$ & $\mathrm{IS}=0.2512 ; 2 \beta^{+} ?$ & \\
\hline${ }^{138} \mathrm{Ce}^{m}$ & -85440 & 10 & 2129.17 & 0.1 & & 8.65 & $\mathrm{~ms}$ & 0.20 & $7^{-}$ & 03 & & & $\mathrm{IT}=100$ & \\
\hline${ }^{138} \mathrm{Pr}$ & -83132 & 14 & & & & 1.45 & $\mathrm{~m}$ & 0.05 & $1^{+}$ & 03 & & & $\beta^{+}=100$ & \\
\hline${ }^{138} \operatorname{Pr}^{m}$ & -82783 & 17 & 348 & 23 & $\mathrm{BD}$ & 2.12 & $\mathrm{~h}$ & 0.04 & $7^{-}$ & 03 & & & $\beta^{+}=100$ & \\
\hline${ }^{138} \mathrm{Nd}$ & -82018 & 12 & & & & 5.04 & $\mathrm{~h}$ & 0.09 & $0^{+}$ & 03 & & & $\beta^{+}=100$ & \\
\hline${ }^{138} \mathrm{Nd}^{m}$ & -78843 & 12 & 3174.9 & 0.4 & & 410 & ns & 50 & $\left(10^{+}\right)$ & 03 & & & $\mathrm{IT}=100$ & \\
\hline${ }^{138} \mathrm{Pm}$ & -74940 & 27 & & & $*$ & 10 & $\mathrm{~s}$ & 2 & $1^{+} \#$ & 03 & & & $\beta^{+}=100$ & \\
\hline${ }^{138} \mathrm{Pm}^{m}$ & -74911 & 13 & 30 & 30 & $\mathrm{BD} *$ & 3.24 & $\mathrm{~m}$ & 0.05 & $5^{-} \#$ & 03 & & & $\beta^{+}=100$ & \\
\hline${ }^{138} \mathrm{Pm}^{n}$ & & & non ex & stent & $\mathrm{EU}$ & 3.24 & $\mathrm{~m}$ & 0.05 & $\left(3^{+}\right)$ & & 81De38 & I & $\beta^{+}=100$ & $*$ \\
\hline${ }^{138} \mathrm{Sm}$ & -71498 & 12 & & & & 3.1 & $\mathrm{~m}$ & 0.2 & $0^{+}$ & 03 & & & $\beta^{+}=100$ & \\
\hline${ }^{138} \mathrm{Eu}$ & -61750 & 28 & & & & 12.1 & $\mathrm{~s}$ & 0.6 & $\left(6^{-}\right)$ & 03 & & & $\beta^{+}=100$ & \\
\hline${ }^{138} \mathrm{Gd}$ & $-55780 \#$ & $200 \#$ & & & & 4.7 & $\mathrm{~s}$ & 0.9 & $0^{+}$ & 03 & & & $\beta^{+}=100$ & \\
\hline${ }^{138} \mathrm{Gd}^{m}$ & $-53550 \#$ & $200 \#$ & 2232.7 & 1.1 & & 6 & $\mu \mathrm{s}$ & 1 & $\left(8^{-}\right)$ & 03 & & & & \\
\hline${ }^{138} \mathrm{~Tb}$ & $-43630 \#$ & $400 \#$ & & & & $800 \#$ & $\mathrm{~ms}$ & $(>200 \mathrm{~ns})$ & & 03 & 00So11 & I & $\beta^{+} ? ; \mathrm{p}=0$ & $*$ \\
\hline${ }^{138}$ Dy & $-34940 \#$ & $600 \#$ & & & & $200 \#$ & $\mathrm{~ms}$ & & $0^{+}$ & & & & $\beta^{+}$? & \\
\hline${ }^{138} \mathrm{La}$ & $\mathrm{D}: \ldots ; \beta^{+}$ & $+=65.6$ & $5 ; \beta^{-}=34$ & & & & & & & & & & & ** \\
\hline${ }^{138} \mathrm{Pm}^{n}$ & $\mathrm{D}:$ argume & ents for & a second & 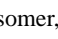 & in & dig & & 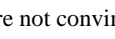 & & & & & & $* *$ \\
\hline$*^{138} \mathrm{~Tb}$ & $\mathrm{D}$ : from 9 & $3 \mathrm{Li} 40$ & & & & & & & & & & & & ** \\
\hline
\end{tabular}

\begin{tabular}{|c|c|c|c|c|c|c|c|c|c|c|c|c|c|c|}
\hline${ }^{139} \mathrm{Sb}$ & $-50320 \#$ & $500 \#$ & & & & $300 \#$ & $\mathrm{~ms}$ & $(>300 \mathrm{~ns})$ & $7 / 2^{+} \#$ & 01 & $94 \mathrm{Be} 24$ & I & $\beta^{-} ?$ & \\
\hline${ }^{139} \mathrm{Te}$ & $-60800 \#$ & $400 \#$ & & & & $500 \#$ & $\mathrm{~ms}$ & $(>300 \mathrm{~ns})$ & $5 / 2^{-} \#$ & 01 & $94 \mathrm{Be} 24$ & I & $\beta^{-} ? ; \beta^{-} \mathrm{n} ?$ & \\
\hline${ }^{139} \mathrm{I}$ & -68840 & 30 & & & & 2.282 & $\mathrm{~s}$ & 0.010 & $7 / 2^{+} \#$ & 01 & 93Ru01 & $\mathrm{T}$ & $\beta^{-}=100 ; \beta^{-} \mathrm{n}=10.03$ & $*$ \\
\hline${ }^{139} \mathrm{Xe}$ & -75644 & 21 & & & & 39.68 & $\mathrm{~s}$ & 0.14 & $3 / 2^{-}$ & 01 & & & $\beta^{-}=100$ & \\
\hline${ }^{139} \mathrm{Cs}$ & -80701 & 3 & & & & 9.27 & $\mathrm{~m}$ & 0.05 & $7 / 2^{+}$ & 01 & & & $\beta^{-}=100$ & \\
\hline${ }^{139} \mathrm{Ba}$ & -84913.7 & 0.4 & & & & 83.1 & $\mathrm{~m}$ & 0.3 & $\left(7 / 2^{-}\right)$ & 01 & & & $\beta^{-}=100$ & \\
\hline${ }^{139} \mathrm{La}$ & -87231.4 & 2.4 & & & & StABLE & & & $7 / 2^{+}$ & 01 & & & $\mathrm{IS}=99.9101$ & \\
\hline${ }^{139} \mathrm{Ce}$ & -86952 & 7 & & & & 137.641 & $\mathrm{~d}$ & 0.020 & $3 / 2^{+}$ & 01 & & & $\varepsilon=100$ & \\
\hline${ }^{139} \mathrm{Ce}^{m}$ & -86198 & 7 & 754.24 & 0.08 & & 56.54 & $\mathrm{~s}$ & 0.13 & $11 / 2^{-}$ & 01 & 94It.A & $\mathrm{T}$ & $\mathrm{IT}=100$ & \\
\hline${ }^{139} \mathrm{Pr}$ & -84823 & 8 & & & & 4.41 & $\mathrm{~h}$ & 0.04 & $5 / 2^{+}$ & 01 & & & $\beta^{+}=100$ & \\
\hline${ }^{139} \mathrm{Nd}$ & -81992 & 26 & & & & 29.7 & $\mathrm{~m}$ & 0.5 & $3 / 2^{+}$ & 01 & & & $\beta^{+}=100$ & \\
\hline${ }^{139} \mathrm{Nd}^{m}$ & -81761 & 26 & 231.15 & 0.05 & & 5.50 & $\mathrm{~h}$ & 0.20 & $11 / 2^{-}$ & 01 & & & $\beta^{+}=88.24 ; \mathrm{IT}=11.84$ & \\
\hline${ }^{139} \mathrm{Pm}$ & -77496 & 13 & & & & 4.15 & $\mathrm{~m}$ & 0.05 & $(5 / 2)^{+}$ & 01 & & & $\beta^{+}=100$ & \\
\hline${ }^{139} \mathrm{Pm}^{m}$ & -77307 & 13 & 188.7 & 0.3 & & 180 & $\mathrm{~ms}$ & 20 & $(11 / 2)^{-}$ & 01 & & & $\mathrm{IT} \approx 100 ; \beta^{+}=0.16 \#$ & \\
\hline${ }^{139} \mathrm{Sm}$ & -72380 & 11 & & & & 2.57 & $\mathrm{~m}$ & 0.10 & $1 / 2^{+}$ & 01 & & & $\beta^{+}=100$ & \\
\hline${ }^{139} \mathrm{Sm}^{m}$ & -71923 & 11 & 457.40 & 0.22 & & 10.7 & $\mathrm{~s}$ & 0.6 & $11 / 2^{-}$ & 01 & & & $\mathrm{IT}=93.75 ; \beta^{+}=6.35$ & \\
\hline${ }^{139} \mathrm{Eu}$ & -65398 & 13 & & & & 17.9 & $\mathrm{~s}$ & 0.6 & $(11 / 2)^{-}$ & 01 & & & $\beta^{+}=100$ & \\
\hline${ }^{139} \mathrm{Gd}$ & $-57530 \#$ & $200 \#$ & & & $*$ & 5.7 & $\mathrm{~s}$ & 0.3 & $9 / 2^{-} \#$ & 01 & 99Xi04 & $\mathrm{T}$ & $\beta^{+}=100 ; \beta^{+} \mathrm{p}=?$ & $*$ \\
\hline${ }^{139} \mathrm{Gd}^{m}$ & $-57280 \#$ & $250 \#$ & $250 \#$ & $150 \#$ & * & 4.8 & $\mathrm{~s}$ & 0.9 & $1 / 2^{+} \#$ & 01 & & & $\beta^{+}=100 ; \beta^{+} \mathrm{p}=?$ & * \\
\hline${ }^{139} \mathrm{~Tb}$ & $-48170 \#$ & $300 \#$ & & & & 1.6 & $\mathrm{~s}$ & 0.2 & $11 / 2^{-} \#$ & 01 & & & $\beta^{+}=100 ; \beta^{+} \mathrm{p} ?$ & \\
\hline${ }^{139} \mathrm{Dy}$ & $-37690 \#$ & $500 \#$ & & & & 600 & $\mathrm{~ms}$ & 200 & $7 / 2^{+} \#$ & 01 & & & $\beta^{+}=100 ; \beta^{+} \mathrm{p} ?$ & \\
\hline$*^{139} \mathrm{I}$ & $\mathrm{T}:$ averag & e $93 \mathrm{Ru} 0$ & $1=2.280($ & $0.011) 80$ & & $.29(0.02)$ & & & & & & & & ** \\
\hline$*^{139} \mathrm{Gd}$ & $\mathrm{T}:$ averag & e $99 \mathrm{Xi0}$ & $4=5.8(0.9$ & ) $88 \mathrm{Be} \cdot \mathrm{A}$ & & 4); other 8 & $83 \mathrm{Ni}$ & $05=4.9(1.0$ & not used & & & & & $k_{*}^{*}$ \\
\hline$*{ }^{139} \mathrm{Gd}$ & $\mathrm{T}$ : & cee it cor & responds & to a mixt & & round-sta & te an & ad isomer & & & & & & ** \\
\hline$*^{139} \mathrm{Gd}^{m}$ & $\mathrm{D}$ : assumi & ing that & he delaye & ed proton & po & d by $83 \mathrm{~N}$ & $\mathrm{Ni05}$ & are from $b c$ & th states & & & & & $<*$ \\
\hline
\end{tabular}




\begin{tabular}{|c|c|c|c|c|c|c|c|c|c|c|c|c|c|c|}
\hline Nuclide & \multicolumn{2}{|c|}{$\begin{array}{l}\text { Mass excess } \\
\quad(\mathrm{keV})\end{array}$} & \multicolumn{3}{|c|}{$\begin{array}{c}\text { Excitation } \\
\text { energy }(\mathrm{keV})\end{array}$} & \multicolumn{3}{|c|}{ Half-life } & \multirow{2}{*}{$\begin{array}{c}J^{\pi} \\
0^{+}\end{array}$} & \multirow{2}{*}{$\begin{array}{c}\text { Ens } \\
98\end{array}$} & \multicolumn{2}{|c|}{ Reference } & \multicolumn{2}{|l|}{$\begin{array}{l}\text { Decay modes and } \\
\text { intensities }(\%)\end{array}$} \\
\hline${ }^{140} \mathrm{Te}$ & $-56960 \#$ & $300 \#$ & & & & $300 \#$ & $\mathrm{~ms}$ & $(>300 \mathrm{~ns})$ & & & $94 \mathrm{Be} 24 \mathrm{I}$ & I & $\beta^{-} ? ; \beta^{-} \mathrm{n} ?$ & \\
\hline${ }^{140} \mathrm{I}$ & $-64270 \#$ & $200 \#$ & & & & 860 & $\mathrm{~ms}$ & 40 & $(3)^{(-\#)}$ & 95 & & & $\beta^{-}=100 ; \beta^{-} \mathrm{n}=9.310$ & \\
\hline${ }^{140} \mathrm{Xe}$ & -72990 & 60 & & & & 13.60 & $\mathrm{~s}$ & 0.10 & $0^{+}$ & 02 & & & $\beta^{-}=100$ & \\
\hline${ }^{140} \mathrm{Cs}$ & -77051 & 8 & & & & 63.7 & $\mathrm{~s}$ & 0.3 & $1^{-}$ & 95 & & & $\beta^{-}=100$ & \\
\hline${ }^{140} \mathrm{Ba}$ & -83271 & 8 & & & & 12.752 & $\mathrm{~d}$ & 0.003 & $0^{+}$ & 98 & & & $\beta^{-}=100$ & \\
\hline${ }^{140} \mathrm{La}$ & -84321.0 & 2.4 & & & & 1.6781 & $\mathrm{~d}$ & 0.0003 & $3^{-}$ & 95 & & & $\beta^{-}=100$ & \\
\hline${ }^{140} \mathrm{Ce}$ & -88083.3 & 2.5 & & & & STABLE & & & $0^{+}$ & 95 & & & $\mathrm{IS}=88.45051$ & \\
\hline${ }^{140} \mathrm{Ce}^{m}$ & -85975.5 & 2.5 & 2107.85 & 0.03 & & 7.3 & $\mu \mathrm{s}$ & 1.5 & $6^{+}$ & & & & & \\
\hline${ }^{140} \mathrm{Pr}$ & -84695 & 6 & & & & 3.39 & $\mathrm{~m}$ & 0.01 & $1^{+}$ & 95 & & & $\beta^{+}=100$ & \\
\hline${ }^{140} \mathrm{Pr}^{m}$ & -83932 & 6 & 763.3 & 0.7 & & 3.05 & $\mu \mathrm{s}$ & 0.20 & $(8)^{-}$ & & & & & \\
\hline${ }^{140} \mathrm{Nd}$ & -84252 & 28 & & & & 3.37 & $\mathrm{~d}$ & 0.02 & $0^{+}$ & 95 & & & $\varepsilon=100$ & \\
\hline${ }^{140} \mathrm{Nd}^{m}$ & -82031 & 28 & 2221.4 & 0.1 & & 600 & $\mu \mathrm{s}$ & 50 & $7^{-}$ & 95 & & & $\mathrm{IT}=100$ & \\
\hline${ }^{140} \mathrm{Pm}$ & -78210 & 40 & & & & 9.2 & $\mathrm{~s}$ & 0.2 & $1^{+}$ & 95 & & & $\beta^{+}=100$ & \\
\hline${ }^{140} \mathrm{Pm}^{m}$ & -77783 & 13 & 420 & 40 & $\mathrm{BD}$ & 5.95 & $\mathrm{~m}$ & 0.05 & $8^{-}$ & 95 & & & $\beta^{+}=100$ & \\
\hline${ }^{140} \mathrm{Sm}$ & -75456 & 12 & & & & 14.82 & $\mathrm{~m}$ & 0.12 & $0^{+}$ & 95 & & & $\beta^{+}=100$ & \\
\hline${ }^{140} \mathrm{Eu}$ & -66990 & 50 & & & & 1.51 & $\mathrm{~s}$ & 0.02 & $1^{+}$ & 95 & & & $\beta^{+}=100$ & \\
\hline${ }^{140} \mathrm{Eu}^{m}$ & -66780 & 50 & 210 & 15 & & 125 & $\mathrm{~ms}$ & 2 & $5^{-} \#$ & 95 & ABBW $I$ & $\mathrm{E}$ & $\mathrm{IT} \approx 100 ; \beta^{+}<1$ & $*$ \\
\hline${ }^{140} \mathrm{Gd}$ & -61782 & 28 & & & & 15.8 & $\mathrm{~s}$ & 0.4 & $0^{+}$ & 95 & $91 \mathrm{Fi} 03$ & $\mathrm{~T}$ & $\beta^{+}=100$ & \\
\hline${ }^{140} \mathrm{~Tb}$ & -50480 & 800 & & & & 2.4 & $\mathrm{~s}$ & 0.2 & 5 & 97 & & & $\beta^{+}=100 ; \beta^{+} \mathrm{p}=0.2613$ & \\
\hline${ }^{140}$ Dy & $-42840 \#$ & $500 \#$ & & & & 700\# & $\mathrm{ms}$ & & $0^{+}$ & 02 & & & $\beta^{+}$? & \\
\hline${ }^{140} \mathrm{Dy}^{m}$ & $-40670 \#$ & $500 \#$ & 2166.1 & 0.5 & & 7.0 & $\mu \mathrm{s}$ & 0.5 & $\left(8^{-}\right)$ & 02 & & & $\beta^{+}$? & \\
\hline${ }^{140} \mathrm{Ho}$ & $-29310 \#$ & $500 \#$ & & & & 6 & $\mathrm{~ms}$ & 3 & $8^{+} \#$ & 02 & & & $\mathrm{p}=? ; \beta^{+}=1 \#$ & \\
\hline${ }^{140} \mathrm{Eu}^{m}$ & $\mathrm{E}:$ less tha & in $50 \mathrm{ke}$ & $\mathrm{V}$ above 18 & $5.3 \mathrm{le}$ & el, fro & ENSDF, tl & hus 1 & $85.3+25(1$ & & & & & & $* *$ \\
\hline
\end{tabular}

\begin{tabular}{|c|c|c|c|c|c|c|c|c|c|c|c|c|c|}
\hline${ }^{141} \mathrm{Te}$ & $-51560 \#$ & $400 \#$ & & & & $100 \#$ & $\mathrm{~ms}$ & $(>300 \mathrm{~ns})$ & $5 / 2^{-} \#$ & 01 & $94 \mathrm{Be} 24$ & I & $\beta^{-} ? ; \beta^{-} \mathrm{n} ?$ \\
\hline${ }^{141} \mathrm{I}$ & $-60520 \#$ & $200 \#$ & & & & 430 & $\mathrm{~ms}$ & 20 & $7 / 2^{+} \#$ & 01 & & & $\beta^{-}=100 ; \beta^{-} \mathrm{n}=213$ \\
\hline${ }^{141} \mathrm{Xe}$ & -68330 & 90 & & & & 1.73 & $\mathrm{~s}$ & 0.01 & $5 / 2^{(-\#)}$ & 01 & & & $\beta^{-}=100 ; \beta^{-} \mathrm{n}=0.0445$ \\
\hline${ }^{141} \mathrm{Cs}$ & -74477 & 11 & & & & 24.84 & s & 0.16 & $7 / 2^{+}$ & 01 & & & $\beta^{-}=100 ; \beta^{-} \mathrm{n}=0.0353$ \\
\hline${ }^{141} \mathrm{Ba}$ & -79726 & 8 & & & & 18.27 & $\mathrm{~m}$ & 0.07 & $3 / 2^{-}$ & 01 & & & $\beta^{-}=100$ \\
\hline${ }^{141} \mathrm{La}$ & -82938 & 5 & & & & 3.92 & $\mathrm{~h}$ & 0.03 & $\left(7 / 2^{+}\right)$ & 01 & & & $\beta^{-}=100$ \\
\hline${ }^{141} \mathrm{Ce}$ & -85440.1 & 2.5 & & & & 32.508 & $\mathrm{~d}$ & 0.013 & $7 / 2^{-}$ & 01 & & & $\beta^{-}=100$ \\
\hline${ }^{141} \mathrm{Pr}$ & -86020.9 & 2.5 & & & & STABLE & & & $5 / 2^{+}$ & 01 & & & $\mathrm{IS}=100$ \\
\hline${ }^{141} \mathrm{Nd}$ & -84198 & 4 & & & & 2.49 & $\mathrm{~h}$ & 0.03 & $3 / 2^{+}$ & 01 & & & $\beta^{+}=100$ \\
\hline${ }^{141} \mathrm{Nd}^{m}$ & -83441 & 4 & 756.51 & 0.05 & & 62.0 & s & 0.8 & $11 / 2^{-}$ & 01 & $70 \mathrm{Ab} 05$ & $\mathrm{D}$ & $\mathrm{IT} \approx 100 ; \beta^{+}=0.0328$ \\
\hline${ }^{141} \mathrm{Pm}$ & -80523 & 14 & & & & 20.90 & $\mathrm{~m}$ & 0.05 & $5 / 2^{+}$ & 01 & & & $\beta^{+}=100$ \\
\hline${ }^{141} \mathrm{Pm}^{m}$ & -79895 & 14 & 628.40 & 0.10 & & 630 & $\mathrm{~ns}$ & 20 & $11 / 2^{-}$ & 01 & & & $\mathrm{IT}=100$ \\
\hline${ }^{141} \mathrm{Sm}$ & -75939 & 9 & & & & 10.2 & $\mathrm{~m}$ & 0.2 & $1 / 2^{+}$ & 01 & & & $\beta^{+}=100$ \\
\hline${ }^{141} \mathrm{Sm}^{m}$ & -75763 & 9 & 176.0 & 0.3 & & 22.6 & $\mathrm{~m}$ & 0.2 & $11 / 2^{-}$ & 01 & & & $\beta^{+} \approx 100 ; \mathrm{IT}=0.313$ \\
\hline${ }^{141} \mathrm{Eu}$ & -69927 & 13 & & & & 40.7 & s & 0.7 & $5 / 2^{+}$ & 01 & & & $\beta^{+}=100$ \\
\hline${ }^{141} \mathrm{Eu}^{m}$ & -69831 & 13 & 96.45 & 0.07 & & 2.7 & s & 0.3 & $11 / 2^{-}$ & 01 & & & $\mathrm{IT}=863 ; \beta^{+}=143$ \\
\hline${ }^{141} \mathrm{Gd}$ & -63224 & 20 & & & & 14 & $\mathrm{~s}$ & 4 & $\left(1 / 2^{+}\right)$ & 01 & & & $\beta^{+}=100 ; \beta^{+} \mathrm{p}=0.031$ \\
\hline${ }^{141} \mathrm{Gd}^{m}$ & -62846 & 20 & 377.8 & 0.2 & & 24.5 & s & 0.5 & $\left(11 / 2^{-}\right)$ & 01 & & & $\beta^{+}=892 ; \mathrm{IT}=112$ \\
\hline${ }^{141} \mathrm{~Tb}$ & -54540 & 110 & & & $*$ & 3.5 & s & 0.2 & $\left(5 / 2^{-}\right)$ & 01 & & & $\beta^{+}=100$ \\
\hline${ }^{141} \mathrm{~Tb}^{m}$ & $-54540 \#$ & $230 \#$ & 0\# & $200 \#$ & $\mathrm{EU} *$ & 7.9 & s & 0.6 & $11 / 2^{-} \#$ & 01 & 88Be.A & I & $\beta^{+}=100$ \\
\hline${ }^{141}$ Dy & $-45320 \#$ & $300 \#$ & & & & 900 & $\mathrm{~ms}$ & 200 & $\left(9 / 2^{-}\right)$ & 01 & & & $\beta^{+}=100 ; \beta^{+} \mathrm{p}=?$ \\
\hline${ }^{141} \mathrm{Ho}$ & $-34370 \#$ & $500 \#$ & & & & 4.1 & $\mathrm{~ms}$ & 0.3 & $\left(7 / 2^{-}\right)$ & 02 & & & $\mathrm{p}=? ; \beta^{+}=1 \#$ \\
\hline${ }^{141} \mathrm{Ho}^{m}$ & $-34300 \#$ & $500 \#$ & 66 & 2 & & 6.4 & $\mu \mathrm{s}$ & 0.8 & $\left(1 / 2^{+}\right)$ & 02 & $01 \mathrm{Se} 03$ & ET & $\mathrm{p}=100$ \\
\hline${ }^{141} \mathrm{~Tb}^{m}$ & $\begin{array}{l}\mathrm{I}: \text { existenc } \\
\mathrm{T} \cdot \text { from } 0\end{array}$ & discu & $\mathrm{d}$ in & A. & visio & acce & & & & & & & \\
\hline
\end{tabular}




\begin{tabular}{|c|c|c|c|c|c|c|c|c|c|c|c|c|c|c|}
\hline Nuclide & \multicolumn{2}{|c|}{$\begin{array}{c}\text { Mass excess } \\
(\mathrm{keV})\end{array}$} & \multicolumn{3}{|c|}{$\begin{array}{l}\text { Excitation } \\
\text { energy }(\mathrm{keV})\end{array}$} & \multicolumn{3}{|c|}{ Half-life } & \multirow{2}{*}{$\begin{array}{l}J^{\pi} \\
0^{+}\end{array}$} & \multirow{2}{*}{$\begin{array}{l}\text { Ens } \\
00\end{array}$} & \multicolumn{2}{|c|}{ Reference } & \multicolumn{2}{|l|}{$\begin{array}{l}\text { Decay modes and } \\
\text { intensities }(\%)\end{array}$} \\
\hline${ }^{142} \mathrm{Te}$ & $-47430 \#$ & $600 \#$ & & & & $50 \#$ & $\mathrm{~ms}$ & $(>300 \mathrm{~ns})$ & & & $94 \mathrm{Be} 24$ & I & $\beta^{-?}$ & \\
\hline${ }^{142} \mathrm{I}$ & $-55720 \#$ & $400 \#$ & & & & 200 & $\mathrm{~ms}$ & & $2^{-} \#$ & 00 & & & $\beta^{-}=100 ; \beta^{-} \mathrm{n}=25 \#$ & \\
\hline${ }^{142} \mathrm{Xe}$ & -65480 & 100 & & & & 1.22 & $\mathrm{~s}$ & 0.02 & $0^{+}$ & 00 & 03Be05 & TD & $\beta^{-}=100 ; \beta^{-} \mathrm{n}=0.363$ & \\
\hline${ }^{142} \mathrm{Cs}$ & -70515 & 11 & & & & 1.689 & $\mathrm{~s}$ & 0.011 & $0^{-}$ & 00 & 93Ru01 & $\mathrm{T}$ & $\beta^{-}=100 ; \beta^{-} \mathrm{n}=0.0904$ & * \\
\hline${ }^{142} \mathrm{Ba}$ & -77823 & 6 & & & & 10.6 & $\mathrm{~m}$ & 0.2 & $0^{+}$ & 00 & & & $\beta^{-}=100$ & * \\
\hline${ }^{142} \mathrm{La}$ & -80035 & 6 & & & & 91.1 & $\mathrm{~m}$ & 0.5 & $2^{-}$ & 00 & & & $\beta^{-}=100$ & \\
\hline${ }^{142} \mathrm{Ce}$ & -84538.5 & 3.0 & & & & STABLE & & $(>50 \mathrm{Py})$ & $0^{+}$ & 00 & & & $\mathrm{IS}=11.11451 ; \alpha ? ; 2 \beta^{-} ?$ & $*$ \\
\hline${ }^{142} \operatorname{Pr}$ & -83792.7 & 2.5 & & & & 19.12 & $\mathrm{~h}$ & 0.04 & $2^{-}$ & 00 & & & $\beta^{-} \approx 100 ; \varepsilon=0.01648$ & \\
\hline${ }^{142} \operatorname{Pr}^{m}$ & -83789.0 & 2.5 & 3.694 & 0.003 & & 14.6 & $\mathrm{~m}$ & 0.5 & $5^{-}$ & 00 & & & $\mathrm{IT}=100$ & \\
\hline${ }^{142} \mathrm{Nd}$ & -85955.2 & 2.3 & & & & STABLE & & & $0^{+}$ & 00 & & & $\mathrm{IS}=27.25$ & \\
\hline${ }^{142} \mathrm{Pm}$ & -81157 & 25 & & & & 40.5 & s & 0.5 & $1^{+}$ & 00 & & & $\beta^{+}=100$ & \\
\hline${ }^{142} \mathrm{Pm}^{m}$ & -80274 & 25 & 883.17 & 0.16 & & 2.0 & $\mathrm{~ms}$ & 0.2 & $(8)^{-}$ & 00 & & & $\mathrm{IT}=100$ & \\
\hline${ }^{142} \mathrm{Sm}$ & -78993 & 6 & & & & 72.49 & $\mathrm{~m}$ & 0.05 & $0^{+}$ & 00 & & & $\beta^{+}=100$ & \\
\hline${ }^{142} \mathrm{Eu}$ & -71320 & 30 & & & & 2.36 & $\mathrm{~s}$ & 0.10 & $1^{+}$ & 00 & 91Fi03 & $\mathrm{T}$ & $\beta^{+}=100$ & * \\
\hline${ }^{142} \mathrm{Eu}^{m}$ & -70856 & 12 & 460 & 30 & $\mathrm{BD}$ & 1.223 & $\mathrm{~m}$ & 0.008 & $8^{-}$ & 00 & & & $\beta^{+}=100$ & \\
\hline${ }^{142} \mathrm{Gd}$ & -66960 & 28 & & & & 70.2 & $\mathrm{~s}$ & 0.6 & $0^{+}$ & 00 & & & $\beta^{+}=100$ & \\
\hline${ }^{142} \mathrm{~Tb}$ & $-57060 \#$ & $300 \#$ & & & & 597 & $\mathrm{~ms}$ & 17 & $1^{+}$ & 00 & & & $\beta^{+}=100 ; \beta^{+} \mathrm{p}=0.002211$ & \\
\hline${ }^{142} \mathrm{~Tb}^{m}$ & $-56780 \#$ & $300 \#$ & 280.2 & 1.0 & & 303 & $\mathrm{~ms}$ & 17 & $\left(5^{-}\right)$ & 00 & & & $\mathrm{IT} \approx 100 ; \beta^{+}<0.5$ & \\
\hline${ }^{142}$ Dy & $-49960 \#$ & $360 \#$ & & & & 2.3 & $\mathrm{~s}$ & 0.3 & $0^{+}$ & 00 & & & $\beta^{+}=100 ; \beta^{+} \mathrm{p}=0.063$ & \\
\hline${ }^{142} \mathrm{Ho}$ & $-37470 \#$ & $500 \#$ & & & & 400 & $\mathrm{~ms}$ & 100 & $(6 t o 9)$ & 02 & & & $\beta^{+} \approx 100 ; \beta^{+} \mathrm{p}=? ; \mathrm{p} \approx 0$ & \\
\hline${ }^{142} \mathrm{Cs}$ & $\mathrm{T}:$ average & e $93 \mathrm{RuC}$ & $1=1.684$ & 014) 77 & $\operatorname{Re} 05=$ & $.70(0.02$ & & & & & & & & $* *$ \\
\hline${ }^{142} \mathrm{Ba}$ & $\mathrm{D}: \beta^{-} \mathrm{n}=0$ & $0.091(0$. & $003) \%$ in & NSDF'O & contr & dicts $Q(\beta$ & $\left.3^{-} n\right)=$ & $=-2955(7)$ & $\mathrm{keV}$ & & & & & $* *$ \\
\hline${ }^{142} \mathrm{Ce}$ & $\mathrm{T}$ : lower $\mathrm{l}$ & limit is & or $\alpha$ deca & ; for $\beta$ & decay & $1 \mathrm{Da} 22>$ & $>260$ & & & & & & & $* *$ \\
\hline${ }^{142} \mathrm{Eu}$ & $\mathrm{T}$ : average & e $91 \mathrm{Fi} 0$ & $3=2.34(0.1$ & 2) $75 \mathrm{Ke}$ & $8=2.4$ & $0.2)$ & & & & & & & & $* *$ \\
\hline
\end{tabular}

\begin{tabular}{|c|c|c|c|c|c|c|c|c|c|c|c|c|c|}
\hline${ }^{143} I$ & $-51640 \#$ & $400 \#$ & & & & $100 \#$ & $\mathrm{~ms}$ & $>300 \mathrm{~ns})$ & $7 / 2^{+} \#$ & 02 & $94 \mathrm{Be} 24$ & I & $\beta^{-} ? ; \beta^{-} \mathrm{n}=40 \#$ \\
\hline${ }^{143} \mathrm{Xe}$ & $-60450 \#$ & $200 \#$ & & & & 511 & $\mathrm{~ms}$ & 6 & $5 / 2^{-}$ & 02 & 03Be05 & TD & $\beta^{-}=100 ; \beta^{-} \mathrm{n}=1.0015$ \\
\hline${ }^{143} \mathrm{Cs}$ & -67671 & 24 & & & & 1.791 & $\mathrm{~s}$ & 0.007 & $3 / 2^{+}$ & 02 & & & $\beta^{-}=100 ; \beta^{-} \mathrm{n}=1.647$ \\
\hline${ }^{143} \mathrm{Ba}$ & -73936 & 13 & & & & 14.5 & s & 0.3 & $5 / 2^{-}$ & 02 & & & $\beta^{-}=100$ \\
\hline${ }^{143} \mathrm{La}$ & -78187 & 15 & & & & 14.2 & $\mathrm{~m}$ & 0.1 & $(7 / 2)^{+}$ & 02 & & & $\beta^{-}=100$ \\
\hline${ }^{143} \mathrm{Ce}$ & -81612.0 & 3.0 & & & & 33.039 & $\mathrm{~h}$ & 0.006 & $3 / 2^{-}$ & 02 & & & $\beta^{-}=100$ \\
\hline${ }^{143} \mathrm{Pr}$ & -83073.5 & 2.6 & & & & 13.57 & $\mathrm{~d}$ & 0.02 & $7 / 2^{+}$ & 02 & & & $\beta^{-}=100$ \\
\hline${ }^{143} \mathrm{Nd}$ & -84007.4 & 2.3 & & & & STABLE & & & $7 / 2^{-}$ & 02 & & & $\mathrm{IS}=12.22$ \\
\hline${ }^{143} \mathrm{Pm}$ & -82966 & 3 & & & & 265 & $\mathrm{~d}$ & 7 & $5 / 2^{+}$ & 02 & & & $\varepsilon=100 ; \mathrm{e}^{+}<5.7 \mathrm{e}-6$ \\
\hline${ }^{143} \mathrm{Pm}^{m}$ & -82006 & 3 & 959.73 & 0.13 & & 24.0 & ns & 0.7 & $11 / 2^{-}$ & 02 & & & $\mathrm{IT}=100$ \\
\hline${ }^{143} \mathrm{Sm}$ & -79523 & 4 & & & & 8.75 & $\mathrm{~m}$ & 0.08 & $3 / 2^{+}$ & 02 & & & $\beta^{+}=100$ \\
\hline${ }^{143} \mathrm{Sm}^{m}$ & -78769 & 4 & 753.99 & 0.16 & & 66 & $\mathrm{~s}$ & 2 & $11 / 2^{-}$ & 02 & & & $\mathrm{IT} \approx 100 ; \beta^{+}=0.246$ \\
\hline${ }^{143} \mathrm{Sm}^{n}$ & -76729 & 4 & 2793.8 & 0.13 & & 30 & $\mathrm{~ms}$ & 3 & $23 / 2^{(-)}$ & 02 & & & $\mathrm{IT}=100$ \\
\hline${ }^{143} \mathrm{Eu}$ & -74242 & 11 & & & & 2.59 & $\mathrm{~m}$ & 0.02 & $5 / 2^{+}$ & 02 & & & $\beta^{+}=100$ \\
\hline${ }^{143} \mathrm{Eu}^{m}$ & -73852 & 11 & 389.51 & 0.04 & & 50.0 & $\mu \mathrm{s}$ & 0.5 & $11 / 2^{-}$ & 02 & & & $\mathrm{IT}=100$ \\
\hline${ }^{143} \mathrm{Gd}$ & -68230 & 200 & & & & 39 & $\mathrm{~s}$ & 2 & $(1 / 2)^{+}$ & 02 & $78 \mathrm{FiO} 2$ & $\mathrm{D}$ & $\beta^{+}=100 ; \beta^{+} \mathrm{p}=? ; \beta^{+} \alpha=?$ \\
\hline${ }^{143} \mathrm{Gd}^{m}$ & -68080 & 200 & 152.6 & 0.5 & & 110.0 & $\mathrm{~s}$ & 1.4 & $\left(11 / 2^{-}\right)$ & 02 & $78 \mathrm{Fi} 02$ & $\mathrm{D}$ & $\beta^{+}=100 ; \beta^{+} \mathrm{p}=? ; \beta^{+} \alpha=$ ? \\
\hline${ }^{143} \mathrm{~Tb}$ & -60430 & 60 & & & * & 12 & $\mathrm{~s}$ & 1 & $\left(11 / 2^{-}\right)$ & 01 & & & $\beta^{+}=100$ \\
\hline${ }^{143} \mathrm{~Tb}^{m}$ & $-60430 \#$ & $120 \#$ & 0\# & $100 \#$ & $*$ & $<21$ & $\mathrm{~s}$ & & $5 / 2^{+} \#$ & 01 & & & $\beta^{+}$? \\
\hline${ }^{143}$ Dy & $-52320 \#$ & $200 \#$ & & & & 5.6 & s & 1.0 & $\left(1 / 2^{+}\right)$ & 01 & 03Xu04 & $\mathrm{TJ}$ & $\beta^{+}=100 ; \beta^{+} \mathrm{p}=?$ \\
\hline${ }^{143} \mathrm{Dy}^{m}$ & $-52010 \#$ & $200 \#$ & 310.7 & 0.6 & & 3.0 & $\mathrm{~s}$ & 0.3 & $\left(11 / 2^{-}\right)$ & 01 & 03Xu04 & JTD & $\beta^{+}=100 ; \beta^{+} \mathrm{p}=$ ? \\
\hline${ }^{143} \mathrm{Ho}$ & $-42280 \#$ & $400 \#$ & & & & $300 \#$ & $\mathrm{~ms}$ & $>200 \mathrm{~ns})$ & $11 / 2^{-} \#$ & 01 & 00So11 & I & $\beta^{+}$? \\
\hline${ }^{143} \mathrm{Er}$ & $-31350 \#$ & $600 \#$ & & & & $200 \#$ & $\mathrm{~ms}$ & & $9 / 2^{-} \#$ & & & & $\beta^{+}$? \\
\hline${ }^{143} \mathrm{Gd}$ & $\mathrm{D}: 78 \mathrm{Fi} 02$ & $2: \beta^{+} \mathrm{p}$ & 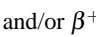 & $f$ & & $i^{m}=0.0$ & $01 \%$, & 0 & es detecte & & & & \\
\hline$*^{143}$ Dy & $\mathrm{T}$ : others: & $84 \mathrm{Ni} 0$ & $3=3.2(0.6$ & $3 \mathrm{Ni05}=$ & $0.3)$ & in two $d$ & differ & t experis & iments & & & & \\
\hline
\end{tabular}




\begin{tabular}{|c|c|c|c|c|c|c|c|c|c|c|c|c|c|c|}
\hline Nuclide & \multicolumn{2}{|c|}{$\begin{array}{l}\text { Mass excess } \\
(\mathrm{keV})\end{array}$} & \multicolumn{3}{|c|}{$\begin{array}{l}\text { Excitation } \\
\text { energy }(\mathrm{keV})\end{array}$} & \multicolumn{3}{|c|}{ Half-life } & \multirow{2}{*}{$\begin{array}{c}J^{\pi} \\
1^{-\#}\end{array}$} & \multirow{2}{*}{$\begin{array}{c}\text { Ens } \\
01\end{array}$} & \multicolumn{2}{|c|}{ Reference } & \multicolumn{2}{|l|}{$\begin{array}{l}\text { Decay modes and } \\
\text { intensities }(\%)\end{array}$} \\
\hline${ }^{144} \mathrm{I}$ & $-46580 \#$ & $500 \#$ & & & & $50 \#$ & $\mathrm{~ms}$ & $(>300 \mathrm{~ns})$ & & & $94 \mathrm{Be} 24$ & I & $\beta^{-} ? ; \beta^{-} \mathrm{n}=40 \#$ & \\
\hline${ }^{144} \mathrm{Xe}$ & $-57280 \#$ & $300 \#$ & & & & 388 & $\mathrm{~ms}$ & 7 & $0^{+}$ & 01 & 03Be05 & TD & $\beta^{-}=100 ; \beta^{-} \mathrm{n}=3.03$ & \\
\hline${ }^{144} \mathrm{Cs}$ & -63270 & 26 & & & * & 994 & $\mathrm{~ms}$ & 4 & $1^{(-\#)}$ & 01 & & & $\beta^{-}=100 ; \beta^{-} \mathrm{n}=3.2021$ & \\
\hline${ }^{144} \mathrm{Cs}^{m}$ & $-62970 \#$ & $200 \#$ & $300 \#$ & $200 \#$ & $*$ & $<1$ & $\mathrm{~s}$ & & $(>3)$ & 01 & & & $\beta^{-}=? ;$ IT $?$ & \\
\hline${ }^{144} \mathrm{Ba}$ & -71769 & 13 & & & & 11.5 & $\mathrm{~s}$ & 0.2 & $0^{+}$ & 01 & & & $\beta^{-}=100$ & $*$ \\
\hline${ }^{144} \mathrm{La}$ & -74890 & 50 & & & & 40.8 & $\mathrm{~s}$ & 0.4 & $\left(3^{-}\right)$ & 01 & & & $\beta^{-}=100$ & \\
\hline${ }^{144} \mathrm{Ce}$ & -80437 & 3 & & & & 284.91 & $\mathrm{~d}$ & 0.05 & $0^{+}$ & 01 & & & $\beta^{-}=100$ & \\
\hline${ }^{144} \operatorname{Pr}$ & -80756 & 3 & & & & 17.28 & $\mathrm{~m}$ & 0.05 & $0^{-}$ & 01 & & & $\beta^{-}=100$ & \\
\hline${ }^{144} \mathrm{Pr}^{m}$ & -80697 & 3 & 59.03 & 0.03 & & 7.2 & $\mathrm{~m}$ & 0.3 & $3^{-}$ & 01 & & & $\mathrm{IT} \approx 100 ; \beta^{-}=0.07$ & \\
\hline${ }^{144} \mathrm{Nd}$ & -83753.2 & 2.3 & & & & 2.29 & Py & 0.16 & $0^{+}$ & 01 & & & $\mathrm{IS}=23.83 ; \alpha=100$ & \\
\hline${ }^{144} \mathrm{Pm}$ & -81421 & 3 & & & & 363 & $\mathrm{~d}$ & 14 & $5^{-}$ & 01 & 94Hi05 & $\mathrm{D}$ & $\varepsilon=100 ; \mathrm{e}^{+}<8 \mathrm{e}-5$ & \\
\hline${ }^{144} \mathrm{Pm}^{m}$ & -80580 & 3 & 840.90 & 0.05 & & 780 & $\mathrm{~ns}$ & 200 & $(9)^{+}$ & 01 & & & $\mathrm{IT}=100$ & \\
\hline${ }^{144} \mathrm{Pm}^{n}$ & -72825 & 4 & 8595.8 & 2.2 & & 2.7 & $\mu \mathrm{s}$ & & $\left(27^{+}\right)$ & 01 & & & $\mathrm{IT}=100$ & \\
\hline${ }^{144} \mathrm{Sm}$ & -81972.0 & 2.8 & & & & STABLE & & & $0^{+}$ & 01 & & & $\mathrm{IS}=3.077 ; 2 \beta^{+} ? ; \alpha ?$ & \\
\hline${ }^{144} \mathrm{Sm}^{m}$ & -79648.4 & 2.8 & 2323.60 & 0.08 & & 880 & ns & 25 & $6^{+}$ & 01 & & & $\mathrm{IT}=100$ & \\
\hline${ }^{144} \mathrm{Eu}$ & -75622 & 11 & & & & 10.2 & $\mathrm{~s}$ & 0.1 & $1^{+}$ & 01 & & & $\beta^{+}=100$ & \\
\hline${ }^{144} \mathrm{Eu}^{m}$ & -74494 & 11 & 1127.6 & 0.6 & & 1.0 & $\mu \mathrm{s}$ & 0.1 & $\left(8^{-}\right)$ & 01 & & & $\mathrm{IT}=100$ & \\
\hline${ }^{144} \mathrm{Gd}$ & -71760 & 28 & & & & 4.47 & $\mathrm{~m}$ & 0.06 & $0^{+}$ & 01 & & & $\beta^{+}=100$ & \\
\hline${ }^{144} \mathrm{~Tb}$ & -62368 & 28 & & & & 1 & $\mathrm{~s}$ & & $1^{+}$ & 01 & & & $\beta^{+}=100 ; \beta^{+} \mathrm{p} ?$ & \\
\hline${ }^{144} \mathrm{~Tb}^{m}$ & -61971 & 28 & 396.9 & 0.5 & & 4.25 & $\mathrm{~s}$ & 0.15 & $\left(6^{-}\right)$ & 01 & & & $\mathrm{IT}=66 ; \beta^{+}=34 ; \beta^{+} \mathrm{p} ?$ & \\
\hline${ }^{144} \mathrm{~Tb}^{n}$ & -61892 & 28 & 476.2 & 0.5 & & 2.8 & $\mu \mathrm{s}$ & 0.3 & $\left(8^{-}\right)$ & 01 & & & $\mathrm{IT}=100$ & \\
\hline${ }^{144} \mathrm{~Tb}^{p}$ & -61851 & 28 & 517.1 & 0.5 & & 670 & ns & 60 & $\left(9^{+}\right)$ & 01 & & & $\mathrm{IT}=100$ & \\
\hline${ }^{144} \mathrm{Dy}$ & -56580 & 30 & & & & 9.1 & $\begin{array}{c}10 \mathrm{~s} \\
\mathrm{~s}\end{array}$ & 0.4 & $0^{+}$ & 01 & & & $\beta^{+}=100 ; \beta^{+} \mathrm{p}=$ ? & \\
\hline${ }^{144} \mathrm{Ho}$ & $-45200 \#$ & $300 \#$ & & & & 700 & $\mathrm{~ms}$ & 100 & & 01 & & & $\beta^{+}=100 ; \beta^{+} \mathrm{p}=$ ? & \\
\hline${ }^{144} \mathrm{Er}$ & $-36910 \#$ & $400 \#$ & & & & $400 \#$ & $\mathrm{~ms}$ & $(>200 \mathrm{~ns})$ & $0^{+}$ & 01 & 00So11 & I & $\beta^{+} ?$ & \\
\hline$*^{144} \mathrm{Ba}$ & $\mathrm{D}: \beta^{-} \mathrm{n}=3$ & $3.67 \mathrm{in}$ & ENSDF'01 & belongs i & fact & to ${ }^{144} \mathrm{Cs}$ & & & & & & & & $* *$ \\
\hline${ }^{145} \mathrm{Xe}$ & $-52100 \#$ & $300 \#$ & & & & 188 & $\mathrm{~ms}$ & 4 & $3 / 2^{-} \#$ & 97 & 03Be05 & $\mathrm{TD}$ & $\beta^{-}=1$ & \\
\hline${ }^{145} \mathrm{Cs}$ & -60057 & 11 & & & & 582 & $\mathrm{~ms}$ & 6 & $3 / 2^{+}$ & 93 & 93Ru01 & TD & $\beta^{-}=100 ; \beta^{-} \mathrm{n}$ & $*$ \\
\hline${ }^{145} \mathrm{Ba}$ & -67410 & 70 & & & & 4.31 & $\mathrm{~s}$ & 0.16 & $5 / 2^{-}$ & 98 & & & $\beta^{-}=100$ & \\
\hline${ }^{145} \mathrm{La}$ & -72990 & 90 & & & & 24.8 & $\mathrm{~s}$ & 2.0 & $\left(5 / 2^{+}\right)$ & 98 & 96Ur02 & $\mathrm{J}$ & $\beta^{-}=100$ & \\
\hline${ }^{145} \mathrm{Ce}$ & -77100 & 40 & & & & 3.01 & $\mathrm{~m}$ & 0.06 & $(3 / 2)^{-}$ & 93 & & & $\beta^{-}=100$ & \\
\hline${ }^{145} \mathrm{Pr}$ & -79632 & 7 & & & & 5.984 & $\mathrm{~h}$ & 0.010 & $7 / 2^{+}$ & 93 & & & $\beta^{-}=100$ & \\
\hline${ }^{145} \mathrm{Nd}$ & -81437.1 & 2.3 & & & & STABLE & & & $7 / 2^{-}$ & 93 & & & $\mathrm{IS}=8.31$ & \\
\hline${ }^{145} \mathrm{Pm}$ & -81274 & 3 & & & & 17.7 & $\mathrm{y}$ & 0.4 & $5 / 2^{+}$ & 93 & & & $\varepsilon=100 ; \alpha=2.8 \mathrm{e}-7$ & \\
\hline${ }^{145} \mathrm{Sm}$ & -80657.7 & 2.8 & & & & 340 & d & 3 & $7 / 2^{-}$ & 02 & & & $\varepsilon=100$ & \\
\hline${ }^{145} \mathrm{Sm}^{m}$ & -71871.5 & 2.9 & 8786.2 & 0.7 & & 990 & ns & 170 & $\left(49 / 2^{+}\right)$ & 02 & & & $\mathrm{IT}=100$ & \\
\hline${ }^{145} \mathrm{Eu}$ & -77998 & 4 & & & & 5.93 & $\mathrm{~d}$ & 0.04 & $5 / 2^{+}$ & 93 & & & $\beta^{+}=100$ & \\
\hline${ }^{145} \mathrm{Eu}^{m}$ & -77282 & 4 & 716.0 & 0.3 & & 490 & $\mathrm{~ns}$ & & $11 / 2^{-}$ & 93 & & & $\mathrm{IT}=100$ & \\
\hline${ }^{145} \mathrm{Gd}$ & -72927 & 19 & & & & 23.0 & $\mathrm{~m}$ & 0.4 & $1 / 2^{+}$ & 01 & & & $\beta^{+}=100$ & \\
\hline${ }^{145} \mathrm{Gd}^{m}$ & -72178 & 19 & 749.1 & 0.2 & & 85 & $\mathrm{~s}$ & 3 & $11 / 2^{-}$ & 01 & & & $\mathrm{IT}=94.35 ; \beta^{+}=5.75$ & \\
\hline${ }^{145} \mathrm{~Tb}$ & -65880 & 60 & & & * & $20 \#$ & $\mathrm{~m}$ & & $\left(3 / 2^{+}\right)$ & 96 & 93То04 & $\mathrm{J}$ & $\beta^{+}$? & \\
\hline${ }^{145} \mathrm{~Tb}^{m}$ & $-65880 \#$ & $120 \#$ & $0 \#$ & $100 \#$ & $*$ & 30.9 & $\mathrm{~s}$ & 0.7 & $\left(11 / 2^{-}\right)$ & 96 & $93 \mathrm{Al} 03$ & $\mathrm{~T}$ & $\beta^{+}=100$ & $*$ \\
\hline${ }^{145}$ Dy & -58290 & 50 & & & & 9.5 & $\mathrm{~s}$ & 1.0 & $\left(1 / 2^{+}\right)$ & 93 & 93A103 & $\mathrm{T}$ & $\beta^{+}=100 ; \beta^{+} \mathrm{p}=$ ? & $*$ \\
\hline${ }^{145} \mathrm{Dy}^{m}$ & -58170 & 50 & 118.2 & 0.2 & & 14.1 & $\mathrm{~s}$ & 0.7 & $\left(11 / 2^{-}\right)$ & 93 & 93Тo04 & $\mathrm{T}$ & $\beta^{+}=100$ & * \\
\hline${ }^{145} \mathrm{Ho}$ & $-49180 \#$ & $300 \#$ & & & $*$ & 2.4 & $\mathrm{~s}$ & 0.1 & $\left(11 / 2^{-}\right)$ & 93 & & & $\beta^{+}=100$ & \\
\hline${ }^{145} \mathrm{Ho}^{m}$ & $-49080 \#$ & $320 \#$ & $100 \#$ & $100 \#$ & $*$ & $100 \#$ & $\mathrm{~ms}$ & & $5 / 2^{+} \#$ & & & & $\beta^{+} ? ;$ IT ? & \\
\hline${ }^{145} \mathrm{Er}$ & $-39690 \#$ & $400 \#$ & & & & 900 & $\mathrm{~ms}$ & 300 & $1 / 2^{+} \#$ & 98 & & & $\beta^{+}=100 ; \beta^{+} \mathrm{p}=$ ? & \\
\hline${ }^{145} \mathrm{Tm}$ & $-27880 \#$ & $400 \#$ & & & & 3.1 & $\mu \mathrm{s}$ & 0.3 & $\left(11 / 2^{-}\right)$ & 02 & 98Ba13 & $\mathrm{TJ}$ & $\mathrm{p}=100$ & * \\
\hline$*^{145} \mathrm{Cs}$ & $\mathrm{T}$ : average & e $93 \mathrm{RuC}$ & $1=579(6)$ & 82Ra13= & & & & & & & & & & $* *$ \\
\hline$*^{145} \mathrm{~Tb}^{m}$ & $\mathrm{~T}:$ average & e $93 \mathrm{Al} 0$ & $3=31.6(0$. & 5) $82 \mathrm{NoO}$ & $=29$ & $5(1.0)$ an & nd 82 & $\mathrm{Al} 07=29.5($ & $(1.5)$ & & & & & $* *$ \\
\hline$*^{145}$ Dy & $\mathrm{T}:$ average & $93 \mathrm{Al} 0$ & $3=10.5(1$. & ) $93 \mathrm{To} 04$ & $=6($ & and $84 \mathrm{~S}$ & Sc. $C=$ & $=10(1)$ & & & & & & $* *$ \\
\hline$*^{145} \mathrm{Dy}^{m}$ & $\mathrm{~T}:$ average & $93 \mathrm{To} 0$ & $4=14.5(1$. & $82 \mathrm{NoO}$ & $=13$ & $6(1.0)$ & & & & & & & & $* *$ \\
\hline$*^{145} \mathrm{Tm}$ & $\mathrm{T}:$ average & $03 \mathrm{KaO}$ & $4=3.1(0.3$ & $98 \mathrm{Ba} 13$ & 3.5( & $1.0)$ & & not adoptec & d by ENSD & DF'02 & & & & $* *$ \\
\hline
\end{tabular}




\begin{tabular}{|c|c|c|c|c|c|c|c|c|c|c|c|c|c|c|}
\hline Nuclide & \multicolumn{2}{|c|}{$\begin{array}{l}\text { Mass excess } \\
(\mathrm{keV})\end{array}$} & \multicolumn{3}{|c|}{$\begin{array}{c}\text { Excitation } \\
\text { energy }(\mathrm{keV})\end{array}$} & \multicolumn{3}{|c|}{ Half-life } & \multirow{2}{*}{$\begin{array}{l}J^{\pi} \\
0^{+}\end{array}$} & \multirow{2}{*}{$\begin{array}{c}\text { Ens } \\
97\end{array}$} & \multicolumn{2}{|c|}{ Reference } & \multicolumn{2}{|l|}{$\begin{array}{l}\text { Decay modes and } \\
\text { intensities }(\%)\end{array}$} \\
\hline${ }^{146} \mathrm{Xe}$ & $-48670 \#$ & $400 \#$ & & & & 146 & $\mathrm{~ms}$ & 6 & & & 03Be05 & $\mathrm{TD}$ & $\beta^{-}=100 ; \beta^{-} \mathrm{n}=6.915$ & \\
\hline${ }^{146} \mathrm{Cs}$ & -55620 & 70 & & & & 323 & $\mathrm{~ms}$ & 6 & $1^{-}$ & 97 & 93Ru01 & $\mathrm{T}$ & $\beta^{-}=100 ; \beta^{-} \mathrm{n}=14.25$ & $*$ \\
\hline${ }^{146} \mathrm{Ba}$ & -65000 & 70 & & & & 2.22 & $\mathrm{~s}$ & 0.07 & $0^{+}$ & 97 & 93Ru01 & $\mathrm{D}$ & $\beta^{-}=100$ & $*$ \\
\hline${ }^{146} \mathrm{La}$ & -69120 & 70 & & & $*$ & 6.27 & $\mathrm{~s}$ & 0.10 & $2^{-}$ & 97 & 93Ru01 & $\mathrm{D}$ & $\beta^{-}=100$ & $*$ \\
\hline${ }^{146} \mathrm{La}^{m}$ & -68990 & 150 & 130 & 130 & $*$ & 10.0 & $\mathrm{~s}$ & 0.1 & $\left(6^{-}\right)$ & 97 & $79 \mathrm{Ke} 02$ & $\mathrm{E}$ & $\beta^{-}=100$ & $*$ \\
\hline${ }^{146} \mathrm{Ce}$ & -75680 & 70 & & & & 13.52 & $\mathrm{~m}$ & 0.13 & $0^{+}$ & 97 & & & $\beta^{-}=100$ & \\
\hline${ }^{146} \mathrm{Pr}$ & -76710 & 60 & & & & 24.15 & $\mathrm{~m}$ & 0.18 & $(2)^{-}$ & 97 & & & $\beta^{-}=100$ & \\
\hline${ }^{146} \mathrm{Nd}$ & -80931.1 & 2.3 & & & & STABle & & & $0^{+}$ & 97 & & & $\mathrm{IS}=17.23 ; 2 \beta^{-} ? ; \alpha ?$ & \\
\hline${ }^{146} \mathrm{Pm}$ & -79460 & 5 & & & & 5.53 & $\mathrm{y}$ & 0.05 & $3^{-}$ & 99 & & & $\varepsilon=66.013 ; \beta^{-}=34.013$ & \\
\hline${ }^{146} \mathrm{Sm}$ & -81002 & 4 & & & & 103 & My & 5 & $0^{+}$ & 97 & & & $\alpha=100$ & \\
\hline${ }^{146} \mathrm{Eu}$ & -77122 & 6 & & & & 4.61 & $\mathrm{~d}$ & 0.03 & $4^{-}$ & 97 & & & $\beta^{+}=100$ & \\
\hline${ }^{146} \mathrm{Eu}^{m}$ & -76456 & 6 & 666.37 & 0.16 & & 235 & $\mu \mathrm{s}$ & 3 & $9^{+}$ & 97 & & & $\mathrm{IT}=100$ & \\
\hline${ }^{146} \mathrm{Gd}$ & -76093 & 5 & & & & 48.27 & $\mathrm{~d}$ & 0.10 & $0^{+}$ & 01 & & & $\varepsilon=100$ & \\
\hline${ }^{146} \mathrm{~Tb}$ & -67770 & 50 & & & $*$ & 8 & $\mathrm{~s}$ & 4 & $1^{+}$ & 97 & & & $\beta^{+}=100$ & \\
\hline${ }^{146} \mathrm{~Tb}^{m}$ & $-67620 \#$ & $110 \#$ & $150 \#$ & $100 \#$ & $*$ & 24.1 & $\mathrm{~s}$ & 0.5 & $5^{-}$ & 97 & $93 \mathrm{Al} 03$ & $\mathrm{~T}$ & $\beta^{+}=100$ & \\
\hline${ }^{146} \mathrm{~Tb}^{n}$ & $-66840 \#$ & $110 \#$ & $930 \#$ & $100 \#$ & & 1.18 & $\mathrm{~ms}$ & 0.02 & $\left(10^{+}\right)$ & 97 & & & $\mathrm{IT}=100$ & $*$ \\
\hline${ }^{146}$ Dy & -62554 & 27 & & & & 33.2 & $\mathrm{~s}$ & 0.7 & $0^{+}$ & 97 & $93 \mathrm{Al} 03$ & $\mathrm{~T}$ & $\beta^{+}=100$ & \\
\hline${ }^{146} \mathrm{Dy}^{m}$ & -59618 & 27 & 2935.7 & 0.6 & & 150 & $\mathrm{~ms}$ & 20 & $10^{+} \#$ & 97 & & & $\mathrm{IT}=100$ & \\
\hline${ }^{146} \mathrm{Ho}$ & $-51570 \#$ & $200 \#$ & & & & 3.6 & $\mathrm{~s}$ & 0.3 & $\left(10^{+}\right)$ & 97 & & & $\beta^{+}=100 ; \beta^{+} \mathrm{p}=?$ & \\
\hline${ }^{146} \mathrm{Er}$ & $-44710 \#$ & $300 \#$ & & & & 1.7 & $\mathrm{~s}$ & 0.6 & $0^{+}$ & 97 & 93To05 & $\mathrm{D}$ & $\beta^{+}=100 ; \beta^{+} \mathrm{p}=$ ? & \\
\hline${ }^{146} \mathrm{Tm}$ & $-31280 \#$ & $400 \#$ & & & & 240 & $\mathrm{~ms}$ & 30 & $\left(6^{-}\right)$ & 02 & & & $\mathrm{p} \approx 100 ; \beta^{+} ?$ & \\
\hline${ }^{146} \mathrm{Tm}^{m}$ & $-31200 \#$ & $400 \#$ & 71 & 6 & $\mathrm{p}$ & 72 & $\mathrm{~ms}$ & 23 & $\left(10^{+}\right)$ & 02 & & & $\mathrm{p}=? ; \beta^{+}=16 \#$ & \\
\hline$*^{146} \mathrm{Cs}$ & $\mathrm{T}$ : average & $93 \mathrm{Ru}$ & $1=321(2)$ & $76 \mathrm{Lu} 02$ & $=343(7$ & & & & & & & & & $* *$ \\
\hline$*^{146} \mathrm{Ba}$ & $\mathrm{D}: 93 \mathrm{Ru} 01$ & $1 \beta^{-} \mathrm{n}<$ & $0.02 \%$ is & not relev & ant sin & $Q\left(\beta^{-} \mathrm{n}\right)$ & is ne & ative & $-190(10$ & & & & & $* *$ \\
\hline$*^{146} \mathrm{La}$ & D : 93Ru01 & $1 \beta^{-} \mathrm{n}<$ & $0.007 \% \mathrm{i}$ & not rele & vant $s$ & $Q\left(\beta^{-} n\right.$ & ) is $n$ & gative & $=-180(8$ & & & & & $* *$ \\
\hline$*^{146} \mathrm{La}^{m}$ & $\mathrm{E}$ : derived & from $C$ & $\left({ }^{146} \mathrm{La}^{m}\right)$ & $=6660(12$ & 0 ) in 7 & $\mathrm{Ke} 02$ & & & & & & & & $* *$ \\
\hline${ }^{146} \mathrm{~Tb}^{n}$ & $\mathrm{E}: 779.6 \mathrm{k}$ & $\mathrm{eV}$ abo & ve ${ }^{146} \mathrm{~Tb}^{m}$ & from $\mathrm{E}$ & NSDF & & & & & & & & & $* *$ \\
\hline
\end{tabular}

\begin{tabular}{|c|c|c|c|c|c|c|c|c|c|c|c|c|c|c|}
\hline${ }^{147} \mathrm{Xe}$ & $-43260 \#$ & 400\# & & & & 130 & $\mathrm{~ms}$ & 80 & $3 / 2^{-} \#$ & 98 & 03Be05 & $\mathrm{TD}$ & $\beta^{-}=100 ; \beta^{-} \mathrm{n}=4.023$ & * \\
\hline${ }^{147} \mathrm{Cs}$ & -52020 & 50 & & & & 225 & $\mathrm{~ms}$ & 5 & $\left(3 / 2^{+}\right)$ & 92 & 93Ru01 & $\mathrm{D}$ & $\beta^{-}=100 ; \beta^{-} \mathrm{n}=28.517$ & \\
\hline${ }^{147} \mathrm{Ba}$ & $-60600 \#$ & $210 \#$ & & & & 893 & $\mathrm{~ms}$ & 1 & $\left(3 / 2^{+}\right)$ & 98 & 93Ru01 & $\mathrm{D}$ & $\beta^{-}=100$ & $*$ \\
\hline${ }^{147} \mathrm{La}$ & -66850 & 50 & & & & 4.015 & $\mathrm{~s}$ & 0.008 & $\left(5 / 2^{+}\right)$ & 98 & 93Ru01 & $\mathrm{D}$ & $\beta^{-}=100 ; \beta^{-} \mathrm{n}=0.0403$ & * \\
\hline${ }^{147} \mathrm{Ce}$ & -72030 & 30 & & & & 56.4 & $\mathrm{~s}$ & 1.0 & $\left(5 / 2^{-}\right)$ & 92 & & & $\beta^{-}=100$ & \\
\hline${ }^{147} \mathrm{Pr}$ & -75455 & 23 & & & & 13.4 & $\mathrm{~m}$ & 0.4 & $\left(3 / 2^{+}\right)$ & 92 & & & $\beta^{-}=100$ & \\
\hline${ }^{147} \mathrm{Nd}$ & -78151.9 & 2.3 & & & & 10.98 & d & 0.01 & $5 / 2^{-}$ & 92 & & & $\beta^{-}=100$ & \\
\hline${ }^{147} \mathrm{Pm}$ & -79047.9 & 2.4 & & & & 2.6234 & $\mathrm{y}$ & 0.0002 & $7 / 2^{+}$ & 96 & & & $\beta^{-}=100$ & \\
\hline${ }^{147} \mathrm{Sm}$ & -79272.1 & 2.4 & & & & 106.0 & Gy & 1.1 & $7 / 2^{-}$ & 92 & $70 \mathrm{Gu} 14$ & $\mathrm{~T}$ & $\mathrm{IS}=14.9918 ; \alpha=100$ & $*$ \\
\hline${ }^{147} \mathrm{Eu}$ & -77550 & 3 & & & & 24.1 & $\mathrm{~d}$ & 0.6 & $5 / 2^{+}$ & 99 & & & $\beta^{+} \approx 100 ; \alpha=0.00226$ & \\
\hline${ }^{147} \mathrm{Gd}$ & -75363 & 3 & & & & 38.06 & $\mathrm{~h}$ & 0.12 & $7 / 2^{-}$ & 99 & & & $\beta^{+}=100$ & \\
\hline${ }^{147} \mathrm{Gd}^{m}$ & -66775 & 3 & 8587.8 & 0.4 & & 510 & $\mathrm{~ns}$ & 20 & $\left(49 / 2^{+}\right)$ & 99 & & & $\mathrm{IT}=100$ & \\
\hline${ }^{147} \mathrm{~Tb}$ & -70752 & 12 & & & & 1.64 & $\mathrm{~h}$ & 0.03 & $1 / 2^{+} \#$ & 99 & $97 \mathrm{Wa04}$ & $\mathrm{T}$ & $\beta^{+}=100$ & \\
\hline${ }^{147} \mathrm{~Tb}^{m}$ & -70701 & 12 & 50.6 & 0.9 & & 1.87 & $\mathrm{~m}$ & 0.05 & $(11 / 2)^{-}$ & 99 & $93 \mathrm{Al} 03$ & $\mathrm{~T}$ & $\beta^{+}=100$ & * \\
\hline${ }^{147}$ Dy & -64188 & 20 & & & & 40 & $\mathrm{~s}$ & 10 & $1 / 2^{+}$ & 92 & $84 \mathrm{To} 07$ & $\mathrm{D}$ & $\beta^{+}=100 ; \beta^{+} \mathrm{p} \approx 0.05$ & \\
\hline${ }^{147} \mathrm{Dy}^{m}$ & -63438 & 20 & 750.5 & 0.4 & & 55 & $\mathrm{~s}$ & 1 & $11 / 2^{-}$ & 92 & & & $\beta^{+}=654 ; \mathrm{IT}=354$ & \\
\hline${ }^{147} \mathrm{Ho}$ & -55837 & 28 & & & & 5.8 & $\mathrm{~s}$ & 0.4 & $\left(11 / 2^{-}\right)$ & 92 & & & $\beta^{+}=100 ; \beta^{+} \mathrm{p} ?$ & \\
\hline${ }^{147} \mathrm{Er}$ & $-47050 \#$ & $300 \#$ & & & $* \&$ & 2.5 & s & & $\left(1 / 2^{+}\right)$ & 92 & & & $\beta^{+}=100 ; \beta^{+} \mathrm{p}=$ ? & \\
\hline${ }^{147} \mathrm{Er}^{m}$ & $-46950 \#$ & $300 \#$ & 100\# & $50 \#$ & $* \&$ & 2.5 & $\mathrm{~s}$ & 0.2 & $\left(11 / 2^{-}\right)$ & 92 & & & $\beta^{+}=100$ & $*$ \\
\hline${ }^{147} \mathrm{Tm}$ & $-36370 \#$ & $300 \#$ & & & & 580 & $\mathrm{~ms}$ & 30 & $11 / 2^{-}$ & 02 & & & $\beta^{+}=855 ; \mathrm{p}=155$ & \\
\hline${ }^{147} \mathrm{Tm}^{m}$ & $-36300 \#$ & $300 \#$ & 60 & 5 & $\mathrm{p}$ & 360 & $\mu \mathrm{s}$ & 40 & $3 / 2^{+}$ & 02 & & & $\mathrm{p}=100$ & \\
\hline$*^{147} \mathrm{Xe}$ & & & & & & & & & & & & & & $* *$ \\
\hline${ }^{147} \mathrm{Ba}$ & \multicolumn{13}{|c|}{$\mathrm{D}: 93 \mathrm{Ru} 01 \beta^{-} \mathrm{n}=0.06(3) \%$ contradicts $Q\left(\beta^{-} \mathrm{n}\right)=-340(120)$} & ** \\
\hline${ }^{*}{ }^{147} \mathrm{La}$ & \multicolumn{13}{|c|}{$\mathrm{J}$ : from $96 \mathrm{Ur} 02$} & ** \\
\hline$*^{147} \mathrm{Sm}$ & \multicolumn{13}{|c|}{$\mathrm{T}:$ average $70 \mathrm{Gu} 14=106(2) 65 \mathrm{Va} 16=108(2) 64 \mathrm{Do} 01=104(3) 61 \mathrm{Wr} 02=105(2)$} & ** \\
\hline${ }^{*}{ }^{147} \mathrm{~Tb}^{m}$ & \multicolumn{13}{|c|}{$\mathrm{T}:$ average $93 \mathrm{Al} 03=1.92(0.07) 73 \mathrm{Bo} 13=1.83(0.06)$} & ** \\
\hline$*^{147} \mathrm{Er}^{m}$ & \multicolumn{13}{|c|}{ E : estimated from $11 / 2^{-}$level in isotones ${ }^{141} \mathrm{Sm}=175^{143} \mathrm{Gd}=152{ }^{145} \mathrm{Dy}=118$} & 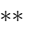 \\
\hline
\end{tabular}




\begin{tabular}{|c|c|c|c|c|c|c|c|c|c|c|c|c|c|c|}
\hline Nuclide & \multicolumn{2}{|c|}{$\begin{array}{l}\text { Mass excess } \\
\quad(\mathrm{keV})\end{array}$} & \multicolumn{2}{|c|}{$\begin{array}{l}\text { Excitation } \\
\text { energy(keV) }\end{array}$} & \multicolumn{4}{|c|}{ Half-life } & \multirow[t]{2}{*}{$J^{\pi}$} & \multirow{2}{*}{$\begin{array}{c}\text { Ens } \\
00\end{array}$} & \multicolumn{2}{|c|}{ Reference } & \multicolumn{2}{|l|}{$\begin{array}{l}\text { Decay modes and } \\
\text { intensities }(\%)\end{array}$} \\
\hline${ }^{148} \mathrm{Cs}$ & -47300 & 580 & & & & 146 & $\mathrm{~ms}$ & 6 & & & & & $\beta^{-}=100 ; \beta^{-} \mathrm{n}=25.125$ & \\
\hline${ }^{148} \mathrm{Ba}$ & -58010 & 80 & & & & 612 & $\mathrm{~ms}$ & 17 & $0^{+}$ & 00 & & & $\beta^{-}=100 ; \beta^{-} \mathrm{n}=0.43$ & \\
\hline${ }^{148} \mathrm{La}$ & -63130 & 60 & & & & 1.26 & $\mathrm{~s}$ & 0.08 & $\left(2^{-}\right)$ & 00 & & & $\beta^{-}=100 ; \beta^{-} \mathrm{n}=0.153$ & \\
\hline${ }^{148} \mathrm{Ce}$ & -70391 & 29 & & & & 56 & $\mathrm{~s}$ & 1 & $0^{+}$ & 00 & & & $\beta^{-}=100$ & \\
\hline${ }^{148} \mathrm{Pr}$ & -72531 & 26 & & & $*$ & 2.29 & $\mathrm{~m}$ & 0.02 & $1^{-}$ & 00 & & & $\beta^{-}=100$ & \\
\hline${ }^{148} \mathrm{Pr}^{m}$ & $-72480 \#$ & $40 \#$ & $50 \#$ & $30 \#$ & $*$ & 2.01 & $\mathrm{~m}$ & 0.07 & (4) & 00 & ABBW & $\mathrm{E}$ & $\beta^{-}=100$ & $*$ \\
\hline${ }^{148} \mathrm{Nd}$ & -77413.4 & 2.8 & & & & STABLE & & $(>3.0 \mathrm{Ey})$ & $0^{+}$ & 00 & $82 \mathrm{Be} 20$ & $\mathrm{~T}$ & $\mathrm{IS}=5.71 ; 2 \beta^{-} ? ; \alpha ?$ & \\
\hline${ }^{148} \mathrm{Pm}$ & -76872 & 6 & & & & 5.368 & $\mathrm{~d}$ & 0.002 & $1^{-}$ & 00 & & & $\beta^{-}=100$ & \\
\hline${ }^{148} \mathrm{Pm}^{m}$ & -76734 & 6 & 137.9 & 0.3 & & 41.29 & $\mathrm{~d}$ & 0.11 & $5^{-}, 6^{-}$ & 00 & & & $\beta^{-}=95.86 ; \mathrm{IT}=4.26$ & \\
\hline${ }^{148} \mathrm{Sm}$ & -79342.2 & 2.4 & & & & 7 & Py & 3 & $0^{+}$ & 00 & & & $\mathrm{IS}=11.2410 ; \alpha=100$ & \\
\hline${ }^{148} \mathrm{Eu}$ & -76302 & 10 & & & & 54.5 & $\mathrm{~d}$ & 0.5 & $5^{-}$ & 00 & & & $\beta^{+}=100 ; \alpha=9.4 \mathrm{e}-728$ & \\
\hline${ }^{148} \mathrm{Gd}$ & -76275.8 & 2.8 & & & & 74.6 & $\mathrm{y}$ & 3.0 & $0^{+}$ & 00 & & & $\alpha=100 ; 2 \beta^{+}$? & \\
\hline${ }^{148} \mathrm{~Tb}$ & -70540 & 14 & & & & 60 & $\mathrm{~m}$ & 1 & $2^{-}$ & 00 & & & $\beta^{+}=100$ & \\
\hline${ }^{148} \mathrm{~Tb}^{m}$ & -70450 & 14 & 90.1 & 0.3 & & 2.20 & $\mathrm{~m}$ & 0.05 & $(9)^{+}$ & 00 & & & $\beta^{+}=100$ & \\
\hline${ }^{148} \mathrm{~Tb}^{n}$ & -61921 & 14 & 8618.6 & 1.0 & & 1.310 & $\mu \mathrm{s}$ & 0.007 & $\left(27^{+}\right)$ & 00 & & & $\mathrm{IT}=100$ & \\
\hline${ }^{148}$ Dy & -67859 & 11 & & & & 3.3 & $\mathrm{~m}$ & 0.2 & $0^{+}$ & 00 & & & $\beta^{+}=100$ & \\
\hline${ }^{148} \mathrm{Ho}$ & -58020 & 130 & & & & 2.2 & $\mathrm{~s}$ & 1.1 & $\left(1^{+}\right)$ & 00 & & & $\beta^{+}=100$ & \\
\hline${ }^{148} \mathrm{Ho}^{m}$ & $-57620 \#$ & $160 \#$ & $400 \#$ & $100 \#$ & & 9.49 & s & 0.12 & $(6)^{-}$ & 00 & 93Al03 & $\mathrm{T}$ & $\beta^{+}=100 ; \beta^{+} \mathrm{p}=0.081$ & $*$ \\
\hline${ }^{148} \mathrm{Ho}^{n}$ & $-57330 \#$ & $160 \#$ & $690 \#$ & $100 \#$ & & 2.35 & $\mathrm{~ms}$ & 0.04 & $\left(10^{+}\right)$ & 00 & & & $\mathrm{IT}=100$ & $*$ \\
\hline${ }^{148} \mathrm{Er}$ & $-51650 \#$ & $200 \#$ & & & & 4.6 & $\mathrm{~s}$ & 0.2 & $0^{+}$ & 00 & & & $\beta^{+}=100 ; \beta^{+} \mathrm{p} \approx 0.15$ & \\
\hline${ }^{148} \mathrm{Tm}$ & $-39270 \#$ & $400 \#$ & & & & 700 & $\mathrm{~ms}$ & 200 & $\left(10^{+}\right)$ & 00 & & & $\beta^{+}=100$ & \\
\hline${ }^{148} \mathrm{Yb}$ & $-30350 \#$ & $600 \#$ & & & & $250 \#$ & $\mathrm{~ms}$ & & $0^{+}$ & & & & $\beta^{+} ?$ & \\
\hline$*^{148} \mathrm{Pr}^{m}$ & $\mathrm{E}:$ derivec & from 1 & ENSDF es & mate $E<9$ & $\mathrm{ke}$ & & & & & & & & & $* *$ \\
\hline$*^{148} \mathrm{Ho}^{m}$ & $\mathrm{~T}$ : averag & e $93 \mathrm{AlC}$ & $03=9.30($ & 20) $89 \mathrm{Ta} 1$ & $=9$ & $59(0.15$ & & & & & & & & $* *$ \\
\hline$*^{148} \mathrm{Ho}^{n}$ & E : $694.4 \mathrm{k}$ & $\mathrm{eV}$ abo & ve ${ }^{148} \mathrm{Ho}$ & , from EN & & & & & & & & & & $* *$ \\
\hline
\end{tabular}

\begin{tabular}{|c|c|c|c|c|c|c|c|c|c|c|c|c|c|}
\hline${ }^{149} \mathrm{Cs}$ & $-43850 \#$ & $200 \#$ & & & 150\# & $\mathrm{ms}$ & $(>50 \mathrm{~ms})$ & $3 / 2^{+} \#$ & 95 & $87 \mathrm{Ra} 12$ & I & $\beta^{-} ? ; \beta^{-} \mathrm{n} ?$ & \\
\hline${ }^{149} \mathrm{Ba}$ & $-53490 \#$ & $200 \#$ & & & 344 & $\mathrm{~ms}$ & 7 & $3 / 2^{-} \#$ & 95 & & & $\beta^{-}=100 ; \beta^{-} \mathrm{n}=0.4312$ & \\
\hline${ }^{149} \mathrm{La}$ & $-60800 \#$ & $320 \#$ & & & 1.05 & $\mathrm{~s}$ & 0.03 & $5 / 2^{+} \#$ & 95 & 93Ru01 & $\mathrm{D}$ & $\beta^{-}=100 ; \beta^{-} \mathrm{n}=1.43$ & \\
\hline${ }^{149} \mathrm{Ce}$ & -66700 & 100 & & & 5.3 & $\mathrm{~s}$ & 0.2 & $3 / 2^{-} \#$ & 98 & & & $\beta^{-}=100$ & \\
\hline${ }^{149} \mathrm{Pr}$ & -71060 & 80 & & & 2.26 & $\mathrm{~m}$ & 0.07 & $\left(5 / 2^{+}\right)$ & 95 & & & $\beta^{-}=100$ & \\
\hline${ }^{149} \mathrm{Nd}$ & -74380.9 & 2.8 & & & 1.728 & $\mathrm{~h}$ & 0.001 & $5 / 2^{-}$ & 95 & & & $\beta^{-}=100$ & \\
\hline${ }^{149} \mathrm{Pm}$ & -76071 & 4 & & & 53.08 & $\mathrm{~h}$ & 0.05 & $7 / 2^{+}$ & 95 & & & $\beta^{-}=100$ & \\
\hline${ }^{149} \mathrm{Pm}^{m}$ & -75831 & 4 & 240.214 & 0.007 & 35 & $\mu \mathrm{s}$ & 3 & $11 / 2^{-}$ & & & & & \\
\hline${ }^{149} \mathrm{Sm}$ & -77141.9 & 2.4 & & & STABLE & & (>2 Py) & $7 / 2^{-}$ & 95 & & & $\mathrm{IS}=13.827 ; \alpha ?$ & \\
\hline${ }^{149} \mathrm{Eu}$ & -76447 & 4 & & & 93.1 & $\mathrm{~d}$ & 0.4 & $5 / 2^{+}$ & 95 & & & $\varepsilon=100$ & \\
\hline${ }^{149} \mathrm{Gd}$ & -75133 & 4 & & & 9.28 & $\mathrm{~d}$ & 0.10 & $7 / 2^{-}$ & 01 & & & $\beta^{+}=100 ; \alpha=4.3 \mathrm{e}-410$ & \\
\hline${ }^{149} \mathrm{~Tb}$ & -71496 & 4 & & & 4.118 & $\mathrm{~h}$ & 0.025 & $1 / 2^{+}$ & 99 & & & $\beta^{+}=83.317 ; \alpha=16.717$ & \\
\hline${ }^{149} \mathrm{~Tb}^{m}$ & -71460 & 4 & 35.78 & 0.13 & 4.16 & $\mathrm{~m}$ & 0.04 & $11 / 2^{-}$ & 99 & & & $\beta^{+} \approx 100 ; \alpha=0.0223$ & \\
\hline${ }^{149}$ Dy & -67715 & 9 & & & 4.20 & $\mathrm{~m}$ & 0.14 & $7 / 2^{(-)}$ & 95 & 88Ah02 & $\mathrm{J}$ & $\beta^{+}=100$ & \\
\hline${ }^{149} \mathrm{Dy}^{m}$ & -65054 & 9 & 2661.1 & 0.4 & 490 & $\mathrm{~ms}$ & 15 & $\left(27 / 2^{-}\right)$ & 95 & & & $\mathrm{IT}=99.33 ; \beta^{+}=0.73$ & \\
\hline${ }^{149} \mathrm{Dy}^{n}$ & -60230 & 30 & 7490 & 30 & 28 & ns & 2 & $\left(47 / 2^{+}\right)$ & 95 & & & $\mathrm{IT}=100$ & $*$ \\
\hline${ }^{149} \mathrm{Ho}$ & -61688 & 18 & & & 21.1 & $\mathrm{~s}$ & 0.2 & $\left(11 / 2^{-}\right)$ & 95 & & & $\beta^{+}=100$ & \\
\hline${ }^{149} \mathrm{Ho}^{m}$ & -61639 & 18 & 48.80 & 0.20 & 56 & s & 3 & $\left(1 / 2^{+}\right)$ & 95 & & & $\beta^{+}=100$ & \\
\hline${ }^{149} \mathrm{Er}$ & -53742 & 28 & & & 4 & $\mathrm{~s}$ & 2 & $\left(1 / 2^{+}\right)$ & 95 & & & $\beta^{+}=100 ; \beta^{+} \mathrm{p}=72$ & \\
\hline${ }^{149} \mathrm{Er}^{m}$ & -53000 & 28 & 741.8 & 0.2 & 8.9 & s & 0.2 & $\left(11 / 2^{-}\right)$ & 95 & & & $\beta^{+}=96.57 ; \mathrm{IT}=3.57 ; \ldots$ & $*$ \\
\hline${ }^{149} \mathrm{Tm}$ & $-44040 \#$ & $300 \#$ & & & 900 & $\mathrm{~ms}$ & 200 & $\left(11 / 2^{-}\right)$ & 95 & & & $\beta^{+}=100 ; \beta^{+} \mathrm{p}=0.2615$ & \\
\hline${ }^{149} \mathrm{Yb}$ & $-33500 \#$ & $500 \#$ & & & 700 & $\mathrm{~ms}$ & 200 & $\left(1 / 2^{+}, 3 / 2^{+}\right)$ & 95 & $01 X u 06$ & $\mathrm{TD}$ & $\beta^{+}=100 ; \beta^{+} \mathrm{p}=?$ & \\
\hline$*^{149} \mathrm{Dy}^{n}$ & E : 7409.9 & above & level at $\approx 8$ & $\mathrm{keV}$ & & & & & & & & & ** \\
\hline$*^{149} \mathrm{Er}^{m}$ & $\mathrm{D}: \ldots ; \beta^{-}$ & ${ }^{+} \mathrm{p}=0.1$ & & & & & & & & & & & ** \\
\hline${ }^{150} \mathrm{Cs}$ & $-38960 \#$ & $300 \#$ & & & $100 \#$ & $\mathrm{~ms}$ & $(>50 \mathrm{~ms})$ & & 97 & 87Ra12 & I & $\beta^{-} ? ; \beta^{-} \mathrm{n} ?$ & \\
\hline${ }^{150} \mathrm{Ba}$ & $-50600 \#$ & $400 \#$ & & & 300 & $\mathrm{~ms}$ & & $0^{+}$ & 95 & & & $\beta^{-}=100 ; \beta^{-} \mathrm{n} ?$ & \\
\hline${ }^{150} \mathrm{La}$ & $-57040 \#$ & $400 \#$ & & & 510 & $\mathrm{~ms}$ & 30 & $\left(3^{+}\right)$ & 97 & $95 \mathrm{Ok} 02$ & $\mathrm{TJ}$ & $\beta^{-}=100 ; \beta^{-} \mathrm{n}=2.73$ & \\
\hline${ }^{150} \mathrm{Ce}$ & -64820 & 50 & & & 4.0 & $\mathrm{~s}$ & 0.6 & $0^{+}$ & 95 & & & $\beta^{-}=100$ & \\
\hline${ }^{150} \mathrm{Pr}$ & -68304 & 26 & & & 6.19 & s & 0.16 & $(1)^{-}$ & 96 & & & $\beta^{-}=100$ & \\
\hline${ }^{150} \mathrm{Nd}$ & -73690 & 3 & & & 6.7 & Ey & 0.7 & $0^{+}$ & 96 & 97De40 & $\mathrm{TD}$ & $\mathrm{IS}=5.62 ; 2 \beta^{-}=100$ & $*$ \\
\hline${ }^{150} \mathrm{Pm}$ & -73603 & 20 & & & 2.68 & $\mathrm{~h}$ & 0.02 & $\left(1^{-}\right)$ & 95 & & & $\beta^{-}=100$ & \\
\hline
\end{tabular}




\begin{tabular}{|c|c|c|c|c|c|c|c|c|c|c|c|c|c|}
\hline Nuclide & \multicolumn{2}{|c|}{$\begin{array}{l}\text { Mass excess } \\
(\mathrm{keV})\end{array}$} & \multicolumn{3}{|c|}{$\begin{array}{l}\text { Excitation } \\
\text { energy }(\mathrm{keV})\end{array}$} & \multicolumn{3}{|c|}{ Half-life } & $J^{\pi}$ & Ens & Reference & \multicolumn{2}{|l|}{$\begin{array}{l}\text { Decay modes and } \\
\text { intensities }(\%)\end{array}$} \\
\hline \multicolumn{14}{|c|}{$\ldots A$-group continued $\ldots$} \\
\hline${ }^{150} \mathrm{Sm}$ & -77057.3 & 2.4 & & & & STABLE & & & $0^{+}$ & 96 & & $\mathrm{IS}=7.381$ & \\
\hline${ }^{150} \mathrm{Eu}$ & -74797 & 6 & & & & 36.9 & $\mathrm{y}$ & 0.9 & $5^{(-)}$ & 95 & & $\beta^{+}=100$ & \\
\hline${ }^{150} \mathrm{Eu}^{m}$ & -74755 & 6 & 42.1 & 0.5 & & 12.8 & $\mathrm{~h}$ & 0.1 & $0^{-}$ & 95 & & $\beta^{-}=892 ; \beta^{+}=112 ; \ldots$ & $*$ \\
\hline${ }^{150} \mathrm{Gd}$ & -75769 & 6 & & & & 1.79 & My & 0.08 & $0^{+}$ & 96 & & $\alpha=100 ; 2 \beta^{+} ?$ & \\
\hline${ }^{150} \mathrm{~Tb}$ & -71111 & 8 & & & & 3.48 & $\mathrm{~h}$ & 0.16 & $\left(2^{-}\right)$ & 96 & & $\beta^{+} \approx 100 ; \alpha<0.05$ & \\
\hline${ }^{150} \mathrm{~Tb}^{m}$ & -70654 & 28 & 457 & 29 & MD & 5.8 & $\mathrm{~m}$ & 0.2 & $9^{+}$ & 96 & & $\beta^{+} \approx 100 ;$ IT ? & \\
\hline${ }^{150} \mathrm{Dy}$ & -69317 & 5 & & & & 7.17 & $\mathrm{~m}$ & 0.05 & $0^{+}$ & 96 & & $\beta^{+}=645 ; \alpha=365$ & \\
\hline${ }^{150} \mathrm{Ho}$ & -61948 & 14 & & & $*$ & 76.8 & $\mathrm{~s}$ & 1.8 & $2^{-}$ & 95 & 93A103 T & $\beta^{+}=100$ & * \\
\hline${ }^{150} \mathrm{Ho}^{m}$ & -61960 & 50 & -10 & 50 & $\mathrm{BD} *$ & 23.3 & s & 0.3 & $(9)^{+}$ & 95 & & $\beta^{+}=100$ & \\
\hline${ }^{150} \mathrm{Ho}^{n}$ & -61960 & 50 & 8000 & & & 751 & $\mathrm{~ns}$ & & & & & & \\
\hline${ }^{150} \mathrm{Er}$ & -57833 & 17 & & & & 18.5 & $\mathrm{~s}$ & 0.7 & $0^{+}$ & 95 & & $\beta^{+}=100$ & \\
\hline${ }^{150} \mathrm{Tm}$ & $-46610 \#$ & $200 \#$ & & & $* \&$ & $3 \#$ & s & & $\left(1^{+}\right)$ & & $88 \mathrm{Ni02} \mathrm{J}$ & $\beta^{+}=100$ & \\
\hline${ }^{150} \mathrm{Tm}^{m}$ & $-46470 \#$ & $240 \#$ & $140 \#$ & $140 \#$ & $* \&$ & 2.20 & $\mathrm{~s}$ & 0.06 & $\left(6^{-}\right)$ & 95 & $96 \mathrm{Ga} 24 \mathrm{~T}$ & $\beta^{+}=100 ; \beta^{+} \mathrm{p}=1.23$ & $*$ \\
\hline${ }^{150} \mathrm{Tm}^{n}$ & $-45800 \#$ & $240 \#$ & $810 \#$ & $140 \#$ & & 5.2 & $\mathrm{~ms}$ & 0.3 & $\left(10^{+}\right)$ & 95 & & $\mathrm{IT}=100$ & $*$ \\
\hline${ }^{150} \mathrm{Yb}$ & $-38730 \#$ & $400 \#$ & & & & $700 \#$ & $\mathrm{~ms}$ & $>200 \mathrm{~ns})$ & $0^{+}$ & 97 & 00So11 I & $\beta^{+} ?$ & \\
\hline${ }^{150} \mathrm{Lu}$ & $-24940 \#$ & $500 \#$ & & & & 46 & $\mathrm{~ms}$ & 6 & $\left(5^{-}, 6^{-}\right)$ & 02 & O0Gi01 J & $\mathrm{p}=? ; \beta^{+}=30 \#$ & \\
\hline${ }^{150} \mathrm{Lu}^{m}$ & $-24900 \#$ & $500 \#$ & 34 & 15 & $\mathrm{p}$ & 80 & $\mu \mathrm{s}$ & 60 & $\left(1^{+}, 2^{+}\right)$ & 02 & 00Gi01 J & $\mathrm{p} \approx 100 ; \beta^{+} ?$ & \\
\hline$*^{150} \mathrm{Nd}$ & \multicolumn{12}{|c|}{$\mathrm{T}:$ from $6.75(+0.37-0.68$ statistics +0.68 systematics $)$} & $* *$ \\
\hline$*^{150} \mathrm{Eu}^{m}$ & \multicolumn{12}{|c|}{$\mathrm{D}: \ldots ; \mathrm{IT} \leq 5 \mathrm{e}-8$} & ** \\
\hline$*{ }^{150} \mathrm{Ho}$ & \multicolumn{12}{|c|}{$\mathrm{T}:$ average $93 \mathrm{Al} 03=78(2) 82 \mathrm{No} 08=72(4)$} & $* *$ \\
\hline$*^{150} \mathrm{Tm}^{m}$ & \multicolumn{12}{|c|}{$\mathrm{T}:$ average $96 \mathrm{Ga} 24=2.22(0.07) 88 \mathrm{Ni} 02=2.15(0.10)$ and $87 \mathrm{To} 05=2.2(0.2)$} & $* *$ \\
\hline$*^{150} \mathrm{Tm}^{m}$ & \multirow{2}{*}{\multicolumn{12}{|c|}{$\begin{array}{l}\mathrm{T}: 82 \mathrm{No} 08=3.5(0.6) \text { at variance, not used } \\
\mathrm{E}: 671.6 \mathrm{keV} \text { above }{ }^{150} \mathrm{Tm}^{m} \text {, from ENSDF }\end{array}$}} & $* *$ \\
\hline$*^{150} \mathrm{Tm}^{n}$ & & & & & & & & & & & & & $* *$ \\
\hline
\end{tabular}

\begin{tabular}{|c|c|c|c|c|c|c|c|c|c|c|c|c|c|c|}
\hline${ }^{151} \mathrm{Cs}$ & $-35220 \#$ & $500 \#$ & & & & & $60 \#$ & $\mathrm{~ms}$ & $>50 \mathrm{~ms})$ & $3 / 2^{+} \#$ & 97 & 87Ra12 I & $\beta^{-} ? ; \beta^{-} \mathrm{n} ?$ & \\
\hline${ }^{151} \mathrm{Ba}$ & $-45820 \#$ & $400 \#$ & & & & & $200 \#$ & $\mathrm{~ms}$ & $>300 \mathrm{~ns})$ & $3 / 2^{-} \#$ & 97 & $94 \mathrm{Be} 24 \mathrm{I}$ & $\beta^{-}$? & \\
\hline${ }^{151} \mathrm{La}$ & $-54290 \#$ & $400 \#$ & & & & & $300 \#$ & $\mathrm{~ms}$ & $>300 \mathrm{~ns})$ & $5 / 2^{+} \#$ & 97 & $94 \mathrm{Be} 24 \mathrm{I}$ & $\beta^{-}$? & \\
\hline${ }^{151} \mathrm{Ce}$ & -61500 & 100 & & & & & 1.02 & $\mathrm{~s}$ & 0.06 & $3 / 2^{-} \#$ & 97 & & $\beta^{-}=100$ & \\
\hline${ }^{151} \mathrm{Pr}$ & -66771 & 23 & & & & & 18.90 & $\mathrm{~s}$ & 0.07 & $(3 / 2)^{(-\#)}$ & 97 & & $\beta^{-}=100$ & \\
\hline${ }^{151} \mathrm{Nd}$ & -70953 & 3 & & & & & 12.44 & $\mathrm{~m}$ & 0.07 & $3 / 2^{+}$ & 97 & & $\beta^{-}=100$ & \\
\hline${ }^{151} \mathrm{Pm}$ & -73395 & 5 & & & & & 28.40 & $\mathrm{~h}$ & 0.04 & $5 / 2^{+}$ & 97 & & $\beta^{-}=100$ & \\
\hline${ }^{151} \mathrm{Sm}$ & -74582.5 & 2.4 & & & & & 90 & $\mathrm{y}$ & 8 & $5 / 2^{-}$ & 97 & & $\beta^{-}=100$ & \\
\hline${ }^{151} \mathrm{Sm}^{m}$ & -74321.4 & 2.4 & 261.13 & 0.04 & & & 1.4 & $\mu \mathrm{s}$ & 0.1 & $(11 / 2)^{-}$ & 97 & & $\mathrm{IT}=100$ & \\
\hline${ }^{151} \mathrm{Eu}$ & -74659.1 & 2.5 & & & & & StABLE & & & $5 / 2^{+}$ & 97 & & $\mathrm{IS}=47.813$ & \\
\hline${ }^{151} \mathrm{Eu}^{m}$ & -74462.9 & 2.5 & 196.245 & 0.010 & & & 58.9 & $\mu \mathrm{s}$ & 0.5 & $11 / 2^{-}$ & 97 & & & \\
\hline${ }^{151} \mathrm{Gd}$ & -74195 & 4 & & & & & 124 & $\mathrm{~d}$ & 1 & $7 / 2^{-}$ & 97 & & $\varepsilon=100 ; \alpha=1.0 \mathrm{e}-66$ & \\
\hline${ }^{151} \mathrm{~Tb}$ & -71630 & 5 & & & & & 17.609 & $\mathrm{~h}$ & 0.001 & $1 / 2^{(+)}$ & 99 & & $\beta^{+} \approx 100 ; \alpha=0.009515$ & \\
\hline${ }^{151} \mathrm{~Tb}^{m}$ & -71530 & 5 & 99.54 & 0.06 & & & 25 & $\mathrm{~s}$ & 3 & $\left(11 / 2^{-}\right)$ & 99 & & $\mathrm{IT}=93.84 ; \beta^{+}=6.24$ & \\
\hline${ }^{151}$ Dy & -68759 & 4 & & & & & 17.9 & $\mathrm{~m}$ & 0.3 & $7 / 2^{(-)}$ & 99 & & $\beta^{+}=? ; \alpha=5.64$ & \\
\hline${ }^{151} \mathrm{Ho}$ & -63632 & 12 & & & & & 35.2 & $\mathrm{~s}$ & 0.1 & $11 / 2^{(-)}$ & 97 & 87Ne.A J & $\beta^{+}=? ; \alpha=223$ & \\
\hline${ }^{151} \mathrm{Ho}^{m}$ & -63591 & 12 & 41.0 & 0.2 & & & 47.2 & $\mathrm{~s}$ & 1.0 & $1 / 2^{(+)}$ & 97 & 87Ne.A J & $\alpha=7718 ; \beta^{+} ?$ & \\
\hline${ }^{151} \mathrm{Er}$ & -58266 & 16 & & & & & 23.5 & $\mathrm{~s}$ & 1.3 & $\left(7 / 2^{-}\right)$ & 97 & & $\beta^{+}=100$ & \\
\hline${ }^{151} \mathrm{Er}^{m}$ & -55681 & 16 & 2585.5 & 0.6 & & & 580 & $\mathrm{~ms}$ & 20 & $\left(27 / 2^{-}\right)$ & 97 & & $\mathrm{IT}=95.33 ; \beta^{+}=4.73$ & \\
\hline${ }^{151} \mathrm{Tm}$ & -50782 & 20 & & & & $\&$ & 4.17 & $\mathrm{~s}$ & 0.10 & $\left(11 / 2^{-}\right)$ & 97 & & $\beta^{+}=100$ & \\
\hline${ }^{151} \mathrm{Tm}^{m}$ & -50690 & 21 & 92 & 7 & $\mathrm{AD}$ & $\&$ & 6.6 & $\mathrm{~s}$ & 1.4 & $\left(1 / 2^{+}\right)$ & 97 & & $\beta^{+}=100$ & \\
\hline${ }^{151} \mathrm{Tm}^{n}$ & -48126 & 20 & 2655.67 & 0.22 & & & 451 & ns & 24 & $\left(27 / 2^{-}\right)$ & 97 & & $\mathrm{IT}=100$ & \\
\hline${ }^{151} \mathrm{Yb}$ & -41540 & 300 & & & & & 1.6 & $\mathrm{~s}$ & 0.5 & $\left(1 / 2^{+}\right)$ & 97 & $86 \mathrm{To} 12 \mathrm{~T}$ & $\beta^{+}=100 ; \beta^{+} \mathrm{p}=?$ & $*$ \\
\hline${ }^{151} \mathrm{Yb}^{m}$ & $-40790 \#$ & $320 \#$ & $750 \#$ & $100 \#$ & & & 1.6 & $\mathrm{~s}$ & 0.5 & $\left(11 / 2^{-}\right)$ & 97 & 86To12 TD & $\beta^{+} \approx 100 ; \beta^{+} \mathrm{p}=? ; \mathrm{IT}=0.4 \#$ & $\# *$ \\
\hline${ }^{151} \mathrm{Yb}^{n}$ & $-39750 \#$ & $580 \#$ & 1790\# & $500 \#$ & & & 2.6 & $\mu \mathrm{s}$ & 0.7 & $19 / 2^{-} \#$ & 97 & & $\mathrm{IT}=100$ & $*$ \\
\hline${ }^{151} \mathrm{Yb}^{p}$ & $-39090 \#$ & $580 \#$ & $2450 \#$ & $500 \#$ & & & 20 & $\mu \mathrm{s}$ & 1 & $27 / 2^{-} \#$ & 97 & & $\mathrm{IT}=100$ & $*$ \\
\hline${ }^{151} \mathrm{Lu}$ & $-30200 \#$ & $400 \#$ & & & & & 80.6 & $\mathrm{~ms}$ & 1.9 & $\left(11 / 2^{-}\right)$ & 02 & $93 \mathrm{Se} 04 \mathrm{D}$ & $\mathrm{p}=? ; \beta^{+}=37 \#$ & $*$ \\
\hline${ }^{151} \mathrm{Lu}^{m}$ & $-30130 \#$ & $400 \#$ & 77 & 5 & $\mathrm{p}$ & & 16 & $\mu \mathrm{s}$ & 1 & $\left(3 / 2^{+}\right)$ & 02 & & $\mathrm{p}=? ; \beta^{+} ?$ & \\
\hline${ }^{151} \mathrm{Yb}$ & $\mathrm{T}:$ deri & ed fron & $.6(0.1)$, & or mixt & e of gr & rol & id-state & and is & ner & th almost & same & half-life & & ** \\
\hline${ }^{151} \mathrm{Yb}^{m}$ & $\mathrm{E}: 740 \neq$ & estim & ed by $90 A$ & K01 (see E & ENSDF & & & & & & & & & $* *$ \\
\hline${ }^{151} \mathrm{Yb}^{n}$ & $\mathrm{E}: 1791.2$ & $2 \mathrm{keV}$ al & bove ${ }^{151} \mathrm{Yb}$ & ${ }^{m}$ (see EN & NSDF'9 & & & & & & & & & $* *$ \\
\hline${ }^{151} \mathrm{Yb}^{p}$ & $\mathrm{E}: 2448 \mathrm{k}$ & $\mathrm{keV}$ abo & $\mathrm{ve}^{151} \mathrm{Yb}^{m}$ & (see ENS & DF'97) & & & & & & & & & $* *$ \\
\hline${ }^{151} \mathrm{Lu}$ & $\mathrm{D}: \mathrm{p}=63$. & $4(0.9) \%$ & 6 in ENSDF' & 02 , based & $d$ on pr & rec & cted bet & ta-de & ay half-lif & ife $\approx 220 \mathrm{~m}$ & & & & k* \\
\hline
\end{tabular}




\begin{tabular}{|c|c|c|c|c|c|c|c|c|c|c|c|c|c|}
\hline Nuclide & \multicolumn{2}{|c|}{$\begin{array}{l}\text { Mass excess } \\
\quad(\mathrm{keV})\end{array}$} & \multicolumn{3}{|c|}{$\begin{array}{l}\text { Excitation } \\
\text { energy }(\mathrm{keV})\end{array}$} & \multicolumn{3}{|c|}{ Half-life } & $J^{\pi}$ & Ens & Reference & \multicolumn{2}{|l|}{$\begin{array}{l}\text { Decay modes and } \\
\text { intensities }(\%)\end{array}$} \\
\hline${ }^{152} \mathrm{Ba}$ & $-42600 \#$ & $500 \#$ & & & & $100 \#$ & $\mathrm{~ms}$ & & $0^{+}$ & 97 & & $\beta^{-} ?$ & \\
\hline${ }^{152} \mathrm{La}$ & $-50070 \#$ & $400 \#$ & & & & $200 \#$ & $\mathrm{~ms}$ & $(>300 \mathrm{~ns})$ & & 97 & $94 \mathrm{Be} 24 \mathrm{I}$ & $\beta^{-} ?$ & \\
\hline${ }^{152} \mathrm{Ce}$ & $-59110 \#$ & $200 \#$ & & & & 1.1 & $\mathrm{~s}$ & 0.3 & $0^{+}$ & 97 & $90 \mathrm{Ta} 07 \mathrm{~T}$ & $\beta^{-}=100$ & $*$ \\
\hline${ }^{152} \mathrm{Pr}$ & -63810 & 120 & & & & 3.63 & $\mathrm{~s}$ & 0.12 & $4^{+}$ & 97 & 99To04 J & $\beta^{-}=100$ & \\
\hline${ }^{152} \mathrm{Nd}$ & -70158 & 25 & & & & 11.4 & $\mathrm{~m}$ & 0.2 & $0^{+}$ & 97 & & $\beta^{-}=100$ & \\
\hline${ }^{152} \mathrm{Pm}$ & -71262 & 26 & & & * & 4.12 & $\mathrm{~m}$ & 0.08 & $1^{+}$ & 97 & & $\beta^{-}=100$ & \\
\hline${ }^{152} \mathrm{Pm}^{m}$ & -71120 & 80 & 140 & 90 & $\mathrm{BD} *$ & 7.52 & $\mathrm{~m}$ & 0.08 & $4^{-}$ & 97 & & $\beta^{-}=100$ & \\
\hline${ }^{152} \mathrm{Pm}^{n}$ & $-71010 \#$ & $150 \#$ & $250 \#$ & $150 \#$ & $*$ & 13.8 & $\mathrm{~m}$ & 0.2 & (8) & 97 & & $\beta^{-} \approx 100 ; \mathrm{IT}=$ ? & $*$ \\
\hline${ }^{152} \mathrm{Sm}$ & -74768.8 & 2.5 & & & & STABLE & & & $0^{+}$ & 97 & & $\mathrm{IS}=26.7516$ & \\
\hline${ }^{152} \mathrm{Eu}$ & -72894.5 & 2.5 & & & & 13.537 & $\mathrm{y}$ & 0.006 & $3^{-}$ & 97 & & $\beta^{+}=72.13 ; \beta^{-}=27.93$ & \\
\hline${ }^{152} \mathrm{Eu}^{m}$ & -72848.9 & 2.5 & 45.5998 & 0.0004 & & 9.3116 & $\mathrm{~h}$ & 0.0013 & $0^{-}$ & 97 & & $\beta^{-}=724 ; \beta^{+}=284$ & \\
\hline${ }^{152} \mathrm{Eu}^{n}$ & -72746.6 & 2.5 & 147.86 & 0.10 & & 96 & $\mathrm{~m}$ & 1 & $8^{-}$ & 97 & & $\mathrm{IT}=100$ & \\
\hline${ }^{152} \mathrm{Gd}$ & -74714.2 & 2.5 & & & & 108 & Ty & 8 & $0^{+}$ & 97 & & $\mathrm{IS}=0.201 ; \alpha=100 ; 2 \beta^{+}$? & \\
\hline${ }^{152} \mathrm{~Tb}$ & -70720 & 40 & & & & 17.5 & $\mathrm{~h}$ & 0.1 & $2^{-}$ & 98 & & $\beta^{+}=100 ; \alpha<7 \mathrm{e}-7$ & \\
\hline${ }^{152} \mathrm{~Tb}^{m}$ & -70220 & 40 & 501.74 & 0.19 & & 4.2 & $\mathrm{~m}$ & 0.1 & $8^{+}$ & 98 & & $\mathrm{IT}=78.88 ; \beta^{+}=21.28$ & \\
\hline${ }^{152}$ Dy & -70124 & 5 & & & & 2.38 & $\mathrm{~h}$ & 0.02 & $0^{+}$ & 99 & & $\varepsilon \approx 100 ; \alpha=0.1007$ & \\
\hline${ }^{152} \mathrm{Ho}$ & -63608 & 14 & & & & 161.8 & $\mathrm{~s}$ & 0.3 & $2^{-}$ & 97 & & $\beta^{+}=883 ; \alpha=123$ & \\
\hline${ }^{152} \mathrm{Ho}^{m}$ & -63448 & 14 & 160 & 1 & & 50.0 & $\mathrm{~s}$ & 0.4 & $9^{+}$ & 97 & & $\beta^{+}=89.217 ; \alpha=10.817$ & \\
\hline${ }^{152} \mathrm{Ho}^{n}$ & -60588 & 14 & 3019.59 & 0.19 & & 8.4 & $\mu \mathrm{s}$ & 0.3 & $19^{-}$ & 97 & & $\mathrm{IT}=100$ & \\
\hline${ }^{152} \mathrm{Er}$ & -60500 & 11 & & & & 10.3 & $\mathrm{~s}$ & 0.1 & $0^{+}$ & 97 & & $\alpha=904 ; \beta^{+}=104$ & \\
\hline${ }^{152} \mathrm{Tm}$ & -51770 & 70 & & & * & 8.0 & $\mathrm{~s}$ & 1.0 & $(2 \#)^{-}$ & 97 & & $\beta^{+}=100$ & \\
\hline${ }^{152} \mathrm{Tm}^{m}$ & $-51670 \#$ & $110 \#$ & $100 \#$ & $80 \#$ & $*$ & 5.2 & $\mathrm{~s}$ & 0.6 & $(9)^{+}$ & 97 & & $\beta^{+}=100$ & \\
\hline${ }^{152} \mathrm{Yb}$ & -46310 & 210 & & & & 3.04 & $\mathrm{~s}$ & 0.06 & $0^{+}$ & 97 & & $\beta^{+}=100 ; \beta^{+} \mathrm{p} ?$ & \\
\hline${ }^{152} \mathrm{Lu}$ & $-33420 \#$ & $200 \#$ & & & & 650 & $\mathrm{~ms}$ & 70 & $\left(5^{-}, 6^{-}\right)$ & 97 & $88 \mathrm{Ni02} \mathrm{T}$ & $\beta^{+}=100 ; \beta^{+} \mathrm{p}=157$ & $*$ \\
\hline${ }^{152} \mathrm{Ce}$ & $\mathrm{T}$ : averag & $90 \mathrm{TaO}$ & $07=1.4(0.2)$ & 91 Ay. $A=0.8$ & $8(0.3)$ & & & & & & & & $* *$ \\
\hline${ }^{152} \mathrm{Pm}^{n}$ & E : ENSDF & :Prob & ably feeds 7 & $.52 \mathrm{~m}$ level & " at 14 & $40 \mathrm{keV}$ & & & & & & & $* *$ \\
\hline${ }^{152} \mathrm{Lu}$ & $\mathrm{T}$ : averag & $88 \mathrm{NiO}$ & $02=600(100$ & $87 \mathrm{To} 02=7$ & $700(100$ & & & & & & & & $* *$ \\
\hline
\end{tabular}

\begin{tabular}{|c|c|c|c|c|c|c|c|c|c|c|c|c|}
\hline${ }^{153} \mathrm{Ba}$ & $-37620 \#$ & $800 \#$ & & & $80 \#$ & $\mathrm{~ms}$ & & $5 / 2^{-} \#$ & & & & $\beta^{-}$? \\
\hline${ }^{153} \mathrm{La}$ & -46930\# & $600 \#$ & & & $150 \#$ & $\mathrm{~ms}($ & $>300 \mathrm{~ns})$ & $5 / 2^{+} \#$ & 98 & $94 \mathrm{Be} 24$ & I & $\beta^{-}$? \\
\hline${ }^{153} \mathrm{Ce}$ & $-55350 \#$ & $400 \#$ & & & $500 \#$ & $\mathrm{~ms}$ & $>300 \mathrm{~ns})$ & $3 / 2^{-} \#$ & 98 & $94 \mathrm{Be} 24$ & I & $\beta^{-}$? \\
\hline${ }^{153} \mathrm{Pr}$ & -61630 & 100 & & & 4.28 & $\mathrm{~s}$ & 0.11 & $5 / 2^{-} \#$ & 98 & & & $\beta^{-}=100$ \\
\hline${ }^{153} \mathrm{Nd}$ & -67349 & 27 & & & 31.6 & $\mathrm{~s}$ & 1.0 & $(3 / 2)^{-}$ & 98 & & & $\beta^{-}=100$ \\
\hline${ }^{153} \mathrm{Pm}$ & -70685 & 11 & & & 5.25 & $\mathrm{~m}$ & 0.02 & $5 / 2^{-}$ & 98 & & & $\beta^{-}=100$ \\
\hline${ }^{153} \mathrm{Sm}$ & -72565.8 & 2.5 & & & 46.284 & $\mathrm{~h}$ & 0.004 & $3 / 2^{+}$ & 98 & & & $\beta^{-}=100$ \\
\hline${ }^{153} \mathrm{Sm}^{m}$ & -72467.4 & 2.5 & 98.37 & 0.10 & 10.6 & $\mathrm{~ms}$ & 0.3 & $11 / 2^{-}$ & 98 & & & $\mathrm{IT}=100$ \\
\hline${ }^{153} \mathrm{Eu}$ & -73373.5 & 2.5 & & & STABLE & & & $5 / 2^{+}$ & 98 & & & $\mathrm{IS}=52.193$ \\
\hline${ }^{153} \mathrm{Gd}$ & -728898 & 2.5 & & & 240.4 & $\mathrm{~d}$ & 10 & $3 / 2^{-}$ & 98 & & & $\varepsilon=100$ \\
\hline${ }^{153} \mathrm{Gd}^{m}$ & -72794.6 & 2.5 & 95.1737 & 0.0012 & 3.5 & $\mu \mathrm{s}$ & 0.4 & $\left(9 / 2^{+}\right)$ & 98 & & & $\mathrm{IT}=100$ \\
\hline${ }^{153} \mathrm{Gd}^{n}$ & -72718.6 & 2.5 & 171.189 & 0.005 & 76.0 & $\mu \mathrm{s}$ & 1.4 & $\left(11 / 2^{-}\right)$ & 98 & & & $\mathrm{IT}=100$ \\
\hline${ }^{153} \mathrm{~Tb}$ & -71320 & 4 & & & 2.34 & $\mathrm{~d}$ & 0.01 & $5 / 2^{+}$ & 98 & & & $\beta^{+}=100$ \\
\hline${ }^{153} \mathrm{~Tb}^{m}$ & -71157 & 4 & 163.175 & 0.005 & 186 & $\mu \mathrm{s}$ & 4 & $11 / 2^{-}$ & 98 & & & $\mathrm{IT}=100$ \\
\hline${ }^{153} \mathrm{Dy}$ & -69150 & 5 & & & 6.4 & $\mathrm{~h}$ & 0.1 & $7 / 2^{(-)}$ & 99 & & & $\beta^{+} \approx 100 ; \alpha=0.009414$ \\
\hline${ }^{153} \mathrm{Ho}$ & -65019 & 6 & & & 2.01 & $\mathrm{~m}$ & 0.03 & $11 / 2^{-}$ & 98 & & & $\beta^{+} \approx 100 ; \alpha=0.05125$ \\
\hline${ }^{153} \mathrm{Ho}^{m}$ & -64950 & 6 & 68.7 & 0.3 & 9.3 & $\mathrm{~m}$ & 0.5 & $1 / 2^{+}$ & 98 & & & $\beta^{+} \approx 100 ; \alpha=0.188$ \\
\hline${ }^{153} \mathrm{Er}$ & -60488 & 9 & & & 37.1 & $\mathrm{~s}$ & 0.2 & $7 / 2^{(-)}$ & 98 & 85Ah.1 & $\mathrm{J}$ & $\alpha=533 ; \beta^{+}=473$ \\
\hline${ }^{153} \mathrm{Tm}$ & -54015 & 18 & & & 1.48 & $\mathrm{~s}$ & 0.01 & $\left(11 / 2^{-}\right)$ & 98 & & & $\alpha=913 ; \beta^{+}=93$ \\
\hline${ }^{153} \mathrm{Tm}^{m}$ & -53972 & 18 & 43.2 & 0.2 & 2.5 & $\mathrm{~s}$ & 0.2 & $\left(1 / 2^{+}\right)$ & 98 & & & $\alpha=923 ; \beta^{+}=$? \\
\hline${ }^{153} \mathrm{Yb}$ & $-47060 \#$ & 200\# & & & 4.2 & $\mathrm{~s}$ & 0.2 & $7 / 2^{-} \#$ & 98 & 88Wi05 & $\mathrm{D}$ & $\beta^{+}=? ; \alpha=50 \# ; \ldots$ \\
\hline${ }^{153} \mathrm{Yb}^{m}$ & $-44360 \#$ & $220 \#$ & 2700 & 100 & 15 & $\mu \mathrm{s}$ & 1 & $\left(27 / 2^{-}\right)$ & 98 & & & \\
\hline${ }^{153} \mathrm{Lu}$ & -38410 & 210 & & & 900 & $\mathrm{~ms}$ & 200 & $11 / 2^{-}$ & 98 & 97Ir01 & $\mathrm{D}$ & $\alpha=70 \# ; \beta^{+}=? ; \mathrm{p}=0$ \\
\hline${ }^{153} \mathrm{Lu}^{m}$ & -38330 & 210 & 80 & 5 & $1 \#$ & $\mathrm{~s}$ & & $1 / 2^{+}$ & 98 & 97Ir01 & $\mathrm{ED}$ & $\beta^{+} ? ; \alpha ? ; p=0$ \\
\hline${ }^{153} \mathrm{Lu}^{n}$ & -35780 & 210 & 2632.9 & 0.5 & 15 & $\mu \mathrm{s}$ & 3 & $27 / 2^{-}$ & 98 & & & \\
\hline${ }^{153} \mathrm{Hf}$ & $-27300 \#$ & $500 \#$ & & & $400 \#$ & $\mathrm{~ms}($ & $>200 \mathrm{~ns})$ & $1 / 2^{+} \#$ & & 00So11 & I & $\beta^{+} ?$ \\
\hline${ }^{153} \mathrm{Hf}^{m}$ & $-26550 \#$ & $510 \#$ & 750\# & $100 \#$ & $500 \#$ & $\mathrm{~ms}$ & & $11 / 2^{-} \#$ & & & & $\beta^{+}$?; IT? \\
\hline${ }^{153} \mathrm{Sm}$ & \multicolumn{12}{|c|}{$\mathrm{T}:$ see also $99 \mathrm{Sc} 12=46.274(7)$} \\
\hline${ }^{153} \mathrm{Er}$ & \multicolumn{12}{|c|}{$\mathrm{J}:$ and 89Ot.A } \\
\hline${ }^{153} \mathrm{Yb}$ & \multicolumn{12}{|c|}{$\mathrm{D}: \ldots ; \beta^{+} \mathrm{p}=0.0082$} \\
\hline${ }^{153} \mathrm{Yb}^{m}$ & \multicolumn{12}{|c|}{$\mathrm{E}:$ in ENSDF $2578.2+\mathrm{x}$} \\
\hline${ }^{153} \mathrm{Lu}$ & \multicolumn{12}{|c|}{$\mathrm{D}: \mathrm{p}$ decay is from $97 \mathrm{Ir} 01$} \\
\hline
\end{tabular}




\begin{tabular}{|c|c|c|c|c|c|c|c|c|c|c|c|c|c|}
\hline Nuclide & \multicolumn{2}{|c|}{$\begin{array}{c}\text { Mass excess } \\
(\mathrm{keV})\end{array}$} & \multicolumn{3}{|c|}{$\begin{array}{l}\text { Excitation } \\
\text { energy }(\mathrm{keV})\end{array}$} & \multicolumn{3}{|c|}{ Half-life } & \multirow[t]{2}{*}{$J^{\pi}$} & \multirow[t]{2}{*}{ Ens } & Reference & \multicolumn{2}{|l|}{$\begin{array}{c}\text { Decay modes and } \\
\text { intensities }(\%)\end{array}$} \\
\hline${ }^{154} \mathrm{La}$ & $-42380 \#$ & $600 \#$ & & & & $100 \#$ & $\mathrm{~ms}$ & & & & & $\beta^{-}$? & \\
\hline${ }^{154} \mathrm{Ce}$ & $-52700 \#$ & $500 \#$ & & & & $300 \#$ & $\mathrm{~ms}$ & $(>300 \mathrm{~ns})$ & $0^{+}$ & 98 & $94 \mathrm{Be} 24 \mathrm{I}$ & $\beta^{-}$? & \\
\hline${ }^{154} \mathrm{Pr}$ & -58200 & 150 & & & & 2.3 & $\mathrm{~s}$ & 0.1 & $\left(3^{+}, 2^{+}\right)$ & 98 & & $\beta^{-}=100$ & \\
\hline${ }^{154} \mathrm{Nd}$ & -65690 & 110 & & & & 25.9 & $\mathrm{~s}$ & 0.2 & $0^{+}$ & 98 & & $\beta^{-}=100$ & \\
\hline${ }^{154} \mathrm{Nd}^{m}$ & $-65210 \#$ & 190\# & $480 \#$ & $150 \#$ & & 1.3 & $\mu \mathrm{s}$ & 0.5 & & 98 & & & \\
\hline${ }^{154} \mathrm{Nd}^{n}$ & -64340 & 110 & 1349 & 10 & & $>1$ & $\mu \mathrm{s}$ & & $\left(5^{-}\right)$ & 98 & & & \\
\hline${ }^{154} \mathrm{Pm}$ & -68500 & 40 & & & $* \&$ & 1.73 & $\mathrm{~m}$ & 0.10 & $(0,1)$ & 98 & & $\beta^{-}=100$ & \\
\hline${ }^{154} \mathrm{Pm}^{m}$ & -68380 & 110 & 120 & 120 & $\mathrm{BD} * \&$ & 2.68 & $\mathrm{~m}$ & 0.07 & $(3,4)$ & 98 & & $\beta^{-}=100$ & \\
\hline${ }^{154} \mathrm{Sm}$ & -72461.6 & 2.5 & & & & STABLE & & (>2.3 Ey) & $0^{+}$ & 98 & & $\mathrm{IS}=22.7529 ; 2 \beta^{-} ?$ & \\
\hline${ }^{154} \mathrm{Eu}$ & -71744.4 & 2.5 & & & & 8.593 & $\mathrm{y}$ & 0.004 & $3^{-}$ & 98 & & $\beta^{-} \approx 100 ; \varepsilon=0.021$ & \\
\hline${ }^{154} \mathrm{Eu}^{m}$ & -71599.1 & 2.5 & 145.3 & 0.3 & & 46.3 & $\mathrm{~m}$ & 0.4 & $\left(8^{-}\right)$ & 98 & & $\mathrm{IT}=100$ & \\
\hline${ }^{154} \mathrm{Gd}$ & -73713.2 & 2.5 & & & & STABLE & & & $0^{+}$ & 98 & & $\mathrm{IS}=2.183$ & \\
\hline${ }^{154} \mathrm{~Tb}$ & -70160 & 50 & & & $*$ & 21.5 & $\mathrm{~h}$ & 0.4 & $0^{(+\#)}$ & 98 & & $\beta^{+} \approx 100 ; \beta^{-}<0.1$ & \\
\hline${ }^{154} \mathrm{~Tb}^{m}$ & -70150 & 50 & 12 & 7 & $*$ & 9.4 & $\mathrm{~h}$ & 0.4 & $3^{-}$ & 98 & ABBW E & $\beta^{+}=78.27 ; \mathrm{IT}=21.87 ; \ldots$ & $*$ \\
\hline${ }^{154} \mathrm{~Tb}^{n}$ & -69960\# & $160 \#$ & $200 \#$ & $150 \#$ & * & 22.7 & $\mathrm{~h}$ & 0.5 & $7^{-}$ & 98 & & $\beta^{+}=98.26 ; \mathrm{IT}=1.86$ & \\
\hline${ }^{154}$ Dy & -70398 & 8 & & & & 3.0 & My & 1.5 & $0^{+}$ & 99 & & $\alpha=100 ; 2 \beta^{+} ?$ & \\
\hline${ }^{154} \mathrm{Ho}$ & -64644 & 8 & & & & 11.76 & $\mathrm{~m}$ & 0.19 & $2^{-}$ & 98 & & $\beta^{+} \approx 100 ; \alpha=0.0195$ & \\
\hline${ }^{154} \mathrm{Ho}^{m}$ & -64406 & 28 & 238 & 30 & $\mathrm{AD}$ & 3.10 & $\mathrm{~m}$ & 0.14 & $8^{+}$ & 98 & & $\beta^{+}=100 ; \alpha<0.001 ; \mathrm{IT} \approx 0$ & \\
\hline${ }^{154} \mathrm{Er}$ & -62612 & 5 & & & & 3.73 & $\mathrm{~m}$ & 0.09 & $0^{+}$ & 01 & & $\beta^{+} \approx 100 ; \alpha=0.4713$ & \\
\hline${ }^{154} \mathrm{Tm}$ & -54429 & 14 & & & $*$ & 8.1 & $\mathrm{~s}$ & 0.3 & $\left(2^{-}\right)$ & 98 & & $\alpha=545 ; \beta^{+}=465$ & \\
\hline${ }^{154} \mathrm{Tm}^{m}$ & -54360 & 50 & 70 & 50 & $\mathrm{BD} *$ & 3.30 & $\mathrm{~s}$ & 0.07 & $\left(9^{+}\right)$ & 98 & & $\alpha=585 ; \beta^{+}=425$ & * \\
\hline${ }^{154} \mathrm{Yb}$ & -49934 & 17 & & & & 409 & $\mathrm{~ms}$ & 2 & $0^{+}$ & 98 & & $\alpha=92.612 ; \beta^{+}=7.412$ & \\
\hline${ }^{154} \mathrm{Lu}$ & $-39570 \#$ & $200 \#$ & & & & $1 \#$ & $\mathrm{~s}$ & & $\left(2^{-}\right)$ & 98 & & $\beta^{+} ?$ & \\
\hline${ }^{154} \mathrm{Lu}^{m}$ & $-39510 \#$ & $200 \#$ & 58 & 13 & $\mathrm{AD}$ & 1.12 & $\mathrm{~s}$ & 0.08 & $\left(9^{+}\right)$ & 98 & $88 \mathrm{Vi02} \mathrm{D}$ & $\beta^{+} \approx 100 ; \beta^{+} \mathrm{p}=? ; \ldots$ & * \\
\hline${ }^{154} \mathrm{Lu}^{n}$ & $-37300 \#$ & $600 \#$ & $>2562$ & & & 35 & $\mu \mathrm{s}$ & 3 & $\left(17^{+}\right)$ & 98 & & $\mathrm{IT}=100$ & \\
\hline${ }^{154} \mathrm{Hf}$ & $-32730 \#$ & $500 \#$ & & & & 2 & $\mathrm{~s}$ & 1 & $0^{+}$ & 98 & & $\beta^{+} \approx 100 ; \alpha \approx 0$ & \\
\hline${ }^{*}{ }^{154} \mathrm{~Tb}^{m}$ & $\mathrm{D}: \ldots ; \beta$ & $-<0.1$ & & & & & & & & & & & $* *$ \\
\hline${ }^{154} \mathrm{~Tb}^{m}$ & $E:$ less th & an $25 \mathrm{k}$ & $\mathrm{eV}$, from $\mathrm{E}$ & ISDF & & & & & & & & & $* *$ \\
\hline${ }^{*}{ }^{154} \mathrm{Tm}^{m}$ & D : IT dec & ay has & not been ob & served & & & & & & & & & $* *$ \\
\hline${ }^{154} \mathrm{Lu}^{m}$ & $\mathrm{D}: \ldots ; \beta$ & $+\alpha=?$ & $\alpha=0.002 \#$ & & & & & & & & & & $* *$ \\
\hline${ }^{154} \mathrm{Lu}^{m}$ & $\mathrm{D}: \beta^{+} \mathrm{p} \mathrm{a}$ & nd $\beta^{+}$ & $x$ modes ob & ervec & $88 \mathrm{ViO} 02$ & $\beta^{+} \mathrm{p} \mathrm{co}$ & onfirm & ned by $90 \mathrm{~S}$ & Sh.A & & & & $* *$ \\
\hline
\end{tabular}

\begin{tabular}{|c|c|c|c|c|c|c|c|c|c|c|c|c|c|c|}
\hline${ }^{155} \mathrm{La}$ & $-38800 \#$ & $800 \#$ & & & & & $60 \#$ & $\mathrm{~ms}$ & & $5 / 2^{+} \#$ & & & $\beta^{-} ?$ & \\
\hline${ }^{155} \mathrm{Ce}$ & $-48400 \#$ & $600 \#$ & & & & & $200 \#$ & $\mathrm{~ms}$ & $(>300 \mathrm{~ns})$ & $5 / 2^{-} \#$ & 97 & $94 \mathrm{Be} 24 \mathrm{I}$ & $\beta^{-} ?$ & \\
\hline${ }^{155} \mathrm{Pr}$ & $-55780 \#$ & $300 \#$ & & & & & $1 \#$ & $\mathrm{~s}$ & $(>300 \mathrm{~ns})$ & $5 / 2^{-} \#$ & 97 & 95Cz.A I & $\beta^{-} ?$ & \\
\hline${ }^{155} \mathrm{Nd}$ & $-62470 \#$ & $150 \#$ & & & & & 8.9 & $\mathrm{~s}$ & 0.2 & $3 / 2^{-} \#$ & 94 & & $\beta^{-}=100$ & \\
\hline${ }^{155} \mathrm{Pm}$ & -66970 & 30 & & & & & 41.5 & $\mathrm{~s}$ & 0.2 & $\left(5 / 2^{-}\right)$ & 94 & & $\beta^{-}=100$ & \\
\hline${ }^{155} \mathrm{Sm}$ & -70197.2 & 2.6 & & & & & 22.3 & $\mathrm{~m}$ & 0.2 & $3 / 2^{-}$ & 94 & & $\beta^{-}=100$ & \\
\hline${ }^{155} \mathrm{Eu}$ & -71824.5 & 2.5 & & & & & 4.7611 & $\mathrm{y}$ & 0.0013 & $5 / 2^{+}$ & 94 & & $\beta^{-}=100$ & \\
\hline${ }^{155} \mathrm{Gd}$ & -72077.1 & 2.5 & & & & & STABLE & & & $3 / 2^{-}$ & 97 & & $\mathrm{IS}=14.8012$ & \\
\hline${ }^{155} \mathrm{Gd}^{m}$ & -71956.1 & 2.5 & 121.05 & 0.19 & & & 32.0 & $\mathrm{~ms}$ & 0.3 & $11 / 2^{-}$ & 97 & & $\mathrm{IT}=100$ & \\
\hline${ }^{155} \mathrm{~Tb}$ & -71254 & 12 & & & & & 5.32 & $\mathrm{~d}$ & 0.06 & $3 / 2^{+}$ & 94 & & $\varepsilon=100$ & \\
\hline${ }^{155}$ Dy & -69160 & 12 & & & & & 9.9 & $\mathrm{~h}$ & 0.2 & $3 / 2^{-}$ & 99 & & $\beta^{+}=100$ & \\
\hline${ }^{155} \mathrm{Dy}^{m}$ & -68926 & 12 & 234.33 & 0.03 & & & 6 & $\mu \mathrm{s}$ & & $11 / 2^{-}$ & 99 & & $\mathrm{IT}=100$ & \\
\hline${ }^{155} \mathrm{Ho}$ & -66040 & 18 & & & & & 48 & $\mathrm{~m}$ & 1 & $5 / 2^{+}$ & 94 & & $\beta^{+}=100$ & \\
\hline${ }^{155} \mathrm{Ho}^{m}$ & -65898 & 18 & 141.97 & 0.11 & & & 880 & $\mu \mathrm{s}$ & 80 & $11 / 2^{-}$ & 94 & & $\mathrm{IT}=100$ & \\
\hline${ }^{155} \mathrm{Er}$ & -62215 & 7 & & & & & 5.3 & $\mathrm{~m}$ & 0.3 & $7 / 2^{-}$ & 94 & & $\beta^{+} \approx 100 ; \alpha=0.0227$ & \\
\hline${ }^{155} \mathrm{Tm}$ & -56635 & 13 & & & & & 21.6 & s & 0.2 & $\left(11 / 2^{-}\right)$ & 95 & & $\beta^{+}=98.13 ; \alpha=1.93$ & \\
\hline${ }^{155} \mathrm{Tm}^{m}$ & -56594 & 14 & 41 & 6 & & & 45 & $\mathrm{~s}$ & 3 & $\left(1 / 2^{+}\right)$ & 95 & & $\beta^{+}>92 ; \alpha<8$ & \\
\hline${ }^{155} \mathrm{Yb}$ & -50503 & 17 & & & & & 1.793 & $\mathrm{~s}$ & 0.019 & $\left(7 / 2^{-}\right)$ & 94 & $96 \mathrm{Pa01} \mathrm{T}$ & $\alpha=894 ; \beta^{+}=114$ & $*$ \\
\hline${ }^{155} \mathrm{Lu}$ & -42554 & 20 & & & & $\&$ & 68.6 & $\mathrm{~ms}$ & 1.6 & $\left(11 / 2^{-}\right)$ & 94 & 97Da07 TD & $\alpha=884 ; \beta^{+} ?$ & $*$ \\
\hline${ }^{155} \mathrm{Lu}^{m}$ & -42534 & 21 & 20 & 6 & $\mathrm{AD}$ & $\&$ & 138 & $\mathrm{~ms}$ & 8 & $\left(1 / 2^{+}\right)$ & 94 & 97Da07 TJD & $\alpha=7616 ; \beta^{+} ?$ & * \\
\hline${ }^{155} \mathrm{Lu}^{n}$ & -40773 & 20 & 1781.0 & 2.0 & $\mathrm{AD}$ & & 2.70 & $\mathrm{~ms}$ & 0.03 & $\left(25 / 2^{-}\right)$ & 94 & $96 \mathrm{~Pa} 01 \mathrm{~T}$ & $\alpha \approx 100 ;$ IT ? & * \\
\hline${ }^{155} \mathrm{Hf}$ & $-34100 \#$ & $400 \#$ & & & & & 890 & $\mathrm{~ms}$ & 120 & $7 / 2^{-} \#$ & 94 & & $\beta^{+} \approx 100 ; \alpha ?$ & \\
\hline${ }^{155} \mathrm{Ta}$ & $-23670 \#$ & $500 \#$ & & & & & 13 & $\mu \mathrm{s}$ & 4 & $\left(11 / 2^{-}\right)$ & 02 & & $\mathrm{p}=100$ & \\
\hline${ }^{155} \mathrm{Yb}$ & \multicolumn{13}{|c|}{$\mathrm{T}:$ average $96 \mathrm{~Pa} 01=1.80(0.02) 91 \mathrm{To} 08=1.75(0.05)$} & $* *$ \\
\hline$*^{155} \mathrm{Lu}$ & \multicolumn{13}{|c|}{$\mathrm{T}:$ average $96 \mathrm{Pa01}=70(1) 97 \mathrm{Da07}=63(2) 91 \mathrm{To} 09=66(7) 79 \mathrm{Ho} 10=70(6)$} & $* *$ \\
\hline$*^{155} \mathrm{Lu}$ & \multicolumn{13}{|c|}{$\mathrm{D}: \alpha:$ average $97 \mathrm{Da} 07=90(2) \% 79 \mathrm{Ho} 10=79(4) \%$ with Birge ratio $B=4.4$} & $* *$ \\
\hline${ }^{155} \mathrm{Lu}^{m}$ & \multicolumn{13}{|c|}{$\mathrm{T}:$ average $97 \mathrm{Da} 07=150(24) 96 \mathrm{~Pa} 01=136(9) 91 \mathrm{To} 09=140(20)$} & $* *$ \\
\hline$*^{155} \mathrm{Lu}^{n}$ & \multicolumn{13}{|c|}{$\mathrm{T}:$ average $96 \mathrm{Pa0} 1=2.71(0.03) 81 \mathrm{Ho} . \mathrm{A}=2.62(0.07)$} & ** \\
\hline
\end{tabular}




\begin{tabular}{|c|c|c|c|c|c|c|c|c|c|c|c|c|c|c|}
\hline Nuclide & \multicolumn{2}{|c|}{$\begin{array}{l}\text { Mass excess } \\
\quad(\mathrm{keV})\end{array}$} & \multicolumn{3}{|c|}{$\begin{array}{c}\text { Excitation } \\
\text { energy }(\mathrm{keV})\end{array}$} & \multicolumn{3}{|c|}{ Half-life } & \multirow{2}{*}{$\begin{array}{c}J^{\pi} \\
0^{+}\end{array}$} & \multirow[t]{2}{*}{ Ens } & \multicolumn{2}{|c|}{ Reference } & \multicolumn{2}{|l|}{$\begin{array}{l}\text { Decay modes and } \\
\text { intensities }(\%)\end{array}$} \\
\hline${ }^{156} \mathrm{Ce}$ & $-45400 \#$ & $600 \#$ & & & & $150 \#$ & $\mathrm{~ms}$ & & & & & & $\beta^{-} ?$ & \\
\hline${ }^{156} \mathrm{Pr}$ & $-51910 \#$ & $400 \#$ & & & & $500 \#$ & $\mathrm{~ms}$ & $(>300 \mathrm{~ns})$ & & & 95Cz.A & & $\beta^{-}$? & \\
\hline${ }^{156} \mathrm{Nd}$ & -60530 & 200 & & & & 5.49 & $\mathrm{~s}$ & 0.07 & $0^{+}$ & 03 & & & $\beta^{-}=100$ & \\
\hline${ }^{156} \mathrm{Nd}^{m}$ & -59100 & 200 & 1432 & 5 & & 135 & ns & & $5^{-}$ & 03 & & & $\mathrm{IT}=100$ & \\
\hline${ }^{156} \mathrm{Pm}$ & -64220 & 30 & & & & 26.70 & $\mathrm{~s}$ & 0.10 & $4^{-}$ & 03 & & & $\beta^{-}=100$ & \\
\hline${ }^{156} \mathrm{Sm}$ & -69370 & 10 & & & & 9.4 & $\mathrm{~h}$ & 0.2 & $0^{+}$ & 03 & & & $\beta^{-}=100$ & \\
\hline${ }^{156} \mathrm{Sm}^{m}$ & -67972 & 10 & 1397.55 & 0.09 & & 185 & ns & 7 & $5^{-}$ & 03 & & & $\mathrm{IT}=100$ & \\
\hline${ }^{156} \mathrm{Eu}$ & -70093 & 6 & & & & 15.19 & $\mathrm{~d}$ & 0.08 & $0^{+}$ & 03 & & & $\beta^{-}=100$ & \\
\hline${ }^{156} \mathrm{Gd}$ & -72542.2 & 2.5 & & & & STABLE & & & $0^{+}$ & 03 & & & $\mathrm{IS}=20.479$ & \\
\hline${ }^{156} \mathrm{Gd}^{m}$ & -70404.6 & 2.5 & 2137.60 & 0.05 & & 1.3 & $\mu \mathrm{s}$ & 0.1 & $7^{-}$ & 03 & & & $\mathrm{IT}=100$ & \\
\hline${ }^{156} \mathrm{~Tb}$ & -70098 & 4 & & & & 5.35 & $\mathrm{~d}$ & 0.10 & $3^{-}$ & 03 & & & $\beta^{+} \approx 100 ; \beta^{-} ?$ & \\
\hline${ }^{156} \mathrm{~Tb}^{m}$ & -70044 & 5 & 54 & 3 & & 24.4 & $\mathrm{~h}$ & 1.0 & $\left(7^{-}\right)$ & 03 & & & $\mathrm{IT}=100$ & $*$ \\
\hline${ }^{156} \mathrm{~Tb}^{n}$ & -70010 & 4 & 88.4 & 0.2 & & 5.3 & $\mathrm{~h}$ & 0.2 & $\left(0^{+}\right)$ & 03 & & & $\mathrm{IT}=? ; \beta^{+}=$? & \\
\hline${ }^{156} \mathrm{Dy}$ & -70530 & 7 & & & & STABLE & & (>1 Ey) & $0^{+}$ & 03 & 58Ri23 & $\mathrm{T}$ & $\mathrm{IS}=0.061 ; \alpha ? ; 2 \beta^{+}$? & $*$ \\
\hline${ }^{156} \mathrm{Ho}$ & -65350 & 40 & & & & 56 & $\mathrm{~m}$ & 1 & $4^{-}$ & 03 & & & $\beta^{+}=100$ & \\
\hline${ }^{156} \mathrm{Ho}^{m}$ & -65300 & 40 & 52.4 & 0.5 & & 9.5 & $\mathrm{~s}$ & 1.5 & $1^{-}$ & 03 & & & $\mathrm{IT}=? ; \beta^{+}$? & \\
\hline${ }^{156} \mathrm{Ho}^{n}$ & $-65250 \#$ & $60 \#$ & $100 \#$ & $50 \#$ & & 7.8 & $\mathrm{~m}$ & 0.3 & $\left(9^{+}\right)$ & 03 & & & $\beta^{+}=75 ;$ IT ? & \\
\hline${ }^{156} \mathrm{Er}$ & -64213 & 24 & & & & 19.5 & $\mathrm{~m}$ & 1.0 & $0^{+}$ & 03 & & & $\beta^{+}=100 ; \alpha=17 \mathrm{e}-64$ & \\
\hline $\begin{array}{l}{ }^{156} \mathrm{Tm} \\
{ }^{2} \mathrm{Tm}\end{array}$ & -56840 & 16 & & & & 83.8 & $\mathrm{~s}$ & 1.8 & $2^{-}$ & 03 & & & $\beta^{+} \approx 100 ; \alpha=0.06410$ & \\
\hline${ }^{156} \mathrm{Tm}^{m}$ & -56636 & 16 & 203.6 & 0.5 & & 400 & $\mathrm{~ns}$ & & $\left(11^{-}\right)$ & 03 & & & $\mathrm{IT}=100$ & \\
\hline${ }^{156} \mathrm{Tm}^{n}$ & & & non exi & stent & $\mathrm{RN}$ & 19 & $\mathrm{~s}$ & 3 & $9^{+}$ & 03 & 91To08 & I & & * \\
\hline${ }^{156} \mathrm{Yb}$ & -53264 & 11 & & & & 26.1 & $\mathrm{~s}$ & 0.7 & $0^{+}$ & 03 & & & $\beta^{+}=902 ; \alpha=102$ & \\
\hline${ }^{156} \mathrm{Lu}$ & -43750 & 70 & & & * & 494 & $\mathrm{~ms}$ & 12 & $(2)^{-}$ & 03 & & & $\alpha=? ; \beta^{+}=5 \#$ & \\
\hline${ }^{156} \mathrm{Lu}^{m}$ & -43530\# & $110 \#$ & $220 \#$ & $80 \#$ & * & 198 & $\mathrm{~ms}$ & 2 & $(9)^{+}$ & 03 & $96 \mathrm{~Pa} 01$ & $\mathrm{D}$ & $\alpha=946 ; \beta^{+}$? & * \\
\hline${ }^{156} \mathrm{Hf}$ & -37850 & 210 & & & & 23 & $\mathrm{~ms}$ & 1 & $0^{+}$ & 03 & $96 \mathrm{~Pa} 01$ & $\mathrm{D}$ & $\alpha=973 ; \beta^{+} ?$ & * \\
\hline${ }^{156} \mathrm{Hf}^{m}$ & -35890 & 210 & 1959.0 & 1.0 & $\mathrm{AD}$ & 480 & $\mu \mathrm{s}$ & 40 & $8^{+}$ & 03 & $96 \mathrm{~Pa} 01$ & $\mathrm{~T}$ & $\alpha=100$ & $*$ \\
\hline${ }^{156} \mathrm{Ta}$ & $-25800 \#$ & $400 \#$ & & & & 144 & $\mathrm{~ms}$ & 24 & $\left(2^{-}\right)$ & 03 & & & $\mathrm{p} \approx 100 ; \beta^{+}$? & \\
\hline${ }^{156} \mathrm{Ta}^{m}$ & $-25700 \#$ & $400 \#$ & 100 & 8 & $\mathrm{AD}$ & 360 & $\mathrm{~ms}$ & 40 & $\left(9^{+}\right)$ & 03 & & & $\beta^{+}=95.89 ; \mathrm{p}=4.29$ & * \\
\hline${ }^{156} \mathrm{~Tb}^{m}$ & $\mathrm{E}:$ derived & d from & E3 $24 \mathrm{~h}$ to & $4^{+} 49$. & $630 \mathrm{lev}$ & land $E(\mathrm{I}$ & $\mathrm{IT})<$ & $B(\mathrm{~L})=9 \mathrm{keV}$ & & & & & & $* *$ \\
\hline${ }^{156} \mathrm{Dy}$ & $\mathrm{T}$ : lower 1 & limit is & for $\alpha$ deca & & & & & & & & & & & $* *$ \\
\hline${ }^{156} \mathrm{Tm}^{n}$ & I : see also & the dis & cussion in & ENSD & F'03 & & & & & & & & & $* *$ \\
\hline${ }^{156} \mathrm{Lu}^{m}$ & $\mathrm{D}:$ derivec & d from & original $\alpha$ & $=98(9)$ & & & & & & & & & & $* *$ \\
\hline${ }^{156} \mathrm{Hf}$ & $\mathrm{D}$ : derivec & d from & original $\alpha$ & $=100($ & & & & & & & & & & $* *$ \\
\hline${ }^{156} \mathrm{Hf}^{m}$ & $\mathrm{~T}:$ averag & $\mathrm{e} 96 \mathrm{~Pa}$ & $1=520(10$ & $81 \mathrm{H}$ & $\mathrm{A}=44$ & (17) & & & & & & & & $* *$ \\
\hline${ }^{156} \mathrm{Ta}^{m}$ & $\mathrm{~T}: 96 \mathrm{PaO} 1$ & $1=375$ & 54) $93 \mathrm{Li} 34$ & $=320$ & & & & & & & & & & $* *$ \\
\hline
\end{tabular}

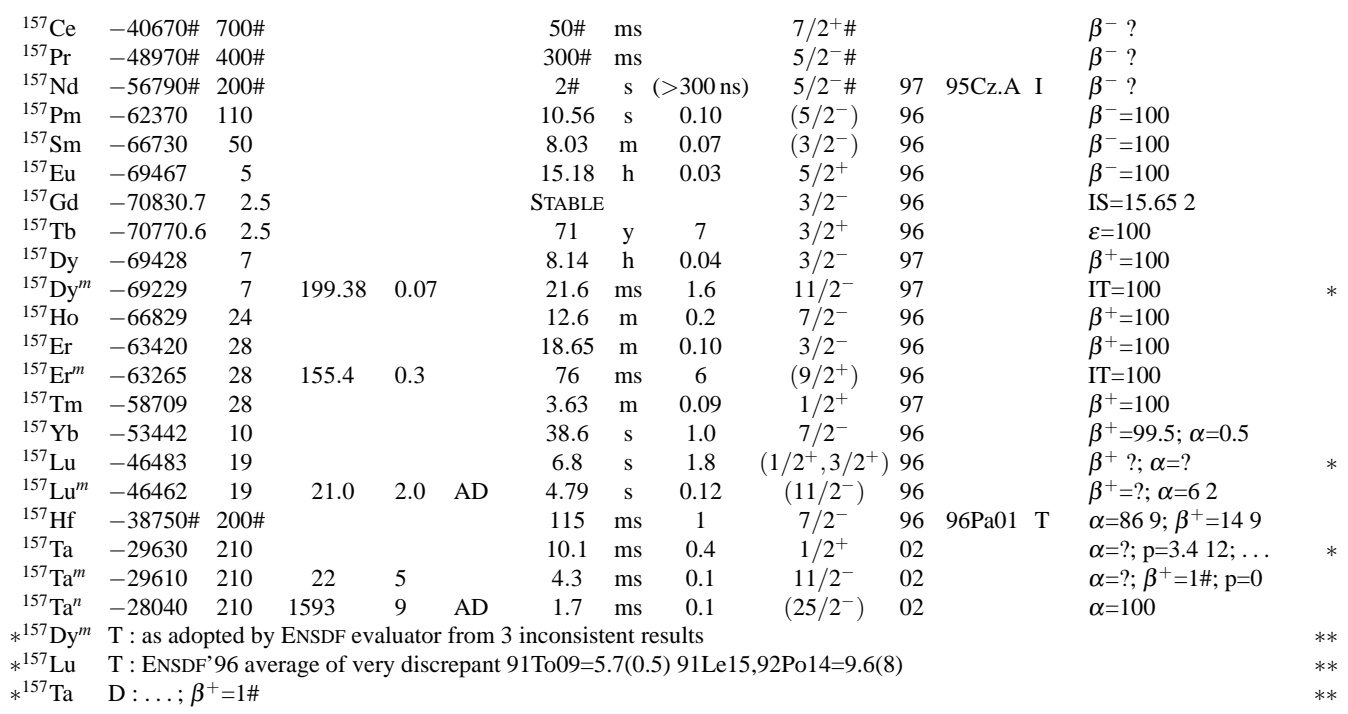




\begin{tabular}{|c|c|c|c|c|c|c|c|c|c|c|c|c|c|c|c|}
\hline Nuclide & \multicolumn{2}{|c|}{$\begin{array}{l}\text { Mass excess } \\
(\mathrm{keV})\end{array}$} & \multicolumn{4}{|c|}{$\begin{array}{l}\text { Excitation } \\
\text { energy }(\mathrm{keV})\end{array}$} & \multicolumn{3}{|c|}{ Half-life } & $J^{\pi}$ & Ens & \multicolumn{2}{|c|}{ Reference } & \multicolumn{2}{|l|}{$\begin{array}{c}\text { Decay modes and } \\
\text { intensities }(\%)\end{array}$} \\
\hline${ }^{158} \operatorname{Pr}$ & $-44730 \#$ & $600 \#$ & & & & & $200 \#$ & $\mathrm{~ms}$ & & & & & & $\beta^{-} ?$ & \\
\hline${ }^{158} \mathrm{Nd}$ & $-54400 \#$ & $400 \#$ & & & & & $700 \#$ & $\mathrm{~ms}$ & $>300 \mathrm{~ns})$ & $0^{+}$ & 97 & 95Cz.A & I & $\beta^{-} ?$ & \\
\hline${ }^{158} \mathrm{Pm}$ & -59090 & 130 & & & & & 4.8 & $\mathrm{~s}$ & 0.5 & & 96 & & & $\beta^{-}=100$ & \\
\hline${ }^{158} \mathrm{Sm}$ & -65210 & 80 & & & & & 5.30 & $\mathrm{~m}$ & 0.03 & $0^{+}$ & 96 & & & $\beta^{-}=100$ & \\
\hline${ }^{158} \mathrm{Eu}$ & -67210 & 80 & & & & & 45.9 & $\mathrm{~m}$ & 0.2 & $\left(1^{-}\right)$ & 96 & & & $\beta^{-}=100$ & \\
\hline${ }^{158} \mathrm{Gd}$ & -70696.8 & 2.5 & & & & & STABLE & & & $0^{+}$ & 96 & & & $\mathrm{IS}=24.847$ & \\
\hline${ }^{158} \mathrm{~Tb}$ & -69477.2 & 2.6 & & & & & 180 & $\mathrm{y}$ & 11 & $3^{-}$ & 96 & & & $\beta^{+}=83.47 ; \beta^{-}=16.67$ & \\
\hline${ }^{158} \mathrm{~Tb}^{m}$ & -69366.9 & 2.9 & 110.3 & 1.2 & & & 10.70 & $\mathrm{~s}$ & 0.17 & $0^{-}$ & 96 & & & $\mathrm{IT} \approx 100 ; \beta^{-}<0.6 ; \ldots$ & * \\
\hline${ }^{158} \mathrm{~Tb}^{n}$ & -69088.8 & 2.6 & 388.37 & 0.1 & & & 395 & $\mu \mathrm{s}$ & & $7^{-}$ & & & & & \\
\hline${ }^{158}$ Dy & -70412 & 3 & & & & & STABLE & & & $0^{+}$ & 96 & & & $\mathrm{IS}=0.101 ; \alpha ? ; 2 \beta^{+} ?$ & \\
\hline${ }^{158} \mathrm{Ho}$ & -66191 & 27 & & & & & 11.3 & $\mathrm{~m}$ & 0.4 & $5^{+}$ & 97 & & & $\beta^{+} \approx 100 ; \alpha ?$ & \\
\hline${ }^{158} \mathrm{Ho}^{m}$ & -66124 & 27 & 67.200 & 0.0 & & & 28 & $\mathrm{~m}$ & 2 & $2^{-}$ & 97 & & & $\mathrm{IT}>81 ; \beta^{+}<19$ & \\
\hline${ }^{158} \mathrm{Ho}^{n}$ & $-66010 \#$ & $80 \#$ & 180\# & $70 \#$ & & & 21.3 & $\mathrm{~m}$ & 2.3 & $\left(9^{+}\right)$ & 97 & & & $\beta^{+}>93 ; \mathrm{IT}<7 \#$ & \\
\hline${ }^{158} \mathrm{Er}$ & -65304 & 25 & & & & & 2.29 & $\mathrm{~h}$ & 0.06 & $0^{+}$ & 96 & & & $\varepsilon=100$ & \\
\hline${ }^{158} \mathrm{Tm}$ & -58703 & 25 & & & & * & 3.98 & $\mathrm{~m}$ & 0.06 & $2^{-}$ & 96 & & & $\beta^{+}=100$ & \\
\hline${ }^{158} \mathrm{Tm}^{m}$ & $-58650 \#$ & $100 \#$ & $50 \#$ & $100 \#$ & & $*$ & 20 & ns & & $\left(5^{+}\right)$ & 96 & 81Dr07 & $\mathrm{T}$ & IT ? & * \\
\hline${ }^{158} \mathrm{Yb}$ & -56015 & 8 & & & & & 1.49 & $\mathrm{~m}$ & 0.13 & $0^{+}$ & 96 & & & $\beta^{+} \approx 100 ; \alpha \approx 0.002112$ & \\
\hline${ }^{158} \mathrm{Lu}$ & -47214 & 15 & & & & & 10.6 & $\mathrm{~s}$ & 0.3 & $2^{-}$ & 96 & 95Ga.A & $\mathrm{J}$ & $\beta^{+}=99.0920 ; \ldots$ & * \\
\hline${ }^{158} \mathrm{Hf}$ & -42104 & 18 & & & & & 2.84 & $\mathrm{~s}$ & 0.07 & $0^{+}$ & 96 & $96 \mathrm{~Pa} 01$ & TD & $\beta^{+}=553 ; \alpha=453$ & * \\
\hline${ }^{158} \mathrm{Ta}$ & $-31020 \#$ & $200 \#$ & & & & $\&$ & 49 & $\mathrm{~ms}$ & 8 & $\left(2^{-}\right)$ & 96 & 97Da07 & TJD & $\alpha=964 ; \beta^{+} ?$ & * \\
\hline${ }^{158} \mathrm{Ta}^{m}$ & $-30880 \#$ & $200 \#$ & 140 & 12 & $\mathrm{AD}$ & $\&$ & 36.0 & $\mathrm{~ms}$ & 0.8 & $\left(9^{+}\right)$ & 96 & 97Da07 & TJE & $\alpha=936 ; \beta^{+}$?; IT ? & $*$ \\
\hline${ }^{158} \mathrm{~W}$ & $-23700 \#$ & $500 \#$ & & & & & 1.37 & $\mathrm{~ms}$ & 0.17 & $0^{+}$ & 96 & $00 \mathrm{Ma} 95$ & $\mathrm{~T}$ & $\alpha=100$ & * \\
\hline${ }^{158} \mathrm{~W}^{m}$ & $-21810 \#$ & $500 \#$ & 1889 & 8 & $\mathrm{AD}$ & & 143 & $\mu \mathrm{s}$ & 19 & $8^{+}$ & & $00 \mathrm{Ma} 95$ & $\mathrm{~T}$ & $\alpha=100$ & * \\
\hline$*^{158} \mathrm{~Tb}^{m}$ & \multicolumn{14}{|c|}{$\mathrm{D}: \ldots ; \beta^{+}<0.01$} & ** \\
\hline$*^{158} \mathrm{Tm}^{m}$ & \multirow{2}{*}{\multicolumn{14}{|c|}{ I : $T \approx 20 \mathrm{~s}$ in $81 \mathrm{Dr} 07$ was a typo. Value in Fig. 2 was correct. See $96 \mathrm{Dr}$.A }} & ** \\
\hline$*^{158} \mathrm{Lu}$ & \multicolumn{2}{|c|}{$\mathrm{D}: \ldots ; \alpha=0.9120$} & & & & & & & & & & & & & ** \\
\hline$*^{158} \mathrm{Hf}$ & \multicolumn{14}{|c|}{$\mathrm{T}:$ average $96 \mathrm{~Pa} 01=2.85(0.07) 73 \mathrm{To} 02=2.8(0.2)$} & ** \\
\hline$*^{158} \mathrm{Ta}$ & \multicolumn{14}{|c|}{$\mathrm{T}:$ average $97 \mathrm{Da} 07=72(12) 96 \mathrm{~Pa} 01=46(4)$ with Birge ratio $B=2$} & ** \\
\hline$*^{158} \mathrm{Ta}$ & \multicolumn{14}{|c|}{$\mathrm{D}$ : derived from original $\alpha \approx 100(8) \%$} & ** \\
\hline$*^{158} \mathrm{Ta}^{m}$ & \multicolumn{14}{|c|}{$\mathrm{T}:$ average $97 \mathrm{Da} 07=37.7(1.5) 96 \mathrm{~Pa} 01=35(1) 79 \mathrm{Ho} 10=36.8(1.6)$} & $* *$ \\
\hline$*{ }^{158} \mathrm{~W}$ & \multicolumn{14}{|c|}{$\mathrm{T}:$ average $00 \mathrm{Ma} 95=1.5(0.2) 96 \mathrm{~Pa} 01=0.9(+0.4-0.3)$} & ** \\
\hline$*^{158} \mathrm{~W}^{m}$ & \multicolumn{14}{|c|}{$\mathrm{T}:$ average $00 \mathrm{Ma} 95=140(20) 96 \mathrm{~Pa} 01=160(50)$} & ** \\
\hline
\end{tabular}

\begin{tabular}{|c|c|c|c|c|c|c|c|c|c|c|c|c|c|c|}
\hline${ }^{159} \mathrm{Pr}$ & $-41450 \#$ & 700\# & & & & $100 \#$ & $\mathrm{~ms}$ & & $5 / 2^{-} \#$ & & & & $\beta^{-} ?$ & \\
\hline${ }^{159} \mathrm{Nd}$ & $-50220 \#$ & $500 \#$ & & & & $500 \#$ & $\mathrm{~ms}$ & & $7 / 2^{+} \#$ & & & & $\beta^{-} ?$ & \\
\hline${ }^{159} \mathrm{Pm}$ & $-56850 \#$ & $200 \#$ & & & & 1.47 & $\mathrm{~s}$ & 0.15 & $5 / 2^{-} \#$ & 03 & & & $\beta^{-}=100$ & \\
\hline${ }^{159} \mathrm{Sm}$ & -62210 & 100 & & & & 11.37 & s & 0.15 & $5 / 2^{-}$ & 03 & & & $\beta^{-}=100$ & \\
\hline${ }^{159} \mathrm{Eu}$ & -66053 & 7 & & & & 18.1 & $\mathrm{~m}$ & 0.1 & $5 / 2^{+}$ & 03 & & & $\beta^{-}=100$ & \\
\hline${ }^{159} \mathrm{Gd}$ & -68568.5 & 2.5 & & & & 18.479 & $\mathrm{~h}$ & 0.004 & $3 / 2^{-}$ & 03 & & & $\beta^{-}=100$ & \\
\hline${ }^{159} \mathrm{~Tb}$ & -69539.0 & 2.6 & & & & StaBle & & & $3 / 2^{+}$ & 03 & & & $\mathrm{IS}=100$ & \\
\hline${ }^{159}$ Dy & -69173.5 & 2.7 & & & & 144.4 & $\mathrm{~d}$ & 0.2 & $3 / 2^{-}$ & 03 & & & $\varepsilon=100$ & \\
\hline${ }^{159} \mathrm{Dy}^{m}$ & -68820.7 & 2.7 & 352.77 & 0.14 & & 122 & $\mu \mathrm{s}$ & 3 & $11 / 2^{-}$ & 03 & & & $\mathrm{IT}=100$ & \\
\hline${ }^{159} \mathrm{Ho}$ & -67336 & 4 & & & & 33.05 & $\mathrm{~m}$ & 0.11 & $7 / 2^{-}$ & 03 & & & $\beta^{+}=100$ & \\
\hline${ }^{159} \mathrm{Ho}^{m}$ & -67130 & 4 & 205.91 & 0.05 & & 8.30 & $\mathrm{~s}$ & 0.08 & $1 / 2^{+}$ & 03 & & & $\mathrm{IT}=100$ & \\
\hline${ }^{159} \mathrm{Er}$ & -64567 & 4 & & & & 36 & $\mathrm{~m}$ & 1 & $3 / 2^{-}$ & 03 & & & $\beta^{+}=100$ & \\
\hline${ }^{159} \mathrm{Er}^{m}$ & -64384 & 4 & 182.602 & 0.02 & & 337 & ns & 14 & $9 / 2^{+}$ & 03 & & & $\mathrm{IT}=100$ & \\
\hline${ }^{159} \mathrm{Er}^{n}$ & -64138 & 4 & 429.05 & 0.03 & & 590 & $\mathrm{~ns}$ & 60 & $11 / 2^{-}$ & 03 & & & $\mathrm{IT}=100$ & \\
\hline${ }^{159} \mathrm{Tm}$ & -60570 & 28 & & & & 9.13 & $\mathrm{~m}$ & 0.16 & $5 / 2^{+}$ & 03 & & & $\beta^{+}=100$ & \\
\hline${ }^{159} \mathrm{Yb}$ & -55843 & 18 & & & & 1.72 & $\mathrm{~m}$ & 0.10 & $5 / 2^{(-)}$ & 03 & $93 \mathrm{Al} 03$ & $\mathrm{~T}$ & $\beta^{+}=100$ & * \\
\hline${ }^{159} \mathrm{Lu}$ & -49710 & 40 & & & $*$ & 12.1 & $\mathrm{~s}$ & 1.0 & $1 / 2^{+} \#$ & 03 & & & $\beta^{+} \approx 100 ; \alpha=0.1 \#$ & \\
\hline${ }^{159} \mathrm{Lu}^{m}$ & $-49610 \#$ & $90 \#$ & $100 \#$ & $80 \#$ & $*$ & $10 \#$ & $\mathrm{~s}$ & & $11 / 2^{-} \#$ & & & & $\beta^{+} ? ;$ IT $? ; \alpha$ ? & \\
\hline${ }^{159} \mathrm{Hf}$ & -42854 & 17 & & & & 5.20 & s & 0.10 & $7 / 2^{-} \#$ & 03 & $96 \mathrm{~Pa} 01$ & $\mathrm{~T}$ & $\beta^{+}=657 ; \alpha=357$ & * \\
\hline${ }^{159} \mathrm{Ta}$ & -34448 & 21 & & & & 1.04 & s & 0.09 & $\left(1 / 2^{+}\right)$ & & 97Da07 & $\mathrm{TJ}$ & $\beta^{+} ? ; \alpha=345$ & * \\
\hline${ }^{159} \mathrm{Ta}^{m}$ & -34385 & 20 & 64 & 5 & $\mathrm{AD}$ & 514 & $\mathrm{~ms}$ & 9 & $\left(11 / 2^{-}\right)$ & 03 & $96 \mathrm{~Pa} 01$ & $\mathrm{~T}$ & $\alpha=551 ; \beta^{+} ?$ & $*$ \\
\hline${ }^{159} \mathrm{~W}$ & $-25230 \#$ & $400 \#$ & & & & 8.2 & $\mathrm{~ms}$ & 0.7 & $7 / 2^{-} \#$ & 03 & $96 \mathrm{~Pa} 01$ & $\mathrm{TD}$ & $\alpha=8216 ; \beta^{+} ?$ & * \\
\hline${ }^{159} \mathrm{Yb}$ & \multicolumn{13}{|c|}{$\mathrm{T}:$ supersedes $80 \mathrm{Al} 14=1.40(0.20)$ from same group } & $* *$ \\
\hline${ }^{159} \mathrm{Hf}$ & \multicolumn{13}{|c|}{$\mathrm{J}: 7 / 2^{-}$is not measured in 00Di18, p.7: "a $7 / 2^{-}$assignment is assumed" } & $* *$ \\
\hline${ }^{159} \mathrm{Ta}$ & \multicolumn{13}{|c|}{$\mathrm{T}:$ average $97 \mathrm{Da} 07=0.83(0.18) 96 \mathrm{~Pa} 01=1.10(0.10)$} & $* *$ \\
\hline${ }^{159} \mathrm{Ta}^{m}$ & \multicolumn{13}{|c|}{$\mathrm{T}:$ average $97 \mathrm{Da} 07=500(11) 96 \mathrm{~Pa} 01=544(16)$; other $02 \mathrm{Ro} 17=620(50)$} & $* *$ \\
\hline${ }^{159} \mathrm{~W}$ & \multicolumn{13}{|c|}{$\mathrm{D}$ : derived from original $\alpha=92(23) \%$} & $* *$ \\
\hline
\end{tabular}




\begin{tabular}{|c|c|c|c|c|c|c|c|c|c|c|c|c|c|c|}
\hline \multirow{2}{*}{$\begin{array}{l}\text { Nuclide } \\
{ }^{160} \mathrm{Nd}\end{array}$} & \multicolumn{2}{|c|}{$\begin{array}{l}\text { Mass excess } \\
\quad(\mathrm{keV})\end{array}$} & \multicolumn{3}{|c|}{$\begin{array}{l}\text { Excitation } \\
\text { energy }(\mathrm{keV})\end{array}$} & \multicolumn{3}{|c|}{ Half-life } & \multirow{2}{*}{$\frac{J^{\pi}}{0^{+}}$} & \multirow[t]{2}{*}{ Ens } & \multicolumn{2}{|c|}{ Reference } & \multicolumn{2}{|l|}{$\begin{array}{l}\text { Decay modes and } \\
\text { intensities }(\%)\end{array}$} \\
\hline & $-47420 \#$ & $600 \#$ & & & & $300 \#$ & $\mathrm{~ms}$ & & & & $85 \mathrm{Si} 25$ & I & $\beta^{-} ?$ & $*$ \\
\hline${ }^{160} \mathrm{Pm}$ & $-53100 \#$ & $300 \#$ & & & & $2 \#$ & $\mathrm{~s}$ & & & & & & $\beta^{-}$? & \\
\hline${ }^{160} \mathrm{Sm}$ & $-60420 \#$ & $200 \#$ & & & & 9.6 & s & 0.3 & $0^{+}$ & 97 & & & $\beta^{-}=100$ & \\
\hline${ }^{160} \mathrm{Eu}$ & $-63370 \#$ & $200 \#$ & & & & 38 & s & 4 & $1^{(-)}$ & 97 & & & $\beta^{-}=100$ & \\
\hline${ }^{160} \mathrm{Gd}$ & -67948.6 & 2.6 & & & & STABLE & & (>31 Ey) & $0^{+}$ & 97 & 01Da22 & $\mathrm{T}$ & IS $=21.8619 ; 2 \beta^{-} ?$ & \\
\hline${ }^{160} \mathrm{~Tb}$ & -67842.9 & 2.6 & & & & 72.3 & d & 0.2 & $3^{-}$ & 97 & & & $\beta^{-}=100$ & \\
\hline${ }^{160}$ Dy & -69678.1 & 2.5 & & & & STABLE & & & $0^{+}$ & 97 & & & $\mathrm{IS}=2.348$ & \\
\hline${ }^{160} \mathrm{Ho}$ & -66388 & 15 & & & & 25.6 & $\mathrm{~m}$ & 0.3 & $5^{+}$ & 97 & & & $\beta^{+}=100$ & \\
\hline${ }^{160} \mathrm{Ho}^{m}$ & -66328 & 15 & 59.98 & 0.03 & & 5.02 & $\mathrm{~h}$ & 0.05 & $2^{-}$ & 97 & & & $\mathrm{IT}=653 ; \beta^{+}=353$ & \\
\hline${ }^{160} \mathrm{Ho}^{n}$ & -66191 & 22 & 197 & 16 & & 3 & $\mathrm{~s}$ & & $\left(9^{+}\right)$ & 97 & ABBW & $\mathrm{E}$ & $\mathrm{IT}=100$ & $*$ \\
\hline${ }^{160} \mathrm{Er}$ & -66058 & 24 & & & & 28.58 & $\mathrm{~h}$ & 0.09 & $0^{+}$ & 97 & & & $\varepsilon=100$ & \\
\hline${ }^{160} \mathrm{Tm}$ & -60300 & 30 & & & & 9.4 & $\mathrm{~m}$ & 0.3 & $1^{-}$ & 97 & & & $\beta^{+}=100$ & \\
\hline${ }^{160} \mathrm{Tm}^{m}$ & -60230 & 40 & 70 & 20 & & 74.5 & $\mathrm{~s}$ & 1.5 & $5^{(+\#)}$ & 97 & & & $\mathrm{IT}=855 ; \beta^{+}=155$ & \\
\hline${ }^{160} \mathrm{Yb}$ & -58170 & 17 & & & & 4.8 & $\mathrm{~m}$ & 0.2 & $0^{+}$ & 97 & & & $\beta^{+}=100$ & \\
\hline${ }^{160} \mathrm{Lu}$ & -50270 & 60 & & & $*$ & 36.1 & $\mathrm{~s}$ & 0.3 & $2^{-} \#$ & 97 & & & $\beta^{+}=100 ; \alpha<1 \mathrm{e}-4$ & \\
\hline${ }^{160} \mathrm{Lu}^{m}$ & $-50270 \#$ & $120 \#$ & 0\# & $100 \#$ & $*$ & 40 & s & 1 & & 97 & & & $\beta^{+} \approx 100 ; \alpha ?$ & \\
\hline${ }^{160} \mathrm{Hf}$ & -45937 & 12 & & & & 13.6 & s & 0.2 & $0^{+}$ & 97 & & & $\beta^{+}=99.32 ; \alpha=0.72$ & \\
\hline${ }^{160} \mathrm{Ta}$ & -35880 & 90 & & & & 1.70 & s & 0.20 & $(2 \#)^{-}$ & & $96 \mathrm{~Pa} 01$ & TJD & $\beta^{+} ? ; \alpha=?$ & $*$ \\
\hline${ }^{160} \mathrm{Ta}^{m}$ & $-35560 \#$ & $110 \#$ & $310 \#$ & $90 \#$ & & 1.55 & s & 0.04 & $(9)^{+}$ & 97 & $96 \mathrm{~Pa} 01$ & $\mathrm{TJ}$ & $\beta^{+}=66 \# ; \alpha=?$ & * \\
\hline${ }^{160} \mathrm{~W}$ & -29360 & 210 & & & & 90 & $\mathrm{~ms}$ & 5 & $0^{+}$ & 97 & $96 \mathrm{~Pa} 01$ & $\mathrm{TD}$ & $\alpha=878 ; \beta^{+} ?$ & $*$ \\
\hline${ }^{160} \mathrm{Re}$ & $-16660 \#$ & $400 \#$ & & & & 860 & $\mu \mathrm{s}$ & 120 & $\left(2^{-}\right)$ & 02 & $92 \mathrm{~Pa} 05$ & $\mathrm{~J}$ & $\mathrm{p}=915 ; \alpha=95$ & * \\
\hline$*^{160} \mathrm{Nd}$ & $\mathrm{I}:$ seen in $\mathrm{t}$ & the ther & mal fissi & $n$ of ${ }^{252} \mathrm{C}$ & & & & & & & & & & $* *$ \\
\hline$*^{160} \mathrm{Ho}^{n}$ & $\mathrm{E}:$ less tha & in $55 \mathrm{ke}$ & $V$ above & $169.55 \mathrm{le}$ & $1, \mathrm{fr}$ & ENSDF & & & & & & & & $* *$ \\
\hline$*^{160} \mathrm{Ta}$ & $\mathrm{J}:$ from $\alpha$ & correla & ion with & ${ }^{56} \mathrm{Lu}$ lin & & & & & & & & & & ** \\
\hline$*^{160} \mathrm{Ta}^{m}$ & $\mathrm{~J}:$ from $\alpha$ & correla & ion with & ${ }^{56} \mathrm{Lu}^{m} 1$ & & & & & & & & & & ** \\
\hline$*^{160} \mathrm{~W}$ & $\mathrm{~T}:$ average & $96 \mathrm{PaO}$ & $1=91(5)$ & $1 \mathrm{Ho} 10=$ & & & & & & & & & & $* *$ \\
\hline${ }^{160} \mathrm{Re}$ & $\mathrm{J}:$ protons & from d & ${ }_{2}$ orbita & & & & & & & & & & & $* *$ \\
\hline
\end{tabular}

\begin{tabular}{|c|c|c|c|c|c|c|c|c|c|c|c|c|c|}
\hline${ }^{161} \mathrm{Nd}$ & $-42960 \#$ & $700 \#$ & & & & $200 \#$ & $\mathrm{~ms}$ & & $1 / 2^{-} \#$ & & & & $\beta^{-} ?$ \\
\hline${ }^{161} \mathrm{Pm}$ & $-50430 \#$ & $500 \#$ & & & & 700\# & $\mathrm{ms}$ & & $5 / 2^{-} \#$ & & & & $\beta^{-}$? \\
\hline${ }^{161} \mathrm{Sm}$ & $-56980 \#$ & $300 \#$ & & & & 4.8 & $\mathrm{~s}$ & 0.8 & $7 / 2^{+} \#$ & 00 & & & $\beta^{-}=100$ \\
\hline${ }^{161} \mathrm{Eu}$ & $-61780 \#$ & $300 \#$ & & & & 26 & s & 3 & $5 / 2^{+} \#$ & 00 & & & $\beta^{-}=100$ \\
\hline${ }^{161} \mathrm{Gd}$ & -65512.7 & 2.7 & & & & 3.646 & $\mathrm{~m}$ & 0.003 & $5 / 2^{-}$ & 00 & 94It.A & $\mathrm{T}$ & $\beta^{-}=100$ \\
\hline${ }^{161} \mathrm{~Tb}$ & -67468.2 & 2.6 & & & & 6.906 & $\mathrm{~d}$ & 0.019 & $3 / 2^{+}$ & 00 & & & $\beta^{-}=100$ \\
\hline${ }^{161}$ Dy & -68061.1 & 2.5 & & & & STABLE & & & $5 / 2^{+}$ & 00 & & & $\mathrm{IS}=18.9124$ \\
\hline${ }^{161} \mathrm{Ho}$ & -67203 & 3 & & & & 2.48 & $\mathrm{~h}$ & 0.05 & $7 / 2^{-}$ & 00 & & & $\varepsilon=100$ \\
\hline${ }^{161} \mathrm{Ho}^{m}$ & -66992 & 3 & 211.16 & 0.03 & & 6.76 & $\mathrm{~s}$ & 0.07 & $1 / 2^{+}$ & 00 & & & $\mathrm{IT}=100$ \\
\hline${ }^{161} \mathrm{Er}$ & -65209 & 9 & & & & 3.21 & $\mathrm{~h}$ & 0.03 & $3 / 2^{-}$ & 00 & & & $\beta^{+}=100$ \\
\hline${ }^{161} \mathrm{Er}^{m}$ & -64813 & 9 & 396.44 & 0.04 & & 7.5 & $\mu \mathrm{s}$ & 0.7 & $11 / 2^{-}$ & 00 & & & $\mathrm{IT}=100$ \\
\hline${ }^{161} \mathrm{Tm}$ & -61899 & 28 & & & & 30.2 & $\mathrm{~m}$ & 0.8 & $7 / 2^{+}$ & 00 & & & $\beta^{+}=100$ \\
\hline${ }^{161} \mathrm{Tm}^{m}$ & -61892 & 28 & 7.4 & 0.2 & & $5 \#$ & $\mathrm{~m}$ & & $1 / 2^{+}$ & 00 & & & $\beta^{+} ? ;$ IT ? \\
\hline${ }^{161} \mathrm{Yb}$ & -57844 & 16 & & & & 4.2 & $\mathrm{~m}$ & 0.2 & $3 / 2^{-}$ & 00 & & & $\beta^{+}=100$ \\
\hline${ }^{161} \mathrm{Lu}$ & -52562 & 28 & & & & 77 & $\mathrm{~s}$ & 2 & $1 / 2^{+}$ & 00 & & & $\beta^{+}=100$ \\
\hline${ }^{161} \mathrm{Lu}^{m}$ & -52400 & 30 & 166 & 18 & & 7.3 & $\mathrm{~ms}$ & 0.4 & $\left(9 / 2^{-}\right)$ & 00 & ABBW & $\mathrm{E}$ & $\mathrm{IT}=100$ \\
\hline${ }^{161} \mathrm{Hf}$ & -46319 & 23 & & & & 18.2 & $\mathrm{~s}$ & 0.5 & $3 / 2^{-} \#$ & 00 & & & $\beta^{+} \approx 100 ; \alpha<0.13$ \\
\hline${ }^{161} \mathrm{Ta}$ & $-38730 \#$ & $60 \#$ & & & $* \&$ & $3 \#$ & $\mathrm{~s}$ & & $1 / 2^{+} \#$ & & & & $\beta^{+}$?; $\alpha$ ? \\
\hline${ }^{161} \mathrm{Ta}^{m}$ & -38684 & 23 & $50 \#$ & $50 \#$ & $* \&$ & 2.89 & s & 0.12 & $11 / 2^{-} \#$ & 00 & & & $\beta^{+}=95 \# ; \alpha=?$ \\
\hline${ }^{161} \mathrm{~W}$ & $-30410 \#$ & $200 \#$ & & & & 409 & $\mathrm{~ms}$ & 16 & $7 / 2^{-} \#$ & 00 & $96 \mathrm{~Pa} 01$ & $\mathrm{~T}$ & $\alpha=733 ; \beta^{+}=273$ \\
\hline${ }^{161} \mathrm{Re}$ & -20880 & 210 & & & & 370 & $\mu \mathrm{s}$ & 40 & $1 / 2^{+}$ & 02 & 97Ir01 & $\mathrm{D}$ & $\mathrm{p}=972 ; \alpha ?$ \\
\hline${ }^{161} \mathrm{Re}^{m}$ & -20750 & 210 & 123.8 & 1.3 & & 15.6 & $\mathrm{~ms}$ & 0.9 & $11 / 2^{-}$ & 02 & & & $\alpha=? ; p=4.86$ \\
\hline${ }^{*}{ }^{161} \mathrm{Lu}^{m}$ & $\mathrm{E}:$ less th & in $\mathrm{K}$ bir & ding energ & $\mathrm{y}(61 \mathrm{ke}$ & above & $135.6 \mathrm{le}$ & evel, & om ENS & & & & & \\
\hline$*^{161} \mathrm{~W}$ & $\mathrm{~T}$ : average & $96 \mathrm{PaO}$ & $1=409(18)$ & 79Ho1 & $410(40$ & & & & & & & & \\
\hline$*^{161} \mathrm{Re}$ & $\mathrm{D}$ : derived & from & riginal $\mathrm{p}=$ & $00(7) \%$ & & & & & & & & & \\
\hline
\end{tabular}




\begin{tabular}{|c|c|c|c|c|c|c|c|c|c|c|c|c|c|c|}
\hline Nuclide & \multicolumn{2}{|c|}{$\begin{array}{l}\text { Mass excess } \\
\quad(\mathrm{keV})\end{array}$} & \multicolumn{3}{|c|}{$\begin{array}{l}\text { Excitation } \\
\text { energy }(\mathrm{keV})\end{array}$} & \multicolumn{3}{|c|}{ Half-life } & \multirow[t]{2}{*}{$J^{\pi}$} & \multirow[t]{2}{*}{ Ens } & \multicolumn{2}{|c|}{ Reference } & \multicolumn{2}{|l|}{$\begin{array}{l}\text { Decay modes and } \\
\text { intensities }(\%)\end{array}$} \\
\hline${ }^{162} \mathrm{Pm}$ & $-46310 \#$ & $700 \#$ & & & & $500 \#$ & $\mathrm{~ms}$ & & & & & & $\beta^{-} ?$ & \\
\hline${ }^{162} \mathrm{Sm}$ & $-54750 \#$ & $500 \#$ & & & & 2.4 & $\mathrm{~s}$ & 0.5 & $0^{+}$ & & 00As.A & TD & $\beta^{-}=100$ & \\
\hline${ }^{162} \mathrm{Eu}$ & $-58650 \#$ & 300\# & & & & 10.6 & $\mathrm{~s}$ & 1.0 & & 99 & & & $\beta^{-}=100$ & \\
\hline${ }^{162} \mathrm{Gd}$ & -64287 & 5 & & & & 8.4 & $\mathrm{~m}$ & 0.2 & $0^{+}$ & 99 & & & $\beta^{-}=100$ & \\
\hline${ }^{162} \mathrm{~Tb}$ & -65680 & 40 & & & & 7.60 & $\mathrm{~m}$ & 0.15 & $1^{-}$ & 99 & & & $\beta^{-}=100$ & \\
\hline${ }^{162}$ Dy & -68186.8 & 2.5 & & & & STABLE & & & $0^{+}$ & 99 & & & $\mathrm{IS}=25.5126$ & \\
\hline${ }^{162} \mathrm{Ho}$ & -66047 & 4 & & & & 15.0 & $\mathrm{~m}$ & 1.0 & $1^{+}$ & 99 & & & $\beta^{+}=100$ & \\
\hline${ }^{162} \mathrm{Ho}^{m}$ & -65941 & 8 & 106 & 7 & & 67.0 & $\mathrm{~m}$ & 0.7 & $6^{-}$ & 99 & & & $\mathrm{IT}=62 ; \beta^{+}=38$ & * \\
\hline${ }^{162} \mathrm{Er}$ & -66343 & 3 & & & & STABLE & & (>140 Ty) & $0^{+}$ & 99 & $56 \mathrm{Po} 16$ & $\mathrm{~T}$ & $\mathrm{IS}=0.141 ; \alpha ? ; 2 \beta^{+} ?$ & $*$ \\
\hline${ }^{162} \mathrm{Tm}$ & -61484 & 26 & & & & 21.70 & $\mathrm{~m}$ & 0.19 & $1^{-}$ & 99 & & & $\beta^{+}=100$ & \\
\hline${ }^{162} \mathrm{Tm}^{m}$ & -61350 & 50 & 130 & 40 & & 24.3 & $\mathrm{~s}$ & 1.7 & $5^{+}$ & 99 & ABBW & $\mathrm{E}$ & IT $? ; \beta^{+}=184$ & * \\
\hline${ }^{162} \mathrm{Yb}$ & -59832 & 16 & & & & 18.87 & $\mathrm{~m}$ & 0.19 & $0^{+}$ & 99 & & & $\beta^{+}=100$ & \\
\hline${ }^{162} \mathrm{Lu}$ & -52840 & 80 & & & & 1.37 & $\mathrm{~m}$ & 0.02 & $1^{(-)}$ & 99 & $98 \mathrm{Ge} 13$ & $\mathrm{~J}$ & $\beta^{+}=100$ & \\
\hline${ }^{162} \mathrm{Lu}^{m}$ & $-52720 \#$ & $220 \#$ & $120 \#$ & $200 \#$ & & 1.5 & $\mathrm{~m}$ & & $4^{-} \#$ & 99 & & & $\beta^{+} \approx 100 ;$ IT ? & \\
\hline${ }^{162} \mathrm{Lu}^{n}$ & $-52540 \#$ & $220 \#$ & $300 \#$ & $200 \#$ & & 1.9 & $\mathrm{~m}$ & & & 99 & & & $\beta^{+} \approx 100 ;$ IT ? & \\
\hline${ }^{162} \mathrm{Hf}$ & -49173 & 10 & & & & 39.4 & $\mathrm{~s}$ & 0.9 & $0^{+}$ & 99 & & & $\beta^{+} \approx 100 ; \alpha=0.0081$ & \\
\hline${ }^{162} \mathrm{Ta}$ & -39780 & 50 & & & & 3.57 & s & 0.12 & $3^{+} \#$ & 99 & & & $\beta^{+} \approx 100 ; \alpha=0.07410$ & \\
\hline${ }^{162} \mathrm{~W}$ & -34002 & 18 & & & & 1.36 & s & 0.07 & $0^{+}$ & 99 & & & $\beta^{+} ? ; \alpha=45.216$ & \\
\hline${ }^{162} \mathrm{Re}$ & $-22350 \#$ & $200 \#$ & & & & 107 & $\mathrm{~ms}$ & 13 & $\left(2^{-}\right)$ & 99 & & & $\alpha=946 ; \beta^{+}$? & \\
\hline${ }^{162} \mathrm{Re}^{m}$ & $-22180 \#$ & $200 \#$ & 173 & 10 & $\mathrm{AD}$ & 77 & $\mathrm{~ms}$ & 9 & $\left(9^{+}\right)$ & 99 & & & $\alpha=915 ; \beta^{+} ?$ & \\
\hline${ }^{162} \mathrm{Os}$ & $-14500 \#$ & $500 \#$ & & & & 1.87 & $\mathrm{~ms}$ & 0.18 & $0^{+}$ & 99 & $00 \mathrm{Ma} 95$ & $\mathrm{~T}$ & $\alpha=100$ & $*$ \\
\hline${ }^{162} \mathrm{Ho}^{m}$ & \multicolumn{13}{|c|}{ E : about $10 \mathrm{keV}$ above level at $96.1(0.1)$, from ENSDF; error from NUBASE } & ** \\
\hline${ }^{162} \mathrm{Er}$ & \multicolumn{13}{|c|}{$\mathrm{T}:$ lower limit is for $\alpha$ decay } & $* *$ \\
\hline${ }^{162} \mathrm{Tm}^{m}$ & \multicolumn{13}{|c|}{ E : above 66.90 level and less than $192 \mathrm{keV}$, from ENSDF } & ** \\
\hline${ }^{162} \mathrm{Os}$ & \multicolumn{13}{|c|}{$\mathrm{T}:$ average $00 \mathrm{Ma} 95=1.9(0.2) 96 \mathrm{Bi07}=1.5(+0.7-0.5) 89 \mathrm{Ho} 12=1.9(0.7)$} & ** \\
\hline
\end{tabular}

\begin{tabular}{|c|c|c|c|c|c|c|c|c|c|}
\hline${ }^{163} \mathrm{Pm}$ & $-43150 \#$ & $800 \#$ & & & & $200 \#$ & $\mathrm{~ms}$ & & $5 / 2^{-} \#$ \\
\hline${ }^{163} \mathrm{Sm}$ & $-50900 \#$ & $700 \#$ & & & & $1 \#$ & $\mathrm{~s}$ & & $1 / 2^{-} \#$ \\
\hline${ }^{163} \mathrm{Eu}$ & $-56630 \#$ & $500 \#$ & & & & $6 \#$ & $\mathrm{~s}$ & & $5 / 2^{+} \#$ \\
\hline${ }^{163} \mathrm{Gd}$ & $-61490 \#$ & $300 \#$ & & & & 68 & $\mathrm{~s}$ & 3 & $7 / 2^{+} \#$ \\
\hline${ }^{163} \mathrm{~Tb}$ & -64601 & 5 & & & & 19.5 & $\mathrm{~m}$ & 0.3 & $3 / 2^{+}$ \\
\hline${ }^{163} \mathrm{Dy}$ & -66386.5 & 2.5 & & & & Stable & & & $5 / 2^{-}$ \\
\hline${ }^{163} \mathrm{Ho}$ & -66383.9 & 2.5 & & & & 4.570 & ky & 0.025 & $7 / 2^{-}$ \\
\hline${ }^{163} \mathrm{Ho}^{m}$ & -66086.0 & 2.5 & 297.88 & 0.07 & & 1.09 & $\mathrm{~s}$ & 0.03 & $1 / 2^{+}$ \\
\hline${ }^{163} \mathrm{Er}$ & -65174 & 5 & & & & 75.0 & $\mathrm{~m}$ & 0.4 & $5 / 2^{-}$ \\
\hline${ }^{163} \mathrm{Er}^{m}$ & -64729 & 5 & 445.5 & 0.6 & & 580 & ns & 100 & $\left(11 / 2^{-}\right)$ \\
\hline${ }^{163} \mathrm{Tm}$ & -62735 & 6 & & & & 1.810 & $\mathrm{~h}$ & 0.005 & $1 / 2^{+}$ \\
\hline${ }^{163} \mathrm{Yb}$ & -59304 & 16 & & & & 11.05 & $\mathrm{~m}$ & 0.25 & $3 / 2^{-}$ \\
\hline${ }^{163} \mathrm{Lu}$ & -54791 & 28 & & & & 3.97 & $\mathrm{~m}$ & 0.13 & $1 / 2^{(+)}$ \\
\hline${ }^{3} \mathrm{Hf}$ & -49286 & 28 & & & & 40.0 & $\mathrm{~s}$ & 0.6 & $3 / 2^{-} \#$ \\
\hline${ }^{3} \mathrm{Ta}$ & -42540 & 40 & & & & 10.6 & $\mathrm{~s}$ & 1.8 & $1 / 2^{+} \#$ \\
\hline${ }^{3} \mathrm{~W}$ & -34910 & 50 & & & & 2.8 & $\mathrm{~s}$ & 0.2 & $3 / 2^{-} \#$ \\
\hline${ }^{3} \mathrm{Re}$ & -26007 & 20 & & & & 390 & $\mathrm{~ms}$ & 70 & $\left(1 / 2^{+}\right)$ \\
\hline${ }^{3} \mathrm{Re}^{m}$ & -25892 & 20 & 115 & 4 & $\mathrm{AD}$ & 214 & $\mathrm{~ms}$ & 5 & $\left(11 / 2^{-}\right)$ \\
\hline${ }^{3} \mathrm{Os}$ & $-16120 \#$ & $400 \#$ & & & & 5.5 & $\mathrm{~ms}$ & 0.6 & $7 / 2^{-} \#$ \\
\hline
\end{tabular}

$\beta^{-} ?$
$\beta^{-} ?$
$\beta^{-} ?$
$\beta^{-}=100$
$\beta^{-}=100$
$\mathrm{IS}=24.9016$
$\varepsilon=100$
$\mathrm{IT}=100$
$\beta^{+}=100$
$\mathrm{IT}=100$
$\beta^{+}=100$
$\beta^{+}=100$
$\beta^{+}=100$
$\beta^{+}=100 ; \alpha<0.0001$
$\beta^{+} \approx 100 ; \alpha \approx 0.2$
$\beta^{+} ? ; \alpha=132$
$\beta^{+} ? ; \alpha=323$
$\alpha=664 ; \beta^{+} ?$
$\alpha \approx 100 ; \beta^{+} ? ; \beta^{+} \mathrm{p} ?$




\begin{tabular}{|c|c|c|c|c|c|c|c|c|c|c|c|c|c|}
\hline Nuclide & \multicolumn{2}{|c|}{$\begin{array}{l}\text { Mass excess } \\
\quad(\mathrm{keV})\end{array}$} & \multicolumn{3}{|c|}{$\begin{array}{l}\text { Excitation } \\
\text { energy(keV) }\end{array}$} & \multicolumn{3}{|c|}{ Half-life } & \multirow[t]{2}{*}{$J^{\pi}$} & \multirow[t]{2}{*}{ Ens } & Reference & \multicolumn{2}{|l|}{$\begin{array}{l}\text { Decay modes and } \\
\text { intensities }(\%)\end{array}$} \\
\hline${ }^{164} \mathrm{Sm}$ & $-48180 \#$ & $800 \#$ & & & & $500 \#$ & $\mathrm{~ms}$ & & & & & $\beta^{-} ?$ & \\
\hline${ }^{164} \mathrm{Eu}$ & $-53100 \#$ & $600 \#$ & & & & $2 \#$ & $\mathrm{~s}$ & & & & & $\beta^{-} ?$ & \\
\hline${ }^{164} \mathrm{Gd}$ & $-59750 \#$ & $400 \#$ & & & & 45 & $\mathrm{~s}$ & 3 & $0^{+}$ & 01 & & $\beta^{-}=100$ & \\
\hline${ }^{164} \mathrm{~Tb}$ & -62080 & 100 & & & & 3.0 & $\mathrm{~m}$ & 0.1 & $\left(5^{+}\right)$ & 01 & & $\beta^{-}=100$ & \\
\hline${ }^{164}$ Dy & -65973.3 & 2.5 & & & & STABLE & & & $0^{+}$ & 01 & & $\mathrm{IS}=28.1837$ & \\
\hline${ }^{164} \mathrm{Ho}$ & -64987.1 & 2.8 & & & & 29 & $\mathrm{~m}$ & 1 & $1^{+}$ & 01 & & $\varepsilon=605 ; \beta^{-}=405$ & \\
\hline${ }^{164} \mathrm{Ho}^{m}$ & -64847.3 & 2.8 & 139.77 & 0.08 & & 38.0 & $\mathrm{~m}$ & 1.0 & $6^{-}$ & 01 & & $\mathrm{IT}=100$ & \\
\hline${ }^{164} \mathrm{Er}$ & -65950 & 3 & & & & StABLE & & & $0^{+}$ & 01 & & $\mathrm{IS}=1.613 ; \alpha ? ; 2 \beta^{+} ?$ & \\
\hline${ }^{164} \mathrm{Tm}$ & -61888 & 28 & & & $*$ & 2.0 & $\mathrm{~m}$ & 0.1 & $1^{+}$ & 01 & & $\varepsilon=611 ; \mathrm{e}^{+}=391$ & \\
\hline${ }^{164} \mathrm{Tm}^{m}$ & -61878 & 29 & 10 & 6 & $*$ & 5.1 & $\mathrm{~m}$ & 0.1 & $6^{-}$ & 01 & ABBW E & $\mathrm{IT} \approx 80 ; \beta^{+} \approx 20$ & * \\
\hline${ }^{164} \mathrm{Yb}$ & -61023 & 16 & & & & 75.8 & $\mathrm{~m}$ & 1.7 & $0^{+}$ & 01 & & $\varepsilon=100$ & \\
\hline${ }^{164} \mathrm{Lu}$ & -54642 & 28 & & & & 3.14 & $\mathrm{~m}$ & 0.03 & $1^{(-)}$ & 01 & & $\beta^{+}=100$ & $*$ \\
\hline${ }^{164} \mathrm{Hf}$ & -51822 & 20 & & & & 111 & $\mathrm{~s}$ & 8 & $0^{+}$ & 01 & & $\beta^{+}=100$ & \\
\hline${ }^{164} \mathrm{Ta}$ & -43283 & 28 & & & & 14.2 & $\mathrm{~s}$ & 0.3 & $\left(3^{+}\right)$ & 01 & & $\beta^{+}=100$ & $*$ \\
\hline${ }^{164} \mathrm{~W}$ & -38234 & 12 & & & & 6.3 & s & 0.2 & $0^{+}$ & 01 & & $\beta^{+}=96.212 ; \alpha=3.812$ & \\
\hline${ }^{164} \mathrm{Re}$ & $-27640 \#$ & $160 \#$ & & & $* \&$ & & & & high & & 95Pa.A J & $\alpha$ ? & \\
\hline${ }^{164} \mathrm{Re}^{m}$ & -27520 & 100 & $120 \#$ & $120 \#$ & $* \&$ & 530 & $\mathrm{~ms}$ & 230 & $(2 \#)^{-}$ & 01 & $96 \mathrm{~Pa} 01 \mathrm{JD}$ & $\alpha=? ; \beta^{+}=42 \#$ & $*$ \\
\hline${ }^{164} \mathrm{Os}$ & -20460 & 210 & & & & 21 & $\mathrm{~ms}$ & 1 & $0^{+}$ & 01 & & $\alpha=? ; \beta^{+}=2 \#$ & \\
\hline${ }^{164} \mathrm{Ir}$ & $-7270 \#$ & $410 \#$ & & & $\&$ & $1 \#$ & $\mathrm{~ms}$ & & $2^{-} \#$ & & & $\mathrm{p} ? ; \alpha ? ; \beta^{+} ?$ & \\
\hline${ }^{164} \mathrm{Ir}^{m}$ & $-7000 \#$ & $400 \#$ & 270\# & $110 \#$ & $\&$ & 94 & $\mu \mathrm{s}$ & 27 & $9^{+} \#$ & 02 & 02Ma61 T & $\mathrm{p}=? ; \alpha ? ; \beta^{+} ?$ & $*$ \\
\hline${ }^{164} \mathrm{Tm}^{m}$ & \multicolumn{12}{|c|}{$\mathrm{E}:$ less than $20 \mathrm{keV}$, from ENSDF } & $* *$ \\
\hline${ }^{164} \mathrm{Lu}$ & \multicolumn{12}{|c|}{$\mathrm{J}$ : negative parity proposed by $98 \mathrm{Ge} 13$; odd-odd ${ }^{160} \mathrm{Tm}{ }^{162} \mathrm{Tm}{ }^{162} \mathrm{Lu}$ have $1^{-}$ground-state } & $* *$ \\
\hline${ }^{164} \mathrm{Ta}$ & \multicolumn{12}{|c|}{$\mathrm{D}$ : was erroneously considered as alpha emitter, instead of ${ }^{163} \mathrm{Ta}$ by $83 \mathrm{Sc} 18$} & ** \\
\hline${ }^{164} \operatorname{Re}^{m}$ & \multicolumn{12}{|c|}{$\mathrm{J}:$ from $\alpha$ correlation with ${ }^{160} \mathrm{Ta}$ line } & $* *$ \\
\hline$*^{164} \operatorname{Ir}^{m}$ & \multicolumn{12}{|c|}{$\mathrm{T}:$ average $02 \mathrm{Ma} 61=58(+46-18) 01 \mathrm{Ke} 05=110(+60-30)$} & $* *$ \\
\hline
\end{tabular}

\begin{tabular}{|c|c|c|c|c|c|c|c|c|c|}
\hline${ }^{165} \mathrm{Sm}$ & $-43800 \#$ & $900 \#$ & & & & $200 \#$ & $\mathrm{~ms}$ & & $5 / 2^{-} \#$ \\
\hline${ }^{165} \mathrm{Eu}$ & $-50560 \#$ & $700 \#$ & & & & $1 \#$ & $\mathrm{~s}$ & & $5 / 2^{+} \#$ \\
\hline${ }^{165} \mathrm{Gd}$ & $-56470 \#$ & $500 \#$ & & & & 10.3 & $\mathrm{~s}$ & 1.6 & $1 / 2^{-} \#$ \\
\hline${ }^{55} \mathrm{~Tb}$ & $-60660 \#$ & $200 \#$ & & & & 2.11 & $\mathrm{~m}$ & 0.10 & $3 / 2^{+} \#$ \\
\hline${ }^{165}$ Dy & -63617.9 & 2.5 & & & & 2.334 & $\mathrm{~h}$ & 0.001 & $7 / 2^{+}$ \\
\hline${ }^{165} \mathrm{Dy}^{m}$ & -63509.7 & 2.5 & 108.160 & 0.003 & & 1.257 & $\mathrm{~m}$ & 0.006 & $1 / 2^{-}$ \\
\hline${ }^{165} \mathrm{Ho}$ & -64904.6 & 2.5 & & & & STABLE & & & $7 / 2^{-}$ \\
\hline${ }^{165} \mathrm{Er}$ & -64528 & 3 & & & & 10.36 & $\mathrm{~h}$ & 0.04 & $5 / 2^{-}$ \\
\hline${ }^{65} \mathrm{Tm}$ & -62936 & 3 & & & & 30.06 & $\mathrm{~h}$ & 0.03 & $1 / 2^{+}$ \\
\hline${ }^{55} \mathrm{Yb}$ & -60287 & 28 & & & & 9.9 & $\mathrm{~m}$ & 0.3 & $5 / 2^{-}$ \\
\hline${ }^{65} \mathrm{Lu}$ & -56442 & 27 & & & $*$ & 10.74 & $\mathrm{~m}$ & 0.10 & $1 / 2^{+}$ \\
\hline${ }^{5} \mathrm{Hf}$ & -51636 & 28 & & & & 76 & $\mathrm{~s}$ & 4 & $\left(5 / 2^{-}\right)$ \\
\hline${ }^{5} \mathrm{Ta}$ & -45855 & 17 & & & & 31.0 & $\mathrm{~s}$ & 1.5 & $5 / 2^{-} \#$ \\
\hline${ }^{5} \mathrm{Ta}^{p}$ & -45800 & 30 & 60 & 30 & $\mathrm{AD}$ & & & & $9 / 2^{-} \#$ \\
\hline${ }^{5} \mathrm{~W}$ & -38862 & 25 & & & & 5.1 & $\mathrm{~s}$ & 0.5 & $3 / 2^{-\#}$ \\
\hline${ }^{5} \mathrm{Re}$ & -30657 & 28 & & & $* \&$ & 1\# & $\mathrm{s}$ & & $1 / 2^{+} \#$ \\
\hline${ }^{165} \mathrm{Re}^{m}$ & -30610 & 23 & 47 & 26 & $\mathrm{AD} * \&$ & 2.1 & $\mathrm{~s}$ & 0.3 & $11 / 2^{-} \#$ \\
\hline${ }^{5} \mathrm{Os}$ & $-21650 \#$ & $200 \#$ & & & & 71 & $\mathrm{~ms}$ & 3 & $\left(7 / 2^{-}\right)$ \\
\hline${ }^{5} \mathrm{Ir}$ & $-11630 \#$ & $220 \#$ & & & & $<1 \#$ & $\mu \mathrm{s}$ & & $1 / 2^{+} \#$ \\
\hline${ }^{65} \mathrm{Ir}^{m}$ & -11440 & 210 & $180 \#$ & $50 \#$ & & 300 & $\mu \mathrm{s}$ & 60 & $11 / 2^{-}$ \\
\hline
\end{tabular}

$\beta^{-} ?$
$\beta^{-} ?$
$\beta^{-}=100$
$\beta^{-}=100$
$\beta^{-}=100$
$\mathrm{IT}=97.7611 ; \beta^{-}=2.2411$
$\mathrm{IS}=100$.
$\varepsilon=100$
$\beta^{+}=100$
$\beta^{+}=100$
$\beta^{+}=100$
$\beta^{+}=100$
$\beta^{+}=100$
$\beta^{+} \approx 100 ; \alpha<0.2$
$\beta^{+} ? ; \alpha ?$
$\beta^{+}=873 ; \alpha=133$
$\alpha>60 ; \beta^{+}<40$
$\mathrm{p} ? ; \alpha ?$
$\mathrm{p}=874 ; \alpha=134$




\begin{tabular}{|c|c|c|c|c|c|c|c|c|c|c|c|c|c|c|}
\hline Nuclide & \multicolumn{2}{|c|}{$\begin{array}{l}\text { Mass excess } \\
\quad(\mathrm{keV})\end{array}$} & \multicolumn{3}{|c|}{$\begin{array}{c}\text { Excitation } \\
\text { energy }(\mathrm{keV})\end{array}$} & \multicolumn{3}{|c|}{ Half-life } & \multirow[t]{2}{*}{$J^{\pi}$} & \multirow[t]{2}{*}{ Ens } & \multicolumn{2}{|c|}{ Reference } & \multicolumn{2}{|l|}{$\begin{array}{l}\text { Decay modes and } \\
\text { intensities }(\%)\end{array}$} \\
\hline${ }^{166} \mathrm{Eu}$ & $-46600 \#$ & 800\# & & & & $400 \#$ & $\mathrm{~ms}$ & & & & & & $\beta^{-?}$ & \\
\hline${ }^{166} \mathrm{Gd}$ & $-54400 \#$ & $600 \#$ & & & & 4.8 & $\mathrm{~s}$ & 1.0 & $0^{+}$ & & 00As.A & $\mathrm{TD}$ & $\beta^{-}=100$ & \\
\hline${ }^{166} \mathrm{~Tb}$ & -57760 & 100 & & & & 25.6 & $\mathrm{~s}$ & 2.2 & & 97 & 00As.A & $\mathrm{T}$ & $\beta^{-}=100$ & $*$ \\
\hline${ }^{166}$ Dy & -62590.1 & 2.6 & & & & 81.6 & $\mathrm{~h}$ & 0.1 & $0^{+}$ & 92 & & & $\beta^{-}=100$ & \\
\hline${ }^{166} \mathrm{Ho}$ & -63076.9 & 2.5 & & & & 26.83 & $\mathrm{~h}$ & 0.02 & $0^{-}$ & 92 & & & $\beta^{-}=100$ & \\
\hline${ }^{166} \mathrm{Ho}^{m}$ & -63070.9 & 2.5 & 5.985 & 0.01 & & 1.20 & ky & 0.18 & $(7)^{-}$ & 92 & & & $\beta^{-}=100$ & \\
\hline${ }^{166} \mathrm{Er}$ & -64931.6 & 2.5 & & & & STABLE & & & $0^{+}$ & 92 & & & $\mathrm{IS}=33.6135$ & \\
\hline${ }^{166} \mathrm{Tm}$ & -61894 & 12 & & & & 7.70 & $\mathrm{~h}$ & 0.03 & $2^{+}$ & 92 & & & $\beta^{+}=100$ & \\
\hline${ }^{166} \mathrm{Tm}^{m}$ & -61772 & 14 & 122 & 8 & & 340 & $\mathrm{~ms}$ & 25 & $6^{-}$ & & $96 \operatorname{Dr} 07$ & TJE & $\mathrm{IT}=100$ & * \\
\hline${ }^{166} \mathrm{Yb}$ & -61588 & 8 & & & & 56.7 & $\mathrm{~h}$ & 0.1 & $0^{+}$ & 92 & & & $\varepsilon=100$ & \\
\hline${ }^{166} \mathrm{Lu}$ & -56021 & 30 & & & & 2.65 & $\mathrm{~m}$ & 0.10 & $6^{(-)}$ & 92 & $98 \mathrm{Ge} 13$ & $\mathrm{~J}$ & $\beta^{+}=100$ & \\
\hline${ }^{166} \mathrm{Lu}^{m}$ & -55990 & 30 & 34.37 & 0.05 & & 1.41 & $\mathrm{~m}$ & 0.10 & $3^{(-)}$ & 92 & $98 \mathrm{Ge} 13$ & $\mathrm{~J}$ & $\beta^{+}=585 ; \mathrm{IT}=425$ & \\
\hline${ }^{166} \mathrm{Lu}^{n}$ & -55980 & 30 & 42.9 & 0.5 & & 2.12 & $\mathrm{~m}$ & 0.10 & $0^{(-)}$ & 92 & $98 \mathrm{Ge} 13$ & $\mathrm{~J}$ & $\beta^{+}>80 ;$ IT $<20$ & \\
\hline${ }^{166} \mathrm{Hf}$ & -53859 & 28 & & & & 6.77 & $\mathrm{~m}$ & 0.30 & $0^{+}$ & 92 & & & $\beta^{+}=100$ & \\
\hline${ }^{166} \mathrm{Ta}$ & -46098 & 28 & & & & 34.4 & $\mathrm{~s}$ & 0.5 & $(2)^{+}$ & 92 & & & $\beta^{+}=100$ & \\
\hline${ }^{166} \mathrm{~W}$ & -41892 & 10 & & & & 19.2 & $\mathrm{~s}$ & 0.6 & $0^{+}$ & 00 & & & $\beta^{+} \approx 100 ; \alpha=0.03512$ & \\
\hline${ }^{166} \mathrm{Re}$ & $-31850 \#$ & $90 \#$ & & & $\&$ & $2 \#$ & $\mathrm{~s}$ & & $2^{-} \#$ & & & & $\beta^{+} ? ; \alpha ?$ & \\
\hline${ }^{166} \mathrm{Re}^{m}$ & -31700 & 70 & $150 \#$ & $50 \#$ & $\&$ & 2.5 & $\mathrm{~s}$ & 0.2 & $9^{+} \#$ & 92 & $92 \mathrm{Me} 10$ & $\mathrm{~T}$ & $\beta^{+} ? ; \alpha=52$ & * \\
\hline${ }^{166} \operatorname{Re}^{p}$ & $-31700 \#$ & $100 \#$ & $150 \#$ & $50 \#$ & & & & & low & & & & & \\
\hline${ }^{166} \mathrm{Os}$ & -25438 & 18 & & & & 216 & $\mathrm{~ms}$ & 9 & $0^{+}$ & 92 & $96 \mathrm{~Pa} 01$ & $\mathrm{~T}$ & $\alpha=7213 ; \beta^{+}=2813$ & * \\
\hline${ }^{166} \mathrm{Ir}$ & $-13210 \#$ & $200 \#$ & & & & 10.5 & $\mathrm{~ms}$ & 2.2 & $\left(2^{-}\right)$ & 02 & & & $\alpha=933 ; \mathrm{p}=73$ & \\
\hline${ }^{166} \mathrm{Ir}^{m}$ & $-13030 \#$ & $200 \#$ & 172 & 6 & $\mathrm{p}$ & 15.1 & $\mathrm{~ms}$ & 0.9 & $\left(9^{+}\right)$ & 02 & & & $\alpha=98.26 ; \mathrm{p}=1.86$ & \\
\hline${ }^{166} \mathrm{Pt}$ & $-4790 \#$ & $500 \#$ & & & & 300 & $\mu \mathrm{s}$ & 100 & $0^{+}$ & 97 & $96 \mathrm{Bi} 07$ & $\mathrm{TD}$ & $\alpha=100$ & \\
\hline$*^{166} \mathrm{~Tb}$ & $\mathrm{~T}$ : superse & edes 94 & s. $A=21(6$ & same & oup & & & & & & & & & $* *$ \\
\hline$*^{166} \mathrm{Tm}^{m}$ & $\mathrm{E}:$ less tha & an $25 \mathrm{ke}$ & $V$ above 1 & 9.341 & & & & & & & & & & $* *$ \\
\hline$*^{166} \operatorname{Re}^{m}$ & $\mathrm{~T}$ : averag & e $92 \mathrm{Me}$ & $10=2.3(0.2$ & $84 \mathrm{Sc}$ & $=2.8(0.3)$ & & & & & & & & & $* *$ \\
\hline$*^{166} \operatorname{Re}^{m}$ & $\mathrm{D}: \alpha$ inter & nsity is & lerived fro & $\mathrm{m} 2 \%$ & $\alpha<8 \%$ & discl & in & ENSDF & & & & & & $* *$ \\
\hline$*^{166} \mathrm{Os}$ & $\mathrm{T}:$ averag & e $96 \mathrm{PaO}$ & $1=220(7)$ & $1 \mathrm{Se} 01$ & 194(17) & & & & & & & & & $* *$ \\
\hline
\end{tabular}

\begin{tabular}{|c|c|c|c|c|c|c|c|c|c|c|c|c|c|}
\hline${ }^{167} \mathrm{Eu}$ & $-43590 \#$ & $800 \#$ & & & & $200 \#$ & $\mathrm{~ms}$ & & $5 / 2^{+} \#$ & & & & $\beta^{-} ?$ \\
\hline${ }^{167} \mathrm{Gd}$ & $-50700 \#$ & $600 \#$ & & & & $3 \#$ & $\mathrm{~s}$ & & $5 / 2^{-} \#$ & & & & $\beta^{-}$? \\
\hline${ }^{167} \mathrm{~Tb}$ & $-55840 \#$ & $400 \#$ & & & & 19 & s & 3 & $3 / 2^{+} \#$ & 00 & 99As03 & $\mathrm{T}$ & $\beta^{-}=100$ \\
\hline${ }^{167}$ Dy & -59940 & 60 & & & & 6.20 & $\mathrm{~m}$ & 0.08 & $\left(1 / 2^{-}\right)$ & 00 & & & $\beta^{-}=100$ \\
\hline${ }^{167} \mathrm{Ho}$ & -62287 & 6 & & & & 3.1 & $\mathrm{~h}$ & 0.1 & $7 / 2^{-}$ & 00 & & & $\beta^{-}=100$ \\
\hline${ }^{167} \mathrm{Ho}^{m}$ & -62028 & 6 & 259.34 & 0.11 & & 6.0 & $\mu \mathrm{s}$ & 1.0 & $3 / 2^{+}$ & 00 & & & $\mathrm{IT}=100$ \\
\hline${ }^{167} \mathrm{Er}$ & -63296.7 & 2.5 & & & & STABLE & & & $7 / 2^{+}$ & 00 & & & IS $=22.9317$ \\
\hline${ }^{167} \mathrm{Er}^{m}$ & -63088.9 & 2.5 & 207.801 & 0.005 & & 2.269 & s & 0.006 & $1 / 2^{-}$ & 00 & & & $\mathrm{IT}=100$ \\
\hline${ }^{167} \mathrm{Tm}$ & -62548.3 & 2.7 & & & & 9.25 & d & 0.02 & $1 / 2^{+}$ & 00 & & & $\varepsilon=100$ \\
\hline${ }^{167} \mathrm{Tm}^{m}$ & -62368.8 & 2.7 & 179.480 & 0.019 & & 1.16 & $\mu \mathrm{s}$ & 0.06 & $(7 / 2)^{+}$ & 00 & & & $\mathrm{IT}=100$ \\
\hline${ }^{167} \mathrm{Tm}^{n}$ & -62255.5 & 2.7 & 292.820 & 0.020 & & 0.9 & $\mu \mathrm{s}$ & 0.1 & $7 / 2^{-}$ & 00 & & & $\mathrm{IT}=100$ \\
\hline${ }^{167} \mathrm{Yb}$ & -60594 & 5 & & & & 17.5 & $\mathrm{~m}$ & 0.2 & $5 / 2^{-}$ & 00 & & & $\beta^{+}=100$ \\
\hline${ }^{167} \mathrm{Lu}$ & -57500 & 30 & & & $*$ & 51.5 & $\mathrm{~m}$ & 1.0 & $7 / 2^{+}$ & 00 & & & $\beta^{+}=100$ \\
\hline${ }^{167} \mathrm{Lu}^{m}$ & $-57500 \#$ & $40 \#$ & O\# & $30 \#$ & * & $>1$ & $\mathrm{~m}$ & & $1 / 2^{(-\#)}$ & 00 & & & IT $? ; \beta^{+} ?$ \\
\hline${ }^{167} \mathrm{Hf}$ & -53468 & 28 & & & & 2.05 & $\mathrm{~m}$ & 0.05 & $(5 / 2)^{-}$ & 00 & & & $\beta^{+}=100$ \\
\hline${ }^{167} \mathrm{Ta}$ & -48351 & 28 & & & & 1.33 & $\mathrm{~m}$ & 0.07 & $\left(3 / 2^{+}\right)$ & 00 & & & $\beta^{+}=100$ \\
\hline${ }^{167} \mathrm{~W}$ & -42089 & 19 & & & & 19.9 & $\mathrm{~s}$ & 0.5 & $3 / 2^{-} \#$ & 00 & & & $\beta^{+}=99.961 ; \alpha=0.041$ \\
\hline${ }^{167} \mathrm{Re}$ & $-34840 \#$ & $50 \#$ & & & $\&$ & 3.4 & s & 0.4 & $9 / 2^{-} \#$ & 00 & & & $\alpha \approx 100 ; \beta^{+} ?$ \\
\hline${ }^{167} \mathrm{Re}^{m}$ & -34710 & 40 & $130 \#$ & $40 \#$ & \& & 5.9 & $\mathrm{~s}$ & 0.3 & $1 / 2^{+} \#$ & 00 & & & $\beta^{+} \approx 99 ; \alpha \approx 1$ \\
\hline${ }^{167} \mathrm{Os}$ & -26500 & 70 & & & & 810 & $\mathrm{~ms}$ & 60 & $3 / 2^{-} \#$ & 00 & & & $\alpha=578 ; \beta^{+}=438$ \\
\hline${ }^{167} \mathrm{Ir}$ & -17079 & 19 & & & & 35.2 & $\mathrm{~ms}$ & 2.0 & $1 / 2^{+}$ & 02 & & & $\alpha=486 ; \mathrm{p}=324 ; \beta^{+} ?$ \\
\hline${ }^{167} \mathrm{Ir}^{m}$ & -16903 & 19 & 175.3 & 2.2 & $\mathrm{p}$ & 30.0 & $\mathrm{~ms}$ & 0.6 & $11 / 2^{-}$ & 02 & & & $\alpha=8010 ; \beta^{+} ? ; \ldots$ \\
\hline${ }^{167} \mathrm{Pt}$ & $-6540 \#$ & $410 \#$ & & & & 700 & $\mu \mathrm{s}$ & 200 & $7 / 2^{-} \#$ & 00 & & & $\alpha=100$ \\
\hline${ }^{167} \mathrm{~W}$ & $\mathrm{~J}$ : lowest o & observed & state by & Th06 & $13 / 2^{+}$ & & & & & & & & \\
\hline${ }^{167} \mathrm{Ir}^{m}$ & $\mathrm{D}: \ldots ; \mathrm{p}=$ & $=0.41$ & & & & & & & & & & & \\
\hline
\end{tabular}




\begin{tabular}{|c|c|c|c|c|c|c|c|c|c|c|c|c|c|c|}
\hline Nuclide & \multicolumn{2}{|c|}{$\begin{array}{l}\text { Mass excess } \\
(\mathrm{keV})\end{array}$} & \multicolumn{3}{|c|}{$\begin{array}{l}\text { Excitation } \\
\text { energy }(\mathrm{keV})\end{array}$} & \multicolumn{3}{|c|}{ Half-life } & $J^{\pi}$ & Ens & \multicolumn{2}{|c|}{ Reference } & \multicolumn{2}{|l|}{$\begin{array}{l}\text { Decay modes and } \\
\text { intensities }(\%)\end{array}$} \\
\hline${ }^{168} \mathrm{Gd}$ & $-48100 \#$ & $700 \#$ & & & & $300 \#$ & $\mathrm{~ms}$ & & $0^{+}$ & & $85 \mathrm{Si} 25$ & I & $\beta^{-} ?$ & $*$ \\
\hline${ }^{168} \mathrm{~Tb}$ & $-52500 \#$ & $500 \#$ & & & & 8.2 & $\mathrm{~s}$ & 1.3 & $4^{-} \#$ & 99 & & & $\beta^{-}=100$ & \\
\hline${ }^{168} \mathrm{Dy}$ & -58560 & 140 & & & & 8.7 & $\mathrm{~m}$ & 0.3 & $0^{+}$ & 99 & & & $\beta^{-}=100$ & \\
\hline${ }^{168} \mathrm{Ho}$ & -60070 & 30 & & & & 2.99 & $\mathrm{~m}$ & 0.07 & $3^{+}$ & 94 & & & $\beta^{-}=100$ & \\
\hline${ }^{168} \mathrm{Ho}^{m}$ & -60010 & 30 & 59 & 1 & & 132 & $\mathrm{~s}$ & 4 & $\left(6^{+}\right)$ & 94 & $90 \mathrm{Ch} 37$ & $\mathrm{E}$ & $\mathrm{IT} \approx 100 ; \beta^{-}<0.5$ & \\
\hline${ }^{168} \mathrm{Er}$ & -62996.7 & 2.5 & & & & StABLE & & & $0^{+}$ & 94 & & & $\mathrm{IS}=26.7826$ & \\
\hline${ }^{168} \mathrm{Tm}$ & -61317.7 & 2.9 & & & & 93.1 & $\mathrm{~d}$ & 0.2 & $3^{+}$ & 94 & & & $\beta^{+} \approx 100 ; \beta^{-}=0.0107$ & \\
\hline${ }^{168} \mathrm{Yb}$ & -61575 & 4 & & & & STABLE & & (>130 Ty) & $0^{+}$ & 94 & 56Рo16 & $\mathrm{T}$ & $\mathrm{IS}=0.131 ; \alpha ? ; 2 \beta^{+} ?$ & * \\
\hline${ }^{168} \mathrm{Lu}$ & -57060 & 50 & & & $*$ & 5.5 & $\mathrm{~m}$ & 0.1 & $6^{(-)}$ & 94 & $98 \mathrm{Ge} 13$ & $\mathrm{~J}$ & $\beta^{+}=100$ & \\
\hline${ }^{168} \mathrm{Lu}^{m}$ & -56880 & 100 & 180 & 110 & $\mathrm{BD} *$ & 6.7 & $\mathrm{~m}$ & 0.4 & $3^{+}$ & 94 & & & $\beta^{+}>95 ;$ IT $<5$ & \\
\hline${ }^{168} \mathrm{Hf}$ & -55361 & 28 & & & & 25.95 & $\mathrm{~m}$ & 0.20 & $0^{+}$ & 01 & & & $\varepsilon \approx 98 ; \mathrm{e}^{+} \approx 2$ & \\
\hline${ }^{168} \mathrm{Ta}$ & -48394 & 28 & & & & 2.0 & $\mathrm{~m}$ & 0.1 & $\left(2^{-}, 3^{+}\right)$ & 94 & & & $\beta^{+}=100$ & \\
\hline${ }^{168} \mathrm{~W}$ & -44890 & 16 & & & & 51 & $\mathrm{~s}$ & 2 & $0^{+}$ & 94 & & & $\beta^{+} \approx 100 ; \alpha=0.003210$ & \\
\hline${ }^{168} \mathrm{Re}$ & -35790 & 30 & & & & 4.4 & s & 0.1 & $\left(5^{+}, 6^{+}, 7^{+}\right)$ & 94 & & & $\beta^{+} \approx 100 ; \alpha \approx 0.005$ & \\
\hline${ }^{168} \operatorname{Re}^{m}$ & & & & existent & RN & 6.6 & s & 1.5 & & & $92 \mathrm{Me} 10$ & & & \\
\hline${ }^{168} \mathrm{Os}$ & -29991 & 12 & & & & 2.06 & s & 0.06 & $0^{+}$ & 94 & $96 \mathrm{~Pa} 01$ & $\mathrm{~T}$ & $\beta^{+}=513 ; \alpha=493$ & * \\
\hline${ }^{168} \mathrm{Ir}$ & $-18740 \#$ & $150 \#$ & & & $*$ & 161 & $\mathrm{~ms}$ & 21 & high & 94 & $96 \mathrm{~Pa} 01$ & TJD & $\alpha=8214$ & \\
\hline${ }^{168} \mathrm{Ir}^{m}$ & -18690 & 110 & $50 \#$ & $100 \#$ & $*$ & 125 & $\mathrm{~ms}$ & 40 & low & 94 & $96 \mathrm{~Pa} 01$ & $\mathrm{TJ}$ & $\alpha=? ; \beta^{+} ?$ & \\
\hline${ }^{168} \mathrm{Pt}$ & -11040 & 210 & & & & 2.00 & $\mathrm{~ms}$ & 0.18 & $0^{+}$ & 94 & $98 \mathrm{Ki} 20$ & $\mathrm{~T}$ & $\alpha \approx 100 ; \beta^{+}=0.7 \#$ & * \\
\hline$*^{168} \mathrm{Gd}$ & I : seen in & the the & rmal fi & sion of 25 & & & & & & & & & & $* *$ \\
\hline$*^{168} \mathrm{Yb}$ & $\mathrm{T}$ : lower $\mathrm{l}$ & limit is & for $\alpha$ & ecay & & & & & & & & & & ** \\
\hline$*^{168}$ Os & $\mathrm{T}:$ averag & $96 \mathrm{~Pa}$ & $1=2.1$ & $0.1) 84 \mathrm{Sc}$ & $=2.0(0$ & 2) $82 \mathrm{En} 03$ & $3=2$. & $2(0.1) 78 \mathrm{C}$ & $\mathrm{Ca} 11=1.9(0.1)$ & & & & & ** \\
\hline${ }^{168} \mathrm{Os}$ & $\mathrm{T}: \quad 84$ & $\mathrm{Sc} 06 \mathrm{~s}$ & persec & es $78 \mathrm{Sc} 2 \mathrm{C}$ & $2.4(0.2$ & from sam & ne gr & oup & & & & & & $* *$ \\
\hline$*^{168} \mathrm{Pt}$ & $\mathrm{T}:$ averag & $98 \mathrm{Ki}$ & $20=2.0$ & $0.2) 96 \mathrm{Bi}$ & $=2.0(0$ & & & & & & & & & $* *$ \\
\hline
\end{tabular}

\begin{tabular}{|c|c|c|c|c|c|c|c|c|c|c|c|c|c|c|}
\hline${ }^{169} \mathrm{Gd}$ & $-43900 \#$ & $800 \#$ & & & & & $1 \#$ & s & & $7 / 2^{-} \#$ & & & & $\beta^{-} ?$ \\
\hline${ }^{169} \mathrm{~Tb}$ & $-50100 \#$ & $600 \#$ & & & & & $2 \#$ & $\mathrm{~s}$ & & $3 / 2^{+} \#$ & & & & $\beta^{-} ?$ \\
\hline${ }^{169}$ Dy & -55600 & 300 & & & & & 39 & $\mathrm{~s}$ & 8 & $\left(5 / 2^{-}\right)$ & 91 & & & $\beta^{-}=100$ \\
\hline${ }^{169} \mathrm{Ho}$ & -58803 & 20 & & & & & 4.7 & $\mathrm{~m}$ & 0.1 & $7 / 2^{-}$ & 91 & & & $\beta^{-}=100$ \\
\hline${ }^{169} \mathrm{Er}$ & -60928.7 & 2.5 & & & & & 9.40 & $\mathrm{~d}$ & 0.02 & $1 / 2^{-}$ & 91 & & & $\beta^{-}=100$ \\
\hline${ }^{169} \mathrm{Tm}$ & -61280.0 & 2.5 & & & & & STABLE & & & $1 / 2^{+}$ & 91 & & & $\mathrm{IS}=100$ \\
\hline${ }^{169} \mathrm{Yb}$ & -60370 & 4 & & & & & 32.026 & $\mathrm{~d}$ & 0.005 & $7 / 2^{+}$ & 91 & & & $\varepsilon=100$ \\
\hline${ }^{169} \mathrm{Yb}^{m}$ & -60346 & 4 & 24.199 & 0.00 & & & 46 & $\mathrm{~s}$ & 2 & $1 / 2^{-}$ & 91 & & & $\mathrm{IT}=100$ \\
\hline${ }^{169} \mathrm{Lu}$ & -58077 & 5 & & & & & 34.06 & $\mathrm{~h}$ & 0.05 & $7 / 2^{+}$ & 91 & & & $\beta^{+}=100$ \\
\hline${ }^{169} \mathrm{Lu}^{m}$ & -58048 & 5 & 29.0 & 0.5 & & & 160 & $\mathrm{~s}$ & 10 & $1 / 2^{-}$ & 91 & & & $\mathrm{IT}=100$ \\
\hline${ }^{169} \mathrm{Hf}$ & -54717 & 28 & & & & & 3.24 & $\mathrm{~m}$ & 0.04 & $(5 / 2)^{-}$ & 91 & & & $\beta^{+}=100$ \\
\hline${ }^{169} \mathrm{Ta}$ & -50290 & 28 & & & & & 4.9 & $\mathrm{~m}$ & 0.4 & $\left(5 / 2^{+}\right)$ & 91 & 98Zh03 & $\mathrm{J}$ & $\beta^{+}=100$ \\
\hline${ }^{169} \mathrm{~W}$ & -44918 & 15 & & & & & 76 & $\mathrm{~s}$ & 6 & $\left(5 / 2^{-}\right)$ & 91 & & & $\beta^{+}=100$ \\
\hline${ }^{169} \mathrm{Re}$ & -38386 & 28 & & & & & 8.1 & s & 0.5 & $9 / 2^{-} \#$ & 91 & $92 \mathrm{Me} 10$ & TD & $\beta^{+}=? ; \alpha=0.0053$ \\
\hline${ }^{169} \mathrm{Re}^{m}$ & -38241 & 17 & 145 & 29 & $\mathrm{AD}$ & & 15.1 & s & 1.6 & $1 / 2^{+} \#$ & 91 & $92 \mathrm{Me} 10$ & TD & $\beta^{+} ? ; \alpha \approx 0.2$ \\
\hline${ }^{169} \mathrm{Os}$ & -30721 & 25 & & & & & 3.46 & $\mathrm{~s}$ & 0.11 & $3 / 2^{-} \#$ & 91 & $96 \mathrm{~Pa} 01$ & $\mathrm{~T}$ & $\beta^{+}=891 ; \alpha=111$ \\
\hline${ }^{169} \mathrm{Ir}$ & -22081 & 26 & & & & $\&$ & 780 & $\mathrm{~ms}$ & 360 & $1 / 2^{+} \#$ & & 99Рo09 & $\mathrm{TD}$ & $\alpha=5018 ; \beta^{+} ?$ \\
\hline${ }^{169} \mathrm{Ir}^{m}$ & -21927 & 22 & 154 & 24 & $\mathrm{AD}$ & $\&$ & 308 & $\mathrm{~ms}$ & 22 & $11 / 2^{-} \#$ & 91 & $96 \mathrm{~Pa} 01$ & $\mathrm{TD}$ & $\alpha=817 ; \beta^{+}=197$ \\
\hline${ }^{169} \mathrm{Pt}$ & $-12380 \#$ & $200 \#$ & & & & & 3.7 & $\mathrm{~ms}$ & 1.5 & $3 / 2^{-} \#$ & 91 & $96 \mathrm{~Pa} 01$ & $\mathrm{~T}$ & $\alpha=? ; \beta^{+}=1 \#$ \\
\hline${ }^{169} \mathrm{Au}$ & $-1790 \#$ & $300 \#$ & & & & & $150 \#$ & $\mu \mathrm{s}$ & & $1 / 2^{+} \#$ & & & & $\alpha ? ; \beta^{+} ?$ \\
\hline${ }^{169} \mathrm{Re}$ & $\mathrm{D}: \alpha=0.0$ & $05(3) \%$ & derived $f$ & om or & inal $\alpha$ & $=0.0$ & $001 \%-0$ & $0.01 \%$ & & & & & & \\
\hline${ }^{169} \mathrm{Re}^{m}$ & $\mathrm{~T}:$ averag & $\mathrm{e} 92 \mathrm{M}$ & $10=16.3$ & .8) 84 & $c 06=1$ & $2.9(1$ & 1.1) & & & & & & & \\
\hline$*^{169} \mathrm{Os}$ & $\mathrm{T}$ : average & e $96 \mathrm{~Pa}$ & $01=3.6(0.2$ & ) $95 \mathrm{Hi}$ & $2=3.2$ & $0.3)$ & $84 \mathrm{Sc} 06$ & $6=3.5$ & $(0.2) 8$ & $3=3.4(0.2$ & & & & \\
\hline${ }^{169} \mathrm{Ir}^{m}$ & $\mathrm{~T}:$ also 99 & $\mathrm{Po} 09=$ & $323(+90$ & & D : & aver & rage 99P & o09= & $84(8) \%$ & $\mathrm{~Pa} 01=72($ & 3) $\%$ & & & \\
\hline${ }^{*}{ }^{169} \mathrm{Pt}$ & $\mathrm{T}$ : averag & e $96 \mathrm{~Pa}$ & $01=5(3) 8$ & Holo= & $2.5(+2$ & $5-1$ & & & & & & & & \\
\hline
\end{tabular}




\begin{tabular}{|c|c|c|c|c|c|c|c|c|c|c|c|c|c|c|}
\hline Nuclide & \multicolumn{2}{|c|}{$\begin{array}{l}\text { Mass excess } \\
(\mathrm{keV})\end{array}$} & \multicolumn{3}{|c|}{$\begin{array}{l}\text { Excitation } \\
\text { energy }(\mathrm{keV})\end{array}$} & \multicolumn{3}{|c|}{ Half-life } & \multirow[t]{2}{*}{$J^{\pi}$} & \multirow[t]{2}{*}{ Ens } & \multicolumn{2}{|c|}{ Reference } & \multicolumn{2}{|l|}{$\begin{array}{l}\text { Decay modes and } \\
\text { intensities }(\%)\end{array}$} \\
\hline${ }^{170} \mathrm{~Tb}$ & $-46340 \#$ & $700 \#$ & & & & $3 \#$ & $\mathrm{~s}$ & & & & & & $\beta^{-?}$ & \\
\hline${ }^{170}$ Dy & $-53660 \#$ & $200 \#$ & & & & $30 \#$ & s & & $0^{+}$ & 02 & & & $\beta^{-} ?$ & \\
\hline${ }^{170} \mathrm{Ho}$ & -56240 & 50 & & & * & 2.76 & $\mathrm{~m}$ & 0.05 & $6^{+} \#$ & 02 & & & $\beta^{-}=100$ & \\
\hline${ }^{170} \mathrm{Ho}^{m}$ & -56140 & 60 & 100 & 80 & $\mathrm{BD} *$ & 43 & $\mathrm{~s}$ & 2 & $\left(1^{+}\right)$ & 02 & & & $\beta^{-}=100$ & \\
\hline${ }^{170} \mathrm{Er}$ & -60114.6 & 2.8 & & & & Stable & & (>320 Py) & $0^{+}$ & 02 & 96De60 & $\mathrm{T}$ & $\mathrm{IS}=14.9327 ; \ldots$ & * \\
\hline${ }^{170} \mathrm{Tm}$ & -59800.6 & 2.5 & & & & 128.6 & $\mathrm{~d}$ & 0.3 & $1^{-}$ & 02 & & & $\beta^{-} \approx 100 ; \varepsilon=0.13110$ & \\
\hline${ }^{170} \mathrm{Tm}^{m}$ & -59617.4 & 2.5 & 183.197 & 0.004 & & 4.12 & $\mu \mathrm{s}$ & 0.13 & $(3)^{+}$ & 02 & & & $\mathrm{IT}=100$ & \\
\hline${ }^{170} \mathrm{Yb}$ & -60769.0 & 2.4 & & & & STABLE & & & $0^{+}$ & 02 & & & $\mathrm{IS}=3.0415$ & \\
\hline${ }^{170} \mathrm{Yb}^{m}$ & -59510.5 & 2.4 & 1258.46 & 0.14 & & 370 & $\mathrm{~ns}$ & 15 & $4^{-}$ & 02 & & & $\mathrm{IT}=100$ & \\
\hline${ }^{170} \mathrm{Lu}$ & -57310 & 17 & & & & 2.012 & $\mathrm{~d}$ & 0.020 & $0^{+}$ & 02 & & & $\beta^{+}=100$ & \\
\hline${ }^{170} \mathrm{Lu}^{m}$ & -57217 & 17 & 92.91 & 0.09 & & 670 & $\mathrm{~ms}$ & 100 & $(4)^{-}$ & 02 & & & $\mathrm{IT}=100$ & \\
\hline${ }^{170} \mathrm{Hf}$ & -56254 & 28 & & & & 16.01 & $\mathrm{~h}$ & 0.13 & $0^{+}$ & 02 & & & $\varepsilon=100$ & \\
\hline${ }^{170} \mathrm{Ta}$ & -50138 & 28 & & & & 6.76 & $\mathrm{~m}$ & 0.06 & $(3)^{(+\#)}$ & 02 & & & $\beta^{+}=100$ & \\
\hline${ }^{170} \mathrm{~W}$ & -47293 & 15 & & & & 2.42 & $\mathrm{~m}$ & 0.04 & $0^{+}$ & 02 & & & $\beta^{+} \approx 100 ; \alpha<1 \#$ & \\
\hline${ }^{170} \mathrm{Re}$ & -38918 & 26 & & & & 9.2 & $\mathrm{~s}$ & 0.2 & $\left(5^{+}\right)$ & 02 & & & $\beta^{+} \approx 100 ; \alpha<0.01 \#$ & \\
\hline${ }^{170} \mathrm{Os}$ & -33928 & 11 & & & & 7.46 & $\mathrm{~s}$ & 0.23 & $0^{+}$ & 02 & & & $\beta^{+}=? ; \alpha=8.618$ & \\
\hline${ }^{170} \mathrm{Ir}$ & $-23320 \#$ & $100 \#$ & & & & 910 & $\mathrm{~ms}$ & 150 & low\# & 02 & & & $\beta^{+} ? ; \alpha=5.217$ & \\
\hline${ }^{170} \mathrm{Ir}^{m}$ & -23050 & 70 & 270\# & $70 \#$ & & 440 & $\mathrm{~ms}$ & 60 & high\# & 02 & & & $\alpha=3610 ; \beta^{+} ? ;$ IT ? & \\
\hline${ }^{170} \mathrm{Pt}$ & -16306 & 19 & & & & 13.8 & $\mathrm{~ms}$ & 0.5 & $0^{+}$ & 02 & & & $\alpha=? ; \beta^{+}=2 \#$ & \\
\hline${ }^{170} \mathrm{Au}$ & $-3610 \#$ & $200 \#$ & & & & 310 & $\mu \mathrm{s}$ & 50 & $\left(2^{-}\right)$ & 02 & & & $\mathrm{p}=8510 ; \alpha=1510$ & \\
\hline${ }^{170} \mathrm{Au}^{m}$ & $-3340 \#$ & $200 \#$ & 274 & 16 & $\mathrm{p}$ & 630 & $\mu \mathrm{s}$ & 60 & $\left(9^{+}\right)$ & 02 & 02Ma61 & $\mathrm{TD}$ & $\mathrm{p}=7515 ; \alpha=? ; \beta^{+} ?$ & * \\
\hline${ }^{170} \mathrm{Er}$ & $\mathrm{D}: \ldots ; 2 \beta$ & $\beta^{-} ? ; \alpha$ & & & & & & & & & & & & $* *$ \\
\hline${ }^{170} \mathrm{Au}^{m}$ & $\mathrm{~T}$ : from 02 & $2 \mathrm{Ke} . \mathrm{C}=$ & $620(+60-$ & other & Ma6 & $70(+$ & $10-10$ & & & & & & & $* *$ \\
\hline
\end{tabular}

\begin{tabular}{|c|c|c|c|c|c|c|c|c|c|c|c|c|c|}
\hline${ }^{171} \mathrm{~Tb}$ & $-43500 \#$ & $800 \#$ & & & & $500 \#$ & $\mathrm{~ms}$ & & $3 / 2^{+} \#$ & & & & $\beta^{-}$? \\
\hline${ }^{171}$ Dy & $-50110 \#$ & $300 \#$ & & & & $6 \#$ & $\mathrm{~s}$ & & $7 / 2^{-} \#$ & & & & $\beta^{-}$? \\
\hline${ }^{171} \mathrm{Ho}$ & -54520 & 600 & & & & 53 & s & 2 & $7 / 2^{-} \#$ & 02 & & & $\beta^{-}=100$ \\
\hline${ }^{171} \mathrm{Er}$ & -57724.9 & 2.8 & & & & 7.516 & $\mathrm{~h}$ & 0.002 & $5 / 2^{-}$ & 02 & & & $\beta^{-}=100$ \\
\hline${ }^{171} \mathrm{Er}^{m}$ & -57526.3 & 2.8 & 198.6 & 0.1 & & 210 & ns & 10 & $1 / 2^{-}$ & 02 & & & $\mathrm{IT}=100$ \\
\hline${ }^{171} \mathrm{Tm}$ & -59215.6 & 2.6 & & & & 1.92 & $\mathrm{y}$ & 0.01 & $1 / 2^{+}$ & 02 & & & $\beta^{-}=100$ \\
\hline${ }^{171} \mathrm{Tm}^{m}$ & -58790.6 & 2.6 & 424.9560 & 0.0015 & & 2.60 & $\mu \mathrm{s}$ & 0.02 & $7 / 2^{-}$ & 02 & & & $\mathrm{IT}=100$ \\
\hline${ }^{171} \mathrm{Yb}$ & -59312.1 & 2.4 & & & & STABLE & & & $1 / 2^{-}$ & 02 & & & $\mathrm{IS}=14.2857$ \\
\hline${ }^{171} \mathrm{Yb}^{m}$ & -59216.8 & 2.4 & 95.282 & 0.002 & & 5.25 & $\mathrm{~ms}$ & 0.24 & $7 / 2^{+}$ & 02 & & & $\mathrm{IT}=100$ \\
\hline${ }^{171} \mathrm{Yb}^{n}$ & -59189.7 & 2.4 & 122.416 & 0.002 & & 265 & $\mathrm{~ns}$ & 20 & $5 / 2^{-}$ & 02 & & & $\mathrm{IT}=100$ \\
\hline${ }^{171} \mathrm{Lu}$ & -57833.5 & 2.8 & & & & 8.24 & $\mathrm{~d}$ & 0.03 & $7 / 2^{+}$ & 02 & & & $\beta^{+}=100$ \\
\hline${ }^{171} \mathrm{Lu}^{m}$ & -57762.4 & 2.8 & 71.13 & 0.08 & & 79 & $\mathrm{~s}$ & 2 & $1 / 2^{-}$ & 02 & & & $\mathrm{IT}=100$ \\
\hline${ }^{171} \mathrm{Hf}$ & -55431 & 29 & & & & 12.1 & $\mathrm{~h}$ & 0.4 & $7 / 2^{(+)}$ & 02 & & & $\beta^{+}=100$ \\
\hline${ }^{171} \mathrm{Hf}^{m}$ & -55409 & 29 & 21.93 & 0.09 & & 29.5 & $\mathrm{~s}$ & 0.9 & $1 / 2^{(-)}$ & 02 & & & $\mathrm{IT} \approx 100 ; \beta^{+} ?$ \\
\hline${ }^{171} \mathrm{Ta}$ & -51720 & 28 & & & & 23.3 & $\mathrm{~m}$ & 0.3 & $\left(5 / 2^{-}\right)$ & 02 & & & $\beta^{+}=100$ \\
\hline${ }^{171} \mathrm{~W}$ & -47086 & 28 & & & & 2.38 & $\mathrm{~m}$ & 0.04 & $\left(5 / 2^{-}\right)$ & 02 & & & $\beta^{+}=100$ \\
\hline${ }^{171} \mathrm{Re}$ & -41250 & 28 & & & & 15.2 & $\mathrm{~s}$ & 0.4 & $\left(9 / 2^{-}\right)$ & 02 & & & $\beta^{+}=100$ \\
\hline${ }^{171} \mathrm{Os}$ & -34293 & 19 & & & & 8.3 & s & 0.2 & $\left(5 / 2^{-}\right)$ & 02 & & & $\beta^{+}$?; $\alpha=1.8021$ \\
\hline${ }^{171} \mathrm{Ir}$ & -26430 & 40 & & & & 3.6 & s & 1.0 & $1 / 2^{+} \#$ & 02 & & & $\alpha \approx 100 ; \beta^{+} ?$ \\
\hline${ }^{171} \mathrm{Ir}^{m}$ & $-26250 \#$ & $50 \#$ & $180 \#$ & $30 \#$ & & 1.40 & $\mathrm{~s}$ & 0.10 & $\left(11 / 2^{-}\right)$ & 02 & 99Ba84 & J & $\alpha=5811 ; \beta^{+} ? ; \mathrm{p} ?$ \\
\hline${ }^{171} \mathrm{Pt}$ & -17470 & 90 & & & & 44 & $\mathrm{~ms}$ & 7 & $3 / 2^{-} \#$ & 02 & & & $\alpha=? ; \beta^{+}=2 \#$ \\
\hline${ }^{171} \mathrm{Au}$ & -7565 & 26 & & & & 30 & $\mu \mathrm{s}$ & 5 & $\left(1 / 2^{+}\right)$ & 02 & 03Ba20 & $\mathrm{T}$ & $\mathrm{p} \approx 100 ; \alpha ?$ \\
\hline${ }^{171} \mathrm{Au}^{m}$ & -7315 & 20 & 250 & 16 & $\mathrm{p}$ & 1.014 & $\mathrm{~ms}$ & 0.019 & $11 / 2^{-}$ & 02 & $03 \mathrm{Ba} 20$ & $\mathrm{TJ}$ & $\alpha=544 ; \mathrm{p}=464$ \\
\hline${ }^{171} \mathrm{Hg}$ & 3500\# & $300 \#$ & & & $\mathrm{p}$ & 80 & $\mu \mathrm{s}$ & 30 & $3 / 2^{-} \#$ & 02 & & & $\alpha \approx 100 ; \beta^{+}=0.01 \#$ \\
\hline${ }^{171} \mathrm{Au}$ & $\mathrm{T}$ : averag & $\mathrm{e} 03 \mathrm{Ba} 2$ & $=37(+7-$ & $99 \mathrm{Po} 0$ & & ); Birge & ratio & $B=2.0$ & & & & & \\
\hline
\end{tabular}




\begin{tabular}{|c|c|c|c|c|c|c|c|c|c|c|c|c|c|c|}
\hline \multirow{2}{*}{$\begin{array}{l}\text { Nuclide } \\
{ }^{172} \mathrm{Dy}\end{array}$} & \multicolumn{2}{|c|}{$\begin{array}{l}\text { Mass excess } \\
\quad(\mathrm{keV})\end{array}$} & \multicolumn{3}{|c|}{$\begin{array}{l}\text { Excitation } \\
\text { energy }(\mathrm{keV})\end{array}$} & \multicolumn{3}{|c|}{ Half-life } & \multirow{2}{*}{$\frac{J^{\pi}}{0^{+}}$} & \multirow{2}{*}{$\begin{array}{c}\text { Ens } \\
\end{array}$} & \multicolumn{2}{|c|}{ Reference } & \multicolumn{2}{|l|}{$\begin{array}{l}\text { Decay modes and } \\
\text { intensities }(\%)\end{array}$} \\
\hline & $-47730 \#$ & $400 \#$ & & & & $3 \#$ & $\mathrm{~s}$ & & & & & & $\beta^{-} ?$ & \\
\hline${ }^{172} \mathrm{Ho}$ & $-51400 \#$ & $400 \#$ & & & & 25 & $\mathrm{~s}$ & 3 & & 95 & & & $\beta^{-}=100$ & \\
\hline${ }^{172} \mathrm{Er}$ & -56489 & 5 & & & & 49.3 & $\mathrm{~h}$ & 0.3 & $0^{+}$ & 95 & & & $\beta^{-}=100$ & \\
\hline${ }^{172} \mathrm{Tm}$ & -57380 & 6 & & & & 63.6 & $\mathrm{~h}$ & 0.2 & $2^{-}$ & 95 & & & $\beta^{-}=100$ & \\
\hline${ }^{172} \mathrm{Yb}$ & -59260.3 & 2.4 & & & & STABLE & & & $0^{+}$ & 95 & & & $\mathrm{IS}=21.8367$ & \\
\hline${ }^{172} \mathrm{Lu}$ & -56741.3 & 3.0 & & & & 6.70 & $\mathrm{~d}$ & 0.03 & $4^{-}$ & 95 & & & $\beta^{+}=100$ & \\
\hline${ }^{172} \mathrm{Lu}^{m}$ & -56699 & 3 & 41.86 & 0.04 & & 3.7 & $\mathrm{~m}$ & 0.5 & $1^{-}$ & 95 & & & $\mathrm{IT}=100$ & \\
\hline${ }^{172} \mathrm{Lu}^{n}$ & -56632 & 3 & 109.41 & 0.10 & & 440 & $\mu \mathrm{s}$ & 12 & $(1)^{+}$ & & & & & \\
\hline${ }^{172} \mathrm{Hf}$ & -56404 & 24 & & & & 1.87 & $\begin{array}{l}\mu_{0} \\
\mathrm{y}\end{array}$ & 0.03 & $0^{+}$ & 95 & & & $\varepsilon=100$ & \\
\hline${ }^{172} \mathrm{Hf}^{m}$ & -54398 & 24 & 2005.58 & 0.11 & & 163 & ns & 3 & $\left(8^{-}\right)$ & & & & & \\
\hline${ }^{172} \mathrm{Ta}$ & -51330 & 28 & & & & 36.8 & $\mathrm{~m}$ & 0.3 & $\left(3^{+}\right)$ & 95 & & & $\beta^{+}=100$ & \\
\hline${ }^{172} \mathrm{~W}$ & -49097 & 28 & & & & 6.6 & $\mathrm{~m}$ & 0.9 & $0^{+}$ & 95 & & & $\beta^{+}=100$ & \\
\hline${ }^{172} \mathrm{Re}$ & -41520 & 50 & & & * & 15 & $\mathrm{~s}$ & 3 & $(5)$ & 95 & & & $\beta^{+}=100$ & \\
\hline${ }^{172} \mathrm{Re}^{m}$ & $-41520 \#$ & $110 \#$ & 0\# & $100 \#$ & * & 55 & $\mathrm{~s}$ & 5 & (2) & 95 & & & $\beta^{+}=100$ & \\
\hline${ }^{172} \mathrm{Os}$ & -37238 & 15 & & & & 19.2 & $\mathrm{~s}$ & 0.9 & $0^{+}$ & 95 & $95 \mathrm{Hi} 02$ & $\mathrm{D}$ & $\beta^{+}=? ; \alpha=1.12$ & \\
\hline${ }^{172} \mathrm{Ir}$ & -27520\# & $110 \#$ & & & & 4.4 & $\mathrm{~s}$ & 0.3 & $\left(3^{+}\right)$ & 95 & & & $\beta^{+}=98 ; \alpha=2$ & \\
\hline${ }^{172} \mathrm{Ir}^{m}$ & -27240 & 30 & $280 \#$ & $100 \#$ & $\mathrm{AD}$ & 2.0 & $\mathrm{~s}$ & 0.1 & $\left(7^{+}\right)$ & 95 & & & $\beta^{+}=773 ; \alpha=233$ & \\
\hline${ }^{172} \mathrm{Pt}$ & -21101 & 13 & & & & 98.4 & $\mathrm{~ms}$ & 2.4 & $0^{+}$ & 95 & 02Ro17 & $\mathrm{T}$ & $\alpha=7721 ; \beta^{+} ?$ & $*$ \\
\hline${ }^{172} \mathrm{Au}$ & $-9280 \#$ & $160 \#$ & & & & 4.7 & $\mathrm{~ms}$ & 1.1 & high & 95 & $96 \mathrm{~Pa} 01$ & $\mathrm{TJ}$ & $\alpha=? ; \mathrm{p}<2$ & * \\
\hline${ }^{172} \mathrm{Hg}$ & -1090 & 210 & & & & 420 & $\mu \mathrm{s}$ & 240 & $0^{+}$ & & $99 \mathrm{Se} 14$ & TD & $\alpha=100$ & \\
\hline$*^{172} \mathrm{Pt}$ & $\mathrm{T}:$ averag & e $02 \mathrm{Ro}$ & $7=104(7)$ & $96 \mathrm{~Pa} 01=$ & 6(3) 82 & $103=90$ & 0) 81 & De22 & (10) a & & & & & $* *$ \\
\hline$*^{172} \mathrm{Pt}$ & $\mathrm{T}: \quad 75 \mathrm{C}$ & $\mathrm{Ga} 25=1$ & $00(10)$ & $\mathrm{D}: \mathrm{d}$ & rived $\mathrm{fr}$ & $n$ origin & $\alpha=$ & $94(32)$ & & & & & & $* *$ \\
\hline$*^{172} \mathrm{Au}$ & $\mathrm{T}:$ average & $96 \mathrm{PaC}$ & $1=6.3(1.5$ & $93 \mathrm{Se} 09=$ & & & & & & & & & & $* *$ \\
\hline$*^{172} \mathrm{Au}$ & $\mathrm{J}:$ from $\alpha$ & correla & tion with ${ }^{1}$ & ${ }^{8} \mathrm{Ir}$ line & & & & & & & & & & $* *$ \\
\hline
\end{tabular}

\begin{tabular}{|c|c|c|c|c|c|c|c|c|c|c|c|c|c|}
\hline${ }^{173}$ Dy & $-43780 \#$ & $500 \#$ & & & & $2 \#$ & $\mathrm{~s}$ & & $9 / 2^{+} \#$ & & & & $\beta^{-} ?$ \\
\hline${ }^{173} \mathrm{Ho}$ & $-49100 \#$ & $400 \#$ & & & & $10 \#$ & s & & $7 / 2^{-} \#$ & & & & $\beta^{-}$? \\
\hline${ }^{173} \mathrm{Er}$ & $-53650 \#$ & $200 \#$ & & & & 1.434 & $\mathrm{~m}$ & 0.017 & $\left(7 / 2^{-}\right)$ & 95 & 94It.A & $\mathrm{T}$ & $\beta^{-}=100$ \\
\hline${ }^{173} \mathrm{Tm}$ & -56259 & 5 & & & & 8.24 & $\mathrm{~h}$ & 0.08 & $\left(1 / 2^{+}\right)$ & 95 & & & $\beta^{-}=100$ \\
\hline${ }^{173} \mathrm{Tm}^{m}$ & -55941 & 5 & 317.73 & 0.20 & & 10 & $\mu \mathrm{s}$ & & $\left(7 / 2^{-}\right)$ & & & & \\
\hline${ }^{173} \mathrm{Yb}$ & -57556.3 & 2.4 & & & & StABLE & & & $5 / 2^{-}$ & 95 & & & $\mathrm{IS}=16.1327$ \\
\hline${ }^{173} \mathrm{Yb}^{m}$ & -57157.4 & 2.5 & 398.9 & 0.5 & & 2.9 & $\mu \mathrm{s}$ & 0.1 & $1 / 2^{-}$ & & & & \\
\hline${ }^{173} \mathrm{Lu}$ & -56885.8 & 2.4 & & & & 1.37 & $\mathrm{y}$ & 0.01 & $7 / 2^{+}$ & 95 & & & $\varepsilon=100$ \\
\hline${ }^{173} \mathrm{Lu}^{m}$ & -56762.1 & 2.4 & 123.672 & 0.013 & & 74.2 & $\mu \mathrm{s}$ & & $5 / 2^{-}$ & & & & \\
\hline${ }^{173} \mathrm{Hf}$ & -55412 & 28 & & & & 23.6 & $h$ & 0.1 & $1 / 2^{-}$ & 95 & & & $\beta^{+}=100$ \\
\hline${ }^{173} \mathrm{Ta}$ & -52397 & 28 & & & & 3.14 & $\mathrm{~h}$ & 0.13 & $5 / 2^{-}$ & 95 & & & $\beta^{+}=100$ \\
\hline${ }^{173} \mathrm{~W}$ & -48727 & 28 & & & & 7.6 & $\mathrm{~m}$ & 0.2 & $5 / 2^{-}$ & 95 & & & $\beta^{+}=100$ \\
\hline${ }^{173} \mathrm{Re}$ & -43554 & 28 & & & & 2.0 & $\mathrm{~m}$ & 0.3 & $\left(5 / 2^{-}\right)$ & 95 & & & $\beta^{+}=100$ \\
\hline${ }^{173} \mathrm{Os}$ & -37438 & 15 & & & & 22.4 & s & 0.9 & $\left(5 / 2^{-}\right)$ & 95 & $95 \mathrm{HiO} 2$ & TD & $\beta^{+} \approx 100 ; \alpha=0.42$ \\
\hline${ }^{173} \mathrm{Ir}$ & -30272 & 14 & & & & 9.0 & $\mathrm{~s}$ & 0.8 & $\left(3 / 2^{+}, 5 / 2^{+}\right)$ & 95 & & & $\beta^{+}>93 ; \alpha<7$ \\
\hline${ }^{173} \mathrm{Ir}^{m}$ & -30019 & 28 & 253 & 27 & $\mathrm{AD}$ & 2.20 & s & 0.05 & $\left(11 / 2^{-}\right)$ & 95 & & & $\beta^{+}=881 ; \alpha=121$ \\
\hline${ }^{173} \mathrm{Pt}$ & -21940 & 60 & & & & 365 & $\mathrm{~ms}$ & 7 & $5 / 2^{-} \#$ & 95 & 02Ro17 & $\mathrm{T}$ & $\alpha=846 ; \beta^{+}=166$ \\
\hline${ }^{173} \mathrm{Au}$ & -12820 & 26 & & & & 25 & $\mathrm{~ms}$ & 1 & $\left(1 / 2^{+}\right)$ & 03 & & & $\alpha=8613 ; \beta^{+}=6 \#$ \\
\hline${ }^{173} \mathrm{Au}^{m}$ & -12606 & 22 & 214 & 23 & $\mathrm{AD}$ & 14.0 & $\mathrm{~ms}$ & 0.9 & $\left(11 / 2^{-}\right)$ & 03 & & & $\alpha=8911 ; \beta^{+}=4 \#$ \\
\hline${ }^{173} \mathrm{Hg}$ & $-2570 \#$ & $210 \#$ & & & & 1.1 & $\mathrm{~ms}$ & 0.4 & $3 / 2^{-} \#$ & 03 & & & $\alpha=100$ \\
\hline${ }^{173} \mathrm{Pt}$ & $\mathrm{T}$ : average & $02 \mathrm{Ro} 1$ & $=370(13$ & $6 \mathrm{~Pa} 01$ & 376( & $2 \mathrm{En} 03=3$ & $360(2$ & 20) and & $81 \mathrm{De} 22=325$ & 20) & & & \\
\hline${ }^{173} \mathrm{Au}$ & D : from 94 & $4(+6-19$ & $\% ;$ and fo & isomer ${ }^{17}$ & ${ }^{73} \mathrm{Au}^{m}$ & $2(+8-13)$ & & & & & & & \\
\hline
\end{tabular}




\begin{tabular}{|c|c|c|c|c|c|c|c|c|c|c|c|c|c|c|c|}
\hline Nuclide & \multicolumn{2}{|c|}{$\begin{array}{l}\text { Mass excess } \\
\quad(\mathrm{keV})\end{array}$} & \multicolumn{4}{|c|}{$\begin{array}{c}\text { Excitation } \\
\text { energy }(\mathrm{keV})\end{array}$} & \multicolumn{3}{|c|}{ Half-life } & \multirow[t]{2}{*}{$J^{\pi}$} & \multirow[t]{2}{*}{ Ens } & \multicolumn{2}{|c|}{ Reference } & \multicolumn{2}{|l|}{$\begin{array}{l}\text { Decay modes and } \\
\text { intensities }(\%)\end{array}$} \\
\hline${ }^{174} \mathrm{Ho}$ & $-45500 \#$ & $500 \#$ & & & & & 8\# & $\mathrm{s}$ & & & & & & $\beta^{-}$? & \\
\hline${ }^{174} \mathrm{Er}$ & $-51950 \#$ & $300 \#$ & & & & & 3.2 & $\mathrm{~m}$ & 0.2 & $0^{+}$ & 99 & & & $\beta^{-}=100$ & \\
\hline${ }^{174} \mathrm{Tm}$ & -53870 & 40 & & & & & 5.4 & $\mathrm{~m}$ & 0.1 & $(4)^{-}$ & 99 & & & $\beta^{-}=100$ & \\
\hline${ }^{174} \mathrm{Yb}$ & -56949.6 & 2.4 & & & & & STABLE & & & $0^{+}$ & 99 & & & $\mathrm{IS}=31.8392$ & \\
\hline${ }^{174} \mathrm{Lu}$ & -55575.3 & 2.4 & & & & & 3.31 & $\mathrm{y}$ & 0.05 & $1^{-}$ & 99 & $98 \mathrm{Ge} 13$ & $\mathrm{~J}$ & $\beta^{+}=100$ & \\
\hline${ }^{174} \mathrm{Lu}^{m}$ & -55404.5 & 2.4 & 170.83 & 0.05 & & & 142 & $\mathrm{~d}$ & 2 & $6^{-}$ & 99 & $98 \mathrm{Ge} 13$ & $\mathrm{~J}$ & $\mathrm{IT}=99.382 ; \varepsilon=0.622$ & \\
\hline${ }^{174} \mathrm{Hf}$ & -55846.6 & 2.8 & & & & & 2.0 & Py & 0.4 & $0^{+}$ & 99 & & & $\mathrm{IS}=0.161 ; \alpha=100 ; 2 \beta^{+} ?$ & \\
\hline${ }^{174} \mathrm{Hf}^{m}$ & -54049 & 3 & 1797.5 & 2.0 & & & 2.39 & $\mu \mathrm{s}$ & 0.04 & $\left(8^{-}\right)$ & 99 & & & $\mathrm{IT}=100$ & \\
\hline${ }^{174} \mathrm{Ta}$ & -51741 & 28 & & & & & 1.14 & $\mathrm{~h}$ & 0.08 & $3^{+}$ & 99 & & & $\beta^{+}=100$ & \\
\hline${ }^{174} \mathrm{~W}$ & -50227 & 28 & & & & & 33.2 & $\mathrm{~m}$ & 2.1 & $0^{+}$ & 99 & & & $\beta^{+}=100$ & \\
\hline${ }^{174} \mathrm{Re}$ & -43673 & 28 & & & & & 2.40 & $\mathrm{~m}$ & 0.04 & & 99 & & & $\beta^{+}=100$ & \\
\hline${ }^{174} \mathrm{Os}$ & -39996 & 11 & & & & & 44 & $\mathrm{~s}$ & 4 & $0^{+}$ & 99 & & & $\beta^{+} \approx 100 ; \alpha=0.0247$ & \\
\hline${ }^{174} \mathrm{Ir}$ & -30869 & 28 & & & & & 7.9 & $\mathrm{~s}$ & 0.6 & $\left(3^{+}\right)$ & 99 & & & $\beta^{+}=99.53 ; \alpha=0.53$ & \\
\hline${ }^{174} \mathrm{Ir}^{m}$ & -30676 & 26 & 193 & 11 & $\mathrm{AD}$ & & 4.9 & s & 0.3 & $\left(7^{+}\right)$ & 99 & & & $\beta^{+}=97.53 ; \alpha=2.53$ & \\
\hline${ }^{174} \mathrm{Pt}$ & -25319 & 12 & & & & & 889 & $\mathrm{~ms}$ & 17 & $0^{+}$ & 99 & & & $\alpha=768 ; \beta^{+} ?$ & \\
\hline${ }^{174} \mathrm{Au}$ & $-14200 \#$ & $100 \#$ & & & & & 139 & $\mathrm{~ms}$ & 3 & low & 99 & 02 Ro17 & $\mathrm{TD}$ & $\alpha=906 ; \beta^{+} ?$ & * \\
\hline${ }^{174} \mathrm{Au}^{m}$ & -13840 & 70 & $360 \#$ & 70\# & & & 171 & $\mathrm{~ms}$ & 29 & high & & $96 \mathrm{~Pa} 01$ & $\mathrm{TJ}$ & $\alpha=? ; \beta^{+} ?$ & \\
\hline${ }^{174} \mathrm{Hg}$ & -6647 & 20 & & & & & 2.0 & $\mathrm{~ms}$ & 0.4 & $0^{+}$ & 99 & $99 \mathrm{Se} 14$ & $\mathrm{~T}$ & $\alpha \approx 100 ; \beta^{+}=0.4 \#$ & \\
\hline$*^{174} \mathrm{Au}$ & $\mathrm{T}:$ others & $96 \mathrm{~Pa} 01$ & $=171(29) 8$ & $83 \mathrm{Sc} 24=$ & $120(2$ & & & & & & & & & & ** \\
\hline${ }^{175} \mathrm{Ho}$ & $-42800 \#$ & $600 \#$ & & & & & $5 \#$ & $\mathrm{~s}$ & & $7 / 2^{-} \#$ & & & & $\beta^{-} ?$ & \\
\hline${ }^{175} \mathrm{Er}$ & $-48650 \#$ & $400 \#$ & & & & & 1.2 & $\mathrm{~m}$ & 0.3 & $\left(9 / 2^{+}\right)$ & 98 & 96Zh03 & $\mathrm{TD}$ & $\beta^{-}=100$ & \\
\hline${ }^{175} \mathrm{Tm}$ & -52320 & 50 & & & & & 15.2 & $\mathrm{~m}$ & 0.5 & $1 / 2^{+}$ & 98 & & & $\beta^{-}=100$ & \\
\hline${ }^{175} \mathrm{Yb}$ & -54700.6 & 2.4 & & & & & 4.185 & d & 0.001 & $7 / 2^{-}$ & 93 & & & $\beta^{-}=100$ & \\
\hline${ }^{175} \mathrm{Yb}^{m}$ & -54185.7 & 2.4 & 514.869 & 0.007 & & & 68.2 & $\mathrm{~ms}$ & 0.3 & $1 / 2^{-}$ & 93 & & & $\mathrm{IT}=100$ & \\
\hline${ }^{175} \mathrm{Lu}$ & -55170.7 & 2.2 & & & & & STABLE & & & $7 / 2^{+}$ & 93 & & & $\mathrm{IS}=97.412$ & \\
\hline${ }^{175} \mathrm{Lu}^{m}$ & -53780 & 4 & 1391 & 3 & & & 930 & $\mu \mathrm{s}$ & 80 & $19 / 2^{+}$ & & 98Wh02 & ETJ & $\mathrm{IT}=100$ & \\
\hline${ }^{175} \mathrm{Hf}$ & -54483.8 & 2.8 & & & & & 70 & $\mathrm{~d}$ & 2 & $5 / 2^{-}$ & 93 & & & $\varepsilon=100$ & \\
\hline${ }^{175} \mathrm{Ta}$ & -52409 & 28 & & & & & 10.5 & $\mathrm{~h}$ & 0.2 & $7 / 2^{+}$ & 93 & & & $\beta^{+}=100$ & \\
\hline${ }^{175} \mathrm{~W}$ & -49633 & 28 & & & & & 35.2 & $\mathrm{~m}$ & 0.6 & $\left(1 / 2^{-}\right)$ & 93 & & & $\beta^{+}=100$ & \\
\hline${ }^{175} \mathrm{Re}$ & -45288 & 28 & & & & & 5.89 & $\mathrm{~m}$ & 0.05 & $\left(5 / 2^{-}\right)$ & 93 & & & $\beta^{+}=100$ & \\
\hline${ }^{175} \mathrm{Os}$ & -40105 & 14 & & & & & 1.4 & $\mathrm{~m}$ & 0.1 & $\left(5 / 2^{-}\right)$ & 93 & & & $\beta^{+}=100$ & \\
\hline${ }^{175} \mathrm{Ir}$ & -33429 & 20 & & & & & 9 & $\mathrm{~s}$ & 2 & $\left(5 / 2^{-}\right)$ & 93 & & & $\beta^{+}=99.1528 ; \alpha=0.8528$ & \\
\hline${ }^{175} \mathrm{Ir}^{p}$ & -33357 & 17 & 72 & 17 & $\mathrm{AD}$ & & & & & am & & & & & \\
\hline${ }^{175} \mathrm{Pt}$ & -25690 & 19 & & & & & 2.52 & $\mathrm{~s}$ & 0.08 & $5 / 2^{-} \#$ & 93 & & & $\alpha=645 ; \beta^{+} ?$ & \\
\hline${ }^{175} \mathrm{Au}$ & -17440 & 40 & & & & $\&$ & $100 \#$ & $\mathrm{~ms}$ & & $1 / 2^{+} \#$ & & 02Ro17 & $\mathrm{D}$ & $\alpha=? ; \beta^{+} ?$ & $*$ \\
\hline${ }^{175} \mathrm{Au}^{m}$ & $-17240 \#$ & $50 \#$ & $200 \#$ & $30 \#$ & & $\&$ & 156 & $\mathrm{~ms}$ & 3 & $11 / 2^{-} \#$ & 93 & 02 Ro17 & $\mathrm{T}$ & $\alpha=8217 ; \beta^{+}$? & $*$ \\
\hline${ }^{175} \mathrm{Hg}$ & -7990 & 100 & & & & & 10.8 & $\mathrm{~ms}$ & 0.4 & $5 / 2^{-} \#$ & 93 & $02 \operatorname{Ro} 17$ & $\mathrm{~T}$ & $\alpha=? ; \beta^{+}=1 \#$ & * \\
\hline$*^{175} \mathrm{Au}$ & $\mathrm{D}$ : from a & nalysis & of data in & $02 \mathrm{Ro}^{17}$ & we as & si & the 64 & 412 lin & e to ${ }^{175}$ & & & & & & $* *$ \\
\hline$*^{175} \mathrm{Au}^{m}$ & $\mathrm{~T}:$ averag & e $02 R o$ & $17=158(3)$ & $01 \mathrm{Ko} 44$ & $=143($ & $8) ; 0$ & others & $96 \mathrm{~Pa} 0$ & $=185(3$ & $83 \mathrm{Sc} 24=$ & $=200$ & $(22)$ & & & $* *$ \\
\hline$*{ }^{175} \mathrm{Hg}$ & $\mathrm{T}:$ others & 97Uu01 & $1=13(+6-4$ & ) $96 \mathrm{~Pa} 0$ & $I=8(8)$ & out & weighe & ed, not & used & & & & & & $* *$ \\
\hline
\end{tabular}

$\begin{array}{lllll}{ }^{176} \mathrm{Er} & -46500 \# & 400 \# & & \\ { }^{176} \mathrm{Tm} & -49370 & 100 & & \\ { }^{176} \mathrm{Yb} & -53494.1 & 2.6 & & \\ { }^{176} \mathrm{Yb}^{m} & -52444.1 & 2.6 & 1050.0 & 0.3 \\ { }^{176} \mathrm{Lu} & -53387.4 & 2.2 & & \\ { }^{176} \mathrm{Lu}^{m} & -53264.5 & 2.2 & 122.855 & 0.006 \\ { }^{176} \mathrm{Hf} & -54577.5 & 2.2 & & \\ { }^{176} \mathrm{Ta} & -51370 & 30 & & \\ { }^{176} \mathrm{Ta}^{m} & -51270 & 30 & 103.0 & 1.0 \\ { }^{176} \mathrm{Ta}^{n} & -48550 & 60 & 2820 & 50 \\ \ldots . \text {-group is continued on next page } \ldots\end{array}$

... A-group is continued on next page ...

\begin{tabular}{|c|c|c|c|c|c|c|c|}
\hline $20 \#$ & s & & $0^{+}$ & & & & $\beta^{-} ?$ \\
\hline 1.85 & $\mathrm{~m}$ & 0.03 & $\left(4^{+}\right)$ & 98 & 94It.A & $\mathrm{T}$ & $\beta^{-}=100$ \\
\hline STABLE & & $(>160 \mathrm{Py})$ & $0^{+}$ & 98 & 96De60 & $\mathrm{T}$ & $\mathrm{IS}=12.7641$; \\
\hline 11.4 & s & 0.3 & $(8)^{-}$ & 98 & & & $\mathrm{IT}=? ; \beta^{-}<10 \#$ \\
\hline 38.5 & Gy & 0.7 & $7^{-}$ & 98 & 03Gr02 & $\mathrm{T}$ & $\mathrm{IS}=2.592 ; \beta^{-}=100$ \\
\hline 3.664 & $\mathrm{~h}$ & 0.019 & $1^{-}$ & 98 & & & $\beta^{-} \approx 100 ; \varepsilon=0.09516$ \\
\hline STABLE & & & $0^{+}$ & 98 & & & $\mathrm{IS}=5.267$ \\
\hline 8.09 & $\mathrm{~h}$ & 0.05 & $(1)^{-}$ & 98 & & & $\beta^{+}=100$ \\
\hline 1.1 & $\mathrm{~ms}$ & 0.1 & $(+)$ & 98 & & & $\mathrm{IT}=100$ \\
\hline 0.97 & $\mathrm{~ms}$ & 0.07 & $\left(20^{-}\right)$ & 98 & & & $\mathrm{IT}=100$ \\
\hline
\end{tabular}




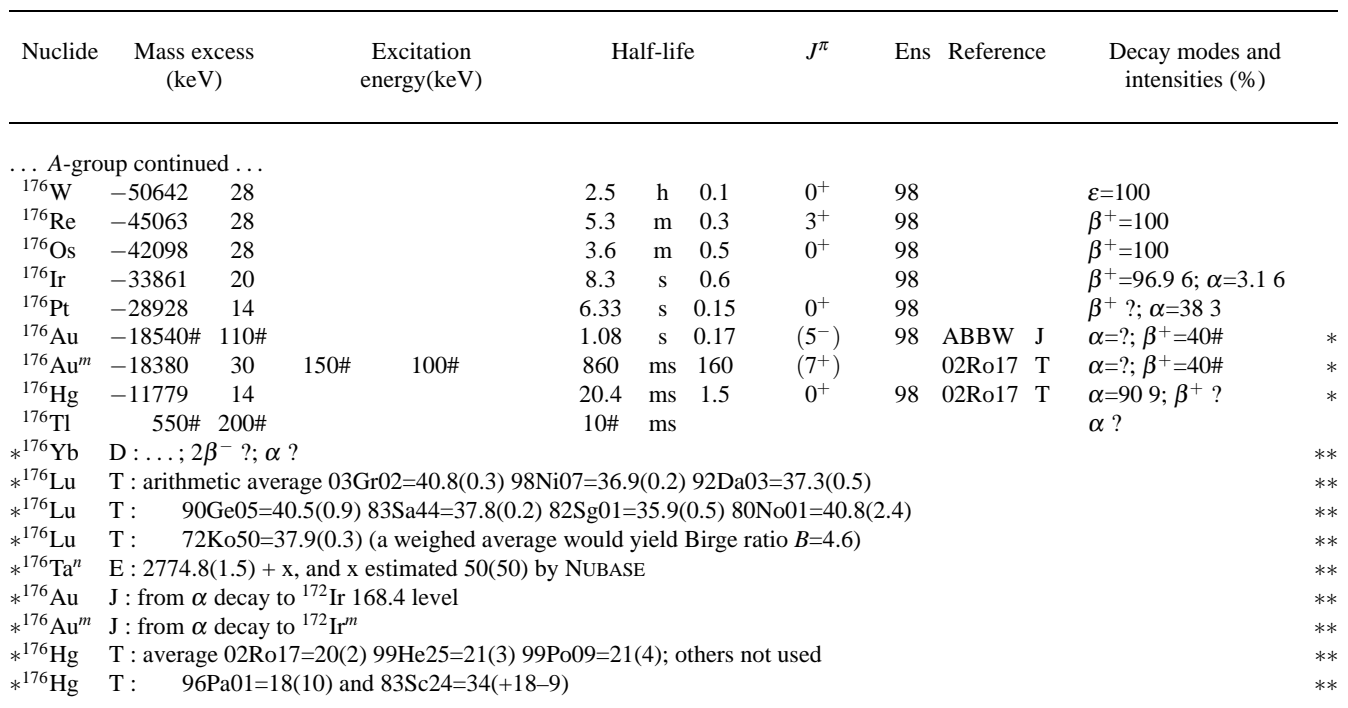

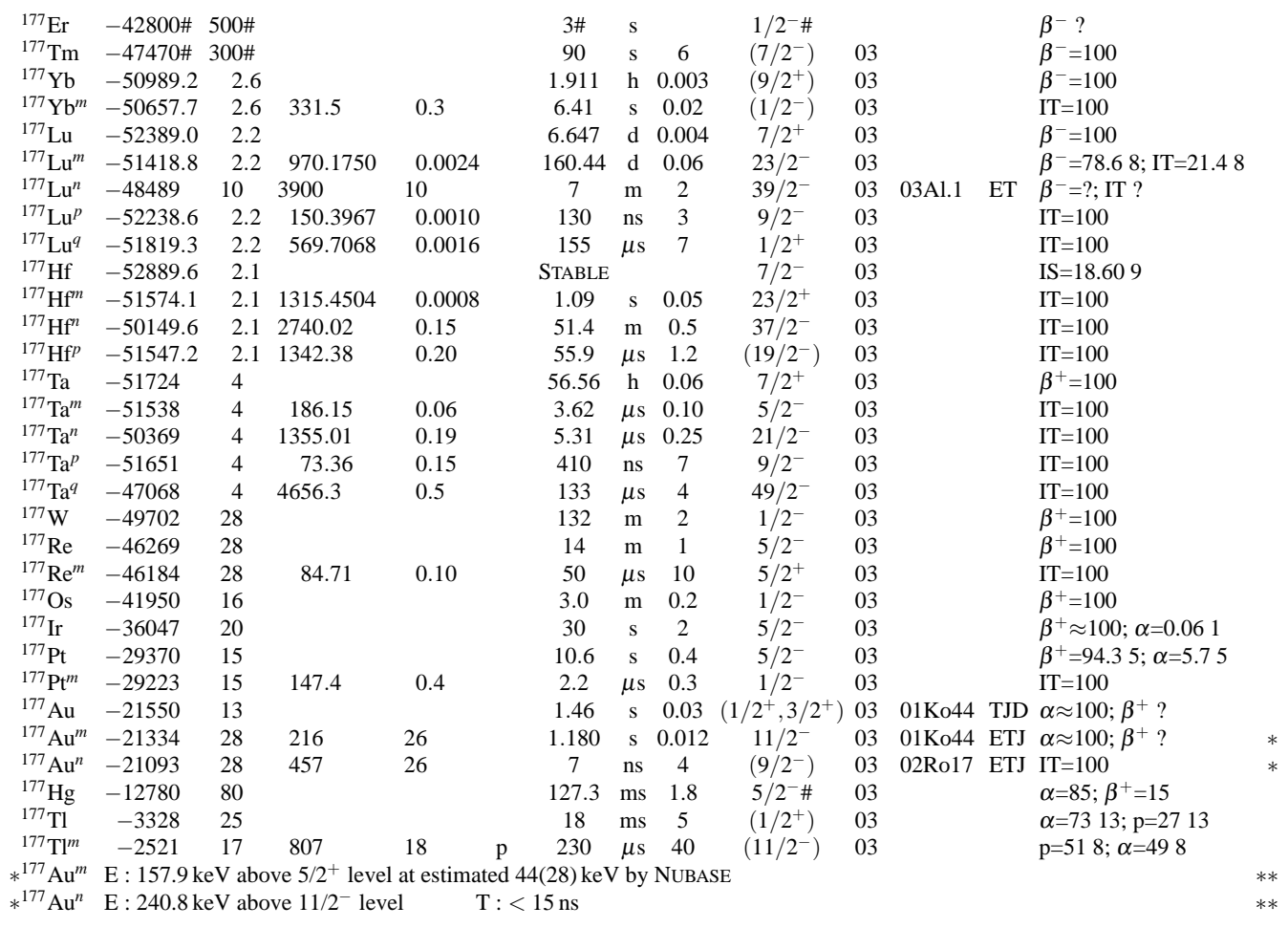




\begin{tabular}{|c|c|c|c|c|c|c|c|c|c|c|c|c|c|c|}
\hline \multirow{2}{*}{$\begin{array}{l}\text { Nuclide } \\
{ }^{178} \mathrm{Tm}\end{array}$} & \multicolumn{2}{|c|}{$\begin{array}{l}\text { Mass excess } \\
\quad(\mathrm{keV})\end{array}$} & \multicolumn{3}{|c|}{$\begin{array}{l}\text { Excitation } \\
\text { energy }(\mathrm{keV})\end{array}$} & \multicolumn{3}{|c|}{ Half-life } & \multirow[t]{2}{*}{$J^{\pi}$} & \multirow[t]{2}{*}{ Ens } & \multicolumn{2}{|c|}{ Reference } & \multicolumn{2}{|l|}{$\begin{array}{l}\text { Decay modes and } \\
\text { intensities }(\%)\end{array}$} \\
\hline & $-44120 \#$ & $400 \#$ & & & & $30 \#$ & $\mathrm{~s}$ & & & & & & $\beta^{-} ?$ & \\
\hline${ }^{178} \mathrm{Yb}$ & -49698 & 10 & & & & 74 & $\mathrm{~m}$ & 3 & $0^{+}$ & 94 & & & $\beta^{-}=100$ & \\
\hline${ }^{178} \mathrm{Lu}$ & -50343.0 & 2.9 & & & & 28.4 & $\mathrm{~m}$ & 0.2 & $1^{(+)}$ & 94 & & & $\beta^{-}=100$ & \\
\hline${ }^{178} \mathrm{Lu}^{m}$ & -50219 & 4 & 123.8 & 2.6 & RQ & 23.1 & $\mathrm{~m}$ & 0.3 & $9^{(-)}$ & 94 & $98 \mathrm{Ge} 13$ & J & $\beta^{-}=100$ & \\
\hline${ }^{178} \mathrm{Hf}$ & -52444.3 & 2.1 & & & & STABLE & & & $0^{+}$ & 94 & & & $\mathrm{IS}=27.287$ & \\
\hline${ }^{178} \mathrm{Hf}^{m}$ & -51296.9 & 2.1 & 1147.423 & 0.005 & & 4.0 & $\mathrm{~s}$ & 0.2 & $8^{-}$ & 94 & & & $\mathrm{IT}=100$ & \\
\hline${ }^{178} \mathrm{Hf}^{n}$ & -49998.6 & 2.1 & 2445.69 & 0.11 & & 31 & $\mathrm{y}$ & 1 & $16^{+}$ & 94 & 94Ki.A & $\mathrm{E}$ & $\mathrm{IT}=100$ & \\
\hline${ }^{178} \mathrm{Hf}^{p}$ & -49870.8 & 2.2 & 2573.5 & 0.5 & & 68 & $\mu \mathrm{s}$ & 2 & $\left(14^{-}\right)$ & 94 & & & $\mathrm{IT}=100$ & \\
\hline${ }^{178} \mathrm{Ta}$ & -50507 & 15 & & & * & 9.31 & $\mathrm{~m}$ & 0.03 & $1^{+}$ & 94 & & & $\beta^{+}=100$ & \\
\hline${ }^{178} \mathrm{Ta}^{m}$ & $-50410 \#$ & $50 \#$ & $100 \#$ & $50 \#$ & * & 2.36 & $\mathrm{~h}$ & 0.08 & $(7)^{-}$ & 94 & & & $\beta^{+}=100$ & \\
\hline${ }^{178} \mathrm{Ta}^{n}$ & $-48940 \#$ & $50 \#$ & $1570 \#$ & $50 \#$ & & 59 & $\mathrm{~ms}$ & 3 & $\left(15^{-}\right)$ & 94 & 96 Ko13 & $\mathrm{T}$ & $\mathrm{IT}=100$ & $*$ \\
\hline${ }^{178} \mathrm{Ta}^{p}$ & $-47510 \#$ & $50 \#$ & $3000 \#$ & $50 \#$ & & 290 & $\mathrm{~ms}$ & 12 & $\left(21^{-}\right)$ & & 96 Ko13 & TJE & & $*$ \\
\hline${ }^{178} \mathrm{~W}$ & -50416 & 15 & & & & 21.6 & $\mathrm{~d}$ & 0.3 & $0^{+}$ & 94 & & & $\varepsilon=100$ & \\
\hline${ }^{178} \mathrm{Re}$ & -45653 & 28 & & & & 13.2 & $\mathrm{~m}$ & 0.2 & $\left(3^{+}\right)$ & 94 & & & $\beta^{+}=100$ & \\
\hline${ }^{178} \mathrm{Os}$ & -43546 & 16 & & & & 5.0 & $\mathrm{~m}$ & 0.4 & $0^{+}$ & 94 & & & $\beta^{+}=100$ & \\
\hline${ }^{178} \mathrm{Ir}$ & -36252 & 20 & & & & 12 & $\mathrm{~s}$ & 2 & & 95 & & & $\beta^{+}=100$ & \\
\hline${ }^{178} \mathrm{Pt}$ & -31998 & 11 & & & & 21.1 & $\mathrm{~s}$ & 0.6 & $0^{+}$ & 94 & & & $\beta^{+}=92.33 ; \alpha=7.73$ & \\
\hline${ }^{178} \mathrm{Au}$ & -22330 & 60 & & & & 2.6 & $\mathrm{~s}$ & 0.5 & & 94 & & & $\beta^{+} \leq 60 ; \alpha>40$ & \\
\hline${ }^{178} \mathrm{Hg}$ & -16317 & 13 & & & & 269 & $\mathrm{~ms}$ & 3 & $0^{+}$ & 94 & 02Ro17 & $\mathrm{T}$ & $\alpha=? ; \beta^{+}=30 \#$ & $*$ \\
\hline${ }^{178} \mathrm{Tl}$ & $-4750 \#$ & $110 \#$ & & & & 255 & $\mathrm{~ms}$ & 10 & & & 02Ro17 & $\mathrm{TD}$ & $\alpha=? ; \beta^{+}=47 \#$ & \\
\hline${ }^{178} \mathrm{~Pb}$ & 3568 & 24 & & & & 230 & $\mu \mathrm{s}$ & 150 & $0^{+}$ & & 01Ro.B & $\mathrm{T}$ & $\alpha \approx 100 ; \beta^{+} ?$ & $*$ \\
\hline$*^{178} \mathrm{Ta}^{n}$ & E : 1470.6 & $\mathrm{keV}$ at & ove ${ }^{178} \mathrm{Ta}^{m}$ & om EN & & & & & & & & & & $* *$ \\
\hline${ }^{178} \mathrm{Ta}^{n}$ & $\mathrm{~T}:$ average & e $96 \mathrm{Ko}$ & $13=58(4) 7$ & $\mathrm{Du} 02=6$ & & & & & & & & & & $* *$ \\
\hline$*^{178} \mathrm{Ta}^{p}$ & $\mathrm{E}: 2902 \mathrm{ke}$ & $\mathrm{eV}$ abo & ve the $(7)^{-}$ & ${ }^{8} \mathrm{Ta}^{m}$ is & & & & & & & & & & $* *$ \\
\hline$*^{178} \mathrm{Hg}$ & $\mathrm{T}:$ others $\mathrm{C}$ & $96 \mathrm{~Pa} 01$ & $=287(23) 9$ & $\mathrm{Se} 01=25$ & (25) a & $9 \mathrm{Ha}$ & -200 & (J) & & & & & & $* *$ \\
\hline$*^{178} \mathrm{~Pb}$ & $\mathrm{~T}:$ two eve & ents at & 202 and 14 & & & & & & & & & & & $* *$ \\
\hline
\end{tabular}

\begin{tabular}{|c|c|c|c|c|c|c|c|c|c|c|c|c|}
\hline${ }^{179} \mathrm{Tm}$ & $-41600 \#$ & $500 \#$ & & & & $20 \#$ & $\mathrm{~s}$ & & $1 / 2^{+} \#$ & & & $\beta^{-} ?$ \\
\hline${ }^{179} \mathrm{Yb}$ & $-46420 \#$ & $300 \#$ & & & & 8.0 & $\mathrm{~m}$ & 0.4 & $\left(1 / 2^{-}\right)$ & 94 & & $\beta^{-}=100$ \\
\hline${ }^{179} \mathrm{Lu}$ & -49064 & 5 & & & & 4.59 & $\mathrm{~h}$ & 0.06 & $7 / 2^{(+)}$ & 94 & & $\beta^{-}=100$ \\
\hline${ }^{179} \mathrm{Lu}^{m}$ & -48472 & 5 & 592.4 & 0.4 & & 3.1 & $\mathrm{~ms}$ & 0.9 & $1 / 2^{(+)}$ & 94 & & $\mathrm{IT}=100$ \\
\hline${ }^{179} \mathrm{Hf}$ & -50471.9 & 2.1 & & & & STABLE & & & $9 / 2^{+}$ & 94 & & $\mathrm{IS}=13.622$ \\
\hline${ }^{179} \mathrm{Hf}^{m}$ & -50096.9 & 2.1 & 375.0367 & 0.0025 & & 18.67 & $\mathrm{~s}$ & 0.04 & $1 / 2^{-}$ & 94 & & $\mathrm{IT}=100$ \\
\hline${ }^{179} \mathrm{Hf}^{n}$ & -49366.1 & 2.1 & 1105.84 & 0.19 & & 25.05 & $\mathrm{~d}$ & 0.25 & $25 / 2^{-}$ & 94 & & $\mathrm{IT}=100$ \\
\hline${ }^{179} \mathrm{Ta}$ & -50366.3 & 2.2 & & & & 1.82 & $\mathrm{y}$ & 0.03 & $7 / 2^{+}$ & 00 & & $\varepsilon=100$ \\
\hline${ }^{179} \mathrm{Ta}^{m}$ & -49049.0 & 2.2 & 1317.3 & 0.4 & & 9.0 & $\mathrm{~ms}$ & 0.2 & $\left(25 / 2^{+}\right)$ & 00 & & $\mathrm{IT}=100$ \\
\hline${ }^{179} \mathrm{Ta}^{n}$ & -47727.0 & 2.3 & 2639.3 & 0.5 & & 54.1 & $\mathrm{~ms}$ & 1.7 & $\left(37 / 2^{+}\right)$ & 00 & & $\mathrm{IT}=100$ \\
\hline${ }^{179} \mathrm{~W}$ & -49304 & 16 & & & & 37.05 & $\mathrm{~m}$ & 0.16 & $(7 / 2)^{-}$ & 94 & & $\beta^{+}=100$ \\
\hline${ }^{179} \mathrm{~W}^{m}$ & -49082 & 16 & 221.926 & 0.008 & & 6.40 & $\mathrm{~m}$ & 0.07 & $(1 / 2)^{-}$ & 94 & & $\mathrm{IT} \approx 100 ; \beta^{+}=0.283$ \\
\hline${ }^{179} \mathrm{Re}$ & -46586 & 24 & & & & 19.5 & $\mathrm{~m}$ & 0.1 & $(5 / 2)^{+}$ & 95 & & $\beta^{+}=100$ \\
\hline${ }^{179} \mathrm{Re}^{m}$ & -46521 & 24 & 65.39 & 0.09 & & 95 & $\mu \mathrm{s}$ & 25 & $\left(5 / 2^{-}\right)$ & & & \\
\hline${ }^{179} \mathrm{Os}$ & -43020 & 18 & & & & 6.5 & $\mathrm{~m}$ & 0.3 & $\left(1 / 2^{-}\right)$ & 94 & & $\beta^{+}=100$ \\
\hline${ }^{179} \mathrm{Ir}$ & -38077 & 11 & & & & 79 & $\mathrm{~s}$ & 1 & $(5 / 2)^{-}$ & 98 & & $\beta^{+}=100$ \\
\hline${ }^{179} \mathrm{Pt}$ & -32264 & 9 & & & & 21.2 & $\mathrm{~s}$ & 0.4 & $1 / 2^{-}$ & 94 & & $\beta^{+} \approx 100 ; \alpha=0.243$ \\
\hline${ }^{179} \mathrm{Au}$ & -24952 & 17 & & & & 7.1 & $\mathrm{~s}$ & 0.3 & $5 / 2^{-} \#$ & 94 & & $\beta^{+}=78.09 ; \alpha=22.09$ \\
\hline${ }^{179} \mathrm{Au}^{p}$ & -24853 & 18 & 99 & 16 & $\mathrm{AD}$ & & & & $\left(11 / 2^{-}\right)$ & & & \\
\hline${ }^{179} \mathrm{Hg}$ & -16922 & 27 & & & & 1.09 & $\mathrm{~s}$ & 0.04 & $5 / 2^{-} \#$ & 94 & 02Ro17 T & $\alpha \approx 53 ; \beta^{+}=? ; \beta^{+} \mathrm{p} \approx 0.15$ \\
\hline${ }^{179} \mathrm{Tl}$ & -8300 & 40 & & & & 270 & $\mathrm{~ms}$ & 30 & $\left(1 / 2^{+}\right)$ & 01 & ABBW J & $\alpha=? ; \beta^{+}=30 \#$ \\
\hline${ }^{179} \mathrm{Tl}^{m}$ & $-7440 \#$ & $50 \#$ & $860 \#$ & $30 \#$ & & 1.60 & $\mathrm{~ms}$ & 0.16 & $\left(9 / 2^{-}\right)$ & 01 & 02Ro17 T & $\alpha \approx 100 ;$ IT $? ; \beta^{+} ?$ \\
\hline${ }^{179} \mathrm{~Pb}$ & $2000 \#$ & $200 \#$ & & & & $3 \#$ & $\mathrm{~ms}$ & & $5 / 2^{-} \#$ & & & $\alpha$ ? \\
\hline$*{ }^{179} \mathrm{Hg}$ & $\mathrm{T}:$ average & e 02 Rol & $17=1.08(0$. & $71 \mathrm{Ha}$ & $=1.0$ & $04)^{3}$ & & & & & & \\
\hline${ }^{179} \mathrm{Tl}$ & $\mathrm{T}$ : average & e 02Ro1 & $17=415(55)$ & $8 \mathrm{To} 14=$ & $30(40)$ & $3 \mathrm{Sc} 24=1$ & $60(+$ & $-90-40$ & & & & \\
\hline$*^{179} \mathrm{Tl}$ & $\mathrm{J}:$ from $\alpha$ & decay t & ${ }^{175} \mathrm{Au}^{m}$ & & & & & & & & & \\
\hline$*^{179} \mathrm{Tl}^{m}$ & $\mathrm{~T}$ : average & e 02Rol & $17=1.7(0.2)$ & 8 7) $14=$ & $8(0.4)$ & $6 \mathrm{~Pa} 01=0$ & $.7(+6$ & $6-4) 8$ & $33 \mathrm{Sc} 24=1$ & $.4(0$. & & \\
\hline
\end{tabular}




\begin{tabular}{|c|c|c|c|c|c|c|c|c|c|c|c|c|c|c|}
\hline Nuclide & \multicolumn{2}{|c|}{$\begin{array}{l}\text { Mass excess } \\
\quad(\mathrm{keV})\end{array}$} & \multicolumn{3}{|c|}{$\begin{array}{l}\text { Excitation } \\
\text { energy }(\mathrm{keV})\end{array}$} & \multicolumn{3}{|c|}{ Half-life } & \multirow{2}{*}{$\begin{array}{l}J^{\pi} \\
0^{+}\end{array}$} & \multirow{2}{*}{$\begin{array}{c}\text { Ens } \\
94\end{array}$} & \multicolumn{2}{|c|}{ Reference } & \multicolumn{2}{|l|}{$\begin{array}{c}\text { Decay modes and } \\
\text { intensities }(\%)\end{array}$} \\
\hline${ }^{180} \mathrm{Yb}$ & $-44400 \#$ & $400 \#$ & & & & 2.4 & $\mathrm{~m}$ & 0.5 & & & & & $\beta^{-}=100$ & \\
\hline${ }^{180} \mathrm{Lu}$ & -46690 & 70 & & & & 5.7 & $\mathrm{~m}$ & 0.1 & $5^{+}$ & 94 & $95 \mathrm{Me} 03$ & $\mathrm{~J}$ & $\beta^{-}=100$ & \\
\hline${ }^{180} \mathrm{Lu}^{m}$ & -46680 & 70 & 13.9 & 0.3 & & 1 & $\mathrm{~s}$ & & $3^{-}$ & & $95 \mathrm{Me} 03$ & EJT & $\beta^{-}$?; IT ? & \\
\hline${ }^{180} \mathrm{Hf}$ & -49788.4 & 2.1 & & & & STABLE & & & $0^{+}$ & 94 & & & $\mathrm{IS}=35.0816$ & \\
\hline${ }^{180} \mathrm{Hf}^{m}$ & -48646.9 & 2.1 & 1141.48 & 0.04 & & 5.5 & $\mathrm{~h}$ & 0.1 & $8^{-}$ & 94 & & & $\mathrm{IT} \approx 100 ; \beta^{-}=0.31$ & \\
\hline${ }^{180} \mathrm{Ta}$ & -48936.2 & 2.2 & & & & 8.152 & $\mathrm{~h}$ & 0.006 & $1^{+}$ & 94 & & & $\varepsilon=863 ; \beta^{-}=143$ & \\
\hline${ }^{180} \mathrm{Ta}^{m}$ & -48860.9 & 1.8 & 75.3 & 1.3 & RQ & STABLE & & $(>1.2 \mathrm{Py})$ & $9^{-}$ & 94 & & & $\mathrm{IS}=0.0122 ; \beta^{-} ?$ & \\
\hline${ }^{180} \mathrm{Ta}^{n}$ & -47485.2 & 2.4 & 1451.0 & 1.0 & & 45 & $\mu \mathrm{s}$ & 2 & $15^{-}$ & & $96 \operatorname{Dr} 02$ & $\mathrm{TE}$ & & \\
\hline${ }^{180} \mathrm{~W}$ & -49644 & 4 & & & & STABLE & & $(>700 \mathrm{Py})$ & $0^{+}$ & 94 & 03Da05 & $\mathrm{T}$ & $\mathrm{IS}=0.121 ; \alpha ? ; 2 \beta^{+} ?$ & $*$ \\
\hline${ }^{180} \mathrm{~W}^{m}$ & -48115 & 4 & 1529.04 & 0.03 & & 5.47 & $\mathrm{~ms}$ & 0.09 & $8^{-}$ & 94 & & & $\mathrm{IT}=100$ & \\
\hline${ }^{180} \mathrm{Re}$ & -45840 & 21 & & & & 2.44 & $\mathrm{~m}$ & 0.06 & $(1)^{-}$ & 94 & & & $\beta^{+}=100$ & \\
\hline${ }^{180} \mathrm{Os}$ & -44359 & 20 & & & & 21.5 & $\mathrm{~m}$ & 0.4 & $0^{+}$ & 94 & & & $\beta^{+}=100$ & \\
\hline${ }^{180} \mathrm{Ir}$ & -37978 & 22 & & & & 1.5 & $\mathrm{~m}$ & 0.1 & $(4,5)^{(+\#)}$ & 94 & & & $\beta^{+}=100$ & \\
\hline${ }^{180} \mathrm{Pt}$ & -34436 & 11 & & & & 52 & s & 3 & $0^{+}$ & 94 & & & $\beta^{+} \approx 100 ; \alpha \approx 0.3$ & \\
\hline${ }^{180} \mathrm{Au}$ & -25596 & 21 & & & & 8.1 & $\mathrm{~s}$ & 0.3 & & 94 & & & $\beta^{+} \leq 98.2 ; \alpha \geq 1.8$ & \\
\hline${ }^{180} \mathrm{Hg}$ & -20245 & 14 & & & & 2.56 & $\mathrm{~s}$ & 0.02 & $0^{+}$ & 94 & 93Wa03 & $\mathrm{T}$ & $\beta^{+}=524 ; \alpha=484$ & \\
\hline${ }^{180} \mathrm{Tl}$ & $-9400 \#$ & $120 \#$ & & & & 1.5 & $\mathrm{~s}$ & 0.2 & & 94 & $98 \mathrm{To} 14$ & $\mathrm{TD}$ & $\beta^{+} ? ; \alpha=73 ; \ldots$ & $*$ \\
\hline${ }^{180} \mathrm{~Pb}$ & -1939 & 21 & & & & 5 & $\mathrm{~ms}$ & 3 & $0^{+}$ & 00 & $96 \mathrm{To} 08$ & TD & $\alpha=100$ & \\
\hline$*^{180} \mathrm{~W}$ & $\mathrm{~T}$ : lower 1 & limit is & for $\alpha$ deca & also 0 & $3 \mathrm{Ce} 01$ & 270 Py 97 & $7 \mathrm{Ge} 15$ & $5>74 \mathrm{Py}$ & & & & & & $* *$ \\
\hline${ }^{180} \mathrm{~W}$ & $\mathrm{~T}$ : ind & lication & in $03 \mathrm{Da} 0$ & for 1.1 & $+0.8-$ & Ey, but & timpo & ortant backg & ground & & & & & $* *$ \\
\hline$*^{180} \mathrm{~W}$ & $\mathrm{~T}: 03 \mathrm{Da} 0$ & $9>80 \mathrm{P}$ & $y$ for $2 \beta^{-}$ & ecay & & & & & & & & & & $* *$ \\
\hline$*^{180} \mathrm{Tl}$ & $\mathrm{D}: \ldots ; \beta$ & $+\mathrm{SF} \approx 1$ & $.0 \mathrm{e}-4$ & & & & & & & & & & & ** \\
\hline$*^{180} \mathrm{Tl}$ & $\mathrm{D}: \alpha=(2-1$ & 12) $\% \mathrm{fr}$ & om 02An. & & & & & & & & & & & $* *$ \\
\hline
\end{tabular}

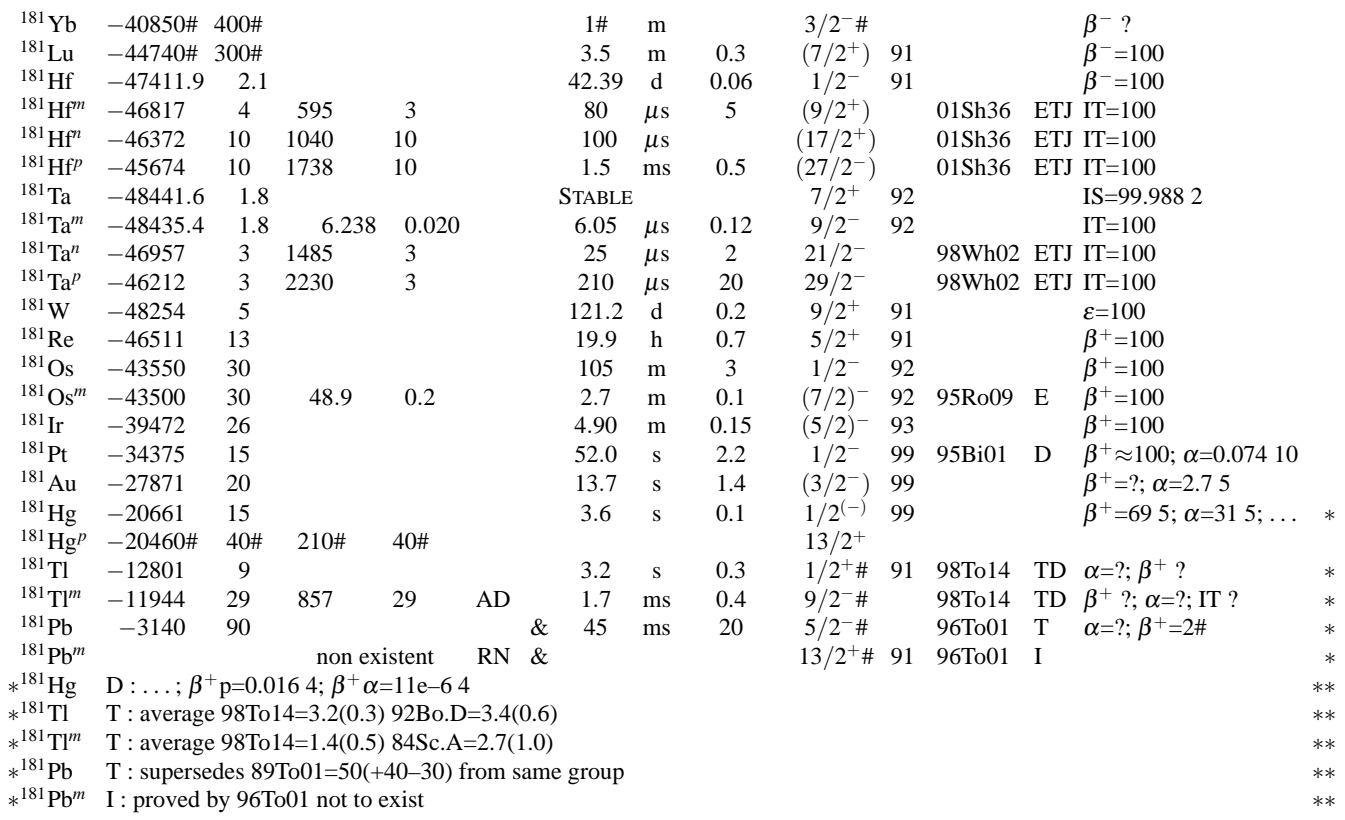




\begin{tabular}{|c|c|c|c|c|c|c|c|c|c|c|c|c|c|c|}
\hline Nuclide & \multicolumn{2}{|c|}{$\begin{array}{c}\text { Mass excess } \\
(\mathrm{keV})\end{array}$} & \multicolumn{3}{|c|}{$\begin{array}{l}\text { Excitation } \\
\text { energy }(\mathrm{keV})\end{array}$} & \multicolumn{3}{|c|}{ Half-life } & $J^{\pi}$ & Ens & \multicolumn{2}{|c|}{ Reference } & \multicolumn{2}{|l|}{$\begin{array}{l}\text { Decay modes and } \\
\text { intensities }(\%)\end{array}$} \\
\hline${ }^{182} \mathrm{Lu}$ & $-41880 \#$ & $200 \#$ & & & & 2.0 & $\mathrm{~m}$ & 0.2 & $(0,1,2)$ & 95 & & & $\beta^{-}=100$ & \\
\hline${ }^{182} \mathrm{Hf}$ & -46059 & 6 & & & & 9 & My & 2 & $0^{+}$ & 95 & & & $\beta^{-}=100$ & \\
\hline${ }^{182} \mathrm{Hf}^{m}$ & -44886 & 6 & 1172.88 & 0.18 & & 61.5 & $\mathrm{~m}$ & 1.5 & $8^{-}$ & 95 & & & $\beta^{-}=583 ; \mathrm{IT}=423$ & \\
\hline${ }^{182} \mathrm{Ta}$ & -46433.3 & 1.8 & & & & 114.43 & $\mathrm{~d}$ & 0.03 & $3^{-}$ & 95 & & & $\beta^{-}=100$ & \\
\hline${ }^{182} \mathrm{Ta}^{m}$ & -46417.0 & 1.8 & 16.263 & 0.00 & & 283 & $\mathrm{~ms}$ & 3 & $5^{+}$ & 95 & & & $\mathrm{IT}=100$ & \\
\hline${ }^{182} \mathrm{Ta}^{n}$ & -45913.7 & 1.8 & 519.572 & 0.01 & & 15.84 & $\mathrm{~m}$ & 0.10 & $10^{-}$ & 95 & & & $\mathrm{IT}=100$ & \\
\hline${ }^{182} \mathrm{~W}$ & -48247.5 & 0.8 & & & & STABLE & & $(>170$ Ey) & $0^{+}$ & 95 & 03Da05 & $\mathrm{T}$ & $\mathrm{IS}=26.5016 ; \alpha ?$ & * \\
\hline${ }^{182} \mathrm{Re}$ & -45450 & 100 & & & $*$ & 64.0 & $\mathrm{~h}$ & 0.5 & $7^{+}$ & 95 & & & $\beta^{+}=100$ & \\
\hline${ }^{182} \mathrm{Re}^{m}$ & -45388 & 20 & 60 & 100 & $\mathrm{BD} *$ & 12.7 & $\mathrm{~h}$ & 0.2 & $2^{+}$ & 95 & & & $\beta^{+}=100$ & \\
\hline${ }^{182} \mathrm{Os}$ & -44609 & 22 & & & & 22.10 & $\mathrm{~h}$ & 0.25 & $0^{+}$ & 95 & & & $\varepsilon=100$ & \\
\hline${ }^{182} \mathrm{Ir}$ & -39052 & 21 & & & & 15 & $\mathrm{~m}$ & 1 & $\left(3^{+}\right)$ & 95 & $95 \mathrm{Sa} 42$ & J & $\beta^{+}=100$ & \\
\hline${ }^{182} \mathrm{Pt}$ & -36169 & 16 & & & & 2.2 & $\mathrm{~m}$ & 0.1 & $0^{+}$ & 95 & & & $\beta^{+} \approx 100 ; \alpha=0.0382$ & \\
\hline${ }^{182} \mathrm{Au}$ & -28301 & 20 & & & & 15.5 & $\mathrm{~s}$ & 0.4 & $\left(2^{+}\right)$ & 95 & 01Ib02 & $\mathrm{J}$ & $\beta^{+} \approx 100 ; \alpha=0.135$ & $*$ \\
\hline${ }^{182} \mathrm{Hg}$ & -23576 & 10 & & & & 10.83 & s & 0.06 & $0^{+}$ & 95 & $97 \mathrm{Ba} 21$ & $\mathrm{D}$ & $\beta^{+}=86.29 ; \alpha=13.89 ;$ & $*$ \\
\hline${ }^{182} \mathrm{Tl}$ & -13350 & 80 & & & $*$ & 2.0 & s & 0.3 & $2^{-} \#$ & 95 & 92Bo.D & $\mathrm{T}$ & $\beta^{+}>96 ; \alpha<4$ & \\
\hline${ }^{182} \mathrm{Tl}^{m}$ & $-13250 \#$ & $130 \#$ & $100 \#$ & $100 \#$ & $*$ & 2.9 & s & 0.5 & $\left(7^{+}\right)$ & & $91 \mathrm{Bo} 22$ & TJ & $\alpha \approx 100 ; \beta^{+} ?$ & $*$ \\
\hline${ }^{182} \mathrm{Tl}^{p}$ & $-12750 \#$ & $160 \#$ & $600 \#$ & $140 \#$ & & & & & $10^{-}$ & & & & & \\
\hline${ }^{182} \mathrm{~Pb}$ & -6826 & 14 & & & & 60 & $\mathrm{~ms}$ & 40 & $0^{+}$ & 95 & & & $\alpha=? ; \beta^{+}=2 \#$ & \\
\hline$*^{182} \mathrm{~W}$ & $\mathrm{~T}:$ also 03 & $3 \mathrm{Ce} 01>$ & $>25 \mathrm{Ey} 970$ & $\mathrm{Ge} 15>8$ & Ey & & & & & & & & & $* *$ \\
\hline$*^{182} \mathrm{Au}$ & $\mathrm{T}$ : average & $95 \mathrm{Bi}$ & $01=14.5(1$. & 3 )(for $\beta$ & ), 15.3 & (1.0)(for & $\mathrm{r} \alpha) \mathrm{a}$ & and $92 \operatorname{Ro} 21$ & $1=15.6(0$ & & & & & $* *$ \\
\hline$*^{182} \mathrm{Hg}$ & $\mathrm{D}: \ldots ; \beta^{+}$ & ${ }^{+} \mathrm{p}<1 \mathrm{e}$ & & & & & & & & & & & & ** \\
\hline$*^{182} \mathrm{Hg}$ & $\mathrm{D}: \alpha$ aver & rage 97 & $\mathrm{Ba} 21=13.3$ & $3(0.5) 8$ & $\mathrm{c} 09=1$ & 5.2(0.8); & $; \beta^{+} \mathrm{p}$ & is from 71 & IHo07 & & & & & $* *$ \\
\hline$*^{182} \mathrm{Tl}^{m}$ & $\mathrm{~T}$ : average & $91 \mathrm{Bo}$ & $22=3.1(1.0$ & 0) $92 \mathrm{Bo}$ & $=2.8(0$ & & & & & & & & & $* *$ \\
\hline
\end{tabular}

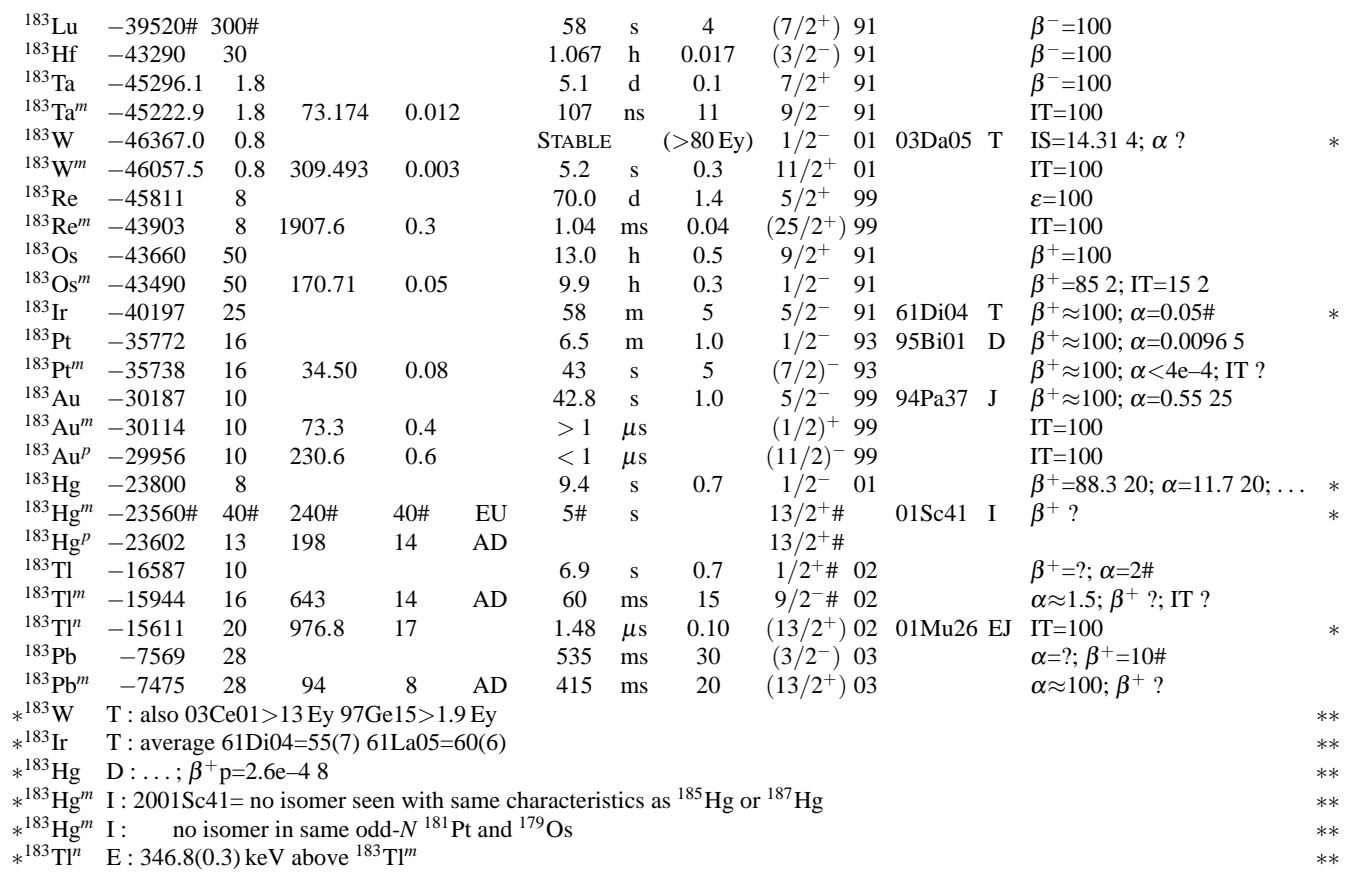




\begin{tabular}{|c|c|c|c|c|c|c|c|c|c|c|c|c|c|c|}
\hline Nuclide & \multicolumn{2}{|c|}{$\begin{array}{c}\text { Mass excess } \\
(\mathrm{keV})\end{array}$} & \multicolumn{3}{|c|}{$\begin{array}{c}\text { Excitation } \\
\text { energy }(\mathrm{keV})\end{array}$} & \multicolumn{3}{|c|}{ Half-life } & $J^{\pi}$ & Ens & \multicolumn{2}{|c|}{ Reference } & \multicolumn{2}{|l|}{$\begin{array}{c}\text { Decay modes and } \\
\text { intensities }(\%)\end{array}$} \\
\hline${ }^{184} \mathrm{Lu}$ & $-36410 \#$ & $400 \#$ & & & & 20 & $\mathrm{~s}$ & 3 & $\left(3^{+}\right)$ & 90 & $95 \mathrm{Kr} 04$ & $\mathrm{TJ}$ & $\beta^{-}=100$ & \\
\hline${ }^{184} \mathrm{Lu}^{m}$ & & & non $\mathrm{e}$ & istent & RN & 20 & $\mathrm{~s}$ & & high & & 95Kr04 & I & & \\
\hline${ }^{184} \mathrm{Hf}$ & -41500 & 40 & & & & 4.12 & $\mathrm{~h}$ & 0.05 & $0^{+}$ & 90 & & & $\beta^{-}=100$ & \\
\hline${ }^{184} \mathrm{Hf}^{m}$ & -40230 & 40 & 1272.4 & 0.4 & & 48 & $\mathrm{~s}$ & 10 & $8^{-}$ & & 95Kr04 & $\mathrm{TE}$ & $\beta^{-}=100$ & \\
\hline${ }^{184} \mathrm{Ta}$ & -42841 & 26 & & & & 8.7 & $\mathrm{~h}$ & 0.1 & $\left(5^{-}\right)$ & 90 & & & $\beta^{-}=100$ & \\
\hline${ }^{184} \mathrm{~W}$ & -45707.3 & 0.9 & & & & STABLE & & $(>180 \mathrm{Ey})$ & $0^{+}$ & 90 & 03Da05 & $\mathrm{T}$ & $\mathrm{IS}=30.642 ; \alpha ?$ & * \\
\hline${ }^{184} \mathrm{Re}$ & -44227 & 4 & & & & 38.0 & $\mathrm{~d}$ & 0.5 & $3^{(-)}$ & 90 & & & $\beta^{+}=100$ & \\
\hline${ }^{184} \mathrm{Re}^{m}$ & -44039 & 4 & 188.01 & 0.04 & & 169 & $\mathrm{~d}$ & 8 & $8^{(+)}$ & 90 & & & $\mathrm{IT}=75.411 ; \varepsilon=24.611$ & \\
\hline${ }^{184} \mathrm{Os}$ & -44256.1 & 1.3 & & & & StABle & & (>56 Ty) & $0^{+}$ & 90 & & & $\mathrm{IS}=0.021 ; \alpha ? ; 2 \beta^{+} ?$ & * \\
\hline${ }^{184} \mathrm{Ir}$ & -39611 & 28 & & & & 3.09 & $\mathrm{~h}$ & 0.03 & $5^{-}$ & 90 & & & $\beta^{+}=100$ & \\
\hline${ }^{184} \mathrm{Ir}^{m}$ & -39385 & 28 & 225.65 & 0.11 & & 470 & $\mu \mathrm{s}$ & & $3^{+}$ & & & & & \\
\hline${ }^{184} \mathrm{Pt}$ & -37332 & 18 & & & & 17.3 & $\mathrm{~m}$ & 0.2 & $0^{+}$ & 90 & 95Bi01 & $\mathrm{D}$ & $\beta^{+} \approx 100 ; \alpha=0.00177$ & \\
\hline${ }^{184} \mathrm{Pt}^{m}$ & -35493 & 18 & 1839.4 & 1.6 & & 1.01 & $\mathrm{~ms}$ & 0.05 & $8^{-}$ & 90 & & & $\mathrm{IT}=100$ & \\
\hline${ }^{184} \mathrm{Au}$ & -30319 & 22 & & & & 20.6 & $\mathrm{~s}$ & 0.9 & $5^{+}$ & 03 & & & $\beta^{+} \approx 100 ; \alpha<0.016$ & \\
\hline${ }^{184} \mathrm{Au}^{m}$ & -30251 & 22 & 68.46 & 0.01 & & 47.6 & $\mathrm{~s}$ & 1.4 & $2^{+}$ & 03 & $94 \mathrm{Ib} 01$ & EJ & $\beta^{+}=$?; IT $=3010 ; \alpha<0.016$ & \\
\hline${ }^{184} \mathrm{Au}^{n}$ & -30091 & 22 & 228.40 & 0.06 & & 69 & ns & 6 & $3^{-}$ & 03 & & & $\mathrm{IT}=100$ & \\
\hline${ }^{184} \mathrm{Hg}$ & -26349 & 10 & & & & 30.6 & $\mathrm{~s}$ & 0.3 & $0^{+}$ & 90 & & & $\beta^{+}=98.896 ; \alpha=1.116$ & \\
\hline${ }^{184} \mathrm{Tl}$ & -16890 & 50 & & & $*$ & 9.7 & $\mathrm{~s}$ & 0.6 & $2^{-} \#$ & 90 & 92Bo.D & $\mathrm{T}$ & $\beta^{+}=97.97 ; \alpha=2.17$ & \\
\hline${ }^{184} \mathrm{Tl}^{m}$ & $-16790 \#$ & $110 \#$ & $100 \#$ & $100 \#$ & $*$ & $10 \#$ & $\mathrm{~s}$ & & $7^{+} \#$ & & & & $\beta^{+}$?; IT ? & \\
\hline${ }^{184} \mathrm{Tl}^{n}$ & $-16390 \#$ & $150 \#$ & $500 \#$ & $140 \#$ & & $>20$ & $\mathrm{~ns}$ & & $\left(10^{-}\right)$ & & 84Sc.A & $\mathrm{T}$ & IT ? & * \\
\hline${ }^{184} \mathrm{~Pb}$ & -11045 & 14 & & & & 490 & $\mathrm{~ms}$ & 25 & $0^{+}$ & 03 & 02An.A & D & $\alpha=8015 ; \beta^{+} ?$ & \\
\hline${ }^{184} \mathrm{Bi}$ & 1050\# & $130 \#$ & & & $*$ & 6.6 & $\mathrm{~ms}$ & 1.5 & $3^{+} \#$ & & 02An.A & $\mathrm{T}$ & $\alpha=$ ? & \\
\hline${ }^{184} \mathrm{Bi}^{m}$ & $1200 \#$ & $160 \#$ & $150 \#$ & $100 \#$ & * & 13 & $\mathrm{~ms}$ & 2 & $10^{-} \#$ & & 02An.A & & $\alpha=$ ? & \\
\hline$*^{184} \mathrm{~W}$ & $\mathrm{~T}:$ also 03 & $3 \mathrm{Ce} 01$; & $>29$ Ey 9 & Ge15> & $.0 \mathrm{Ey}$ & & & & & & & & & $* *$ \\
\hline$*^{184} \mathrm{Os}$ & $\mathrm{T}$ : lower & limit is & for $\alpha \mathrm{de}$ & & & & & & & & & & & $* *$ \\
\hline$*{ }^{184} \mathrm{Tl}^{n}$ & $\mathrm{~T}:$ alpha & decay $f$ & rom ${ }^{188} \mathrm{~B}$ & ${ }^{m}$ not & . & ith & 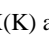 & & & & & & & $* *$ \\
\hline${ }^{* 184} \mathrm{Tl}^{n}$ & I : identifi & ed by $c$ & $2 \mathrm{Sc} . \mathrm{A}$ & & & & & & & & & & & $* *$ \\
\hline
\end{tabular}

\begin{tabular}{|c|c|c|c|c|c|c|c|c|c|c|c|c|c|}
\hline${ }^{185} \mathrm{Hf}$ & $-38360 \#$ & $200 \#$ & & & & 3.5 & $\mathrm{~m}$ & 0.6 & $3 / 2^{-} \#$ & 95 & & & $\beta^{-}=100$ \\
\hline${ }^{185} \mathrm{Ta}$ & -41396 & 14 & & & & 49.4 & $\mathrm{~m}$ & 1.5 & $7 / 2^{+} \#$ & 95 & & & $\beta^{-}=100$ \\
\hline${ }^{185} \mathrm{Ta}^{m}$ & -40090 & 30 & 1308 & 29 & & $>1$ & $\mathrm{~ms}$ & & $\left(21 / 2^{-}\right)$ & & 99Wh03 & TJD & $\mathrm{IT}=100$ \\
\hline${ }^{185} \mathrm{~W}$ & -43389.7 & 0.9 & & & & 75.1 & $\mathrm{~d}$ & 0.3 & $3 / 2^{-}$ & 95 & & & $\beta^{-}=100$ \\
\hline${ }^{185} \mathrm{~W}^{m}$ & -43192.3 & 0.9 & 197.43 & 0.05 & & 1.597 & $\mathrm{~m}$ & 0.004 & $11 / 2^{+}$ & 95 & 94It.A & $\mathrm{T}$ & $\mathrm{IT}=100$ \\
\hline${ }^{185} \mathrm{Re}$ & -43822.2 & 1.2 & & & & STABLE & & & $5 / 2^{+}$ & 95 & & & $\mathrm{IS}=37.402$ \\
\hline${ }^{185} \mathrm{Re}^{m}$ & -41698.2 & 2.3 & 2124 & 2 & & 123 & $\mathrm{~ns}$ & 23 & $(21 / 2)$ & & 97Sh37 & $\mathrm{T}$ & $\mathrm{IT}=100$ \\
\hline${ }^{185} \mathrm{Os}$ & -42809.4 & 1.3 & & & & 93.6 & $\mathrm{~d}$ & 0.5 & $1 / 2^{-}$ & 95 & & & $\varepsilon=100$ \\
\hline${ }^{185} \mathrm{Os}^{m}$ & -42707.1 & 1.5 & 102.3 & 0.7 & & 3.0 & $\mu \mathrm{s}$ & 0.4 & $7 / 2^{-} \#$ & 95 & & & IT ? \\
\hline${ }^{185} \mathrm{Ir}$ & -40336 & 28 & & & & 14.4 & $\mathrm{~h}$ & 0.1 & $5 / 2^{-}$ & 95 & & & $\beta^{+}=100$ \\
\hline${ }^{185} \mathrm{Pt}$ & -36680 & 40 & & & & 70.9 & $\mathrm{~m}$ & 2.4 & $\left(9 / 2^{+}\right)$ & 95 & & & $\beta^{+} \approx 100 ; \alpha=0.005020$ \\
\hline${ }^{185} \mathrm{Pt}^{m}$ & -36580 & 40 & 103.4 & 0.2 & & 33.0 & $\mathrm{~m}$ & 0.8 & $\left(1 / 2^{-}\right)$ & 95 & & & $\beta^{+}=$?; IT $<2$ \\
\hline${ }^{185} \mathrm{Au}$ & -31867 & 26 & & & $*$ & 4.25 & $\mathrm{~m}$ & 0.06 & $5 / 2^{-}$ & 95 & & & $\beta^{+} \approx 100 ; \alpha=0.266$ \\
\hline${ }^{185} \mathrm{Au}^{m}$ & $-31770 \#$ & $100 \#$ & $100 \#$ & $100 \#$ & * & 6.8 & $\mathrm{~m}$ & 0.3 & $1 / 2^{+} \#$ & 95 & & & $\beta^{+}<100 ;$ IT ? \\
\hline${ }^{185} \mathrm{Hg}$ & -26176 & 16 & & & & 49.1 & $\mathrm{~s}$ & 1.0 & $1 / 2^{-}$ & 95 & & & $\beta^{+}=941 ; \alpha=61$ \\
\hline${ }^{185} \mathrm{Hg}^{m}$ & -26072 & 16 & 103.8 & 1.0 & & 21.6 & $\mathrm{~s}$ & 1.5 & $13 / 2^{+}$ & 95 & 87Ki.A & $\mathrm{E}$ & $\mathrm{IT}=5410 ; \beta^{+}=4610 ; \alpha \approx 0.03$ \\
\hline${ }^{185} \mathrm{Tl}$ & -19760 & 50 & & & & 19.5 & $\mathrm{~s}$ & 0.5 & $1 / 2^{+} \#$ & 95 & & & $\beta^{+}=? ; \alpha ?$ \\
\hline${ }^{185} \mathrm{Tl}^{m}$ & -19300 & 50 & 452.8 & 2.0 & & 1.83 & $\mathrm{~s}$ & 0.12 & $9 / 2^{-} \#$ & 95 & $77 \mathrm{Sc} 03$ & $\mathrm{E}$ & $\mathrm{IT} \approx 100 ; \alpha=0.103 ; \beta^{+} ?$ \\
\hline${ }^{185} \mathrm{Tl}^{n}$ & -18760 & 50 & 1003.0 & 2.0 & & 8.3 & $\mathrm{~ns}$ & 1.4 & $\left(13 / 2^{+}\right)$ & & 95La08 & $\mathrm{T}$ & \\
\hline${ }^{185} \mathrm{~Pb}$ & -11541 & 16 & & & * & 6.3 & $\mathrm{~s}$ & 0.4 & $3 / 2^{-}$ & 95 & $02 \mathrm{An} 15$ & TJD & $\alpha=5025 ; \beta^{+} ?$ \\
\hline${ }^{185} \mathrm{~Pb}^{m}$ & $-11480 \#$ & $40 \#$ & $60 \#$ & $40 \#$ & $*$ & 4.07 & $\mathrm{~s}$ & 0.15 & $13 / 2^{+}$ & & $02 \mathrm{An} 15$ & TJD & $\alpha=5025 ; \beta^{+} ?$ \\
\hline${ }^{185} \mathrm{Bi}$ & $-2210 \#$ & $50 \#$ & & & $* \&$ & $2 \#$ & $\mathrm{~ms}$ & & $9 / 2^{-} \#$ & & $96 \mathrm{Da} 06$ & $\mathrm{~J}$ & $\mathrm{p} ? ; \alpha$ ? \\
\hline${ }^{185} \mathrm{Bi}^{m}$ & -2143 & 18 & 70\# & $50 \#$ & $* \&$ & 49 & $\mu \mathrm{s}$ & 7 & $1 / 2^{+}$ & 02 & 01Po05 & $\mathrm{T}$ & $\mathrm{p}=856 ; \alpha=156$ \\
\hline$*^{185} \mathrm{Ta}^{m}$ & $\mathrm{E}$ : from 9 & $9 \mathrm{Wh} 0$ & $3:$ less th & an $100 \mathrm{k}$ & abov & e 12581 & level & & : assuming & g gro & ound-state & $=7 / 2$ & \\
\hline${ }^{*}{ }^{185} \mathrm{Pt}$ & $\mathrm{D}:$ if the & $4444(1$ & 0) $\operatorname{keV} \alpha$ & line is $\mathrm{f}$ & grou & Ind-state & ; oth & rwise $c$ & $=0.0010(4$ & 4) $\%$ & from isom & & \\
\hline$*^{185} \mathrm{Hg}^{m}$ & E : ENSDF & gives & $99.3(0.5)$ & plus " 8 - & unce & ertainty' & , but & missed & 87Ki.A wc & & & & \\
\hline$*^{185} \mathrm{~Pb}$ & $\mathrm{~T}$ : averag & $02 \mathrm{Ar}$ & $\mathrm{n} 15=6.3(\mathrm{C}$ & .4) $80 \mathrm{~S}$ & $=6.1(1$ & 1.1) & & & & & & & \\
\hline$*^{185} \mathrm{~Pb}^{m}$ & $\mathrm{~T}:$ averag & $02 \mathrm{Ar}$ & $\mathrm{n} 15=4.3(\mathrm{C}$ & .2) $80 \mathrm{~S}$ & $=3.73$ & $(0.24)($ & exclu & ding the & $5.1 \mathrm{~s}$ activ & ) & & & \\
\hline$*^{185} \mathrm{Bi}$ & $\mathrm{T}$ : estima & ted fro & $\mathrm{m} 9 / 2^{-}$is & omers is & $\mathrm{d} \mathrm{Bi}$ & and $\mathrm{Tl} \mathrm{i}$ & sotop & & & & & & \\
\hline${ }^{185} \mathrm{Bi}^{m}$ & $\mathrm{~T}:$ averag & ge $01 \mathrm{Po}$ & $5=50(\varepsilon$ & $6 \mathrm{Da} 0 \mathrm{c}$ & (16) & & & & & & & & \\
\hline
\end{tabular}




\begin{tabular}{|c|c|c|c|c|c|c|c|c|c|c|c|c|c|c|}
\hline Nuclide & \multicolumn{2}{|c|}{$\begin{array}{l}\text { Mass excess } \\
\quad(\mathrm{keV})\end{array}$} & \multicolumn{3}{|c|}{$\begin{array}{l}\text { Excitation } \\
\text { energy }(\mathrm{keV})\end{array}$} & \multicolumn{3}{|c|}{ Half-life } & $J^{\pi}$ & Ens & \multicolumn{2}{|c|}{ Reference } & \multicolumn{2}{|l|}{$\begin{array}{c}\text { Decay modes and } \\
\text { intensities }(\%)\end{array}$} \\
\hline${ }^{186} \mathrm{Hf}$ & $-36430 \#$ & $300 \#$ & & & & 2.6 & $\mathrm{~m}$ & 1.2 & $0^{+}$ & 03 & & & $\beta^{-}=100$ & \\
\hline${ }^{186} \mathrm{Ta}$ & -38610 & 60 & & & & 10.5 & $\mathrm{~m}$ & 0.3 & $\left(2^{-}, 3^{-}\right)$ & 03 & & & $\beta^{-}=100$ & \\
\hline${ }^{186} \mathrm{~W}$ & -42509.5 & 1.7 & & & & STABLE & & $(>4.1 \mathrm{Ey})$ & $0^{+}$ & 03 & 03Da09 & $\mathrm{T}$ & $\mathrm{IS}=28.4319 ; 2 \beta^{-} ? ; \alpha$ ? & $*$ \\
\hline${ }^{186} \mathrm{~W}^{m}$ & -40992.3 & 1.8 & 1517.2 & 0.6 & & 18 & $\mu \mathrm{s}$ & 1 & $\left(7^{-}\right)$ & 03 & & & $\mathrm{IT}=100$ & \\
\hline${ }^{186} \mathrm{~W}^{n}$ & -38966.7 & 2.7 & 3542.8 & 2.1 & & $>3$ & $\mathrm{~ms}$ & & $\left(16^{+}\right)$ & 03 & & & $\mathrm{IT}=100$ & * \\
\hline${ }^{186} \mathrm{Re}$ & -41930.2 & 1.2 & & & & 3.7183 & $\mathrm{~d}$ & 0.0011 & $1^{-}$ & 03 & & & $\beta^{-}=92.5310 ; \varepsilon=7.4710$ & \\
\hline${ }^{186} \mathrm{Re}^{m}$ & -41781 & 7 & 149 & 7 & & 200 & ky & 50 & $\left(8^{+}\right)$ & 03 & & & $\mathrm{IT}=? ; \beta^{-}<10$ & * \\
\hline${ }^{186} \mathrm{Os}$ & -42999.5 & 1.4 & & & & 2.0 & Py & 1.1 & $0^{+}$ & 03 & & & $\mathrm{IS}=1.593 ; \alpha=100$ & \\
\hline${ }^{186} \mathrm{Ir}$ & -39173 & 17 & & & & 16.64 & $\mathrm{~h}$ & 0.03 & $5^{+}$ & 03 & & & $\beta^{+}=100$ & \\
\hline${ }^{186} \mathrm{Ir}^{m}$ & -39172 & 17 & 0.8 & 0.4 & & 1.92 & $\mathrm{~h}$ & 0.05 & $2^{-}$ & 03 & $91 \mathrm{Be} 25$ & ET & $\beta^{+} \approx 75 ; \mathrm{IT} \approx 25$ & * \\
\hline${ }^{186} \mathrm{Pt}$ & -37864 & 22 & & & & 2.08 & $\mathrm{~h}$ & 0.05 & $0^{+}$ & 03 & & & $\beta^{+}=100 ; \alpha \approx 1.4 \mathrm{e}-4$ & \\
\hline${ }^{186} \mathrm{Au}$ & -31715 & 21 & & & & 10.7 & $\mathrm{~m}$ & 0.5 & $3^{-}$ & 03 & & & $\beta^{+}=100 ; \alpha=0.00082$ & \\
\hline${ }^{186} \mathrm{Au}^{m}$ & -31487 & 21 & 227.77 & 0.07 & & 110 & ns & 10 & $2^{+}$ & 03 & & & $\mathrm{IT}=100$ & \\
\hline${ }^{186} \mathrm{Au}^{p}$ & & & non e & istent & $\mathrm{RN}$ & $<2$ & $\mathrm{~m}$ & & & & $83 \mathrm{Po} 10$ & I & & \\
\hline${ }^{186} \mathrm{Hg}$ & -28539 & 11 & & & & 1.38 & $\mathrm{~m}$ & 0.06 & $0^{+}$ & 03 & & & $\beta^{+} \approx 100 ; \alpha=0.0165$ & \\
\hline${ }^{186} \mathrm{Hg}^{m}$ & -26322 & 11 & 2217.3 & 0.4 & & 82 & $\mu \mathrm{s}$ & 5 & $\left(8^{-}\right)$ & 03 & & & $\mathrm{IT}=100$ & \\
\hline${ }^{186} \mathrm{Tl}$ & -20190 & 180 & & & $* \&$ & $40 \#$ & s & & $\left(2^{-}\right)$ & 03 & $91 \mathrm{Va} 04$ & I & $\beta^{+} ?$ & * \\
\hline${ }^{186} \mathrm{Tl}^{m}$ & -19874 & 9 & 320 & 180 & $\mathrm{AD} * \&$ & 27.5 & s & 1.0 & $\left(7^{+}\right)$ & 03 & & & $\beta^{+} \approx 100 ; \alpha \approx 0.006$ & \\
\hline${ }^{186} \mathrm{Tl}^{n}$ & -19501 & 9 & 690 & 180 & $\mathrm{AD}$ & 2.9 & s & 0.2 & $\left(10^{-}\right)$ & 03 & & & $\mathrm{IT}=100$ & * \\
\hline${ }^{186} \mathrm{~Pb}$ & -14681 & 11 & & & & 4.82 & $\mathrm{~s}$ & 0.03 & $0^{+}$ & 03 & & & $\beta^{+} ? ; \alpha=408$ & \\
\hline${ }^{186} \mathrm{Bi}$ & -3170 & 80 & & & $*$ & 14.8 & $\mathrm{~ms}$ & 0.7 & $\left(3^{+}\right)$ & 03 & 02An.A & & $\alpha \approx 100 ; \beta^{+}$? & * \\
\hline${ }^{186} \mathrm{Bi}^{m}$ & $-2900 \#$ & $160 \#$ & $270 \#$ & $140 \#$ & $*$ & 9.8 & $\mathrm{~ms}$ & 0.4 & $\left(10^{-}\right)$ & 03 & 02An.A & $\mathrm{T}$ & $\alpha \approx 100 ; \beta^{+} ?$ & \\
\hline$*^{186} \mathrm{~W}$ & $\mathrm{~T}:$ limit is & $2 \beta^{-}$ & lecay; 03 & $\mathrm{a} 05>17$ & 0 Ey $03 \mathrm{Ce}$ & $e 1>27 \mathrm{E}$ & Ey 97 & $7 \mathrm{Ge} 15>6$. & 5 Ey for $C$ & $\alpha \mathrm{dec}$ & & & & $* *$ \\
\hline$*^{186} \mathrm{~W}^{n}$ & $\mathrm{~T}$ : lower $\mathrm{l}$ & limit is & $3 \mathrm{~ms} ;$ up & er limit & & & & & & & & & & $* *$ \\
\hline$*^{186} \mathrm{Re}^{m}$ & $\mathrm{~T}$ : uncert & ainty es & timated $\mathrm{t}$ & y ENSDF & 89 evalua & & & & & & & & & $* *$ \\
\hline$*^{186} \mathrm{Ir}^{m}$ & $\mathrm{~T}$ : averag & e $91 \mathrm{Be}$ & $25=1.90$ & $.05) 70 \mathrm{I}$ & i. $A=2.0(0$. & & & & & & & & & $* *$ \\
\hline$*^{186} \mathrm{Ir}^{m}$ & $\mathrm{E}: \mathrm{E}$ is po & sitive : & and belov & $1.5 \mathrm{keV}$ & & & & & & & & & & $* *$ \\
\hline$*^{186} \mathrm{Tl}$ & I : identifi & $\mathrm{ed}$ as $\mathrm{d}$ & ecay leve & from 190 & $\mathrm{Bi}$ in $91 \mathrm{Va}$ & $\mathrm{a} 04$ & & & & & & & & ** \\
\hline$*^{186} \mathrm{Tl}^{n}$ & E : 374.0 & $0.2) \mathrm{ke}$ & $V$ above & ${ }^{6} \mathrm{Tl}^{m}$ & & & & & & & & & & $* *$ \\
\hline$*^{186} \mathrm{Bi}$ & $\mathrm{T}$ : averag & e $02 \mathrm{An}$ & $\mathrm{A}=14.8$ & .8) $97 \mathrm{~B}$ & $21=15.0$ & 1.7) & & & & & & & & $* *$ \\
\hline
\end{tabular}

\begin{tabular}{|c|c|c|c|c|c|c|c|c|c|c|c|c|c|c|}
\hline${ }^{187} \mathrm{Hf}$ & $-32980 \#$ & $400 \#$ & & & & & $30 \#$ & $\mathrm{~s}$ & (>300 ns) & $3 / 2^{-} \#$ & & 99Be63 & & $\beta^{-} ?$ \\
\hline${ }^{187} \mathrm{Ta}$ & $-36770 \#$ & $200 \#$ & & & & & 2\# & $\mathrm{m}$ & $(>300 \mathrm{~ns})$ & $7 / 2^{+} \#$ & & $99 \mathrm{Be} 63$ & I & $\beta^{-}$? \\
\hline${ }^{187} \mathrm{~W}$ & -39904.8 & 1.7 & & & & & 23.72 & $\mathrm{~h}$ & 0.06 & $3 / 2^{-}$ & 92 & & & $\beta^{-}=100$ \\
\hline${ }^{187} \mathrm{Re}$ & -41215.7 & 1.4 & & & & & 41.2 & Gy & 0.2 & $5 / 2^{+}$ & 91 & $01 \mathrm{Ga} 01$ & $\mathrm{~T}$ & $\mathrm{IS}=62.602 ; \beta^{-}=100$ \\
\hline${ }^{187} \mathrm{Os}$ & -41218.2 & 1.4 & & & & & StABLE & & & $1 / 2^{-}$ & 92 & & & $\mathrm{IS}=1.962$ \\
\hline${ }^{187} \mathrm{Ir}$ & -39716 & 6 & & & & & 10.5 & $\mathrm{~h}$ & 0.3 & $3 / 2^{+}$ & 91 & & & $\beta^{+}=100$ \\
\hline${ }^{187} \mathrm{Ir}^{m}$ & -39530 & 6 & 186.15 & 0.04 & & & 30.3 & $\mathrm{~ms}$ & 0.6 & $9 / 2^{-}$ & 91 & & & $\mathrm{IT}=100$ \\
\hline${ }^{187} \mathrm{Pt}$ & -36713 & 28 & & & & & 2.35 & $\mathrm{~h}$ & 0.03 & $3 / 2^{-}$ & 91 & & & $\beta^{+}=100$ \\
\hline${ }^{187} \mathrm{Au}$ & -33005 & 25 & & & & & 8.4 & $\mathrm{~m}$ & 0.3 & $1 / 2^{+}$ & 91 & & & $\beta^{+} \approx 100 ; \alpha=0.003 \#$ \\
\hline${ }^{187} \mathrm{Au}^{m}$ & -32884 & 25 & 120.51 & 0.16 & & & 2.3 & $\mathrm{~s}$ & 0.1 & $9 / 2^{-}$ & 91 & & & $\mathrm{IT}=100$ \\
\hline${ }^{187} \mathrm{Hg}$ & -28118 & 14 & & & & $\&$ & 1.9 & $\mathrm{~m}$ & 0.3 & $3 / 2^{-}$ & 91 & & & $\beta^{+}=100 ; \alpha>1.2 \mathrm{e}-4$ \\
\hline${ }^{187} \mathrm{Hg}^{m}$ & -28059 & 20 & 59 & 16 & MD & $\&$ & 2.4 & $\mathrm{~m}$ & 0.3 & $13 / 2^{+}$ & 91 & & & $\beta^{+}=100 ; \alpha>2.5 \mathrm{e}-4$ \\
\hline${ }^{187} \mathrm{Tl}$ & -22444 & 8 & & & & & 51 & $\mathrm{~s}$ & & $\left(1 / 2^{+}\right)$ & 99 & & & $\beta^{+}<100 ; \alpha ?$ \\
\hline${ }^{187} \mathrm{Tl}^{m}$ & -22109 & 8 & 335 & 3 & $\mathrm{AD}$ & & 15.60 & $\mathrm{~s}$ & 0.12 & $\left(9 / 2^{-}\right)$ & 99 & & & $\mathrm{IT}=? ; \beta^{+} ? ; \alpha=0.155$ \\
\hline${ }^{187} \mathrm{~Pb}$ & -14980 & 8 & & & $*$ & $*$ & 15.2 & $\mathrm{~s}$ & 0.3 & $\left(3 / 2^{-}\right)$ & 00 & & & $\beta^{+}=932 ; \alpha=72$ \\
\hline${ }^{187} \mathrm{~Pb}^{m}$ & -14969 & 11 & 11 & 11 & $\mathrm{AD} *$ & & 18.3 & $\mathrm{~s}$ & 0.3 & $\left(13 / 2^{+}\right)$ & 00 & & & $\beta^{+}=882 ; \alpha=122$ \\
\hline${ }^{187} \mathrm{Bi}$ & -6373 & 15 & & & & & 32 & $\mathrm{~ms}$ & 3 & $9 / 2^{-} \#$ & 01 & & & $\alpha>50 ; \beta^{+} ?$ \\
\hline${ }^{187} \mathrm{Bi}^{m}$ & -6272 & 18 & 101 & 20 & $\mathrm{AD}$ & & 320 & $\mu \mathrm{s}$ & 70 & $1 / 2^{+} \#$ & 01 & & & $\alpha>50 ; \beta^{+} ?$ \\
\hline${ }^{187} \mathrm{Bi}^{n}$ & -6121 & 15 & 252 & 1 & & & 7 & $\mu \mathrm{s}$ & 5 & $\left(13 / 2^{+}\right)$ & & $02 \mathrm{Hu} 14$ & ETJ & $\mathrm{IT}=100$ \\
\hline${ }^{187} \mathrm{Re}$ & $\mathrm{D}: \ldots ; \alpha$ & $<0.000$ & & & & & & & & & & & & \\
\hline${ }^{187} \mathrm{Re}$ & $\mathrm{T}$ : others: & $89 \operatorname{Li} 30$ & 2.3( & uty & hec & an & me gr & roup, & $0,86 \mathrm{Li11}$ & $5(1.3)$ & & & & \\
\hline
\end{tabular}




\begin{tabular}{|c|c|c|c|c|c|c|c|c|c|c|c|c|c|}
\hline Nuclide & \multicolumn{2}{|c|}{$\begin{array}{l}\text { Mass excess } \\
\quad(\mathrm{keV})\end{array}$} & \multicolumn{3}{|c|}{$\begin{array}{l}\text { Excitation } \\
\text { energy }(\mathrm{keV})\end{array}$} & \multicolumn{3}{|c|}{ Half-life } & \multirow{2}{*}{$\begin{array}{c}J^{\pi} \\
0^{+}\end{array}$} & \multirow{2}{*}{$\begin{array}{l}\text { Ens } \\
02\end{array}$} & Reference & \multicolumn{2}{|l|}{$\begin{array}{l}\text { Decay modes and } \\
\text { intensities (\%) }\end{array}$} \\
\hline${ }^{188} \mathrm{Hf}$ & $-30880 \#$ & $500 \#$ & & & & $20 \#$ & $\mathrm{~s}$ & $(>300 \mathrm{~ns})$ & & & $99 \mathrm{Be} 63 \mathrm{I}$ & $\beta^{-}$? & \\
\hline${ }^{188} \mathrm{Ta}$ & $-33810 \#$ & $200 \#$ & & & & $20 \#$ & $\mathrm{~s}$ & $(>300 \mathrm{~ns})$ & & 02 & $99 \mathrm{Be} 63 \mathrm{I}$ & $\beta^{-}$? & \\
\hline${ }^{188} \mathrm{~W}$ & -38667 & 3 & & & & 69.78 & $\mathrm{~d}$ & 0.05 & $0^{+}$ & 02 & & $\beta^{-}=100$ & \\
\hline${ }^{188} \mathrm{Re}$ & -39016.1 & 1.4 & & & & 17.0040 & $\mathrm{~h}$ & 0.0022 & $1^{-}$ & 02 & & $\beta^{-}=100$ & \\
\hline${ }^{188} \operatorname{Re}^{m}$ & -38844.0 & 1.4 & 172.069 & 0.00 & & 18.59 & $\mathrm{~m}$ & 0.04 & $(6)^{-}$ & 02 & & $\mathrm{IT}=100$ & \\
\hline${ }^{188} \mathrm{Os}$ & -41136.4 & 1.4 & & & & STABLE & & & $0^{+}$ & 02 & & $\mathrm{IS}=13.248$ & \\
\hline${ }^{188} \mathrm{Ir}$ & -38328 & 7 & & & & 41.5 & $\mathrm{~h}$ & 0.5 & $1^{-}$ & 02 & & $\beta^{+}=100$ & \\
\hline${ }^{188} \mathrm{Ir}^{m}$ & -37360 & 30 & 970 & 30 & & 4.2 & $\mathrm{~ms}$ & 0.2 & $7^{+} \#$ & 02 & ABBW E & $\mathrm{IT} \approx 100 ; \beta^{+} ?$ & * \\
\hline${ }^{188} \mathrm{Pt}$ & -37823 & 5 & & & & 10.2 & $\mathrm{~d}$ & 0.3 & $0^{+}$ & 02 & & $\varepsilon=100 ; \alpha=2.6 \mathrm{e}-53$ & \\
\hline${ }^{188} \mathrm{Au}$ & -32301 & 20 & & & & 8.84 & $\mathrm{~m}$ & 0.06 & $1^{(-)}$ & 02 & & $\beta^{+}=100$ & \\
\hline${ }^{188} \mathrm{Hg}$ & -30202 & 12 & & & & 3.25 & $\mathrm{~m}$ & 0.15 & $0^{+}$ & 02 & & $\beta^{+}=100 ; \alpha=3.7 \mathrm{e}-58$ & \\
\hline${ }^{188} \mathrm{Hg}^{m}$ & -27478 & 12 & 2724.3 & 0.4 & & 134 & ns & 15 & $\left(12^{+}\right)$ & 02 & & $\mathrm{IT}=100$ & \\
\hline${ }^{188} \mathrm{Tl}$ & -22350 & 30 & & & $*$ & 71 & $\mathrm{~s}$ & 2 & $\left(2^{-}\right)$ & 02 & & $\beta^{+}=100$ & \\
\hline${ }^{188} \mathrm{Tl}^{m}$ & -22307 & 10 & 40 & 30 & $\mathrm{MD} *$ & 71 & $\mathrm{~s}$ & 1 & $\left(7^{+}\right)$ & 02 & & $\beta^{+}=100$ & \\
\hline${ }^{188} \mathrm{Tl}^{n}$ & -22038 & 10 & 310 & 30 & MD & 41 & $\mathrm{~ms}$ & 4 & $\left(9^{-}\right)$ & 02 & & $\mathrm{IT} \approx 100 ; \beta^{+} ?$ & * \\
\hline${ }^{188} \mathrm{~Pb}$ & -17815 & 11 & & & & 25.5 & $\mathrm{~s}$ & 0.1 & $0^{+}$ & 02 & & $\beta^{+}=? ; \alpha=9.38$ & \\
\hline${ }^{188} \mathrm{~Pb}^{m}$ & -15237 & 11 & 2578.2 & 0.7 & & 830 & ns & 210 & $\left(8^{-}\right)$ & 02 & & $\mathrm{IT}=100$ & \\
\hline${ }^{188} \mathrm{~Pb}^{n}$ & -15102 & 11 & 2713.0 & 0.6 & & 94 & ns & & $\left(11^{-}\right)$ & 02 & & $\mathrm{IT}=100$ & \\
\hline${ }^{188} \mathrm{~Pb}^{p}$ & -15020 & 50 & 2800 & 50 & & 797 & ns & 21 & & 02 & & $\mathrm{IT}=100$ & $*$ \\
\hline${ }^{188} \mathrm{Bi}$ & -7200 & 50 & & & $* \&$ & 44 & $\mathrm{~ms}$ & 3 & $3^{+} \#$ & 02 & $97 \mathrm{Wa} 05 \mathrm{~T}$ & $\alpha=? ; \beta^{+} ?$ & * \\
\hline${ }^{188} \mathrm{Bi}^{m}$ & $-7000 \#$ & $150 \#$ & $210 \#$ & $140 \#$ & $* \&$ & 220 & $\mathrm{~ms}$ & 40 & $\left(10^{-}\right)$ & 02 & $97 \mathrm{Wa05} \mathrm{T}$ & $\alpha=? ; \beta^{+} ?$ & $*$ \\
\hline${ }^{188} \mathrm{Po}$ & -538 & 19 & & & & 430 & $\mu \mathrm{s}$ & 180 & $0^{+}$ & 02 & & $\alpha=? ; \beta^{+}$? & \\
\hline$*^{188} \mathrm{Ir}^{m}$ & $\mathrm{E}:$ less th & an 1001 & keV above & 923.51 & el, from EN & NSDF & & & & & & & $* *$ \\
\hline$*^{188} \mathrm{Tl}^{n}$ & E : 268.8 & $0.5) \mathrm{ke}$ & $V$ above ${ }^{188}$ & $\mathrm{Tl}^{m}, \mathrm{fr}$ & $91 \mathrm{Va} 04$ & & & & & & & & $* *$ \\
\hline$*^{188} \mathrm{~Pb}^{p}$ & $E: 2700.5$ & above & unknown 1 & evel, se & ENSDF'02 & & & & & & & & $* *$ \\
\hline$*^{188} \mathrm{Bi}$ & $\mathrm{T}:$ averag & e $97 \mathrm{Wa}$ & $05=46(7)$ & $4 \mathrm{Sc} . \mathrm{A}=$ & $4(3)$ & & & & & & & & $* *$ \\
\hline$*^{188} \mathrm{Bi}^{m}$ & $\mathrm{~T}:$ averag & e $97 \mathrm{Wa}$ & $05=218(50$ & ) $84 \mathrm{Sc}$. & $=210(90)$ & & & & & & & & $* *$ \\
\hline
\end{tabular}

\begin{tabular}{|c|c|c|c|c|c|c|c|c|c|c|c|c|c|}
\hline${ }^{189} \mathrm{Ta}$ & $-31830 \#$ & $300 \#$ & & & & 3\# & $\mathrm{s}$ & $(>300 \mathrm{~ns})$ & $7 / 2^{+} \#$ & & $99 \mathrm{Be} 63$ & I & $\beta^{-} ?$ \\
\hline${ }^{189} \mathrm{~W}$ & -35480 & 200 & & & & 11.6 & $\mathrm{~m}$ & 0.3 & $\left(3 / 2^{-}\right)$ & 91 & 97Ya03 & $\mathrm{T}$ & $\beta^{-}=100$ \\
\hline${ }^{189} \mathrm{Re}$ & -37978 & 8 & & & & 24.3 & $\mathrm{~h}$ & 0.4 & $5 / 2^{+}$ & 91 & & & $\beta^{-}=100$ \\
\hline${ }^{189} \mathrm{Os}$ & -38985.4 & 1.5 & & & & STABLE & & & $3 / 2^{-}$ & 91 & & & $\mathrm{IS}=16.155$ \\
\hline${ }^{189} \mathrm{Os}^{m}$ & -38954.6 & 1.5 & 30.814 & 0.018 & & 5.8 & $\mathrm{~h}$ & 0.1 & $9 / 2^{-}$ & 91 & & & $\mathrm{IT}=100$ \\
\hline${ }^{189} \mathrm{Ir}$ & -38453 & 13 & & & & 13.2 & $\mathrm{~d}$ & 0.1 & $3 / 2^{+}$ & 91 & & & $\varepsilon=100$ \\
\hline${ }^{189} \mathrm{Ir}^{m}$ & -38081 & 13 & 372.18 & 0.04 & & 13.3 & $\mathrm{~ms}$ & 0.3 & $11 / 2^{-}$ & 91 & & & $\mathrm{IT}=100$ \\
\hline${ }^{189} \mathrm{Ir}^{n}$ & -36120 & 13 & 2333.3 & 0.4 & & 3.7 & $\mathrm{~ms}$ & 0.2 & $(25 / 2)^{+}$ & 91 & & & $\mathrm{IT}=100$ \\
\hline${ }^{189} \mathrm{Pt}$ & -36483 & 11 & & & & 10.87 & $\mathrm{~h}$ & 0.12 & $3 / 2^{-}$ & 92 & & & $\beta^{+}=100$ \\
\hline${ }^{189} \mathrm{Pt}^{m}$ & -36291 & 11 & 191.6 & 0.4 & & 143 & $\mu \mathrm{s}$ & & $\left(13 / 2^{+}\right)$ & & & & \\
\hline${ }^{189} \mathrm{Au}$ & -33582 & 20 & & & & 28.7 & $\mathrm{~m}$ & 0.3 & $1 / 2^{+}$ & 92 & & & $\beta^{+}=100 ; \alpha<3 \mathrm{e}-5$ \\
\hline${ }^{189} \mathrm{Au}^{m}$ & -33335 & 20 & 247.23 & 0.17 & & 4.59 & $\mathrm{~m}$ & 0.11 & $11 / 2^{-}$ & 92 & & & $\beta^{+} \approx 100 ; \mathrm{IT}=$ ? \\
\hline${ }^{189} \mathrm{Hg}$ & -29630 & 30 & & & & 7.6 & $\mathrm{~m}$ & 0.1 & $3 / 2^{-}$ & 96 & & & $\beta^{+}=100 ; \alpha<3 \mathrm{e}-5$ \\
\hline${ }^{189} \mathrm{Hg}^{m}$ & -29549 & 18 & 80 & 30 & MD & 8.6 & $\mathrm{~m}$ & 0.1 & $13 / 2^{+}$ & 96 & $01 \mathrm{Sc} 41$ & $\mathrm{E}$ & $\beta^{+}=100 ; \alpha<3 \mathrm{e}-5$ \\
\hline${ }^{189} \mathrm{Tl}$ & -24602 & 11 & & & & 2.3 & $\mathrm{~m}$ & 0.2 & $\left(1 / 2^{+}\right)$ & 99 & & & $\beta^{+}=100$ \\
\hline${ }^{189} \mathrm{Tl}^{m}$ & -24319 & 10 & 283 & 6 & $\mathrm{AD}$ & 1.4 & $\mathrm{~m}$ & 0.1 & $9 / 2^{(-)}$ & 99 & $85 \mathrm{Bo} 46$ & J & $\beta^{+} \approx 100 ;$ IT $<4$ \\
\hline${ }^{189} \mathrm{~Pb}$ & -17880 & 30 & & & * & 51 & $\mathrm{~s}$ & 3 & $\left(3 / 2^{-}\right)$ & 91 & ABBW & $\mathrm{J}$ & $\beta^{+}>99 ; \alpha \approx 0.4$ \\
\hline${ }^{189} \mathrm{~Pb}^{m}$ & $-17840 \#$ & $50 \#$ & $40 \#$ & $30 \#$ & * & $1 \#$ & $\mathrm{~m}$ & & $\left(13 / 2^{+}\right)$ & & ABBW & $\mathrm{J}$ & $\beta^{+} ? ;$ IT ? \\
\hline${ }^{189} \mathrm{Bi}$ & -10060 & 50 & & & & 674 & $\mathrm{~ms}$ & 11 & $\left(9 / 2^{-}\right)$ & 98 & $95 \mathrm{Ba} 75$ & $\mathrm{~J}$ & $\alpha>50 ; \beta^{+}<50$ \\
\hline${ }^{189} \mathrm{Bi}^{m}$ & -9880 & 50 & 181 & 6 & $\mathrm{AD}$ & 6.6 & $\mathrm{~ms}$ & 0.6 & $\left(1 / 2^{+}\right)$ & 98 & $95 \mathrm{Ba} 75$ & TJ & $\alpha>50 ; \beta^{+}<50$ \\
\hline${ }^{189} \mathrm{Bi}^{n}$ & -9700 & 50 & 357 & 1 & & 880 & ns & 50 & $\left(13 / 2^{+}\right)$ & & 01 An 11 & ETJ & $\mathrm{IT}=100$ \\
\hline${ }^{189} \mathrm{Po}$ & -1415 & 22 & & & & 5 & $\mathrm{~ms}$ & 1 & $3 / 2^{-} \#$ & & 99An52 & TD & $\alpha=? ; \beta^{+} ?$ \\
\hline${ }^{189} \mathrm{~W}$ & \multicolumn{13}{|c|}{$\mathrm{T}:$ average $97 \mathrm{Ya} 03=11.7(0.5) 65 \mathrm{Ka} 07=11.5(0.3)$} \\
\hline${ }^{189} \mathrm{~Pb}$ & \multicolumn{13}{|c|}{$\mathrm{J}$ : from $\alpha$ decay to ${ }^{185} \mathrm{Hg}$} \\
\hline${ }^{189} \mathrm{~Pb}^{m}$ & \multicolumn{13}{|c|}{$\mathrm{J}:$ from $\alpha$ decay from ${ }^{193} \mathrm{Po}^{m}$} \\
\hline${ }^{189} \mathrm{Bi}$ & \multicolumn{13}{|c|}{$\mathrm{T}:$ average $02 \mathrm{Hu} 14=667(13) 97 \mathrm{Wa} 05=728(40) 85 \mathrm{Co} 06=680(30)$} \\
\hline${ }^{189} \mathrm{Bi}^{m}$ & \multicolumn{13}{|c|}{$\mathrm{T}:$ average $97 \mathrm{An} 09=4.8(0.5) 97 \mathrm{Wa} 05=5.2(0.6) 95 \mathrm{Ba} 75=7.0(0.2)$} \\
\hline${ }^{39} \mathrm{Bi}^{n}$ & \multicolumn{13}{|c|}{$\mathrm{T}$ : from $02 \mathrm{Hu} 14$; also $01 \mathrm{An} 11>360(120)$} \\
\hline
\end{tabular}




\begin{tabular}{|c|c|c|c|c|c|c|c|c|c|c|c|c|c|c|}
\hline Nuclide & \multicolumn{2}{|c|}{$\begin{array}{l}\text { Mass excess } \\
(\mathrm{keV})\end{array}$} & \multicolumn{3}{|c|}{$\begin{array}{l}\text { Excitation } \\
\text { energy }(\mathrm{keV})\end{array}$} & \multicolumn{3}{|c|}{ Half-life } & \multirow[t]{2}{*}{$J^{\pi}$} & \multirow[t]{2}{*}{ Ens } & \multicolumn{2}{|c|}{ Reference } & \multicolumn{2}{|l|}{$\begin{array}{l}\text { Decay modes and } \\
\text { intensities }(\%)\end{array}$} \\
\hline${ }^{190} \mathrm{Ta}$ & $-28660 \#$ & $400 \#$ & & & & $300 \#$ & $\mathrm{~ms}$ & & & & & & $\beta^{-} ?$ & \\
\hline${ }^{190} \mathrm{~W}$ & -34300 & 160 & & & & 30.0 & $\mathrm{~m}$ & 1.5 & $0^{+}$ & 03 & & & $\beta^{-}=100$ & \\
\hline${ }^{190} \mathrm{~W}^{m}$ & -31920 & 160 & 2381 & 5 & & $<3.1$ & $\mathrm{~ms}$ & & $\left(10^{-}\right)$ & 03 & & & $\mathrm{IT}=100$ & \\
\hline${ }^{190} \mathrm{Re}$ & -35570 & 150 & & & & 3.1 & $\mathrm{~m}$ & 0.3 & $(2)^{-}$ & 03 & & & $\beta^{-}=100$ & \\
\hline${ }^{190} \mathrm{Re}^{m}$ & -35360 & 160 & 210 & 60 & & 3.2 & $\mathrm{~h}$ & 0.2 & $\left(6^{-}\right)$ & 03 & ABBW & $\mathrm{E}$ & $\beta^{-}=54.420 ;$ IT ? & * \\
\hline${ }^{190} \mathrm{Os}$ & -38706.3 & 1.5 & & & & StABLE & & & $0^{+}$ & 03 & & & $\mathrm{IS}=26.262$ & \\
\hline${ }^{190} \mathrm{Os}^{m}$ & -37000.9 & 1.5 & 1705.4 & 0.2 & & 9.9 & $\mathrm{~m}$ & 0.1 & $(10)^{-}$ & 03 & & & $\mathrm{IT}=100$ & \\
\hline${ }^{190} \mathrm{Ir}$ & -36751.2 & 1.7 & & & & 11.78 & d & 0.10 & $4^{-}$ & 03 & & & $\beta^{+}=100 ; \mathrm{e}^{+}<0.002$ & \\
\hline${ }^{190} \mathrm{Ir}^{m}$ & -36725.1 & 1.7 & 26.1 & 0.1 & & 1.120 & $\mathrm{~h}$ & 0.003 & $\left(1^{-}\right)$ & 03 & & & $\mathrm{IT}=100$ & \\
\hline${ }^{190} \operatorname{Ir}^{n}$ & -36374.8 & 1.7 & 376.4 & 0.1 & & 3.087 & $\mathrm{~h}$ & 0.012 & $(11)^{-}$ & 03 & & & $\beta^{+}=91.42 ; \mathrm{IT}=8.62$ & \\
\hline${ }^{190} \operatorname{Ir}^{p}$ & -36715.0 & 1.7 & 36.154 & 0.02 & & $>2$ & $\mu \mathrm{s}$ & & $(4)^{+}$ & 03 & & & $\mathrm{IT}=100$ & \\
\hline${ }^{190} \mathrm{Ir}^{q}$ & -36433.6 & 1.7 & 317.56 & 0.04 & & 90 & ns & & $\left(5^{-}\right)$ & 03 & & & $\mathrm{IT}=100$ & \\
\hline${ }^{190} \mathrm{Pt}$ & -37323 & 6 & & & & 650 & Gy & 30 & $0^{+}$ & 03 & & & $\mathrm{IS}=0.0141 ; \alpha=100 ; \ldots$ & * \\
\hline${ }^{190} \mathrm{Au}$ & -32881 & 16 & & & & 42.8 & $\mathrm{~m}$ & 1.0 & $1^{-}$ & 03 & & & $\beta^{+}=100 ; \alpha<1 \mathrm{e}-6$ & \\
\hline${ }^{190} \mathrm{Au}^{m}$ & $-32680 \#$ & $150 \#$ & $200 \#$ & $150 \#$ & & * 125 & $\mathrm{~ms}$ & 20 & $11^{-} \#$ & 03 & & & $\mathrm{IT} \approx 100 ; \beta^{+} ?$ & \\
\hline${ }^{190} \mathrm{Hg}$ & -31370 & 16 & & & & 20.0 & $\mathrm{~m}$ & 0.5 & $0^{+}$ & 03 & & & $\varepsilon \approx 100 ; \mathrm{e}^{+}<1 ; \ldots$ & * \\
\hline${ }^{190} \mathrm{Tl}$ & -24330 & 50 & & & & 2.6 & $\mathrm{~m}$ & 0.3 & $2^{(-)}$ & 03 & & & $\beta^{+}=100$ & \\
\hline${ }^{190} \mathrm{Tl}^{m}$ & $-24200 \#$ & $70 \#$ & $130 \#$ & $90 \#$ & & 3.7 & $\mathrm{~m}$ & 0.3 & $7^{(+\#)}$ & 03 & & & $\beta^{+}=100$ & \\
\hline${ }^{190} \mathrm{Tl}^{n}$ & $-24040 \#$ & $90 \#$ & $290 \#$ & $70 \#$ & & 750 & $\mu \mathrm{s}$ & 40 & $\left(8^{-}\right)$ & 03 & & & $\mathrm{IT}=100$ & * \\
\hline${ }^{190} \mathrm{Tl}^{p}$ & $-23920 \#$ & $90 \#$ & $410 \#$ & $70 \#$ & & $>1$ & $\mu \mathrm{s}$ & & $9^{-}$ & 03 & $91 \mathrm{Va} 04$ & ET & IT ? & * \\
\hline${ }^{190} \mathrm{~Pb}$ & -20417 & 12 & & & & 71 & $\mathrm{~s}$ & 1 & $0^{+}$ & 03 & & & $\beta^{+} ? ; \alpha=0.404$ & \\
\hline${ }^{190} \mathrm{~Pb}^{m}$ & -17802 & 12 & 2614.8 & 0.8 & & 150 & $\mathrm{~ns}$ & & $(10)^{+}$ & 03 & & & $\mathrm{IT}=100$ & \\
\hline${ }^{190} \mathrm{~Pb}^{n}$ & -17799 & 23 & 2618 & 20 & & 25 & $\mu \mathrm{s}$ & & $\left(12^{+}\right)$ & 03 & & & IT ? & * \\
\hline${ }^{190} \mathrm{~Pb}^{p}$ & -17759 & 12 & 2658.2 & 0.8 & & 7.2 & $\mu \mathrm{s}$ & 0.6 & $(11)^{-}$ & 03 & & & $\mathrm{IT}=100$ & \\
\hline${ }^{190} \mathrm{Bi}$ & -10900 & 180 & & & & 6.3 & $\mathrm{~s}$ & 0.1 & $\left(3^{+}\right)$ & 03 & $91 \mathrm{Va} 04$ & $\mathrm{~J}$ & $\alpha=7721 ; \beta^{+}=?$ & \\
\hline${ }^{190} \mathrm{Bi}^{m}$ & -10483 & 10 & 420 & 180 & MD & 6.2 & s & 0.1 & $\left(10^{-}\right)$ & 03 & $91 \mathrm{Va} 04$ & $\mathrm{~J}$ & $\alpha=709 ; \beta^{+} ?$ & \\
\hline${ }^{190} \mathrm{Bi}^{n}$ & -10210 & 10 & 690 & 180 & MD & $>500$ & ns & 100 & $7^{+} \#$ & 03 & 01An11 & ET & $\mathrm{IT}=100$ & * \\
\hline${ }^{190} \mathrm{Po}$ & -4563 & 13 & & & & 2.46 & $\mathrm{~ms}$ & 0.05 & $0^{+}$ & 03 & & & $\alpha \approx 100 ; \beta^{+}=0.1 \#$ & \\
\hline${ }^{1}{ }^{190} \mathrm{Re}^{m}$ & \multicolumn{13}{|c|}{ E : from lower limit 119.12 and calculated 173 and 220 (see ENSDF'90) } & ** \\
\hline${ }^{*}{ }^{190} \mathrm{Re}^{m}$ & \multicolumn{13}{|c|}{ E : 210(290) from difference in beta-decay } & ** \\
\hline${ }^{1}{ }^{190} \mathrm{Pt}$ & \multicolumn{13}{|c|}{$\mathrm{D}: \ldots ; 2 \beta^{+} ?$} & $* *$ \\
\hline${ }^{190} \mathrm{Hg}$ & \multicolumn{13}{|c|}{$\mathrm{D}: \ldots ; \alpha<3.4 \mathrm{e}-7$} & ** \\
\hline${ }^{190} \mathrm{Tl}^{n}$ & \multicolumn{13}{|c|}{$\mathrm{E}: 161.9 \mathrm{keV}$ above ${ }^{190} \mathrm{Tl}^{m}$} & $* *$ \\
\hline${ }^{190} \mathrm{Tl}^{p}$ & \multicolumn{13}{|c|}{$\mathrm{E}: 236.2 \mathrm{keV}$ above ${ }^{190} \mathrm{Tl}^{m}$} & $* *$ \\
\hline${ }^{1}{ }^{190} \mathrm{~Pb}^{n}$ & \multicolumn{13}{|c|}{$\mathrm{E}:$ above ${ }^{190} \mathrm{~Pb}^{m}$, see ENSDF'03 } & $* *$ \\
\hline${ }^{1}{ }^{190} \mathrm{Bi}^{n}$ & \multicolumn{13}{|c|}{$\mathrm{E}: 273(1) \mathrm{keV}$ above the $\left(10^{-}\right)$isomer } & ** \\
\hline
\end{tabular}

\begin{tabular}{|c|c|c|c|c|c|c|c|c|c|c|c|c|c|c|}
\hline${ }^{191} \mathrm{~W}$ & $-31110 \#$ & $200 \#$ & & & & 20\# & $\mathrm{s}$ & $(>300 \mathrm{~ns})$ & $3 / 2^{-} \#$ & & $99 \mathrm{Be} 63$ & I & $\beta^{-} ?$ & \\
\hline${ }^{191} \mathrm{Re}$ & -34349 & 10 & & & & 9.8 & $\mathrm{~m}$ & 0.5 & $\left(3 / 2^{+}, 1 / 2^{+}\right)$ & 95 & & & $\beta^{-}=100$ & \\
\hline${ }^{191}$ Os & -36393.7 & 1.5 & & & & 15.4 & $\mathrm{~d}$ & 0.1 & $9 / 2^{-}$ & 95 & & & $\beta^{-}=100$ & \\
\hline${ }^{191} \mathrm{Os}^{m}$ & -36319.3 & 1.5 & 74.382 & 0.003 & & 13.10 & $\mathrm{~h}$ & 0.05 & $3 / 2^{-}$ & 95 & & & $\mathrm{IT}=100$ & \\
\hline${ }^{191} \mathrm{Ir}$ & -36706.4 & 1.7 & & & & StABLE & & & $3 / 2^{+}$ & 95 & & & $\mathrm{IS}=37.32$ & \\
\hline${ }^{191} \mathrm{Ir}^{m}$ & -36535.2 & 1.7 & 171.24 & 0.05 & & 4.94 & $\mathrm{~s}$ & 0.03 & $11 / 2^{-}$ & 95 & & & $\mathrm{IT}=100$ & \\
\hline${ }^{191} \mathrm{Ir}^{n}$ & -34590 & 40 & 2120 & 40 & & 5.5 & $\mathrm{~s}$ & 0.7 & & 95 & ABBW & E & $\mathrm{IT}=100$ & * \\
\hline${ }^{191} \mathrm{Pt}$ & -35698 & 4 & & & & 2.802 & d & 0.025 & $3 / 2^{-}$ & 96 & & & $\varepsilon=100$ & \\
\hline${ }^{191} \mathrm{Pt}^{m}$ & -35549 & 4 & 149.04 & 0.02 & & 95 & $\mu \mathrm{s}$ & & $13 / 2^{+}$ & & & & & \\
\hline${ }^{191} \mathrm{Au}$ & -33810 & 40 & & & & 3.18 & $\mathrm{~h}$ & 0.08 & $3 / 2^{+}$ & 99 & & & $\beta^{+}=100$ & \\
\hline${ }^{191} \mathrm{Au}^{m}$ & -33540 & 40 & 266.2 & 0.5 & & 920 & $\mathrm{~ms}$ & 110 & $\left(11 / 2^{-}\right)$ & 99 & & & $\mathrm{IT}=100$ & \\
\hline${ }^{191} \mathrm{Hg}$ & -30593 & 23 & & & & 49 & $\mathrm{~m}$ & 10 & $3 / 2^{(-)}$ & 00 & 86Ul02 & $\mathbf{J}$ & $\beta^{+}=100 ; \alpha<5 \mathrm{e}-6$ & \\
\hline${ }^{191} \mathrm{Hg}^{m}$ & -30470 & 30 & 128 & 22 & & 50.8 & $\mathrm{~m}$ & 1.5 & $13 / 2^{+}$ & 00 & $01 \mathrm{Sc} 41$ & E & $\beta^{+}=100 ; \alpha<5 \mathrm{e}-6$ & * \\
\hline${ }^{191} \mathrm{Tl}$ & -26281 & 8 & & & & $20 \#$ & $\mathrm{~m}$ & & $\left(1 / 2^{+}\right)$ & 95 & & & $\beta^{+}$? & \\
\hline${ }^{191} \mathrm{Tl}^{m}$ & -25984 & 7 & 297 & 7 & BD & 5.22 & $\mathrm{~m}$ & 0.16 & $9 / 2^{(-)}$ & 95 & & & $\beta^{+}=100$ & \\
\hline${ }^{191} \mathrm{~Pb}$ & -20250 & 40 & & & $*$ & 1.33 & $\mathrm{~m}$ & 0.08 & $\left(3 / 2^{-}\right)$ & 95 & & & $\beta^{+} \approx 100 ; \alpha=0.0135$ & \\
\hline${ }^{191} \mathrm{~Pb}^{m}$ & -20231 & 28 & 20 & 50 & $\mathrm{MD} *$ & 2.18 & $\mathrm{~m}$ & 0.08 & $13 / 2^{(+)}$ & 95 & 88Me.A & $\mathrm{J}$ & $\beta^{+} \approx 100 ; \alpha \approx 0.02$ & \\
\hline${ }^{191} \mathrm{Bi}$ & -13240 & 7 & & & & 12.3 & $\mathrm{~s}$ & 0.3 & $\left(9 / 2^{-}\right)$ & 00 & $03 \mathrm{Ke} 04$ & $\mathrm{~T}$ & $\alpha=6020 ; \beta^{+}=4020$ & * \\
\hline${ }^{191} \mathrm{Bi}^{m}$ & -13000 & 9 & 240 & 4 & $\mathrm{AD}$ & 124 & $\mathrm{~ms}$ & 5 & $\left(1 / 2^{+}\right)$ & 00 & $03 \mathrm{Ke} 04$ & $\mathrm{~T}$ & $\alpha=7525 ; \beta^{+} \approx 25$ & * \\
\hline${ }^{191} \mathrm{Po}$ & -5054 & 11 & & & & 22 & $\mathrm{~ms}$ & 1 & $3 / 2^{-} \#$ & 00 & & & $\alpha \approx 100 ; \beta^{+} ?$ & \\
\hline${ }^{191} \mathrm{Po}^{m}$ & -5020 & 10 & 34 & 12 & $\mathrm{AD}$ & 98 & $\mathrm{~ms}$ & 8 & $\left(13 / 2^{+}\right)$ & 00 & & & $\alpha \approx 100 ; \beta^{+} ?$ & \\
\hline${ }^{191} \operatorname{Ir}^{n}$ & E : estima & ted less & than $150 \mathrm{k}$ & eV above & 2047.1 & 1 level, fr & om E & ENSDF & & & & & & * \\
\hline${ }^{91} \mathrm{Hg}^{m}$ & E : origin & 1 error & $(8 \mathrm{keV})$ inc & reased by & 20 for & isomer+ & grour & ind-state & in trap & & & & & 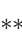 \\
\hline${ }^{11} \mathrm{Bi}$ & $\mathrm{T}$ : averag & e $03 \mathrm{Ke}$ & $04=12.4(c$ & $85 \mathrm{CoO}$ & $6=12(1)$ & ) $74 \mathrm{Le} 02$ & $=13($ & (1) $72 \mathrm{Ga} 2$ & $=12.0(0.7)$ & & & & & $*$ \\
\hline${ }^{91} \mathrm{Bi}^{m}$ & $\mathrm{~T}$ : average & e $03 \mathrm{Ke}$ & $04=121(+8$ & -5) $99 \mathrm{An}$ & $136=115$ & $5(10) 81 \mathrm{I}$ & Le23= & $=150(15)$ & & & & & & \\
\hline
\end{tabular}




\begin{tabular}{|c|c|c|c|c|c|c|c|c|c|c|c|c|c|}
\hline Nuclide & \multicolumn{2}{|c|}{$\begin{array}{l}\text { Mass excess } \\
\quad(\mathrm{keV})\end{array}$} & \multicolumn{3}{|c|}{$\begin{array}{l}\text { Excitation } \\
\text { energy }(\mathrm{keV})\end{array}$} & \multicolumn{3}{|c|}{ Half-life } & \multirow[t]{2}{*}{$J^{\pi}$} & \multirow[t]{2}{*}{ Ens } & Reference & \multicolumn{2}{|l|}{$\begin{array}{l}\text { Decay modes and } \\
\text { intensities }(\%)\end{array}$} \\
\hline${ }^{192} \mathrm{~W}$ & $-29650 \#$ & $600 \#$ & & & & $10 \#$ & $\mathrm{~s}$ & $(>300 \mathrm{~ns})$ & & & $99 B e 63 \mathrm{I}$ & $\beta^{-} ?$ & \\
\hline${ }^{192} \mathrm{Re}$ & $-31710 \#$ & $200 \#$ & & & & 16 & $\mathrm{~s}$ & 1 & & 98 & & $\beta^{-}=100$ & \\
\hline${ }^{192} \mathrm{Os}$ & -35880.5 & 2.6 & & & & StABLE & & (>9.8 Ty) & $0^{+}$ & 98 & & $\mathrm{IS}=40.7819 ; 2 \beta^{-} ? ; \alpha ?$ & $*$ \\
\hline${ }^{192} \mathrm{Os}^{m}$ & -33865.1 & 2.6 & 2015.40 & 0.11 & & 5.9 & $\mathrm{~s}$ & 0.1 & $\left(10^{-}\right)$ & 98 & & $\mathrm{IT}>87 ; \beta^{-}<13$ & \\
\hline${ }^{192} \mathrm{Ir}$ & -34833.2 & 1.7 & & & & 73.827 & $\mathrm{~d}$ & 0.013 & $4^{+}$ & 98 & & $\beta^{-}=95.1314 ; \varepsilon=4.8714$ & \\
\hline${ }^{192} \operatorname{Ir}^{m}$ & -34776.5 & 1.7 & 56.720 & 0.005 & & 1.45 & $\mathrm{~m}$ & 0.05 & $1^{-}$ & 98 & & $\mathrm{IT} \approx 100 ; \beta^{-}=0.0175$ & \\
\hline${ }^{192} \operatorname{Ir}^{n}$ & -34665.1 & 1.7 & 168.14 & 0.12 & & 241 & $\mathrm{y}$ & 9 & $\left(11^{-}\right)$ & 98 & & $\mathrm{IT}=100$ & \\
\hline${ }^{192} \mathrm{Pt}$ & -36292.9 & 2.5 & & & & StABLE & & & $0^{+}$ & 98 & & IS $=0.7827$ & \\
\hline${ }^{192} \mathrm{Au}$ & -32777 & 16 & & & & 4.94 & $\mathrm{~h}$ & 0.09 & $1^{-}$ & 98 & & $\beta^{+}=100$ & \\
\hline${ }^{192} \mathrm{Au}^{m}$ & -32642 & 16 & 135.41 & 0.25 & & 29 & $\mathrm{~ms}$ & & $5 \#^{+}$ & 98 & & $\mathrm{IT}=100$ & \\
\hline${ }^{192} \mathrm{Au}^{n}$ & -32345 & 16 & 431.6 & 0.5 & & 160 & $\mathrm{~ms}$ & 20 & $\left(11^{-}\right)$ & 98 & & $\mathrm{IT}=100$ & \\
\hline${ }^{192} \mathrm{Hg}$ & -32011 & 16 & & & & 4.85 & $\mathrm{~h}$ & 0.20 & $0^{+}$ & 00 & & $\varepsilon=100 ; \alpha<4 \mathrm{e}-6$ & \\
\hline${ }^{192} \mathrm{Tl}$ & -25870 & 30 & & & & 9.6 & $\mathrm{~m}$ & 0.4 & $\left(2^{-}\right)$ & 99 & & $\beta^{+}=100$ & \\
\hline${ }^{192} \mathrm{Tl}^{m}$ & -25710 & 60 & 160 & 50 & & 10.8 & $\mathrm{~m}$ & 0.2 & $\left(7^{+}\right)$ & 99 & $91 \mathrm{Va} 04 \mathrm{E}$ & $\beta^{+}=100$ & \\
\hline${ }^{192} \mathrm{Tl}^{p}$ & -25694 & 25 & 180 & 40 & $\mathrm{AD}$ & & & & $\left(3^{+}\right)$ & & $91 \mathrm{Va} 04 \mathrm{E}$ & & \\
\hline${ }^{192} \mathrm{~Pb}$ & -22556 & 13 & & & & 3.5 & $\mathrm{~m}$ & 0.1 & $0^{+}$ & 98 & & $\beta^{+} \approx 100 ; \alpha=0.00597$ & \\
\hline${ }^{192} \mathrm{~Pb}^{m}$ & -19975 & 13 & 2581.1 & 0.1 & & 164 & ns & 7 & $(10)^{+}$ & 98 & & $\mathrm{IT}=100$ & \\
\hline${ }^{192} \mathrm{~Pb}^{n}$ & -19931 & 13 & 2625.1 & 1.1 & & 1.1 & $\mu \mathrm{s}$ & 0.5 & $\left(12^{+}\right)$ & 98 & & $\mathrm{IT}=100$ & \\
\hline${ }^{192} \mathrm{~Pb}^{p}$ & -19813 & 13 & 2743.5 & 0.4 & & 756 & ns & 21 & $(11)^{-}$ & 98 & & $\mathrm{IT}=100$ & \\
\hline${ }^{192} \mathrm{Bi}$ & -13550 & 30 & & & & 34.6 & $\mathrm{~s}$ & 0.9 & $\left(3^{+}\right)$ & 98 & & $\beta^{+}=885 ; \alpha=125$ & \\
\hline${ }^{192} \mathrm{Bi}^{m}$ & -13399 & 9 & 150 & 30 & MD & 39.6 & $\mathrm{~s}$ & 0.4 & $\left(10^{-}\right)$ & 98 & & $\beta^{+}=903 ; \alpha=103$ & \\
\hline${ }^{192} \mathrm{Po}$ & -8071 & 12 & & & & 32.2 & $\mathrm{~ms}$ & 0.3 & $0^{+}$ & 98 & $99 \mathrm{He} 32 \mathrm{~T}$ & $\alpha=? ; \beta^{+}=0.5 \#$ & * \\
\hline${ }^{192} \mathrm{Po}^{m}$ & $-5470 \#$ & $500 \#$ & $2600 \#$ & $500 \#$ & & 1 & $\mu \mathrm{s}$ & & $12^{+} \#$ & & $99 \mathrm{He} 32 \mathrm{~T}$ & $\mathrm{IT}=100$ & \\
\hline$*^{192} \mathrm{Os}$ & $\mathrm{T}$ : lower $\mathrm{l}$ & limit is & for $0 v-2 \beta$ & decay & & & & & & & & & F* \\
\hline$*^{192} \mathrm{Po}$ & $\mathrm{T}$ : others & 98Al2 & $7=31(4) 96$ & $\mathrm{Bi} 17=33.2$ & 2(1.4) & $1 \mathrm{Le} 23=3$ & $34(3)$ & ) outweigh & d, not use & & & & $* *$ \\
\hline
\end{tabular}

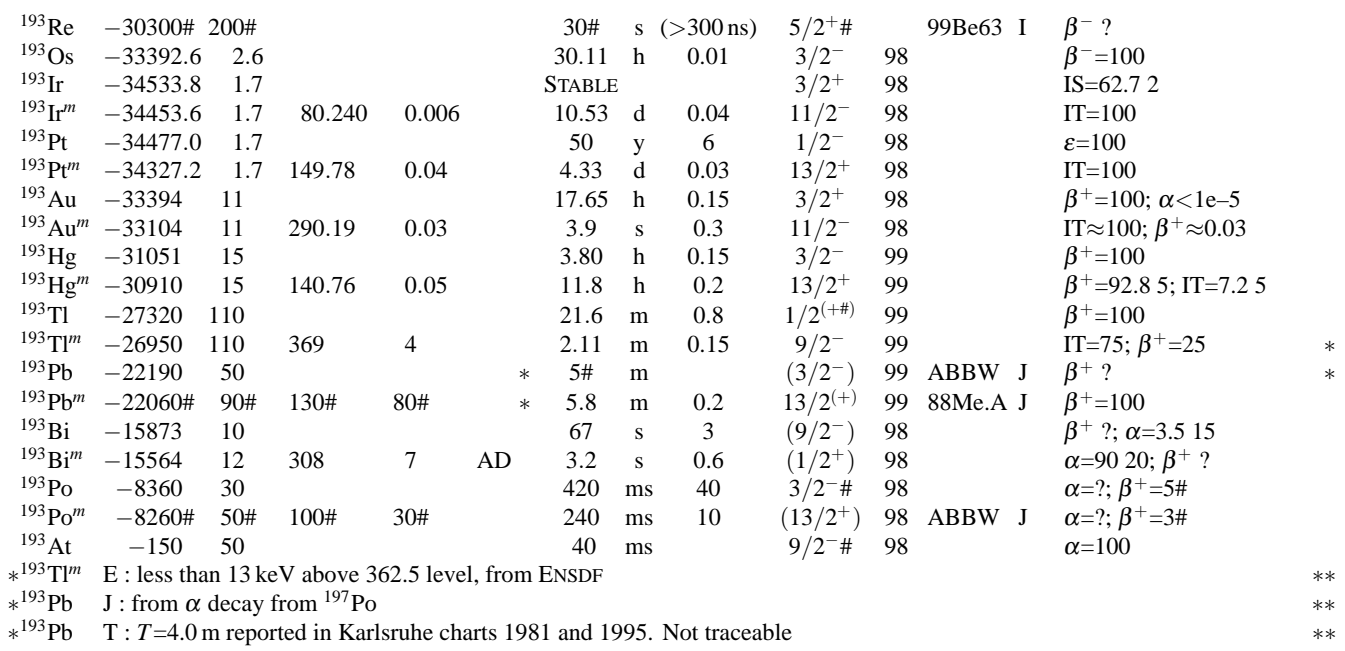

\begin{tabular}{|c|c|c|c|c|c|c|c|c|c|c|c|}
\hline${ }^{194} \mathrm{Re}$ & $-27550 \#$ & 300\# & & & & $2 \#$ & $\mathrm{~s}$ & $(>300 \mathrm{~ns})$ & & 99Be63 I & $\beta^{-} ?$ \\
\hline${ }^{194} \mathrm{Os}$ & -32432.7 & 2.6 & & & & 6.0 & $\mathrm{y}$ & 0.2 & $0^{+}$ & 96 & $\beta^{-}=100$ \\
\hline${ }^{194} \mathrm{Ir}$ & -32529.3 & 1.7 & & & & 19.28 & $\mathrm{~h}$ & 0.13 & $1^{-}$ & 96 & $\beta^{-}=100$ \\
\hline${ }^{194} \mathrm{Ir}^{m}$ & -32382.2 & 1.7 & 147.078 & 0.005 & & 31.85 & $\mathrm{~ms}$ & 0.24 & $\left(4^{+}\right)$ & 96 & $\mathrm{IT}=100$ \\
\hline${ }^{194} \operatorname{Ir}^{n}$ & -32160 & 70 & 370 & 70 & $\mathrm{BD}$ & 171 & $\mathrm{~d}$ & 11 & $(10,11)^{(-\#)}$ & 96 & $\beta^{-}=100$ \\
\hline${ }^{194} \mathrm{Pt}$ & -34763.1 & 0.9 & & & & STABLE & & & $0^{+}$ & 96 & $\mathrm{IS}=32.96799$ \\
\hline${ }^{194} \mathrm{Au}$ & -32262 & 10 & & & & 38.02 & $\mathrm{~h}$ & 0.10 & $1^{-}$ & 96 & $\beta^{+}=100$ \\
\hline${ }^{194} \mathrm{Au}^{m}$ & -32155 & 10 & 107.4 & 0.5 & & 600 & $\mathrm{~ms}$ & 8 & $\left(5^{+}\right)$ & 96 & $\mathrm{IT}=100$ \\
\hline${ }^{194} \mathrm{Au}^{n}$ & -31786 & 10 & 475.8 & 0.6 & & 420 & $\mathrm{~ms}$ & 10 & $\left(11^{-}\right)$ & 96 & $\mathrm{IT}=100$ \\
\hline${ }^{194} \mathrm{Hg}$ & -32193 & 13 & & & & 440 & $\mathrm{y}$ & 80 & $0^{+}$ & 01 & $\varepsilon=100$ \\
\hline
\end{tabular}




\begin{tabular}{|c|c|c|c|c|c|c|c|c|c|c|c|c|c|c|c|}
\hline Nuclide & \multicolumn{2}{|c|}{$\begin{array}{l}\text { Mass excess } \\
(\mathrm{keV})\end{array}$} & \multicolumn{4}{|c|}{$\begin{array}{c}\text { Excitation } \\
\text { energy }(\mathrm{keV})\end{array}$} & \multicolumn{3}{|c|}{ Half-life } & \multirow[t]{2}{*}{$J^{\pi}$} & \multirow[t]{2}{*}{ Ens } & \multicolumn{2}{|c|}{ Reference } & \multicolumn{2}{|l|}{$\begin{array}{l}\text { Decay modes and } \\
\text { intensities }(\%)\end{array}$} \\
\hline \multicolumn{14}{|c|}{$A$-group continued } & & \\
\hline${ }^{194} \mathrm{Tl}$ & -26830 & 140 & & & * & & 33.0 & $\mathrm{~m}$ & 0.5 & $2^{-}$ & 99 & & & $\beta^{+}=100 ; \alpha<1 \mathrm{e}-7$ & \\
\hline${ }^{194} \mathrm{Tl}^{m}$ & $-26530 \#$ & $240 \#$ & $300 \#$ & $200 \#$ & * & & 32.8 & $\mathrm{~m}$ & 0.2 & $\left(7^{+}\right)$ & 99 & & & $\beta^{+}=100$ & \\
\hline${ }^{194} \mathrm{~Pb}$ & -24208 & 17 & & & & & 12.0 & $\mathrm{~m}$ & 0.5 & $0^{+}$ & 99 & & & $\beta^{+}=100 ; \alpha=7.3 \mathrm{e}-629$ & \\
\hline${ }^{194} \mathrm{Bi}$ & -15990 & 50 & & & $*$ & & 95 & $\mathrm{~s}$ & 3 & $\left(3^{+}\right)$ & 96 & & & $\beta^{+} \approx 100 ; \alpha=0.4625$ & \\
\hline${ }^{194} \mathrm{Bi}^{m}$ & -15880 & 50 & 110 & 70 & $\mathrm{MD} *$ & & 125 & $\mathrm{~s}$ & 2 & $\left(6^{+}, 7^{+}\right)$ & 96 & & & $\beta^{+} \approx 100 ; \alpha ?$ & \\
\hline${ }^{194} \mathrm{Bi}^{n}$ & $-15760 \#$ & $70 \#$ & 230\# & $90 \#$ & & & 115 & s & 4 & $\left(10^{-}\right)$ & 96 & & & $\beta^{+} \approx 100 ; \alpha=0.207$ & \\
\hline${ }^{194} \mathrm{Po}$ & -11005 & 13 & & & & & 392 & $\mathrm{~ms}$ & 4 & $0^{+}$ & 96 & & & $\alpha \approx 100 ; \beta^{+} ?$ & \\
\hline${ }^{194} \mathrm{Po}^{m}$ & -8480 & 13 & 2525 & 2 & & & 15 & $\mu \mathrm{s}$ & 2 & $\left(11^{-}\right)$ & & $99 \mathrm{He} 32$ & TJD & $\mathrm{IT}=100$ & \\
\hline${ }^{194} \mathrm{At}$ & -1190 & 190 & & & & & 40 & $\mathrm{~ms}$ & & $3^{+} \#$ & 96 & & & $\alpha \approx 100 ; \beta^{+} ?$ & \\
\hline${ }^{194} \mathrm{At}^{m}$ & -711 & 17 & 480 & 190 & $\mathrm{AD}$ & & 250 & $\mathrm{~ms}$ & & $10^{-} \#$ & 96 & & & $\alpha \approx 100 ;$ IT ? & \\
\hline${ }^{195} \mathrm{Os}$ & -29690 & 500 & & & & & 6.5 & $\mathrm{~m}$ & & $3 / 2^{-} \#$ & 99 & & & $\beta^{-}=100$ & * \\
\hline${ }^{195} \mathrm{Ir}$ & -31689.8 & 1.7 & & & & & 2.5 & $\mathrm{~h}$ & 0.2 & $3 / 2^{+}$ & 99 & & & $\beta^{-}=100$ & \\
\hline${ }^{195} \mathrm{Ir}^{m}$ & -31590 & 5 & 100 & 5 & & & 3.8 & $\mathrm{~h}$ & 0.2 & $11 / 2^{-}$ & 99 & & & $\beta^{-}=955 ; \mathrm{IT}=55$ & \\
\hline${ }^{195} \mathrm{Pt}$ & -32796.8 & 0.9 & & & & & STABLE & & & $1 / 2^{-}$ & 99 & & & $\mathrm{IS}=33.83210$ & \\
\hline${ }^{195} \mathrm{Pt}^{m}$ & -32537.5 & 0.9 & 259.30 & 0.08 & & & 4.02 & d & 0.01 & $13 / 2^{+}$ & 99 & & & $\mathrm{IT}=100$ & \\
\hline${ }^{195} \mathrm{Au}$ & -32570.0 & 1.3 & & & & & 186.10 & $d$ & 0.05 & $3 / 2^{+}$ & 99 & & & $\varepsilon=100$ & \\
\hline${ }^{195} \mathrm{Au}^{m}$ & -32251.4 & 1.3 & 318.58 & 0.04 & & & 30.5 & $\mathrm{~s}$ & 0.2 & $11 / 2^{-}$ & 99 & & & $\mathrm{IT}=100$ & \\
\hline${ }^{195} \mathrm{Hg}$ & -31000 & 23 & & & & & 10.53 & $\mathrm{~h}$ & 0.03 & $1 / 2^{-}$ & 99 & 01Li17 & $\mathrm{T}$ & $\beta^{+}=100$ & \\
\hline${ }^{195} \mathrm{Hg}^{m}$ & -30824 & 23 & 176.07 & 0.04 & & & 41.6 & $\mathrm{~h}$ & 0.8 & $13 / 2^{+}$ & 99 & & & $\mathrm{IT}=54.220 ; \beta^{+}=45.820$ & \\
\hline${ }^{195} \mathrm{Tl}$ & -28155 & 14 & & & & & 1.16 & $\mathrm{~h}$ & 0.05 & $1 / 2^{+}$ & 99 & & & $\beta^{+}=100$ & \\
\hline${ }^{195} \mathrm{Tl}^{m}$ & -27672 & 14 & 482.63 & 0.17 & & & 3.6 & s & 0.4 & $9 / 2^{-}$ & 99 & & & $\mathrm{IT}=100$ & \\
\hline${ }^{195} \mathrm{~Pb}$ & -23714 & 23 & & & & & 15 & $\mathrm{~m}$ & & $3 / 2 \#^{-}$ & 99 & & & $\beta^{+}=100$ & \\
\hline${ }^{195} \mathrm{~Pb}^{m}$ & -23511 & 23 & 202.9 & 0.7 & & & 15.0 & $\mathrm{~m}$ & 1.2 & $13 / 2^{+}$ & 99 & & & $\beta^{+}=100$ & \\
\hline${ }^{195} \mathrm{Bi}$ & -18024 & 6 & & & & & 183 & $\mathrm{~s}$ & 4 & $\left(9 / 2^{-}\right)$ & 99 & ABBW & $\mathrm{J}$ & $\beta^{+} \approx 100 ; \alpha=0.032$ & \\
\hline${ }^{195} \mathrm{Bi}^{m}$ & -17624 & 8 & 399 & 6 & $\mathrm{AD}$ & & 87 & $\mathrm{~s}$ & 1 & $\left(1 / 2^{+}\right)$ & 99 & ABBW & $\mathrm{J}$ & $\beta^{+}=6717 ; \alpha=3317$ & * \\
\hline${ }^{195} \mathrm{Po}$ & -11070 & 40 & & & & & 4.64 & $\mathrm{~s}$ & 0.09 & $3 / 2^{-} \#$ & 99 & & & $\alpha=7515 ; \beta^{+}=2515$ & \\
\hline${ }^{195} \mathrm{Po}^{m}$ & -10964 & 28 & 110 & 50 & $\mathrm{AD}$ & & 1.92 & $\mathrm{~s}$ & 0.02 & $13 / 2^{+} \#$ & 99 & & & $\alpha \approx 90 ; \beta^{+} \approx 10 ;$ IT $<0.01$ & \\
\hline${ }^{195} \mathrm{At}$ & -3476 & 9 & & & & $\&$ & 328 & $\mathrm{~ms}$ & 20 & $\left(1 / 2^{+}\right)$ & 00 & $03 \mathrm{Ke} 04$ & $\mathrm{~T}$ & $\alpha \approx 100 ; \beta^{+} ?$ & \\
\hline${ }^{195} \mathrm{At}^{m}$ & -3443 & 8 & 34 & 7 & $\mathrm{AD}$ & $\&$ & 147 & $\mathrm{~ms}$ & 5 & $9 / 2^{-} \#$ & 00 & $03 \mathrm{Ke} 04$ & $\mathrm{~T}$ & $\alpha=? ; \beta^{+}<25 \#$ & \\
\hline${ }^{195} \mathrm{Rn}$ & 5070 & 50 & & & * & & 6 & $\mathrm{~ms}$ & & $3 / 2^{-} \#$ & & $01 \mathrm{Ke} 06$ & TD & $\alpha=?$ & \\
\hline${ }^{195} \mathrm{Rn}^{m}$ & 5118 & 15 & 50 & 50 & 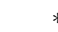 & & 6 & $\mathrm{~ms}$ & & $13 / 2^{+} \#$ & & 01Ke06 & TD & $\alpha=?$ & \\
\hline${ }^{195} \mathrm{Os}$ & I : identifi & cation & of this 1 & lide ha & een qu & e & ioned, $\mathrm{s}$ & see El & F'99 & & & & & & $* *$ \\
\hline${ }^{195} \mathrm{Bi}^{m}$ & $\mathrm{~J}:$ spins of & f groun & id-state an & $\mathrm{d}$ of isom & er deri & ved & from al & lpha & ecay & & & & & & $* *$ \\
\hline
\end{tabular}

\begin{tabular}{|c|c|c|c|c|c|c|c|c|c|c|c|c|c|}
\hline${ }^{196} \mathrm{Os}$ & -28280 & 40 & & & & 34.9 & $\mathrm{~m}$ & 0.2 & $0^{+}$ & 98 & & & $\beta^{-}=100$ \\
\hline${ }^{196} \mathrm{Ir}$ & -29440 & 40 & & & & 52 & $\mathrm{~s}$ & 1 & $\left(0^{-}\right)$ & 98 & & & $\beta^{-}=100$ \\
\hline${ }^{196} \mathrm{Ir}^{m}$ & -29229 & 20 & 210 & 40 & $\mathrm{BD}$ & 1.40 & $\mathrm{~h}$ & 0.02 & $\left(10,11^{-}\right)$ & 98 & & & $\beta^{-} \approx 100 ; \mathrm{IT}<0.3$ \\
\hline${ }^{196} \mathrm{Pt}$ & -32647.4 & 0.9 & & & & STABLE & & & $0^{+}$ & 98 & & & $\mathrm{IS}=25.24241$ \\
\hline${ }^{196} \mathrm{Au}$ & -31140.0 & 3.0 & & & & 6.1669 & $\mathrm{~d}$ & 0.0006 & $2^{-}$ & 98 & 01Li17 & $\mathrm{T}$ & $\beta^{+}=92.88 ; \beta^{-}=7.28$ \\
\hline${ }^{196} \mathrm{Au}^{m}$ & -31055 & 3 & 84.660 & 0.020 & & 8.1 & $\mathrm{~s}$ & 0.2 & $5^{+}$ & 98 & & & IT $=100$ \\
\hline${ }^{196} \mathrm{Au}^{n}$ & -30544 & 3 & 595.66 & 0.04 & & 9.6 & $\mathrm{~h}$ & 0.1 & $12^{-}$ & 98 & & & $\mathrm{IT}=100$ \\
\hline${ }^{196} \mathrm{Hg}$ & -31826.7 & 2.9 & & & & STABLE & & $(>2.5$ Ey) & $0^{+}$ & 98 & $90 \mathrm{Bu} 28$ & $\mathrm{~T}$ & $\mathrm{IS}=0.151 ; 2 \beta^{+} ?$ \\
\hline${ }^{196} \mathrm{Tl}$ & -27497 & 12 & & & & 1.84 & $\mathrm{~h}$ & 0.03 & $2^{-}$ & 98 & & & $\beta^{+}=100$ \\
\hline${ }^{196} \mathrm{Tl}^{m}$ & -27103 & 12 & 394.2 & 0.5 & & 1.41 & $\mathrm{~h}$ & 0.02 & $\left(7^{+}\right)$ & 98 & & & $\beta^{+}=95.5 ; \mathrm{IT}=4.5$ \\
\hline${ }^{196} \mathrm{~Pb}$ & -25361 & 14 & & & & 37 & $\mathrm{~m}$ & 3 & $0^{+}$ & 01 & & & $\beta^{+}=100 ; \alpha \leq 3 \mathrm{e}-5$ \\
\hline${ }^{196} \mathrm{~Pb}^{m}$ & -23623 & 14 & 1738.27 & 0.12 & & $<1$ & $\mu \mathrm{s}$ & & $4^{+}$ & 01 & & & $\mathrm{IT}=100$ \\
\hline${ }^{196} \mathrm{Bi}$ & -18009 & 24 & & & & 5.1 & $\mathrm{~m}$ & 0.2 & $\left(3^{+}\right)$ & 99 & & & $\beta^{+} \approx 100 ; \alpha=0.0011534$ \\
\hline${ }^{196} \mathrm{Bi}^{m}$ & -17842 & 25 & 166.6 & 3.0 & $\mathrm{AD}$ & 0.6 & $\mathrm{~s}$ & 0.5 & $\left(7^{+}\right)$ & 99 & & & $\mathrm{IT}=? ; \beta^{+} ?$ \\
\hline${ }^{196} \mathrm{Bi}^{n}$ & -17739 & 25 & 270 & 3 & $\mathrm{AD}$ & 4.00 & $\mathrm{~m}$ & 0.05 & $\left(10^{-}\right)$ & 99 & & & $\beta^{+}=74.225 ; \mathrm{IT}=25.825 ; \ldots$ \\
\hline${ }^{196} \mathrm{Po}$ & -13474 & 13 & & & & 5.56 & $\mathrm{~s}$ & 0.12 & $0^{+}$ & 98 & $93 \mathrm{Wa} 04$ & TD & $\alpha=945 ; \beta^{+}=65$ \\
\hline${ }^{196} \mathrm{Po}^{m}$ & -10984 & 13 & 2490.5 & 1.7 & & 850 & ns & 90 & $\left(11^{-}\right)$ & 98 & & & $\mathrm{IT}=100$ \\
\hline${ }^{196} \mathrm{At}$ & -3920 & 60 & & & * & 253 & $\mathrm{~ms}$ & 9 & $3^{+} \#$ & 98 & 97Pu01 & $\mathrm{T}$ & $\alpha=? ; \beta^{+}=4 \#$ \\
\hline${ }^{196} \mathrm{At}^{m}$ & -3950 & 50 & -30 & 80 & $\mathrm{AD} *$ & $20 \#$ & $\mathrm{~ms}$ & & $10^{-} \#$ & & $96 \mathrm{En} 01$ & $\mathrm{D}$ & IT ? \\
\hline${ }^{196} \mathrm{At}^{n}$ & -3760 & 60 & 157.9 & 0.1 & & 11 & $\mu \mathrm{s}$ & & $5^{+} \#$ & & 00Sm06 & ET & IT ? \\
\hline${ }^{196} \mathrm{Rn}$ & 1970 & 15 & & & & 4.7 & $\mathrm{~ms}$ & 1.1 & $0^{+}$ & 98 & $01 \mathrm{Ke} 06$ & $\mathrm{~T}$ & $\alpha \approx 100 ; \beta^{+}=0.2 \#$ \\
\hline$*^{196} \mathrm{Bi}^{n}$ & $\mathrm{D}: \ldots ; \alpha$ & $=0.00$ & 3810 & & & & & & & & & & \\
\hline${ }_{*}{ }^{196} \mathrm{Po}$ & $\mathrm{T}$ : averag & $97 \mathrm{P}$ & $01=5.5(0$ & $33 \mathrm{Wa}$ & $=5.8$ & & & & & & & & \\
\hline
\end{tabular}




\begin{tabular}{|c|c|c|c|c|c|c|c|c|c|c|c|c|c|}
\hline Nuclide & \multicolumn{2}{|c|}{$\begin{array}{l}\text { Mass excess } \\
\quad(\mathrm{keV})\end{array}$} & \multicolumn{3}{|c|}{$\begin{array}{l}\text { Excitation } \\
\text { energy }(\mathrm{keV})\end{array}$} & \multicolumn{3}{|c|}{ Half-life } & $J^{\pi}$ & Ens & Reference & \multicolumn{2}{|l|}{$\begin{array}{l}\text { Decay modes and } \\
\text { intensities }(\%)\end{array}$} \\
\hline${ }^{197} \mathrm{Ir}$ & -28268 & 20 & & & & 5.8 & $\mathrm{~m}$ & 0.5 & $3 / 2^{+}$ & 96 & & $\beta^{-}=100$ & \\
\hline${ }^{197} \mathrm{Ir}^{m}$ & -28153 & 21 & 115 & 5 & & 8.9 & $\mathrm{~m}$ & 0.3 & $11 / 2^{-}$ & 96 & & $\beta^{-} \approx 100 ; \mathrm{IT}=0.2510$ & \\
\hline${ }^{197} \mathrm{Pt}$ & -30422.4 & 0.8 & & & & 19.8915 & $\mathrm{~h}$ & 0.0019 & $1 / 2^{-}$ & 96 & & $\beta^{-}=100$ & \\
\hline${ }^{197} \mathrm{Pt}^{m}$ & -30022.8 & 0.8 & 399.59 & 0.20 & & 95.41 & $\mathrm{~m}$ & 0.18 & $13 / 2^{+}$ & 96 & & $\mathrm{IT}=96.74 ; \beta^{-}=3.34$ & \\
\hline${ }^{197} \mathrm{Au}$ & -31141.1 & 0.6 & & & & STABLE & & & $3 / 2^{+}$ & 96 & & $\mathrm{IS}=100$. & \\
\hline${ }^{197} \mathrm{Au}^{m}$ & -30732.0 & 0.6 & 409.15 & 0.08 & & 7.73 & s & 0.06 & $11 / 2^{-}$ & 96 & & $\mathrm{IT}=100$ & \\
\hline${ }^{197} \mathrm{Hg}$ & -30541 & 3 & & & & 64.94 & $\mathrm{~h}$ & 0.07 & $1 / 2^{-}$ & 96 & 01Li17 T & $\varepsilon=100$ & * \\
\hline${ }^{197} \mathrm{Hg}^{m}$ & -30242 & 3 & 298.93 & 0.08 & & 23.8 & $\mathrm{~h}$ & 0.1 & $13 / 2^{+}$ & 96 & & $\mathrm{IT}=91.47 ; \varepsilon=8.67$ & \\
\hline${ }^{197} \mathrm{Tl}$ & -28341 & 16 & & & & 2.84 & $\mathrm{~h}$ & 0.04 & $1 / 2^{+}$ & 96 & & $\beta^{+}=100$ & \\
\hline${ }^{197} \mathrm{Tl}^{m}$ & -27733 & 16 & 608.22 & 0.08 & & 540 & $\mathrm{~ms}$ & 10 & $9 / 2^{-}$ & 96 & & $\mathrm{IT}=100$ & \\
\hline${ }^{197} \mathrm{~Pb}$ & -24749 & 6 & & & & 8 & $\mathrm{~m}$ & 2 & $3 / 2^{-}$ & 01 & & $\beta^{+}=100$ & \\
\hline${ }^{197} \mathrm{~Pb}^{m}$ & -24429 & 6 & 319.31 & 0.11 & & 43 & $\mathrm{~m}$ & 1 & $13 / 2^{+}$ & 01 & & $\beta^{+}=812 ; \mathrm{IT}=192 ; \ldots$ & * \\
\hline${ }^{197} \mathrm{~Pb}^{n}$ & -22835 & 6 & 1914.10 & 0.25 & & 1.15 & $\mu \mathrm{s}$ & 0.20 & $21 / 2^{-}$ & 01 & & $\mathrm{IT}=100$ & \\
\hline${ }^{197} \mathrm{Bi}$ & -19688 & 8 & & & & 9.3 & $\mathrm{~m}$ & 0.5 & $\left(9 / 2^{-}\right)$ & 99 & & $\beta^{+}=100 ; \alpha=1 \mathrm{e}-4 \#$ & \\
\hline${ }^{197} \mathrm{Bi}^{m}$ & -19000 & 110 & 690 & 110 & $\mathrm{AD}$ & 5.04 & $\mathrm{~m}$ & 0.16 & $\left(1 / 2^{+}\right)$ & 99 & & $\alpha=5540 ; \beta^{+}=4540 ;$. & * \\
\hline${ }^{197} \mathrm{Po}$ & -13360 & 50 & & & & 53.6 & s & 1.0 & $\left(3 / 2^{-}\right)$ & 96 & & $\beta^{+} ? ; \alpha=447$ & \\
\hline${ }^{197} \mathrm{Po}^{m}$ & $-13120 \#$ & $90 \#$ & $230 \#$ & $80 \#$ & & 25.8 & s & 0.1 & $\left(13 / 2^{+}\right)$ & 96 & & $\alpha=849 ; \beta^{+} ? ; \mathrm{IT}=0.01 \#$ & \\
\hline${ }^{197} \mathrm{At}$ & -6340 & 50 & & & $*$ & 350 & $\mathrm{~ms}$ & 40 & $\left(9 / 2^{-}\right)$ & 96 & & $\alpha=964 ; \beta^{+}=44$ & \\
\hline${ }^{197} \mathrm{At}^{m}$ & -6293 & 13 & 50 & 50 & $\mathrm{AD} *$ & 3.7 & $\mathrm{~s}$ & 2.5 & $\left(1 / 2^{+}\right)$ & 96 & & $\alpha \approx 100 ; \beta^{+} ? ;$ IT $<0.004$ & \\
\hline${ }^{197} \mathrm{Rn}$ & 1480 & 60 & & & & 66 & $\mathrm{~ms}$ & 16 & $3 / 2^{-} \#$ & 98 & 96En02 T & $\alpha \approx 100 ; \beta^{+}$? & $*$ \\
\hline${ }^{197} \mathrm{Rn}^{m}$ & $1670 \#$ & $50 \#$ & $200 \#$ & $60 \#$ & & 21 & $\mathrm{~ms}$ & 5 & $\left(13 / 2^{+}\right)$ & 98 & $96 \mathrm{En} 02 \mathrm{~T}$ & $\alpha \approx 100 ; \beta^{+} ?$ & $*$ \\
\hline$*^{197} \mathrm{Hg}$ & $\mathrm{T}:$ other 6 & $6 \mathrm{E} 109=$ & $=64.14(0.0$ & ) at strong & variance & e: Birge & ratio & would be & $B=9.3$ & & & & ** \\
\hline${ }^{1}{ }^{197} \mathrm{~Pb}^{m}$ & $\mathrm{D}: \ldots ; \alpha$ & $<3 e-4$ & & & & & & & & & & & ** \\
\hline$*^{197} \mathrm{Bi}^{m}$ & $\mathrm{D}: \ldots ; \mathrm{IT}$ & $<0.3$ & & & & & & & & & & & ** \\
\hline$*^{197} \mathrm{Rn}$ & $\mathrm{T}:$ averag & e $96 \mathrm{En}$ & $02=65(+2$ & $-14) 95 \mathrm{Mc}$ & $14=51(+$ & $+35-15)$ & & & & & & & $* *$ \\
\hline${ }^{* 197} \mathrm{Rn}^{m}$ & $\mathrm{~T}$ : averag & e $96 \mathrm{En}$ & $02=19(+8$ & $95 \mathrm{Mol}$ & $=18(+9$ & $-5)$ & $\mathrm{J}:$ & from $\alpha \mathrm{d}$ & ecay to 19 & ${ }^{3} \mathrm{Po}^{m}$ & & & ** \\
\hline
\end{tabular}

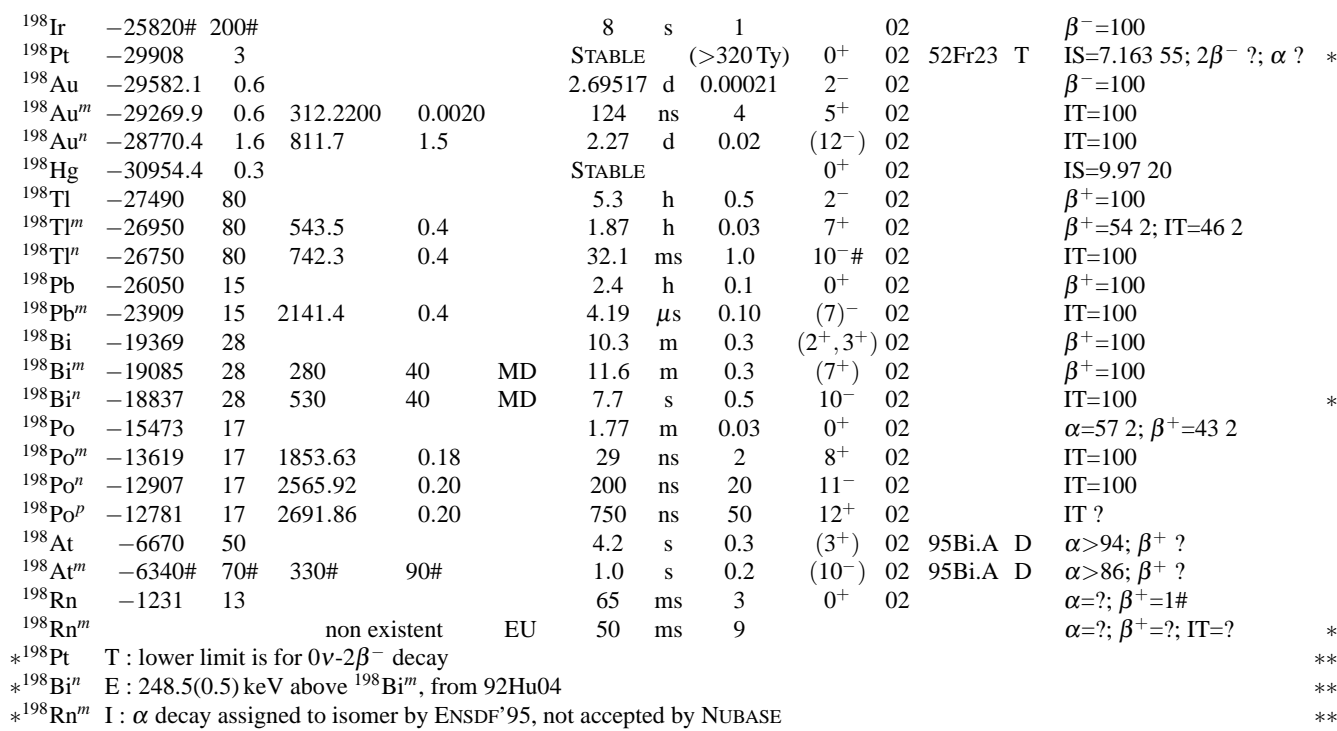

\begin{tabular}{|c|c|c|c|c|c|c|c|c|c|c|}
\hline${ }^{199} \mathrm{Ir}$ & -24400 & 40 & & & $20 \#$ & $\mathrm{~s}$ & & $3 / 2^{+} \# 01$ & & $\beta^{-} ?$ \\
\hline${ }^{199} \mathrm{Pt}$ & -27392 & 3 & & & 30.80 & $\mathrm{~m}$ & 0.21 & $5 / 2^{-} \quad 94$ & & $\beta^{-}=100$ \\
\hline${ }^{199} \mathrm{Pt}^{m}$ & -26968 & 4 & 424 & 2 & 13.6 & $\mathrm{~s}$ & 0.4 & $(13 / 2)^{+} 94$ & & $\mathrm{IT}=100$ \\
\hline${ }^{199} \mathrm{Au}$ & -29095.0 & 0.6 & & & 3.139 & $\mathrm{~d}$ & 0.007 & $3 / 2^{+} \quad 94$ & & $\beta^{-}=100$ \\
\hline${ }^{199} \mathrm{Au}^{m}$ & -28546.1 & 0.6 & 548.9368 & 0.0021 & 440 & $\mu \mathrm{s}$ & 30 & $(11 / 2)^{-} 94$ & & $\mathrm{IT}=100$ \\
\hline${ }^{199} \mathrm{Hg}$ & -29547.1 & 0.4 & & & Stable & & & $1 / 2^{-} \quad 94$ & & $\mathrm{IS}=16.8722$ \\
\hline${ }^{199} \mathrm{Hg}^{m}$ & -29014.6 & 0.4 & 532.48 & 0.10 & 42.66 & $\mathrm{~m}$ & 0.08 & $13 / 2^{+} 94$ & 01Li17 T & $\mathrm{IT}=100$ \\
\hline
\end{tabular}




\begin{tabular}{|c|c|c|c|c|c|c|c|c|c|c|c|c|c|c|}
\hline Nuclide & \multicolumn{2}{|c|}{$\begin{array}{l}\text { Mass excess } \\
\quad(\mathrm{keV})\end{array}$} & \multicolumn{3}{|c|}{$\begin{array}{c}\text { Excitation } \\
\text { energy }(\mathrm{keV})\end{array}$} & \multicolumn{3}{|c|}{ Half-life } & $J^{\pi}$ & Ens & \multicolumn{2}{|c|}{ Reference } & \multicolumn{2}{|l|}{$\begin{array}{l}\text { Decay modes and } \\
\text { intensities }(\%)\end{array}$} \\
\hline \multicolumn{15}{|c|}{ A-group continued ... } \\
\hline${ }^{199} \mathrm{Tl}$ & -28059 & 28 & & & & 7.42 & $\mathrm{~h}$ & 0.08 & $1 / 2^{+}$ & 94 & & & $\beta^{+}=100$ & \\
\hline${ }^{199} \mathrm{Tl}^{m}$ & -27309 & 28 & 749.7 & 0.3 & & 28.4 & $\mathrm{~ms}$ & 0.2 & $9 / 2^{-}$ & 94 & & & $\mathrm{IT}=100$ & \\
\hline${ }^{199} \mathrm{~Pb}$ & -25228 & 26 & & & & 90 & $\mathrm{~m}$ & 10 & $3 / 2^{-}$ & 01 & & & $\beta^{+}=100$ & \\
\hline${ }^{199} \mathrm{~Pb}^{m}$ & -24799 & 26 & 429.5 & 2.7 & & 12.2 & $\mathrm{~m}$ & 0.3 & $\left(13 / 2^{+}\right)$ & 01 & ABBW & $\mathrm{E}$ & $\mathrm{IT}=93 ; \beta^{+}=7$ & $*$ \\
\hline${ }^{199} \mathrm{~Pb}^{n}$ & -22664 & 26 & 2563.8 & 2.7 & & 10.1 & $\mu \mathrm{s}$ & 0.2 & $\left(29 / 2^{-}\right)$ & 01 & ABBW & $\mathrm{E}$ & $\mathrm{IT}=100$ & $*$ \\
\hline${ }^{199} \mathrm{Bi}$ & -20798 & 12 & & & & 27 & $\mathrm{~m}$ & 1 & $9 / 2^{-}$ & 94 & & & $\beta^{+}=100$ & \\
\hline${ }^{199} \mathrm{Bi}^{m}$ & -20131 & 12 & 667 & 4 & & 24.70 & $\mathrm{~m}$ & 0.15 & $\left(1 / 2^{+}\right)$ & 94 & & & $\beta^{+}=? ;$ IT $<2 ; \alpha \approx 0.01$ & \\
\hline${ }^{199} \mathrm{Po}$ & -15215 & 23 & & & & 5.48 & $\mathrm{~m}$ & 0.16 & $\left(3 / 2^{-}\right)$ & 94 & & & $\beta^{+}=92.53 ; \alpha=7.53$ & \\
\hline${ }^{199} \mathrm{Po}^{m}$ & -14903 & 23 & 312.0 & 2.8 & $\mathrm{AD}$ & 4.17 & $\mathrm{~m}$ & 0.04 & $13 / 2^{+}$ & 94 & & & $\beta^{+}=73.510 ; \alpha=241 ; \mathrm{IT}=2.5$ & \\
\hline${ }^{199} \mathrm{At}$ & -8820 & 50 & & & & 7.2 & $\mathrm{~s}$ & 0.5 & $\left(9 / 2^{-}\right)$ & 94 & & & $\alpha=896 ; \beta^{+} ?$ & \\
\hline${ }^{199} \mathrm{Rn}$ & -1520 & 60 & & & & 620 & $\mathrm{~ms}$ & 30 & $3 / 2^{-} \#$ & 98 & & & $\alpha=? ; \beta^{+}=6 \#$ & \\
\hline${ }^{199} \mathrm{Rn}^{m}$ & -1334 & 29 & 180 & 70 & $\mathrm{AD}$ & 320 & $\mathrm{~ms}$ & 20 & $13 / 2^{+} \#$ & 98 & & & $\alpha=? ; \beta^{+}=3 \#$ & \\
\hline${ }^{199} \mathrm{Fr}$ & 6760 & 40 & & & & 16 & $\mathrm{~ms}$ & 7 & $1 / 2^{+} \#$ & 01 & 99Ta20 & $\mathrm{T}$ & $\alpha \approx 100 ; \beta^{+} ?$ & \\
\hline$*^{199} \mathrm{Hg}^{m}$ & \multicolumn{10}{|c|}{$\mathrm{T}:$ average $01 \mathrm{Li} 17=42.67(0.09) 69 \mathrm{~K} 106=42.6(0.2)$} & & & & $* *$ \\
\hline$*{ }^{199} \mathrm{~Pb}^{m}$ & \multicolumn{10}{|c|}{ E : $424.8 \gamma$ to level lower than $9.3 \mathrm{keV}$, from ENSDF } & & & & $* *$ \\
\hline$*^{199} \mathrm{~Pb}^{n}$ & \multicolumn{10}{|c|}{ E : 2559.1 to level lower than $9.3 \mathrm{keV}$, from ENSDF } & & & & $* *$ \\
\hline
\end{tabular}

\begin{tabular}{|c|c|c|c|c|c|c|c|c|c|c|c|c|c|}
\hline${ }^{200} \mathrm{Pt}$ & -26603 & 20 & & & & 12.5 & $\mathrm{~h}$ & 0.3 & $0^{+}$ & 95 & & & $\beta^{-}=100$ \\
\hline${ }^{200} \mathrm{Au}$ & -27270 & 50 & & & & 48.4 & $\mathrm{~m}$ & 0.3 & $1^{(-)}$ & 95 & & & $\beta^{-}=100$ \\
\hline${ }^{200} \mathrm{Au}^{m}$ & -26300 & 50 & 970 & 70 & $\mathrm{BD}$ & 18.7 & $\mathrm{~h}$ & 0.5 & $12^{-}$ & 95 & & & $\beta^{-}=822 ; \mathrm{IT}=182$ \\
\hline${ }^{200} \mathrm{Hg}$ & -29504.1 & 0.4 & & & & STABLE & & & $0^{+}$ & 95 & & & $\mathrm{IS}=23.1019$ \\
\hline${ }^{200} \mathrm{Tl}$ & -27048 & 6 & & & & 26.1 & $\mathrm{~h}$ & 0.1 & $2^{-}$ & 95 & & & $\beta^{+}=100$ \\
\hline${ }^{200} \mathrm{Tl}^{m}$ & -26294 & 6 & 753.6 & 0.2 & & 34.3 & $\mathrm{~ms}$ & 1.0 & $7^{+}$ & 95 & & & $\mathrm{IT}=100$ \\
\hline${ }^{200} \mathrm{~Pb}$ & -26243 & 11 & & & & 21.5 & $\mathrm{~h}$ & 0.4 & $0^{+}$ & 95 & & & $\varepsilon=100$ \\
\hline${ }^{200} \mathrm{Bi}$ & -20370 & 24 & & & * & 36.4 & $\mathrm{~m}$ & 0.5 & $7^{+}$ & 95 & & & $\beta^{+}=100$ \\
\hline${ }^{200} \mathrm{Bi}^{m}$ & $-20270 \#$ & 70\# & $100 \#$ & 70\# & * & 31 & $\mathrm{~m}$ & 2 & $\left(2^{+}\right)$ & 95 & & & $\beta^{+}>90 ; \mathrm{IT}<10$ \\
\hline${ }^{200} \mathrm{Bi}^{n}$ & -19942 & 24 & 428.20 & 0.10 & & 400 & $\mathrm{~ms}$ & 50 & $\left(10^{-}\right)$ & 95 & & & $\mathrm{IT}=100$ \\
\hline${ }^{200} \mathrm{Po}$ & -16954 & 14 & & & & 11.5 & $\mathrm{~m}$ & 0.1 & $0^{+}$ & 95 & & & $\beta^{+}=88.93 ; \alpha=11.13$ \\
\hline${ }^{200} \mathrm{At}$ & -8988 & 24 & & & & 43.2 & $\mathrm{~s}$ & 0.9 & $\left(3^{+}\right)$ & 95 & 96Ta18 & $\mathrm{T}$ & $\alpha=576 ; \beta^{+}=436$ \\
\hline${ }^{200} \mathrm{At}^{m}$ & -8875 & 25 & 112.7 & 3.0 & $\mathrm{AD}$ & 47 & $\mathrm{~s}$ & 1 & $\left(7^{+}\right)$ & 95 & & & $\alpha=437 ; \beta^{+}=$?; IT ? \\
\hline${ }^{200} \mathrm{At}^{n}$ & -8644 & 24 & 344 & 3 & $\mathrm{AD}$ & 3.5 & s & 0.2 & $\left(10^{-}\right)$ & 95 & & & $\mathrm{IT} \approx 84 ; \alpha \approx 10.5 ; \beta^{+} \approx 4.5$ \\
\hline${ }^{200} \mathrm{Rn}$ & -4006 & 13 & & & & 1.03 & $\mathrm{~s}$ & 0.05 & $0^{+}$ & 98 & 96Ta18 & $\mathrm{T}$ & $\alpha=? ; \beta^{+}=2 \#$ \\
\hline${ }^{200} \mathrm{Fr}$ & 6120 & 80 & & & * & 24 & $\mathrm{~ms}$ & 10 & $3^{+} \#$ & 97 & 96En01 & TD & $\alpha=100$ \\
\hline${ }^{200} \mathrm{Fr}^{m}$ & 6180 & 70 & 60 & 110 & $\mathrm{AD} *$ & 650 & $\mathrm{~ms}$ & 210 & $10^{-} \#$ & 97 & $95 \mathrm{Mo} 14$ & $\mathrm{TD}$ & $\alpha \approx 100 ;$ IT ? \\
\hline${ }^{200} \mathrm{At}$ & $\mathrm{T}$ : average & e 96T & $8=44(2)$ & $92 \mathrm{Hu} 04$ & $=43(1)$ & & & & & & & & \\
\hline${ }^{200} \mathrm{At}^{n}$ & $\mathrm{E}: 230.9(\mathrm{C}$ & $0.2) \mathrm{k}$ & above $^{21}$ & ${ }^{00} \mathrm{At}^{m}$ & om EN & & & & & & & & \\
\hline${ }^{200} \mathrm{Rn}$ & $\mathrm{T}$ : average & e 96Ta & $8=0.96(0$ & $.03) 840$ & $\mathrm{Ca} 32=1$ & $06(0.02)$ & & & & & & & \\
\hline
\end{tabular}

\begin{tabular}{|c|c|c|c|c|c|c|c|c|c|c|c|c|}
\hline${ }^{201} \mathrm{Pt}$ & -23740 & 50 & & & 2.5 & $\mathrm{~m}$ & 0.1 & $\left(5 / 2^{-}\right)$ & 94 & & & $\beta^{-}=100$ \\
\hline${ }^{201} \mathrm{Au}$ & -26401 & 3 & & & 26 & $\mathrm{~m}$ & 1 & $3 / 2^{+}$ & 94 & & & $\beta^{-}=100$ \\
\hline${ }^{201} \mathrm{Hg}$ & -27663.3 & 0.6 & & & STABLE & & & $3 / 2^{-}$ & 94 & & & $\mathrm{IS}=13.189$ \\
\hline${ }^{201} \mathrm{Hg}^{m}$ & -26897.1 & 0.6 & 766.23 & 0.15 & 94 & $\mu \mathrm{s}$ & & $13 / 2^{+}$ & & & & \\
\hline${ }^{201} \mathrm{Tl}$ & -27182 & 15 & & & 72.912 & h & 0.017 & $1 / 2^{+}$ & 94 & & & $\varepsilon=100$ \\
\hline${ }^{201} \mathrm{Tl}^{m}$ & -26263 & 15 & 919.50 & 0.09 & 2.035 & $\mathrm{~ms}$ & 0.007 & $\left(9 / 2^{-}\right)$ & 94 & & & $\mathrm{IT}=100$ \\
\hline${ }^{201} \mathrm{~Pb}$ & -25258 & 22 & & & 9.33 & $\mathrm{~h}$ & 0.03 & $5 / 2^{-}$ & 94 & & & $\beta^{+}=100$ \\
\hline${ }^{201} \mathrm{~Pb}^{m}$ & -24629 & 22 & 629.14 & 0.17 & 61 & $\mathrm{~s}$ & 2 & $13 / 2^{+}$ & 94 & & & IT $>99 ; \beta^{+}<1$ \\
\hline${ }^{201} \mathrm{Bi}$ & -21416 & 15 & & & 108 & $\mathrm{~m}$ & 3 & $9 / 2^{-}$ & 94 & & & $\beta^{+}=100 ; \alpha<1 \mathrm{e}-4$ \\
\hline${ }^{201} \mathrm{Bi}^{m}$ & -20570 & 15 & 846.34 & 0.21 & 59.1 & $\mathrm{~m}$ & 0.6 & $1 / 2^{+}$ & 94 & & & $\beta^{+}=92.9 \# ;$ IT $<6.8 ; \alpha=$ ? \\
\hline${ }^{201} \mathrm{Po}$ & -16525 & 6 & & & 15.3 & $\mathrm{~m}$ & 0.2 & $3 / 2^{-}$ & 94 & & & $\beta^{+}=98.43 ; \alpha=1.63$ \\
\hline${ }^{201} \mathrm{Po}^{m}$ & -16101 & 6 & 424.1 & $2.4 \quad \mathrm{AD}$ & 8.9 & $\mathrm{~m}$ & 0.2 & $13 / 2^{+}$ & 94 & & & $\mathrm{IT}=5614 ; \beta^{+}=4110 ; \alpha \approx 2.9$ \\
\hline${ }^{201} \mathrm{At}$ & -10789 & 8 & & & 85 & $\mathrm{~s}$ & 3 & $\left(9 / 2^{-}\right)$ & 94 & 96Ta18 & $\mathrm{T}$ & $\alpha=717 ; \beta^{+}=297$ \\
\hline${ }^{201} \mathrm{Rn}$ & -4070 & 70 & & & 7.0 & $\mathrm{~s}$ & 0.4 & $\left(3 / 2^{-}\right)$ & 94 & 96Ta18 & $\mathrm{T}$ & $\alpha=? ; \beta^{+}=20 \#$ \\
\hline${ }^{201} \mathrm{Rn}^{m}$ & $-3790 \#$ & $90 \#$ & $280 \#$ & $90 \#$ & 3.8 & $\mathrm{~s}$ & 0.1 & $\left(13 / 2^{+}\right)$ & 94 & 96Ta18 & $\mathrm{T}$ & $\alpha=? ; \beta^{+}=10 \# ; \mathrm{IT}=0.01 \#$ \\
\hline${ }^{201} \mathrm{Fr}$ & 3600 & 70 & & & 61 & $\mathrm{~ms}$ & 12 & $\left(9 / 2^{-}\right)$ & 94 & 96En01 & $\mathrm{T}$ & $\alpha \approx 100 ; \beta^{+}<1$ \\
\hline$*^{201} \mathrm{Bi}^{m}$ & $\mathrm{D}: \alpha$ deca & ay is o & served. I & branching ra & is estime & ated $\mathrm{c}$ & $0.3 \% \#$ & in ENSDI & & & & \\
\hline$*^{201} \mathrm{At}$ & $\mathrm{T}$ : average & e $96 \mathrm{~T}$ & $18=83(2)$ & and two result & ENSDF= & $=89(3$ & & & & & & \\
\hline$*^{201} \mathrm{Rn}$ & $\mathrm{T}$ : average & e $96 \mathrm{Ta}$ & $18=7.1(0$. & 3) $71 \mathrm{Ho} 01=7$ & & & & & & & & \\
\hline$*^{201} \mathrm{Fr}$ & $\mathrm{T}$ : average & e $96 \mathrm{Er}$ & $1=69(+1$ & 6-11) $80 \mathrm{Ew} 0$ & $48(15)$ & & & & & & & \\
\hline
\end{tabular}




\begin{tabular}{|c|c|c|c|c|c|c|c|c|c|c|c|c|c|}
\hline Nuclide & \multicolumn{2}{|c|}{$\begin{array}{l}\text { Mass excess } \\
\quad(\mathrm{keV})\end{array}$} & \multicolumn{3}{|c|}{$\begin{array}{l}\text { Excitation } \\
\text { energy }(\mathrm{keV})\end{array}$} & \multicolumn{3}{|c|}{ Half-life } & \multirow[t]{2}{*}{$J^{\pi}$} & \multirow{2}{*}{$\begin{array}{c}\text { Ens } \\
97\end{array}$} & Reference & \multicolumn{2}{|l|}{$\begin{array}{c}\text { Decay modes and } \\
\text { intensities }(\%)\end{array}$} \\
\hline${ }^{202} \mathrm{Pt}$ & $-22600 \#$ & $300 \#$ & & & & 44 & $\mathrm{~h}$ & 15 & & & & $\beta^{-}=100$ & \\
\hline${ }^{202} \mathrm{Au}$ & -24400 & 170 & & & & 28.8 & s & 1.9 & $\left(1^{-}\right)$ & 97 & & $\beta^{-}=100$ & \\
\hline${ }^{202} \mathrm{Hg}$ & -27345.9 & 0.6 & & & & STABLE & & & $0^{+}$ & 97 & & IS $=29.8626$ & * \\
\hline${ }^{202} \mathrm{Tl}$ & -25983 & 15 & & & & 12.23 & d & 0.02 & $2^{-}$ & 97 & & $\beta^{+}=100$ & \\
\hline${ }^{202} \mathrm{Tl}^{m}$ & -25033 & 15 & 950.19 & 0.10 & & 572 & $\mu \mathrm{s}$ & 7 & $7^{+}$ & 97 & & & \\
\hline${ }^{202} \mathrm{~Pb}$ & -25934 & 8 & & & & 52.5 & ky & 2.8 & $0^{+}$ & 97 & & $\varepsilon \approx 100 ; \alpha<1 \#$ & \\
\hline${ }^{202} \mathrm{~Pb}^{m}$ & -23764 & 8 & 2169.83 & 0.07 & & 3.53 & $\mathrm{~h}$ & 0.01 & $9^{-}$ & 97 & & $\mathrm{IT}=90.55 ; \beta^{+}=9.55$ & \\
\hline${ }^{202} \mathrm{Bi}$ & -20733 & 20 & & & & 1.72 & $\mathrm{~h}$ & 0.05 & $5^{(+\#)}$ & 97 & & $\beta^{+}=100 ; \alpha<1 \mathrm{e}-5$ & $*$ \\
\hline${ }^{202} \mathrm{Bi}^{m}$ & -20118 & 21 & 615 & 7 & & 3.04 & $\mu \mathrm{s}$ & 0.06 & $(10 \#)^{-}$ & 97 & & & \\
\hline${ }^{202} \mathrm{Po}$ & -17924 & 15 & & & & 44.7 & $\mathrm{~m}$ & 0.5 & $0^{+}$ & 97 & & $\beta^{+}=? ; \alpha=1.927$ & \\
\hline${ }^{202} \mathrm{Po}^{m}$ & -15297 & 15 & 2626.7 & 0.7 & & $>200$ & ns & & $11^{-}$ & 97 & & $\mathrm{IT}=100$ & \\
\hline${ }^{202} \mathrm{At}$ & -10591 & 28 & & & & 184 & $\mathrm{~s}$ & 1 & $(2,3)^{+}$ & 97 & & $\beta^{+}=? ; \alpha=183$ & \\
\hline${ }^{202} \mathrm{At}^{m}$ & -10401 & 28 & 190 & 40 & MD & 182 & $\mathrm{~s}$ & 2 & $\left(7^{+}\right)$ & 97 & & IT ?; $\beta^{+}$?; $\alpha=8.715$ & \\
\hline${ }^{202} \mathrm{At}^{n}$ & -10010 & 28 & 580 & 40 & MD & 460 & $\mathrm{~ms}$ & 50 & $\left(10^{-}\right)$ & 97 & 92Hu04 E & $\mathrm{IT} \approx 100 ; \beta^{+}=0.25 \# ; \ldots$ & $*$ \\
\hline${ }^{202} \mathrm{Rn}$ & -6275 & 18 & & & & 9.94 & $\mathrm{~s}$ & 0.18 & $0^{+}$ & 97 & $96 \mathrm{Ta} 18 \mathrm{~T}$ & $\alpha=? ; \beta^{+}=14 \#$ & $*$ \\
\hline${ }^{202} \mathrm{Fr}$ & 3140 & 50 & & & & 290 & $\mathrm{~ms}$ & 30 & $\left(3^{+}\right)$ & 97 & $96 \mathrm{En01} \mathrm{T}$ & $\alpha=? ; \beta^{+}=3 \#$ & $*$ \\
\hline${ }^{202} \mathrm{Fr}^{m}$ & 3470\# & $70 \#$ & $330 \#$ & $90 \#$ & & 340 & $\mathrm{~ms}$ & 40 & $\left(10^{-}\right)$ & 97 & & $\alpha=? ; \beta^{+}=3 \#$ & \\
\hline${ }^{202} \mathrm{Ra}$ & 9210 & 60 & & & & 2.6 & $\mathrm{~ms}$ & 2.1 & $0^{+}$ & 98 & 96Le09 TD & $\alpha=100$ & \\
\hline$*^{202} \mathrm{Hg}$ & \multicolumn{12}{|c|}{ D : lower half-life limit for ${ }^{24} \mathrm{Ne}$ decay $T>3.7 \mathrm{Zy}$, from $90 \mathrm{Bu} 28$} & $* *$ \\
\hline$*^{202} \mathrm{Bi}$ & \multicolumn{12}{|c|}{$\mathrm{J}$ : re-evaluation to a possible $6^{+}$is discussed in $96 \mathrm{Ca} 02$} & $* *$ \\
\hline$*^{202} \mathrm{At}^{n}$ & \multicolumn{12}{|c|}{$\mathrm{D}: \ldots ; \alpha=0.09611$} & $* *$ \\
\hline$*^{202} \mathrm{At}^{n}$ & \multicolumn{12}{|c|}{$\mathrm{E}: 391.7(0.5) \mathrm{keV}$ above ${ }^{202} \mathrm{At}^{m}$} & ** \\
\hline$*^{202} \mathrm{Rn}$ & \multicolumn{12}{|c|}{$\mathrm{T}:$ average $96 \mathrm{Ta} 18=10.3(0.4) 71 \mathrm{Ho} 01=9.85(0.20)$} & $* *$ \\
\hline$*^{202} \mathrm{Fr}$ & \multicolumn{12}{|c|}{$\mathrm{T}:$ average $96 \mathrm{En} 01=230(+80-40) 95 \mathrm{Bi} . \mathrm{A}=300(40)$} & $* *$ \\
\hline
\end{tabular}

\begin{tabular}{|c|c|c|c|c|c|c|c|c|c|c|c|c|c|}
\hline${ }^{203} \mathrm{Au}$ & -23143 & 3 & & & & 53 & $\mathrm{~s}$ & 2 & $3 / 2^{+}$ & 93 & & & $\beta^{-}=100$ \\
\hline${ }^{203} \mathrm{Hg}$ & -25269.1 & 1.7 & & & & 46.612 & d & 0.018 & $5 / 2^{-}$ & 93 & & & $\beta^{-}=100$ \\
\hline${ }^{203} \mathrm{Hg}^{m}$ & -24336.0 & 2.0 & 933.1 & 1.0 & & 24 & $\mu \mathrm{s}$ & & $\left(13 / 2^{+}\right)$ & & & & \\
\hline${ }^{203} \mathrm{Tl}$ & -25761.2 & 1.3 & & & & STABLE & & & $1 / 2^{+}$ & 93 & & & $\mathrm{IS}=29.52414$ \\
\hline${ }^{203} \mathrm{Tl}^{m}$ & -22360 & 300 & 3400 & 300 & & 7.7 & $\mu \mathrm{s}$ & 0.5 & $\left(25 / 2^{+}\right)$ & & 98Pf02 & $\mathrm{TJ}$ & $\mathrm{IT}=100$ \\
\hline${ }^{203} \mathrm{~Pb}$ & -24787 & 7 & & & & 51.873 & $\mathrm{~h}$ & 0.009 & $5 / 2^{-}$ & 93 & & & $\varepsilon=100$ \\
\hline${ }^{203} \mathrm{~Pb}^{m}$ & -23962 & 7 & 825.20 & 0.09 & & 6.3 & $\mathrm{~s}$ & 0.2 & $13 / 2^{+}$ & 93 & & & $\mathrm{IT}=100$ \\
\hline${ }^{203} \mathrm{~Pb}^{n}$ & -21838 & 7 & 2949.47 & 0.22 & & 480 & $\mathrm{~ms}$ & 20 & $29 / 2^{-}$ & 93 & & & $\mathrm{IT}=100$ \\
\hline${ }^{203} \mathrm{Bi}$ & -21540 & 22 & & & & 11.76 & $\mathrm{~h}$ & 0.05 & $9 / 2^{-}$ & 93 & & & $\beta^{+}=100 ; \alpha \approx 1 \mathrm{e}-5$ \\
\hline${ }^{203} \mathrm{Bi}^{m}$ & -20442 & 22 & 1098.14 & 0.07 & & 303 & $\mathrm{~ms}$ & 5 & $1 / 2^{+}$ & 93 & & & $\mathrm{IT}=100$ \\
\hline${ }^{203} \mathrm{Po}$ & -17307 & 26 & & & & 36.7 & $\mathrm{~m}$ & 0.5 & $5 / 2^{-}$ & 93 & & & $\beta^{+} \approx 100 ; \alpha=0.112$ \\
\hline${ }^{203} \mathrm{Po}^{m}$ & -16666 & 26 & 641.49 & 0.17 & & 45 & $\mathrm{~s}$ & 2 & $13 / 2^{+}$ & 93 & & & $\mathrm{IT} \approx 100 ; \alpha=0.04 \#$ \\
\hline${ }^{203} \mathrm{At}$ & -12163 & 12 & & & & 7.4 & $\mathrm{~m}$ & 0.2 & $9 / 2^{-}$ & 93 & & & $\beta^{+}=693 ; \alpha=313$ \\
\hline${ }^{203} \mathrm{Rn}$ & -6160 & 24 & & & & 43.5 & $\mathrm{~s}$ & 2.1 & $(3 / 2,5 / 2)^{-}$ & 93 & 96Ta18 & $\mathrm{T}$ & $\alpha=669 ; \beta^{+}=349$ \\
\hline${ }^{203} \mathrm{Rn}^{m}$ & -5798 & 24 & 363 & 4 & $\mathrm{AD}$ & 26.7 & $\mathrm{~s}$ & 0.5 & $13 / 2^{(+)}$ & 93 & $87 \mathrm{Bo} 29$ & $\mathrm{~J}$ & $\alpha=? ; \beta^{+}=20 \#$ \\
\hline${ }^{203} \mathrm{Fr}$ & 861 & 16 & & & & 550 & $\mathrm{~ms}$ & 20 & $9 / 2^{-} \#$ & 98 & & & $\alpha=? ; \beta^{+}=5 \#$ \\
\hline${ }^{203} \mathrm{Ra}$ & 8640 & 80 & & & & 4 & $\mathrm{~ms}$ & 3 & $\left(3 / 2^{-}\right)$ & 98 & 96Le09 & TJD & $\alpha \approx 100 ; \beta^{+} ?$ \\
\hline${ }^{203} \mathrm{Ra}^{m}$ & 8860 & 40 & 220 & 90 & $\mathrm{AD}$ & 41 & $\mathrm{~ms}$ & 17 & $\left(13 / 2^{+}\right)$ & 98 & 96Le09 & TJD & $\alpha \approx 100 ; \beta^{+} ?$ \\
\hline$*^{203} \mathrm{Rn}$ & \multicolumn{13}{|c|}{$\mathrm{T}:$ average $96 \mathrm{Ta} 18=42(3) 71 \mathrm{Ho} 01=45(3)$} \\
\hline$*^{203} \mathrm{Rn}^{m}$ & \multicolumn{13}{|c|}{$\mathrm{T}$ : from $96 \mathrm{Ta} 18$} \\
\hline${ }^{204} \mathrm{Au}$ & $-20750 \#$ & $200 \#$ & & & & 39.8 & $\mathrm{~s}$ & 0.9 & $\left(2^{-}\right)$ & 94 & & & $\beta^{-}=100$ \\
\hline${ }^{204} \mathrm{Hg}$ & -24690.2 & 0.3 & & & & STABLE & & & $0^{+}$ & 94 & & & $\mathrm{IS}=6.8715 ; 2 \beta^{-} ?$ \\
\hline${ }^{204} \mathrm{Tl}$ & -24346.0 & 1.3 & & & & 3.78 & $\mathrm{y}$ & 0.02 & $2^{-}$ & 94 & & & $\beta^{-}=97.1012 ; \varepsilon=2.9012$ \\
\hline${ }^{204} \mathrm{Tl}^{m}$ & -23242.0 & 1.4 & 1104.0 & 0.4 & & 63 & $\mu \mathrm{s}$ & 2 & $(7)^{+}$ & 94 & & & $\mathrm{IT}=100$ \\
\hline${ }^{204} \mathrm{Tl}^{n}$ & -21850 & 500 & 2500 & 500 & & 2.6 & $\mu \mathrm{s}$ & 0.2 & $\left(12^{-}\right)$ & & 98Pf02 & $\mathrm{TJ}$ & $\mathrm{IT}=100$ \\
\hline${ }^{204} \mathrm{Tl}^{p}$ & -20850 & 500 & 3500 & 500 & & 1.6 & $\mu \mathrm{s}$ & 0.2 & $\left(20^{+}\right)$ & & 98Pf02 & $\mathrm{TJ}$ & $\mathrm{IT}=100$ \\
\hline${ }^{204} \mathrm{~Pb}$ & -25109.7 & 1.2 & & & & STABLE & & $(>140 \mathrm{Py})$ & $0^{+}$ & 94 & & & $\mathrm{IS}=1.41 ; \alpha ?$ \\
\hline${ }^{204} \mathrm{~Pb}^{m}$ & -22923.9 & 1.2 & 2185.79 & 0.05 & & 67.2 & $\mathrm{~m}$ & 0.3 & $9^{-}$ & 94 & & & $\mathrm{IT}=100$ \\
\hline${ }^{204} \mathrm{Bi}$ & -20667 & 26 & & & & 11.22 & $\mathrm{~h}$ & 0.10 & $6^{+}$ & 94 & & & $\beta^{+}=100$ \\
\hline${ }^{204} \mathrm{Bi}^{m}$ & -19862 & 26 & 805.5 & 0.3 & & 13.0 & $\mathrm{~ms}$ & 0.1 & $10^{-}$ & 94 & & & $\mathrm{IT}=100$ \\
\hline${ }^{204} \mathrm{Bi}^{n}$ & -17834 & 26 & 2833.4 & 1.1 & & 1.07 & $\mathrm{~ms}$ & 0.03 & $\left(17^{+}\right)$ & 94 & & & $\mathrm{IT}=100$ \\
\hline${ }^{204} \mathrm{Po}$ & -18334 & 11 & & & & 3.53 & $\mathrm{~h}$ & 0.02 & $0^{+}$ & 94 & & & $\beta^{+}=99.341 ; \alpha=0.661$ \\
\hline
\end{tabular}




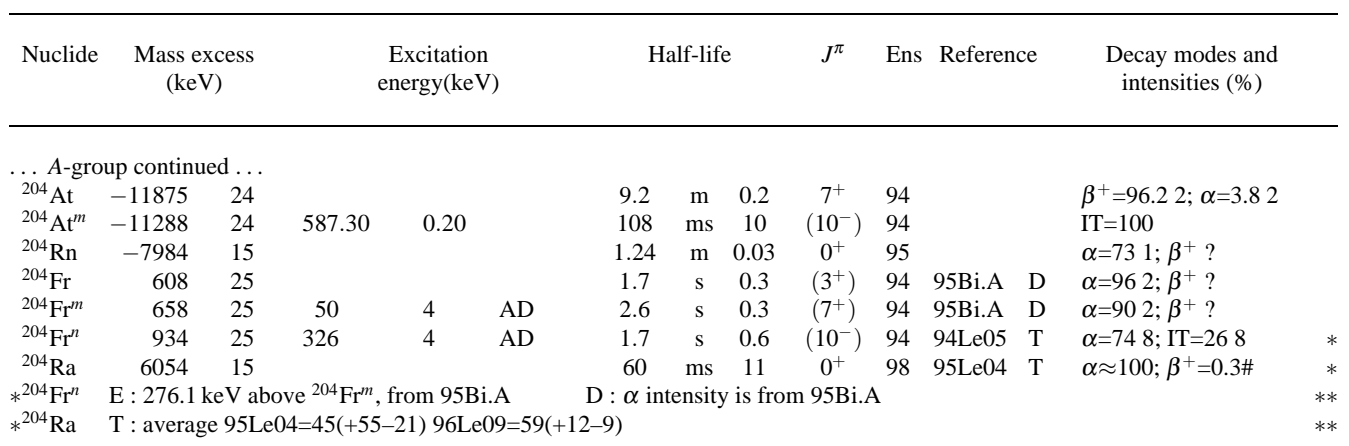

\begin{tabular}{|c|c|c|c|c|c|c|c|c|c|c|c|c|c|c|}
\hline${ }^{205} \mathrm{Au}$ & $-18750 \#$ & $300 \#$ & & & & 31 & $\mathrm{~s}$ & 2 & $3 / 2^{+}$ & 97 & $94 \mathrm{We} 02$ & $\mathrm{~T}$ & $\beta^{-}=100$ & \\
\hline${ }^{205} \mathrm{Hg}$ & -22287 & 4 & & & & 5.2 & $\mathrm{~m}$ & 0.1 & $1 / 2^{-}$ & 98 & & & $\beta^{-}=100$ & \\
\hline${ }^{205} \mathrm{Hg}^{m}$ & -20730 & 4 & 1556.53 & 0.24 & & 1.10 & $\mathrm{~ms}$ & 0.04 & $\left(13 / 2^{+}\right)$ & 98 & & & $\mathrm{IT}=100$ & \\
\hline${ }^{205} \mathrm{Tl}$ & -23820.6 & 1.3 & & & & StABLE & & & $1 / 2^{+}$ & 93 & & & $\mathrm{IS}=70.47614$ & \\
\hline${ }^{205} \mathrm{Tl}^{m}$ & -20530.0 & 1.3 & 3290.63 & 0.17 & & 2.6 & $\mu \mathrm{s}$ & 0.2 & $25 / 2^{+}$ & 93 & & & $\mathrm{IT}=100$ & \\
\hline${ }^{205} \mathrm{~Pb}$ & -23770.1 & 1.2 & & & & 15.3 & Мy & 0.7 & $5 / 2^{-}$ & 93 & & & $\varepsilon=100$ & \\
\hline${ }^{205} \mathrm{~Pb}^{m}$ & -22756.3 & 1.2 & 1013.839 & 0.013 & & 5.54 & $\mathrm{~ms}$ & 0.10 & $13 / 2^{+}$ & 93 & & & $\mathrm{IT}=100$ & \\
\hline${ }^{205} \mathrm{~Pb}^{n}$ & -20574.5 & 1.4 & 3195.6 & 0.8 & & 217 & $\mathrm{~ns}$ & 5 & $25 / 2^{-}$ & 93 & & & $\mathrm{IT}=100$ & \\
\hline${ }^{205} \mathrm{Bi}$ & -21062 & 7 & & & & 15.31 & $\mathrm{~d}$ & 0.04 & $9 / 2^{-}$ & 93 & & & $\beta^{+}=100$ & \\
\hline${ }^{205} \mathrm{Po}$ & -17509 & 20 & & & & 1.66 & $\mathrm{~h}$ & 0.02 & $5 / 2^{-}$ & 93 & & & $\beta^{+} \approx 100 ; \alpha=0.041$ & \\
\hline${ }^{205} \mathrm{Po}^{m}$ & -16048 & 20 & 1461.20 & 0.21 & & 58 & $\mathrm{~ms}$ & 1 & $19 / 2^{-}$ & 93 & & & $\mathrm{IT}=100$ & \\
\hline${ }^{205} \mathrm{Po}^{n}$ & -16629 & 20 & 880.30 & 0.04 & & 645 & $\mu \mathrm{s}$ & & $13 / 2^{+}$ & & & & & \\
\hline${ }^{205} \mathrm{At}$ & -12972 & 15 & & & & 26.2 & $\mathrm{~m}$ & 0.5 & $9 / 2^{-}$ & 93 & & & $\beta^{+}=902 ; \alpha=102$ & \\
\hline${ }^{205} \mathrm{At}^{m}$ & -10909 & 15 & 2062.57 & 0.25 & & 67.9 & $\mathrm{~ns}$ & & $25 / 2^{+}$ & & & & & \\
\hline${ }^{205} \mathrm{At}^{n}$ & -10632 & 15 & 2339.60 & 0.25 & & 7.8 & $\mu \mathrm{s}$ & & $29 / 2^{+}$ & & & & & \\
\hline${ }^{205} \mathrm{Rn}$ & -7710 & 50 & & & & 2.8 & $\mathrm{~m}$ & 0.1 & $5 / 2^{-}$ & 93 & & & $\beta^{+}=774 ; \alpha=234$ & \\
\hline${ }^{205} \mathrm{Fr}$ & -1310 & 8 & & & & 3.85 & $\mathrm{~s}$ & 0.10 & $\left(9 / 2^{-}\right)$ & 93 & & & $\alpha \approx 100 ; \beta^{+}<1$ & \\
\hline${ }^{205} \mathrm{Ra}$ & 5840 & 90 & & & & 220 & $\mathrm{~ms}$ & 40 & $\left(3 / 2^{-}\right)$ & 93 & 96Le09 & $\mathrm{TJ}$ & $\alpha=? ; \beta^{+} ?$ & $*$ \\
\hline${ }^{205} \mathrm{Ra}^{m}$ & $6150 \#$ & $100 \#$ & $310 \#$ & $110 \#$ & & 180 & $\mathrm{~ms}$ & 50 & $\left(13 / 2^{+}\right)$ & & 96Le09 & TJD & $\alpha=? ;$ IT ? & \\
\hline$*^{205} \mathrm{Ra}$ & $\mathrm{T}:$ average & e $96 \mathrm{Le} 0$ & $9=210(+60$ & 0-40) 871 & $\mathrm{Ie} 10=220(60$ & & & & & & & & & $* *$ \\
\hline${ }^{206} \mathrm{Hg}$ & -20946 & 20 & & & & 8.15 & $\mathrm{~m}$ & 0.10 & $0^{+}$ & 99 & & & $\beta^{-}=100$ & \\
\hline${ }^{206} \mathrm{Tl}$ & -22253.1 & 1.4 & & & & 4.200 & $\mathrm{~m}$ & 0.017 & $0^{-}$ & 99 & & & $\beta^{-}=100$ & \\
\hline${ }^{206} \mathrm{Tl}^{m}$ & -19610.0 & 1.4 & 2643.11 & 0.19 & & 3.74 & $\mathrm{~m}$ & 0.03 & $\left(12^{-}\right)$ & 99 & & & $\mathrm{IT}=100$ & \\
\hline${ }^{206} \mathrm{~Pb}$ & -23785.4 & 1.2 & & & & StABLE & & & $0^{+}$ & 99 & & & $\mathrm{IS}=24.11$ & \\
\hline${ }^{206} \mathrm{~Pb}^{m}$ & -21585.3 & 1.2 & 2200.14 & 0.04 & & 125 & $\mu \mathrm{s}$ & 2 & $7^{-}$ & 99 & & & $\mathrm{IT}=100$ & \\
\hline${ }^{206} \mathrm{~Pb}^{n}$ & -19758.1 & 1.4 & 4027.3 & 0.7 & & 202 & ns & 3 & $12^{+}$ & 99 & & & $\mathrm{IT}=100$ & \\
\hline${ }^{206} \mathrm{Bi}$ & -20028 & 8 & & & & 6.243 & $\mathrm{~d}$ & 0.003 & $6^{(+)}$ & 99 & & & $\beta^{+}=100$ & \\
\hline${ }^{206} \mathrm{Bi}^{m}$ & -19968 & 8 & 59.897 & 0.017 & & 7.7 & $\mu \mathrm{s}$ & 0.2 & $\left(4^{+}\right)$ & 99 & & & $\mathrm{IT}=100$ & \\
\hline${ }^{206} \mathrm{Bi}^{n}$ & -18983 & 8 & 1044.8 & 0.5 & & 890 & $\mu \mathrm{s}$ & 10 & $\left(10^{-}\right)$ & 99 & & & $\mathrm{IT}=100$ & \\
\hline${ }^{206} \mathrm{Po}$ & -18182 & 8 & & & & 8.8 & $\mathrm{~d}$ & 0.1 & $0^{+}$ & 99 & & & $\beta^{+}=94.555 ; \alpha=5.455$ & \\
\hline${ }^{206} \mathrm{Po}^{m}$ & -16596 & 8 & 1585.85 & 0.11 & & 222 & ns & 10 & $8^{+} \#$ & 99 & & & $\mathrm{IT}=100$ & $*$ \\
\hline${ }^{206} \mathrm{Po}^{n}$ & -15920 & 8 & 2262.22 & 0.14 & & 1.05 & $\mu \mathrm{s}$ & 0.06 & $9^{-} \#$ & 99 & & & $\mathrm{IT}=100$ & \\
\hline${ }^{206} \mathrm{At}$ & -12420 & 20 & & & & 30.6 & $\mathrm{~m}$ & 1.3 & $(5)^{+}$ & 99 & & & $\beta^{+}=99.118 ; \alpha=0.898$ & \\
\hline${ }^{206} \mathrm{At}^{m}$ & -11613 & 20 & 807 & 3 & & 410 & ns & 80 & $(10)^{-}$ & & $99 \mathrm{Fe} 10$ & ETJ & $\mathrm{IT}=100$ & \\
\hline${ }^{206} \mathrm{Rn}$ & -9116 & 15 & & & & 5.67 & $\mathrm{~m}$ & 0.17 & $0^{+}$ & 99 & & & $\alpha=623 ; \beta^{+}=383$ & \\
\hline${ }^{206} \mathrm{Fr}$ & -1243 & 28 & & & & 16 & $\mathrm{~s}$ & & $\left(2^{+}, 3^{+}\right)$ & 99 & $92 \mathrm{Hu} 04$ & $\mathrm{D}$ & $\beta^{+}=? ; \alpha=4224$ & $*$ \\
\hline${ }^{206} \mathrm{Fr}^{m}$ & -1048 & 28 & 190 & 40 & MD & 15.9 & $\mathrm{~s}$ & 0.1 & $\left(7^{+}\right)$ & 99 & $92 \mathrm{Hu} 04$ & $\mathrm{D}$ & $\alpha=4224 ; \beta^{+}$?; IT ? & \\
\hline${ }^{206} \mathrm{Fr}^{n}$ & -517 & 28 & 730 & 40 & MD & 700 & $\mathrm{~ms}$ & 100 & $\left(10^{-}\right)$ & 99 & & & $\mathrm{IT}=? ; \alpha \approx 12 \#$ & * \\
\hline${ }^{206} \mathrm{Ra}$ & 3565 & 18 & & & & 240 & $\mathrm{~ms}$ & 20 & $0^{+}$ & 99 & & & $\alpha=100$ & \\
\hline${ }^{206} \mathrm{Ac}$ & 13510 & 70 & & & $* \&$ & 25 & $\mathrm{~ms}$ & 7 & $\left(3^{+}\right)$ & 99 & & & $\alpha \approx 100 ; \beta^{+}=0.2 \#$ & \\
\hline${ }^{206} \mathrm{Ac}^{m}$ & 13590 & 90 & 80 & 50 & $* \&$ & 15 & $\mathrm{~ms}$ & 6 & & 99 & & & $\alpha \approx 100$ & \\
\hline${ }^{206} \mathrm{Ac}^{n}$ & $13800 \#$ & $80 \#$ & $290 \#$ & $110 \#$ & $\&$ & 41 & $\mathrm{~ms}$ & 16 & $\left(10^{-}\right)$ & 99 & & & $\alpha \approx 100$ & \\
\hline$*^{206} \mathrm{Po}^{m}$ & $\mathrm{E}:$ less tha & an $40 \mathrm{ke}$ & $\mathrm{V}$ above 1 & 573.4 lev & , from ENS & & & & & & & & & ** \\
\hline$*^{206} \mathrm{Fr}$ & $\mathrm{D}: \alpha=84$ & $2) \%$ for & mixture of & $\mathrm{f}^{206} \mathrm{Fr}$ an & ${ }^{206} \mathrm{Fr}^{m}$, in & $92 \mathrm{Hu} 0$ & $\mathrm{~V}$ & re & ced by & & & & & $* *$ \\
\hline$*^{206} \mathrm{Fr}$ & $\mathrm{D}$ : uni & iform di & istribution & $0 \%-84 \%$ & for each ison & & & & & & & & & $* *$ \\
\hline$*^{206} \mathrm{Fr}^{n}$ & $\mathrm{E}: 531 \mathrm{keV}$ & V above & ${ }^{206} \mathrm{Fr}^{m}, \mathrm{fr}$ & om ENSD & & & & & & & & & & ** \\
\hline
\end{tabular}




\begin{tabular}{|c|c|c|c|c|c|c|c|c|c|c|c|c|c|c|}
\hline Nuclide & \multicolumn{2}{|c|}{$\begin{array}{l}\text { Mass excess } \\
\quad(\mathrm{keV})\end{array}$} & \multicolumn{3}{|c|}{$\begin{array}{l}\text { Excitation } \\
\text { energy }(\mathrm{keV})\end{array}$} & \multicolumn{3}{|c|}{ Half-life } & $J^{\pi}$ & Ens & \multicolumn{2}{|c|}{ Reference } & \multicolumn{2}{|l|}{$\begin{array}{l}\text { Decay modes and } \\
\text { intensities }(\%)\end{array}$} \\
\hline${ }^{207} \mathrm{Hg}$ & -16220 & 150 & & & & 2.9 & $\mathrm{~m}$ & 0.2 & $\left(9 / 2^{+}\right)$ & 94 & & & $\beta^{-}=100$ & \\
\hline${ }^{207} \mathrm{Tl}$ & -21034 & 5 & & & & 4.77 & $\mathrm{~m}$ & 0.02 & $1 / 2^{+}$ & 94 & & & $\beta^{-}=100$ & \\
\hline${ }^{207} \mathrm{Tl}^{m}$ & -19686 & 5 & 1348.1 & 0.3 & & 1.33 & $\mathrm{~s}$ & 0.11 & $11 / 2^{-}$ & 94 & & & $\mathrm{IT} \approx 100 ; \beta^{-}<0.1 \#$ & \\
\hline${ }^{207} \mathrm{~Pb}$ & -22451.9 & 1.2 & & & & STABLE & & & $1 / 2^{-}$ & 94 & & & $\mathrm{IS}=22.11$ & \\
\hline${ }^{207} \mathrm{~Pb}^{m}$ & -20818.5 & 1.2 & 1633.368 & 0.005 & & 806 & $\mathrm{~ms}$ & 6 & $13 / 2^{+}$ & 94 & & & $\mathrm{IT}=100$ & \\
\hline${ }^{207} \mathrm{Bi}$ & -20054.4 & 2.4 & & & & 32.9 & $\mathrm{y}$ & 1.4 & $9 / 2^{-}$ & 94 & & & $\beta^{+}=100$ & \\
\hline${ }^{207} \mathrm{Bi}^{m}$ & -17952.9 & 2.4 & 2101.49 & 0.16 & & 182 & $\mu \mathrm{s}$ & 6 & $21 / 2^{+}$ & 94 & & & $\mathrm{IT}=100$ & \\
\hline${ }^{207} \mathrm{Po}$ & -17146 & 7 & & & & 5.80 & $\mathrm{~h}$ & 0.02 & $5 / 2^{-}$ & 94 & & & $\beta^{+} \approx 100 ; \alpha=0.0212$ & \\
\hline${ }^{207} \mathrm{Po}^{m}$ & -15763 & 7 & 1383.15 & 0.06 & & 2.79 & $\mathrm{~s}$ & 0.08 & $19 / 2^{-}$ & 94 & & & $\mathrm{IT}=100$ & \\
\hline${ }^{207} \mathrm{Po}^{n}$ & -16031 & 7 & 1115.073 & 0.016 & & 49 & $\mu \mathrm{s}$ & & $13 / 2^{+}$ & & & & & \\
\hline${ }^{207}$ At & -13243 & 21 & & & & 1.80 & $\mathrm{~h}$ & 0.04 & $9 / 2^{-}$ & 94 & & & $\beta^{+}=91.410 ; \alpha=8.610$ & \\
\hline${ }^{207} \mathrm{Rn}$ & -8631 & 26 & & & & 9.25 & $\mathrm{~m}$ & 0.17 & $5 / 2^{-}$ & 94 & & & $\beta^{+}=793 ; \alpha=213$ & \\
\hline${ }^{207} \mathrm{Rn}^{m}$ & -7732 & 26 & 899.0 & 1.0 & & 181 & $\mu \mathrm{s}$ & 18 & $\left(13 / 2^{+}\right)$ & 94 & & & $\mathrm{IT}=100$ & \\
\hline${ }^{207} \mathrm{Fr}$ & -2840 & 50 & & & & 14.8 & $\mathrm{~s}$ & 0.1 & $9 / 2^{-}$ & 94 & & & $\alpha=952 ; \beta^{+}=52$ & \\
\hline${ }^{207} \mathrm{Ra}$ & 3540 & 60 & & & & 1.3 & s & 0.2 & $\left(5 / 2^{-}, 3 / 2^{-}\right)$ & 94 & & & $\alpha \approx 90 ; \beta^{+} \approx 10$ & \\
\hline${ }^{207} \mathrm{Ra}^{m}$ & 4095 & 25 & 560 & 50 & $\mathrm{AD}$ & 57 & $\mathrm{~ms}$ & 8 & $\left(13 / 2^{+}\right)$ & 94 & 96Le09 & $\mathrm{T}$ & $\mathrm{IT}=85 \# ; \alpha=? ; \ldots$ & $*$ \\
\hline${ }^{207} \mathrm{Ac}$ & 11130 & 50 & & & & 31 & $\mathrm{~ms}$ & 8 & $9 / 2^{-} \#$ & 98 & 94Le05 & $\mathrm{TD}$ & $\alpha=100$ & $*$ \\
\hline${ }^{207} \mathrm{Ra}^{m}$ & \multicolumn{13}{|c|}{$\mathrm{D}: \ldots ; \beta^{+}=0.55 \#$} & $* *$ \\
\hline$*^{207} \mathrm{Ra}^{m}$ & & $* *$ \\
\hline$*^{207} \mathrm{Ac}$ & & & & & & & \multicolumn{8}{|c|}{$\mathrm{T}:$ average $98 \mathrm{Es} 02=27(+11-6) 94 \mathrm{Le} 05=22(+40-9)$} \\
\hline${ }^{208} \mathrm{Hg}$ & $-13100 \#$ & $300 \#$ & & & & 42 & $\mathrm{~m}$ & 5 & $0^{+}$ & 98 & $98 Z h 22$ & $\mathrm{~T}$ & $\beta^{-}=100$ & $*$ \\
\hline${ }^{208} \mathrm{Tl}$ & -16749.5 & 2.0 & & & & 3.053 & $\mathrm{~m}$ & 0.004 & $5^{(+)}$ & 98 & & & $\beta^{-}=100$ & \\
\hline${ }^{208} \mathrm{~Pb}$ & -21748.5 & 1.2 & & & & STABLE & & & $0^{+}$ & 96 & & & $\mathrm{IS}=52.41$ & \\
\hline${ }^{208} \mathrm{~Pb}^{m}$ & -16853.5 & 2.3 & 4895 & 2 & & 500 & ns & 10 & $10^{+}$ & 86 & 98Pf02 & $\mathrm{T}$ & $\mathrm{IT}=100$ & \\
\hline${ }^{208} \mathrm{Bi}$ & -18870.0 & 2.4 & & & & 368 & ky & 4 & $(5)^{+}$ & 86 & & & $\beta^{+}=100$ & \\
\hline${ }^{208} \mathrm{Bi}^{m}$ & -17298.9 & 2.4 & 1571.1 & 0.4 & & 2.58 & $\mathrm{~ms}$ & 0.04 & $(10)^{-}$ & 86 & & & $\mathrm{IT}=100$ & \\
\hline${ }^{208} \mathrm{Po}$ & -17469.5 & 1.8 & & & & 2.898 & $\mathrm{y}$ & 0.002 & $0^{+}$ & 86 & & & $\alpha \approx 100 ; \beta^{+}=0.0022323$ & \\
\hline${ }^{208} \mathrm{At}$ & -12491 & 26 & & & & 1.63 & $\mathrm{~h}$ & 0.03 & $6^{+}$ & 86 & & & $\beta^{+}=99.456 ; \alpha=0.556$ & \\
\hline${ }^{208} \mathrm{Rn}$ & -9648 & 11 & & & & 24.35 & $\mathrm{~m}$ & 0.14 & $0^{+}$ & 86 & & & $\alpha=627 ; \beta^{+}=387$ & \\
\hline${ }^{208} \mathrm{Fr}$ & -2670 & 50 & & & & 59.1 & $\mathrm{~s}$ & 0.3 & $7^{+}$ & 86 & & & $\alpha=904 ; \beta^{+}=104$ & \\
\hline${ }^{208} \mathrm{Ra}$ & 1714 & 15 & & & & 1.3 & s & 0.2 & $0^{+}$ & 86 & & & $\alpha=? ; \beta^{+}=5 \#$ & \\
\hline${ }^{208} \mathrm{Ra}^{m}$ & 3510 & 200 & 1800 & 200 & & 270 & $\mathrm{~ns}$ & & $\left(8^{+}\right)$ & & 98Le.A & ETJ & & \\
\hline${ }^{208} \mathrm{Ac}$ & 10760 & 60 & & & & 97 & $\mathrm{~ms}$ & 16 & $\left(3^{+}\right)$ & 96 & 96Ik01 & $\mathrm{T}$ & $\alpha=? ; \beta^{+}=1 \#$ & $*$ \\
\hline${ }^{208} \mathrm{Ac}^{m}$ & 11258 & 28 & 500 & 50 & $\mathrm{AD}$ & 28 & $\mathrm{~ms}$ & 7 & $\left(10^{-}\right)$ & 96 & 96Ik01 & $\mathrm{T}$ & $\alpha=? ;$ IT $<10 \# ; \beta^{+}=1 \#$ & * \\
\hline$*^{208} \mathrm{Hg}$ & \multicolumn{13}{|c|}{$\mathrm{T}: 98 \mathrm{Zh} 22=41(+5-4)$ supersedes $94 \mathrm{Zh} 02=42(+23-12)$ of same group } & $* *$ \\
\hline$*^{208} \mathrm{Ac}$ & \multicolumn{13}{|c|}{$\mathrm{T}:$ average $96 \mathrm{Ik} 01=83(+34-19) 94 \mathrm{Le} 05=95(+24-16)$} & $* *$ \\
\hline$*^{208} \mathrm{Ac}^{m}$ & \multicolumn{13}{|c|}{ E : if $\alpha$ decay goes to $\left(7^{+}\right)^{204} \mathrm{Fr}^{m}$, instead of $\left(10^{-}\right)$as assumed in AME, then } & $* *$ \\
\hline$*^{208} \mathrm{Ac}^{m}$ & \multirow{2}{*}{\multicolumn{13}{|c|}{$\begin{array}{l}\text { E : E will become } 234(22) \mathrm{keV} \\
\mathrm{T}: \text { average } 96 \mathrm{Kk} 01=21(+28-8) 94 \mathrm{Le} 05=25(+9-5)\end{array}$}} & ** \\
\hline$*^{208} \mathrm{Ac}^{m}$ & & & & & & & & & & & & & & $* *$ \\
\hline
\end{tabular}

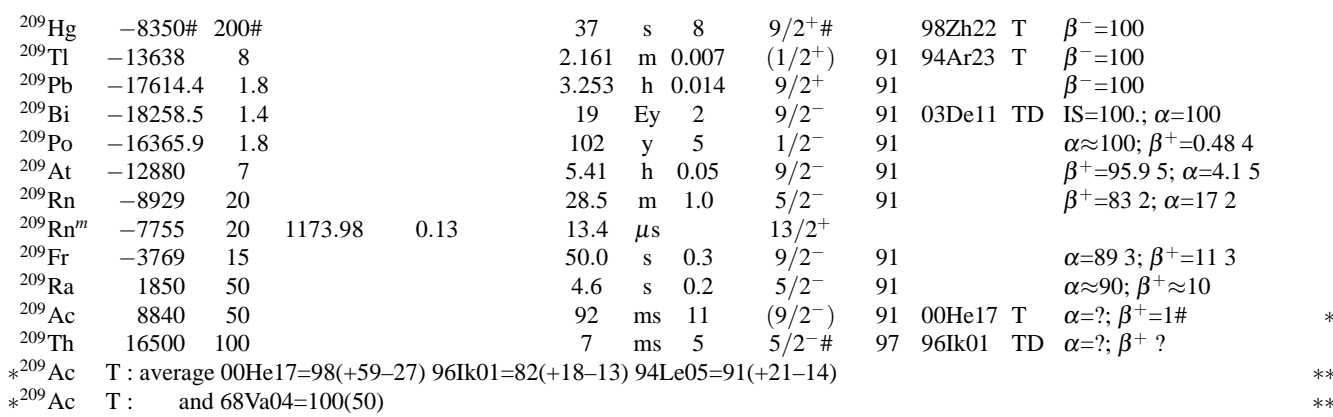




\begin{tabular}{|c|c|c|c|c|c|c|c|c|c|c|c|}
\hline Nuclide & \multicolumn{2}{|c|}{$\begin{array}{l}\text { Mass excess } \\
\quad(\mathrm{keV})\end{array}$} & \multicolumn{2}{|c|}{$\begin{array}{c}\text { Excitation } \\
\text { energy(keV) }\end{array}$} & \multicolumn{3}{|c|}{ Half-life } & $J^{\pi}$ & Ens & Reference & \multirow[t]{2}{*}{$\begin{array}{l}\text { Decay modes and } \\
\text { intensities }(\%)\end{array}$} \\
\hline${ }^{210} \mathrm{Hg}$ & $-5110 \#$ & $300 \#$ & & & $10 \#$ & $\mathrm{~m}$ & $(>300 \mathrm{~ns})$ & $0^{+}$ & 03 & 98Pf02 I & \\
\hline${ }^{210} \mathrm{Tl}$ & -9246 & 12 & & & 1.30 & $\mathrm{~m}$ & 0.03 & $5^{+} \#$ & 03 & & $\beta^{-}=100 ; \beta^{-} \mathrm{n}=0.0096$ \\
\hline${ }^{210} \mathrm{~Pb}$ & -14728.3 & 1.5 & & & 22.20 & $\mathrm{y}$ & 0.22 & $0^{+}$ & 03 & & $\beta^{-}=100 ; \alpha=1.9 \mathrm{e}-64$ \\
\hline${ }^{210} \mathrm{~Pb}^{m}$ & -13450 & 5 & 1278 & 5 & 201 & $\mathrm{~ns}$ & 17 & $8^{+}$ & 03 & & $\mathrm{IT}=100$ \\
\hline${ }^{210} \mathrm{Bi}$ & -14791.8 & 1.4 & & & 5.012 & $\mathrm{~d}$ & 0.005 & $1^{-}$ & 03 & & $\beta^{-}=100 ; \alpha=13.2 \mathrm{e}-510$ \\
\hline${ }^{210} \mathrm{Bi}^{m}$ & -14520.5 & 1.4 & 271.31 & 0.11 & 3.04 & My & 0.06 & $9^{-}$ & 03 & & $\alpha=100$ \\
\hline${ }^{210} \mathrm{Bi}^{n}$ & -14358.3 & 1.4 & 433.49 & 0.10 & 57.5 & $\mathrm{~ns}$ & 10 & $7^{-}$ & 03 & & $\mathrm{IT}=100$ \\
\hline${ }^{210} \mathrm{Po}$ & -15953.1 & 1.2 & & & 138.376 & $\mathrm{~d}$ & 0.002 & $0^{+}$ & 03 & & $\alpha=100$ \\
\hline${ }^{210} \mathrm{Po}^{m}$ & -14396.1 & 1.2 & 1556.96 & 0.03 & 98.9 & $\mathrm{~ns}$ & 2.5 & $8^{+}$ & 03 & & $\mathrm{IT}=100$ \\
\hline${ }^{210} \mathrm{At}$ & -11972 & 8 & & & 8.1 & $\mathrm{~h}$ & 0.4 & $(5)^{+}$ & 03 & & $\beta^{+} \approx 100 ; \alpha=0.17520$ \\
\hline${ }^{210} \mathrm{At}^{m}$ & -9422 & 8 & 2549.6 & 0.2 & 482 & $\mu \mathrm{s}$ & 6 & $(15)^{-}$ & 03 & & $\mathrm{IT}=100$ \\
\hline${ }^{210} \mathrm{At}^{n}$ & -7944 & 8 & 4027.7 & 0.2 & 5.66 & $\mu \mathrm{s}$ & 0.07 & $(19)^{+}$ & 03 & & $\mathrm{IT}=100$ \\
\hline${ }^{210} \mathrm{At}^{p}$ & -5013 & 8 & 6959.3 & 0.6 & 98 & ns & 2 & $\left(26^{-}\right)$ & 03 & & $\mathrm{IT}=100$ \\
\hline${ }^{210} \mathrm{Rn}$ & -9598 & 9 & & & 2.4 & $\mathrm{~h}$ & 0.1 & $0^{+}$ & 03 & & $\alpha=961 ; \beta^{+} ?$ \\
\hline${ }^{210} \mathrm{Rn}^{m}$ & -7908 & 17 & 1690 & 15 & 644 & ns & 40 & $8^{+} \#$ & 03 & & IT? \\
\hline${ }^{210} \mathrm{Rn}^{n}$ & -5761 & 17 & 3837 & 15 & 1.06 & $\mu \mathrm{s}$ & 0.05 & $(17)^{-}$ & 03 & & $\mathrm{IT}=100$ \\
\hline${ }^{210} \mathrm{Rn}^{p}$ & -3105 & 17 & 6493 & 15 & 1.04 & $\mu \mathrm{s}$ & 0.07 & $(22)^{+}$ & 03 & & $\mathrm{IT}=100$ \\
\hline${ }^{210} \mathrm{Fr}$ & -3346 & 22 & & & 3.18 & $\mathrm{~m}$ & 0.06 & $6^{+}$ & 03 & & $\alpha=6030 ; \beta^{+}=4030$ \\
\hline${ }^{210} \mathrm{Ra}$ & 461 & 15 & & & 3.7 & $\mathrm{~s}$ & 0.2 & $0^{+}$ & 03 & & $\alpha=? ; \beta^{+}=4 \#$ \\
\hline${ }^{210} \mathrm{Ra}^{m}$ & 2260 & 200 & 1800 & 200 & 2.24 & $\mu \mathrm{s}$ & & $\left(8^{+}\right)$ & 03 & 98Le.A EJ & \\
\hline${ }^{210} \mathrm{Ac}$ & 8790 & 60 & & & 350 & $\mathrm{~ms}$ & 40 & $7^{+} \#$ & 03 & $00 \mathrm{He} 17 \mathrm{~T}$ & $\alpha=? ; \beta^{+}=9 \#$ \\
\hline${ }^{210} \mathrm{Th}$ & 14043 & 25 & & & 17 & $\mathrm{~ms}$ & 11 & $0^{+}$ & 03 & & $\alpha=? ; \beta^{+}=1 \#$ \\
\hline${ }^{210} \mathrm{Rn}^{m}$ & E : ENSDF2 & 2003: & ess than & $0 \mathrm{keV}$ at & $664.6 \mathrm{lev}$ & & & & & & \\
\hline${ }^{210} \mathrm{Ac}$ & $\mathrm{T}:$ average & $\mathrm{e} 0 \mathrm{H}$ & $17=335$ & $64-46)$ & $4=350(50$ & & & & & & \\
\hline
\end{tabular}

\begin{tabular}{|c|c|c|c|c|c|c|c|c|c|c|c|c|c|c|}
\hline${ }^{211} \mathrm{Tl}$ & $-6080 \#$ & $200 \#$ & & & & $1 \#$ & $\mathrm{~m}$ & $(>300 \mathrm{~ns})$ & $1 / 2^{+} \#$ & & 98Pf02 & I & $\beta^{-} ?$ & \\
\hline${ }^{211} \mathrm{~Pb}$ & -10491.4 & 2.7 & & & & 36.1 & $\mathrm{~m}$ & 0.2 & $9 / 2^{+}$ & 91 & & & $\beta^{-}=100$ & \\
\hline${ }^{211} \mathrm{Bi}$ & -11858 & 6 & & & & 2.14 & $\mathrm{~m}$ & 0.02 & $9 / 2^{-}$ & 91 & & & $\alpha \approx 100 ; \beta^{-}=0.2764$ & \\
\hline${ }^{211} \mathrm{Bi}^{m}$ & -10631 & 6 & 1227.2 & 0.3 & & 70 & $\mathrm{~ns}$ & 5 & $\left(21 / 2^{-}\right)$ & 91 & & & $\mathrm{IT}=100$ & \\
\hline${ }^{211} \mathrm{Bi}^{n}$ & -10601 & 12 & 1257 & 10 & & 1.4 & $\mu \mathrm{s}$ & 0.3 & $\left(25 / 2^{-}\right)$ & 91 & 98Pf02 & $\mathrm{T}$ & $\mathrm{IT}=100$ & \\
\hline${ }^{211} \mathrm{Po}$ & -12432.5 & 1.3 & & & & 516 & $\mathrm{~ms}$ & 3 & $9 / 2^{+}$ & 91 & & & $\alpha=100$ & \\
\hline${ }^{211} \mathrm{Po}^{m}$ & -10970 & 5 & 1462 & 5 & $\mathrm{AD}$ & 25.2 & $\mathrm{~s}$ & 0.6 & $\left(25 / 2^{+}\right)$ & 91 & & & $\alpha \approx 100 ; \mathrm{IT}=0.0164$ & \\
\hline${ }^{211} \mathrm{Po}^{n}$ & -10298 & 5 & 2135 & 5 & & 0.25 & $\mu \mathrm{s}$ & 0.07 & $\left(31 / 2^{-}\right)$ & & 98Fo04 & ETJ & $\mathrm{IT} \approx 100 ; \alpha ?$ & \\
\hline${ }^{211} \mathrm{Po}^{p}$ & -7559 & 5 & 4874 & 5 & & 2 & $\mu \mathrm{s}$ & 1 & $\left(43 / 2^{+}\right)$ & & 98Fo04 & ETJ & $\mathrm{IT} \approx 100 ; \alpha ?$ & \\
\hline${ }^{211} \mathrm{At}$ & -11647.1 & 2.8 & & & & 7.214 & $\mathrm{~h}$ & 0.007 & $9 / 2^{-}$ & 96 & & & $\varepsilon=58.208 ; \alpha=41.808$ & \\
\hline${ }^{211} \mathrm{Rn}$ & -8756 & 7 & & & & 14.6 & $\mathrm{~h}$ & 0.2 & $1 / 2^{-}$ & 96 & & & $\beta^{+}=72.617 ; \alpha=27.417$ & \\
\hline${ }^{211} \mathrm{Fr}$ & -4158 & 21 & & & & 3.10 & $\mathrm{~m}$ & 0.02 & $9 / 2^{-}$ & 91 & & & $\alpha>80 ; \beta^{+}<20$ & \\
\hline${ }^{211} \mathrm{Ra}$ & 836 & 26 & & & & 13 & $\mathrm{~s}$ & 2 & $5 / 2^{(-)}$ & 91 & & & $\alpha>93 ; \beta^{+}<7$ & \\
\hline${ }^{211} \mathrm{Ac}$ & 7200 & 70 & & & & 213 & $\mathrm{~ms}$ & 25 & $9 / 2^{-} \#$ & 91 & $00 \mathrm{He} 17$ & $\mathrm{~T}$ & $\alpha \approx 100 ; \beta^{+}<0.2$ & $*$ \\
\hline${ }^{211} \mathrm{Th}$ & 13910 & 70 & & & & 48 & $\mathrm{~ms}$ & 20 & $5 / 2^{-} \#$ & 96 & 95Uu01 & $\mathrm{T}$ & $\alpha=? ; \beta^{+}=0.5 \#$ & \\
\hline$*^{211} \mathrm{Ac}$ & $\mathrm{T}:$ averag & $\mathrm{e} 0 \mathrm{He}$ & $17=200(2$ & 29) $68 \mathrm{~V}$ & $04=25$ & $(50)$ & & & & & & & & ** \\
\hline${ }^{212} \mathrm{Tl}$ & $-1650 \#$ & $300 \#$ & & & & $30 \#$ & $\mathrm{~s}$ & $(>300 \mathrm{~ns})$ & $5^{+} \#$ & & 98Pf02 & I & $\beta^{-} ?$ & \\
\hline${ }^{212} \mathrm{~Pb}$ & -7547.4 & 2.2 & & & & 10.64 & $\mathrm{~h}$ & 0.01 & $0^{+}$ & 92 & & & $\beta^{-}=100$ & \\
\hline${ }^{212} \mathrm{~Pb}^{m}$ & -6212 & 10 & 1335 & 10 & & 5 & $\mu \mathrm{s}$ & 1 & $\left(8^{+}\right)$ & 92 & 98Pf02 & $\mathrm{T}$ & $\mathrm{IT}=100$ & \\
\hline${ }^{212} \mathrm{Bi}$ & -8117.3 & 2.0 & & & & 60.55 & $\mathrm{~m}$ & 0.06 & $1^{(-)}$ & 92 & 89Hа.А & $\mathrm{D}$ & $\beta^{-}=64.066 ; \alpha=35.946 ;$ & $*$ \\
\hline${ }^{212} \mathrm{Bi}^{m}$ & -7870 & 30 & 250 & 30 & $\mathrm{AD}$ & 25.0 & $\mathrm{~m}$ & 0.2 & $\left(9^{-}\right)$ & 92 & & & $\alpha=671 ; \beta^{-}=331 ; \beta^{-} \alpha=301$ & \\
\hline${ }^{212} \mathrm{Bi}^{n}$ & $-5920 \#$ & $200 \#$ & $2200 \#$ & $200 \#$ & & 7.0 & $\mathrm{~m}$ & 0.3 & $>15$ & 92 & & & $\beta^{-} \approx 100 ;$ IT ? & $*$ \\
\hline${ }^{212} \mathrm{Po}$ & -10369.4 & 1.2 & & & & 299 & $\mathrm{~ns}$ & 2 & $0^{+}$ & 92 & & & $\alpha=100$ & \\
\hline${ }^{212} \mathrm{Po}^{m}$ & -7459 & 12 & 2911 & 12 & $\mathrm{AD}$ & 45.1 & $\mathrm{~s}$ & 0.6 & $\left(18^{+}\right)$ & 92 & & & $\alpha \approx 100 ; \mathrm{IT}=0.072$ & \\
\hline${ }^{212} \mathrm{At}$ & -8621 & 7 & & & & 314 & $\mathrm{~ms}$ & 2 & $\left(1^{-}\right)$ & 92 & & & $\alpha \approx 100 ; \beta^{+}<0.03 ; \beta^{-}<2 \mathrm{e}-6$ & \\
\hline${ }^{212} \mathrm{At}^{m}$ & -8395 & 6 & 226 & 9 & $\mathrm{AD}$ & 119 & $\mathrm{~ms}$ & 3 & $\left(9^{-}\right)$ & 92 & & & $\alpha>99 ; \mathrm{IT}<1$ & \\
\hline${ }^{212} \mathrm{At}^{n}$ & -3849 & 8 & 4772 & 3 & & 152 & $\mu \mathrm{s}$ & 5 & $\left(25^{-}\right)$ & & 98By01 & ETJ & $\mathrm{IT}=100$ & \\
\hline${ }^{212} \mathrm{Rn}$ & -8660 & 3 & & & & 23.9 & $\mathrm{~m}$ & 1.2 & $0^{+}$ & 92 & & & $\alpha=100 ; 2 \beta^{+} ?$ & \\
\hline${ }^{212} \mathrm{Fr}$ & -3538 & 26 & & & & 20.0 & $\mathrm{~m}$ & 0.6 & $5^{+}$ & 92 & & & $\beta^{+}=572 ; \alpha=432$ & \\
\hline${ }^{212} \mathrm{Ra}$ & -191 & 11 & & & & 13.0 & $\mathrm{~s}$ & 0.2 & $0^{+}$ & 92 & & & $\alpha=? ; \beta^{+}=15 \#$ & \\
\hline${ }^{212} \mathrm{Ra}^{m}$ & 1767 & 11 & 1958.4 & 0.5 & & 10.9 & $\mu \mathrm{s}$ & 0.4 & $(8)^{+}$ & 92 & & & $\mathrm{IT}=100$ & \\
\hline
\end{tabular}




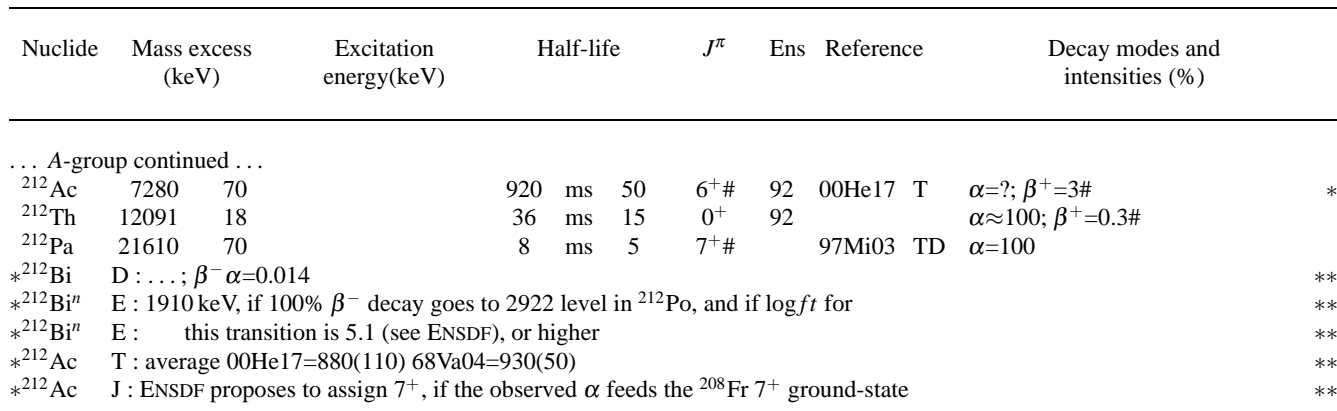

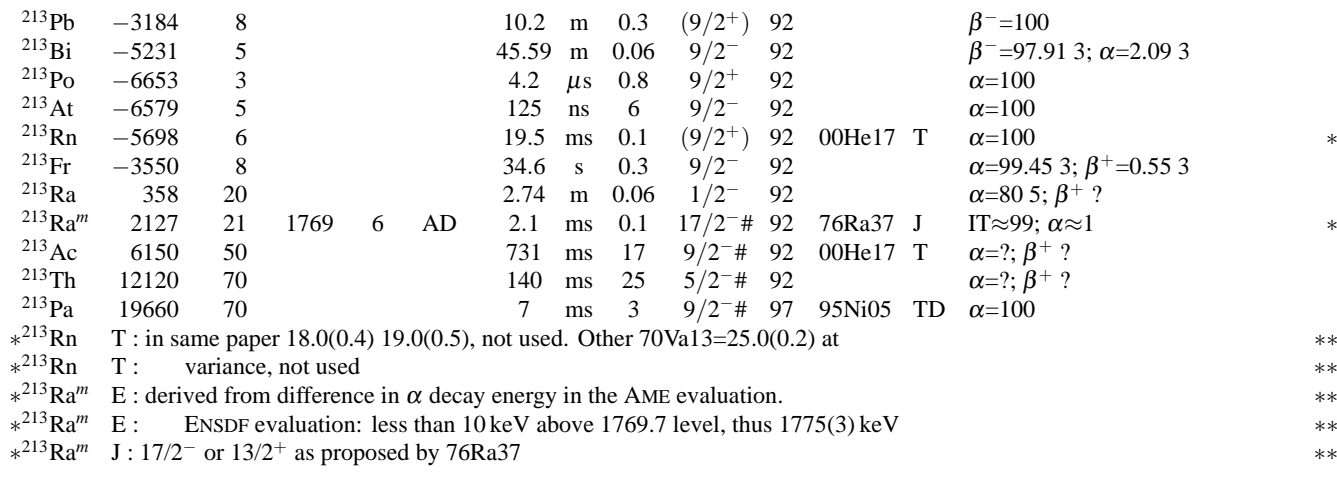

\begin{tabular}{|c|c|c|c|c|c|c|c|c|c|c|c|c|c|}
\hline${ }^{214} \mathrm{~Pb}$ & -181.3 & 2.4 & & & & 26.8 & $\mathrm{~m}$ & 0.9 & $0^{+}$ & 95 & & & $\beta^{-}=100$ \\
\hline${ }^{214} \mathrm{Bi}$ & -1200 & 11 & & & & 19.9 & $\mathrm{~m}$ & 0.4 & $1^{-}$ & 95 & 89Hа.А & $\mathrm{D}$ & $\beta^{-} \approx 100 ; \alpha=0.0211 ; \beta^{-} \alpha=0.003$ \\
\hline${ }^{214} \mathrm{Po}$ & -4469.9 & 1.5 & & & & 164.3 & $\mu \mathrm{s}$ & 2.0 & $0^{+}$ & 95 & & & $\alpha=100$ \\
\hline${ }^{214} \mathrm{At}$ & -3380 & 4 & & & & 558 & ns & 10 & $1^{-}$ & 95 & & & $\alpha=100$ \\
\hline${ }^{214} \mathrm{At}^{m}$ & -3320 & 8 & 59 & 9 & $\mathrm{AD}$ & 268 & ns & & & & & & \\
\hline${ }^{214} \mathrm{At}^{n}$ & -3146 & 5 & 234 & 6 & $\mathrm{AD}$ & 760 & $\mathrm{~ns}$ & & $9^{-}$ & & & & \\
\hline${ }^{214} \mathrm{Rn}$ & -4320 & 9 & & & & 270 & ns & 20 & $0^{+}$ & 95 & & & $\alpha=100 ; 2 \beta^{+} ?$ \\
\hline${ }^{214} \mathrm{Rn}^{m}$ & -2695 & 9 & 1625.1 & 0.5 & & 6.5 & $\mathrm{~ns}$ & 3.0 & $8^{+}$ & & & & \\
\hline${ }^{214} \mathrm{Fr}$ & -958 & 9 & & & & 5.0 & $\mathrm{~ms}$ & 0.2 & $\left(1^{-}\right)$ & 95 & & & $\alpha=100$ \\
\hline${ }^{214} \mathrm{Fr}^{m}$ & -835 & 9 & 123 & 6 & $\mathrm{AD}$ & 3.35 & $\mathrm{~ms}$ & 0.05 & $\left(8^{-}\right)$ & 95 & & & $\alpha=100$ \\
\hline${ }^{214} \mathrm{Ra}$ & 101 & 9 & & & & 2.46 & $\mathrm{~s}$ & 0.03 & $0^{+}$ & 95 & & & $\alpha \approx 100 ; \beta^{+}=0.0594$ \\
\hline${ }^{214} \mathrm{Ac}$ & 6429 & 22 & & & & 8.2 & $\mathrm{~s}$ & 0.2 & $5^{+} \#$ & 95 & & & $\alpha \geq 893 ; \beta^{+} \leq 113$ \\
\hline${ }^{214} \mathrm{Th}$ & 10712 & 17 & & & & 100 & $\mathrm{~ms}$ & 25 & $0^{+}$ & 95 & & & $\alpha \approx 100 ; \beta^{+}=0.1 \#$ \\
\hline${ }^{214} \mathrm{~Pa}$ & 19490 & 80 & & & & 17 & $\mathrm{~ms}$ & 3 & & 95 & $95 \mathrm{Ni} 05$ & $\mathrm{D}$ & $\alpha=100$ \\
\hline${ }^{215} \mathrm{~Pb}$ & $4480 \#$ & $410 \#$ & & & & 36 & $\mathrm{~s}$ & 1 & $5 / 2^{+} \#$ & & 96Ry.B & $\mathrm{T}$ & $\beta^{-}=100$ \\
\hline${ }^{215} \mathrm{Bi}$ & 1649 & 15 & & & & 7.6 & $\mathrm{~m}$ & 0.2 & $\left(9 / 2^{-}\right)$ & 01 & & & $\beta^{-}=100$ \\
\hline${ }^{215} \mathrm{Bi}^{m}$ & 2997 & 15 & 1347.5 & 2.5 & & 36.4 & $\mathrm{~m}$ & 2.5 & $\left(25 / 2^{-}\right)$ & 01 & 02Fr.B & $\mathrm{D}$ & $\mathrm{IT}=? ; \beta^{-}=$? \\
\hline${ }^{215} \mathrm{Po}$ & -540.3 & 2.5 & & & & 1.781 & $\mathrm{~ms}$ & 0.004 & $9 / 2^{+}$ & 01 & & & $\alpha=100 ; \beta^{-}=2.3 \mathrm{e}-42$ \\
\hline${ }^{215} \mathrm{At}$ & -1255 & 7 & & & & 100 & $\mu \mathrm{s}$ & 20 & $9 / 2^{-}$ & 01 & & & $\alpha=100$ \\
\hline${ }^{215} \mathrm{Rn}$ & -1169 & 8 & & & & 2.30 & $\mu \mathrm{s}$ & 0.10 & $9 / 2^{+}$ & 01 & & & $\alpha=100$ \\
\hline${ }^{215} \mathrm{Fr}$ & 318 & 7 & & & & 86 & ns & 5 & $9 / 2^{-}$ & 01 & & & $\alpha=100$ \\
\hline${ }^{215} \mathrm{Ra}$ & 2534 & 8 & & & & 1.55 & $\mathrm{~ms}$ & 0.07 & $9 / 2^{+} \#$ & 01 & & & $\alpha=100$ \\
\hline${ }^{215} \mathrm{Ra}^{m}$ & 4412 & 8 & 1877.8 & 0.5 & & 7.1 & $\mu \mathrm{s}$ & 0.2 & $\left(25 / 2^{+}\right)$ & 01 & & & $\mathrm{IT}=100$ \\
\hline${ }^{215} \mathrm{Ra}^{n}$ & 4781 & 8 & 2246.9 & 0.5 & & 1.39 & $\mu \mathrm{s}$ & 0.07 & $\left(29 / 2^{-}\right)$ & 01 & & & $\mathrm{IT}=100$ \\
\hline${ }^{215} \mathrm{Ac}$ & 6012 & 21 & & & & 170 & $\mathrm{~ms}$ & 10 & $9 / 2^{-}$ & 01 & & & $\alpha \approx 100 ; \beta^{+}=0.092$ \\
\hline${ }^{215} \mathrm{Th}$ & 10927 & 27 & & & & 1.2 & $\mathrm{~s}$ & 0.2 & $\left(1 / 2^{-}\right)$ & 01 & & & $\alpha=100$ \\
\hline${ }^{215} \mathrm{~Pa}$ & 17870 & 90 & & & & 14 & $\mathrm{~ms}$ & 2 & $9 / 2^{-} \#$ & 01 & & & $\alpha=100$ \\
\hline${ }^{215} \mathrm{~Pb}$ & $\mathrm{~T}$ : othe & relin & y re & 1t 02 & $\mathrm{Fr} . \mathrm{B}=$ & (12) s & & & & & & & \\
\hline${ }^{215} \mathrm{Bi}^{m}$ & $\mathrm{~T}:$ othe & elim & y resu & It $02 \mathrm{~F}$ & 11 & $9(0.6) \mathrm{s}$ & & & & & & & \\
\hline
\end{tabular}




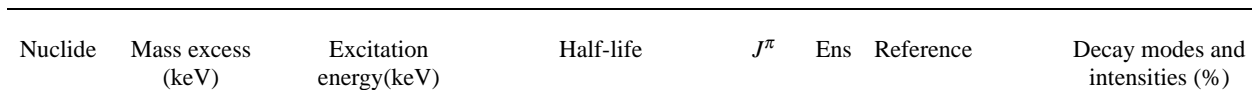

\begin{tabular}{|c|c|c|c|c|c|c|c|c|c|c|c|}
\hline${ }^{219} \mathrm{Po}$ & $12800 \#$ & $360 \#$ & $2 \#$ & $\mathrm{~m}$ & $(>300 \mathrm{~ns})$ & $7 / 2^{+} \#$ & & 98Pf02 & I & $\beta^{-} ? ; \alpha ?$ & \\
\hline${ }^{219} \mathrm{At}$ & 10397 & 4 & 56 & $\mathrm{~s}$ & 3 & $5 / 2^{-} \#$ & 01 & & & $\alpha \approx 97 ; \beta^{-} \approx 3$ & \\
\hline${ }^{219} \mathrm{Rn}$ & 8830.8 & 2.5 & 3.96 & $\mathrm{~s}$ & 0.01 & $5 / 2^{+}$ & 01 & & & $\alpha=100$ & \\
\hline${ }^{219} \mathrm{Fr}$ & 8618 & 7 & 20 & $\mathrm{~ms}$ & 2 & $9 / 2^{-}$ & 01 & & & $\alpha=100$ & \\
\hline${ }^{219} \mathrm{Ra}$ & 9394 & 8 & 10 & $\mathrm{~ms}$ & 3 & $(7 / 2)^{+}$ & 01 & & & $\alpha=100$ & \\
\hline${ }^{219} \mathrm{Ac}$ & 11570 & 50 & 11.8 & $\mu \mathrm{s}$ & 1.5 & $9 / 2^{-}$ & 01 & & & $\alpha=100 ; \beta^{+}=1 \mathrm{e}-6 \#$ & \\
\hline${ }^{219} \mathrm{Th}$ & 14470 & 50 & 1.05 & $\mu \mathrm{s}$ & 0.03 & $9 / 2^{+} \#$ & 01 & & & $\alpha=100 ; \beta^{+}=1 \mathrm{e}-7 \#$ & \\
\hline${ }^{219} \mathrm{~Pa}$ & 18520 & 50 & 53 & ns & 10 & $9 / 2^{-}$ & 01 & & & $\alpha=100 ; \beta^{+}=5 \mathrm{e}-9 \#$ & \\
\hline${ }^{219} \mathrm{U}$ & 23210 & 60 & 55 & $\mu \mathrm{s}$ & 25 & $9 / 2^{+} \#$ & 01 & & & $\alpha=100 ; \beta^{+}=1.4 \mathrm{e}-5 \#$ & \\
\hline${ }^{220} \mathrm{Po}$ & 15470\# & $360 \#$ & $40 \#$ & $\mathrm{~s}$ & $(>300 \mathrm{~ns})$ & $0^{+}$ & & 98Pf02 & I & $\beta^{-} ?$ & \\
\hline${ }^{220} \mathrm{At}$ & 14350 & 50 & 3.71 & $\mathrm{~m}$ & 0.04 & $3^{(-\#)}$ & 97 & & & $\beta^{-}=922 ; \alpha=82$ & \\
\hline${ }^{220} \mathrm{Rn}$ & 10613.4 & 2.2 & 55.6 & $\mathrm{~s}$ & 0.1 & $0^{+}$ & 97 & & & $\alpha=100 ; 2 \beta^{-} ?$ & \\
\hline${ }^{220} \mathrm{Fr}$ & 11483 & 4 & 27.4 & $\mathrm{~s}$ & 0.3 & $1^{+}$ & 97 & & & $\alpha \approx 100 ; \beta^{-}=0.355$ & \\
\hline${ }^{220} \mathrm{Ra}$ & 10273 & 9 & 17.9 & $\mathrm{~ms}$ & 1.4 & $0^{+}$ & 97 & $00 \mathrm{He} 17$ & $\mathrm{~T}$ & $\alpha=100$ & * \\
\hline${ }^{220} \mathrm{Ac}$ & 13752 & 15 & 26.36 & $\mathrm{~ms}$ & 0.19 & $\left(3^{-}\right)$ & 97 & 90An19 & $\mathrm{T}$ & $\alpha=100 ; \beta^{+}=5 \mathrm{e}-4 \#$ & * \\
\hline${ }^{220} \mathrm{Th}$ & 14669 & 22 & 9.7 & $\mu \mathrm{s}$ & 0.6 & $0^{+}$ & 97 & & & $\alpha=100 ; \varepsilon=2 \mathrm{e}-7 \#$ & \\
\hline${ }^{220} \mathrm{~Pa}$ & 20380 & 60 & 780 & ns & 160 & $1^{-} \#$ & 97 & & & $\alpha=100 ; \beta^{+}=3 \mathrm{e}-7 \#$ & \\
\hline${ }^{220} \mathrm{U}$ & $23030 \#$ & $200 \#$ & $60 \#$ & ns & & $0^{+}$ & & & & $\alpha ? ; \beta^{+} ?$ & \\
\hline${ }^{220} \mathrm{Ra}$ & $\mathrm{T}:$ avera & ge $00 \mathrm{H}$ & 7(2) 611 & $06=$ & $=23(5)$ & & & & & & $* *$ \\
\hline${ }^{220} \mathrm{Ac}$ & $\mathrm{T}:$ avera & ge $90 \mathrm{Al}$ & $=26.1($ & & & & & & & & ** \\
\hline
\end{tabular}

\begin{tabular}{|c|c|c|c|c|c|c|c|c|c|c|}
\hline${ }^{221} \mathrm{At}$ & 16810\# & $200 \#$ & 2.3 & $\mathrm{~m}$ & 0.2 & $3 / 2^{-} \#$ & 90 & & & $\beta^{-}=100$ \\
\hline${ }^{221} \mathrm{Rn}$ & 14472 & 6 & 25 & $\mathrm{~m}$ & 2 & $7 / 2^{(+)}$ & 90 & & & $\beta^{-}=781 ; \alpha=221$ \\
\hline${ }^{221} \mathrm{Fr}$ & 13278 & 5 & 4.9 & $\mathrm{~m}$ & 0.2 & $5 / 2^{-}$ & 90 & 97Ch53 & $\mathrm{D}$ & $\alpha \approx 100 ; \beta^{-}=0.004815 ; \ldots$ \\
\hline${ }^{221} \mathrm{Ra}$ & 12964 & 5 & 28 & $\mathrm{~s}$ & 2 & $5 / 2^{+}$ & 90 & $94 \mathrm{Bo} 28$ & $\mathrm{D}$ & $\alpha=100 ;{ }^{14} \mathrm{C}=1.2 \mathrm{e}-109$ \\
\hline${ }^{221} \mathrm{Ac}$ & 14520 & 50 & 52 & $\mathrm{~ms}$ & 2 & $9 / 2^{-} \#$ & 90 & & & $\alpha=100$ \\
\hline${ }^{221} \mathrm{Th}$ & 16938 & 9 & 1.68 & $\mathrm{~ms}$ & 0.06 & $\left(7 / 2^{+}\right)$ & 90 & & & $\alpha=100$ \\
\hline${ }^{221} \mathrm{~Pa}$ & 20380 & 50 & 5.9 & $\mu \mathrm{s}$ & 1.7 & $9 / 2^{-}$ & 90 & & & $\alpha=100$ \\
\hline${ }^{221} \mathrm{U}$ & 24590\# & $100 \#$ & 700\# & ns & & $9 / 2^{+} \#$ & & & & $\alpha ? ; \beta^{+} ?$ \\
\hline${ }^{221} \mathrm{Fr}$ & $\mathrm{D}: \ldots ;$ & ${ }^{14} \mathrm{C}=8.8 \mathrm{e}-1111$ & & & & & & & & \\
\hline
\end{tabular}

$*^{221} \mathrm{Fr} \quad \mathrm{D}: \beta^{-}$intensity is from $97 \mathrm{Ch} 53 ;{ }^{14} \mathrm{C}$ intensity is from $94 \mathrm{Bo} 28$

$*^{221} \mathrm{Th} \quad \mathrm{T}$ : also $00 \mathrm{He} 17=2.0(+0.3-0.2)$

\begin{tabular}{|c|c|c|c|c|c|c|c|}
\hline${ }^{222} \mathrm{At}$ & 20800\# & $300 \#$ & & & & 54 & $\mathrm{~s}$ \\
\hline${ }^{222} \mathrm{Rn}$ & 16373.6 & 2.4 & & & & 3.8235 & $\mathrm{~d}$ \\
\hline${ }^{222} \mathrm{Fr}$ & 16349 & 21 & & & & 14.2 & $\mathrm{~m}$ \\
\hline${ }^{222} \mathrm{Ra}$ & 14321 & 5 & & & & 38.0 & $\mathrm{~s}$ \\
\hline${ }^{222} \mathrm{Ac}$ & 16621 & 5 & & & * & 5.0 & $\mathrm{~s}$ \\
\hline${ }^{222} \mathrm{Ac}^{m}$ & $16820 \#$ & $150 \#$ & 200\# & $150 \#$ & $*$ & 1.05 & $\mathrm{~m}$ \\
\hline${ }^{222} \mathrm{Th}$ & 17203 & 12 & & & & 2.05 & $\mathrm{~ms}$ \\
\hline${ }^{222} \mathrm{~Pa}$ & 22120\# & $70 \#$ & & & & 3.2 & $\mathrm{~ms}$ \\
\hline${ }^{222} \mathrm{U}$ & $24300 \#$ & $100 \#$ & & & & 1.4 & $\mu \mathrm{s}$ \\
\hline$*^{222} \mathrm{Ac}^{m}$ & \multicolumn{7}{|c|}{$\mathrm{D}:$ derived from $0.7 \%<\beta^{+}<2 \%$, in ENSDF } \\
\hline$*^{222} \mathrm{Th}$ & \multicolumn{7}{|c|}{$\mathrm{T}:$ average $00 \mathrm{He} 17=2.0(0.1) 99 \mathrm{Gr} 28=2.1(0.1)$} \\
\hline$*^{222} \mathrm{~Pa}$ & \multicolumn{7}{|c|}{$\mathrm{T}:$ average $95 \mathrm{Ni} . \mathrm{A}=3.3(0.3) 79 \mathrm{Sc} 09=2.9(+0.6-0.4)$} \\
\hline$*^{222} \mathrm{~Pa}$ & \multicolumn{7}{|c|}{$\mathrm{T}: 70 \mathrm{Bo} 13=5.7(0.5)$ at variance, not used } \\
\hline
\end{tabular}

$\begin{array}{ccc}10 & & 96 \\ 0.0003 & 0^{+} & 96 \\ 0.3 & 2^{-} & 96 \\ 0.5 & 0^{+} & 96 \\ 0.5 & 1^{-} & 96 \\ 0.07 & \text { high } & 96 \\ 0.07 & 0^{+} & 96 \\ 0.3 & & 96 \\ 0.7 & 0^{+} & 9\end{array}$

$\begin{array}{lll} & & \\ 6 & & \\ 6 & & \\ 6 & 00 \mathrm{He} 17 & \mathrm{~T} \\ & 95 \mathrm{Ni} . \mathrm{A} & \mathrm{T}\end{array}$

$\beta^{-}=100$

$\alpha=100$

$\beta^{-}=100$

$\alpha=100 ;{ }^{14} \mathrm{C}=3.0 \mathrm{e}-810$

$\alpha=991 ; \beta^{+}=11$

$\alpha=$ ?; IT $<10 ; \beta^{+}=1.44$

$\alpha=100 ; \varepsilon<1.3 \mathrm{e}-8 \#$

$\alpha=100$

$\alpha=100 ; \beta^{+}<1 \mathrm{e}-6 \#$

$\begin{array}{llr}{ }^{223} \mathrm{At} & 23460 \# & 400 \# \\ { }^{223} \mathrm{Rn} & 20300 \# & 300 \# \\ { }^{223} \mathrm{Fr} & 18383.8 & 2.4 \\ { }^{223} \mathrm{Ra} & 17234.7 & 2.5 \\ { }^{223} \mathrm{Ac} & 17826 & 7 \\ { }^{223} \mathrm{Th} & 19386 & 9 \\ { }^{223} \mathrm{~Pa} & 22320 & 70 \\ { }^{223} \mathrm{U} & 25840 & 70\end{array}$

$\begin{array}{ccccc}50 & \mathrm{~s} & 7 & 3 / 2^{-} \# & 01 \\ 24.3 & \mathrm{~m} & 0.4 & 7 / 2 & 01 \\ 22.00 & \mathrm{~m} & 0.07 & 3 / 2^{(-)} & 01 \\ 11.43 & \mathrm{~d} & 0.05 & 3 / 2^{+} & 01 \\ 2.10 & \mathrm{~m} & 0.05 & \left(5 / 2^{-}\right) & 01 \\ 600 & \mathrm{~ms} & 20 & (5 / 2)^{+} & 01 \\ 5.1 & \mathrm{~ms} & 0.3 & 9 / 2^{-} \# & 01 \\ 21 & \mu \mathrm{s} & 8 & 7 / 2^{+} \# & 01\end{array}$

$\beta^{-} \approx 100 ; \alpha=0.008 \#$

$\beta^{-}=100 ; \alpha=0.0004 \#$

$\beta^{-} \approx 100 ; \alpha=0.006$

$\alpha=100 ;{ }^{14} \mathrm{C}=8.9 \mathrm{e}-84$

$\alpha=99 ; \varepsilon=1$

$\alpha=100$

99Ho28 Т $\quad \alpha=100 ; \beta^{+}<0.001 \#$

$*^{223} \mathrm{~Pa} \quad \mathrm{~T}:$ average $99 \mathrm{Ho} 28=4.9(0.4) 95 \mathrm{Ni} . \mathrm{A}=5.0(1.0) 70 \mathrm{Bo} 13=6.5(1.0)$

$\alpha \approx 100 ; \beta^{+}=0.2 \#$ 


\begin{tabular}{|c|c|c|c|c|c|c|c|c|c|c|c|c|}
\hline Nuclide & \multicolumn{2}{|c|}{$\begin{array}{l}\text { Mass excess } \\
\quad(\mathrm{keV})\end{array}$} & $\begin{array}{l}\text { Excitation } \\
\text { energy }(\mathrm{keV})\end{array}$ & \multicolumn{3}{|c|}{ Half-life } & $J^{\pi}$ & Ens & \multicolumn{2}{|c|}{ Reference } & \multicolumn{2}{|l|}{$\begin{array}{l}\text { Decay modes and } \\
\text { intensities }(\%)\end{array}$} \\
\hline${ }^{224} \mathrm{Rn}$ & 22440\# & $300 \#$ & & 107 & $\mathrm{~m}$ & 3 & $0^{+}$ & 97 & & & $\beta^{-}=100$ & \\
\hline${ }^{224} \mathrm{Fr}$ & 21660 & 50 & & 3.33 & $\mathrm{~m}$ & 0.10 & $1^{-}$ & 97 & & & $\beta^{-}=100$ & \\
\hline${ }^{224} \mathrm{Ra}$ & 18827.2 & 2.2 & & 3.66 & $\mathrm{~d}$ & 0.04 & $0^{+}$ & 97 & & & $\alpha=100 ;{ }^{14} \mathrm{C}=4.0 \mathrm{e}-912$ & \\
\hline${ }^{224} \mathrm{Ac}$ & 20235 & 4 & & 2.78 & $\mathrm{~h}$ & 0.17 & $0^{-}$ & 97 & & & $\beta^{+}=90.617 ; \alpha=9.417 ; \beta^{-}<1.6 \#$ & \\
\hline${ }^{224} \mathrm{Th}$ & 19996 & 11 & & 1.05 & s & 0.02 & $0^{+}$ & 97 & & & $\alpha=100 ; 2 \beta^{+} ?$ & \\
\hline${ }^{224} \mathrm{~Pa}$ & 23870 & 16 & & 844 & $\mathrm{~ms}$ & 19 & $5^{-} \#$ & 97 & $96 \mathrm{Li} 05$ & $\mathrm{~T}$ & $\alpha \approx 100 ; \beta^{+}=0.1 \#$ & $*$ \\
\hline${ }^{224} U$ & 25714 & 25 & & 940 & $\mu \mathrm{s}$ & 270 & $0^{+}$ & 97 & $92 \mathrm{To} 02$ & $\mathrm{~T}$ & $\alpha=100 ; \beta^{+}<1.2 \mathrm{e}-4 \#$ & * \\
\hline$*^{224} \mathrm{~Pa}$ & $\mathrm{~T}:$ averas & ge $96 \mathrm{Li}$ & $=790(60) 96 \mathrm{~W}$ & $=850(2$ & & & & & & & & ** \\
\hline$*^{224} \mathrm{U}$ & $\mathrm{T}:$ averas & ge $92 \mathrm{To}$ & $=1000(400) 91$ & $10=70$ & $0(+50$ & $-200)$ & & & & & & ** \\
\hline
\end{tabular}

$\begin{array}{lllllllllll}{ }^{225} \mathrm{Rn} & 26490 \# & 300 \# & 4.66 & \mathrm{~m} & 0.04 & 7 / 2^{-} & 90 & 97 \mathrm{Bu} 03 & \mathrm{~T} & \beta^{-}=100 \\ { }^{225} \mathrm{Fr} & 23810 & 30 & 4.0 & \mathrm{~m} & 0.2 & 3 / 2^{-} & 90 & & & \beta^{-}=100 \\ { }^{225} \mathrm{Ra} & 21994.0 & 3.0 & 14.9 & \mathrm{~d} & 0.2 & 1 / 2^{+} & 90 & & & \beta^{-}=100 \\ { }^{225} \mathrm{Ac} & 21638 & 5 & 10.0 & \mathrm{~d} & 0.1 & \left(3 / 2^{-}\right) & 90 & 93 \mathrm{Bo} 26 & \mathrm{D} & \alpha=100 ;{ }^{14} \mathrm{C}=6.0 \mathrm{e}-1013 \\ { }^{225} \mathrm{Th} & 22310 & 5 & 8.72 & \mathrm{~m} & 0.04 & (3 / 2)^{+} & 90 & & & \alpha \approx 90 ; \varepsilon \approx 10 \\ { }^{225} \mathrm{~Pa} & 24340 & 70 & 1.7 & \mathrm{~s} & 0.2 & 5 / 2^{-} \# & 90 & & & \alpha=100 \\ { }^{225} \mathrm{U} & 27377 & 12 & 61 & \mathrm{~ms} & 4 & 5 / 2^{+} \# & 90 & 00 \mathrm{He} 17 & \mathrm{~T} & \alpha=100 \\ { }^{225} \mathrm{~Np} & 31590 & 70 & 3 \# & \mathrm{~ms}(>2 \mu \mathrm{s}) & 9 / 2^{-} \# & 97 & 94 \mathrm{Ye} 08 & \mathrm{ID} & \alpha=100 \\ *^{225} \mathrm{U} & \mathrm{T}: 00 \mathrm{He} 17=59(+5-2) ; \text { others } 94 \mathrm{An} 02=68(+45-20) & 92 \mathrm{To} 02=95(15) \text { and } & & & \\ *^{225} \mathrm{U} & \mathrm{T}: \quad 89 \mathrm{He} 13=80(+40-10) \text { outweighed, not used } & & & & \end{array} * *$

\begin{tabular}{|c|c|c|c|c|c|c|c|c|c|c|}
\hline${ }^{226} \mathrm{Rn}$ & $28770 \#$ & $400 \#$ & 7.4 & $\mathrm{~m}$ & 0.1 & $0^{+}$ & 96 & & & $\beta^{-}=100$ \\
\hline${ }^{226} \mathrm{Fr}$ & 27370 & 100 & 49 & $\mathrm{~s}$ & 1 & $1^{-}$ & 96 & & & $\beta^{-}=100$ \\
\hline${ }^{226} \mathrm{Ra}$ & 23669.1 & 2.3 & 1.600 & ky & 0.007 & $0^{+}$ & 96 & 90We01 & $\mathrm{D}$ & $\alpha=100 ;{ }^{14} \mathrm{C}=2.6 \mathrm{e}-96 ; 2 \beta^{-} ?$ \\
\hline${ }^{226} \mathrm{Ac}$ & 24310 & 3 & 29.37 & $\mathrm{~h}$ & 0.12 & $(1)^{(-\#)}$ & 96 & & & $\beta^{-}=833 ; \varepsilon=173 ; \alpha=0.0062$ \\
\hline${ }^{226} \mathrm{Th}$ & 23197 & 5 & 30.57 & $\mathrm{~m}$ & 0.10 & $0^{+}$ & 96 & 01Bo11 & D & $\alpha=100 ;{ }^{18} \mathrm{O}<3.2 \mathrm{e}-12$ \\
\hline${ }^{226} \mathrm{~Pa}$ & 26033 & 11 & 1.8 & $\mathrm{~m}$ & 0.2 & & 96 & & & $\alpha=745 ; \beta^{+}=265$ \\
\hline${ }^{226} \mathrm{U}$ & 27329 & 13 & 269 & $\mathrm{~ms}$ & 6 & $0^{+}$ & 96 & 01Ca.B & $\mathrm{T}$ & $\alpha=100$ \\
\hline${ }^{226} \mathrm{~Np}$ & $32740 \#$ & $90 \#$ & 35 & $\mathrm{~ms}$ & 10 & & 96 & & & $\alpha=100 ; \beta^{+}=0.003 \#$ \\
\hline$*^{226} \mathrm{Ra}$ & $\mathrm{D}:{ }^{14} \mathrm{C}:$ & average & $6 \mathrm{Ba} 26=$ & $2.9(1$ & .0) $85 \mathrm{H}$ & $21=3.2($ & & & & \\
\hline${ }^{226} \mathrm{U}$ & $\mathrm{T}:$ aver & ge $01 \mathrm{C}$ & $7=281(9$ & ) 990 & $\mathrm{j} 28=26$ & (10) & & & & \\
\hline
\end{tabular}

\begin{tabular}{|c|c|c|c|c|c|c|c|c|c|c|}
\hline${ }^{227} \mathrm{Rn}$ & 32980\# & $420 \#$ & 20.8 & $\mathrm{~s}$ & 0.7 & $5 / 2^{(+\#)}$ & 01 & $97 \mathrm{Ku} 20$ & $\mathrm{~J}$ & $\beta^{-}=100$ \\
\hline${ }^{227} \mathrm{Fr}$ & 29650 & 100 & 2.47 & $\mathrm{~m}$ & 0.03 & $1 / 2^{+}$ & 01 & & & $\beta^{-}=100$ \\
\hline${ }^{227} \mathrm{Ra}$ & 27179.0 & 2.4 & 42.2 & $\mathrm{~m}$ & 0.5 & $3 / 2^{+}$ & 01 & & & $\beta^{-}=100$ \\
\hline${ }^{227} \mathrm{Ac}$ & 25850.9 & 2.4 & 21.772 & $\mathrm{y}$ & 0.003 & $3 / 2^{-}$ & 01 & & & $\beta^{-}=98.6236 ; \alpha=1.3836$ \\
\hline${ }^{227} \mathrm{Th}$ & 25806.2 & 2.5 & 18.68 & $\mathrm{~d}$ & 0.09 & $1 / 2^{+}$ & 01 & & & $\alpha=100$ \\
\hline${ }^{227} \mathrm{~Pa}$ & 26832 & 7 & 38.3 & $\mathrm{~m}$ & 0.3 & $\left(5 / 2^{-}\right)$ & 01 & & & $\alpha=852 ; \varepsilon=152$ \\
\hline${ }^{227} \mathrm{U}$ & 29022 & 17 & 1.1 & $\mathrm{~m}$ & 0.1 & $\left(3 / 2^{+}\right)$ & 01 & & & $\alpha=100 ; \beta^{+}<0.001 \#$ \\
\hline${ }^{227} \mathrm{~Np}$ & 32560 & 70 & 510 & $\mathrm{~ms}$ & 60 & $5 / 2^{-} \#$ & 01 & & & $\alpha \approx 100 ; \beta^{+}=0.05 \#$ \\
\hline${ }^{228} \mathrm{Rn}$ & 35380\# & $410 \#$ & 65 & $\mathrm{~s}$ & 2 & $0^{+}$ & 97 & & & $\beta^{-}=100$ \\
\hline${ }^{228} \mathrm{Fr}$ & 33280\# & $200 \#$ & 38 & $\mathrm{~s}$ & 1 & $2^{-}$ & 97 & & & $\beta^{-}=100$ \\
\hline${ }^{228} \mathrm{Ra}$ & 28941.8 & 2.4 & 5.75 & $\mathrm{y}$ & 0.03 & $0^{+}$ & 97 & & & $\beta^{-}=100$ \\
\hline${ }^{228} \mathrm{Ac}$ & 28896.0 & 2.5 & 6.15 & $\mathrm{~h}$ & 0.02 & $3^{+}$ & 97 & & & $\beta^{-}=100$ \\
\hline${ }^{228} \mathrm{Th}$ & 26772.2 & 2.2 & 1.9116 & $\mathrm{y}$ & 0.0016 & $0^{+}$ & 97 & & & $\alpha=100 ;{ }^{20} \mathrm{O}=1.13 \mathrm{e}-1122$ \\
\hline${ }^{228} \mathrm{~Pa}$ & 28924 & 4 & 22 & $\mathrm{~h}$ & 1 & $3^{+}$ & 97 & & & $\beta^{+}=98.02 ; \alpha=2.02$ \\
\hline${ }^{228} \mathrm{U}$ & 29225 & 15 & 9.1 & $\mathrm{~m}$ & 0.2 & $0^{+}$ & 97 & & & $\alpha>95 ; \varepsilon<5$ \\
\hline${ }^{228} \mathrm{~Np}$ & 33700\# & $200 \#$ & 61.4 & $\mathrm{~s}$ & 1.4 & & 97 & $94 \mathrm{Kr} 13$ & $\mathrm{D}$ & $\varepsilon=607 ; \alpha=407 ; \beta^{+} \mathrm{SF}=0.0126$ \\
\hline${ }^{228} \mathrm{Pu}$ & 36090 & 30 & $10 \#$ & $\mathrm{~ms}$ & $(>2 \mu \mathrm{s})$ & $0^{+}$ & 97 & $94 \mathrm{An} 02$ & ID & $\alpha \approx 100 ; \beta^{+}=0.1 \#$ \\
\hline${ }^{228} \mathrm{~Np}$ & $\mathrm{D}: \beta^{+} \mathrm{S}$ & $=0.0$ & $\mathrm{r} 13 \mathrm{re}$ & lative & to $\varepsilon$, thu & 0.012 & $\%$ & f total & & \\
\hline
\end{tabular}




\begin{tabular}{|c|c|c|c|c|c|c|c|c|c|c|c|c|}
\hline Nuclide & \multicolumn{2}{|c|}{$\begin{array}{l}\text { Mass excess } \\
\quad(\mathrm{keV})\end{array}$} & \multicolumn{2}{|c|}{$\begin{array}{l}\text { Excitation } \\
\text { energy }(\mathrm{keV})\end{array}$} & \multicolumn{3}{|c|}{ Half-life } & $J^{\pi}$ & Ens & \multicolumn{2}{|c|}{ Reference } & $\begin{array}{l}\text { Decay modes and } \\
\text { intensities }(\%)\end{array}$ \\
\hline${ }^{229} \mathrm{Fr}$ & 35820 & 40 & & & 50.2 & $\mathrm{~s}$ & 0.4 & $1 / 2^{+} \#$ & 90 & 92Bo05 & $\mathrm{T}$ & $\beta^{-}=100$ \\
\hline${ }^{229} \mathrm{Ra}$ & 32563 & 19 & & & 4.0 & $\mathrm{~m}$ & 0.2 & $5 / 2^{(+)}$ & 90 & & & $\beta^{-}=100$ \\
\hline${ }^{229} \mathrm{Ac}$ & 30750 & 30 & & & 62.7 & $\mathrm{~m}$ & 0.5 & $\left(3 / 2^{+}\right)$ & 90 & & & $\beta^{-}=100$ \\
\hline${ }^{229} \mathrm{Th}$ & 29586.5 & 2.8 & & & 7.34 & ky & 0.16 & $5 / 2^{+}$ & 90 & & & $\alpha=100$ \\
\hline${ }^{229} \mathrm{Th}^{m}$ & 29586.5 & 2.8 & 0.0035 & 0.0010 & 70 & $\mathrm{~h}$ & 50 & $3 / 2^{+}$ & & $94 \mathrm{He} 08$ & TEJ & IT ? \\
\hline${ }^{229} \mathrm{~Pa}$ & 29898.0 & 2.7 & & & 1.50 & $\mathrm{~d}$ & 0.05 & $\left(5 / 2^{+}\right)$ & 90 & & & $\varepsilon \approx 100 ; \alpha=0.485$ \\
\hline${ }^{229} \mathrm{~Pa}^{m}$ & 29909.6 & 2.7 & 11.6 & 0.3 & 420 & ns & 30 & $3 / 2^{-}$ & & 98Le15 & EJD & $\mathrm{IT}=100$ \\
\hline${ }^{229} \mathrm{U}$ & 31211 & 6 & & & 58 & $\mathrm{~m}$ & 3 & $\left(3 / 2^{+}\right)$ & 90 & & & $\beta^{+} \approx 80 ; \alpha \approx 20$ \\
\hline${ }^{229} \mathrm{~Np}$ & 33780 & 90 & & & 4.0 & $\mathrm{~m}$ & 0.2 & $5 / 2^{+} \#$ & 90 & & & $\alpha>50 ; \beta^{+}<50$ \\
\hline${ }^{229} \mathrm{~Np}^{p}$ & 33850\# & $100 \#$ & 70\# & $50 \#$ & & & & $5 / 2^{-} \#$ & & & & \\
\hline${ }^{229} \mathrm{Pu}$ & 37400 & 50 & & & 120 & $\mathrm{~s}$ & 50 & $3 / 2^{+} \#$ & 97 & 01Ca.B & TD & $\alpha=100$ \\
\hline$*^{229} \mathrm{Th}^{m}$ & \multicolumn{12}{|c|}{ D : ultraviolet $\gamma$-ray emission assigned by $97 \mathrm{Ir} 02$ and $98 \mathrm{Ri} 03$ to IT decay is } \\
\hline$*^{229} \mathrm{Th}^{m}$ & \multicolumn{12}{|c|}{ D : proved by $99 \mathrm{Sh} 12$ to be due to $\mathrm{N}_{2}$ discharge emission. $99 \mathrm{Ut} 01$ sees } \\
\hline$*^{229} \mathrm{Th}^{m}$ & \multicolumn{12}{|c|}{ D : no UV in vacuo. } \\
\hline
\end{tabular}

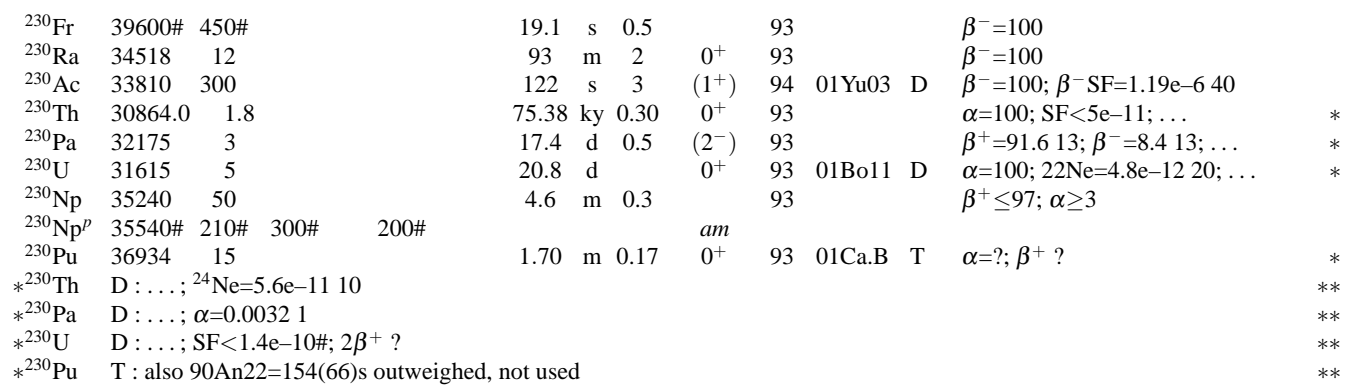

\begin{tabular}{|c|c|c|c|c|c|c|c|c|c|c|c|c|c|}
\hline${ }^{231} \mathrm{Fr}$ & 42330\# & $470 \#$ & & & 17.6 & $\mathrm{~s}$ & 0.6 & $1 / 2^{+} \#$ & 01 & & & $\beta^{-}=100$ & \\
\hline${ }^{231} \mathrm{Ra}$ & 38400\# & $300 \#$ & & & 103 & $\mathrm{~s}$ & 3 & $\left(5 / 2^{+}\right)$ & 01 & & & $\beta^{-}=100$ & \\
\hline${ }^{231} \mathrm{Ra}^{m}$ & 38470\# & $300 \#$ & 66.21 & 0.09 & 53 & $\mu \mathrm{s}$ & & $\left(1 / 2^{+}\right)$ & 01 & & & $\mathrm{IT}=100$ & \\
\hline${ }^{231} \mathrm{Ac}$ & 35920 & 100 & & & 7.5 & $\mathrm{~m}$ & 0.1 & $\left(1 / 2^{+}\right)$ & 01 & & & $\beta^{-}=100$ & \\
\hline${ }^{231} \mathrm{Th}$ & 33817.3 & 1.8 & & & 25.52 & $\mathrm{~h}$ & 0.01 & $5 / 2^{+}$ & 01 & & & $\beta^{-}=100 ; \alpha=4 \mathrm{e}-11 \#$ & \\
\hline${ }^{231} \mathrm{~Pa}$ & 33425.7 & 2.3 & & & 32.76 & ky & 0.11 & $3 / 2^{-}$ & 01 & & & $\alpha=100 ; \mathrm{SF} \leq 3 \mathrm{e}-10 ; \ldots$ & $*$ \\
\hline${ }^{231} \mathrm{U}$ & 33807 & 3 & & & 4.2 & d & 0.1 & $(5 / 2)^{(+\#)}$ & 01 & & & $\varepsilon \approx 100 ; \alpha=0.0041$ & \\
\hline${ }^{231} \mathrm{~Np}$ & 35630 & 50 & & & 48.8 & $\mathrm{~m}$ & 0.2 & $(5 / 2)^{(+\#)}$ & 01 & & & $\beta^{+}=981 ; \alpha=21$ & \\
\hline${ }^{231} \mathrm{~Np}^{p}$ & 35690\# & $60 \#$ & $60 \#$ & $40 \#$ & & & & $5 / 2^{-} \#$ & & & & & \\
\hline${ }^{231} \mathrm{Pu}$ & 38285 & 26 & & & 8.6 & $\mathrm{~m}$ & 0.5 & $3 / 2^{+} \#$ & 01 & 99La14 & $\mathrm{D}$ & $\beta^{+}=875 ; \alpha=135$ & \\
\hline${ }^{231} \mathrm{Am}$ & 42440\# & $300 \#$ & & & $30 \#$ & $\mathrm{~s}$ & & & & & & $\beta^{+} ? ; \alpha ?$ & \\
\hline$*^{231} \mathrm{~Pa}$ & $\mathrm{D}: \ldots$ & ${ }^{24} \mathrm{Ne}=1$ & $3.4 \mathrm{e}-10$ & ${ }^{23} \mathrm{~F}=9.9 \mathrm{e}-13$ & & & & & & & & & $* *$ \\
\hline${ }^{232} \mathrm{Fr}$ & $46360 \#$ & $640 \#$ & & & 5 & s & 1 & & 97 & $90 \mathrm{Me} 13$ & $\mathrm{~T}$ & $\beta^{-}=100$ & \\
\hline${ }^{232} \mathrm{Ra}$ & 40650\# & $280 \#$ & & & 250 & $\mathrm{~s}$ & 50 & $0^{+}$ & 91 & & & $\beta^{-}=100$ & \\
\hline${ }^{232} \mathrm{Ac}$ & 39150 & 100 & & & 119 & $\mathrm{~s}$ & 5 & $\left(1^{+}\right)$ & 91 & & & $\beta^{-}=100$ & \\
\hline${ }^{232} \mathrm{Th}$ & 35448.3 & 2.0 & & & 14.05 & Gy & 0.06 & $0^{+}$ & 91 & 95Bo18 & $\mathrm{D}$ & $\mathrm{IS}=100 . ; \alpha=100 ; \mathrm{SF}=11 \mathrm{e}-103 ;$ & $*$ \\
\hline${ }^{232} \mathrm{~Pa}$ & 35948 & 8 & & & 1.31 & d & 0.02 & $\left(2^{-}\right)$ & 91 & & & $\beta^{-} \approx 100 ; \varepsilon=0.0031$ & \\
\hline${ }^{232} \mathrm{U}$ & 34610.7 & 2.2 & & & 68.9 & $\mathrm{y}$ & 0.4 & $0^{+}$ & 91 & 90 Bo16 & $\mathrm{D}$ & $\alpha=100 ;{ }^{24} \mathrm{Ne}=8.9 \mathrm{e}-107 ;$. & $*$ \\
\hline${ }^{232} \mathrm{~Np}$ & 37360\# & $100 \#$ & & & 14.7 & $\mathrm{~m}$ & 0.3 & $\left(4^{+}\right)$ & 91 & & & $\beta^{+} \approx 100 ; \alpha \approx 0.003$ & \\
\hline${ }^{232} \mathrm{Pu}$ & 38366 & 18 & & & 33.7 & $\mathrm{~m}$ & 0.5 & $0^{+}$ & 91 & ABBW & $\mathrm{D}$ & $\varepsilon=? ; \alpha=11 \#$ & $*$ \\
\hline${ }^{232} \mathrm{Am}$ & $43400 \#$ & $300 \#$ & & & 1.31 & $\mathrm{~m}$ & 0.04 & & 91 & & & $\beta^{+}=? ; \alpha=2 \# ; \beta^{+} \mathrm{SF}=0.06910$ & \\
\hline$*^{232} \mathrm{Th}$ & $\mathrm{D}: \ldots$ & ${ }^{24} \mathrm{Ne}+{ }^{26}$ & $\mathrm{Ne}<2.7$ & $-10 ; 2 \beta^{-} ?$ & & & & & & & & & $* *$ \\
\hline$*^{232} \mathrm{U}$ & $\mathrm{D}: \ldots$ & ${ }^{28} \mathrm{Mg}<$ & $5 e-12 ; S$ & $1 e-12$ & & & & & & & & & $* *$ \\
\hline$*^{232} \mathrm{U}$ & D : ${ }^{24} \mathrm{Ne}$ & : avera & e, as ado & ed by $91 \mathrm{Bo} 2$ & 0 , of 2 & resul & lts fro & m their & roup & & & & $* *$ \\
\hline$*^{232} \mathrm{Pu}$ & $\mathrm{T}:$ avera & age $00 \mathrm{~L}$ & $25=33.1$ & 8) $73 \mathrm{Ja} 06=3$ & $4.1(0.7$ & & & & & & & & $* *$ \\
\hline$*{ }^{232} \mathrm{Pu}$ & $\mathrm{D}:$ deriv & ved from & $1.6 \% \#$ & $\alpha<20 \% \#$, il & n ENSD & & & & & & & & $* *$ \\
\hline
\end{tabular}




\begin{tabular}{|c|c|c|c|c|c|c|c|c|c|c|c|c|}
\hline Nuclide & \multicolumn{2}{|c|}{$\begin{array}{l}\text { Mass excess } \\
\quad(\mathrm{keV})\end{array}$} & \multicolumn{2}{|c|}{$\begin{array}{l}\text { Excitation } \\
\text { energy }(\mathrm{keV})\end{array}$} & \multicolumn{3}{|c|}{ Half-life } & $J^{\pi}$ & Ens & \multicolumn{2}{|c|}{ Reference } & $\begin{array}{l}\text { Decay modes and } \\
\text { intensities }(\%)\end{array}$ \\
\hline${ }^{233} \mathrm{Ra}$ & 44770\# & $470 \#$ & & & 30 & s & 5 & $1 / 2^{+} \#$ & 97 & $90 \mathrm{Me} 13$ & $\mathrm{~T}$ & $\beta^{-}=100$ \\
\hline${ }^{233} \mathrm{Ac}$ & 41500\# & $300 \#$ & & & 145 & s & 10 & $\left(1 / 2^{+}\right)$ & 90 & & & $\beta^{-}=100$ \\
\hline${ }^{233} \mathrm{Th}$ & 38733.2 & 2.0 & & & 22.3 & $\mathrm{~m}$ & 0.1 & $1 / 2^{+}$ & 90 & & & $\beta^{-}=100$ \\
\hline${ }^{233} \mathrm{~Pa}$ & 37490.1 & 2.2 & & & 26.967 & $\mathrm{~d}$ & 0.002 & $3 / 2^{-}$ & 90 & & & $\beta^{-}=100$ \\
\hline${ }^{233} \mathrm{U}$ & 36920.0 & 2.7 & & & 159.2 & ky & 0.2 & $5 / 2^{+}$ & 96 & $91 \operatorname{Pr} 02$ & $\mathrm{D}$ & $\alpha=100 ; \mathrm{SF}<6 \mathrm{e}-9 ; \ldots$ \\
\hline${ }^{233} \mathrm{~Np}$ & 37950 & 50 & & & 36.2 & $\mathrm{~m}$ & 0.1 & $\left(5 / 2^{+}\right)$ & 90 & & & $\beta^{+} \approx 100 ; \alpha \leq 0.001$ \\
\hline${ }^{233} \mathrm{~Np}^{p}$ & $38000 \#$ & $60 \#$ & $50 \#$ & $30 \#$ & & & & $\left(5 / 2^{-}\right)$ & 90 & & & \\
\hline${ }^{233} \mathrm{Pu}$ & 40050 & 50 & & & 20.9 & $\mathrm{~m}$ & 0.4 & $5 / 2^{+} \#$ & 90 & & & $\beta^{+} \approx 100 ; \alpha=0.125$ \\
\hline${ }^{233} \mathrm{Am}$ & 43170\# & $100 \#$ & & & 3.2 & $\mathrm{~m}$ & 0.8 & & & $00 \mathrm{Sa} 52$ & TD & $\beta^{+} ? ; \alpha>3$ \\
\hline${ }^{233} \mathrm{Cm}$ & 47290 & 70 & & & $1 \#$ & $\mathrm{~m}$ & & $3 / 2^{+} \#$ & & 01Ca.B & $\mathrm{D}$ & $\alpha=? ; \beta^{+} ?$ \\
\hline$*^{233} \mathrm{U}$ & $\mathrm{D}: \ldots ;^{2}$ & ${ }^{24} \mathrm{Ne}=7$ & $7.2 \mathrm{e}-11 \mathrm{~S}$ & ${ }^{8} \mathrm{Mg}<1.3 \mathrm{e}-13$ & & & & & & & & \\
\hline${ }^{234} \mathrm{Ra}$ & 47230\# & $490 \#$ & & & 30 & $\mathrm{~s}$ & 10 & $0^{+}$ & 94 & & & $\beta^{-}=100$ \\
\hline${ }^{234} \mathrm{Ac}$ & 45100\# & $400 \#$ & & & 44 & s & 7 & & 94 & & & $\beta^{-}=100$ \\
\hline${ }^{234} \mathrm{Th}$ & 40614 & 3 & & & 24.10 & d & 0.03 & $0^{+}$ & 94 & & & $\beta^{-}=100$ \\
\hline${ }^{234} \mathrm{~Pa}$ & 40341 & 5 & & & 6.70 & $\mathrm{~h}$ & 0.05 & $4^{+}$ & 94 & $78 \mathrm{Ga} 07$ & $\mathrm{D}$ & $\beta^{-}=100 ; \mathrm{SF}<3 \mathrm{e}-10$ \\
\hline${ }^{234} \mathrm{~Pa}^{m}$ & 40419 & 4 & 78 & 3 & 1.17 & $\mathrm{~m}$ & 0.03 & $\left(0^{-}\right)$ & 94 & $78 \mathrm{Ga} 07$ & $\mathrm{D}$ & $\beta^{-} \approx 100 ; \mathrm{IT}=0.164 ; \mathrm{SF}<1 \mathrm{e}-10$ \\
\hline${ }^{234} \mathrm{U}$ & 38146.6 & 1.8 & & & 245.5 & ky & 0.6 & $0^{+}$ & 94 & & & IS $=0.00552 ; \alpha=100 ; \ldots$ \\
\hline${ }^{234} \mathrm{U}^{m}$ & 39567.9 & 1.8 & 1421.32 & 0.10 & 33.5 & $\mu \mathrm{s}$ & 2.0 & $6^{-}$ & & & & \\
\hline${ }^{234} \mathrm{~Np}$ & 39956 & 9 & & & 4.4 & $\mathrm{~d}$ & 0.1 & $\left(0^{+}\right)$ & 94 & & & $\beta^{+}=100$ \\
\hline${ }^{234} \mathrm{Pu}$ & 40350 & 7 & & & 8.8 & $\mathrm{~h}$ & 0.1 & $0^{+}$ & 94 & & & $\varepsilon \approx 94 ; \alpha \approx 6$ \\
\hline${ }^{234} \mathrm{Am}$ & $44530 \#$ & $210 \#$ & & & 2.32 & $\mathrm{~m}$ & 0.08 & & 94 & $90 \mathrm{Ha} 02$ & $\mathrm{D}$ & $\beta^{+} \approx 100 ; \alpha=0.03912 ;$. \\
\hline${ }^{234} \mathrm{Cm}$ & 46724 & 18 & & & 51 & $\mathrm{~s}$ & 12 & $0^{+}$ & & 01Ca.B & TD & $\alpha=? ; \beta^{+}=47 \# ; \mathrm{SF}=3$ \\
\hline$*^{234} \mathrm{U}$ & $\mathrm{D}: \ldots ; \mathrm{S}$ & $\mathrm{SF}=1.7$ & $3 \mathrm{e}-910$ & $\mathrm{Ig}=1.4 \mathrm{e}-113$ & $\mathrm{Ne}+{ }^{26} \mathrm{~N}$ & $\mathrm{e}=9$ & $e-127$ & & & & & \\
\hline
\end{tabular}

\begin{tabular}{|c|c|c|c|c|c|c|c|c|c|c|c|c|}
\hline${ }^{235} \mathrm{Ac}$ & 47720\# & $360 \#$ & & & & $40 \#$ & $\mathrm{~s}$ & & $1 / 2^{+} \#$ & & & $\beta^{-} ?$ \\
\hline${ }^{235} \mathrm{Th}$ & 44260 & 50 & & & & 7.2 & $\mathrm{~m}$ & 0.1 & $1 / 2^{+} \#$ & 03 & & $\beta^{-}=100$ \\
\hline${ }^{235} \mathrm{~Pa}$ & 42330 & 50 & & & & 24.44 & $\mathrm{~m}$ & 0.11 & $\left(3 / 2^{-}\right)$ & 03 & & $\beta^{-}=100$ \\
\hline${ }^{235} \mathrm{U}$ & 40920.5 & 1.8 & & & & 704 & My & 1 & $7 / 2^{-}$ & 03 & & IS $=0.720051 ; \alpha=100 ; \ldots$ \\
\hline${ }^{235} \mathrm{U}^{m}$ & 40920.6 & 1.8 & 0.0765 & 0.0004 & & 26 & $\mathrm{~m}$ & & $1 / 2^{+}$ & 03 & & $\mathrm{IT}=100$ \\
\hline${ }^{235} \mathrm{~Np}$ & 41044.7 & 2.0 & & & & 396.1 & $\mathrm{~d}$ & 1.2 & $5 / 2^{+}$ & 03 & & $\varepsilon \approx 100 ; \alpha=0.0026013$ \\
\hline${ }^{235} \mathrm{Pu}$ & 42184 & 21 & & & & 25.3 & $\mathrm{~m}$ & 0.5 & $\left(5 / 2^{+}\right)$ & 03 & & $\beta^{+} \approx 100 ; \alpha=0.00287$ \\
\hline${ }^{235} \mathrm{Am}$ & $44660 \#$ & $120 \#$ & & & & 9.9 & $\mathrm{~m}$ & 0.5 & $5 / 2^{-} \#$ & 03 & & $\beta^{+} \approx 100 ; \alpha=0.405$ \\
\hline${ }^{235} \mathrm{Cm}$ & 47910\# & $200 \#$ & & & & $5 \#$ & $\mathrm{~m}$ & & $5 / 2^{+} \#$ & 03 & & $\beta^{+} ? ; \alpha ?$ \\
\hline${ }^{235} \mathrm{Cm}^{p}$ & 47960\# & $210 \#$ & $50 \#$ & $50 \#$ & & & & & am & & & \\
\hline${ }^{235} \mathrm{Bk}$ & $52700 \#$ & $400 \#$ & & & & $20 \#$ & $\mathrm{~s}$ & & & & & $\beta^{+} ? ; \alpha ?$ \\
\hline$*^{235} \mathrm{U}$ & $\mathrm{D}: \ldots$ & $\mathrm{SF}=7 \mathrm{e}-$ & $2 ;{ }^{20} \mathrm{Ne}$ & $-104 ;^{2}$ & ${ }^{25} \mathrm{Ne} \approx 8 \mathrm{e}$ & $-10 ;{ }^{28}$ & $\mathrm{Mg}=8$ & $=8 \mathrm{e}-10$ & & & & \\
\hline${ }^{236} \mathrm{Ac}$ & $51510 \#$ & $500 \#$ & & & & $2 \#$ & $\mathrm{~m}$ & & & & & $\beta^{-} ?$ \\
\hline${ }^{236} \mathrm{Th}$ & $46450 \#$ & $200 \#$ & & & & 37.5 & $\mathrm{~m}$ & 0.2 & $0^{+}$ & 91 & & $\beta^{-}=100$ \\
\hline${ }^{236} \mathrm{~Pa}$ & 45350 & 200 & & & & 9.1 & $\mathrm{~m}$ & 0.1 & $1^{(-)}$ & 91 & & $\beta^{-}=100 ; \beta^{-} \mathrm{SF}=6 \mathrm{e}-84$ \\
\hline${ }^{236} \mathrm{U}$ & 42446.3 & 1.8 & & & & 23.42 & My & 0.03 & $0^{+}$ & 91 & & $\alpha=100 ; \mathrm{SF}=9.6 \mathrm{e}-86$ \\
\hline${ }^{236} \mathrm{U}^{m}$ & 45196 & 10 & 2750 & 10 & & 115 & $\mathrm{~ns}$ & & $0^{+}$ & & & \\
\hline${ }^{236} \mathrm{~Np}$ & 43380 & 50 & & & * & 154 & ky & 6 & $\left(6^{-}\right)$ & 91 & & $\varepsilon=87.35 ; \beta^{-}=12.55 ; \alpha=0.164$ \\
\hline${ }^{236} \mathrm{~Np}^{m}$ & 43439 & 7 & 60 & 50 & $*$ & 22.5 & $\mathrm{~h}$ & 0.4 & 1 & 91 & & $\varepsilon=521 ; \beta^{-}=481$ \\
\hline${ }^{236} \mathrm{~Np}^{p}$ & 43618 & 14 & 240 & 50 & $\mathrm{AD}$ & & & & $3^{-}$ & & & \\
\hline${ }^{236} \mathrm{Pu}$ & 42902.7 & 2.2 & & & & 2.858 & y & 0.008 & $0^{+}$ & 91 & $90 \operatorname{Og} 01 \mathrm{D}$ & $\alpha=100 ; \mathrm{SF}=1.36 \mathrm{e}-74$ \\
\hline${ }^{236} \mathrm{Am}$ & $46180 \#$ & $100 \#$ & & & & $30 \#$ & $\mathrm{~m}$ & & & 91 & & $\beta^{+} ? ; \alpha ?$ \\
\hline${ }^{236} \mathrm{Cm}$ & 47890\# & $200 \#$ & & & & $10 \#$ & $\mathrm{~m}$ & & $0^{+}$ & 91 & & $\beta^{+} ? ; \alpha ?$ \\
\hline${ }^{236} \mathrm{Bk}$ & $53400 \#$ & $400 \#$ & & & & $1 \#$ & $\mathrm{~m}$ & & & & & $\beta^{+} ? ; \alpha ?$ \\
\hline$*^{236} \mathrm{~Pa}$ & $\mathrm{D}: \beta^{-} \mathrm{S}$ & F decay & questione & by $90 \mathrm{HaC}$ & & & & & & & & \\
\hline$*^{236} \mathrm{U}$ & $\mathrm{D}:$ and $\mathrm{I}$ & $\mathrm{Ne}+\mathrm{Mg}$ & $<4 \mathrm{e}-10 \%$ & from $89 N$ & Mi.A & & & & & & & \\
\hline$*{ }^{236} \mathrm{Pu}$ & $\mathrm{D}: \ldots$ & ${ }^{28} \mathrm{Mg}=$ & $-12 ; 2 \beta$ & & & & & & & & & \\
\hline
\end{tabular}




\begin{tabular}{|c|c|c|c|c|c|c|c|c|c|c|c|c|}
\hline \multirow{2}{*}{$\begin{array}{l}\text { Nuclide } \\
{ }^{237} \mathrm{Th}\end{array}$} & $\begin{array}{l}\text { Mass excess } \\
(\mathrm{keV})\end{array}$ & \multicolumn{3}{|c|}{$\begin{array}{l}\text { Excitation } \\
\text { energy }(\mathrm{keV})\end{array}$} & \multicolumn{3}{|c|}{ Half-life } & \multirow[t]{2}{*}{$J^{\pi}$} & \multirow[t]{2}{*}{ Ens } & Reference & \multicolumn{2}{|l|}{$\begin{array}{l}\text { Decay modes and } \\
\text { intensities }(\%)\end{array}$} \\
\hline & $50200 \#$ 360\# & & & & 4.8 & $\mathrm{~m}$ & 0.5 & & & 00Xu02 T & $\beta^{-}=100$ & * \\
\hline${ }^{237} \mathrm{~Pa}$ & $47640 \quad 100$ & & & & 8.7 & $\mathrm{~m}$ & 0.2 & $\left(1 / 2^{+}\right)$ & 95 & & $\beta^{-}=100$ & \\
\hline${ }^{237} \mathrm{U}$ & $45391.9 \quad 1.9$ & & & & 6.75 & $\mathrm{~d}$ & 0.01 & $1 / 2^{+}$ & 95 & & $\beta^{-}=100$ & \\
\hline${ }^{237} \mathrm{~Np}$ & $44873.3 \quad 1.8$ & & & & 2.144 & $\mathrm{My}$ & 0.007 & $5 / 2^{+}$ & 95 & 89Pr.A D & $\alpha=100 ; \mathrm{SF} \leq 2 \mathrm{e}-10 ;{ }^{30} \mathrm{Mg}<4 \mathrm{e}-12$ & $*$ \\
\hline${ }^{237} \mathrm{Pu}$ & $45093.3 \quad 2.2$ & & & & 45.2 & $\mathrm{~d}$ & 0.1 & $7 / 2^{-}$ & 95 & & $\varepsilon \approx 100 ; \alpha=0.00424$ & \\
\hline${ }^{237} \mathrm{Pu}^{m}$ & $45238.8 \quad 2.2$ & 145.544 & 0.010 & & 180 & $\mathrm{~ms}$ & 20 & $1 / 2^{+}$ & 95 & & $\mathrm{IT}=100$ & \\
\hline${ }^{237} \mathrm{Am}$ & 46570\# 60\# & & & & 73.0 & $\mathrm{~m}$ & 1.0 & $5 / 2^{(-)}$ & 95 & & $\beta^{+} \approx 100 ; \alpha=0.0253$ & \\
\hline${ }^{237} \mathrm{Cm}$ & 49280\# 210\# & & & & $20 \#$ & $\mathrm{~m}$ & & $5 / 2^{+} \#$ & 95 & & $\beta^{+} ? ; \alpha ?$ & \\
\hline${ }^{237} \mathrm{Cm}^{p}$ & 49480\# 260\# & $200 \#$ & $150 \#$ & & & & & $7 / 2^{-}$ & & & & \\
\hline${ }^{237} \mathrm{Bk}$ & $53100 \#$ 220\# & & & & $1 \#$ & $\mathrm{~m}$ & & $7 / 2^{+} \#$ & & & $\beta^{+} ? ; \alpha ?$ & \\
\hline${ }^{237} \mathrm{Bk}^{p}$ & $53170 \#$ 230\# & 70\# & $30 \#$ & $\mathrm{Nm}$ & & & & $\left(3 / 2^{-}\right)$ & & & & \\
\hline${ }^{237} \mathrm{Cf}$ & $57820 \# 500 \#$ & & & & 2.1 & $\mathrm{~s}$ & 0.3 & $5 / 2^{+} \#$ & 98 & 95La09 TD & $\alpha ? ; \mathrm{SF} \approx 10 ; \beta^{+} ?$ & \\
\hline$*^{237} \mathrm{Th}$ & $\mathrm{T}:$ average 002 & $\mathrm{Xu} 02=4.69$ & $0.60) 93 \mathrm{Y}$ & $u 03=5$ & $.0(0.9)$ & & & & & & & $* *$ \\
\hline$*^{237} \mathrm{~Np}$ & $\mathrm{D}:$ and cluster & $(Z=10-14)$ & $<1.8 \mathrm{e}-12$ & $2 \%$, frc & $\mathrm{m} 92 \mathrm{M}$ & 1o03 & & & & & & ** \\
\hline${ }^{238} \mathrm{Th}$ & $52630 \# 280 \#$ & & & & 9.4 & $\mathrm{~m}$ & 2.0 & $0^{+}$ & 02 & & $\beta^{-}=100$ & \\
\hline${ }^{238} \mathrm{~Pa}$ & $50770 \quad 60$ & & & & 2.27 & $\mathrm{~m}$ & 0.09 & $3^{-} \#$ & 02 & 85Ba57 D & $\beta^{-}=100 ; \beta^{-} \mathrm{SF}<2.6 \mathrm{e}-6$ & \\
\hline${ }^{238} \mathrm{U}$ & $47308.9 \quad 1.9$ & & & & 4.468 & Gy ( & 0.003 & $0^{+}$ & 02 & 91Tu02 D & IS=99.2745 106; $\alpha=100 ;$. & * \\
\hline${ }^{238} \mathrm{U}^{m}$ & $49866.8 \quad 2.0$ & 2557.9 & 0.5 & & 280 & $\mathrm{~ns}$ & 6 & $0^{+}$ & 02 & & $\mathrm{IT}=? ; \mathrm{SF}=2.64 ; \alpha<0.5$ & \\
\hline${ }^{238} \mathrm{~Np}$ & $47456.3 \quad 1.8$ & & & & 2.117 & $\mathrm{~d}$ & 0.002 & $2^{+}$ & 02 & & $\beta^{-}=100$ & \\
\hline${ }^{238} \mathrm{~Np}^{m}$ & 49760\# 200\# & $2300 \#$ & 200\# & & 112 & $\mathrm{~ns}$ & 39 & & 02 & & $\mathrm{SF} \approx 100 ; \mathrm{IT} ?$ & \\
\hline${ }^{238} \mathrm{Pu}$ & $46164.7 \quad 1.8$ & & & & 87.7 & $\mathrm{y}$ & 0.1 & $0^{+}$ & 02 & 89Wa10 D & $\alpha=100 ; \mathrm{SF}=1.9 \mathrm{e}-71 ; \ldots$ & $*$ \\
\hline${ }^{238} \mathrm{Am}$ & $48420 \quad 50$ & & & & 98 & $\mathrm{~m}$ & 2 & $1^{+}$ & 02 & & $\beta^{+}=100 ; \alpha=1.0 \mathrm{e}-44$ & \\
\hline${ }^{238} \mathrm{Am}^{m}$ & $50920 \# 210 \#$ & $2500 \#$ & $200 \#$ & & 35 & $\mu \mathrm{s}$ & 10 & & 02 & & $\mathrm{SF} \approx 100 ; \mathrm{IT} ?$ & \\
\hline${ }^{238} \mathrm{Cm}$ & $49400 \quad 40$ & & & & 2.4 & $\mathrm{~h}$ & 0.1 & $0^{+}$ & 02 & & $\varepsilon ? ; \alpha \leq 10$ & \\
\hline${ }^{238} \mathrm{Bk}$ & $54290 \#$ 290\# & & & & 2.40 & $\mathrm{~m}$ & 0.08 & & 02 & 94Kr03 D & $\beta^{+} \approx 100 ; \alpha ? ; \beta^{+} \mathrm{SF}=0.0482$ & \\
\hline${ }^{238} \mathrm{Bk}^{p}$ & 54490\# 330\# & 200\# & $150 \#$ & & & & & $a m$ & & & & \\
\hline${ }^{238} \mathrm{Cf}$ & $57200 \# 400 \#$ & & & & 21.1 & $\mathrm{~ms}$ & 1.3 & $0^{+}$ & 02 & $01 \mathrm{Og} 08 \mathrm{TD}$ & $\mathrm{SF} \approx 100 ; \alpha \approx 0.2 ; \beta^{+} ?$ & $*$ \\
\hline$*^{238} \mathrm{U}$ & $\mathrm{D}: \ldots ; \mathrm{SF}=5$ & $45 \mathrm{e}-57 ; 2$ & $3^{-}=2.2 \mathrm{e}-1$ & 07 & & & & & & & & $* *$ \\
\hline$*^{238} \mathrm{U}$ & $\mathrm{D}: 2 \beta^{-}=2.2(7$ & e- $-10 \%$ de & ived from & $2 \beta^{-1}$ & alf-life & $T=2$ & $2.0(0$ & ) $Z y$ & $1 \mathrm{TuC}$ & & & ** \\
\hline$*^{238} \mathrm{Pu}$ & $\mathrm{D}: \ldots ;{ }^{32} \mathrm{Si} \approx$ & $1.4 \mathrm{e}-14 ;{ }^{28}$ & $\mathrm{Mg}+{ }^{30} \mathrm{Mg}$ & $\approx 6 \mathrm{e}-1$ & & & & & & & & $* *$ \\
\hline$*^{238} \mathrm{Cf}$ & $\mathrm{T}:$ average 01 & $\mathrm{Og} 08=21.1$ & $(+1.9-1.7)$ & $95 \mathrm{La}$ & $9=21(2$ & & & & & & & $* *$ \\
\hline
\end{tabular}

\begin{tabular}{|c|c|c|c|c|c|c|c|c|c|c|c|c|}
\hline${ }^{239} \mathrm{~Pa}$ & $53340 \#$ & $200 \#$ & & & & 1.8 & $\mathrm{~h}$ & 0.5 & $(3 / 2)^{(-\#)}$ & 03 & & $\beta^{-}=100$ \\
\hline${ }^{239} \mathrm{U}$ & 50573.9 & 1.9 & & & & 23.45 & $\mathrm{~m}$ & 0.02 & $5 / 2^{+}$ & 03 & & $\beta^{-}=100$ \\
\hline${ }^{239} \mathrm{U}^{m}$ & 50594\# & $20 \#$ & 20\# & 20\# & & $>250$ & ns & & $\left(5 / 2^{+}\right)$ & 03 & & $\beta^{-}=100$ \\
\hline${ }^{239} \mathrm{U}^{n}$ & 50707.7 & 1.9 & 133.7990 & 0.0010 & & 780 & ns & 40 & $1 / 2^{+}$ & 03 & & $\mathrm{IT}=100$ \\
\hline${ }^{239} \mathrm{~Np}$ & 49312.4 & 2.1 & & & & 2.356 & $\mathrm{~d} 0$ & 0.003 & $5 / 2^{+}$ & 03 & & $\beta^{-}=100 ; \alpha=5 \mathrm{e}-10 \#$ \\
\hline${ }^{239} \mathrm{Pu}$ & 48589.9 & 1.8 & & & & 24.11 & ky & 0.03 & $1 / 2^{+}$ & 03 & & $\alpha=100 ; \mathrm{SF}=3.1 \mathrm{e}-106$ \\
\hline${ }^{239} \mathrm{Pu}^{m}$ & 48981.5 & 1.8 & 391.584 & 0.003 & & 193 & ns & 4 & $7 / 2^{-}$ & 03 & & $\mathrm{IT}=100$ \\
\hline${ }^{239} \mathrm{Am}$ & 49392.0 & 2.4 & & & & 11.9 & $\mathrm{~h}$ & 0.1 & $(5 / 2)^{-}$ & 03 & & $\varepsilon \approx 100 ; \alpha=0.0101$ \\
\hline${ }^{239} \mathrm{Am}^{m}$ & 51890 & 200 & 2500 & 200 & & 163 & ns & 12 & $\left(7 / 2^{+}\right)$ & 03 & & $\mathrm{SF} \approx 100 ; \mathrm{IT} ?$ \\
\hline${ }^{239} \mathrm{Cm}$ & 51190\# & $100 \#$ & & & & 2.9 & $\mathrm{~h}$ & & $\left(7 / 2^{-}\right)$ & 03 & & $\beta^{+} \approx 100 ; \alpha<0.1$ \\
\hline${ }^{239} \mathrm{Cm}^{p}$ & $51340 \#$ & $140 \#$ & $150 \#$ & $100 \#$ & & & & & $1 / 2^{+}$ & & & \\
\hline${ }^{239} \mathrm{Bk}$ & 54290\# & $230 \#$ & & & & $3 \#$ & $\mathrm{~m}$ & & $7 / 2^{+} \#$ & 03 & & $\beta^{+} ? ; \alpha ?$ \\
\hline${ }^{239} \mathrm{Bk}^{p}$ & $54330 \#$ & $230 \#$ & 41 & 11 & $\mathrm{AD}$ & & & & $\left(3 / 2^{-}\right)$ & & & \\
\hline${ }^{239} \mathrm{Cf}$ & 58150\# & $210 \#$ & & & & 60 & $\mathrm{~s}$ & 30 & $5 / 2^{+} \#$ & 03 & & $\alpha=? ; \beta^{+} ?$ \\
\hline${ }^{240} \mathrm{~Pa}$ & $56800 \#$ & $300 \#$ & & & & $2 \#$ & $\mathrm{~m}$ & & & & & $\beta^{-} ?$ \\
\hline${ }^{240} \mathrm{U}$ & 52715 & 5 & & & & 14.1 & $\mathrm{~h}$ & 0.1 & $0^{+}$ & 96 & & $\beta^{-}=100 ; \alpha<1 \mathrm{e}-10$ \\
\hline${ }^{240} \mathrm{~Np}$ & 52315 & 15 & & & * & 61.9 & $\mathrm{~m}$ & 0.2 & $\left(5^{+}\right)$ & 96 & & $\beta^{-}=100$ \\
\hline${ }^{240} \mathrm{~Np}^{m}$ & 52335 & 21 & 20 & 15 & * & 7.22 & $\mathrm{~m}$ & 0.02 & $1^{(+)}$ & 96 & $81 \mathrm{Hs} 02 \mathrm{E}$ & $\beta^{-} \approx 100 ; \mathrm{IT}=0.113$ \\
\hline${ }^{240} \mathrm{Pu}$ & 50127.0 & 1.8 & & & & 6.564 & ky 0 & 0.011 & $0^{+}$ & 01 & 89Pr.A D & $\alpha=100 ; \mathrm{SF}=5.7 \mathrm{e}-62 ; 34 \mathrm{Si}<1.3 \mathrm{e}-13$ \\
\hline${ }^{240} \mathrm{Am}$ & 51512 & 14 & & & & 50.8 & $\mathrm{~h}$ & 0.3 & $\left(3^{-}\right)$ & 96 & & $\beta^{+}=100 ; \alpha \approx 1.9 \mathrm{e}-4$ \\
\hline${ }^{240} \mathrm{Cm}$ & 51725.4 & 2.3 & & & & 27 & $\mathrm{~d}$ & 1 & $0^{+}$ & 96 & & $\alpha \approx 100 ; \varepsilon<0.5 ; \mathrm{SF}=3.9 \mathrm{e}-68$ \\
\hline${ }^{240} \mathrm{Bk}$ & 55670\# & $150 \#$ & & & & 4.8 & $\mathrm{~m}$ & 0.8 & & 96 & & $\beta^{+}$?; $\alpha=10 \# ; \beta^{+} \mathrm{SF}=0.002013$ \\
\hline${ }^{240} \mathrm{Bk}^{p}$ & $55910 \#$ & $180 \#$ & $240 \#$ & $100 \#$ & & & & & $a m$ & & & \\
\hline${ }^{240} \mathrm{Cf}$ & 58030\# & $200 \#$ & & & & 1.06 & $\mathrm{~m}$ & 0.15 & $0^{+}$ & 96 & 95La09 D & $\alpha \approx 98 ; \mathrm{SF} \approx 2 ; \beta^{+} ?$ \\
\hline${ }^{240} \mathrm{Es}$ & 64200\# & $400 \#$ & & & & $1 \#$ & $\mathrm{~s}$ & & & & & $\alpha ? ; \beta^{+} ?$ \\
\hline
\end{tabular}




\begin{tabular}{|c|c|c|c|c|c|c|c|c|c|c|c|c|c|c|}
\hline \multirow{2}{*}{$\begin{array}{l}\text { Nuclide } \\
{ }^{241} \mathrm{U}\end{array}$} & \multicolumn{2}{|c|}{$\begin{array}{l}\text { Mass excess } \\
\quad(\mathrm{keV})\end{array}$} & \multicolumn{3}{|c|}{$\begin{array}{c}\text { Excitation } \\
\text { energy }(k e V)\end{array}$} & \multicolumn{3}{|c|}{ Half-life } & \multirow{2}{*}{$\begin{array}{c}J^{\pi} \\
7 / 2^{+} \#\end{array}$} & \multirow[t]{2}{*}{ Ens } & \multicolumn{2}{|c|}{ Reference } & \multicolumn{2}{|l|}{$\begin{array}{l}\text { Decay modes and } \\
\text { intensities }(\%)\end{array}$} \\
\hline & $56200 \#$ & $300 \#$ & & & & $5 \#$ & $\mathrm{~m}$ & & & & & & $\beta^{-}$? & \\
\hline${ }^{241} \mathrm{~Np}$ & 54260 & 70 & & & & 13.9 & $\mathrm{~m}$ & 0.2 & $\left(5 / 2^{+}\right)$ & 94 & & & $\beta^{-}=100$ & \\
\hline${ }^{241} \mathrm{Pu}$ & 52956.8 & 1.8 & & & & 14.35 & $\mathrm{y}$ & 0.10 & $5 / 2^{+}$ & 96 & & & $\beta^{-} \approx 100 ; \alpha=0.002452 ; \ldots$ & $*$ \\
\hline${ }^{241} \mathrm{Pu}^{m}$ & 53118.4 & 1.8 & 161.60 & 0.10 & & 880 & $\mathrm{~ns}$ & & $1 / 2^{+}$ & & & & & \\
\hline${ }^{241} \mathrm{Pu}^{n}$ & 55160 & 200 & 2200 & 200 & & 21 & $\mu \mathrm{s}$ & 3 & & & & & & \\
\hline${ }^{241} \mathrm{Am}$ & 52936.0 & 1.8 & & & & 432.2 & $\mathrm{y}$ & 0.7 & $5 / 2^{-}$ & 94 & & & $\alpha=100 ; \mathrm{SF}=4.3 \mathrm{e}-1018 ; \ldots$ & * \\
\hline${ }^{241} \mathrm{Am}^{m}$ & 55140 & 100 & 2200 & 100 & & 1.5 & $\mu \mathrm{s}$ & & & & & & & \\
\hline${ }^{241} \mathrm{Cm}$ & 53703.4 & 2.2 & & & & 32.8 & $\mathrm{~d}$ & 0.2 & $1 / 2^{+}$ & 94 & & & $\varepsilon=99.01 ; \alpha=1.01$ & \\
\hline${ }^{241} \mathrm{Bk}$ & $56100 \#$ & $200 \#$ & & & & 4.6 & $\mathrm{~m}$ & 0.4 & $\left(7 / 2^{+}\right)$ & 94 & 03As01 & $\mathrm{T}$ & $\alpha ? ; \beta^{+} ?$ & \\
\hline${ }^{241} \mathrm{Bk}^{p}$ & $56150 \#$ & $200 \#$ & 51 & 3 & $\mathrm{AD}$ & & & & $3 / 2^{-}$ & & & & & \\
\hline${ }^{241} \mathrm{Cf}$ & 59360\# & $260 \#$ & & & & 3.8 & $\mathrm{~m}$ & 0.7 & $7 / 2^{-} \#$ & 94 & & & $\beta^{+} \approx 75 ; \alpha \approx 25$ & \\
\hline${ }^{241} \mathrm{Cf}^{p}$ & 59510\# & $270 \#$ & $150 \#$ & $100 \#$ & $\mathrm{Nm}$ & & & & $\left(1 / 2^{+}\right)$ & & & & & \\
\hline${ }^{241}$ Es & 63840\# & $230 \#$ & & & & 10 & s & 5 & $\left(3 / 2^{-}\right)$ & 97 & $96 \mathrm{Ni09}$ & TJD & $\alpha=? ; \beta^{+} ?$ & \\
\hline${ }^{241} \mathrm{Es}^{p}$ & $64240 \#$ & $300 \#$ & $400 \#$ & $200 \#$ & & & & & $\left(7 / 2^{+}\right)$ & & & & & \\
\hline$*^{241} \mathrm{Pu}$ & $\mathrm{D}: \ldots$; & $\mathrm{SF}<2.4$ & te-14 & & & & & & & & & & & $* *$ \\
\hline$*^{241} \mathrm{Am}$ & $\mathrm{D}: \ldots$; & ${ }^{34} \mathrm{Si}<7$ & $.4 \mathrm{e}-14$ & & & & & & & & & & & $* *$ \\
\hline${ }^{242} \mathrm{U}$ & $58620 \#$ & $200 \#$ & & & & 16.8 & $\mathrm{~m}$ & 0.5 & $0^{+}$ & 02 & & & $\beta^{-}=100$ & \\
\hline${ }^{242} \mathrm{~Np}$ & 57420 & 200 & & & * & 2.2 & $\mathrm{~m}$ & 0.2 & $\left(1^{+}\right)$ & 02 & & & $\beta^{-}=100$ & \\
\hline${ }^{242} \mathrm{~Np}^{m}$ & $57420 \#$ & $210 \#$ & 0\# & $50 \#$ & $*$ & 5.5 & $\mathrm{~m}$ & 0.1 & $6^{+} \#$ & 02 & & & $\beta^{-}=100$ & \\
\hline${ }^{242} \mathrm{Pu}$ & 54718.4 & 1.9 & & & & 375 & ky & 2 & $0^{+}$ & 02 & & & $\alpha=100 ; \mathrm{SF}=5.50 \mathrm{e}-46$ & \\
\hline${ }^{242} \mathrm{Am}$ & 55469.7 & 1.8 & & & & 16.02 & $\mathrm{~h}$ & 0.02 & $1^{-}$ & 02 & & & $\beta^{-}=82.73 ; \varepsilon=17.33$ & \\
\hline${ }^{242} \mathrm{Am}^{m}$ & 55518.3 & 1.8 & 48.60 & 0.05 & & 141 & $\mathrm{y}$ & 2 & $5^{-}$ & 02 & & & $\mathrm{IT} \approx 100 ; \alpha=0.452 ; \mathrm{SF}<4.7 \mathrm{e}-9$ & \\
\hline${ }^{242} \mathrm{Am}^{n}$ & 57670 & 80 & 2200 & 80 & & 14.0 & $\mathrm{~ms}$ & 1.0 & $\left(2^{+}, 3^{-}\right)$ & 02 & & & $\mathrm{SF} \approx 100 ; \mathrm{IT}=? ; \alpha$ ? & \\
\hline${ }^{242} \mathrm{Cm}$ & 54805.2 & 1.8 & & & & 162.8 & $\mathrm{~d}$ & 0.2 & $0^{+}$ & 02 & & & $\alpha=100 ; \mathrm{SF}=6.2 \mathrm{e}-63 ; \ldots$ & $*$ \\
\hline${ }^{242} \mathrm{Bk}$ & $57740 \#$ & $200 \#$ & & & & 7.0 & $\mathrm{~m}$ & 1.3 & $2^{-} \#$ & 02 & $80 \mathrm{Ga} 07$ & $\mathrm{D}$ & $\beta^{+} \approx 100 ; \beta^{+} \mathrm{SF}<3 \mathrm{e}-5 ; \alpha ?$ & \\
\hline${ }^{242} \mathrm{Bk}^{m}$ & $57940 \#$ & $280 \#$ & $200 \#$ & $200 \#$ & & 600 & $\mathrm{~ns}$ & 100 & & 02 & & & $\mathrm{SF} \approx 100 ;$ IT ? & \\
\hline${ }^{242} \mathrm{Bk}^{p}$ & 57990\# & $220 \#$ & $250 \#$ & $100 \#$ & & & & & $4^{-}$ & & & & & \\
\hline${ }^{242} \mathrm{Cf}$ & 59340 & 40 & & & & 3.49 & $\mathrm{~m}$ & 0.15 & $0^{+}$ & 02 & $70 S i 19$ & $\mathrm{~T}$ & $\alpha=8020 ; \beta^{+} ? ; \mathrm{SF}<0.014$ & * \\
\hline${ }^{242} \mathrm{Es}$ & 64970\# & $330 \#$ & & & & 13.5 & $\mathrm{~s}$ & 2.5 & & 02 & 94Ke.B & $\mathrm{D}$ & $\alpha=? ; \beta^{+}=? ; \beta^{+} \mathrm{SF}=0.6$ & $*$ \\
\hline${ }^{242} \mathrm{Fm}$ & $68400 \#$ & $400 \#$ & & & & 800 & $\mu \mathrm{s}$ & 200 & $0^{+}$ & 02 & & & $\mathrm{SF}=? ; \alpha$ ? & \\
\hline$*^{242} \mathrm{Cm}$ & $\mathrm{D}: \ldots$; & ${ }^{34} \mathrm{Si}=1$. & $1 \mathrm{e}-144 ; 2$ & $\beta^{+} ?$ & & & & & & & & & & $* *$ \\
\hline$*^{242} \mathrm{Cf}$ & $\mathrm{T}:$ aver & age $70 \mathrm{~S}$ & $\mathrm{i} 19=3.68($ & .44) 67 & $77=3.4($ & $(0.2) 671$ & $\mathrm{FiO4}=$ & $=3.2(0$ & .5) $67 \mathrm{II} 0$ & $1=3.7$ & $7(0.3)$ & & & $* *$ \\
\hline$*^{242} \mathrm{Es}$ & $\mathrm{D}: \beta^{+} \mathrm{S}$ & $\mathrm{F}=0.6 \%$ & $b$ assumin & $\alpha$ and $\beta$ & are eq & ual & & & & & & & & $* *$ \\
\hline
\end{tabular}

\begin{tabular}{|c|c|c|c|c|c|c|c|c|c|c|c|c|}
\hline${ }^{243} \mathrm{~Np}$ & 59880\# & $30 \#$ & & & & 1.85 & $\mathrm{~m}$ & 0.15 & $\left(5 / 2^{-}\right)$ & 93 & & $\beta^{-}=100$ \\
\hline${ }^{243} \mathrm{~Np}^{p}$ & 59925 & 11 & $50 \#$ & $30 \#$ & $\mathrm{Nm}$ & & & & $\left(5 / 2^{-}\right)$ & & & \\
\hline${ }^{243} \mathrm{Pu}$ & 57756 & 3 & & & & 4.956 & $\mathrm{~h}$ & 0.003 & $7 / 2^{+}$ & 93 & & $\beta^{-}=100$ \\
\hline${ }^{243} \mathrm{Pu}^{m}$ & 58140 & 3 & 383.6 & 0.4 & & 330 & ns & 30 & $\left(1 / 2^{+}\right)$ & 93 & & $\mathrm{IT}=100$ \\
\hline${ }^{243} \mathrm{Am}$ & 57176.1 & 2.3 & & & & 7.37 & ky & 0.04 & $5 / 2^{-}$ & 93 & & $\alpha=100 ; \mathrm{SF}=3.7 \mathrm{e}-92$ \\
\hline${ }^{243} \mathrm{Cm}$ & 57183.6 & 2.1 & & & & 29.1 & $\mathrm{y}$ & 0.1 & $5 / 2^{+}$ & 93 & & $\alpha \approx 100 ; \varepsilon=0.293 ; \mathrm{SF}=5.3 \mathrm{e}-9$ \\
\hline${ }^{243} \mathrm{Cm}^{p}$ & 57312 & 10 & 129 & 9 & $\mathrm{AD}$ & & & & $7 / 2^{+}$ & & & \\
\hline${ }^{243} \mathrm{Bk}$ & 58691 & 5 & & & & 4.5 & $\mathrm{~h}$ & 0.2 & $\left(3 / 2^{-}\right)$ & 93 & & $\beta^{+} \approx 100 ; \alpha \approx 0.15$ \\
\hline${ }^{243} \mathrm{Bk}^{p}$ & $58740 \#$ & $30 \#$ & $50 \#$ & $30 \#$ & & & & & $\left(7 / 2^{-}\right)$ & & & \\
\hline${ }^{243} \mathrm{Cf}$ & $60950 \#$ & $140 \#$ & & & & 10.7 & $\mathrm{~m}$ & 0.5 & $\left(1 / 2^{+}\right)$ & 93 & & $\beta^{+} \approx 86 ; \alpha \approx 14$ \\
\hline${ }^{243} \mathrm{Es}$ & $64780 \#$ & $230 \#$ & & & & 21 & $\mathrm{~s}$ & 2 & $3 / 2^{-} \#$ & 93 & & $\beta^{+} \leq 70 ; \alpha \geq 30$ \\
\hline${ }^{243} \mathrm{Es}^{p}$ & $65180 \#$ & $310 \#$ & $400 \#$ & $200 \#$ & & & & & am & & & \\
\hline${ }^{243} \mathrm{Fm}$ & $69260 \#$ & $220 \#$ & & & & 210 & $\mathrm{~ms}$ & 60 & $7 / 2^{-} \#$ & 93 & ABBW D & $\alpha=6040 ; \beta^{+}$?; $\mathrm{SF}=0.57 \#$ \\
\hline${ }^{243} \mathrm{Fm}$ & $\mathrm{D}: \alpha=40$ & $0(20) \%$ & $\alpha \mathrm{b}$ & ig of & $C f$ & $0 \%, \mathrm{se}$ & ee Es & NSDF & & & & \\
\hline
\end{tabular}

\begin{tabular}{|c|c|c|c|c|c|c|c|c|c|c|c|c|}
\hline${ }^{244} \mathrm{~Np}$ & 63200\# & $300 \#$ & & & & 2.29 & $\mathrm{~m}$ & 0.16 & $\left(7^{-}\right)$ & 03 & & $\beta^{-}=100$ \\
\hline${ }^{244} \mathrm{Pu}$ & 59806 & 5 & & & & 80.0 & My & 0.9 & $0^{+}$ & 03 & $92 \mathrm{Mo} 25 \mathrm{D}$ & $\alpha \approx 100 ; \mathrm{SF}=0.1214$ \\
\hline${ }^{244} \mathrm{Am}$ & 59881.0 & 2.1 & & & & 10.1 & $\mathrm{~h}$ & 0.1 & $6^{-} \#$ & 03 & & $\beta^{-}=100$ \\
\hline${ }^{244} \mathrm{Am}^{m}$ & 59969.5 & 2.3 & 88.6 & 1.7 & RQ & 26 & $\mathrm{~m}$ & 1 & $1^{+}$ & 03 & & $\beta^{-} \approx 100 ; \varepsilon=0.036113$ \\
\hline${ }^{244} \mathrm{Cm}$ & 58453.7 & 1.8 & & & & 18.10 & $y$ & 0.02 & $0^{+}$ & 03 & & $\alpha=100 ; \mathrm{SF}=1.37 \mathrm{e}-43$ \\
\hline${ }^{244} \mathrm{Cm}^{m}$ & 59493.9 & 1.8 & 1040.188 & 0.012 & & 34 & $\mathrm{~ms}$ & 2 & $6^{+}$ & 03 & & $\mathrm{IT}=100$ \\
\hline
\end{tabular}




\begin{tabular}{|c|c|c|c|c|c|c|c|c|c|c|c|}
\hline Nuclide & \multicolumn{2}{|c|}{$\begin{array}{l}\text { Mass excess } \\
(\mathrm{keV})\end{array}$} & & $\begin{array}{l}\text { Excitation } \\
\text { energy }(\mathrm{keV})\end{array}$ & \multicolumn{3}{|c|}{ Half-life } & $J^{\pi}$ & Ens & Reference & $\begin{array}{l}\text { Decay modes and } \\
\text { intensities }(\%)\end{array}$ \\
\hline \multicolumn{12}{|c|}{$\ldots A$-group continued $\ldots$} \\
\hline${ }^{244} \mathrm{Bk}$ & 60716 & 14 & & & 4.35 & $\mathrm{~h}$ & 0.15 & $4^{-} \#$ & 03 & & $\beta^{+}$?; $\alpha=0.0063$ \\
\hline${ }^{244} \mathrm{Bk}^{p}$ & 60860\# & $50 \#$ & $140 \#$ & $50 \#$ & & & & $a m$ & & & \\
\hline${ }^{244} \mathrm{Cf}$ & 61479.2 & 2.9 & & & 19.4 & $\mathrm{~m}$ & 0.6 & $0^{+}$ & 03 & & $\alpha \approx 100 ; \varepsilon ?$ \\
\hline${ }^{244} \mathrm{Es}$ & $66030 \#$ & $180 \#$ & & & 37 & $\mathrm{~s}$ & 4 & & 03 & & $\beta^{+}=? ; \alpha=53 ; \beta^{+} \mathrm{SF}=0.01$ \\
\hline${ }^{244} \mathrm{Es}^{p}$ & 66230\# & $240 \#$ & $200 \#$ & 150\# & & & & $a m$ & & & \\
\hline${ }^{244} \mathrm{Fm}$ & 69010\# & $280 \#$ & & & 3.3 & $\mathrm{~ms}$ & 0.5 & $0^{+}$ & 03 & & $\mathrm{SF} \approx 100 ; \alpha=0.4 \#$ \\
\hline$*^{244} \mathrm{Pu}$ & \multicolumn{11}{|c|}{$\mathrm{D}: \ldots ; 2 \beta^{-}<7.3 \mathrm{e}-9$} \\
\hline$*^{244} \mathrm{Pu}$ & \multicolumn{11}{|c|}{$\mathrm{T}:$ and $T\left(2 \beta^{-}\right)>1.1 \mathrm{Ey}$, from $92 \mathrm{Mo} 25$; thus $2 \beta^{-}<7.3 \mathrm{e}-9 \%$} \\
\hline
\end{tabular}

\begin{tabular}{|c|c|c|c|c|c|c|c|c|c|c|c|c|c|}
\hline${ }^{245} \mathrm{Pu}$ & 63106 & 14 & & & & 10.5 & $\mathrm{~h}$ & 0.1 & $\left(9 / 2^{-}\right)$ & 93 & & & $\beta^{-}=100$ \\
\hline${ }^{245} \mathrm{Am}$ & 61900 & 3 & & & & 2.05 & $\mathrm{~h}$ & 0.01 & $(5 / 2)^{+}$ & 93 & & & $\beta^{-}=100$ \\
\hline${ }^{245} \mathrm{Cm}$ & 61004.7 & 2.1 & & & & 8.5 & ky & 0.1 & $7 / 2^{+}$ & 93 & & & $\alpha=100 ; \mathrm{SF}=6.1 \mathrm{e}-79$ \\
\hline${ }^{245} \mathrm{Cm}^{m}$ & 61360.6 & 2.1 & 355.90 & 0.10 & & 290 & $\mathrm{~ns}$ & 20 & $1 / 2^{+}$ & 93 & & & $\mathrm{IT}=100$ \\
\hline${ }^{245} \mathrm{Bk}$ & 61815.4 & 2.3 & & & & 4.94 & d & 0.03 & $3 / 2^{-}$ & 93 & & & $\varepsilon \approx 100 ; \alpha=0.121$ \\
\hline${ }^{245} \mathrm{Bk}^{p}$ & 61870\# & $30 \#$ & $50 \#$ & $30 \#$ & & & & & $\left(7 / 2^{-}\right)$ & & & & \\
\hline${ }^{245} \mathrm{Cf}$ & 63386.9 & 2.9 & & & & 45.0 & $\mathrm{~m}$ & 1.5 & $\left(5 / 2^{+}\right)$ & 93 & & & $\beta^{+}=643 ; \alpha=363$ \\
\hline${ }^{245} \mathrm{Cf} p$ & $63540 \#$ & $100 \#$ & $150 \#$ & $100 \#$ & & & & & $7 / 2^{+}$ & & & & \\
\hline${ }^{245} \mathrm{Es}$ & $66440 \#$ & $200 \#$ & & & & 1.1 & $\mathrm{~m}$ & 0.1 & $\left(3 / 2^{-}\right)$ & 93 & & & $\beta^{+}=6010 ; \alpha=4010$ \\
\hline${ }^{245} \mathrm{Es}^{p}$ & $66740 \#$ & $220 \#$ & $300 \#$ & $100 \#$ & & & & & am & & & & \\
\hline${ }^{245} \mathrm{Es}^{q}$ & 66790\# & $250 \#$ & $350 \#$ & $140 \#$ & & & & & $a m$ & & & & \\
\hline${ }^{245} \mathrm{Fm}$ & 70220\# & $280 \#$ & & & & 4.2 & $\mathrm{~s}$ & 1.3 & $1 / 2^{+} \#$ & 93 & & & $\alpha=? ; \beta^{+}=4.2 \# ; \mathrm{SF}=0.13 \#$ \\
\hline${ }^{245} \mathrm{Md}$ & 75290\# & $320 \#$ & & & * & 900 & $\mu \mathrm{s}$ & 250 & $1 / 2^{-} \#$ & 97 & $96 \mathrm{Ni} 09$ & TJD & $\mathrm{SF}=? ; \alpha ?$ \\
\hline${ }^{245} \mathrm{Md}^{m}$ & 75490\# & $310 \#$ & 200\# & $100 \#$ & * & 400 & $\mathrm{~ms}$ & 200 & $\left(7 / 2^{+}\right)$ & 97 & $96 \mathrm{Ni09}$ & TJD & $\alpha=? ; \beta^{+} ?$ \\
\hline${ }^{246} \mathrm{Pu}$ & 65395 & 15 & & & & 10.84 & d & 0.02 & $0^{+}$ & 98 & & & $\beta^{-}=100$ \\
\hline${ }^{246} \mathrm{Am}$ & 64995 & 18 & & & & 39 & $\mathrm{~m}$ & 3 & $\left(7^{-}\right)$ & 98 & & & $\beta^{-}=100$ \\
\hline${ }^{246} \mathrm{Am}^{m}$ & 65025 & 15 & 30 & 10 & & 25.0 & $\mathrm{~m}$ & 0.2 & $2^{(-)}$ & 98 & & & $\beta^{-} \approx 100 ; \mathrm{IT}<0.02$ \\
\hline${ }^{246} \mathrm{Cm}$ & 62618.4 & 2.1 & & & & 4.76 & ky & 0.04 & $0^{+}$ & 98 & & & $\alpha \approx 100 ; \mathrm{SF}=0.026157$ \\
\hline${ }^{246} \mathrm{Bk}$ & 63970 & 60 & & & & 1.80 & d & 0.02 & $2^{(-)}$ & 98 & & & $\beta^{+} \approx 100 ; \alpha=0.1 \#$ \\
\hline${ }^{246} \mathrm{Cf}$ & 64091.7 & 2.1 & & & & 35.7 & $\mathrm{~h}$ & 0.5 & $0^{+}$ & 98 & & & $\alpha=100 ; \mathrm{SF}=2.5 \mathrm{e}-42 ; \varepsilon<4 \mathrm{e}-3$ \\
\hline${ }^{246} \mathrm{Es}$ & 67900\# & $220 \#$ & & & & 7.7 & $\mathrm{~m}$ & 0.5 & $4^{-} \#$ & 98 & & & $\beta^{+}=90.118 ; \alpha=9.918 ; \ldots$ \\
\hline${ }^{246} \mathrm{Es}^{p}$ & $68250 \#$ & $300 \#$ & $350 \#$ & $200 \#$ & & & & & $a m$ & & & & \\
\hline${ }^{246} \mathrm{Fm}$ & 70140 & 40 & & & & 1.1 & $\mathrm{~s}$ & 0.2 & $0^{+}$ & 98 & $96 \mathrm{Ni09}$ & $\mathrm{D}$ & $\alpha=? ; \beta^{+}>10 ; \mathrm{SF}=4.513 ; \ldots$ \\
\hline${ }^{246} \mathrm{Md}$ & 76280\# & 330\# & & & & 1.0 & $\mathrm{~s}$ & 0.4 & & 98 & & & $\alpha=? ; \beta^{+} ? ; \mathrm{SF}$ ? \\
\hline${ }^{246} \mathrm{Md}^{m}$ & $76490 \#$ & $340 \#$ & 210 & 70 & $\mathrm{EU}$ & 1.0 & $\mathrm{~s}$ & 0.4 & & & $96 \mathrm{Ni09}$ & TD & $\alpha=? ; \beta^{+}$? \\
\hline${ }^{246} \mathrm{Es}$ & $\mathrm{D}: \ldots ;$ & $\beta^{+} \mathrm{SF} \approx$ & 0.003 & & & & & & & & & & \\
\hline${ }^{246} \mathrm{Fm}$ & $\mathrm{D}: \ldots ;$ & $\beta^{+} \mathrm{SF}=$ & 105 & & & & & & & & & & \\
\hline${ }^{246} \mathrm{Md}^{m}$ & I : no lon & ger co & 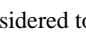 & & & & & & & & & & \\
\hline
\end{tabular}

\begin{tabular}{|c|c|c|c|c|c|c|c|c|c|c|c|c|c|}
\hline${ }^{247} \mathrm{Pu}$ & $69000 \#$ & $300 \#$ & & & & 2.27 & d & 0.23 & $1 / 2^{+} \#$ & 93 & & & $\beta^{-}=100$ \\
\hline${ }^{247} \mathrm{Am}$ & $67150 \#$ & $100 \#$ & & & & 23.0 & $\mathrm{~m}$ & 1.3 & $5 / 2 \#$ & 93 & & & $\beta^{-}=100$ \\
\hline${ }^{247} \mathrm{Cm}$ & 65534 & 4 & & & & 15.6 & My & 0.5 & $9 / 2^{-}$ & 93 & & & $\alpha=100$ \\
\hline${ }^{247} \mathrm{Bk}$ & 65491 & 6 & & & & 1.38 & ky & 0.25 & $\left(3 / 2^{-}\right)$ & 93 & & & $\alpha \approx 100 ;$ SF ? \\
\hline${ }^{247} \mathrm{Cf}$ & 66137 & 8 & & & & 3.11 & $\mathrm{~h}$ & 0.03 & $7 / 2^{+} \#$ & 93 & & & $\varepsilon \approx 100 ; \alpha=0.0355$ \\
\hline${ }^{247}$ Es & $68610 \#$ & $30 \#$ & & & & 4.6 & $\mathrm{~m}$ & 0.3 & $7 / 2^{+} \#$ & 93 & & & $\beta^{+} \approx 93 ; \alpha \approx 7 ; \mathrm{SF} \approx 9 \mathrm{e}-5 \#$ \\
\hline${ }^{247} \mathrm{Es}^{p}$ & 68930\# & $200 \#$ & $320 \#$ & $200 \#$ & & & & & am & & & & \\
\hline${ }^{247} \mathrm{Fm}$ & 71580\# & $140 \#$ & & & & 35 & $\mathrm{~s}$ & 4 & $5 / 2^{+} \#$ & 93 & & & $\alpha \geq 50 ; \beta^{+} \leq 50$ \\
\hline${ }^{247} \mathrm{Fm}^{m}$ & & & non & xistent & EU & 9.2 & $\mathrm{~s}$ & 2.3 & & 93 & 67 Fl15 & I & $\alpha \approx 100 ;$ IT ? \\
\hline${ }^{247} \mathrm{Fm}^{p}$ & 71730\# & $170 \#$ & $150 \#$ & $100 \#$ & $\mathrm{Nm}$ & & & & $\left(7 / 2^{+}\right)$ & & & & \\
\hline${ }^{247} \mathrm{Fm}^{q}$ & 71980\# & $210 \#$ & $400 \#$ & $150 \#$ & & & & & & & & & \\
\hline${ }^{247} \mathrm{Md}$ & 76040\# & $320 \#$ & & & $*$ & 270 & $\mathrm{~ms}$ & 160 & $1 / 2^{-\#}$ & 93 & 93Но.А & TD & $\mathrm{SF}=? ; \alpha ?$ \\
\hline${ }^{247} \mathrm{Md}^{m}$ & 76170\# & $310 \#$ & $130 \#$ & $100 \#$ & $\mathrm{Nm} *$ & 1.12 & $\mathrm{~s}$ & 0.22 & $\left(7 / 2^{+}\right)$ & & 93Но.А & $\mathrm{TD}$ & $\alpha=100 ; \mathrm{SF}=0.0001 \#$ \\
\hline
\end{tabular}




\begin{tabular}{|c|c|c|c|c|c|c|c|c|c|c|c|c|c|}
\hline Nuclide & \multicolumn{2}{|c|}{$\begin{array}{l}\text { Mass excess } \\
\quad(\mathrm{keV})\end{array}$} & \multicolumn{3}{|c|}{$\begin{array}{l}\text { Excitation } \\
\text { energy }(\mathrm{keV})\end{array}$} & \multicolumn{3}{|c|}{ Half-life } & $J^{\pi}$ & Ens & Reference & \multicolumn{2}{|l|}{$\begin{array}{l}\text { Decay modes and } \\
\text { intensities }(\%)\end{array}$} \\
\hline${ }^{248} \mathrm{Am}$ & $70560 \#$ & $200 \#$ & & & & $3 \#$ & $\mathrm{~m}$ & & & 99 & & $\beta^{-} ?$ & \\
\hline${ }^{248} \mathrm{Cm}$ & 67392 & 5 & & & & 348 & ky & 6 & $0^{+}$ & 99 & & $\alpha=91.6116 ; \mathrm{SF}=8.3916 ; \ldots$ & * \\
\hline${ }^{248} \mathrm{Bk}$ & $68080 \#$ & 70\# & & & & $*>9$ & $\mathrm{y}$ & & $6^{+} \#$ & 99 & & $\alpha ?$ & \\
\hline${ }^{248} \mathrm{Bk}^{m}$ & 68110 & 21 & $30 \#$ & 70\# & & $* \quad 23.7$ & $\mathrm{~h}$ & 0.2 & $1^{(-)}$ & 99 & & $\beta^{-}=705 ; \varepsilon=305 ; \alpha=0.001 \#$ & \\
\hline${ }^{248} \mathrm{Bk}^{p}$ & 68130 & 50 & $50 \#$ & $50 \#$ & & & & & $\left(5^{-}\right)$ & & & & \\
\hline${ }^{248} \mathrm{Cf}$ & 67240 & 5 & & & & 334 & $\mathrm{~d}$ & 3 & $0^{+}$ & 99 & & $\alpha \approx 100 ; \mathrm{SF}=0.00293$ & \\
\hline${ }^{248}$ Es & $70300 \#$ & $50 \#$ & & & & 27 & $\mathrm{~m}$ & 5 & $2^{-} \#, 0^{+} \#$ & 99 & & $\beta^{+} \approx 100 ; \alpha \approx 0.25 ; \beta^{+} \mathrm{SF}=3 \mathrm{e}-5$ & \\
\hline${ }^{248} \mathrm{Es}^{m}$ & & & \multicolumn{2}{|c|}{ non existent } & $\mathrm{RN}$ & 41 & $\mathrm{~m}$ & & & & $89 \mathrm{Ha} 27 \mathrm{I}$ & & \\
\hline${ }^{248} \mathrm{Fm}$ & 71906 & 12 & & & & 36 & $\mathrm{~s}$ & 3 & $0^{+}$ & 99 & & $\alpha=937 ; \beta^{+}=77 ; \mathrm{SF}=0.105$ & \\
\hline${ }^{248} \mathrm{Md}$ & $77150 \#$ & $240 \#$ & & & & 7 & s & 3 & & 99 & & $\beta^{+}=8010 ; \alpha=2010 ; \ldots$ & * \\
\hline${ }^{248} \mathrm{Md}^{p}$ & 77250\# & $250 \#$ & $100 \#$ & 70\# & & & & & & & & & \\
\hline${ }^{248} \mathrm{No}$ & 80660\# & $300 \#$ & & & & $<2$ & $\mu \mathrm{s}$ & & $0^{+}$ & & 03Be18 I & SF ? & \\
\hline$*^{248} \mathrm{Cm}$ & \multicolumn{5}{|c|}{$\mathrm{D}: \ldots ; 2 \beta^{-} ?$} & & & & & & & & ** \\
\hline$*^{248} \mathrm{Md}$ & \multicolumn{5}{|c|}{$\mathrm{D}: \ldots ; \beta^{+} \mathrm{SF}<0.05$} & & & & & & & & ** \\
\hline${ }^{249} \mathrm{Am}$ & 73100\# & $300 \#$ & & & & $1 \#$ & $\mathrm{~m}$ & & & & & $\beta^{-} ?$ & \\
\hline${ }^{249} \mathrm{Cm}$ & 70750 & 5 & & & & 64.15 & $\mathrm{~m}$ & 0.03 & $1 / 2^{(+)}$ & 99 & & $\beta^{-}=100$ & \\
\hline${ }^{249} \mathrm{Cm}^{m}$ & 70799 & 5 & 48.758 & 0.017 & & 23 & $\mu \mathrm{s}$ & & $\left(7 / 2^{+}\right)$ & 99 & & $\alpha=100$ & \\
\hline${ }^{249} \mathrm{Bk}$ & 69849.6 & 2.6 & & & & 330 & $\mathrm{~d}$ & 4 & $7 / 2^{+}$ & 99 & & $\beta^{-} \approx 100 ; \alpha=0.001458$ & * \\
\hline${ }^{249} \mathrm{Bk}^{m}$ & 69858.4 & 2.6 & 8.80 & 0.10 & & 300 & $\mu \mathrm{s}$ & & $\left(3 / 2^{-}\right)$ & 99 & & $\mathrm{IT}=100$ & \\
\hline${ }^{249} \mathrm{Cf}$ & 69725.6 & 2.2 & & & & 351 & $\mathrm{y}$ & 2 & $9 / 2^{-}$ & 99 & & $\alpha=100 ; \mathrm{SF}=5.0 \mathrm{e}-74$ & \\
\hline${ }^{249} \mathrm{Cf}^{m}$ & 69870.6 & 2.2 & 144.98 & 0.05 & & 45 & $\mu \mathrm{s}$ & 5 & $5 / 2^{+}$ & 99 & & $\mathrm{IT}=100$ & \\
\hline${ }^{249} \mathrm{Es}$ & $71180 \#$ & $30 \#$ & & & & 102.2 & $\mathrm{~m}$ & 0.6 & $7 / 2^{+}$ & 99 & & $\beta^{+} \approx 100 ; \alpha=0.578$ & \\
\hline${ }^{249} \mathrm{Fm}$ & $73620 \#$ & $100 \#$ & & & & 2.6 & $\mathrm{~m}$ & 0.7 & $7 / 2^{+} \#$ & 99 & & $\beta^{+} ? ; \alpha=339$ & \\
\hline${ }^{249} \mathrm{Md}$ & $77330 \#$ & $220 \#$ & & & & 24 & $\mathrm{~s}$ & 4 & $\left(7 / 2^{-}\right)$ & 99 & $01 \mathrm{He} 35 \mathrm{~J}$ & $\alpha>60 ; \beta^{+} ?$ & \\
\hline${ }^{249} \mathrm{Md}^{m}$ & $77430 \#$ & $250 \#$ & $100 \#$ & $100 \#$ & & 1.9 & $\mathrm{~s}$ & 0.9 & $\left(1 / 2^{-}\right)$ & & $01 \mathrm{He} 35$ TJD & $\alpha=100$ & \\
\hline${ }^{249}$ No & $81820 \#$ & $340 \#$ & & & & 57 & $\mu \mathrm{s}$ & 12 & $5 / 2^{+} \#$ & 99 & 03Be18 T & $\beta^{+} ? ; \alpha ?$ & \\
\hline
\end{tabular}

\begin{tabular}{|c|c|c|c|c|c|c|c|c|c|c|c|c|}
\hline${ }^{250} \mathrm{Cm}$ & 72989 & 11 & & & & 8300\# & $y$ & & $0^{+}$ & 01 & & $\mathrm{SF} \approx 74 ; \alpha \approx 18 ; \beta^{-} \approx 8$ \\
\hline${ }^{250} \mathrm{Bk}$ & 72951 & 4 & & & & 3.212 & $\mathrm{~h}$ & 0.005 & $2^{-}$ & 01 & & $\beta^{-}=100$ \\
\hline${ }^{250} \mathrm{Bk}^{m}$ & 72987 & 4 & 35.59 & 0.05 & & 29 & $\mu \mathrm{s}$ & 1 & $\left(4^{+}\right)$ & 01 & & $\mathrm{IT}=100$ \\
\hline${ }^{250} \mathrm{Bk}^{n}$ & 73036 & 5 & 84.1 & 2.1 & $\mathrm{AD}$ & 213 & $\mu \mathrm{s}$ & 8 & $\left(7^{+}\right)$ & 01 & & IT ? \\
\hline${ }^{250} \mathrm{Cf}$ & 71171.8 & 2.1 & & & & 13.08 & $\mathrm{y}$ & 0.09 & $0^{+}$ & 01 & & $\alpha \approx 100 ; \mathrm{SF}=0.0773$ \\
\hline${ }^{250} \mathrm{Es}$ & 73230\# & $100 \#$ & & & * & 8.6 & $\mathrm{~h}$ & 0.1 & $\left(6^{+}\right)$ & 01 & & $\beta^{+}>97 ; \alpha ?$ \\
\hline${ }^{250} \mathrm{Es}^{m}$ & $73430 \#$ & $180 \#$ & $200 \#$ & $150 \#$ & $*$ & 2.22 & $\mathrm{~h}$ & 0.05 & $1^{(-)}$ & 01 & & $\beta^{+} \approx 100 ; \alpha ?$ \\
\hline${ }^{250} \mathrm{Fm}$ & 74074 & 12 & & & & 30 & $\mathrm{~m}$ & 3 & $0^{+}$ & 01 & & $\alpha>90 ; \varepsilon<10 ; \mathrm{SF}=0.006910$ \\
\hline${ }^{250} \mathrm{Fm}^{m}$ & 75570\# & $300 \#$ & $1500 \#$ & $300 \#$ & & 1.8 & $\mathrm{~s}$ & 0.1 & $7,8 \#$ & 01 & & $\mathrm{IT}>80 ; \alpha<20 ; \beta^{+} ? ; \ldots$ \\
\hline${ }^{250} \mathrm{Md}$ & 78640\# & $300 \#$ & & & & 52 & $\mathrm{~s}$ & 6 & & 01 & & $\beta^{+}=933 ; \alpha=73 ; \beta^{+} \mathrm{SF}=0.02$ \\
\hline${ }^{250} \mathrm{Md}^{p}$ & 78830\# & $340 \#$ & $190 \#$ & $150 \#$ & & & & & am & & & \\
\hline${ }^{250} \mathrm{No}$ & $81520 \#$ & $200 \#$ & & & & 5.7 & $\mu \mathrm{s}$ & 0.8 & $0^{+}$ & 01 & 03Be18 T & $\mathrm{SF} \approx 100 ; \alpha=0.1 \# ; \ldots$ \\
\hline$*^{250} \mathrm{Fm}^{m}$ & $\mathrm{D}: \ldots$ & $\mathrm{SF}<8$ & $2 \mathrm{E}-5$ & & & & & & & & & \\
\hline$*^{250} \mathrm{No}$ & $\mathrm{D}: \ldots$ & $\beta^{+}=0$. & $0025 \#$ & & & & & & & & & \\
\hline$*^{250} \mathrm{No}$ & $\mathrm{T}:$ also & $010 \mathrm{~g} 08$ & $3=36(+11-c$ & & & & & & & & & \\
\hline${ }^{251} \mathrm{Cm}$ & 76648 & 23 & & & & 16.8 & $\mathrm{~m}$ & 0.2 & $\left(1 / 2^{+}\right)$ & 99 & & $\beta^{-}=100$ \\
\hline${ }^{251} \mathrm{Bk}$ & 75228 & 11 & & & & 55.6 & $\mathrm{~m}$ & 1.1 & $3 / 2^{-} \#$ & 99 & & $\beta^{-}=100$ \\
\hline${ }^{251} \mathrm{Bk}^{m}$ & 75264 & 11 & 35.5 & 1.3 & & 58 & $\mu \mathrm{s}$ & 4 & $7 / 2^{+} \#$ & 99 & & $\mathrm{IT}=100$ \\
\hline${ }^{251} \mathrm{Cf}$ & 74135 & 4 & & & & 900 & $\mathrm{y}$ & 40 & $1 / 2^{+}$ & 99 & & $\alpha \approx 100 ;$ SF ? \\
\hline${ }^{251}$ Es & 74512 & 6 & & & & 33 & $\mathrm{~h}$ & 1 & $\left(3 / 2^{-}\right)$ & 99 & & $\varepsilon ? ; \alpha=0.52$ \\
\hline${ }^{251} \mathrm{Fm}$ & 75987 & 8 & & & & 5.30 & $\mathrm{~h}$ & 0.08 & $\left(9 / 2^{-}\right)$ & 99 & & $\beta^{+}=98.2013 ; \alpha=1.8013$ \\
\hline${ }^{251} \mathrm{Fm}^{m}$ & 76178 & 8 & 191 & 2 & & 15.2 & $\mu \mathrm{s}$ & 2.3 & $\left(5 / 2^{+}\right)$ & 99 & & $\mathrm{IT}=100$ \\
\hline${ }^{251} \mathrm{Md}$ & 79030\# & $200 \#$ & & & & 4.0 & $\mathrm{~m}$ & 0.5 & $7 / 2^{-} \#$ & 99 & & $\beta^{+}=95 \# ; \alpha=$ ? \\
\hline${ }^{251} \mathrm{Md}^{p}$ & 79080\# & $210 \#$ & $50 \#$ & $30 \#$ & & & & & am & & & \\
\hline${ }^{251}$ No & 82910\# & $180 \#$ & & & $*$ & 760 & $\mathrm{~ms}$ & 30 & $7 / 2^{+} \#$ & 99 & $01 \mathrm{He} 35 \mathrm{TD}$ & $\alpha=8316 ; \beta^{+}$?; $\mathrm{SF}<0.3$ \\
\hline${ }^{251} \mathrm{No}^{m}$ & 83030\# & $210 \#$ & $110 \#$ & $180 \#$ & $*$ & 1.7 & $\mathrm{~s}$ & 1.0 & $9 / 2^{-} \#$ & & 97He29 ETD & $\alpha=100$ \\
\hline${ }^{251} \mathrm{Lr}$ & 87900\# & $300 \#$ & & & & $150 \#$ & $\mu \mathrm{s}$ & & & & & $\beta^{+} ? ; \alpha ?$ \\
\hline$*^{251} \mathrm{No}^{m}$ & I : tentat & tive assi & ment & $97 \mathrm{He} 2$ & uld no & ot be co & onfirn & med i & 01He35 & & & \\
\hline
\end{tabular}




\begin{tabular}{|c|c|c|c|c|c|c|c|c|c|c|c|c|c|c|}
\hline \multirow{2}{*}{$\begin{array}{l}\text { Nuclide } \\
{ }^{252} \mathrm{Cm}\end{array}$} & \multicolumn{2}{|c|}{$\begin{array}{l}\text { Mass excess } \\
(\mathrm{keV})\end{array}$} & \multicolumn{4}{|c|}{$\begin{array}{c}\text { Excitation } \\
\text { energy }(\mathrm{keV})\end{array}$} & \multicolumn{3}{|c|}{ Half-life } & \multirow{2}{*}{$\frac{J^{\pi}}{0^{+}}$} & \multirow{2}{*}{$\begin{array}{l}\text { Ens } \\
99\end{array}$} & Reference & \multicolumn{2}{|l|}{$\begin{array}{l}\text { Decay modes and } \\
\text { intensities (\%) }\end{array}$} \\
\hline & 79060\# & $300 \#$ & & & & & $<1$ & $\mathrm{~d}$ & & & & & $\beta^{-}$? & \\
\hline${ }^{252} \mathrm{Bk}$ & $78530 \#$ & $200 \#$ & & & & & 1.8 & $\mathrm{~m}$ & 0.5 & & 99 & 92Kr.A TD & $\beta^{-}=? ; \alpha ?$ & \\
\hline${ }^{252} \mathrm{Cf}$ & 76034 & 5 & & & & & 2.645 & $\mathrm{y}$ & 0.008 & $0^{+}$ & 99 & & $\alpha=96.9088 ; \mathrm{SF}=3.0928$ & \\
\hline${ }^{252} \mathrm{Es}$ & 77290 & 50 & & & & & 471.7 & $\mathrm{~d}$ & 1.9 & $\left(5^{-}\right)$ & 99 & & $\alpha=782 ; \varepsilon=222$ & \\
\hline${ }^{252} \mathrm{Fm}$ & 76817 & 6 & & & & & 25.39 & $\mathrm{~h}$ & 0.04 & $0^{+}$ & 99 & & $\alpha \approx 100 ; \mathrm{SF}=0.00232 ; 2 \beta^{+}$? & \\
\hline${ }^{252} \mathrm{Md}$ & 80630\# & $200 \#$ & & & & & 2.3 & $\mathrm{~m}$ & 0.8 & & 99 & & $\beta^{+}>50 ; \alpha<50$ & \\
\hline${ }^{252} \mathrm{Md}^{p}$ & $80670 \#$ & $220 \#$ & $40 \#$ & $100 \#$ & & & & & & $a m$ & & & & \\
\hline${ }^{252} \mathrm{No}$ & 82881 & 13 & & & & & 2.44 & $\mathrm{~s}$ & 0.04 & $0^{+}$ & 99 & $01 \mathrm{Og} 08 \mathrm{TD}$ & $\alpha \approx 67 ; \mathrm{SF}=32.25 ; \beta^{+} ?$ & $*$ \\
\hline${ }^{252} \mathrm{Lr}$ & 88840\# & $250 \#$ & & & & & 390 & $\mathrm{~ms}$ & 90 & & 99 & $01 \mathrm{He} 35 \mathrm{TD}$ & $\beta^{+}=71 \# ; \alpha=? ; \mathrm{SF}<1$ & \\
\hline${ }^{252} \mathrm{Lr}^{p}$ & 89140\# & $290 \#$ & $300 \#$ & $150 \#$ & & & & & & & & & & \\
\hline$*^{252} \mathrm{No}$ & $\mathrm{T}$ : other & 03Be18 & $=2.38(+$ & $+0.26-0$ & $.22)$ & & $D: S$ & SF fro & om $01 \mathrm{c}$ & $\mathrm{Og} 08 ; \alpha \mathrm{e}$ & estima & ated by NUBASE & & $* *$ \\
\hline${ }^{253} \mathrm{Bk}$ & 80930\# & $360 \#$ & & & & & $10 \#$ & $\mathrm{~m}$ & & & & 91Kr.A I & $\beta^{-} ?$ & * \\
\hline${ }^{253} \mathrm{Cf}$ & 79301 & 6 & & & & & 17.81 & $\mathrm{~d}$ & 0.08 & $\left(7 / 2^{+}\right)$ & 99 & & $\beta^{-} \approx 100 ; \alpha=0.314$ & \\
\hline${ }^{253} \mathrm{Es}$ & 79013.7 & 2.6 & & & & & 20.47 & $\mathrm{~d}$ & 0.03 & $7 / 2^{+}$ & 99 & & $\alpha=100 ; \mathrm{SF}=8.7 \mathrm{e}-63$ & \\
\hline${ }^{253} \mathrm{Fm}$ & 79350 & 4 & & & & & 3.00 & $\mathrm{~d}$ & 0.12 & $(1 / 2)^{+}$ & 99 & & $\varepsilon=881 ; \alpha=121$ & \\
\hline${ }^{253} \mathrm{Md}$ & $81300 \#$ & $210 \#$ & & & & & 12 & $\mathrm{~m}$ & 8 & $7 / 2^{-} \#$ & 99 & & $\beta^{+} \approx 100 ; \alpha=0.6 \#$ & \\
\hline${ }^{253} \mathrm{Md}^{p}$ & $81300 \#$ & $210 \#$ & $0 \#$ & $30 \#$ & & & & & & $a m$ & & & & \\
\hline${ }^{253} \mathrm{No}$ & 84470\# & $100 \#$ & & & & & 1.62 & $\mathrm{~m}$ & 0.15 & 9/2-\# & 99 & & $\alpha=? ; \beta^{+}=20 \# ; \mathrm{SF}=0.001 \#$ & \\
\hline${ }^{253} \mathrm{No}^{m}$ & $84590 \#$ & $100 \#$ & 129 & 19 & $\mathrm{AD}$ & & 31 & $\mu \mathrm{s}$ & & $5 / 2^{+} \#$ & & & $\alpha=?$ & \\
\hline${ }^{253} \mathrm{Lr}$ & $88690 \#$ & $220 \#$ & & & & $* \&$ & 580 & $\mathrm{~ms}$ & 70 & $\left(7 / 2^{-}\right)$ & 99 & 01He35 TJD & $\alpha=9010 ; \mathrm{SF}=2.621 ; \beta^{+}=1 \#$ & \\
\hline${ }^{253} \mathrm{Lr}^{m}$ & $88710 \#$ & $250 \#$ & $30 \#$ & $100 \#$ & & $* \&$ & 1.5 & $\mathrm{~s}$ & 0.3 & $\left(1 / 2^{-}\right)$ & 99 & $01 \mathrm{He} 35$ TJD & $\alpha=9010 ; \mathrm{SF}=85 ; \beta^{+}=1 \#$ & \\
\hline${ }^{253} \mathrm{Rf}$ & $93790 \#$ & $450 \#$ & & & & $*$ & 13 & $\mathrm{~ms}$ & 5 & $(7 / 2)^{(+\#)}$ & & 95Ho.B TJ & $\mathrm{SF} \approx 50 ; \alpha \approx 50$ & * \\
\hline${ }^{253} \mathrm{Rf}^{m}$ & 93990\# & $470 \#$ & $200 \#$ & $150 \#$ & & $*$ & 52 & $\mu \mathrm{s}$ & 14 & $(1 / 2)^{(-\#)}$ & 99 & $97 \mathrm{He} 29 \mathrm{~J}$ & $\mathrm{SF}=? ; \alpha=5 \#$ & \\
\hline$*^{253} \mathrm{Bk}$ & I : possib & le identi & fication & , in 91 & Kr.A. & Need & ds conf & firma & ation & & & & & $* *$ \\
\hline$*^{253} \mathrm{Rf}$ & I : the sta & te with & $\approx 1.8 \mathrm{~s} \mathrm{r}$ & reporte & in $\mathrm{El}$ & NSDF & is not & $t \operatorname{conf}$ & firmed & & & & & ** \\
\hline
\end{tabular}

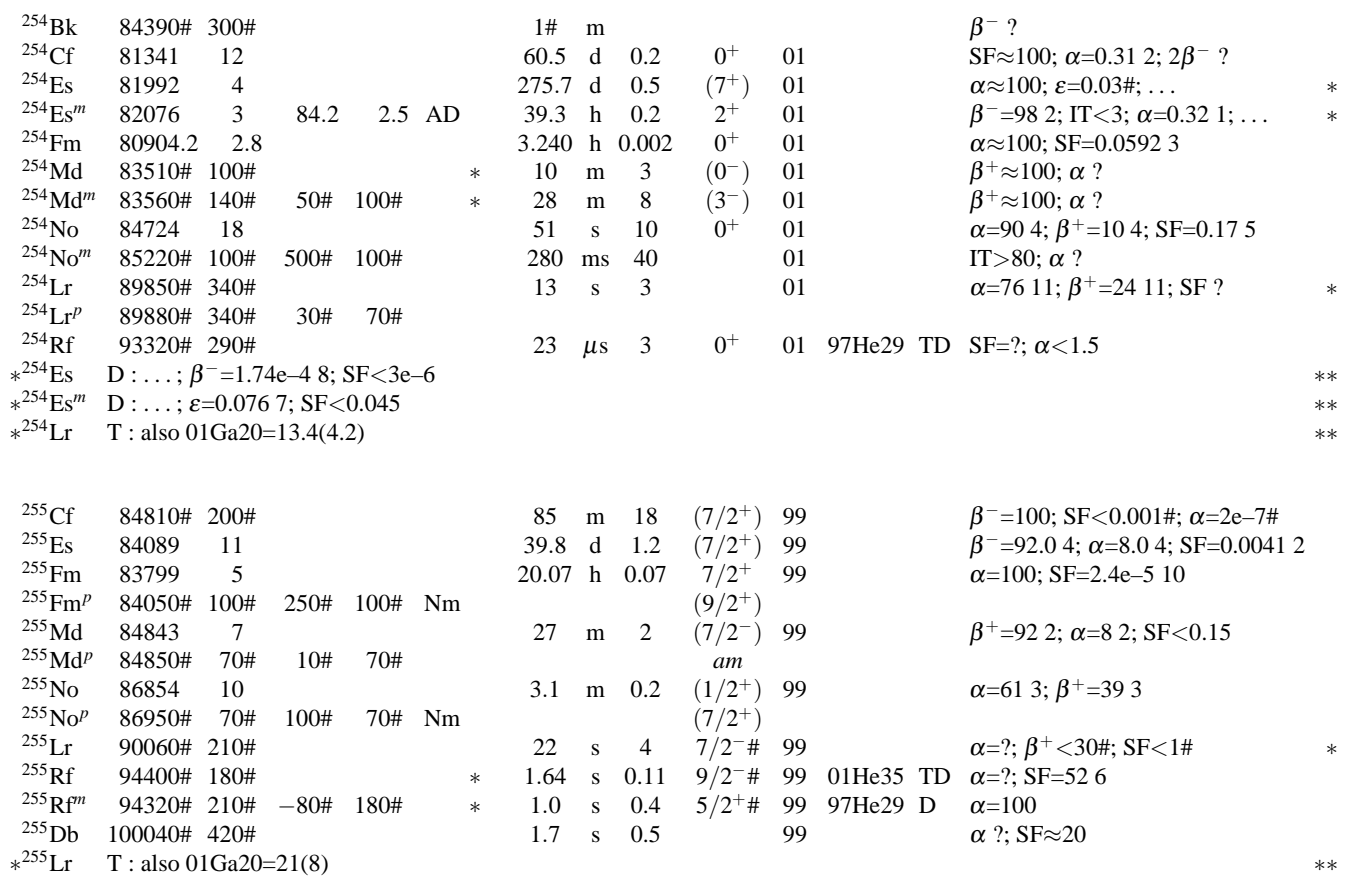




\begin{tabular}{|c|c|c|c|c|c|c|c|c|c|c|c|c|c|}
\hline Nuclide & \multicolumn{2}{|c|}{$\begin{array}{l}\text { Mass excess } \\
(\mathrm{keV})\end{array}$} & \multicolumn{3}{|c|}{$\begin{array}{l}\text { Excitation } \\
\text { energy }(\mathrm{keV})\end{array}$} & \multicolumn{3}{|c|}{ Half-life } & $J^{\pi}$ & Ens & Reference & \multicolumn{2}{|l|}{$\begin{array}{c}\text { Decay modes and } \\
\text { intensities (\%) }\end{array}$} \\
\hline${ }^{256} \mathrm{Cf}$ & $87040 \#$ & $300 \#$ & & & & 12.3 & $\mathrm{~m}$ & 1.2 & $0^{+}$ & 99 & & $\mathrm{SF}=100 ; \alpha=6.2 \mathrm{e}-7 \# ; 2 \beta^{-} ?$ & \\
\hline${ }^{256}$ Es & 87190\# & $100 \#$ & & & * & 25.4 & $\mathrm{~m}$ & 2.4 & $\left(1^{+}, 0^{-}\right)$ & 99 & & $\beta^{-}=100$ & \\
\hline${ }^{256} \mathrm{Es}^{m}$ & 87190\# & $140 \#$ & $0 \#$ & $100 \#$ & * & 7.6 & $\mathrm{~h}$ & & $\left(8^{+}\right)$ & 99 & & $\beta^{-} \approx 100 ; \beta^{-} \mathrm{SF}=0.002$ & \\
\hline${ }^{256} \mathrm{Fm}$ & 85486 & 7 & & & & 157.6 & $\mathrm{~m}$ & 1.3 & $0^{+}$ & 99 & & $\mathrm{SF}=91.93 ; \alpha=8.13$ & \\
\hline${ }^{256} \mathrm{Md}$ & 87620 & 50 & & & & 77 & $\mathrm{~m}$ & 2 & $\left(1^{-}\right)$ & 99 & & $\beta^{+}=? ; \alpha=9.27 ; \mathrm{SF}<3$ & \\
\hline${ }^{256} \mathrm{Md}^{p}$ & $87700 \#$ & $110 \#$ & $80 \#$ & $100 \#$ & & & & & am & & & & \\
\hline${ }^{256} \mathrm{No}$ & 87824 & 8 & & & & 2.91 & $\mathrm{~s}$ & 0.05 & $0^{+}$ & 99 & & $\alpha \approx 100 ; \mathrm{SF}=0.536 ; \varepsilon<0.01 \#$ & \\
\hline${ }^{256} \mathrm{Lr}$ & $91870 \#$ & $220 \#$ & & & & 27 & $\mathrm{~s}$ & 3 & & 99 & & $\alpha=8510 ; \beta^{+}=1510 ; \mathrm{SF}<0.03$ & \\
\hline${ }^{256} \mathrm{Lr}^{p}$ & 91970\# & $230 \#$ & 100 & 70 & XL & & & & & & & & \\
\hline${ }^{256} \mathrm{Rf}$ & 94236 & 24 & & & & 6.45 & $\mathrm{~ms}$ & 0.14 & $0^{+}$ & 99 & $97 \mathrm{He} 29$ TD & $\mathrm{SF}=? ; \alpha=0.3217$ & * \\
\hline${ }^{256} \mathrm{Db}$ & 100720\# & $290 \#$ & & & & 1.9 & $\mathrm{~s}$ & 0.4 & & 99 & $01 \mathrm{He} 35 \mathrm{TD}$ & $\alpha=? ; \beta^{+}=3612 ; \mathrm{SF}=?$ & * \\
\hline${ }^{256} \mathrm{Rf}$ & $\mathrm{T}:$ averas & $97 \mathrm{H}$ & $29=6$ & $2(0.2) 8$ & $4 \mathrm{Og} 02$ & $.7(0.2)$ & & & & & & & $* *$ \\
\hline$k^{256} \mathrm{Db}$ & $\mathrm{T}:$ averas & ge $01 \mathrm{H}$ & $35=1.6$ & $6(+0.5-$ & $0.3) 83$ & $A=2.6$ & $(+1.4$ & $4-0.8)$ & & & & & $* *$ \\
\hline
\end{tabular}

\begin{tabular}{|c|c|c|c|c|c|c|c|c|c|c|c|c|c|c|}
\hline${ }^{257}$ Es & 89400\# & $410 \#$ & & & & & 7.7 & $\mathrm{~d}$ & 0.2 & $7 / 2^{+} \#$ & 99 & & & $\beta^{-}=100 ; \alpha=4 \mathrm{e}-4 \#$ \\
\hline${ }^{257} \mathrm{Fm}$ & 88589 & 6 & & & & & 100.5 & d & 0.2 & $\left(9 / 2^{+}\right)$ & 99 & & & $\alpha \approx 100 ; \mathrm{SF}=0.2104$ \\
\hline${ }^{257} \mathrm{Md}$ & 88996.2 & 2.8 & & & & & 5.52 & $\mathrm{~h}$ & 0.05 & $\left(7 / 2^{-}\right)$ & 99 & & & $\varepsilon=853 ; \alpha=153 ; \mathrm{SF}<4$ \\
\hline${ }^{257}$ No & 90241 & 22 & & & & & 25 & $\mathrm{~s}$ & 2 & $\left(7 / 2^{+}\right)$ & 99 & $02 \mathrm{Ho} 11$ & $\mathrm{D}$ & $\alpha=? ; \beta^{+}=158$ \\
\hline${ }^{257} \mathrm{No}^{p}$ & 90550\# & $110 \#$ & $310 \#$ & $100 \#$ & & & & & & am & & & & \\
\hline${ }^{257} \mathrm{Lr}$ & $92740 \#$ & $210 \#$ & & & & & 646 & $\mathrm{~ms}$ & 25 & $9 / 2^{+} \#$ & 99 & & & $\alpha \approx 100 ; \beta^{+}=0.01 \# ; \mathrm{SF}=0.001 \#$ \\
\hline${ }^{257} \mathrm{Lr}^{p}$ & 92890\# & $230 \#$ & $150 \#$ & $100 \#$ & & & & & & $a m$ & & & & \\
\hline${ }^{257} \mathrm{Rf}$ & 95930\# & $100 \#$ & & & & & 4.7 & $\mathrm{~s}$ & 0.3 & $\left(1 / 2^{+}\right)$ & 99 & $97 \mathrm{He} 29$ & JD & $\alpha=? ; \beta^{+}=111 ; \mathrm{SF}<1.4$ \\
\hline${ }^{257} \mathrm{Rf}^{m}$ & $96050 \#$ & $100 \#$ & 114 & 17 & $\mathrm{AD}$ & & 3.9 & s & 0.4 & $\left(11 / 2^{-}\right)$ & 99 & $97 \mathrm{He} 29$ & EJ & $\alpha \approx 100 ; \mathrm{SF}=0.7 \# ; \beta^{+} ?$ \\
\hline${ }^{257} \mathrm{Rf}^{p}$ & 96030\# & $120 \#$ & $100 \#$ & 70\# & & & & & & $\left(7 / 2^{+}\right)$ & & & & \\
\hline${ }^{257} \mathrm{Db}$ & 100340\# & $230 \#$ & & & & $* \&$ & 1.53 & $\mathrm{~s}$ & 0.17 & $\left(9 / 2^{+}\right)$ & 99 & $01 \mathrm{He} 35$ & TJD & $\alpha>94 ; \mathrm{SF}<6 ; \beta^{+}=1 \#$ \\
\hline${ }^{257} \mathrm{Db}^{m}$ & 100450\# & $250 \#$ & $100 \#$ & $100 \#$ & & $* \&$ & 790 & $\mathrm{~ms}$ & 130 & $\left(1 / 2^{-}\right)$ & 99 & $01 \mathrm{He} 35$ & TJD & $\alpha>87 ; \mathrm{SF}<13 ; \beta^{+}=1 \#$ \\
\hline
\end{tabular}

${ }^{257} \mathrm{Rf}^{m} \quad \mathrm{E}: 97 \mathrm{He} 29=118(4) \mathrm{keV}$ form direct comparison of two alpha lines

\begin{tabular}{|c|c|c|c|c|c|c|c|c|c|c|c|c|c|c|}
\hline${ }^{258} \mathrm{Es}$ & $92700 \#$ & $300 \#$ & & & & 3\# & $\mathrm{m}$ & & & & & & $\beta^{-} ? ; \alpha ?$ & \\
\hline${ }^{258} \mathrm{Fm}$ & 90430\# & $200 \#$ & & & & 370 & $\mu \mathrm{s}$ & 14 & $0^{+}$ & 01 & $86 \mathrm{Hu} 05$ & $\mathrm{~T}$ & $\mathrm{SF} \approx 100 ; \alpha ?$ & * \\
\hline${ }^{258} \mathrm{Md}$ & 91688 & 5 & & & $*$ & 51.5 & $\mathrm{~d}$ & 0.3 & $8^{-} \#$ & 01 & $93 \mathrm{Mo18}$ & $\mathrm{D}$ & $\alpha \approx 100 ; \beta^{+}<0.0015 ; \beta^{-}<0.0015$ & * \\
\hline${ }^{258} \mathrm{Md}^{m}$ & $91690 \#$ & $200 \#$ & $0 \#$ & $200 \#$ & * & 57.0 & $\mathrm{~m}$ & 0.9 & $1^{-} \#$ & 01 & $93 \mathrm{Mo18}$ & $\mathrm{D}$ & $\varepsilon=? ; \mathrm{SF}<20 ; \beta^{-}<10 \# ; \alpha<1.2$ & $*$ \\
\hline${ }^{258}$ No & 91480\# & $200 \#$ & & & & 1.2 & $\mathrm{~ms}$ & 0.2 & $0^{+}$ & 01 & & & $\mathrm{SF} \approx 100 ; \alpha=0.001 \# ; 2 \beta^{+} ?$ & \\
\hline${ }^{258} \mathrm{Lr}$ & $94840 \#$ & $100 \#$ & & & & 4.1 & $\mathrm{~s}$ & 0.3 & & 01 & & & $\alpha>95 ; \beta^{+}<5$ & \\
\hline${ }^{258} \mathrm{Lr}^{p}$ & $95040 \#$ & $180 \#$ & $200 \#$ & $150 \#$ & & & & & $a m$ & & & & & \\
\hline${ }^{258} \mathrm{Rf}$ & $96400 \#$ & $200 \#$ & & & & 12 & $\mathrm{~ms}$ & 2 & $0^{+}$ & 01 & & & $\mathrm{SF}=872 ; \alpha=132$ & \\
\hline${ }^{258} \mathrm{Db}$ & 101750\# & $340 \#$ & & & * & 4.5 & $\mathrm{~s}$ & 0.6 & & 01 & & & $\alpha=647 ; \beta^{+}=367 ; \mathrm{SF}<1 \#$ & \\
\hline${ }^{258} \mathrm{Db}^{m}$ & 101810\# & $350 \#$ & $60 \#$ & $100 \#$ & * & 20 & $\mathrm{~s}$ & 10 & & 01 & & & $\beta^{+} \approx 100 ;$ IT ? & \\
\hline${ }^{258} \mathrm{Sg}$ & $105420 \#$ & $410 \#$ & & & & 3.3 & $\mathrm{~ms}$ & 1.0 & $0^{+}$ & 01 & & & $\mathrm{SF}=? ; \alpha<20$ & \\
\hline$*^{258} \mathrm{Fm}$ & $\mathrm{T}:$ avera & ge $86 \mathrm{H}$ & $105=36$ & $0(20) 7$ & $1 \mathrm{Hu} 03=$ & $0(20)$ & (all 1 & $\sigma) \mathrm{EN}$ & NSDF give & es $3 \sigma$ & & & & ** \\
\hline$*^{258} \mathrm{Md}$ & $\mathrm{D}$ : deriv & ed from & "the $\mathrm{s}$ & um of & $\mathrm{SF}, \varepsilon$ an & $3^{-} \mathrm{dec}$ & ay br & ranche & $s<0.00$ & $3 \% "$ & & & & $* *$ \\
\hline$*^{258} \mathrm{Md}$ & D : & 3Мo18 & and $T(S$ & $\mathrm{SF})>1$ & $50000 \mathrm{y}$, & om 86I & Lo16 & , thus & $\mathrm{SF}<1 \mathrm{e}-$ & $4 \% \#$ & & & & $* *$ \\
\hline$*^{258} \mathrm{Md}^{m}$ & $\mathrm{D}: \mathrm{SF}<2$ & $0 \%$ der & ived fro & om 93N & Мo18 "th & sum of & $\mathrm{SF}$ & and $\beta$ & decay $b$ & rancl & hes $<30 \%$ & & & $* *$ \\
\hline${ }^{259} \mathrm{Fm}$ & 93700\# & $280 \#$ & & & & 1.5 & $\mathrm{~s}$ & 0.3 & $3 / 2^{+} \#$ & 99 & & & $\mathrm{SF}=100$ & \\
\hline${ }^{259} \mathrm{Md}$ & $93620 \#$ & $200 \#$ & & & & 1.60 & $\mathrm{~h}$ & 0.06 & $7 / 2^{-} \#$ & 99 & 93 Mo18 & $\mathrm{T}$ & $\mathrm{SF}=? ; \alpha<1.3$ & \\
\hline${ }^{259} \mathrm{No}$ & $94110 \#$ & $100 \#$ & & & & 58 & $\mathrm{~m}$ & 5 & $9 / 2^{+} \#$ & 99 & & & $\alpha=754 ; \varepsilon=254 ; \mathrm{SF}<10$ & \\
\hline${ }^{259} \mathrm{No}^{p}$ & 94390\# & $180 \#$ & $280 \#$ & $150 \#$ & & & & & & & & & & \\
\hline${ }^{259} \mathrm{Lr}$ & 95850\# & 70\# & & & & 6.2 & $\mathrm{~s}$ & 0.3 & $9 / 2^{+} \#$ & 99 & & & $\alpha=782 ; \mathrm{SF}=222 ; \beta^{+}=0.6 \#$ & \\
\hline${ }^{259} \mathrm{Lr}^{p}$ & $96200 \#$ & $170 \#$ & $350 \#$ & $150 \#$ & & & & & & & & & & \\
\hline${ }^{259} \mathrm{Rf}$ & 98400\# & $70 \#$ & & & & 2.8 & $\mathrm{~s}$ & 0.4 & $7 / 2^{+} \#$ & 99 & 94Gr08 & $\mathrm{T}$ & $\alpha=922 ; \mathrm{SF}=82 ; \beta^{+}=0.3 \#$ & $*$ \\
\hline${ }^{259} \mathrm{Rf}^{p}$ & 98500\# & $100 \#$ & $100 \#$ & 70\# & $\mathrm{Nm}$ & & & & $\left(3 / 2^{+}\right)$ & & & & & \\
\hline${ }^{259} \mathrm{Rf}^{q}$ & $98610 \#$ & $130 \#$ & $210 \#$ & $110 \#$ & $\mathrm{Nm}$ & & & & $\left(9 / 2^{+}\right)$ & & & & & \\
\hline${ }^{259} \mathrm{Db}$ & 102100\# & $210 \#$ & & & & 510 & $\mathrm{~ms}$ & 160 & & 99 & $01 \mathrm{Ga} 20$ & TD & $\alpha=100$ & \\
\hline${ }^{259} \mathrm{Sg}$ & 106660\# & $180 \#$ & & & & 580 & $\mathrm{~ms}$ & 210 & $1 / 2^{+} \#$ & 99 & & & $\alpha=9010 ; \mathrm{SF}<20$ & \\
\hline$*{ }^{259} \mathrm{Rf}$ & $\mathrm{T}$ : avera & ge $94 \mathrm{G}$ & $08=1.7$ & $(+0.8-$ & $0.5) 85$ & $3=3.4($ & (1.7) & $81 \mathrm{Be}$ & $03=3 \cdot 0(1$ & & & & & $* *$ \\
\hline${ }^{259} \mathrm{Rf}$ & $\mathrm{T}:$ & $3 \operatorname{Dr} 10=$ & $3.2(0.8)$ & and 6 & Gh01 $=$ & $(0.8)$ & & & & & & & & * \\
\hline
\end{tabular}




\begin{tabular}{|c|c|c|c|c|c|c|c|c|c|c|c|c|c|}
\hline \multirow{2}{*}{$\begin{array}{l}\text { Nuclide } \\
{ }^{260} \mathrm{Fm}\end{array}$} & \multicolumn{3}{|c|}{$\begin{array}{l}\text { Mass excess } \\
\quad(\mathrm{keV})\end{array}$} & \multirow{2}{*}{$\begin{array}{r}\begin{array}{c}\text { Excitation } \\
\text { energy(keV) }\end{array} \\
\text { EU }\end{array}$} & \multicolumn{3}{|c|}{ Half-life } & \multirow{2}{*}{$\begin{array}{l}J^{\pi} \\
0^{+}\end{array}$} & \multirow[t]{2}{*}{ Ens } & \multicolumn{2}{|c|}{ Reference } & \multicolumn{2}{|l|}{$\begin{array}{l}\text { Decay modes and } \\
\text { intensities }(\%)\end{array}$} \\
\hline & 95640\# & $500 \#$ & & & $1 \#$ & $\mathrm{~m}$ & & & & & & SF ? & $*$ \\
\hline${ }^{260} \mathrm{Md}$ & $96550 \#$ & $320 \#$ & & & 27.8 & $\mathrm{~d}$ & 0.8 & & 99 & 92Lo.B & TD & $\mathrm{SF}=? ; \alpha<5 ; \varepsilon<5 ; \beta^{-}<3.5$ & $*$ \\
\hline${ }^{260}$ No & 95610\# & $200 \#$ & & & 106 & $\mathrm{~ms}$ & 8 & $0^{+}$ & 99 & & & $\mathrm{SF}=100$ & \\
\hline${ }^{260} \mathrm{Lr}$ & $98280 \#$ & $120 \#$ & & & 3.0 & $\mathrm{~m}$ & 0.5 & & 99 & & & $\alpha=8020 ; \beta^{+}=2020$ & \\
\hline${ }^{260} \mathrm{Rf}$ & 99150\# & $200 \#$ & & & 21 & $\mathrm{~ms}$ & 1 & $0^{+}$ & 99 & & & $\mathrm{SF}=? ; \alpha=2 \# ; \varepsilon=0.01 \#$ & \\
\hline${ }^{260} \mathrm{Db}$ & 103680\# & $230 \#$ & & & 1.52 & $\mathrm{~s}$ & 0.13 & & 99 & & & $\alpha>90.46 ; \mathrm{SF}<9.66 ; \beta^{+}<2.5$ & \\
\hline${ }^{260} \mathrm{Db}^{p}$ & 103880\# & 280\# & $200 \#$ & $150 \#$ & & & & & & & & & \\
\hline${ }^{260} \mathrm{Sg}$ & 106580 & 40 & & & 3.8 & $\mathrm{~ms}$ & 0.8 & $0^{+}$ & 99 & & & $\mathrm{SF}=6030 ; \alpha=4030$ & \\
\hline${ }^{260} \mathrm{Bh}$ & $113610 \#$ & $580 \#$ & & & $300 \#$ & $\mu \mathrm{s}$ & & & 99 & & & $\alpha=100$ & \\
\hline$*^{260} \mathrm{Fm}$ & I : half-lif & $\mathrm{fe} \approx 4 \mathrm{n}$ & as and $s$ & $\mathrm{SF}=100$ mode & reporte & ed in & the 92 & o.B i & ernal & & & & $* *$ \\
\hline$*^{260} \mathrm{Fm}$ & I : rep & oort. N & ot conf & rmed in subse & experi & imen & t by sa & he grc & p $(97$ & Lo.A) & & & $* *$ \\
\hline$*^{260} \mathrm{Fm}$ & Dis & scover & $y$ of thi & nuclide is co & red unp & brove & & & & & & & $* *$ \\
\hline$*^{260} \mathrm{Md}$ & $\mathrm{T}$ : supers & sedes 8 & $6 \mathrm{Hu} 01=$ & $=31.8(0.5)$ of $s$ & group & & & & & & & & $* *$ \\
\hline
\end{tabular}

\begin{tabular}{|c|c|c|c|c|c|c|c|c|c|c|c|c|c|}
\hline${ }^{11} \mathrm{Md}$ & 98480\# & $650 \#$ & & & & & $40 \#$ & $\mathrm{~m}$ & & $7 / 2^{-} \#$ & & & $\alpha ?$ \\
\hline${ }^{61}$ No & $98500 \#$ & $300 \#$ & & & & & $3 \#$ & $\mathrm{~h}$ & & $3 / 2^{+} \#$ & & & $\alpha ?$ \\
\hline${ }^{61} \mathrm{Lr}$ & 99560\# & $200 \#$ & & & & & 39 & $\mathrm{~m}$ & 12 & & 99 & & $\mathrm{SF}=? ; \alpha ?$ \\
\hline${ }^{61} \mathrm{Rf}$ & 101315 & 29 & & & & $* \&$ & 5.5 & $\mathrm{~s}$ & 2.5 & $3 / 2^{+} \#$ & 99 & 02Ho11 T & $\alpha=? ; \mathrm{SF}=40$ \\
\hline${ }^{61} \mathrm{Rf}^{m}$ & 101390\# & $100 \#$ & $70 \#$ & $100 \#$ & & $* \&$ & 81 & $\mathrm{~s}$ & 9 & $9 / 2^{+} \#$ & & 02Ho11 TD & $\alpha=? ; \beta^{+}<15 ; \mathrm{SF}<10$ \\
\hline${ }^{61} \mathrm{Rf}^{p}$ & 101420 & 70 & 100 & 60 & $\mathrm{AD}$ & & & & & $3 / 2^{+} \#$ & & & \\
\hline $261 \mathrm{Db}$ & 104380\# & $230 \#$ & & & & & 1.8 & $\mathrm{~s}$ & 0.4 & & 99 & & $\alpha>82 ; \mathrm{SF}<18$ \\
\hline${ }^{261} \mathrm{Sg}$ & 108160\# & $130 \#$ & & & & & 230 & $\mathrm{~ms}$ & 60 & $7 / 2^{+} \#$ & 99 & & $\alpha \approx 100 ; \mathrm{SF}<1$ \\
\hline${ }^{261} \mathrm{Sg}^{p}$ & 108290\# & $140 \#$ & 130 & 50 & $\mathrm{AD}$ & & & & & $\left(9 / 2^{+}\right)$ & & & \\
\hline${ }^{261} \mathrm{Sg}^{q}$ & 108320\# & $140 \#$ & 160 & 50 & $\mathrm{AD}$ & & & & & $\left(3 / 2^{+}\right)$ & & & \\
\hline${ }^{261} \mathrm{Bh}$ & 113330\# & $230 \#$ & & & & & 13 & $\mathrm{~ms}$ & 4 & & 99 & & $\alpha=955 ; \mathrm{SF}<10$ \\
\hline
\end{tabular}

\begin{tabular}{|c|c|c|c|c|c|c|c|c|c|c|c|c|c|c|c|}
\hline${ }^{262} \mathrm{Md}$ & 101410\# & $580 \#$ & & & & & 3\# & $\mathrm{m}$ & & & & & & $\mathrm{SF} ? ; \alpha$ ? & \\
\hline${ }^{262} \mathrm{No}$ & 99950\# & $450 \#$ & & & & & 5 & $\mathrm{~ms}$ & & $0^{+}$ & 01 & & & $\mathrm{SF} \approx 100 ; \alpha ?$ & \\
\hline${ }^{262} \mathrm{Lr}$ & $102120 \#$ & $200 \#$ & & & & & 4 & $\mathrm{~h}$ & & & 01 & & & $\beta^{+}=? ; \mathrm{SF}<10 ; \alpha ?$ & \\
\hline${ }^{262} \mathrm{Rf}$ & 102390\# & $280 \#$ & & & & $*$ & 2.3 & s & 0.4 & $0^{+}$ & 01 & & & $\mathrm{SF} \approx 100 ; \alpha<0.8$ & \\
\hline${ }^{262} \mathrm{Rf}^{m}$ & 102990\# & $490 \#$ & $600 \#$ & $400 \#$ & & $*$ & 47 & $\mathrm{~ms}$ & 5 & high & & 96La11 & I & $\mathrm{SF}=100$ & $*$ \\
\hline${ }^{262} \mathrm{Db}$ & 106270\# & $180 \#$ & & & & & 35 & $\mathrm{~s}$ & 5 & & 01 & & & $\alpha \approx 67 ; \mathrm{SF} \approx 30 ; \beta^{+}=3 \#$ & \\
\hline${ }^{262} \mathrm{Db}^{p}$ & $106390 \#$ & $200 \#$ & $120 \#$ & 70\# & & & & & & & & & & $\alpha ?$ & \\
\hline${ }^{262} \mathrm{Sg}$ & 108420\# & $280 \#$ & & & & & 8 & $\mathrm{~ms}$ & 3 & $0^{+}$ & 01 & 01Ho06 & TD & $\mathrm{SF}=? ; \alpha<22$ & \\
\hline${ }^{262} \mathrm{Bh}$ & $114470 \#$ & $350 \#$ & & & & & 290 & $\mathrm{~ms}$ & 160 & & 01 & 97 Ho14 & $\mathrm{T}$ & $\alpha=? ; \mathrm{SF}<20$ & * \\
\hline${ }^{262} \mathrm{Bh}^{m}$ & $114780 \#$ & $350 \#$ & 300 & 60 & $\mathrm{AD}$ & & 14 & $\mathrm{~ms}$ & 4 & & 01 & 97Ho14 & $\mathrm{T}$ & $\alpha=? ; \mathrm{SF}<10$ & * \\
\hline$*^{262} \mathrm{Rf}^{m}$ & $\mathrm{I}:$ assign & ed by 9 & 6La11 & $\mathrm{o}$-is & meric & sta & & & & & & & & & $* *$ \\
\hline$*^{262} \mathrm{Bh}$ & $\mathrm{T}: 3$ ever & ts at 22 & 25,255 & and 27 & $8 \mathrm{~ms} \mathrm{y}$ & jield & 175( & +240 & $-64)$, & see $84 \mathrm{~S}$ & & & & & $* *$ \\
\hline$*^{262} \mathrm{Bh}^{m}$ & $\mathrm{~T}: 11 \mathrm{eve}$ & ents yie & lding 1 & $2.2(+5$ & $5-2.8)$ & & & & & & & & & & $* *$ \\
\hline${ }^{263} \mathrm{No}$ & 102980\# & $490 \#$ & & & & & $20 \#$ & $\mathrm{~m}$ & & & & & & $\alpha ? ; \mathrm{SF} ?$ & \\
\hline${ }^{263} \mathrm{Lr}$ & 103670\# & $360 \#$ & & & & & $5 \#$ & $\mathrm{~h}$ & & & & & & $\alpha ?$ & \\
\hline${ }^{263} \mathrm{Rf}$ & 104840\# & $180 \#$ & & & & & 11 & $\mathrm{~m}$ & 3 & $3 / 2^{+} \#$ & 99 & 93Gr.C & TD & $\mathrm{SF}=? ; \alpha=30$ & $*$ \\
\hline${ }^{263} \mathrm{Db}$ & 107110\# & $170 \#$ & & & & & 29 & s & 9 & & 99 & $92 \mathrm{Kr} 01$ & $\mathrm{D}$ & $\mathrm{SF}=5614 ; \alpha=? ; \beta^{+}=6.916$ & $*$ \\
\hline${ }^{263} \mathrm{Db}^{p}$ & 107510\# & $260 \#$ & $400 \#$ & $200 \#$ & & & & & & & & & & & \\
\hline${ }^{263} \mathrm{Sg}$ & $110220 \#$ & $120 \#$ & & & & $*$ & 1.0 & $\mathrm{~s}$ & 0.2 & $9 / 2^{+} \#$ & 99 & & & $\alpha>70 ; \mathrm{SF} ?$ & \\
\hline${ }^{263} \mathrm{Sg}^{m}$ & $110320 \#$ & $100 \#$ & $100 \#$ & 70\# & $\mathrm{Nm}$ & * & 120 & $\mathrm{~ms}$ & & $3 / 2^{+} \#$ & 99 & & & $\alpha=? ;$ IT ? & \\
\hline${ }^{263} \mathrm{Bh}$ & 114610\# & $370 \#$ & & & & & $200 \#$ & $\mathrm{~ms}$ & & & 99 & & & $\alpha ?$ & \\
\hline${ }^{263} \mathrm{Hs}$ & 119750\# & $350 \#$ & & & & & $1 \#$ & $\mathrm{~ms}$ & & $7 / 2^{+} \#$ & 99 & & & $\alpha=100$ & \\
\hline${ }^{263} \mathrm{Hs}^{p}$ & $120250 \#$ & $360 \#$ & $500 \#$ & $100 \#$ & & & & & & am & & & & $\alpha$ ?; SF ? & \\
\hline$*^{263} \mathrm{Rf}$ & $\mathrm{T}:$ avera & ge $03 \mathrm{KI}$ & $1=24($ & $+19-7$ & m 930 & Gr.C & $00(+3$ & $00-2$ & 00) $\mathrm{s}$ & $2 \mathrm{Cz} . \mathrm{A}=$ & 600( & $+300-200)$ & & & $* *$ \\
\hline$*^{263} \mathrm{Db}$ & D : SF fro & $\mathrm{m} 92 \mathrm{~K}$ & $\mathrm{r} 01=57$ & $(+13-$ & 5); $\beta^{+}$ & + av & age 03 & $\mathrm{Kr} .1=$ & $=3(+4$ & -1) $93 \mathrm{Gr}$ & $\mathrm{C}=8$ & & & & $* *$ \\
\hline$*^{263} \mathrm{Db}$ & $\mathrm{T}$ : Possib & bly a ca & ndidate & for th & $54(+9$ & $98-2-2=1-2 \cdot 1$ & $\mathrm{~s} \mathrm{SF}$ & lecay & obser & ved by & $8 \mathrm{Ik0}$ & & & & $* *$ \\
\hline
\end{tabular}




\begin{tabular}{|c|c|c|c|c|c|c|c|c|c|c|c|c|c|c|}
\hline \multirow{2}{*}{$\begin{array}{l}\text { Nuclide } \\
{ }^{264} \mathrm{No}\end{array}$} & \multicolumn{2}{|c|}{$\begin{array}{l}\text { Mass excess } \\
(\mathrm{keV})\end{array}$} & \multicolumn{3}{|c|}{$\begin{array}{c}\text { Excitation } \\
\text { energy(keV) }\end{array}$} & \multicolumn{3}{|c|}{ Half-life } & \multirow{2}{*}{$\begin{array}{l}J^{\pi} \\
0^{+}\end{array}$} & \multirow[t]{2}{*}{ Ens } & \multicolumn{2}{|c|}{ Reference } & \multicolumn{2}{|l|}{$\begin{array}{c}\text { Decay modes and } \\
\text { intensities }(\%)\end{array}$} \\
\hline & 104650\# & $640 \#$ & & & & $1 \#$ & $\mathrm{~m}$ & & & & & & $\alpha ? ; \mathrm{SF} ?$ & \\
\hline${ }^{264} \mathrm{Lr}$ & $106230 \#$ & $440 \#$ & & & & $10 \#$ & $\mathrm{~h}$ & & & & & & $\alpha ? ; \mathrm{SF} ?$ & \\
\hline${ }^{264} \mathrm{Rf}$ & $106180 \#$ & $450 \#$ & & & & $1 \#$ & $\mathrm{~h}$ & & $0^{+}$ & & & & $\alpha ?$ & \\
\hline${ }^{264} \mathrm{Db}$ & 109360\# & 230\# & & & & 3\# & $\mathrm{m}$ & & & & & & $\alpha ?$ & \\
\hline${ }^{264} \mathrm{Sg}$ & $110780 \#$ & $280 \#$ & & & & $400 \#$ & $\mathrm{~ms}$ & & $0^{+}$ & 99 & & & $\alpha ?$ & \\
\hline${ }^{264} \mathrm{Bh}$ & 116070\# & 280\# & & & & 1.3 & $\mathrm{~s}$ & 0.5 & & 99 & 02Ho11 & $\mathrm{T}$ & $\alpha=? ; \beta^{+} ?$ & * \\
\hline${ }^{264} \mathrm{Bh}^{p}$ & $116370 \#$ & $310 \#$ & $300 \#$ & $150 \#$ & & & & & $a m$ & & & & & \\
\hline${ }^{264} \mathrm{Hs}$ & 119600 & 40 & & & & 540 & $\mu \mathrm{s}$ & 300 & $0^{+}$ & 99 & 95Ho.B & $\mathrm{T}$ & $\alpha \approx 50 ; \mathrm{SF} \approx 50$ & * \\
\hline$*^{264} \mathrm{Bh}$ & \multicolumn{13}{|c|}{$\mathrm{T}:$ mean lifetime of 6 events $1.5 \mathrm{~s}$} & $* *$ \\
\hline$*^{264} \mathrm{Hs}$ & \multirow{2}{*}{\multicolumn{13}{|c|}{$\mathrm{T}: 95 \mathrm{Ho} . \mathrm{B}(2$ events $76 \mu \mathrm{s}$ and $825 \mu \mathrm{s}) \quad 87 \mathrm{Mu} 15(1$ event }} & $* *$ \\
\hline$*^{264} \mathrm{Hs}$ & & & & & & \multicolumn{8}{|c|}{$\mathrm{T}: \quad$ the 3 events: $327(+448-120) \mu \mathrm{s}$, see $84 \mathrm{Sc} 13$} & $* *$ \\
\hline${ }^{265} \mathrm{Lr}$ & 107900\# & $710 \#$ & & & & $10 \#$ & $\mathrm{~h}$ & & & & & & $\alpha ? ; \mathrm{SF} ?$ & \\
\hline${ }^{265} \mathrm{Rf}$ & $108710 \#$ & $420 \#$ & & & & 13 & $\mathrm{~h}$ & & $3 / 2^{+} \#$ & 00 & 990g.A & TD & $\alpha ?$ & * \\
\hline${ }^{265} \mathrm{Db}$ & $110480 \#$ & $280 \#$ & & & & $15 \#$ & $\mathrm{~m}$ & & & & & & $\alpha ?$ & \\
\hline${ }^{265} \mathrm{Sg}$ & 112820 & 60 & & & & 8 & $\mathrm{~s}$ & 3 & $3 / 2^{+} \#$ & 99 & & & $\alpha>50 ;$ SF? & \\
\hline${ }^{265} \mathrm{Sg}^{p}$ & $113120 \#$ & $120 \#$ & $300 \#$ & $100 \#$ & & & & & $11 / 2^{-} \#$ & & & & & \\
\hline${ }^{265} \mathrm{Bh}$ & $116570 \#$ & $380 \#$ & & & & $500 \#$ & $\mathrm{~ms}$ & & & & & & $\alpha ?$ & \\
\hline${ }^{265} \mathrm{Hs}$ & $121170 \#$ & $140 \#$ & & & & 2.1 & $\mathrm{~ms}$ & 0.3 & $9 / 2^{+} \#$ & 99 & & & $\alpha \approx 100 ; \mathrm{SF}<1$ & \\
\hline${ }^{265} \mathrm{Hs}^{m}$ & $121480 \#$ & $140 \#$ & 300 & 70 & $\mathrm{AD}$ & 780 & $\mu \mathrm{s}$ & 150 & $3 / 2^{+} \#$ & 99 & & & $\alpha \approx 100 ;$ IT ? & \\
\hline${ }^{265} \mathrm{Mt}$ & $126820 \#$ & $460 \#$ & & & & $2 \#$ & $\mathrm{~ms}$ & & & & & & $\alpha$ ? & \\
\hline$*^{265} \mathrm{Rf}$ & \multicolumn{13}{|c|}{$\mathrm{T}:$ one case only after a $1.3 \mathrm{~h}$ measurement } & $* *$ \\
\hline${ }^{266} \mathrm{Lr}$ & 111130\# & $660 \#$ & & & & $1 \#$ & $\mathrm{~h}$ & & & & & & $\alpha$ ?; SF? & \\
\hline${ }^{266} \mathrm{Rf}$ & 109880\# & $540 \#$ & & & & $10 \#$ & $\mathrm{~h}$ & & $0^{+}$ & & & & $\alpha ? ; \mathrm{SF} ?$ & \\
\hline${ }^{266} \mathrm{Db}$ & $112740 \#$ & $360 \#$ & & & & $20 \#$ & $\mathrm{~m}$ & & & & & & $\alpha$ ?; SF ? & \\
\hline${ }^{266} \mathrm{Sg}$ & $113700 \#$ & $290 \#$ & & & & 21 & s & 6 & $0^{+}$ & 01 & 98Tu01 & $\mathrm{T}$ & $\alpha=349 ; \mathrm{SF}=669$ & * \\
\hline${ }^{266} \mathrm{Bh}$ & 118250\# & $200 \#$ & & & & $\begin{array}{c}21 \\
5\end{array}$ & $\mathrm{~s}$ & 3 & & 01 & & & $\alpha \approx 100 ; \beta^{+}$?; SF ? & * \\
\hline${ }^{266} \mathrm{Hs}$ & 121190\# & $280 \#$ & & & & 2.7 & $\mathrm{~ms}$ & 1.0 & $0^{+}$ & 01 & 01Нo06 & $\mathrm{TD}$ & $\alpha=? ; \mathrm{SF} \approx 1.4 \#$ & \\
\hline${ }^{266} \mathrm{Mt}$ & $127890 \#$ & $350 \#$ & & & & 1.2 & $\mathrm{~ms}$ & 0.4 & & 01 & $84 \mathrm{Og} 03$ & $\mathrm{D}$ & $\alpha=? ; \mathrm{SF}<5.5$ & * \\
\hline${ }^{266} \mathrm{Mt}^{m}$ & 129120\# & $350 \#$ & 1230 & 80 & $\mathrm{AD}$ & 6 & $\mathrm{~ms}$ & 3 & & 01 & $97 \mathrm{Ho} 14$ & $\mathrm{TD}$ & $\alpha=100$ & $*$ \\
\hline$*^{266} \mathrm{Sg}$ & \multicolumn{13}{|c|}{$\mathrm{T}:$ average $98 \mathrm{Tu} 01=21(+20-12) 94 \mathrm{La} 22=10-30 \quad \mathrm{D}:$ from $18 \%<\alpha<50 \% 50 \%<\mathrm{SF}<82 \%$} & $* *$ \\
\hline$*^{266} \mathrm{Bh}$ & \multicolumn{13}{|c|}{$\mathrm{T}:$ from $T=1-10 ;$ estimated $1 \# \mathrm{~s}$ from systematics } & $* *$ \\
\hline$*^{266} \mathrm{Mt}$ & \multirow{2}{*}{\multicolumn{13}{|c|}{$\mathrm{T}: 10$ events yielding $1.01(+0.47-0.24)$}} & $* *$ \\
\hline$*^{266} \mathrm{Mt}^{m}$ & & & & & & & & & & & & & & $* *$ \\
\hline
\end{tabular}

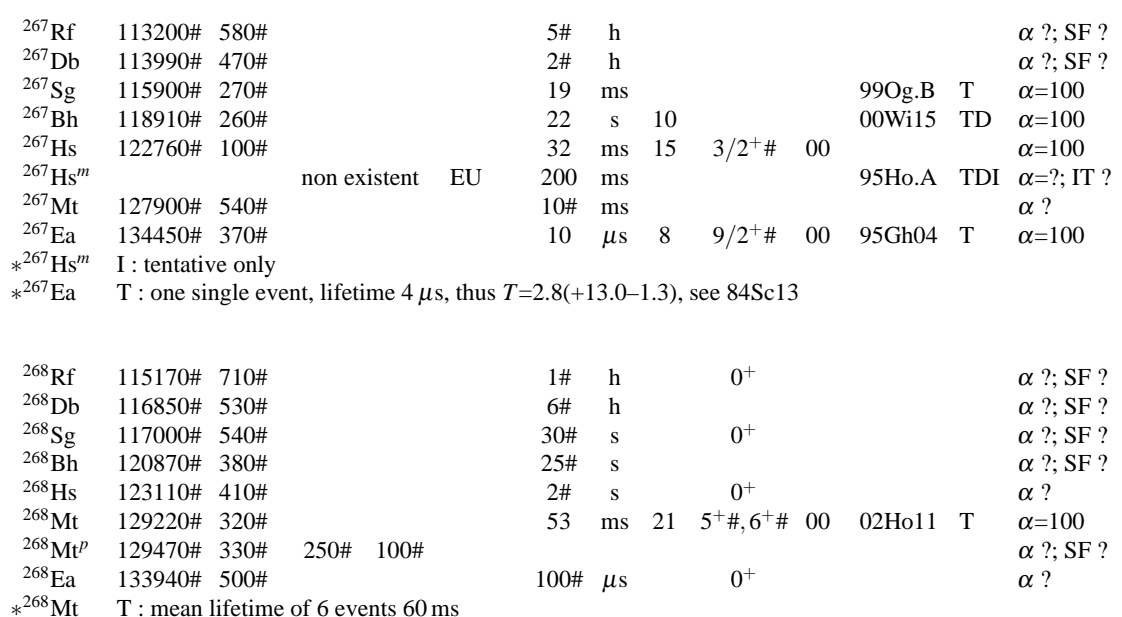




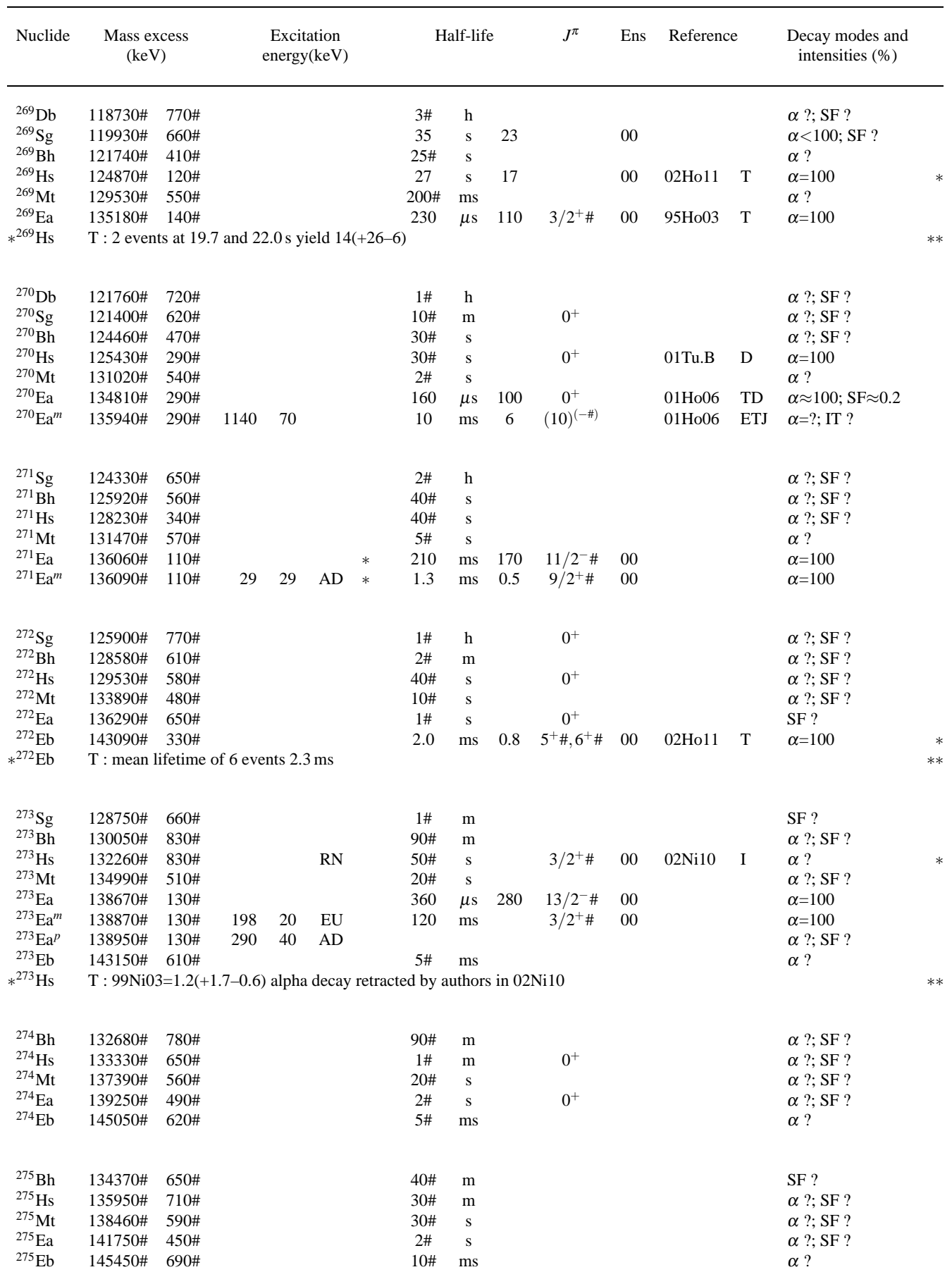




\begin{tabular}{|c|c|c|c|c|c|c|c|c|c|c|c|}
\hline Nuclide & \multicolumn{2}{|c|}{$\begin{array}{l}\text { Mass excess } \\
\quad(\mathrm{keV})\end{array}$} & $\begin{array}{l}\text { Excitation } \\
\text { energy }(\mathrm{keV})\end{array}$ & \multicolumn{3}{|c|}{ Half-life } & $J^{\pi}$ & Ens & \multicolumn{2}{|c|}{ Reference } & $\begin{array}{l}\text { Decay modes and } \\
\text { intensities }(\%)\end{array}$ \\
\hline${ }^{276} \mathrm{Hs}$ & 137120\# & $820 \#$ & & $1 \#$ & $\mathrm{~h}$ & & $0^{+}$ & & & & $\alpha ? ; \mathrm{SF} ?$ \\
\hline${ }^{276} \mathrm{Mt}$ & 140800\# & $680 \#$ & & $40 \#$ & $\mathrm{~s}$ & & & & & & $\alpha ? ; \mathrm{SF} ?$ \\
\hline${ }^{276} \mathrm{Ea}$ & 142550\# & $610 \#$ & & $5 \#$ & $\mathrm{~s}$ & & $0^{+}$ & & & & $\alpha$ ?; SF ? \\
\hline${ }^{276} \mathrm{~Eb}$ & 147640\# & $630 \#$ & & $100 \#$ & $\mathrm{~ms}$ & & & & & & $\alpha ? ; \mathrm{SF} ?$ \\
\hline${ }^{277} \mathrm{Hs}$ & 139580\# & 730\# & & 40 & $\mathrm{~m}$ & 30 & $3 / 2^{+} \#$ & 00 & $99 \operatorname{Og} 10$ & TD & $\mathrm{SF}=100$ \\
\hline${ }^{277} \mathrm{Mt}$ & 141980\# & $880 \#$ & & $1 \#$ & $\mathrm{~m}$ & & & & & & $\alpha ? ; \mathrm{SF} ?$ \\
\hline${ }^{277} \mathrm{Ea}$ & 144980\# & $960 \#$ & $\mathrm{RN}$ & $5 \#$ & $\mathrm{~s}$ & & $11 / 2^{+} \#$ & 00 & $02 \mathrm{Ni} 10$ & I & $\alpha ?$ \\
\hline${ }^{277} \mathrm{~Eb}$ & $148590 \#$ & $620 \#$ & & $1 \#$ & $\mathrm{~s}$ & & & & & & $\alpha ? ; \mathrm{SF} ?$ \\
\hline${ }^{277} \mathrm{Ec}$ & $152710 \#$ & $130 \#$ & & 1.1 & $\mathrm{~ms}$ & 0.7 & $3 / 2^{+} \#$ & 00 & 02Ho11 & $\mathrm{T}$ & $\alpha=100$ \\
\hline$*^{277} \mathrm{Hs}$ & $\mathrm{T}:$ one sin & le ever & yields $11(+5$ & & & & & & & & \\
\hline$*^{277} \mathrm{Ea}$ & $\mathrm{T}: 99 \mathrm{Ni03}$ & $=3.0(+4$ & ) alpha decay & ed by a & thors & $\ln 02$ & Ni10 & & & & \\
\hline$*^{277} \mathrm{Ec}$ & $\mathrm{T}:$ two ev & nts at 0 & $\mathrm{~s}$ and $1.406 \mathrm{~ms}$ & & & & & & & & \\
\hline
\end{tabular}

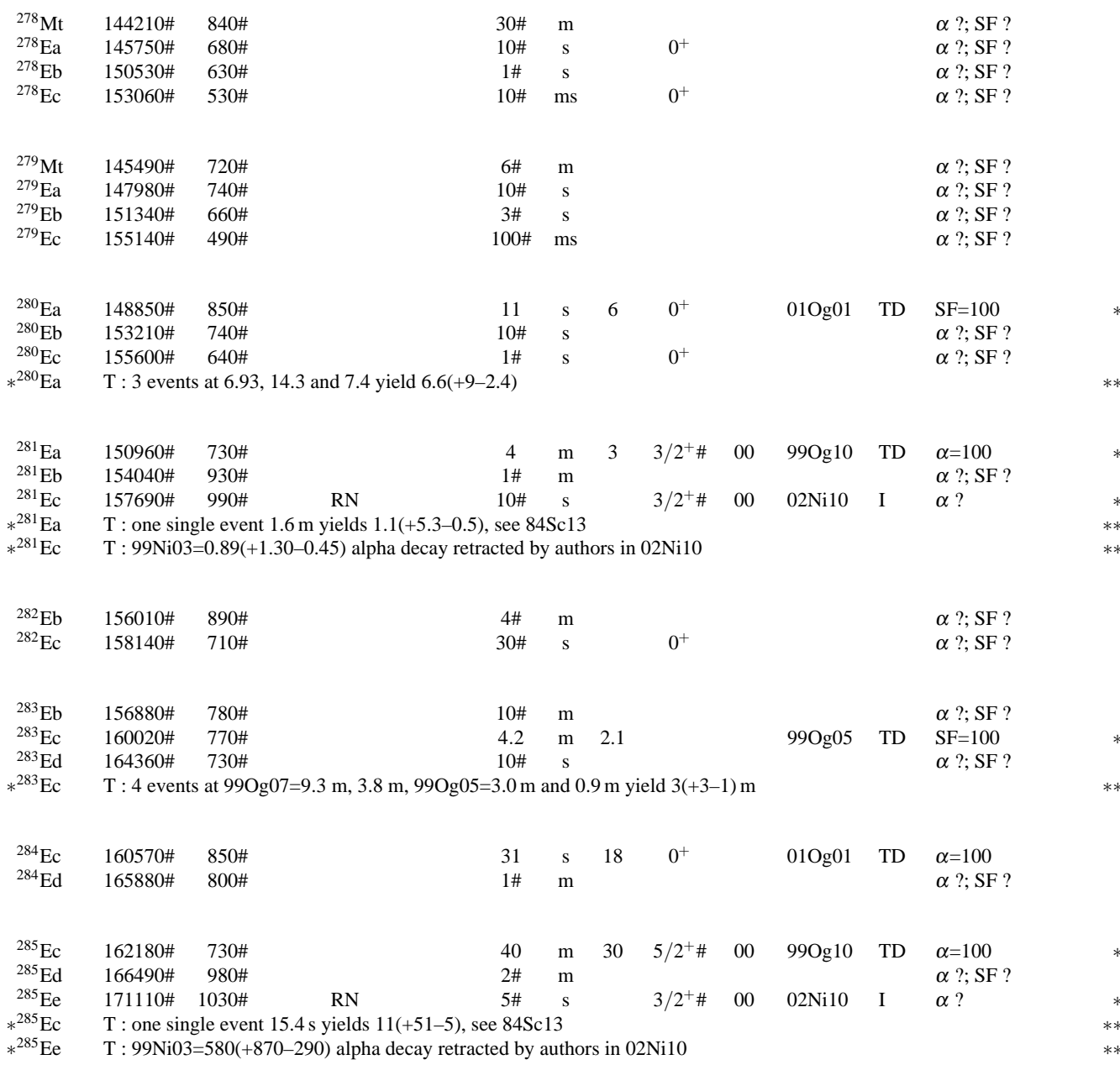




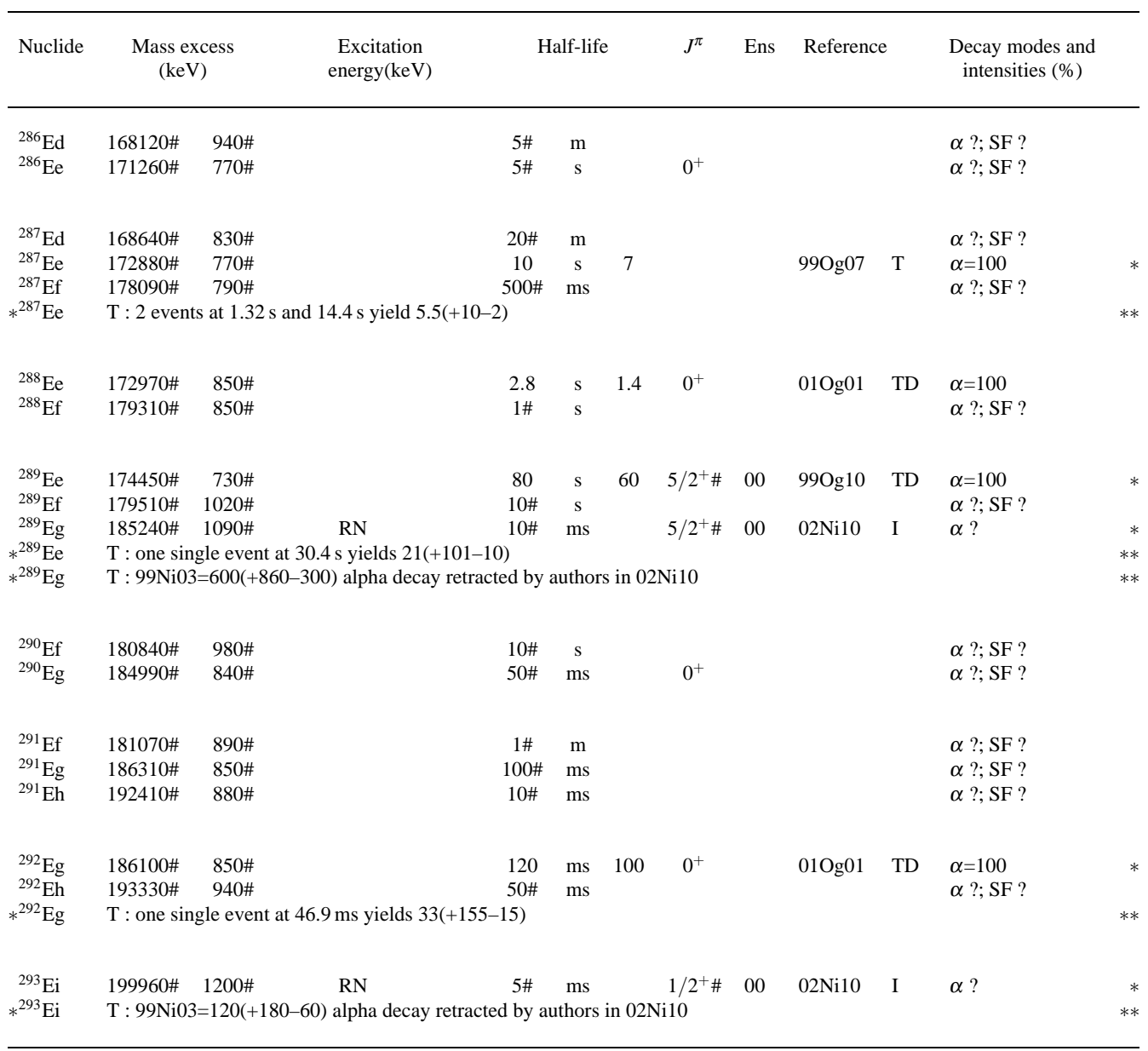

